\title{
Total synthesis of (+)-Madangamine D
}

\author{
Roberto Ballette
}

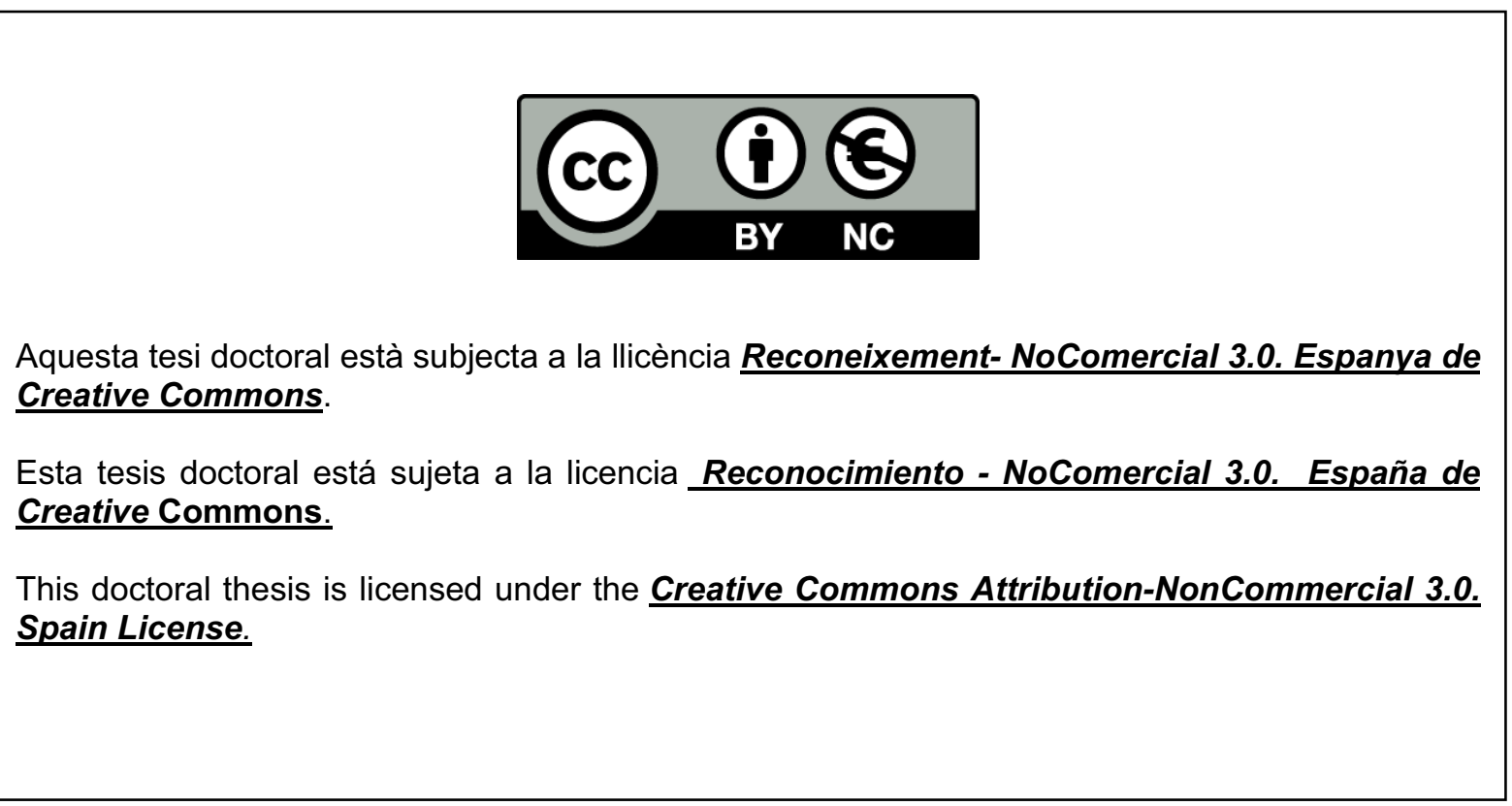


UNIVERSITAT DE BARCELONA

FACULTAD DE FARMACIA

DEPARTAMENTO DE FARMACOLOGÍA Y QUÍMICA TERAPEUTICA

TOTAL SYNTHESIS OF (+)-MADANGAMINE D

Roberto Ballette 2014 

UNIVERSITAD DE BARCELONA

FACULTAD DE FARMÀCIA

DEPARTAMENTO DE FARMACOLOGÍA Y QUÍMICA TERAPEUTICA

PROGRAMA DE DOCTORAT:

Química Orgánica Experimental e Industrial

TOTAL SYNTHESIS OF (+)-MADANGAMINE D

Memòria presentada per Roberto Ballette per optar al títol de doctor per la universitat de Barcelona

Prof. Merceded Amat Tusón

Dirigida por:

Dra. Maria Pérez Bosch

Roberto Ballette

Roberto Ballette 2014 



\section{TABLE OF CONTENTS}

\section{Acknowledgements}

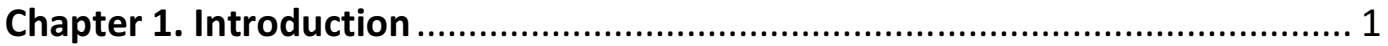

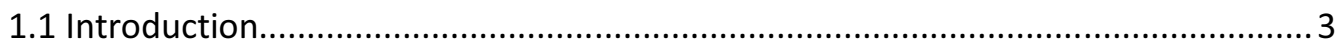

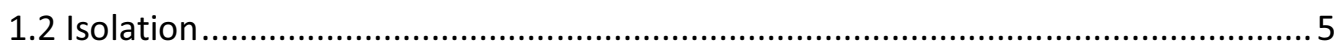

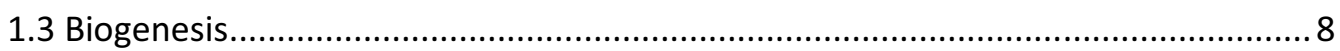

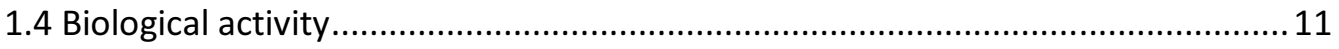

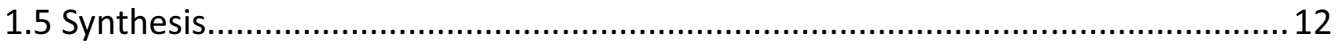

1.5.1 Construction of $A B C$ diazatricyclic core ….................................................. 12

1.5.1.1 Weinreb's approach............................................................................ 12

1.5.1.2. Kibayashi's approach .......................................................................... 14

1.5.1.3. Biomimetic Marazano approach........................................................... 15

1.5.1.4. Bonjoch's approach ........................................................................ 17

1.5.2 Construction of ACE system ................................................................... 18

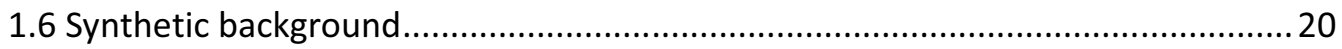

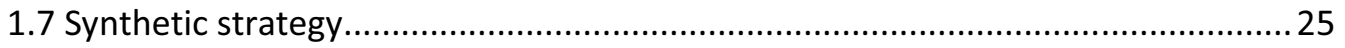

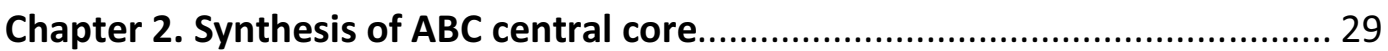

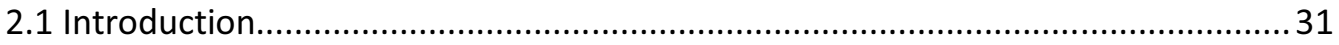

2.2 Synthetic strategy toward the $A B C$ core of madangamine .................................. 31

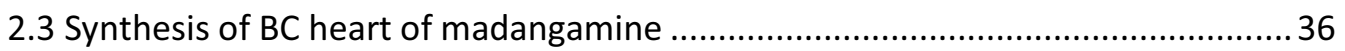

2.3.1. Synthesis of the enantiopure bicyclic lactam:

Cyclocondensation reaction

2.3.2. Generation of the $\mathrm{C}-12$ stereocenter:

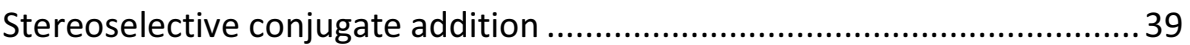

2.3.3. First generation synthesis of $B C$ bicyclic heart of madangamines................ 42

2.3.3.1 Synthesis of cis-hexahydroisoquinolone scaffold ..................................4

2.3.4. Generation of the quaternary C-9 stereocenter ...........................................46 
2.4 An alternative route to the $B C$ system

2.4.1. Formulation of the alternative strategy ............................................50

2.4.2. Cleavage of phenylethanol moiety from amine derivatives .......................5 52

2.4.2.1. Oxidative cleavage with lead tetraacetate and CAN ........................53

2.4.2.2. Acid mediated elimination of the chiral inductor................................ 55

2.4.2.3. Trans-acylating reagents ...................................................... 56

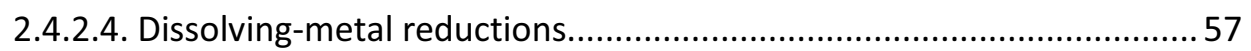

2.4.2.5. Cleavage of chiral inductor by mesylation/elimination ......................60

2.4.3. Removal of the chiral inductor from tricyclic lactams ...............................61

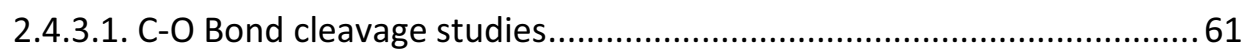

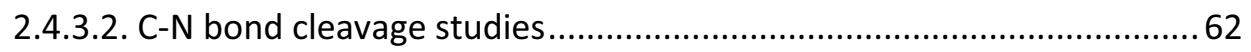

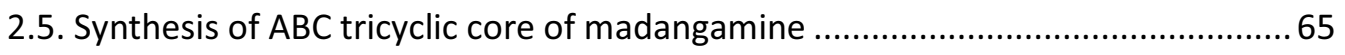

Chapter 3. Construction of macrocyclic rings ......................................... 71

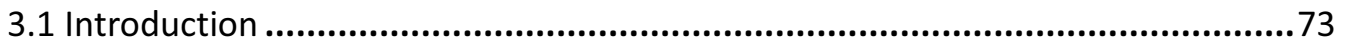

3.2. Contribution from previous work .......................................................... 75

3.2.1. Macroclactamization approach to the ABCD tetracyclic system .................76

3.3. Assembly of the $D$ ring: annulation studies ................................................... 78

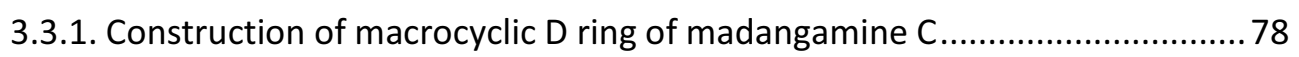

3.3.1.1. Intramolecular B-Alkyl Suzuky-Miyaura approach: model studies...... 80

3.3.2. Ring-closing metathesis strategy:

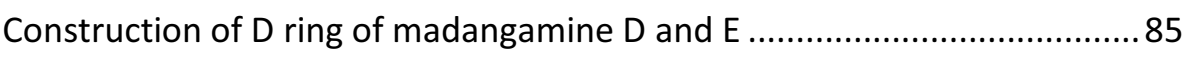

3.3.2.1. Ring-closing metathesis approach: model studies ........................... 87

3.3.2.2. Ring-closing metathesis strategy: flow chemistry approach ...............89

3.3.2.3. Flow reactors structure and main principles ............................... 90

3.3.2.4. Continuous flow RCM macrocyclization: model studies ....................992

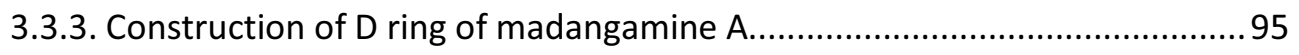

3.3.3.1. Synthesis of Z-skipped D ring of madangamine A:

First synthetic approach.................................................. 96

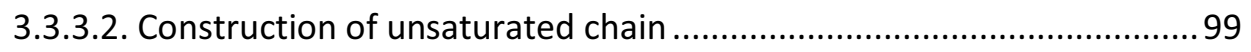

3.3.3.3. Incorporation of the unsaturated chain

into the piperidinic model compound 102

3.3.3.4. Synthesis of Z-skipped D ring of madangamine A:

Second synthetic approach..... 102 
3.3.3.5. Model studies toward the construction of the $D$ ring of madangamine $\mathrm{A}$ : Path $\mathrm{A}$ 104

3.3.3.6. Model studies toward the construction

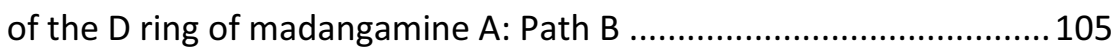

3.3.3.7. Closure of the macrocyclic $D$ ring of madangamine $A$.........................106

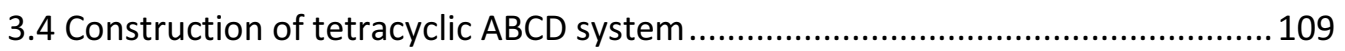

3.4.1. Assembly of the 14-membered ring of madangamine D............................ 110

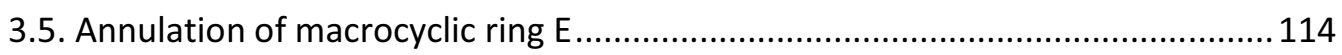

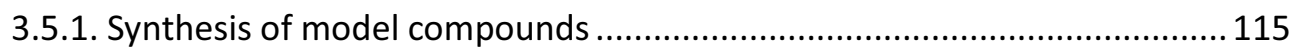

3.5.2. Annulation of $E$ ring on model compounds .............................................117

3.5.2.1 Still-Gennary strategy ................................................................. 117

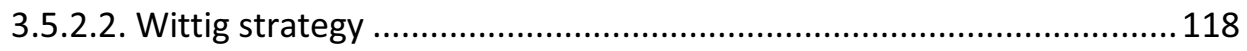

3.5.3. Synthesis ABCDE pentacyclic system of madangamine D........................... 121

3.6 Primera síntesis total de la (+)-madangamina ................................................ 124

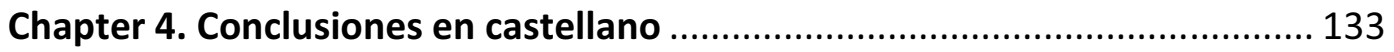

Chapter 5. Experimental data and spectra ............................................. 137 



\section{ABBREVIATIONS AND ACRONYMS}

\begin{tabular}{|c|c|}
\hline$[\alpha]^{22} \mathrm{D}$ & specific optical rotatory power at $\lambda=589 \mathrm{~nm}$ \\
\hline${ }^{13} \mathrm{C}-\mathrm{NMR}$ & carbon-13 nuclear magnetic resonance \\
\hline${ }^{1} \mathrm{H}-\mathrm{NMR}$ & proton nuclear magnetic resonance \\
\hline 9-BBN & 9-borabicyclo[3.3.1]nonane \\
\hline ACE-Cl & 1-Chloroethyl chloroformate \\
\hline Alloc-Cl & Allyl chloroformate \\
\hline Anal. & elemental analysis \\
\hline aq. & aqueous \\
\hline ax & axial \\
\hline Boc & $t$-butoxycarbonyl \\
\hline $\mathrm{bp}$ & boiling point \\
\hline br & broad \\
\hline c & concentration \\
\hline calcd. & calculated \\
\hline CAN & ammonium cerium(IV) nitrate \\
\hline $\mathrm{Cbz}$ & benzyloxycarbonyl \\
\hline Celite ${ }^{\circledR}$ & filtration agent $\left(90 \% \mathrm{SiO}_{2}, 4 \% \mathrm{Al}_{2} \mathrm{O}_{3}, 3.3 \% \mathrm{Na}_{2} \mathrm{O}\right.$ and \\
\hline & $\left.\mathrm{K}_{2} \mathrm{O}, 1.3 \% \mathrm{Fe}_{2} \mathrm{O}_{3}, 0.5 \% \mathrm{CaO}\right)$ \\
\hline $\mathrm{CI}$ & chemical ionization \\
\hline COSY & H-H correlation spectroscopy \\
\hline$d$ & chemical shift \\
\hline DBU & 1,8-diazabicyclo[5.4.0]undec-7-ene \\
\hline DCE & 1,2 dichloroethane \\
\hline dd & doublet of doublets \\
\hline DEAD & diethyl azodicarboxylate \\
\hline DIBAL-H & diisobutylaluminium hydride \\
\hline $\mathrm{dm}$ & doublet of multiplets \\
\hline DMAP & 4-dimethylaminopyridine \\
\hline DMF & $\mathrm{N}, \mathrm{N}$-dimethylformamide \\
\hline DMP & Dess-Martin Periodinane \\
\hline DNP & 2,4-dinitrophenylhydrazine \\
\hline $\mathrm{dt}$ & doublet of triplets \\
\hline $\mathrm{dt}$ & doublet of triplets \\
\hline EDA & ethylendiamina \\
\hline $\mathrm{EDCl}$ & $\mathrm{N}$-(3-Dimethylaminopropyl)- $\mathrm{N}^{\prime}$-ethylcarbodiimide hydrochloride \\
\hline $\mathrm{ED}_{50}$ & dose effective in $50 \%$ of test subjects \\
\hline EI & electron impact \\
\hline
\end{tabular}




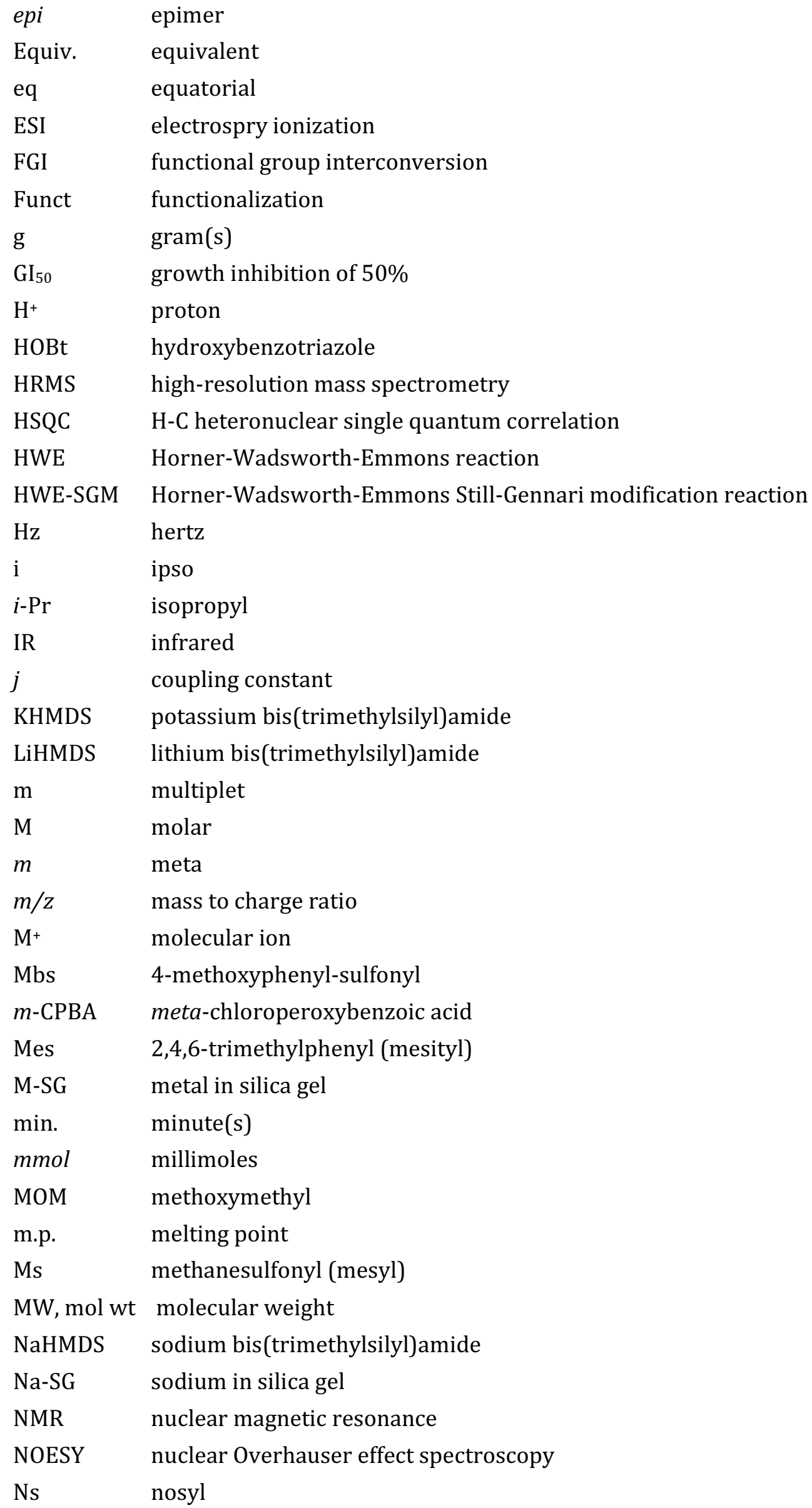




$\begin{array}{ll}\mathrm{Nu} & \text { nucleophile } \\ o & \text { ortho } \\ p & \text { para } \\ \text { PCC } & \text { pyridinium chlorochromate } \\ \text { PDC } & \text { pyridinium dichromate } \\ \text { PG } & \text { protecting group } \\ \text { PhtnNH } & \text { phthalimide } \\ \text { PMB } & p \text {-methoxybenzyl } \\ \text { Poc-Cl } & \text { propargyl chloroformate } \\ \text { ppm } & \text { part(s) per million } \\ \text { PPTS } & \text { pyridinium } p \text {-toluenesulfonate } \\ \text { q } & \text { quartet } \\ \text { R } & \text { generalized alkyl group or substituent } \\ \text { R } & \text { retention factor } \\ \text { RCM } & \text { ring-closing metathesis } \\ \text { r.t. } & \text { room temperature } \\ \text { sat. } & \text { saturated } \\ \text { SE } & \text { sinthetic equivalent } \\ \text { SES } & \text { b-(trimethylsilyl)ethanesulfonyl } \\ t & \text { tertiary } \\ \text { TBAF } & \text { tetra- } n \text {-butylammonium fluoride } \\ \text { TBAI } & \text { tetra- } n \text {-butylammonium iodide } \\ \text { TBDMS } & t \text {-butyldimethylsilyl } \\ \text { TBDPS } & t \text {-butyldiphenylsilyl } \\ \text { td } & \text { triplet of doublets } \\ \text { Teoc-Cl } & 2 \text {-(trimethylsilyl)-ethyl chloroformate } \\ \text { Teoc-Osu } & \mathrm{N} \text {-[2-(Trimethylsilyl)ethoxycarbonyloxy]succinimide } \\ \text { TFA } & \text { trifluoroacetic acid } \\ \text { Troc-Cl } & 2 \text {,2,2-Trichloroethyl chloroformate } \\ & \end{array}$




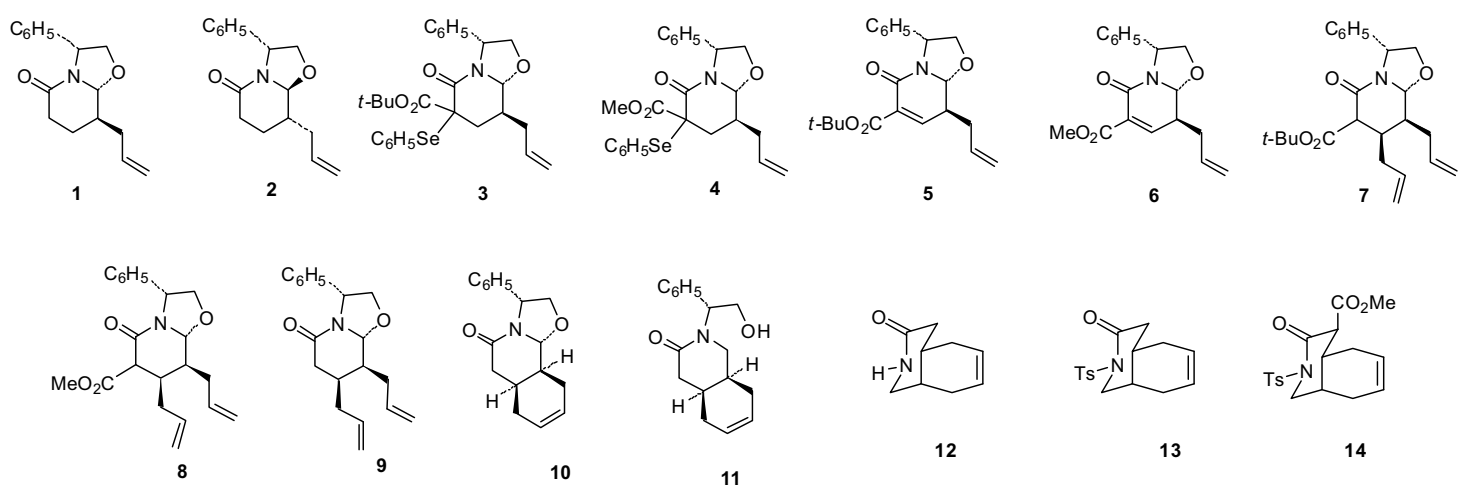

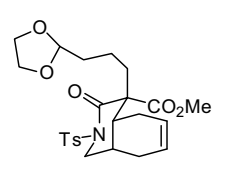

15

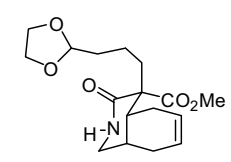

16

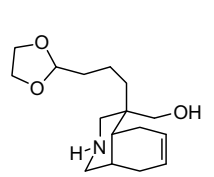

17

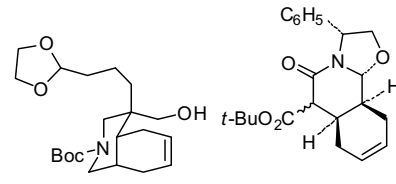

18

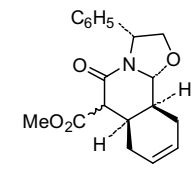

19

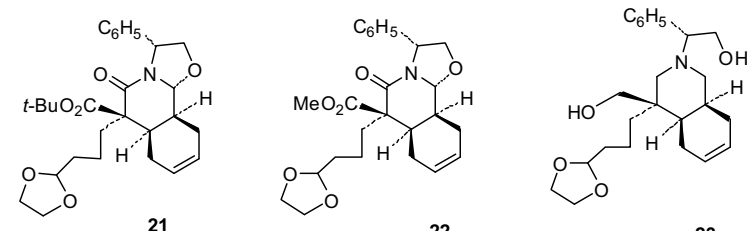

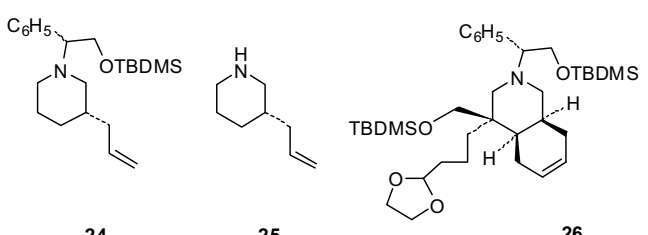

23

25

26

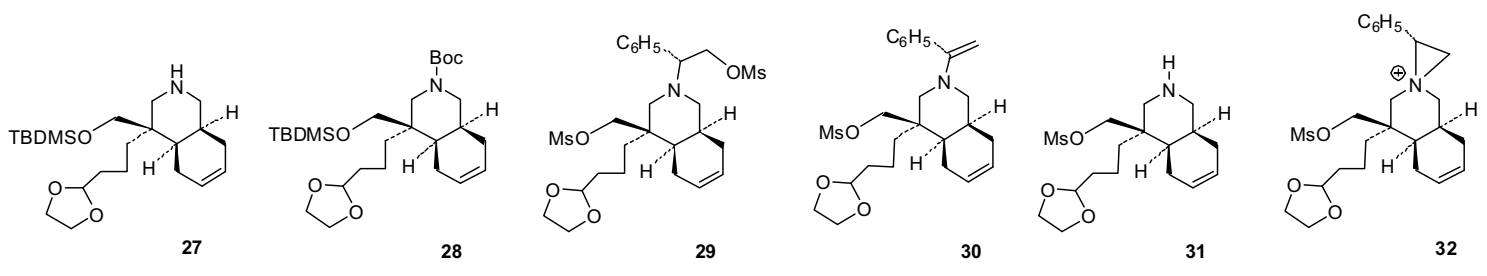

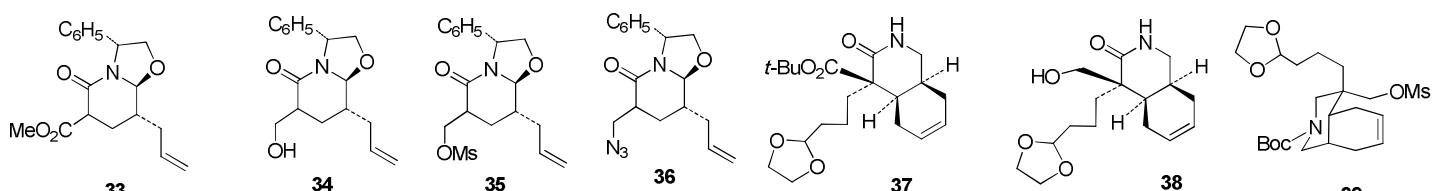

33

34

35

37

39

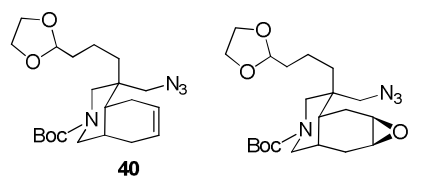

41

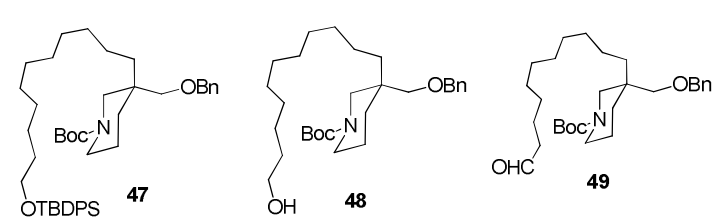

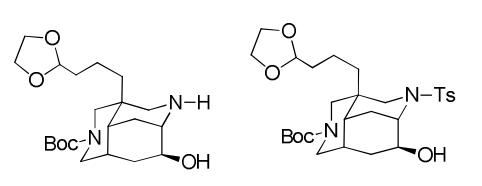

43

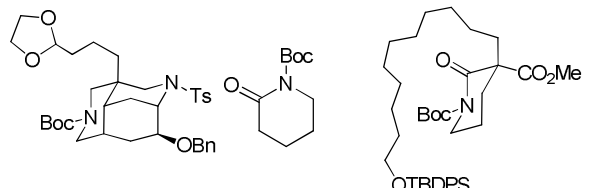

44

$45 \quad 46$

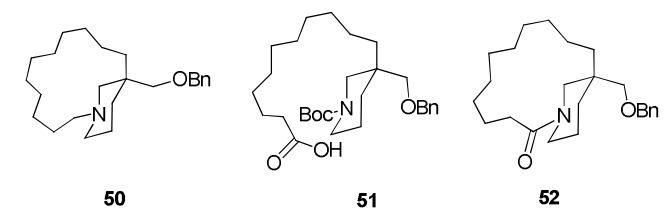



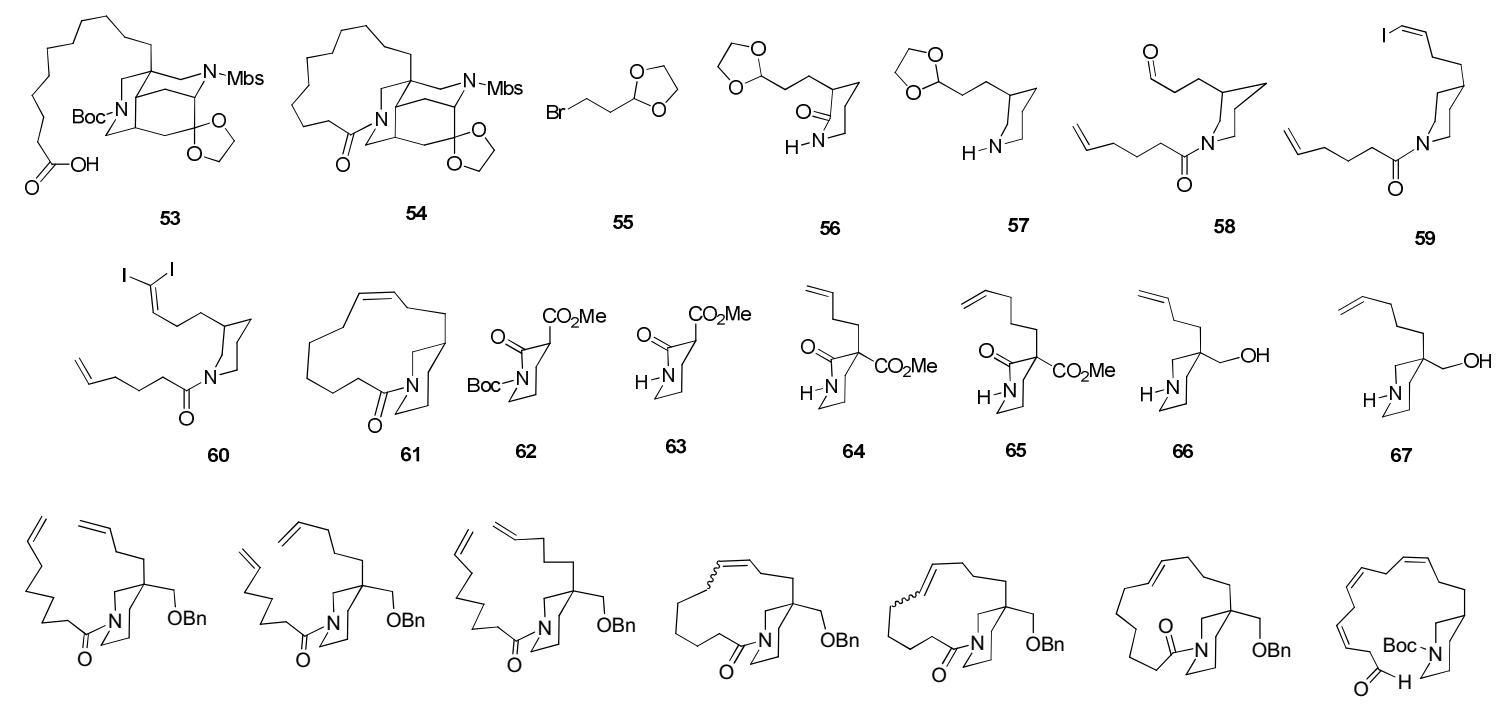
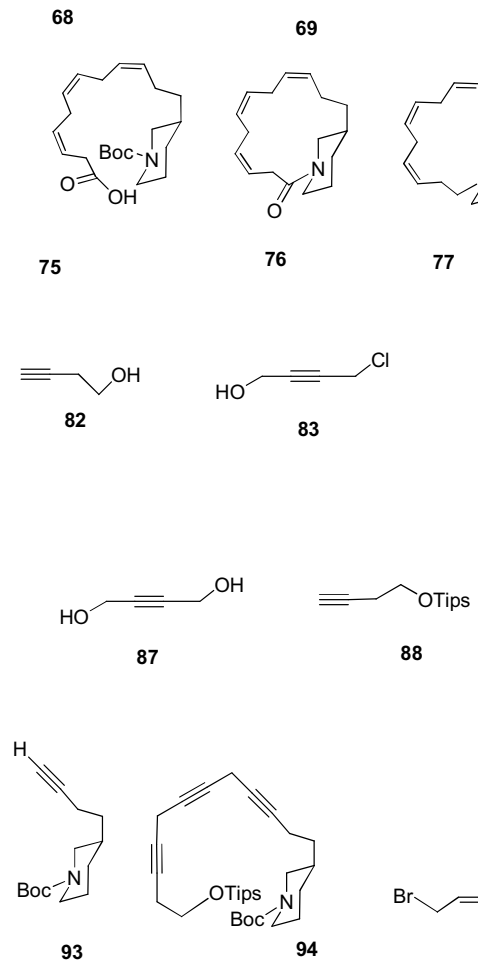

93

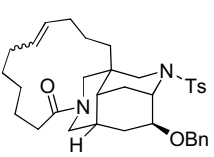

99

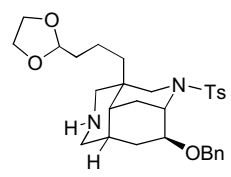

100

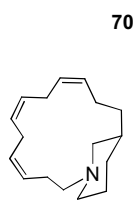

77 71

72

73

74

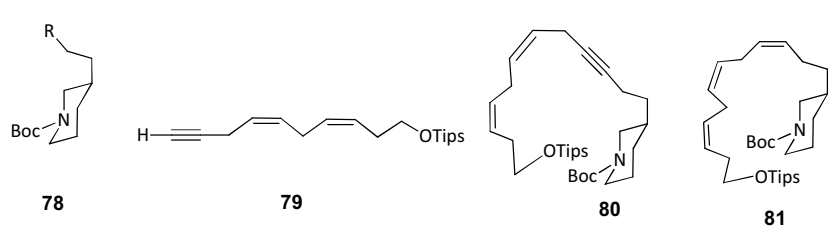

81

TMS $\frac{\overline{\overline{84}}}{84}$

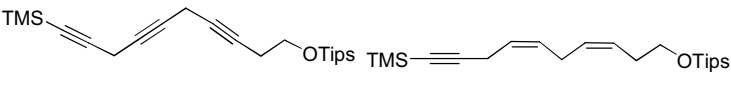

86<smiles>OCC#CC#CCC[OH2+]</smiles>

89

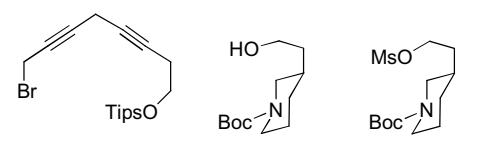

90

92

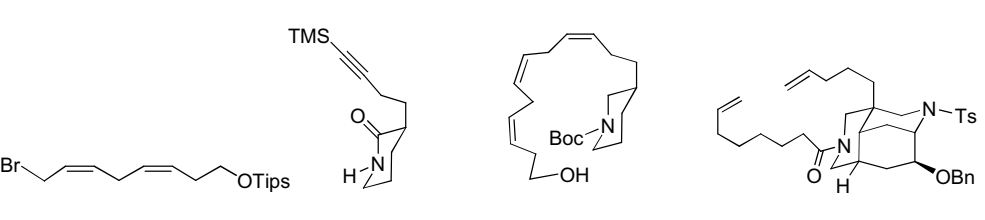

96

97

98

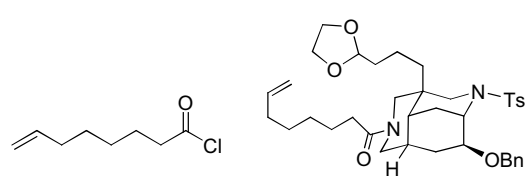

101
102 


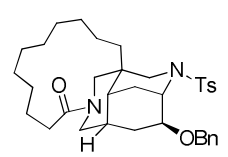

104

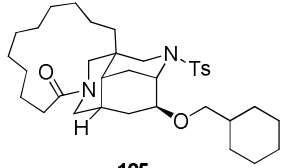

105

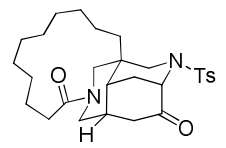

106<smiles>C=CC1CC=CCC1</smiles>

107

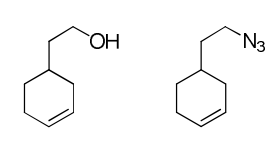

108

109
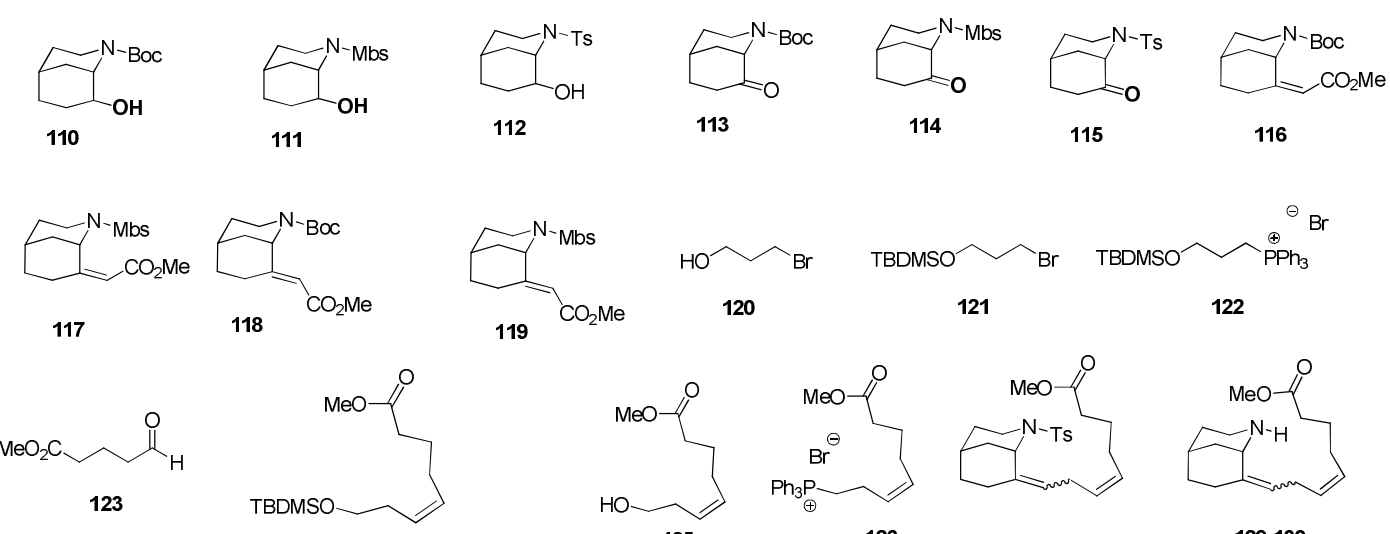

119

121
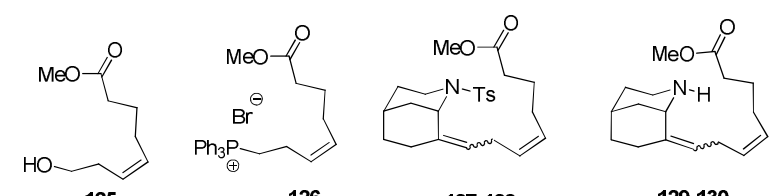

124

126

127-128

129-130

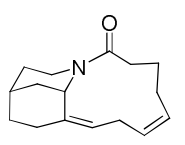

131

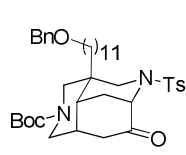

132

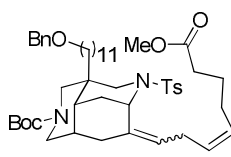

133-134

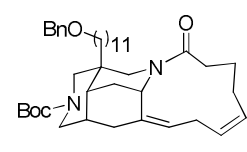

135

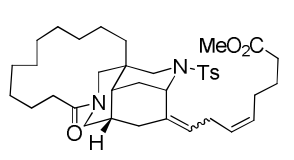

136-137

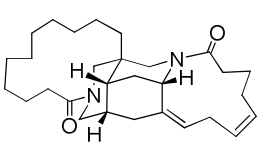

138

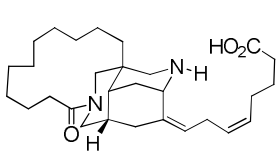

139

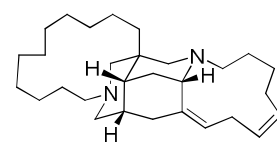

Madangamine $D$ 

Chapter 1 



\subsection{INTRODUCTION}

Although relatively unexplored until quite late in the last century, marine natural products have become the subject of increasing interest from the biological, ecological, pharmacological, and chemical standpoints. ${ }^{1,2,3}$ Many of the marine secondary metabolites possess complex structures not found in terrestrial organisms, and display significant biological activities. The structural diversity of marine natural products makes them attractive candidates as lead compounds for drug discovery. ${ }^{4,5}$

About one third of all marine natural products and more than half of all known alkaloids of marine origin have been isolated from sponges (Phylum Porifera), which are among the oldest and most primitive multicellular animals. In particular, sponges in the order Haplosclerida are the source of a great number of structurally diverse but biogenetically related alkaloids, the socalled 3-alkylpiperidine (or 3-alkylpyridine) alkaloids, ${ }^{6,7}$ which exhibit a broad spectrum of bioactivity profiles. They include a great variety of skeletal types, such as monomers and oligomers of 3-alkylpyridines, macrocycles containing bis-3-alkylpiperidine, bis-quinolizidine, bis-1-oxaquinolizidine and 3,4'-bipiperidine moieties, and an array of complex polycyclic diamine structures bearing macrocyclic rings (Figure 1.1). These alkaloids usually incorporate a saturated or unsaturated C-8 to C-16 carbon chain attached at the 3-position of the sixmembered nitrogen ring, either in the piperidine, tetrahydropyridine, or pyridine oxidation state. 3-Alkylpiperidine alkaloids are also referred to as "manzamine alkaloids" .,9 Although this term is usually restricted to $\beta$-carboline-containing alkaloids, in some cases its usage encompasses other biogenetically related complex polycyclic diamine alkaloids lacking the $\beta$ carboline moiety. ${ }^{10,11}$

\footnotetext{
${ }^{1}$ Kornprobst, J-M. Encyclopedia of Marine Natural Products; Wiley-VCH: Weinheim, 2010.

2 Blunt, J. W.; Copp, B. R.; Keyzers, R. A.; Munro, M. H. G.; Prinsep, M. R. Nat. Prod. Rep. 2013, 30, 237323 and previous reviews in this series.

${ }^{3}$ Morris, J. C. Nat. Prod. Rep. 2013, 30, 783-805 and previous reviews in this series.

${ }^{4}$ Thakur, N. L.; Thakur, A. N.; Müller, W. E. G. Nat. Prod. Rad. 2005, 46, 471-477.

${ }^{5}$ Gerwick, W. H.; Moore, B. S. Chem. Biol. 2012, 19, 85-98.

${ }^{6}$ Andersen, R. J.; Van Soest, R. W. M.; Kong, F. In Alkaloids: Chemical and Biological Perspectives; Pelletier, S. W., Ed.; Pergamon Press: New York, 1996; Vol. 10, pp 301-355.

${ }^{7}$ Becking, L. E.; Nakao, Y.; de Voogd, N. J.; van Soest, R. W. M.; Fusetani, N.; Matsunaga, S. In Porifera Research: Biodiversity, Innovation and Sustainability; Custódio, M. R.; Lôbo-Hajdu, G.; Hajdu, E.; Muricy, G., Eds.; Museu Nacional: Rio De Janeiro, Brazil, 2007; pp 173-178.

${ }^{8}$ Kaiser, A.; Billot, X.; Gateau-Olesker, A.; Marazano, C.; Das, B. C. J. Am. Chem. Soc. 1998, 120, 80268064.

${ }^{9}$ Duval, R.; Poupon, E. In Biomimetic Organic Synthesis; Poupon, E.; Nay, B. Eds.; Wiley-VCH: Weinheim, 2011, Chap 6, pp 181-224.

${ }^{10}$ Peng, J.; Rao, K. V.; Choo, Y.-M.; Hamann, M. T. Modern Alkaloids: Structure, Isolation, Synthesis and Biology; Fattorusso, E.; Tagliatela-Scafali, O. Eds.; Wiley-VCH: Weinheim, 2008, Chap 8, pp 189-232.

${ }^{11}$ Hu, J.-F.; Hamann, M. T.; Hill. R.; Kelly, M. In The Alkaloids: Chemistry and Biology; Cordell G. A., Ed.; Elsevier: San Diego, CA, 2003; Vol. 60, pp 207-285.
} 


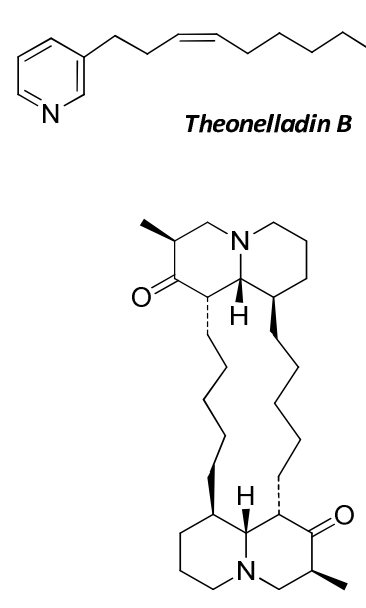

Petrosin

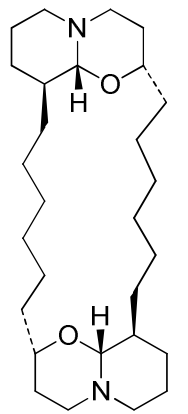

Xestospongin A

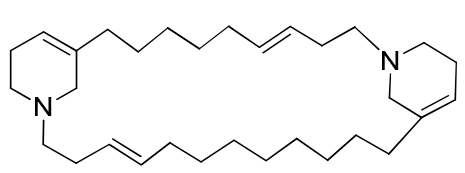

Haliclamine B

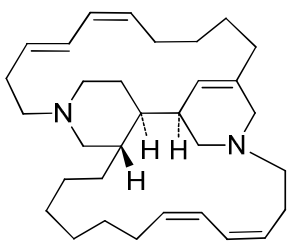

Halicyclamine A

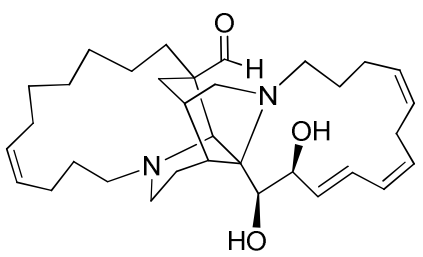

Saraine B

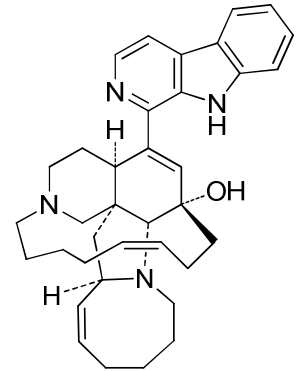

Manzamine A

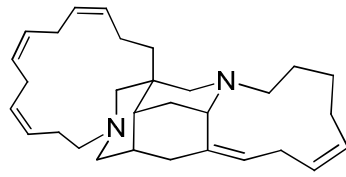

Madangamine A

Figure 1.1 Representative 3-alkylpiperidine alkaloids.

The 3-alkylpiperidine alkaloids were first classified according to their structural types by Andersen and coworkers in an excellent review, ${ }^{6}$ which also covers biogenetic proposals and phylogenetic distribution. Other general reviews of these marine sponge alkaloids, additionally dealing with synthetic aspects, have been written by Weinreb, ${ }^{12}$ Berlinck, ${ }^{13,14}$ Rodríguez, ${ }^{15}$ and Poupon. ${ }^{9}$ Reviews of specific groups of these alkaloids, for instance, simple 3-alkylpyridine (or pyridinium) alkaloids ${ }^{16}$ or manzamine-related alkaloids, ${ }^{10,11,17,18}$ have also been published.

\footnotetext{
${ }^{12}$ Matzanke, N.; Gregg, R. J.; Weinreb, S. M. Org. Prep. Proced. Int. 1998, 30, 1-51.

${ }^{13}$ Almeida, A. M. P.; Berlinck, R. G. S. Quim. Nova, 1997, 20, 170-185.

${ }^{14}$ Berlinck, R. G. S. Top. Heterocycl. Chem. 2007, 10, 211-238.

${ }^{15}$ Rodríguez, J. In Studies in Natural Products Chemistry; Rahman A.-U., Ed.; Elsevier: Oxford, U.K., 2000; Vol. 24, pp 573-681.

${ }^{16}$ Turk, T.; Sepcic, K.; Mancini, I.; Guella, G. In Studies in Natural Products Chemistry; Rahman A.-U., Ed.; Elsevier: Oxford, U.K., 2008; Vol. 35, pp 355-397.

${ }^{17}$ Magnier, E.; Langlois, Y. Tetrahedron 1998, 54, 6201-6258.

${ }^{18}$ Nishida, A.; Nagata, T.; Nakagawa, M. Top. Heterocycl. Chem. 2006, 5, 255-280.
} 
The present work is focused on madangamines (Figure 1.2), a small group of complex pentacyclic diamine alkaloids isolated from marine sponges belonging to the order Haplosclerida biogenetically derived from 3-alkylpiperidine precursors. Structurally, madangamines are characterized by a diazatricyclic core (ABC rings), unprecedented among natural products, and two linear carbon bridges connecting N-7 to C-9 (D ring) and N-1 to C-3 ( $E$ ring). The peripheral macrocyclic ring $D$ varies in each madangamine both in size (13 to 15membered) and in the position and degree of unsaturation, whereas $E$ ring is identical in madangamines A-E (two unsaturations; 11-membered) but different in madangamine $F$ (four unsaturations; 13-membered). In addition madangamine $\mathrm{F}$ incorporates an alcohol function at $\mathrm{C}-4$, being the first alkaloid of this group to bear a hydroxy substituent.

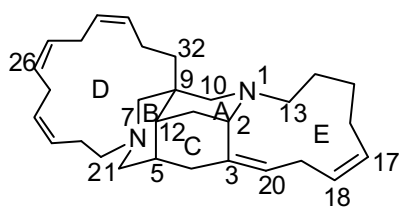

Madangamine $A$

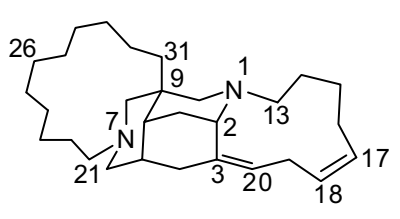

Madangamine D

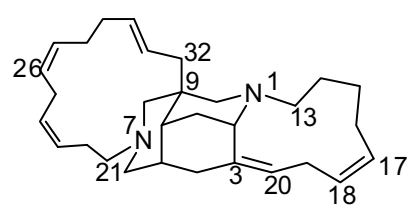

Madangamine B

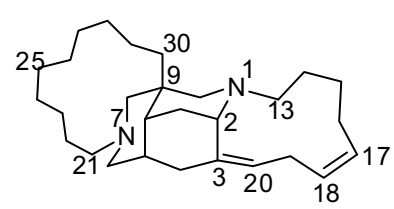

Madangamine E

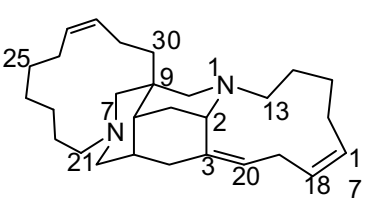

Madangamine C

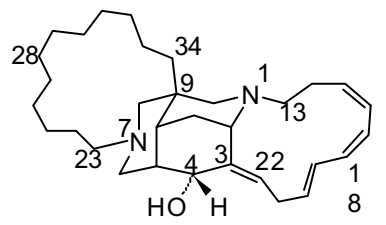

Madangamine $F$

Figure 1.2 Alkaloids of the madangamine group.

\subsection{ISOLATION}

As part of a program for the search of bioactive metabolites in extracts of tropical marine sponges, the Andersen group found that the crude extracts of Xestospongia ingens Van Soest showed in vitro cytotoxicity. ${ }^{19}$ This sponge (order Haplosclerida, family Petrosiidae) was collected by hand using scuba on reefs at 15-20 m depth off Madang, in Papua New Guinea. A bioassay-guided fractionation of the extracts resulted in the isolation of madangamines $A-E, a$ new class of pentacyclic alkaloids with an unprecedented skeletal type. The first isolation of an alkaloid of this group, madangamine A, was reported in $1994,{ }^{20}$ and four years later the same team reported ${ }^{21}$ the isolation of madangamines B-E. Freshly collected sponge samples were deep frozen on site and transported over dry ice to the laboratory in Vancouver for extraction. Once thawed, the sponges were repeatedly extracted with methanol. The extracts were concentrated to an aqueous suspension, which was diluted with distilled water and then

\footnotetext{
${ }^{19}$ Kong, F.; Andersen, R. J.; Allen, T. M. Tetrahedron Lett. 1994, 35, 1643-1646.

${ }^{20}$ Kong, F.; Andersen, R. J.; Allen, T. M. J. Am. Chem. Soc. 1994, 116, 6007-6008.

${ }^{21}$ Kong, F.; Graziani, E. I.; Andersen, R. J. Nat. Prod. 1998, 61, 267-271.
} 
partitioned sequentially against hexanes and EtOAc. The alkaloids were isolated as colorless glass by repeated fractionation of the hexane-soluble fraction using silica gel flash chromatography and then normal-phase HPLC (hexanes, EtOAc, $i-\mathrm{Pr}_{2} \mathrm{NH}$ ). From $200 \mathrm{~g}$ wet weight of sponge, the procedure afforded $50-60 \mathrm{mg}$ of madangamine $\mathrm{A}, 5.5 \mathrm{mg}$ of madangamine $B, 11 \mathrm{mg}$ of madangamine $C$, and $5 \mathrm{mg}$ of an inseparable mixture of madangamines $D$ and $E$. Other biogenetically related alkaloids isolated from Xestospongia ingens were ingenamine, ${ }^{19}$ ingamines $A$ and $B,{ }^{22}$ ingenamines $B-F,{ }^{23}$ xestocyclamines $A^{24}$ and $\mathrm{B}^{23,24}$ and keramaphidin $\mathrm{B}^{23}$ (Figure 1.3).

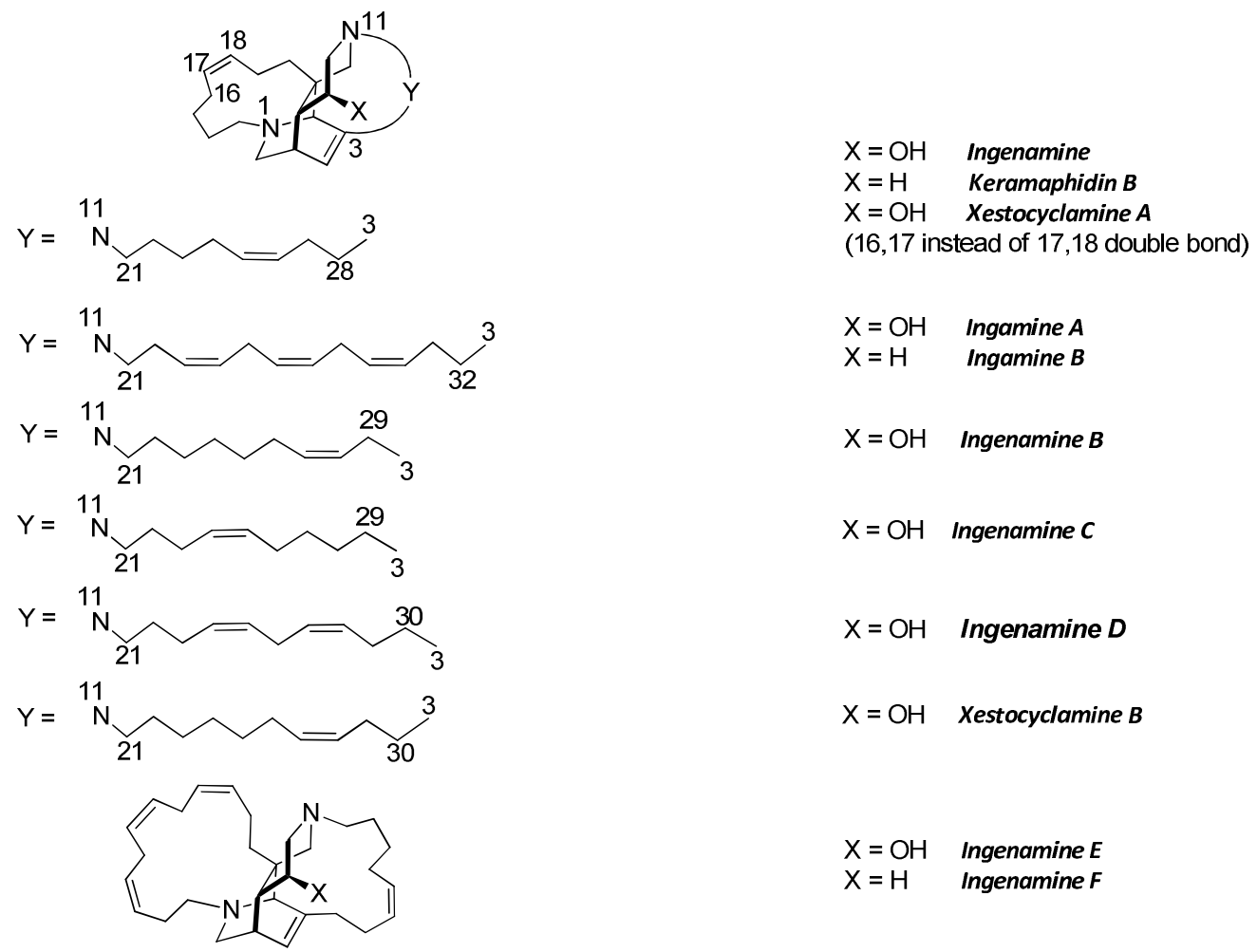

Figure 1.3 Other alkaloids isolated from Xestospongia ingens.

Pure madangamines $\mathrm{A}-\mathrm{C}$ and $\mathrm{F}$ were isolated as optically active. Although the absolute configuration of the alkaloids has not been unambiguously established, it may be tentatively deduced by correlation with the putative precursor ingenamines.

An intriguing property of madangamines A-E is their low polarity. Thus, during their isolation madangamines dissolve in the hexane layer, in contrast with ingenamine $F$, an alkaloid with the same molecular formula, which remains in the aqueous layer. This dramatic difference in polarity can be explained taking into account that the tricyclic $A B C$ core of madangamines adopts a rigid chair-chair-chair conformation, in which the nitrogen atom at the 7-position

\footnotetext{
${ }^{22}$ Kong, F.; Andersen, R. J.; Allen, T. M. Tetrahedron 1994, 50, 6137-6144.

${ }^{23}$ Kong, F.; Andersen, R. J. Tetrahedron 1995, 51, 2895-2906.

${ }^{24}$ Rodríguez, J.; Crews, P. Tetrahedron Lett. 1994, 35, 4719-4722.
} 
cannot undergo inversion since the cavity of the central core is too small to accommodate the C-21 alkyl substituent. For this reason, the N-7 lone pair is hidden within the tricyclic core, where it is inaccessible for protonation and hydrogen bonding.

In 2007, Berlinck et al. reported ${ }^{25}$ the isolation of a new alkaloid of this group, madangamine $F$, from the marine sponge Pachychalina alcaloidifera (order Haploscherida, family Niphatidae), which was collected at 10-15 m depth in Ilha do Pai (Father's Island), Nisterói, Rio de Janeiro. Once retrieved, the sponge was immediately immersed in $\mathrm{EtOH}$ and shipped to the laboratory at the Universidade de São Paulo, where it was extracted with $\mathrm{MeOH}$. The crude extracts contained a very complex mixture of alkaloids, which were difficult to separate. Starting from 2 $\mathrm{Kg}$ of sponge, only a minute amount $(4 \mathrm{mg}$ ) of madangamine $\mathrm{F}$ was isolated. Besides madangamine $F$, other 3-alkylpiperidine alkaloids isolated from this sponge were haliclonacyclamine $F$, arenosclerins $D$ and $E$, ingenamine $G$, and several cyclostellettamines ${ }^{26}$ (Figure 1.4).

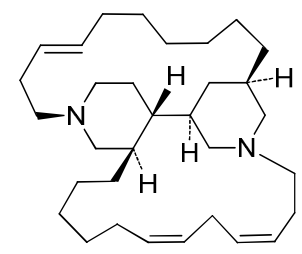

Haliclonacyclamine $F$

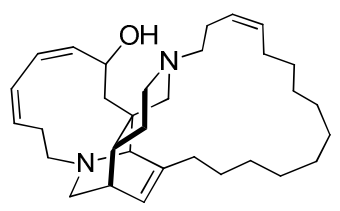

Ingenamine $G$

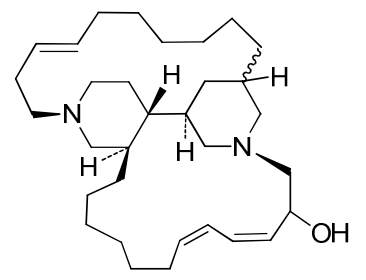

Arenosclerin D

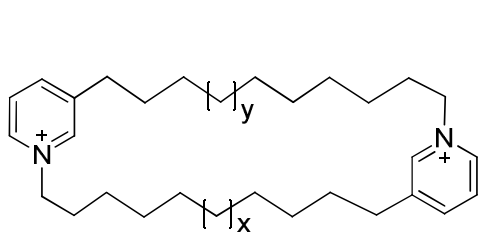

Cyclostellettamines

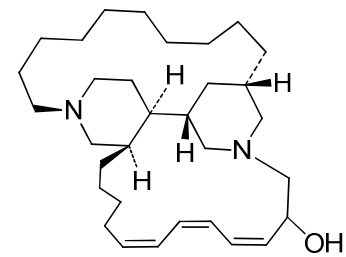

Arenosclerin E

Figure 1.4 Other alkaloids isolated from Pachychalina alcaloidifera.

\footnotetext{
${ }^{25}$ De Oliveira, J. H. H. L.; Nascimento, A. M.; Kossuga, M. H.; Cavalcanti, B. C.; Pessoa, C. O.; Moraes, M. O.; Macedo, M. L.; Ferreira, A. G.; Hajdu, E.; Pinheiro, U. S.; Berlinck, R. G. S. J. Nat. Prod. 2007, 70, 538543.

${ }^{26}$ De Oliveira, J. H. H. L.; Grube, A.; Köck, M.; Berlinck, R. G. S.; Macedo, M. L.; Ferreira, A. G.; Hajdu, E. J. Nat. Prod., 2004, 67, 1685-1689.
} 


\subsection{BIOGENESIS}

The imaginative proposal of Baldwin and Whitehead to explain the biogenesis of manzamines, ${ }^{27,28,29,30}$ formulated in 1992, also provided the groundwork for a mechanistic rationale for the biosynthesis of 3-alkylpiperidine alkaloids. Despite their structural diversity, all 3-alkylpiperidine alkaloids can biogenetically derive from similar simple building blocks: ammonia, a three carbon acrolein equivalent, and saturated or unsaturated linear long-chain dialdehydes. These units are first assembled into partially reduced 3-alkylpyridine monomers, which can then either polymerize to give oligomers or dimerize to give bis-3-alkylpiperidine macrocycles (Scheme 1.1). Subsequent transformations, including intramolecular [4+2] cycloaddition reactions, would lead to the variety of polycyclic skeletons found in these alkaloids. ${ }^{6,9,12,15,31,32,33}$

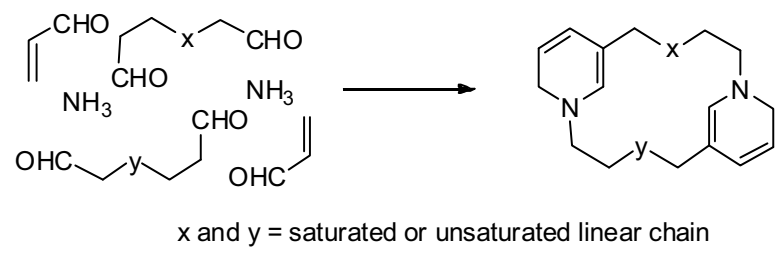

Scheme 1.1 Proposed biogenesis of dimeric bis-3-alkylpiperidine macrocyclic alkaloids.

A plausible biosynthetic pathway to madangamines from partially reduced bis-3-alkylpyridine macrocycles is outlined in Scheme 1.2. ${ }^{6,20}$ A distinguishing feature of this pathway compared to those leading to other 3-alkylpiperidine polycyclic alkaloids is that it involves a skeletal rearrangement.

\footnotetext{
${ }^{27}$ Baldwin, J. E.; Whitehead, R. C. Tetrahedron Lett. 1992, 2059-2062.

${ }^{28}$ Baldwin, J. E.; Bischoff, L.; Claridge, T. D. W.; Heupel, F. A.; Spring, D. R.; Whitehead, R. C. Tetrahedron 1997, 53, 2271-2290.

${ }^{29}$ Baldwin, J. E.; Claridge, T. D. W.; Culshaw, A. J.; Heupel, F. A.; Lee, V.; Spring, D. R.; Whitehead, R. C.; Boughtflower, R. J.; Mutton, I. M.; Upton, R. J. Angew. Chem. Int. Ed. 1998, 37, 2661-2663.

${ }^{30}$ Baldwin, J. E.; Claridge, T. D. W.; Culshaw, A. J.; Heupel, F. A.; Lee, V.; Spring, D. R.; Whitehead, R. C. Chem. Eur. J. 1999, 5, 3154-3161.

${ }^{31}$ Crews, P.; Cheng, X.-C.; Adamczeski, M.; Rodríguez, J.; Jaspars, M.; Schmitz, F. J.; Traeger, S. C.; Pordesimo, E. O. Tetrahedron 1994, 50, 13567-13574.

${ }^{32}$ Tsuda, M.; Kobayashi, J. Heterocycles 1997, 46, 765-793.Whitehead, R. Annu. Rep. Prog. Chem., Sect. B 1999, 95, 183-205.

${ }^{33}$ Whitehead, R. Annu. Rep. Prog. Chem., Sect. B 1999, 95, 183-205.
} 


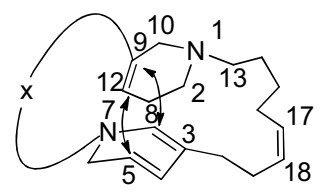

$1 \mathrm{a}$

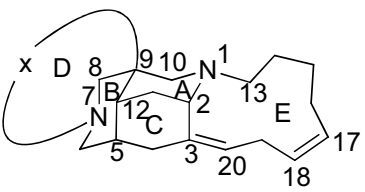

Madangamines A-E

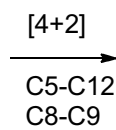

C2-C3

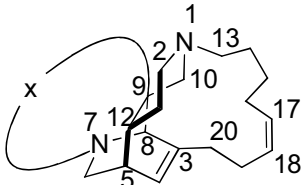

$2 a$

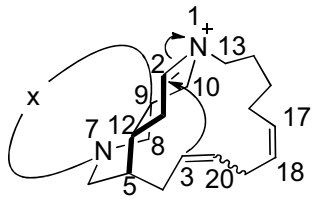

$5 a$

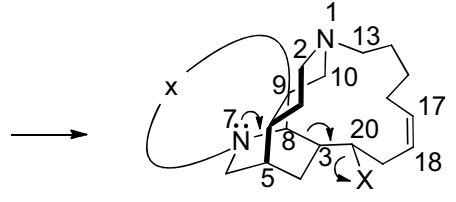

3a

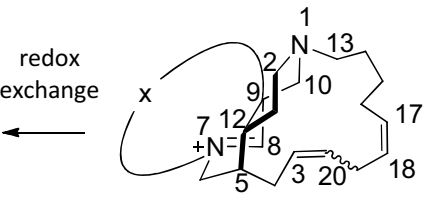

4a

Scheme 1.2 Proposed biogenesis for the madangamine alkaloids.

(For clarity, the madangamine numbering is used throughout the scheme)

Thus, the partially reduced bis-3-alkylpyridine macrocycle 1a would undergo an intramolecular endo Diels Alder cycloaddition reaction to generate an ingenamine-type pentacyclic intermediate 2a. After activation of the carbon 20 and reduction of the tetrahydropyridine double bond, fragmentation of intermediate $3 a$, followed by a redox exchange between the two nitrogen atoms of the resulting tetracyclic iminium ion $\mathbf{4 a}$ would give the regioisomeric iminium ion 5a. A final transannular cyclization would lead to the rearranged skeleton of madangamines. ${ }^{6,20}$

An alternative biosynthetic proposal, suggesting the participation of malonaldehyde, instead of acrolein, as the three carbon unit to generate 3-alkylpyridinium salts via 5-amino-2,4pentadienal derivatives, has been proposed by Marazano (Scheme 1.3). ${ }^{8,34}$

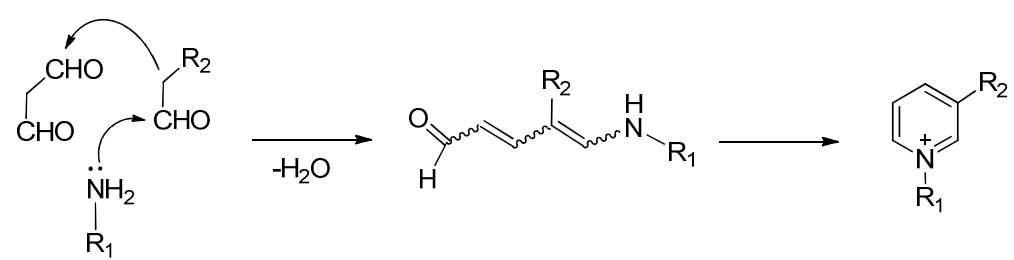

Scheme 1.3 Alternative biosynthetic pathway to sponge pyridines and pyridinium salts.

\footnotetext{
${ }^{34}$ Jakubowicz, K.; Abdeljelil, K. B.; Herdemann, M.; Martin, M.-T.; Gateau-Olesker, A.; Al Mourabit, A.; Marazano, C.; Das, B. C. J. Org. Chem. 1999, 64, 7381-7387.
} 
In a similar manner, condensation of malonaldehyde, ammonia, and appropriate dialdehydes could produce macrocyclic aminopentadienal derivatives, which can be envisaged as alternative intermediates in the biosynthesis of complex polycyclic 3-alkylpiperidine alkaloids, including the alkaloids of the madangamine group. For instance, the open-chain aminopentadienal moiety in dihydropyridinium derivatives 6 a could participate as the diene in intramolecular Diels-Alder reactions, leading to tetracyclic ircinal-type derivatives $\mathbf{7 a}$, from which madangamine alkaloids would eventually be generated as outlined in Scheme 1.5, via a vinylogous retro-Mannich fragmentation, an intramolecular redox transfer, cyclization of the resulting amino aldehyde $\mathbf{8 a}$, and finally, a vinylogous aza-Mannich reaction. ${ }^{9,35}$

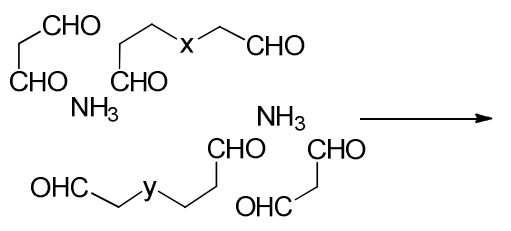<smiles>[X]CC1=CCC[N+]2=C1CC[Y]CCC(/C=C\C=O)=C/NCC2</smiles>

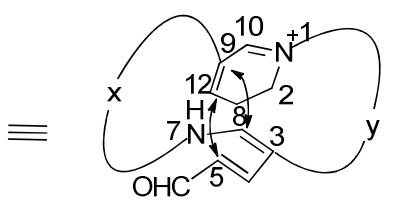

$6 a$ $\mathrm{OHC}$

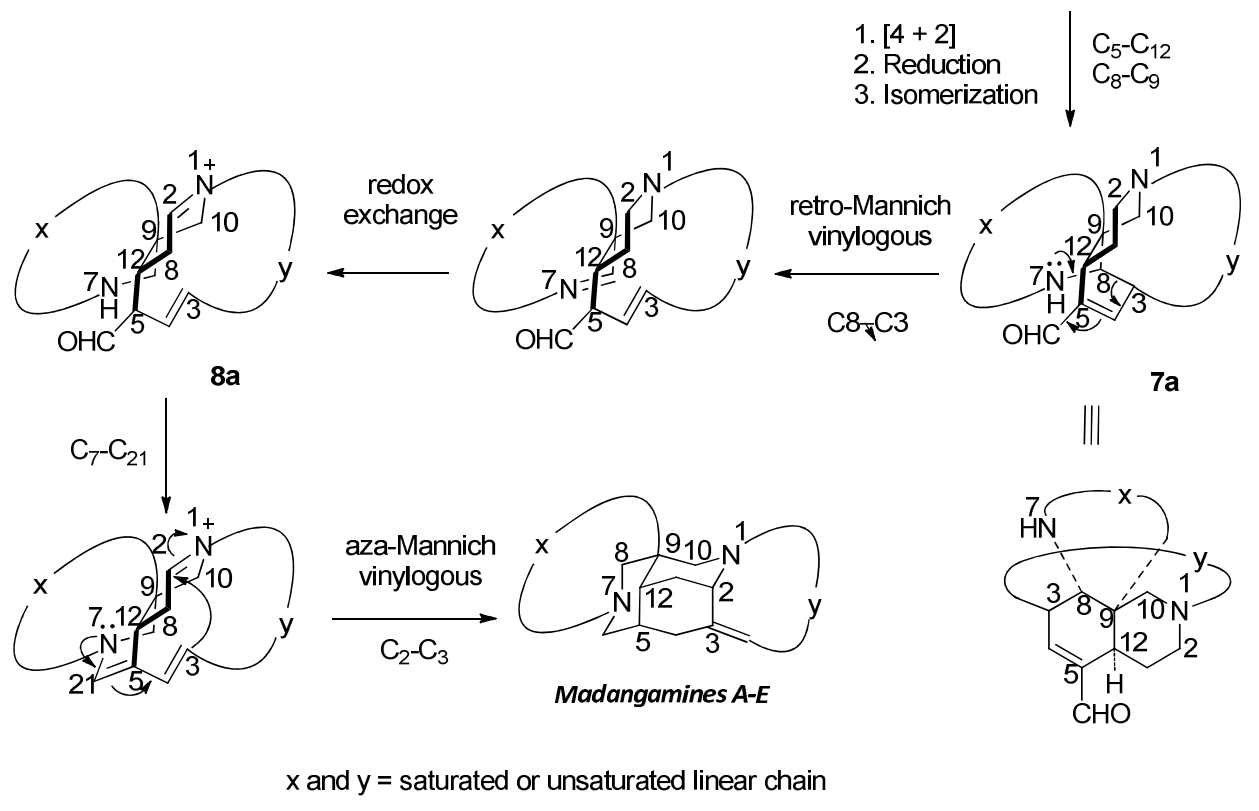

Scheme 1.4 Alternative biosynthetic scheme towards madangamine alkaloids.

(For clarity, the madangamine numbering is used throughout the scheme)

The fact that ingenamine-type intermediates are biosynthetic precursors of madangamines (see Scheme 1.2) allows the absolute configuration of the latter to be tentatively assigned. Thus, madangamine A would biogenetically derive from ingenamine $F$, an alkaloid with the same carbon skeleton and sign of specific rotation as ingenamine $E$, whose absolute configuration was determined by Mosher ester methodology as $2 R, 5 S, 7 S, 8 R$, $9 S$ (ingenamine numbering). ${ }^{23}$ Taking into account that madangamine carbons 5, 9, and 12 correspond to

\footnotetext{
${ }^{35}$ Tong, H. M.; Martin, M.-T.; Chiaroni, A.; Benechie, M.; Marazano, C. Org. Lett. 2005, 7, 2437-2440.
} 
ingenamine carbons 5, 7, and 9, respectively, and that the configuration of C-2 in madangamines is determined by its bridgehead character, the absolute configuration of madangamines can be assumed to be $2 S, 5 S, 9 R, 12 R$ (Scheme 1.5).
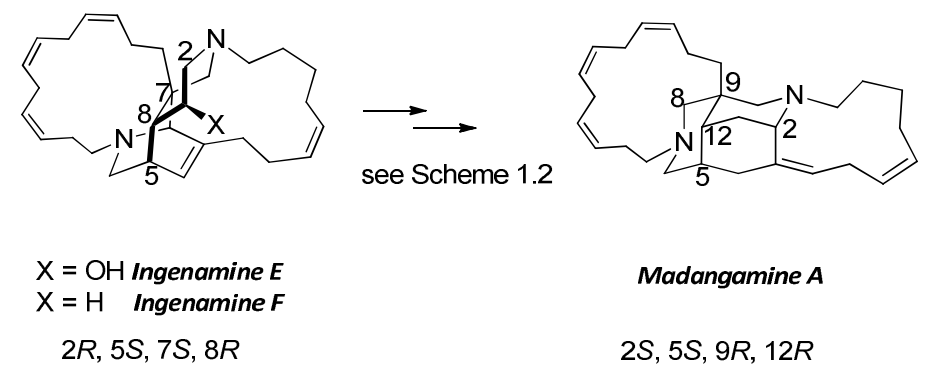

Scheme 1.5 Tentative absolute configuration of madangamine A.

\subsection{BIOLOGICAL ACTIVITY}

Marine sponges are recognized to be one of the richest sources of pharmacologically active chemicals among marine organisms. ${ }^{36}$ In particular, the alkaloids of the 3-alkylpiperidine group, isolated from Haplosclerida sponges, have been found to exhibit a wide range of biological activities, suggesting potential for drug development.

In the case of madangamine alkaloids, extensive pharmacological research has been hampered by the low quantities of samples available from natural sources. Madangamine A has shown significant in vitro cytotoxic activity against murine leukemia $P 388\left(E_{50} 0.93 \mu \mathrm{g} / \mathrm{mL}\right)$ and human lung A549 (ED $5014 \mu \mathrm{g} / \mathrm{mL})$, brain U373 (ED $50.1 \mu \mathrm{g} / \mathrm{mL})$, and breast MCF-7 (ED 50.7 $\mu \mathrm{g} / \mathrm{mL}$ ) cancer cell lines. ${ }^{20}$ In turn, madangamine $\mathrm{F}$ displays weak cytotoxicity against human CNS SF-295 (ED $5019.8 \mu \mathrm{g} / \mathrm{mL})$, human breast MDA-MB-435 (ED $\left.{ }_{50} 16.2 \mu \mathrm{g} / \mathrm{mL}\right)$, colon HCT-8 $\left(E_{50}>25 \mu \mathrm{g} / \mathrm{mL}\right)$, and leukemia $\mathrm{HL}-60\left(\mathrm{ED}_{50} 16.7 \mu \mathrm{g} / \mathrm{mL}\right)$ cancer cell lines using the $\mathrm{MTT}$ cell proliferation assay. No bioactivity data have been reported for madangamines $B-E$, so these alkaloids are attractive challenges for synthetic organic chemists, not only due to their unprecedented molecular architecture but also as targets for pharmaceutical research.

\subsection{SYNTHESIS}

The synthesis of alkaloids of the madangamine group has been little explored. The most logical approach to the pentacyclic skeleton of these alkaloids consists in the initial

\footnotetext{
${ }^{36}$ Laport, M. S.; Santos, O. C. S.; Muricy, G. Curr. Pharm. Biotechnol. 2009, 10, 86-105.
} 
construction of the bridged diazatricyclic $A B C$ core common to all madangamines, with the appropriate substitution and functionality to allow the subsequent building of the peripheral macrocyclic $D$ and $E$ rings.

Previous to our work in this field, only four synthetic approaches to the diazatricyclic madangamine $A B C$ core $e^{35,37,38,39}$ and the construction of the 11-membered $E$ macrocycle common to madangamines A-E from a model azabicyclic derivative ${ }^{40}$ had been reported. All these studies were performed in the racemic series.

\subsubsection{Construction of the Diazatricyclic ABC Core}

\subsubsection{Weinreb's Approach}

In 1997 Weinreb reported ${ }^{37}$ the first synthesis of the tricyclic core of madangamines. The synthesis started from the SES-protected furfurylamine, which was converted to dihydropyridone 9a (ring B) by an oxidation-rearrangement sequence (Scheme 1.6). A DielsAlder reaction with 1,3-butadiene under high pressure conditions produced cisoctahydroisoquinolone 10a (rings $A B$ ), from which the quaternary $C-9$ stereocenter (madangamine numbering) was stereoselectively installed. After a one-carbon homologation with TosMIC and reduction of the resulting nitrile 11a to aldehyde 12a (1:1 mixture of epimers), a reaction with diallylamine and $\mathrm{Pd}\left(\mathrm{OCOCF}_{3}\right)_{2}-\mathrm{PPh}_{3}$, followed by acidic hydrolysis furnished the $\alpha$-allyl substituted aldehyde 13a. This transformation involves the initial generation of an $\mathrm{N}$-allyl enamine, which undergoes an in situ stereospecific 3-aza-Cope rearrangement from the less congested convex face, and a final hydrolytic step from the resulting $\alpha$-allyl imine. The formyl group in 13a was used to construct the piperidine A ring. Thus, once 13a was converted into an $O$-benzyl oxime, a hydroboration-oxidation reaction led to alcohol 14a. Protection of the hydroxy group with $p$-methoxybenzyl chloride also produced the elimination of the $O$-benzyl oxime moiety to give a nitrile, which was then reduced to the primary amine 15a. Finally, closure of the piperidine A ring was accomplished by an intramolecular aminomercuriation with mercuric trifluoroacetate, followed by an oxidative demercuriation process with oxygen and $\mathrm{NaBH}_{4}$. The resulting diazatricyclic derivative 16a possesses suitable functionality both at $\mathrm{C}-3$ and the $\mathrm{C}-9$ chain to allow the building of the macrocyclic $D$ and $E$ rings of madangamines.

\footnotetext{
${ }^{37}$ Matzanke, N.; Gregg, R. J.; Weinreb R. J.; Parvez, M. J. Org. Chem. 1997, 62, 1920-1921.

${ }^{38}$ Yamazaki, N.; Kusanagi, T.; Kibayashi, C. Tetrahedron Lett. 2004, 45, 6509-6512.

${ }^{39}$ Quirante, J.; Paloma, L.; Diaba, F.; Vila, X.; Bonjoch, J. J. Org. Chem. 2008, 73, 768-771.

${ }^{40}$ Yoshimura, Y.; Inoue, J.; Yamazaki, N.; Aoyagi, S.; Kibayashi, C. Tetrahedron Lett. 2006, 47, 3489-3492.
} 

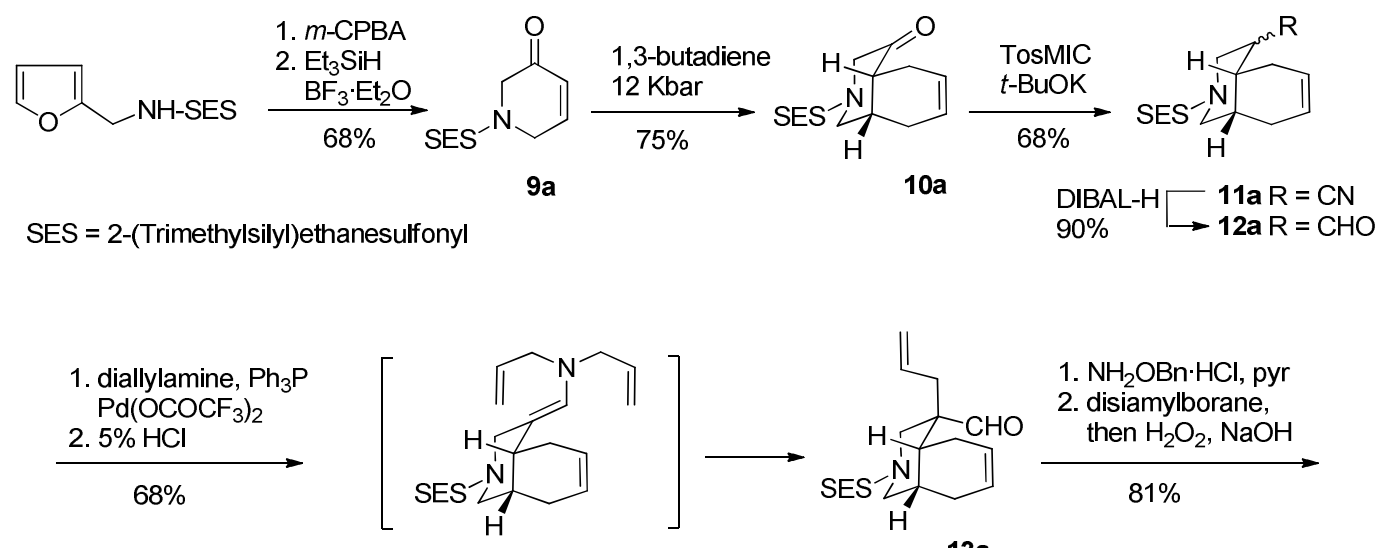

$13 a$

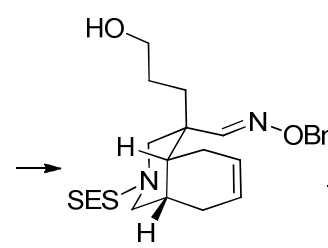

$14 a$
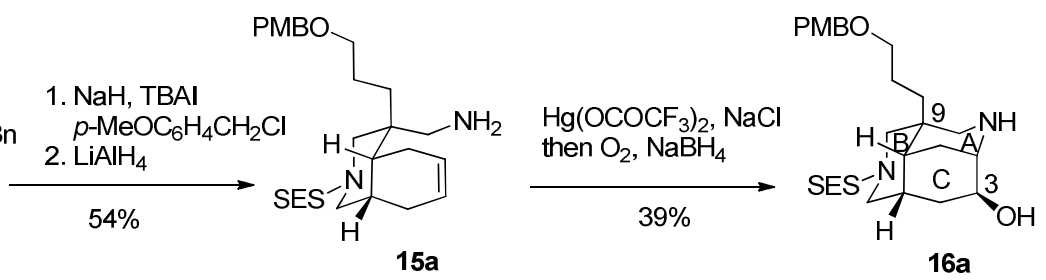

Scheme 1.6 Weinreb's synthetic approach to the ABC diazatricyclic core.

\subsubsection{Kibayashi's Approach}

The second approach to the diazatricyclic $A B C$ core of madangamines was reported by Kibayashi in 2004. ${ }^{40,41}$ The synthesis starts from cyclohexenone 17a (ring B) and involves the initial construction of the piperidine $A$ ring to give a functionalized 2-azabicyclo[3.3.1]nonane derivative (rings $A B$ ) and the closure of the piperidine $C$ ring in the last synthetic step (Scheme 1.7).

A Michael addition of ethyl cyanoacetate, followed by protection of the cyclohexanone carbonyl group in 18a as a cyclic acetal led to cyano ester 19a. Alkylation with formaldehyde, with subsequent protection of the hydroxy group as a MOM-ether provided the substituted cyclohexane derivative 20a as a mixture of diastereoisomers. Successive reduction of the ester function and, after protection of the resulting alcohol as a MOM-ether, of the cyano group gave the primary amine 21a as a single diastereoisomer. Reductive amination of 21a with salicylaldehyde, followed by treatment of the resulting aminophenol $22 \mathrm{a}$ with $p$ toluenesulfonic acid in acetone- $\mathrm{H}_{2} \mathrm{O}$ brought about the deprotection of the ketone function and the closure of the piperidine $\mathrm{A}$ ring by intramolecular $\mathrm{N}, \mathrm{O}$-acetalization to give the tetracyclic derivative 23a. Once the 2-azabicyclo[3.3.1]nonane system was assembled, the auxiliary 2-hydroxybenzyl moiety was removed by a Lewis acid-mediated reductive cleavage of the $\mathrm{C}-\mathrm{O}$ bond, which took place with simultaneous selective deprotection of the axial MOMether substituent, followed by $\mathrm{N}$-debenzylation by hydrogenolysis.

\footnotetext{
${ }^{41}$ Yoshimura, Y., Kusanagi, T.; Kibayashi, C.; Yamazaki, N.; Aoyagi, S. Heterocycles 2008, 75, 1329-1354.
} 
After a sequential protection of the amino and hydroxy groups, a TBAF-induced deprotection of the silylated alcohol and oxidation of the resulting secondary hydroxy group led to ketone 25a. Tebbe methylenation of $\mathbf{2 5 a}$, followed by a hydroboration-oxidation reaction from the most accessible face of the exocyclic methylene substituent gave alcohol $\mathbf{2 6} \mathbf{a}$ as a single diastereoisomer, from which the nitrogen atom required to construct the piperidine $B$ ring was introduced by a Mitsunobu reaction using phthalimide. Then, a sequence of $N$ and $O$ protection-deprotection steps led to the functionalized bicyclic derivatives 27a and 28a. Finally, mesylation of the hydroxy group of $\mathbf{2 8 a}$, followed by exposure of the resulting mesylate to $t$-BuOK completed the synthesis of the model diazatricyclic derivatives 29a, which bear the quaternary C-9 center present in madangamines and a functionalized substituent at this position, but lack the functionalization required at $\mathrm{C}-3$ (madangamine numbering) to assemble the macrocyclic E ring.

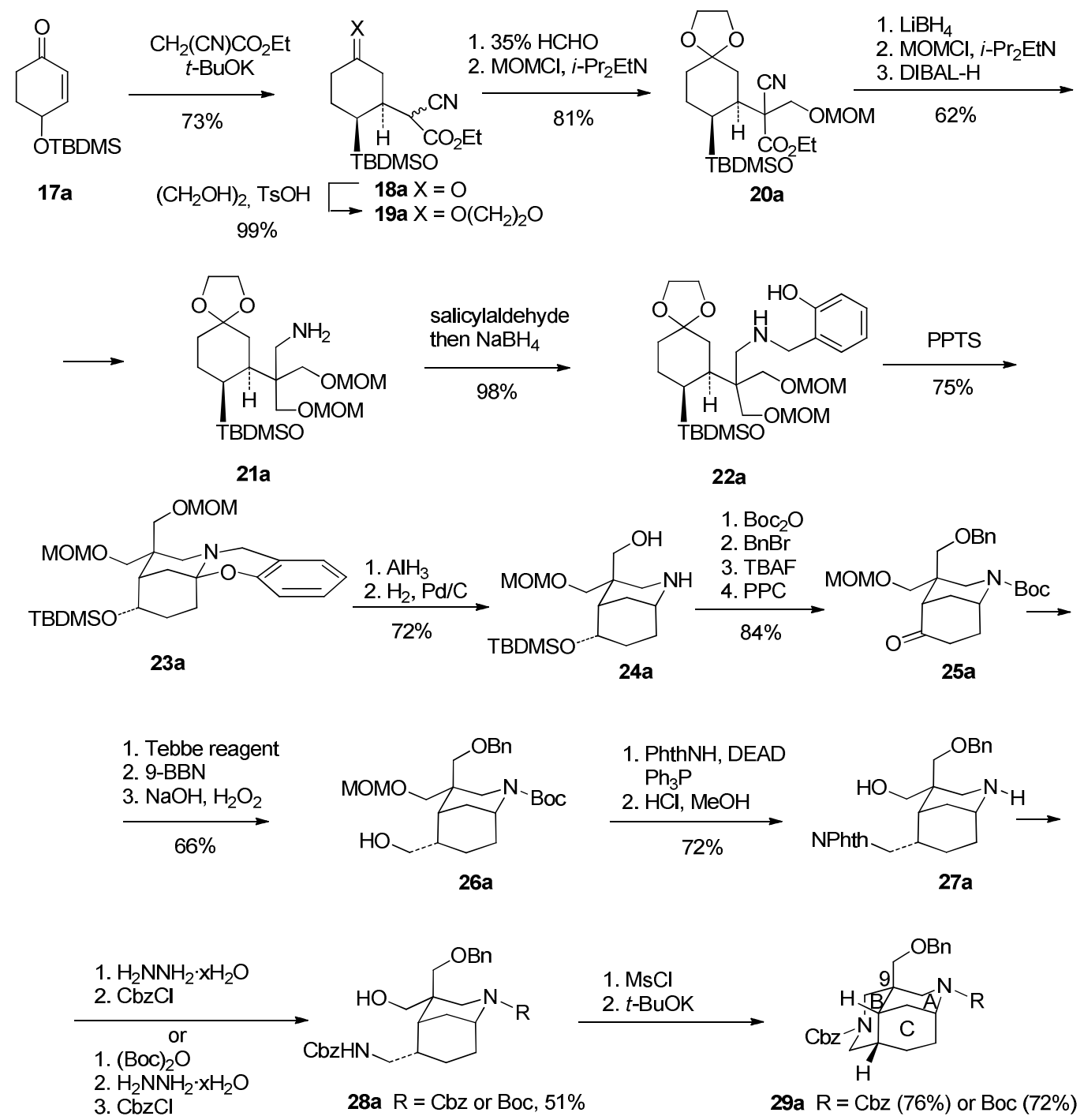

Scheme 1.7 Kibayashi's synthetic approach to the ABC diazatricyclic system. 


\subsubsection{Biomimetic Marazano Approach}

A totally different approach to the tricyclic core of madangamines was reported ${ }^{35}$ in 2005 by Marazano, based upon his biogenetic proposal ${ }^{8,34}$ linking these alkaloids to ircinals (see Scheme 1.4). On the assumption that the hypothetical biogenetic intermediate $8 \mathbf{a}$ is in equilibrium with a dihydropyridinium species, to access the madangamine core skeleton the authors envisaged a biomimetic strategy involving the reaction of an appropriate dihydropyridinium salt with a bis-nuclephile such as an acetonedicarboxylate, as outlined in Scheme 1.8.
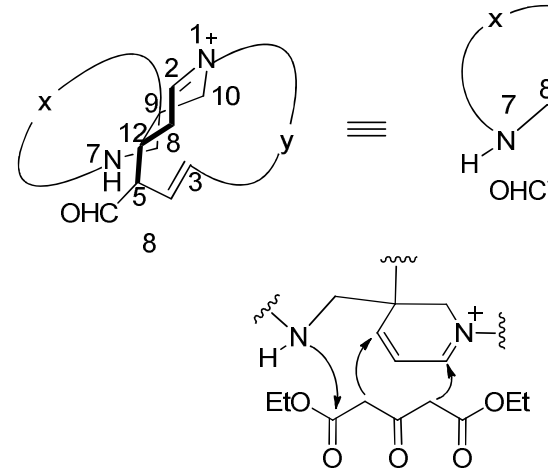
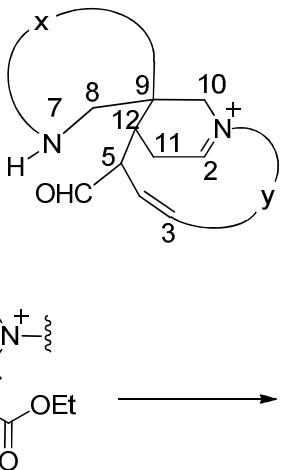

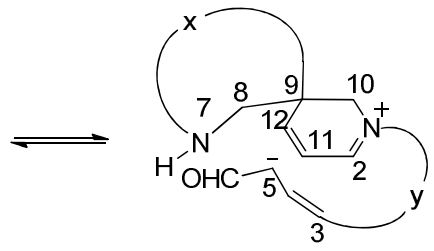

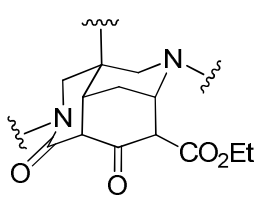

Scheme 1.8 Biomimetic strategy.

Dihydropyridinium salt 31a was initially selected to check the viability of the proposal. This salt was prepared from tetrahydropyridine 30a, which was accessible in six steps from methyl nicotinate, by treatment of the corresponding $\mathrm{N}$-oxide derivative (mixture of two diastereisomers) under Polonovski-Potier reaction conditions (Scheme 1.9) ${ }^{42}$. Reaction of the crude salt 31a with the sodium salt of diethyl 1,3-acetonedicarboxylate gave a mixture of two diastereoisomeric adducts 32a (two isolable enol forms) and 33a in $87 \%$ overall yield, the undesired stereoisomers 32a (axial aminomethyl chain) predominating (69:31 ratio).

\footnotetext{
${ }^{42}$ Grierson, D. S. Org. React. 1990, 39, 85-295.
} 

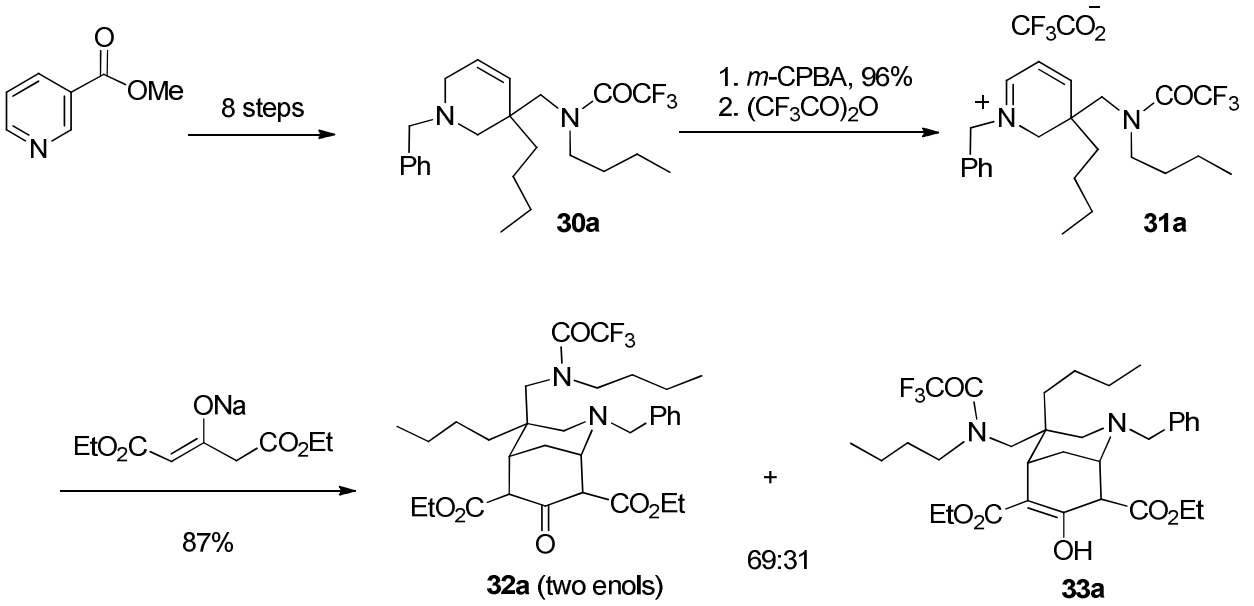

Scheme 1.9 Marazano's biomimetic approach.

This stereochemical problem was overcome using dihydropyridinium salt 35a, which lacks the $\mathrm{N}$-butyl substituent (Scheme 1.10). This salt was generated, as in the above series, from the corresponding tetrahydropyridine derivative 34a, which, in turn, was prepared in eight steps from methyl nicotinate. Condensation of the crude salt 35a with the sodium salt of diethyl acetonedicarboxylate resulted in the formation of a mixture of adducts 36 and $37 a$, which were difficult to separate. However, this crude mixture could directly be cyclized to the madangamine tricyclic core models 38a and 39a by treatment with $\mathrm{K}_{2} \mathrm{CO}_{3}$ in $\mathrm{EtOH}-\mathrm{H}_{2} \mathrm{O}$ at reflux. The formation of $\mathbf{3 8 a}$ from $\mathbf{3 6 a}$ involves the hydrolysis of the trifluoroacetamide group followed by lactamization, with concomitant hydrolysis-decarboxylation of the remaining ester group.

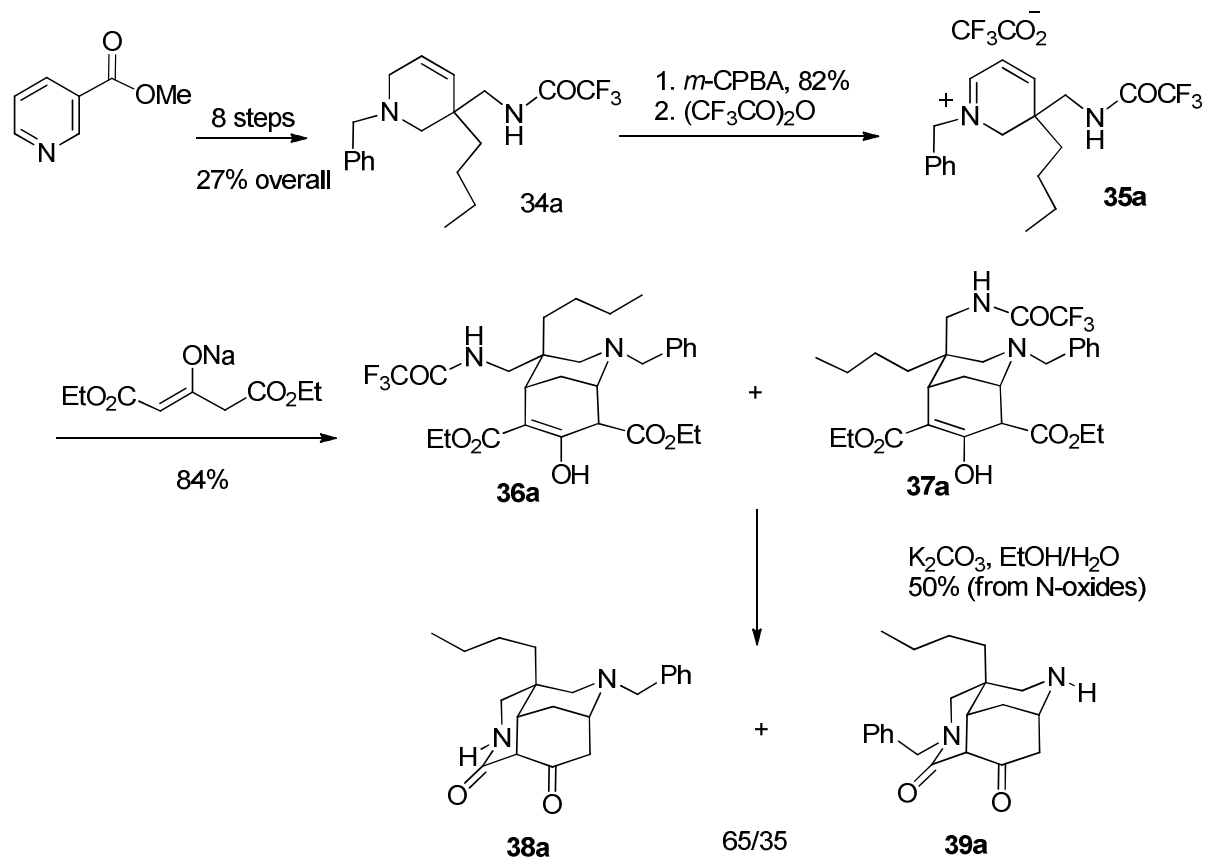

Scheme 1.10 Marazano's biomimetic approach to the ABC diazatricyclic core. 
The isolation of 39a indicates that the "wrong" stereosiomer 37a, bearing an axial amino chain, undergoes a rearrangement via a retro-Michael process, followed by a Michael addition and lactamization, as outlined in Scheme 1.11.

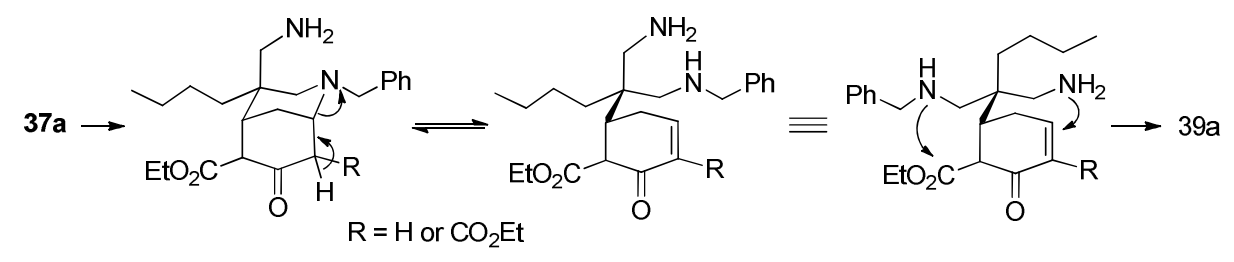

Scheme 1.11 Proposed mechanism for the formation of tricyclic derivative 39a.

\subsubsection{Bonjoch's Approach}

A new synthetic entry to the diazatricyclic core of madangamines was reported by Bonjoch in $2008 .^{41}$ The synthesis starts from 4-(aminomethyl)anisole derivatives 40a ( $R=$ Me or $\mathrm{Bn}$ ), which incorporate the carbocyclic $\mathrm{C}$ ring and the $\mathrm{C}_{6}-\mathrm{N}_{7}$ atoms of the target alkaloids, and involves the successive construction of the piperidine $B$ and $A$ rings (Scheme 1.12). A Birch reduction of 40a, followed by acylation of the amino group in the resulting dihydro derivatives 41a with cyanoacetic acid and subsequent hydrolysis of the enol ether moiety gave cyclohexenones 42a. Treatment of 42a with a substoichiometric amount of NaOEt caused the isomerization of the carbon-carbon double bond to give an $\alpha, \beta$-enone and the closure of the piperidine $B$ ring by an intramolecular Michael addition to give the cis-fused perhydroisoquinoline derivatives 43a as mixtures of C-9 epimers (madangamine numbering). A stereoselective allylation from the most accessible face of $\mathbf{4 3 a}$ installed the quaternary C-9 stereocenter in $\mathbf{4 4 a}$. The cyano group not only provides activation towards the Michael addition and allylation reactions, but is also the precursor of the aminomethyl chain required for the closure of the piperidine A ring. This was accomplished, after the activation of the primary amino group of 45a as a nosylamide, hydrolysis of the acetal, and reduction of the resulting ketone, by an intramolecular Mitsunobu-type $N$-alkylation from alcohols 46a. The resulting diazatricyclic derivatives 47 a incorporate a functionalized chain at the quaternary C-9 center but lack functionalization at the carbocyclic ring. 


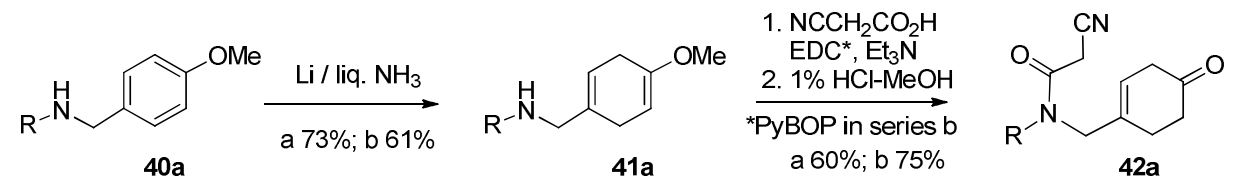

Series a $\mathrm{R}=\mathrm{Me}$; Series $\mathrm{b} \mathrm{R}=\mathrm{Bn}$
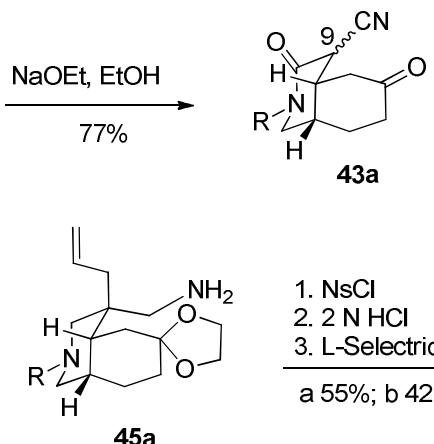

$45 a$
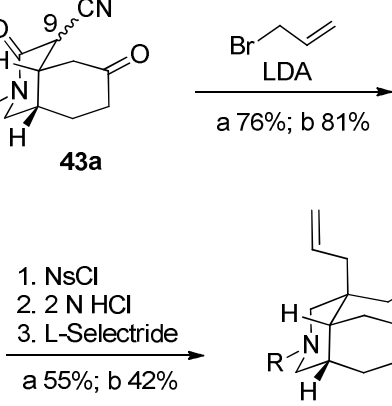
a $55 \%$; b $42 \%$

Scheme 1.12 Bonjoch's synthetic approach to the ABC diazatricyclic core.

\subsubsection{Construction of the ACE System}

In 2006 Kibayashi and coworkers reported the first synthesis of the ABE system of madangamine which includes the construction of the unsaturated 11-membered ring bearing a $Z, Z, Z$ skipped system of olefins. ${ }^{40}$<smiles>O=C1C=CCCC1</smiles>

1. $\mathrm{CH}_{2}(\mathrm{CN}) \mathrm{CO}_{2} \mathrm{Et}, \mathrm{NaOEt}$

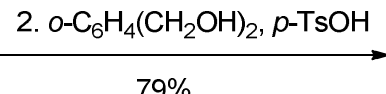

$48 a$

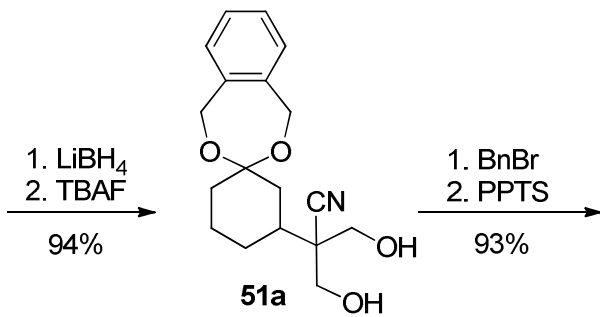

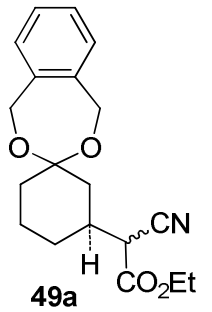
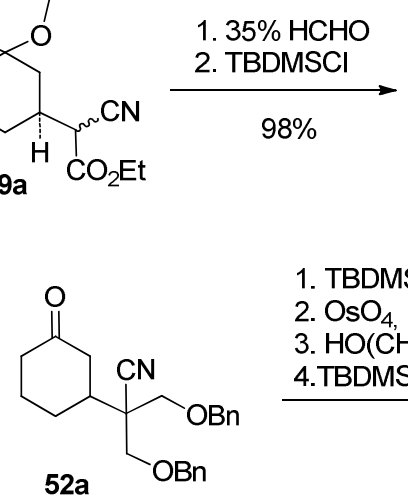

1. TBDMSCI

2. $\mathrm{OsO}_{4} \mathrm{NMO}$

3. $\mathrm{HO}\left(\mathrm{CH}_{2}\right)_{2} \mathrm{OH}, \mathrm{TMSCl}$

4.TBDMSOTf, 2,6-lutidine

$51 \%$

$47 a$

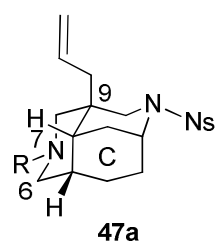

$46 a$

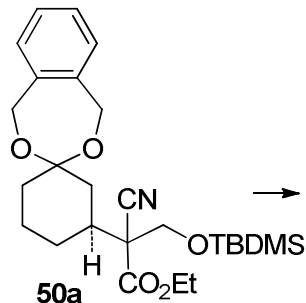



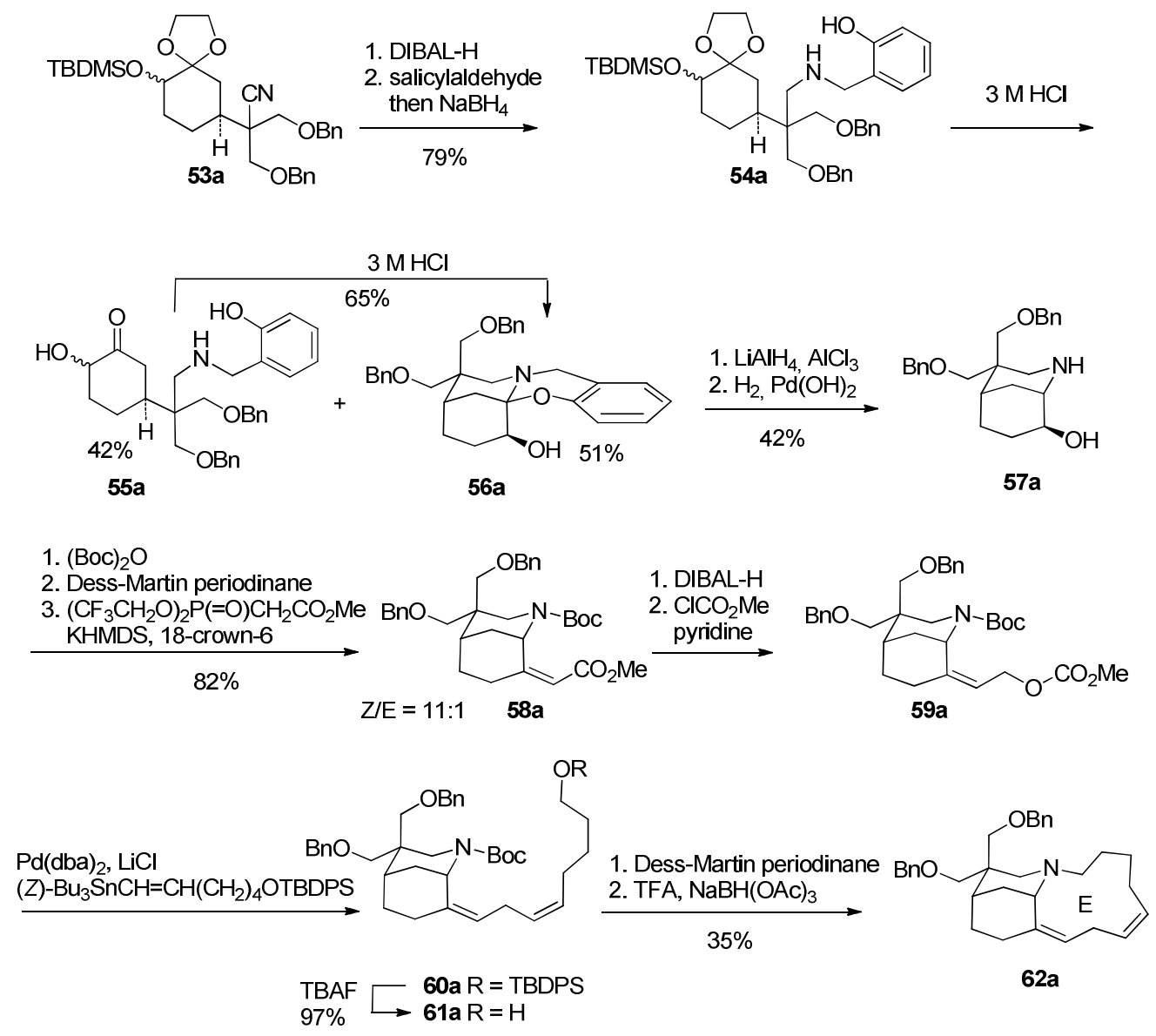

Scheme 1.13 Kibayashi's synthetic approach to the $E$ ring of madangamines A-E.

The methodology adopts an intramolecular $\mathrm{N}, \mathrm{O}$-acetalization for the AC-ring construction while the assembly of the $E$ ring was accomplished using a Still's Z-selective Wittig-Horner olefination followed by a cross-coupling reaction and an intramolecular reductive amination. Michael addition of ethyl cyanoacetate to ketone 48a, followed by protection of the carbonylic group gave cyclic acetal 49a in a 1:1 diastereomeric mixture. The hydroxymethylation reaction led to the formation of the quaternary carbon center while the protection of the resulting primary alcohol afforded compound 50a. After the reduction of the ester moiety the two hydroxy groups were protected as benzyl ethers while the hydrolysis of the acetal gave ketone 52a. After the oxidation of 52a the resulting $\alpha$-hydroxylated cyclohexanone was converted in TBDMS- ether while the carbonyl moiety was protected as a cyclic acetal 53a. Reduction of the nitrile group followed by reaction of the resulting primary amine with salicylaldehyde and $\mathrm{NaBH}_{4}$ gave tetracyclic $\mathrm{N}, \mathrm{O}$-acetal 54a. Treatment of compound 54a with $\mathrm{HCl}$ followed by removal of the $\mathrm{N}, \mathrm{O}$-acetal provided the morphan derivative 57a which was $\mathrm{N}$-protected and oxidize to ketone with Dess-Martin periodinane. A Still-Gennari Wittig-Horner olefination afforded the desired Z-isomer of the related ester. A subsequent cross coupling reaction between compound 61a and an appropriate (Z)-vinylstannane derivative gave the skipped diene $\mathbf{6 2} \mathrm{a}$ as a single isomer. Finally, the 11-membered E ring was constructed through an intramolecular reductive amination that provided the expected ACE system. 


\subsection{SYNTHETIC BACKGROUND}

The development of new methodologies for the enantioselective synthesis of chiral compounds has undergone huge improvements in the last decades. This is of great significance in the field of the synthesis of natural products and biologically active compounds since chirality is crucial for the expression of their bioactivity. In this context, in the last years, we have focused our attention on the search of novel, general methods for the preparation of chiral non-racemic compounds in an enantiopure form with the final aim of applying them to the total synthesis of natural products and biologically active compounds.

Functionalized piperidine structures embody some of the key features found in numerous alkaloids (more than half of the known alkaloids bear a piperidine ring), biologically active compounds, and drugs. This observation prompted us to focus our interest in the search of general procedures for the enantioselective synthesis of piperidine derivatives with a high variety of substitution patterns. ${ }^{43}$ With this purpose in mind, we decided to explore the potential of chiral aminoalcohol-derived bicyclic lactams as enantiomeric scaffolds for the stereocontrolled construction of complex piperidine derivatives. Bicyclic lactams were originally developed by A. I. Meyers as chiral templates for the enantioselective synthesis of cycloalkenones and carboxylic acids containing quaternary stereocenters. In subsequent work Meyers also reported some applications to the synthesis of simple $\alpha$-susbtituted piperidine and tetrahydroquinoline alkaloids, as well as imino-sugars. This seminal work was summarized in three excellent reviews. ${ }^{44}$

Chiral oxazolopiperidone lactams, easily available in a single step by cyclocondensation of oxoacid derivatives and enantiopure amino-alcohols, bear a tactically versatile functionalized piperidine ring embedded in a conformationally rigid bicyclic system. These structural properties enable the introduction of substituents at virtually all the carbon positions of the heterocycle in a high degree of stereoselectivity giving access to a broad variety of either simple or complex piperidine derivatives.

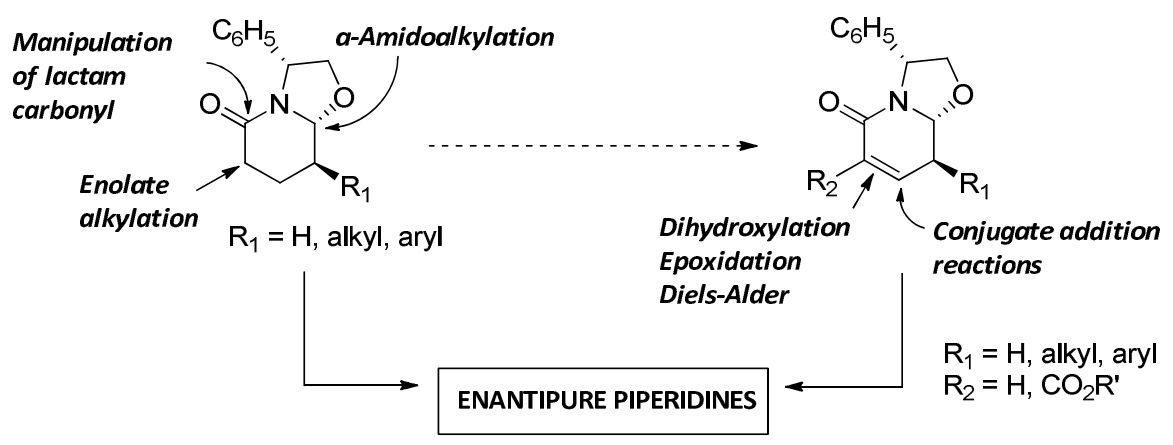

Figure 1.5 Chiral lactams as enantiomeric scaffolds.

\footnotetext{
${ }^{43}$ (a) Escolano, C.; Amat, M.; Bosch, J. Chem. Eur. J. 2006, 12, 8198-8207. (b) Amat, M.; Pérez, M.; Bosch, J. Synlett 2011, 143-160. (c) Amat, M.; Pérez, M.; Bosch, J. Chem. Eur. J. 2011, 17, 7724-7732.

${ }^{44}$ (a) Romo, D.; Meyers, A. I. Tetrahedron 1991, 47, 9503-9569. (b) Meyers, A. I.; Brengel, G. P. Chem. Commun. 1997, 1-8. (c) Groaning, M. D.; Meyers, A. I. Tetrahedron 2000, 56, 9843-9873.
} 
More recently, we have expanded the potential of the methodology to the synthesis of enantiopure cis-decahydroquinolines from tricyclic lactams prepared by cyclocondensation of 2-cyclohexanonepropionic acid derivatives and chiral enantiopure amino-alcohols. In most cases phenylglycinol has been used as chiral amino-alcohol due to its availability in both enantiomeric forms and to the benzylic nature of the carbon-nitrogen bond which facilitates the removal of the phenylethanol moiety at an appropriate stage of the sequence. For the synthesis of indole and benzo[a]quinolizidine alkaloids we have also used (S)-tryptophanol and (S)-(3,4-dimethoxyphenyl)alaninol which not only act as source of chirality but also incorporate the aryl ethylamine moiety of the target compound. More recently, we have explored an efficient reductive ring opening of bicyclic lactams leading to chiral aminoalcohols.

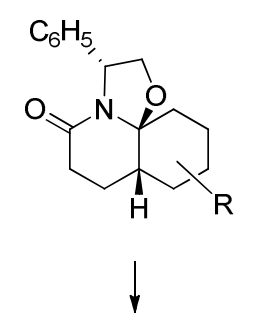

DECAHYDROQUINOLINES

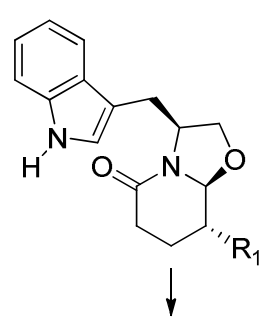

INDOLE ALKALOIDS

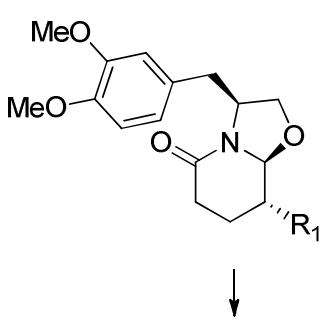

BENZO[a]QUINOLIZIDINE ALKALOIDS

Figure 1.6 Amino-alcohol-derived oxazolopiperidone lactams.

Therefore, we have now in hand a flexible and versatile tool for the preparation in an enantiopure form a broad range of piperidine, cis-decahydroquinoline and aminoalcohol derivatives which allows the generation of new stereocenters on the heterocyclic ring with a high degree of stereoselectivity and predictable absolute and relative configuration..$^{45}$ The potential of the approach based on the use of chiral bicyclic and tricyclic lactams as enantiomeric scaffolds for the preparation of natural products has been demonstrated in our research group with the synthesis of a variety of alkaloids, some of them depicted in Fig. 1.7.

\footnotetext{
${ }^{45}$ For recent works, see: (a) Amat, M.; Griera, R.; Fabregat, R.; Bosch, J. Angew. Chem. 2008, 120, 33963399; Angew. Chem. Int. Ed. 2008, 47, 3348-3351. (b) Amat, M.; Gómez-Esqué, A.; Escolano, C.; Santos, M. M. M.; Molins, E.; Bosch, J. J. Org. Chem. 2009, 74, 1205-1211. (c) Amat, M.; Llor, N.; Checa, B.; Molins, E.; Bosch, J. J. Org. Chem. 2010, 75, 178-189. (d) Amat, M.; Pérez, M.; Bosch, J. Synlett 2011, 2, 143-160. (e) Amat, M.; Elias, V.; Llor, N.; Subrizi, F.; Molins, E.; Bosch, J. Eur. J. Org. Chem. 2010, 41, 4017-4026. (f) Pérez, M.; Arioli, F.; Rigacci, G.; Santos, M. M. M.; Gómez-Esqué, A.; Escolano, C.; Florindo, P.; Ramos, C.; Bosch, J.; Amat, M. Eur. J. Org. Chem. 2011, 3858-3863 (g) Amat, M.; Navío, L.; Llor, N.; Molins, E.; Bosch J. Org. Lett. 2012, 14, 210-213 (h) Amat, M.; Subrizi, F.; Elias, V.; Llor, N.; Molins E.; Bosch, J. Eur. J. Org. Chem. 2012, 1835-1842 (i) Amat, M.; Subrizi, F.; Elias, V.; Llor, N.; Molins E.; Bosch, J. Eur. J. Org. Chem. 2012, 5497-5497 (I) Amat, M.; Ramos, C.; Pérez, M.; Molins, E.; Florindo, P.; Bosch J. Chem. Commun. 2013, 49, 1954-1956 (m) Amat, M.; Llor, N.; Subrizi, F.; Pérez, M.; Molins, E.; Bosch, J. Eur. J. Org. Chem. 2013, 1246-1252 (n) Amat, M.; Ghirardi, E.; Navío, L.; Griera, R.; Llor, N.; Molins, E.; Bosch. J. Chem. Eur. J. 2013, 19, 16044-16049.
} 
Piperidine derived alkaloids<smiles>CCC[C@@H]1CCCCN1</smiles>

(S)-(+)-Coniine (both enantiomers)<smiles>c1cncc([C@H]2CCCCN2)c1</smiles>

(S)-anabasine

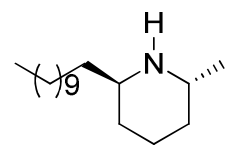

(-)-Solenopsin A<smiles>C[C@H]1CCC[C@@H](C)N1</smiles>

(-)-Lupetidine<smiles>CCC[C@@H]1CCC[C@H](C)N1</smiles>

(2R,6S)-Dihydropinidine

\section{Quinolizidine}<smiles>C[C@H]1C[C@@H](C)N2CCCC[C@@H]2C1</smiles>

(-)-Cermicine C<smiles>CC1=[N+]2CCCC[C@H]2C[C@H](C)C1</smiles>

(-)-Senepodine G<smiles>C=CC[C@@H]1CCC(CC)[C@H]2CCCCN12</smiles>

Quinolizidine-4-epi-207I

Indolizidine<smiles>CCC[C@@H]1CCC[C@H]2CCCN21</smiles>

(-)-Indolizidine 167B<smiles>CCCC[C@@H]1CC[C@H]2CCC[C@@H](C)N12</smiles>

(+)-Monomorine I

Iminosugars<smiles>OC[C@H]1NC[C@@H](O)[C@H](O)[C@@H]1O</smiles>

Deoxy-D-gulonojirimycin (1-Deoxy-3,4-diepinojirimycin)<smiles>OC[C@H]1CNC[C@H](O)[C@H]1O</smiles>

Epiisofagomine (Isogalactofagomine) (selective and potent inhibitor of $\beta$-galactosidases)<smiles>O[C@H]1CNC[C@@H](O)[C@H]1O</smiles>

Nor-fucodeoxynojirimycin

Biologically active compounds<smiles>CCCN1CCC[C@H](c2cccc(O)c2)C1</smiles><smiles>Fc1ccc([C@H]2CCNC[C@H]2COc2ccc3c(c2)OCO3)cc1</smiles>

(-)-Paroxetine (antidepressant)<smiles>COc1ccc(OC[C@H]2CCNCC2c2ccccc2)cc1</smiles>

(+)-Femoxetine (antidepressant) 
Tetrahydroisoquinoline alkaloids<smiles>COc1cc2c(cc1OC)C(C)NCC2</smiles>

(-)-Salsolidine

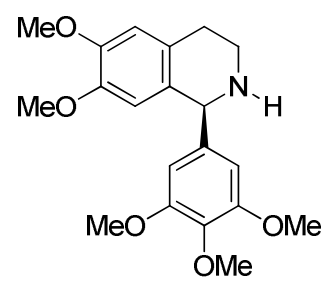

(-)-Norcryptostiline III<smiles>COc1ccc(CC2NCCc3cc(OC)c(OC)cc32)cc1</smiles>

(-)-O,O-Dimethylcoclaurine<smiles>COc1cc(OC)c2c(c1-c1c(C)cc(OC)c3c(OC)cccc13)CC(C)N(C)[C@H]2C</smiles>

O-Methylancistrocline (Formal synthesis)

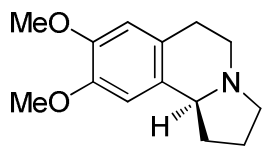

(-)-Crispine A

Indole alkaloids

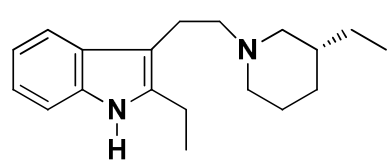

(+)-Decarbomethoxytetrahydrosecodine

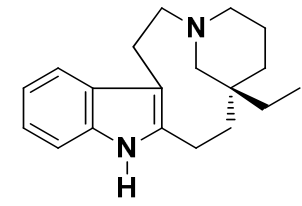

(-)-Quebrachamine<smiles>CC[C@H]1C[C@@H](CCc2[nH]c3ccccc3c2CCN2CC2)CN1</smiles>

(-)-20S-Dihydrocleavamine

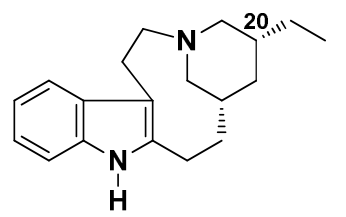

(+)-20R-Dihydrocleavamine

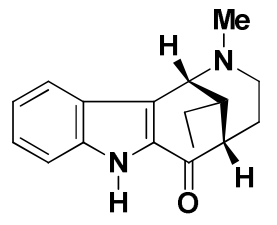

(+)-Dasycarpidone

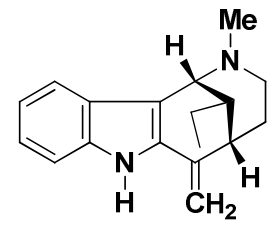

(+)-Uleine<smiles>CC[C@H]1CN2CC[C@@]34C[C@H]2[C@H]1CC3=Nc1ccccc14</smiles>

(-)-Tubifoline<smiles>CC[C@H]1CN(C)C[C@H]2Cc3c([nH]c4ccccc34)C(=O)C[C@@H]21</smiles>

(-)-16-Episilicine

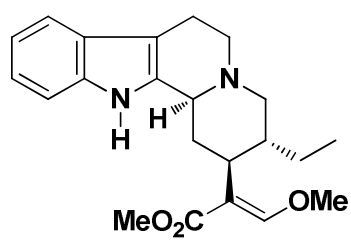

(+)-Dihydrocorynantheine 


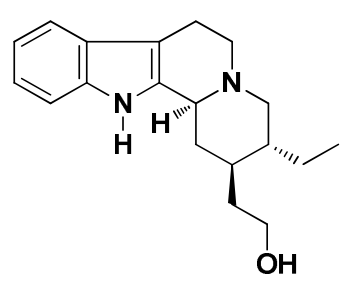

(-)-Dihydrocorynantheol

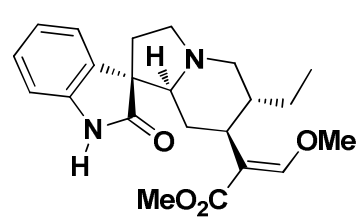

(-)-Rhynchophylline

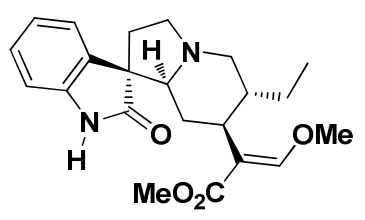

(+)-Isorhynchophylline

Haliclorensin marine alkaloids

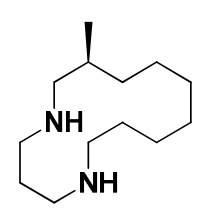

Haliclorensin

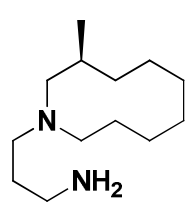

Isohaliclorensin

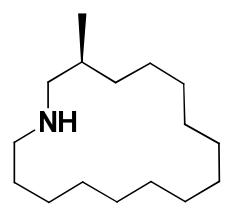

Haliclorensin C

Myrioneuron alkaloids

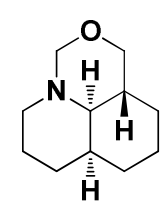

Myrioxazine A

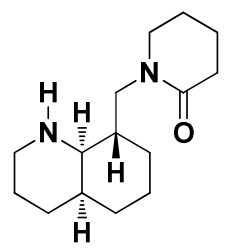

Myrionine

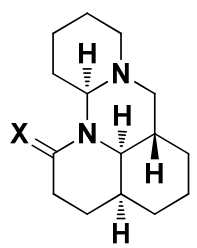

Schoberine $X=H . H$ Myrionamide $X=O$

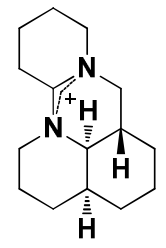

Myrionidine

Amphibian and marine cis-decahydroquinoline alkaloids

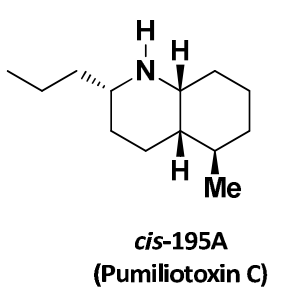

(Pumiliotoxin C)

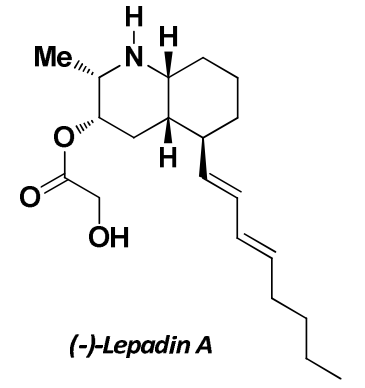

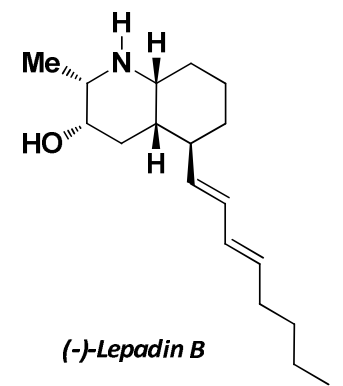

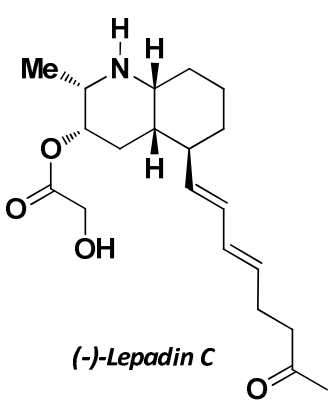

Figure 1.7 Alkaloids prepared from chiral amino-alcohol derived lactams.

As an evolution of this work, we decided to undertake the synthesis of more complex targets in order to demonstrate the scope and synthetic potential of the strategy based on the use of chiral aminoalcohol-derived lactams as enantiomeric scaffolds. ${ }^{46}$ With this purpose in mind, we selected the alkaloids of the madangamine group due to their attractive structural architecture

${ }^{46}$ Enantiomeric scaffolding strategy: (a) Coombs, T. C.; Lee, M. D., IV, Wong, H.; Armstrong, M.; Cheng, B.; Chen, W.; Moretto, A. F.; Liebeskind, L. S. J. Org. Chem. 2008, 73, 882-888. (b) Wong, H.; GarnierAmblard, E. C.; Liebeskind, L. S. J. Am. Chem. Soc. 2011, 133, 7517-7527. 
and because there was no total synthesis for this group of natural products described in the literature.

This work was initiated by Dr. Stefano Proto in his Ph. D. Thesis which was defended in March 2012. Due to the complexity inherent to the total synthesis of challenging natural products, such as the alkaloids of the madangamine group, the final goal could not be reached in this Thesis.

The aim of this work was to conclude the total synthesis of madangamine $D$, which involve:

- to scale up and optimize the procedures for the preparation of starting materials in order to provide enough amounts to reach the target compound.

- to improve the synthetic sequence developed in previous work by reducing the number of steps in the preparation of the diazatricyclic core of madangamines.

- to explore different synthetic pathways that could potentially permit the assembly the macrocyclic $D$ ring of various members of madangamine's family, which are different in size as well in degree and position of unsaturations.

- to set up efficient procedures to solve the final steps of the synthesis.

\subsection{SYNTHETIC STRATEGY}

The synthetic strategy we devised for the synthesis of madangamines involves the initial construction of the fully functionalized diazatricyclic core common to all madangamines $A$ to $E$, and the subsequent annulations of the macrocyclic $D$ and $E$ rings.

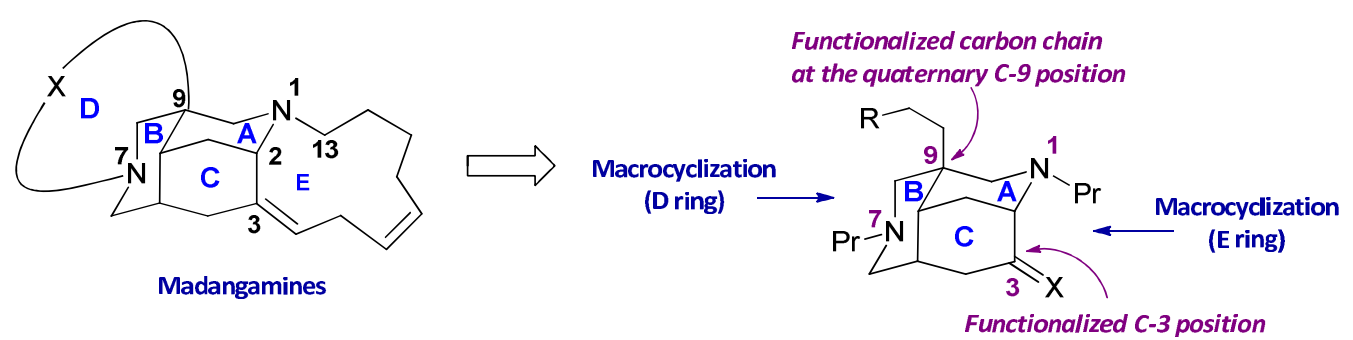

Figure 1.8 General synthetic strategy.

The stereoselective construction of the diazatricyclic core in enantiopure form relies on the use of an allyl-substituted chiral phenylglycinol-derived lactam as enantiomeric scaffold and in our experience in the generation of stereocenters of well-defined configuration at the carbon positions of the piperidine ring of these lactams. Accordingly, the allyl-substituted lactam 1, with the appropriate absolute configuration at the stereocenter next to the allyl group 
(corresponding to the 5-position of madangamines) was selected as starting material. A stereoselective conjugate addition on the corresponding unsaturated derivative, in which the double bond is activated by an alcoxycarbonyl group, is used for the introduction of an allyl substituent at C-12, cis with respect to the allyl group of the next position (C-5). A RCM of the resulting diallyl derivative is performed for the closure of the carbocyclic ring $C$ of madangamines. The generation of the quaternary stereocenter at the C-9 position by alkylation of an enolate takes advantage of the acidity of the hydrogen next to the carbonyl of the ester and the amide-lactam and of the bended shape of the cis-octahydroisoquinoline which allows the selective approach of the alkylating reagent from the less hindered face. In this step a carbon chain with the appropriate functionalization at the terminal position for the closure of the $D$ ring is introduced. The removal of the phenylethanol moiety of the chiral inductor (phenylglycinol) and the closure of the piperidine A ring by an intramolecular aminohydroxylation constitute the final strategic transformations needed to complete the synthesis of the diazatriciclic core of madangamines. ${ }^{47 .}$

For the closure of the macrocyclic $D$ ring of madangamines classical macrolactamizations or the more recent but well-established procedure based on ring-closing metathesis (RCM) constitute two of the most popular methods used in the construction of medium and large rings. ${ }^{45}$ Ring-closing alkyne metathesis (RCAM) and other palladium-(0) cross-coupling reactions have also proven to be invaluable tools for the intramolecular carbon-carbon formation in the construction of macrocycles with control of the E/Z selectivity. Finally, the elaboration of the unsaturated $E$ ring requires the incorporation at the 3-position of an 8carbon chain, containing a Z-configurated double bond, followed by a macrolactamization. The first step involves a Wittig olefination in which a carbon-carbon double bond with the $Z$ configuration has to be stereoselectively generated. ${ }^{48}$

\footnotetext{
${ }^{47}$ Amat, M.; Ballette, R.; Proto, S.; Pérez, M.; Bosch, J. Chem. Commun. 2013, 49, 3149-3151.

${ }^{48}$ Ballette, R.; Pérez, M.; Proto, S.; Amat, M.; Bosch, J. Angew. Chem. Int. Ed. 2014, accepted.
} 
A. The starting enantiomeric scaffold
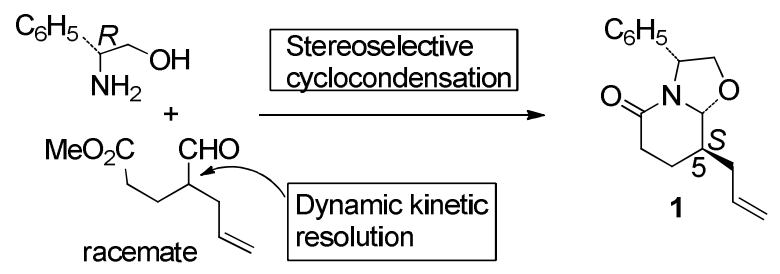

B. Construction of the diazatricyclic core

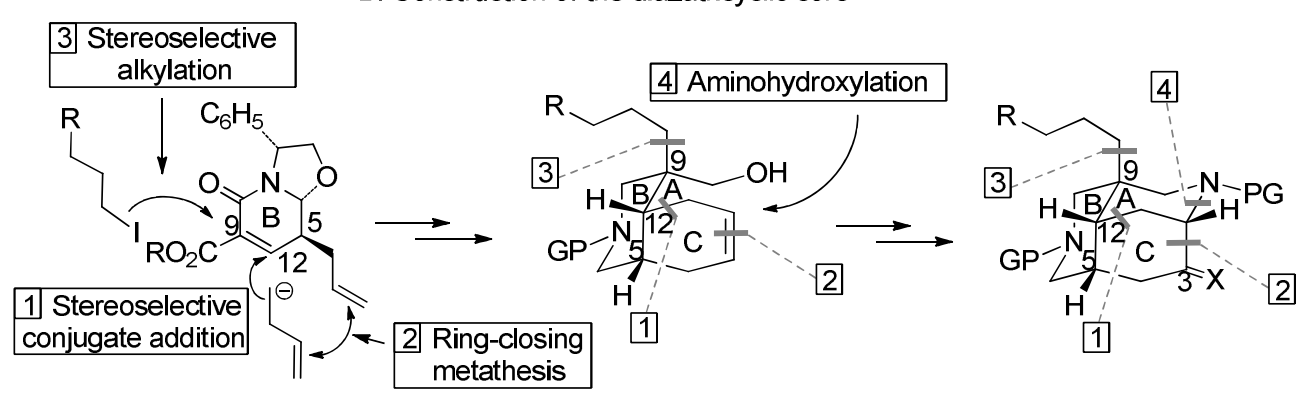

C. Assembly of the macrocyclic rings

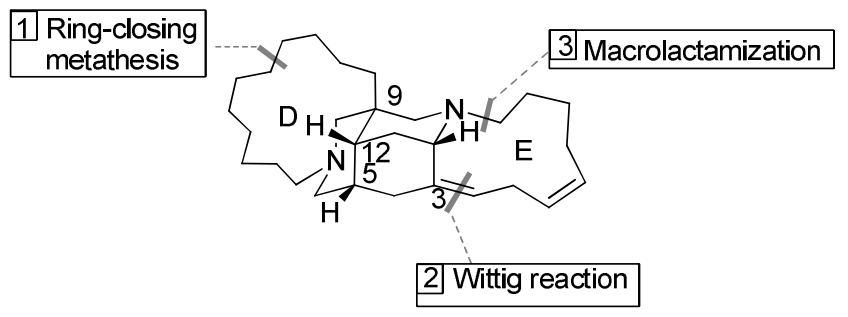

Figure 1.9 Synthetic strategy. 
Chapter 2 



\subsection{INTRODUCTION}

In this chapter, our efforts on the synthesis of the diazatricyclic core common to all madangamines (rings A-B-C) from the allyl-substituted phenylglycinol-derived bicyclic lactam 1 are described. This scaffold (1) bears the piperidine B ring of madangamines and the stereocenter corresponding to the C-5 position of these natural products. For the sake of clarity, the numbering of madangamines is used for the compounds appearing throughout the text.
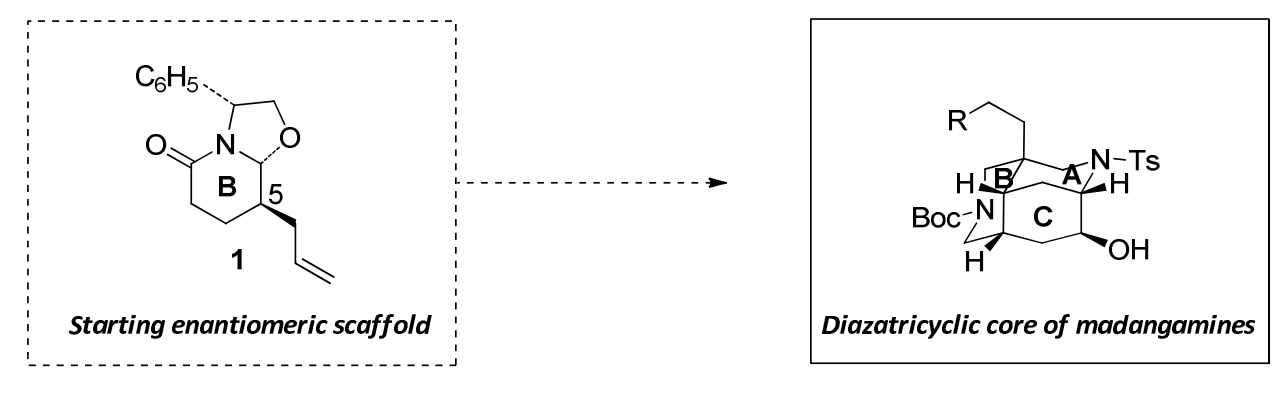

Figure 2.1 Enantiomeric scaffolding strategy.

The synthetic strategy originally devised for the diazatricyclic core of madangamines is depicted in Scheme 2.1. The first stages of the synthesis are oriented to the generation of a cisoctahydroisoquinoline $(A)$ with a quaternary stereocenter at the C-9 position holding a hydroxymethyl substituent and a carbon chain appropriately functionalized for the closure of the macrocyclic $D$ ring in subsequent stages of the synthesis.

\subsection{SYNTHETIC STRATEGY TOWARD THE ABC CORE OF MADANGAMINE}

The key steps in the preparation of this cis-octahydroisoquinoline (A) include i) a stereoselective conjugate addition of an allyl group to the unsaturated lactam derived from 1, ii) a RCM for the closure of the carbocyclic $C$ ring, iii) the alkylation of the enolate of the $\beta$ keto-lactam, iv) the removal of the phenylethanol moiety of the chiral inductor, and v) the reduction of the carbonylic groups. In this intermediate $A$ the key stereocenters at the C-5, C-9, and $\mathrm{C}-12$ positions are installed, two of them generated in the conjugate addition ( $\mathrm{C}-12$ ) and in the enolate alkylation (C-9) reactions. Starting from compound A, only the closure of the piperidine $A$ ring is needed to complete the target structure. This transformation can be carried out by conversion of the hydroxymethyl on C-9 into an aminomethyl group followed by an intramolecular amino-hydroxylation of the carbon-carbon double bond present into the cisoctahydroisoquinoline system. ${ }^{47}$ 


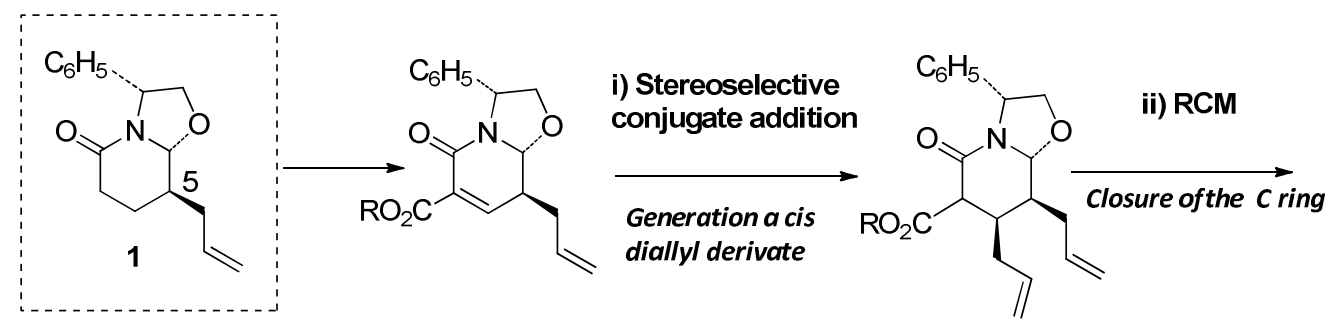

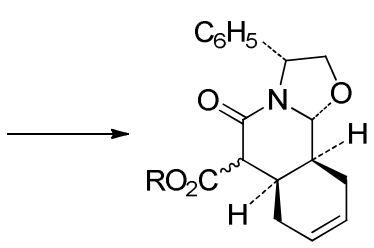

iii) Stereoselective enolate alkylation

Generation of the quaternary stereocenter

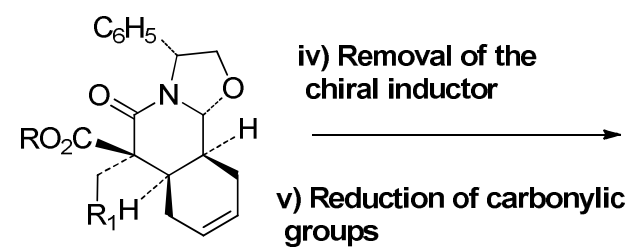

v) Reduction of carbonylic
groups
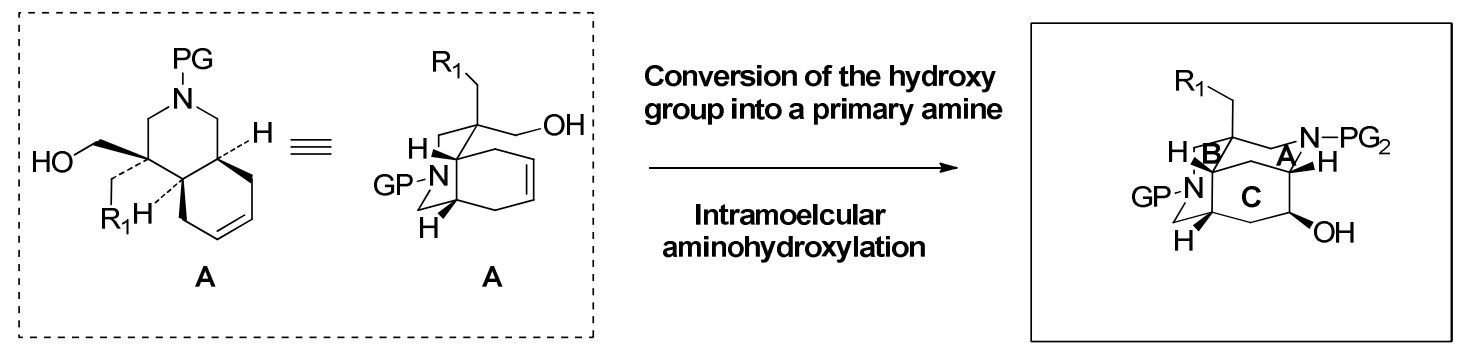

Scheme 2.1 General synthetic plan.

In previous work we prepared a variety of tricyclic lactams II, bearing the cisoctahydroisoquinoline system and the quaternary stereocenter at C-9, starting from the allylsubstituted bicyclic lactam 1 as illustrated in Scheme 2.2. The reactions involved in this sequence took place in good yields and excellent stereoselectivity. Compounds I and II were isolated as the only stereoisomers detectable by spectroscopic methods. However, all attempts to remove the phenylethanol moiety of the $(R)$-phenylglycinol, which had played the role of chiral inductor, resulted in failure or provided the expected compound in moderate yields. For the $\mathrm{N}$-debenzylation of compounds III, the common catalytic hydrogenation could not be used due to the presence of a carbon-carbon double bond. On the other hand, the presence of the hydroxymethyl substituent in III made difficult the selective manipulation of both alcohols. Alternatively, all attempts to provoke the reductive ring opening of the oxazolidine ring present in II by using $\mathrm{Et}_{3} \mathrm{SiH}$ and Lewis acids did not afford the expected 
compounds IV.$^{49}$ However, in previous work we found that this reaction works well on bicyclic lactams lacking functional groups such as a methyl carboxylate.
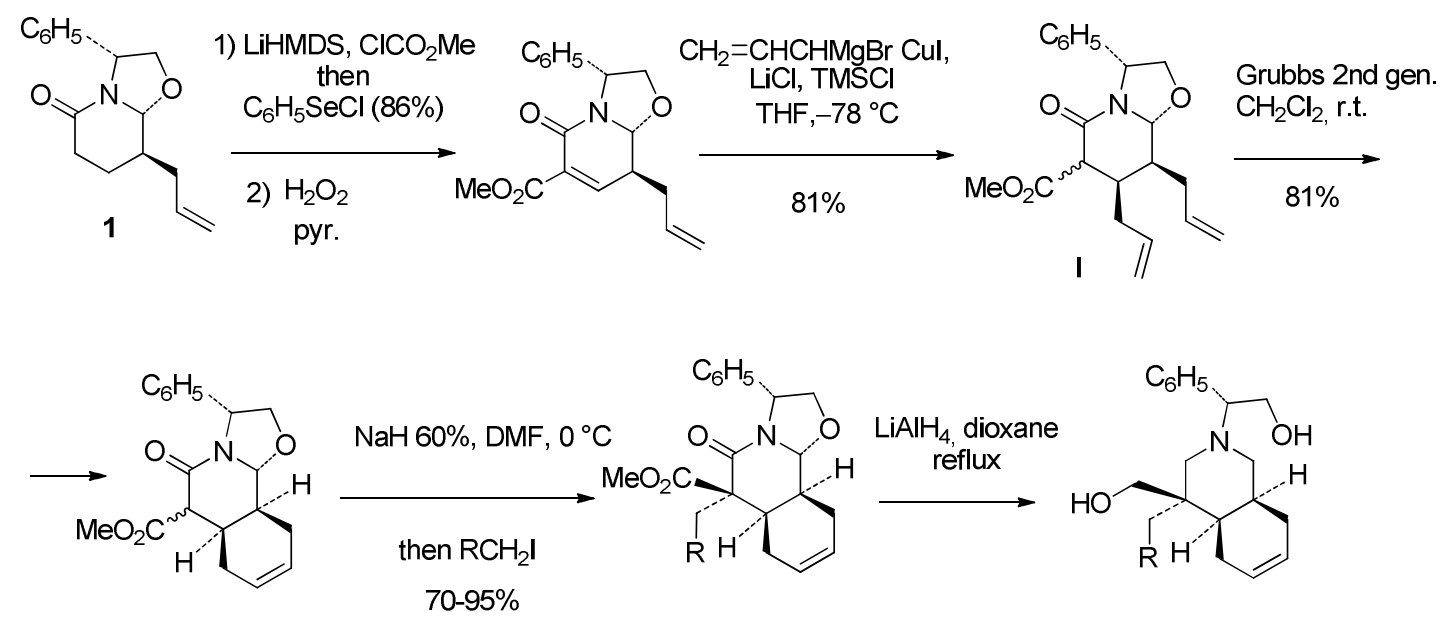

II

III
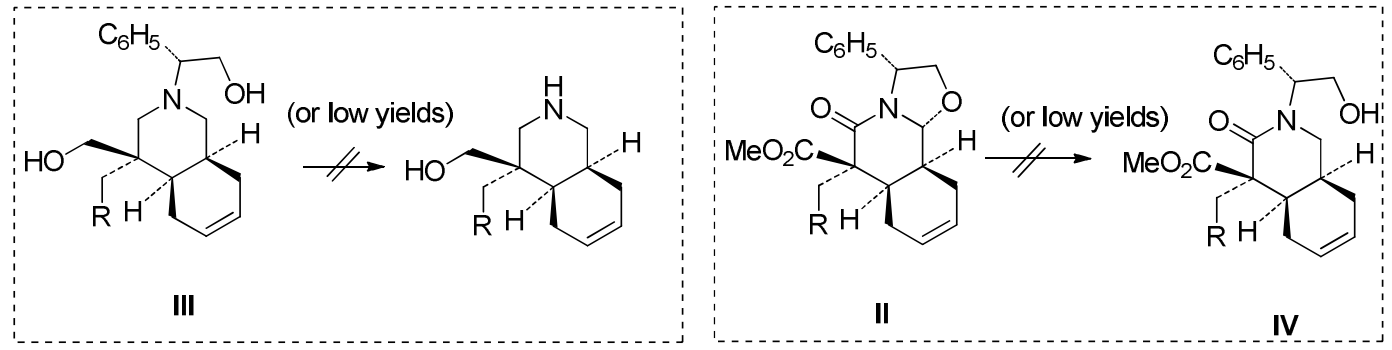

Scheme 2.2 First synthetic plan.

The above results prompted us to modify the synthetic sequence to the diazatricyclic core of madangamines $(\mathbf{X I})$ by removing in earlier stages of the synthesis the phenylethanol moiety of the chiral inductor. When tricyclic lactam $\mathbf{V}$, lacking the methoxycarbonyl substituent at the C9 position, was subjected to treatment with $\mathrm{Et}_{3} \mathrm{SiH}$ and $\mathrm{TiCl}_{4}$ followed by $\mathrm{N}$-debenzylation with sodium in liquid ammonia, the expected compound VI was obtained in an excellent $82 \%$ yield for the two steps. It is worth mentioning that the presence of an activating group, such an alkyl carboxylate, on the unsaturated lactam is required to obtain good yields in the conjugate addition reaction. The generation of the quaternary stereocenter at the C-9 position by alkylation of the enolate of VII took place in excellent stereoselectivity to give compound VIII although, in this case, small amounts of the C-9 diastereomer could be detected by spectroscopic methods. On the other hand, the presence of a robust electron withdrawing

\footnotetext{
${ }^{49}$ A detailed explanation of all the efforts in the removal of the phenylethanol moiety of the $(R)$ phenylglycinol is included in paragraph 2.4 .
} 
group, such as tosyl, afforded better results in the enolate alkylation reaction. ${ }^{50}$ The final key transformation, involving the closure of the piperidine A ring, was carried out from intermediate IX by conversion of the hydroxy group into an azide, stereoselective epoxydation of the alkene to give $\mathbf{X}$ followed by treatment with trimethylphosphine, which provoked the reduction of the azide and the subsequent intramolecular opening of the epoxide in a one pot reaction. Protection of the resulting secondary amine conducted to the target compound $\mathbf{X I .}{ }^{51}$
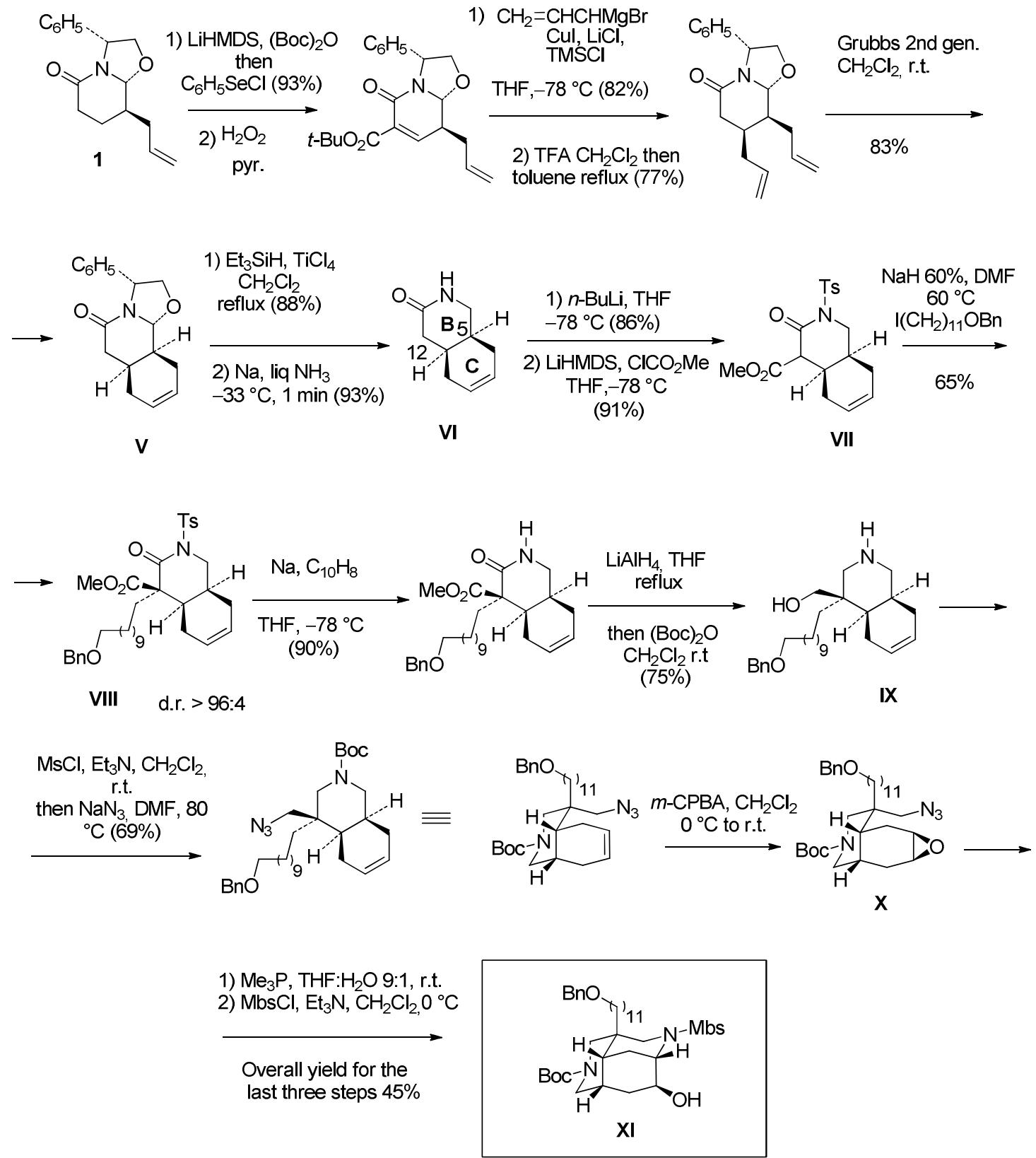

Scheme 2.3 Revised synthetic plan.

\footnotetext{
${ }^{50}$ The use of $\mathrm{N}$-Boc derivatives of VII afforded mixtures of the desired compound along with variable amount of the corresponding unprotected compound.

${ }^{51}$ Amat, M.; Pérez, M.; Proto, S.; Gatti, T.; Bosch, J. Chem. Eur. J. 2010, 16, 9438-9441.
} 
Although the above synthesis allowed us to reach the target compound $\mathbf{X I}$, this sequence includes more steps than the originally one (see Scheme 2.3), which is a limitation under a synthetic standpoint considering that the two macrocycles $D$ and $E$ had to be installed yet.

Two main drawbacks can be identified in this sequence:

- The alkyl carboxylate introduced at the beginning of the synthesis to activate the unsaturated lactam has to be taken out for the removal of the chiral inductor and reintroduced later to activate the C-9 position in the enolate alkylation step and as precursor of the aminomethyl substituent needed for the closure of the A ring.

- On the other hand, the protecting $N$-tosyl group present in VIII has to be removed before the $\mathrm{LiAlH}_{4}$ reduction of the lactam carbonyl and ester functions and the resulting secondary amine has to be reprotected with an $\mathrm{N}$-Boc group. In previous work we observed that treatment of the $N$ - $p$-methoxybenzenesulfonyl lactam XII with $\mathrm{LiAlH}_{4}$ followed by addition of an excess of mesyl chloride and sodium azide afforded a mixture of the expected compound XIIIa and its diastereomer at the C-9 quaternary center XIIIb.

This stereochemical result can be accounted for by considering that the reaction of XII with $\mathrm{LiAlH}_{4}$ provokes the reduction of the ester and the reductive ring opening of the lactam ring affording a compound with two diastereotopic hydroxymethyl groups. Treatment of this diol with mesyl chloride gives a dimesyltate which undergoes cyclization leading a mixture of epimeric C-9 cis-octahydroisoquinolines which are precursors of the isolated azides XIIIa and XIIIb. 


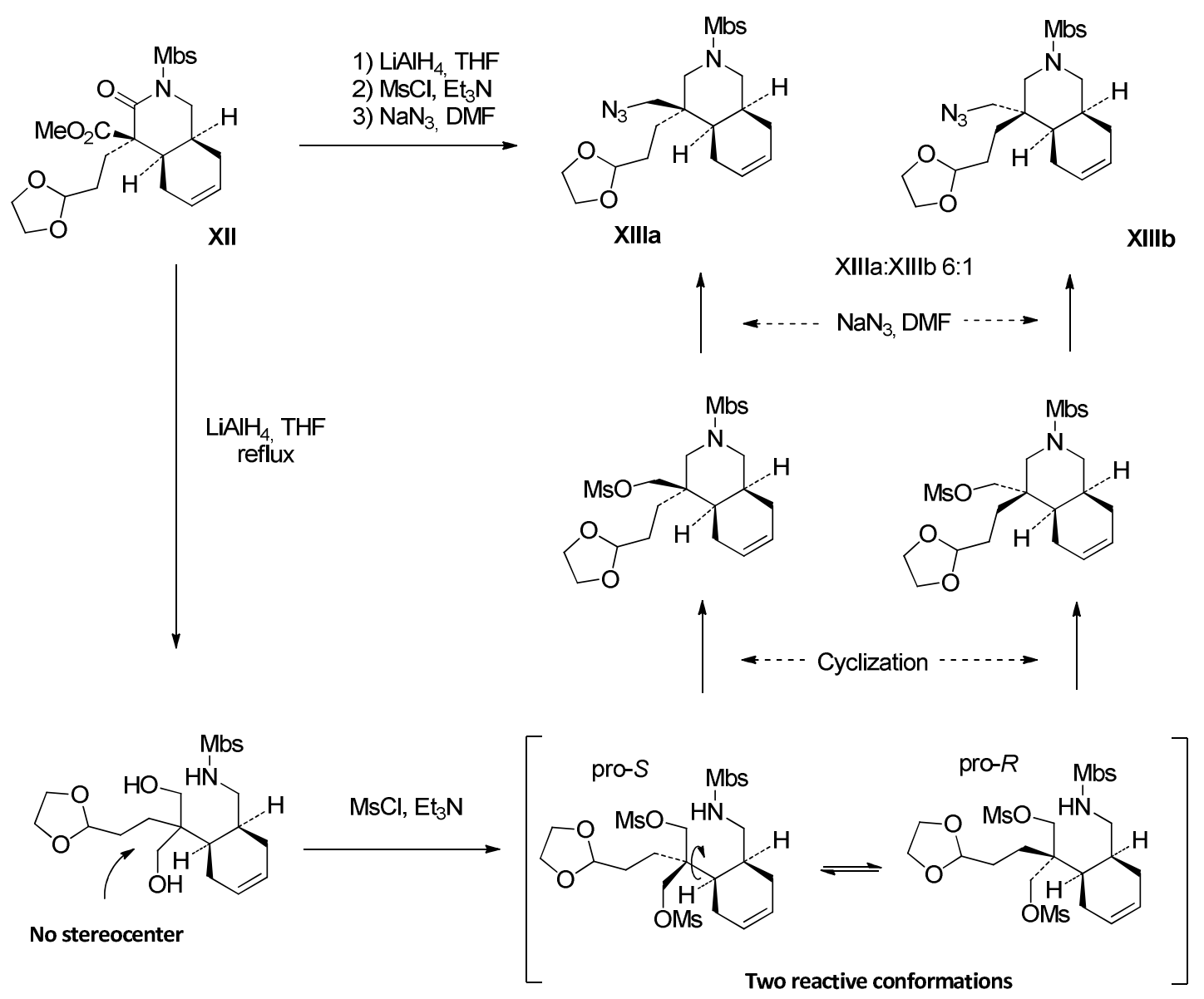

Scheme 2.4 Isomerization of C-9 stereocenter.

On the grounds of the experience acquired in previous work on the synthesis of marine alkaloids of the madangamine group, mentioned above, in the present chapter we decided to focus our efforts on two main points:

- To scale up and optimize the reactions included in the sequence leading to the diazatricyclic core of madangamines. This was crucial in order to dispose enough amounts to undertake the studies about the construction of rings $D$ and $E$ of madangamines.

- To review the original synthetic sequence trying to find an efficient solution for the removal of the phenylethanol moiety of the chiral inductor avoiding the drawbacks of the synthesis depicted in Scheme 2.2.

\subsection{SYNTHESIS OF BC HEART OF MADANGAMINE}

As described above the first challenges of our synthesis were represented by the construction of the chiral nonracemic allyl oxazolopiperidone scaffold $\mathbf{1}$ and its transformation into the 
fully-functionalized BC azabicyclic heart of madangamines (A). The main features of this synthon include a cis-convex isoquinolic structure embodying three contiguous stereocenters and bearing the endocyclic double bond among the $\mathrm{C}$ ring. This functionalization, together with the presence at the C-9 position of a hydroxymethyl chain, synthetic equivalent of the aminomethyl fragment, forms the ideal platform for the construction of the piperidinic $A$ ring. On the other hand a suitable carbon chain, installed at the quaternary stereocenter, guarantees the access to the western macrocyclic ring $D$ of madangamines.

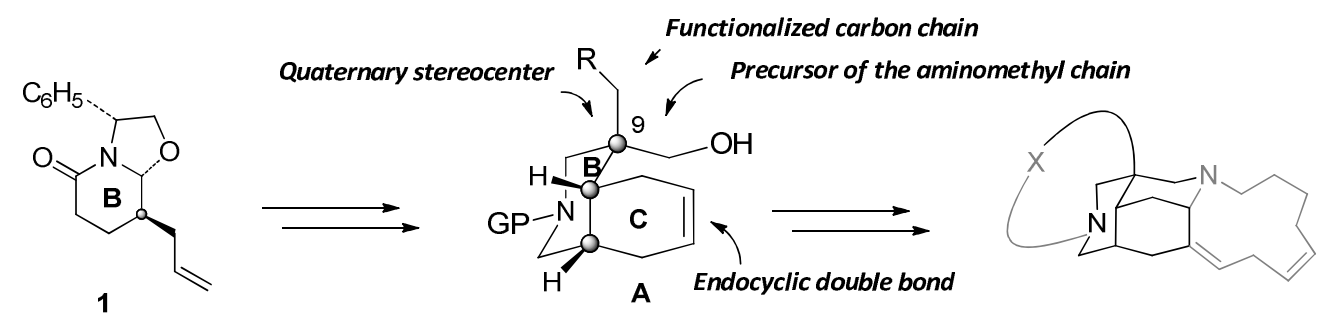

Figure 2.2 BC system of madangamines.

The construction of the BC azabicyclic heart involves as key steps (i) the generation of the first enantiopure intermediate, the bicyclic lactam 1 , by cyclocondensation of $(R)$-phenylglycinol with racemic $\gamma$-substituted $\delta$-oxoester, in a process that involves a dynamic kinetic resolution; (ii) a highly stereoselective conjugate addition to unsaturated lactam; (iii) the closure of the carbocyclic ring by a ring-closing olefin metathesis (RCM); and (iv) the stereoselective generation of the C-9 quaternary stereocenter.

\subsubsection{Synthesis of the Enantiopure Bicyclic Lactam: Cyclocondensation reaction}

Our route toward the total synthesis of the alkaloid started with the construction of the appropriate chiral oxazolopiperidone lactam 1 which represents a versatile building block for the stereocontrolled construction of the bridged diazatricyclic $A B C$ core of madangamine.

The required bicyclic lactam $\mathbf{1}$ was prepared in a straightforward way through a cyclondensation reaction of $(R)$-phenylglycinol with racemic 4 -formyl-6-heptenoate, ${ }^{52}$ which incorporates a labile stereocenter capable of undergoing in situ racemization or epimerization during the reaction. Thus, the starting enantiopure allyl-substituted lactam 1 was obtained in $71 \%$ yield, with only a minor amount of the corresponding $(85,8 \mathrm{aS})$-diastereoisomer $\mathbf{2}$.

\footnotetext{
${ }^{52}$ For the synthesis of 4-formyl-6-heptenoate, see: (a) Lawton, G.; Saxton, J. E.; Smith, A. J. Tetrahedron 1977, 33, 1641-1653. (b) Costello, G.; Saxton, J. E. Tetrahedron 1986, 42, 6047-6069.
} 


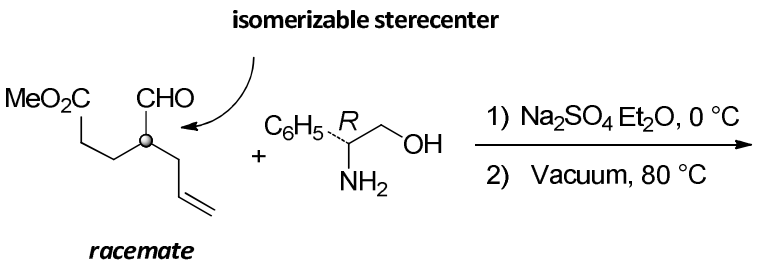

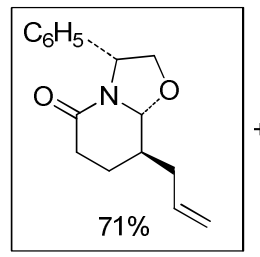

1

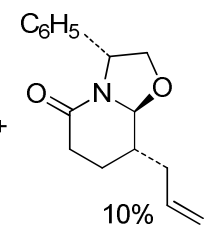

2

Scheme 2.5 Construction of piperidine B ring.

The high stereoselectivity observed in the reaction can be justified considering that the cyclodehydration involves a dynamic kinetic resolution (DKR) of the racemic substrate. ${ }^{53}$ As shown in Scheme 2.6, the mixture of the four diastereomeric oxazolidines (C-F) formed initially by reaction between the $(R)$-phenylglycinol and the racemic aldehyde ester, is in equilibrium through the corresponding imines/enamines (A-B), and the subsequent lactamization takes place through a chairlike transition state in which the substituent allyl is equatorial. The preferential formation of isomers $\mathrm{H}-3 / \mathrm{H}-8$ a cis indicates that lactamization occurs faster from the diastereomeric oxazolidine (D) that allows the approach of the carboxylate group to the nitrogen atom by the most accessible face, that is anti with respect to $\mathrm{C}_{6} \mathrm{H}_{5}$ (from aldehyde esters).

\footnotetext{
${ }^{53}$ For related examples, see: (a) Amat, M.; Cantó, M.; Llor, N.; Ponzo, V.; Pérez, M.; Bosch, J. Angew. Chem. 2002, 114, 345-348; Angew. Chem. Int. Ed. 2002, 41, 335-338. (b) Amat, M.; Cantó, M.; Escolano, C.; Molins, E.; Espinosa, E.; Bosch, J. J. Org. Chem. 2002, 67, 5343-5351. (c) Amat, M.; Bassas, O.; Pericàs, M.; Pastó, M.; Bosch, J. Chem. Comm. 2005, 1327-1329. (d) For a review, see: Pellissier, H. Tetrahedron 2003, 59, 8291-8327.
} 


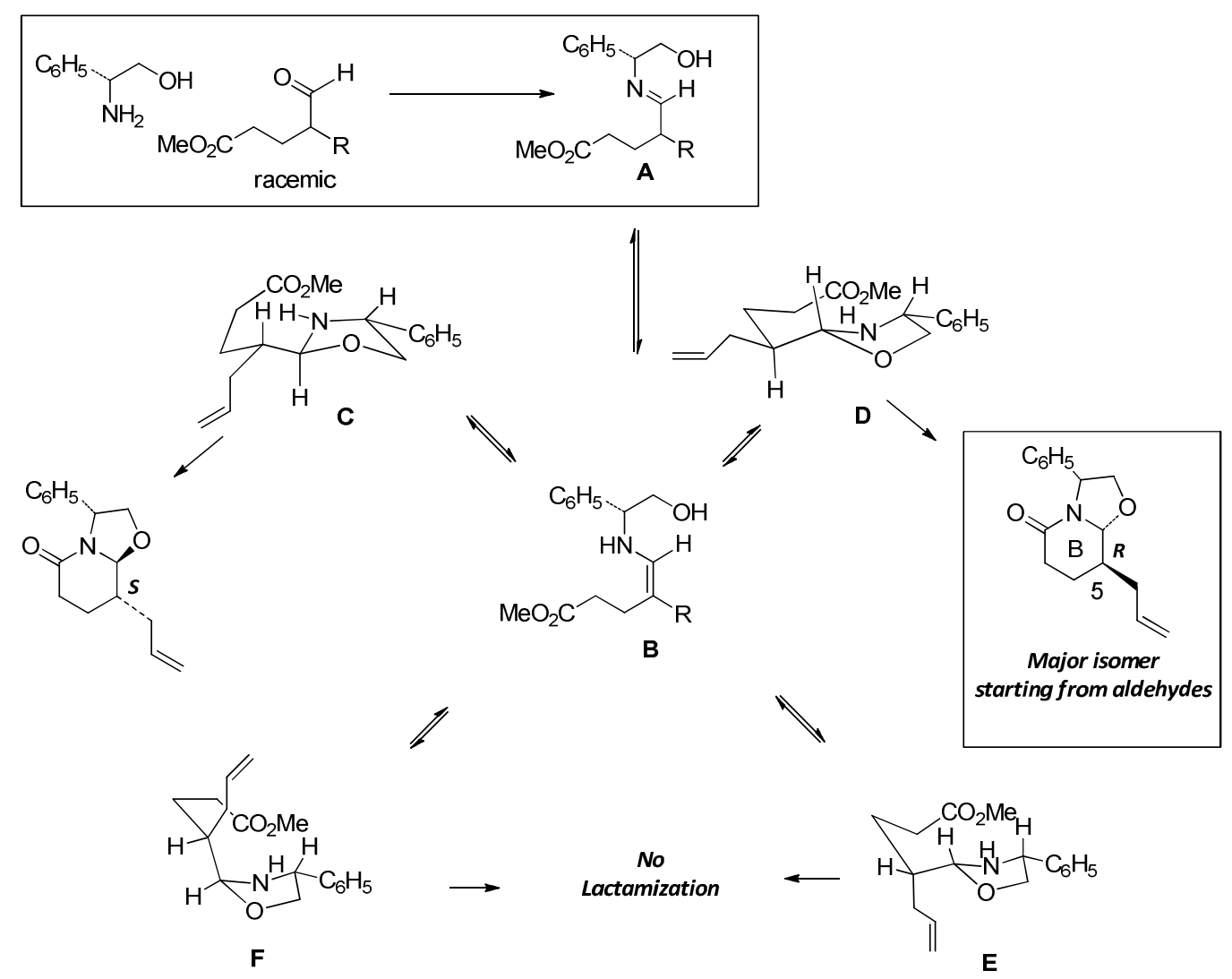

Scheme 2.6 Dynamic kinetic resolution.

The highly stereoselective process reported herein allowed not only the successful assembly of the piperidine ring $B$ but also the generation of the first stereocenter at C-5 position of the alkaloid. This strategy has proven to be an efficient method to synthesize our starting chiral building block for the enantioselective synthesis of madangamines alkaloids as it can be generated in high quantity and excellent yields.

\subsubsection{Generation of the C-12 Stereocenter: Stereoselective Conjugate Addition}

With lactam 1 in hand we decided to pursue our synthetic route toward the synthesis of the BC azabicyclic heart of madangamine using, as a key reaction, a highly stereoselective conjugated addition of an organocuprate to the unsaturated lactams $\mathbf{5}$ and $\mathbf{6}$. This strategy can be applied to accomplish the preparation of the required diallyl compound, bearing the appropriate $B / C$ cis ring junction, which represents a useful platform for the enantioselective construction of the enantiopure cis-isoquinolinic framework (see Scheme 2.8). 
Since $\alpha, \beta$-unsaturated lactams are poor Michael acceptors, has been necessary the introduction of an activating electron-withdrawing group ${ }^{54}$ at the C-9 position in order to improve the reactivity of these compounds towards the addition of organocuprates. ${ }^{55}$ In this work we selected the easily removable tert-butoxycarbonyl (Boc) group, since can be easily removed under nonreductive conditions without affecting the carbon-carbon double bonds, and the methoxycarbonyl group as activating electron-withdrawing groups.

Thus, the activated allyl unsaturated compounds $\mathbf{5}$ and $\mathbf{6}$ were prepared from lactam $\mathbf{1}$ by a sequential treatment with 2.2 equivalents of LiHMDS, di-tert-buty carbonate or the appropriate chloroformate and phenylselenyl chloride. The resulting mixture of selenides epimers $\mathbf{3}$ and $\mathbf{4}$ were then oxidized with $\mathrm{H}_{2} \mathrm{O}_{2}$ in presence of pyridine affording compounds $\mathbf{5}$ and 6 . These unsaturated lactams are unstable and very sensitive to both mild acid and basic conditions which would provoke the formation of 2-pyridones. For this reason they have to use in the next reaction immediately without further purification.

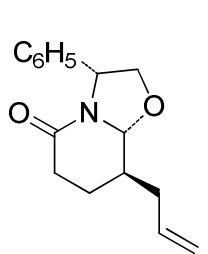

1

\section{1) LiHMDS}

2) $(\mathrm{Boc})_{2} \mathrm{O}$ or $\mathrm{ClCO}_{2} \mathrm{Me}$

3) $\mathrm{C}_{6} \mathrm{H}_{5} \mathrm{SeCl}$

$\mathrm{THF},-78^{\circ} \mathrm{C}$

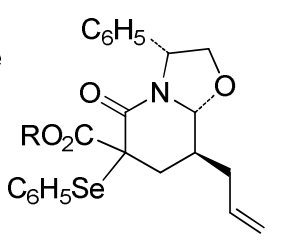

$3 \mathrm{R}=t-\mathrm{Bu} 93 \%$

$4 \mathrm{R}=\mathrm{Me} 86 \%$

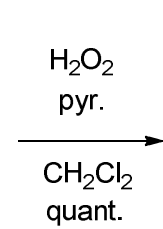

quant.

$$
\begin{aligned}
& 5 \mathrm{R}=t-\mathrm{Bu} \\
& 6 \mathrm{R}=\mathrm{Me}
\end{aligned}
$$

Scheme 2.7 Preparation of unsaturated lactams.

The conjugate addition of an allyl group to the $\alpha, \beta$ unsaturated lactams $\mathbf{5}$ and $\mathbf{6}$ was satisfactorily accomplished, with allylmagnesium bromide in the presence of $\mathrm{Cul}, \mathrm{LiCl}$, and $\mathrm{TMSCl}$, in excellent chemical yields and high exo-facial diastereoselectivities, ${ }^{56}$ leading to cisdiallyl lactams $\mathbf{7}$ and $\mathbf{8}$ as mixtures of epimers at the isomerizable C-9 stereocenter.

\footnotetext{
${ }^{54}$ (a) Nagashima, H.; Ozaki, N; Washiyama, M.; Itoh, K. Tetrahedron Lett. 1985, 26, 657-660. (b) Hagen, T. J. Synlett 1990, 63-66.

${ }^{55}$ (a) Overman, L. E.; Robichaud, A. J. J. Am. Chem. Soc. 1989, 111, 300-308. (b) Herdeis, C.; Kaschinski, C.; Karla, R.; Lotter, H. Tetrahedron: Asymmetry 1996, 7, 867-884. (c) Muller, M.; Schoenfelder, A.; Didier, B.; Mann, A.; Wermuth, C.-G. Chem. Commun. 1999, 683-684. (d) Lerchner, A.; Carreira, E. M. J. Am. Chem. Soc. 2002, 124, 14826-14827. (e) Hanessian, S.; van Otterlo, W. A. L.; Nilsson, I.; Bauer, U. Tetrahedron Lett. 2002, 43, 1995-1998. (f) Cossy, J.; Mirguet, O.; Gomez Pardo, D.; Desmurs, J.-R. New J. Chem. 2003, 27, 475-482. (g) Pineschi, M.; Del Moro, F.; Gini, F.; Minnaard, A. J.; Feringa, B. L. Chem. Commun. 2004, 1244-1245. (h) Deiters, A.; Pettersson, M.; Martin, S. F. J. Org. Chem. 2006, 71, 65476561.

For the stereochemical outcome of the conjugate addition of organocuprates to phenylglycinol-derived lactams, see: Amat, M.; Pérez, M.; Bosch, J. Chem. Eur. J. 2011, 17, 7724-7732.and references therein.
} 


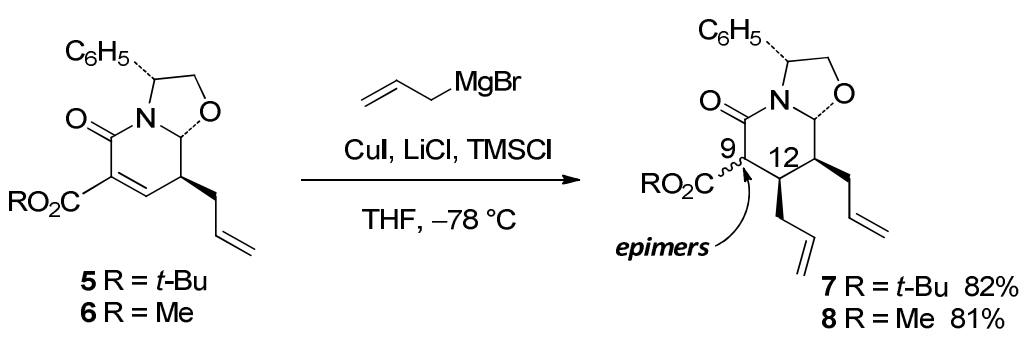

Scheme 2.8 Stereoselective conjugate addition.

The stereoselectivity in the attack of organocuprates to the unsaturated lactams can be rationalized by considering that these compounds possess a lock structure, as a result of the presence of an amide bond in a bicyclic system, and the conformation of the six-membered ring is determined by the configuration of the angular position of $\mathrm{C}-8 \mathrm{a}$. As a consequence, they adopt the pseudochair conformations described in Scheme 2.9, which implies the pseudoequatorial disposition of the allyl substituent.

The nucleophilic attack occurs under stereoelectronic control, axial to the conjugated double bond, preferentially via a chair-like transition state generating the cis isomer, kinetically more favored than the trans one that would be obtained through a boat-like transition state. ${ }^{57}$

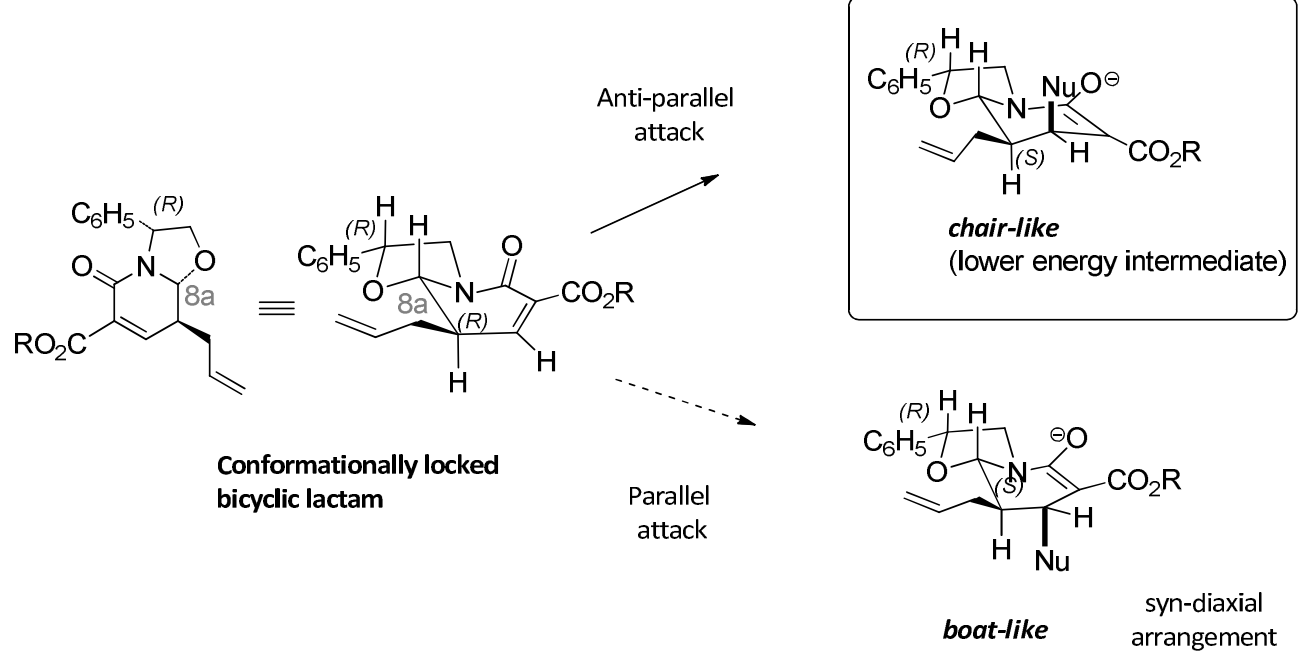

Scheme 2.9 Stereoelectronic control in conjugate addition.

\footnotetext{
57 (a) Deslongchamps, P. Stereoelectronic Effects in Organic Chemistry; Pergamon Press: Oxford, 1983, p. 221. (b) Perlmutter, P. Conjugate Addition Reactions in Organic Synthesis; Pergamon Press: Oxford, 1992, p. 25.
} 
As described earlier, the cis-diallyl lactam $\mathbf{7}$ and $\mathbf{8}$ were isolated as mixture of epimers at the isomerizable stereocenter C-9 adjacent to both carbonyl groups, but with a complete stereoselectivity in the formation of the new bond. Nevertheless, this result was thought to be inconsequential from the synthetic standpoint, since in the next step, we hoped to remove the activating tert-butoxycarbonyl moiety present in $\mathbf{7}$ or, alternatively, to generate an enolate for the alkylation step in the route that involves the methoxycarbonyl derivative (8). The conjugate addition, thus, provides a practical and efficient access to an important compound, direct precursor of the $\mathrm{BC}$ ring system, and permits the installation of the second contiguous stereocenter at the $\mathrm{C}-12$ position of madangamines.

\subsubsection{First Generation Synthesis of $B C$ bicyclic Heart of Madangamines}

Cis-diallyl lactams $\mathbf{7}$ and $\mathbf{8}$ seem to be the perfect candidates to allow the entry to the substituted tricyclic lactam $\mathbf{X}$, which represents an easy access to BC azabicyclic system of madangamines. Furthermore, as discussed in Section 2.2, we have already developed a general synthetic route which would permit, starting from conjugated adducts $\mathbf{7}$ and $\mathbf{8}$, to reach the construction of a variety of enantiopure tricyclic lactams bearing the crucial quaternary stereocenter at C-9 position in only few synthetic steps and excellent chemical yields.

Unfortunately, at this point, we had to face the main limitation of our original synthetic plan; in fact removal of the chiral inductor from our tricyclic compound resulted in a poor yields or degradation of the starting material under the classical triethylsilane- $\mathrm{TiCl}_{4}$ conditions.

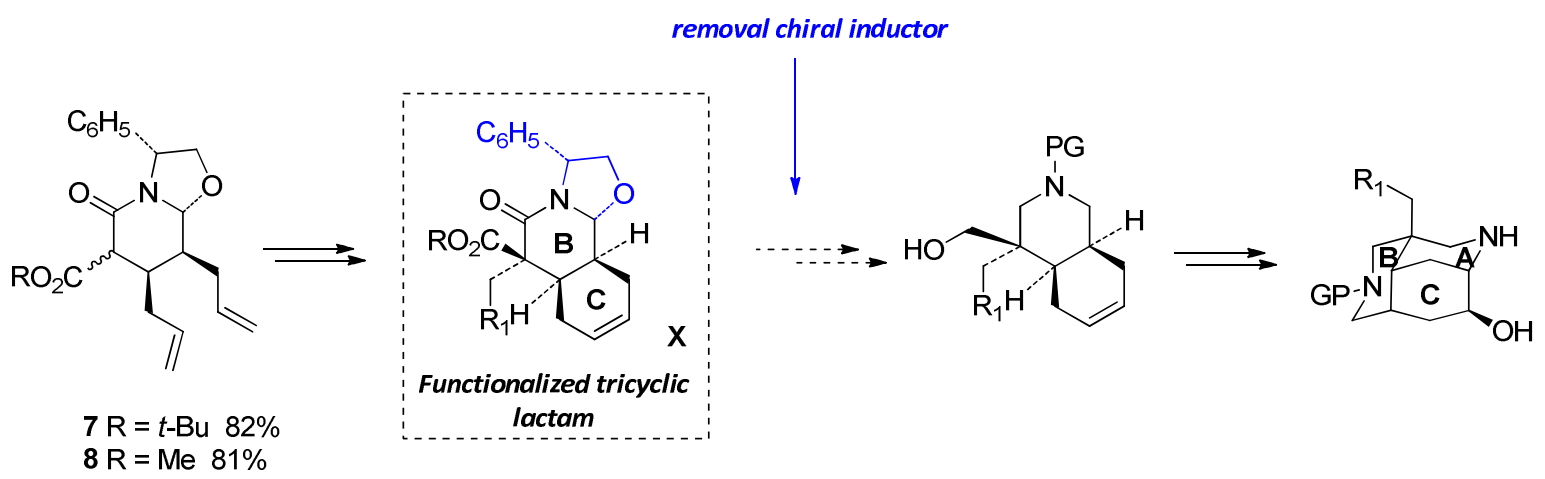

Figure 2.3 Limitation reaction of the original plan.

After a careful analysis, validated by many experimental procedures, we found that the responsible of this restriction was the presence of the electron withdrawing ester moiety at C9 position of the lactam which prevents the oxazolidine opening reaction, needed to cleavage 
the chiral inductor. ${ }^{58}$ On the other hand its presence is fundamental for the activation of the unsaturated lactams toward the stereoselective conjugate addition. Keeping in mind this limitation we were obliged to extend our synthetic path to permit to reach our synthetic target.

The adapted route involved the use of compound $\mathbf{5}$, holding a tert-butyl carbonyl ester, which act as activating electron withdrawing substituent and could be easy removed under mild conditions. The cleavage of the chiral inductor in these substrates, lacking of substituents, proceeded smoothly in a two steps sequence which involved the reductive opening oxazolidine reaction followed by $\mathrm{N}$-debenzylation of the resulting amido compound.

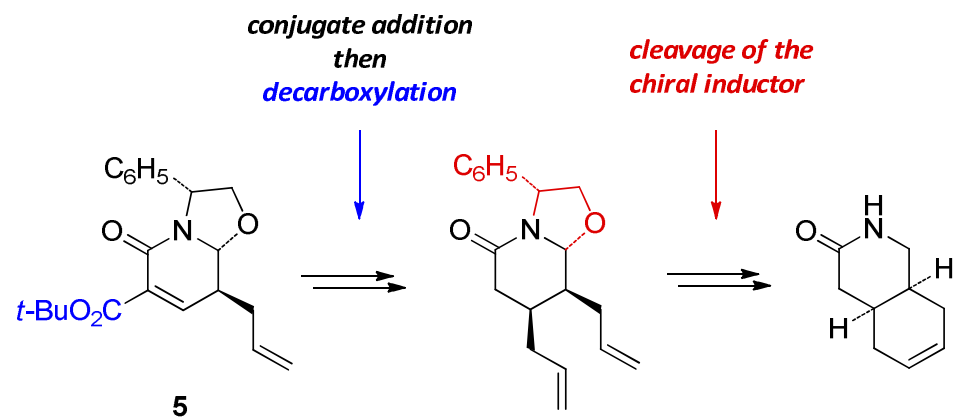

Figure 2.4 Adapted synthetic route.

This modified synthetic approach permitted the generation of the enantiopure cis-isoquinolic scaffold which required the subsequent installation of the functionalizations to form the quaternary stereocenter at C-9 position. Finally the construction of the substituted BC ring system of madangamine was accomplished by reductions of the both amide and ester moieties.

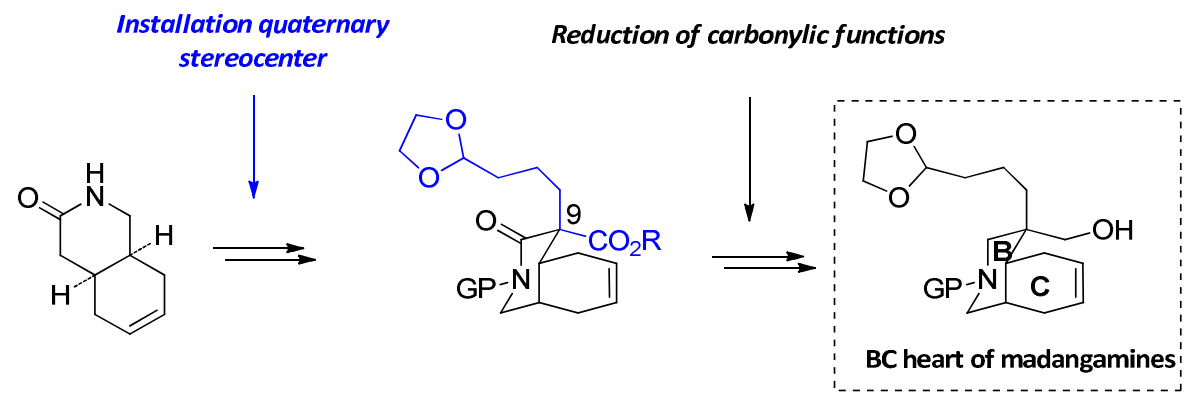

Figure 2.5 Synthesis of BC heart of madangamines.

\footnotetext{
${ }^{58}$ Annamaria Tania Minaglia Ph.D thesis, University of Barcelona, 2008.
} 


\subsubsection{Synthesis of Cis-hexahydroisoquinolone Scaffold}

The synthesis of the enantiopure isoquinolinic BC ring system started with the removal the tert-butyl carbonyl ester from epimeric compounds $\mathbf{7}$, which worked as activating group in the stereoselective conjugate addition. Therefore dienes 7 were treated with a 2:1 mixture solution of DCM/TFA providing the corresponding carboxylic acids. The resulting mixture was finally heat to reflux in toluene to perform the decarboxylation which permitted the access to the enantiopure desired cis di-allylic compounds 9.

It is worth to mention that in most cases the strong acidic reaction conditions provoked the isomerization of the C-8a and C-5 sterecenters affording a mixture of two cis-isomers $9 \mathrm{a}$ and $\mathbf{9 b}$, epimers in the $\mathrm{C}-8 \mathrm{a}$ position, togheter with the undesired more stable trans-isomer $\mathbf{9 c}$ which result useless in our syntesis (approximate ratio 2:2:1).

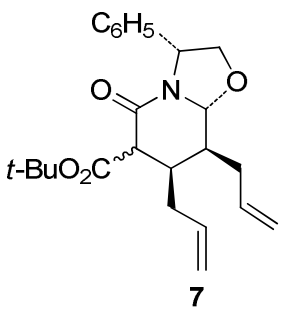

1) $\mathrm{TFA} / \mathrm{CH}_{2} \mathrm{Cl}_{2}(1: 2)$

2) Toluene, reflux $77 \%$ overall<smiles>C=CCC1CC(=O)N(CC)CCO1</smiles><smiles>C=CCC1CC(=O)N2C(C)CO[C@@H]2C1CC=C</smiles><smiles>C=CCC1CC(=O)N2C(CC=C)CO[C@H]12</smiles>

Scheme 2.10 Removal of alkoxycarbonyl moiety.

The cis-dienes $9 \mathrm{a}$ and $\mathbf{9 b}$ were finally subjected to a ring-closing metathesis reaction with the 2nd generation Grubbs catalyst which allowed the formation of the unsaturated six-membered $\mathrm{C}$ ring ${ }^{59}$ and consequentially the synthesis $\mathbf{1 0}$ which form the cis-isoquinolinic $\mathrm{BC}$ system of madangamines.

\footnotetext{
${ }^{59}$ For reviews of the alkene-metathesis reaction, see: (a) Grubbs, R. H.; Chang, S. Tetrahedron 1998, 54, 4413-4450. (b) Fürstner, A. Angew. Chem. 2000, 112, 3140-3172; Angew. Chem. Int. Ed. 2000, 39, 3012-3043. (c) Schmidt, B.; Hermanns, J. Top. Organomet. Chem. 2004, 13, 223-267. (d) Connon, S. J.; Blechert, S. Top. Organomet. Chem. 2004, 11, 93-124. For a review of olefin and enyne metathesis, see: Prunet, J.; Grimauld, L. Comprehensive Organic Functional Group Transformations II; Katritzky, A. R.; Taylor, R. J. K., Eds.; Elsevier: Oxford, 2005, Vol. 1, pp. 669-722.
} 


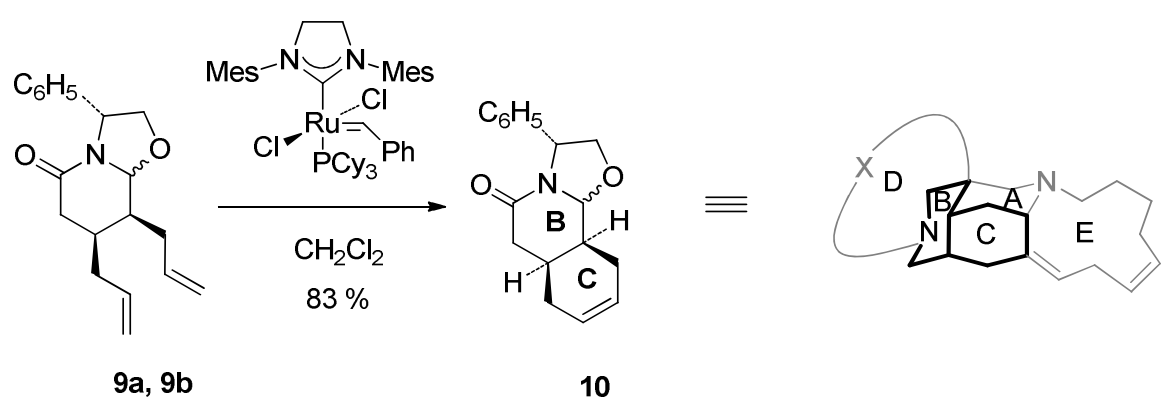

Scheme 2.11 Construction of $\mathrm{C}$ ring.

With tricyclic lactam 10 in hand the next issue was represented by the removal of the chiral inductor. Conversion of tricyclic lactam $\mathbf{1 0}$ into the desired bicyclic piperidine derivative $\mathbf{1 2}$ was accomplished removing the phenylethanol moiety of the chiral inductor by classical reductive opening reductive cleavage of the $\mathrm{C} 8 \mathrm{a}-\mathrm{O}$ and $\mathrm{C}-\mathrm{N}$ bonds of the oxazolidine ring. The process was divided in two synthetic steps and the formation of lactam $\mathbf{1 2}$ was carried out treating compound $\mathbf{1 0}$ with triethylsilane and $\mathrm{TiCl}_{4}$ followed by removal of the benzylic substituent from compound $\mathbf{1 1}$ with sodium in liquid ammonia. The outcome of the process demonstrates the efficiency of this methodology in C-9 unsubstituted lactams.

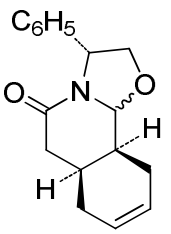

10

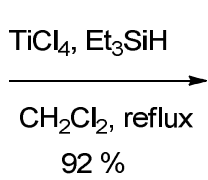
$92 \%$

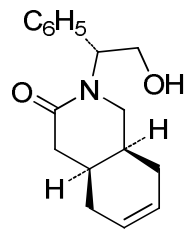

11

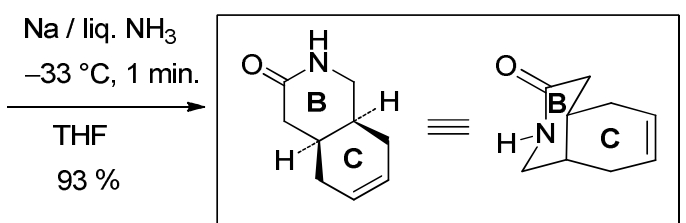

12

Scheme 2.12 Removal of phenylethanol moiety.

The reaction proceeded with excellent yield affording the desired bicyclic system $\mathbf{1 2}$ bearing the endocyclic double bond required for assembly the $A B C$ system of madangamines.

Unfortunately one of the limitations of this process was represented by the difficulty to scale up the debenzylation step. Indeed the reaction works, in an almost quantitative yield, when a maximum of $\mathbf{3 0 0 ~} \mathrm{mg}$ of starting material $\mathbf{1 1}$ was used while, an increase of quantity coincide unequivocally with a dramatic decrease of the yield due to the formation of complex byproducts.

It is easy to understand that the impossibility to scale this crucial reaction, at this early stage of the synthesis, represents an important drawback of our synthetic strategy. 


\subsubsection{Generation of the Quaternary C-9 Stereocenter}

Once assembled the cis-hydroisoquinolone compound $\mathbf{1 2}$ the subsequent challenging was represented by the construction of the advanced $B C$ heart of madangamine bearing the crucial quaternary stereocenter at C-9 position.

The functionalization of the $\mathrm{BC}$ ring system started with the protection of the bicyclic compound $\mathbf{1 2}$ as $\mathrm{N}$-tosyl derivate $\mathbf{1 3}$ and the re-installation of an ester moiety at the C-9 position which afforded compound 14. This group acts as a precursor of the desired aminomethyl chain and permits the subsequent alkylation reaction, enhancing the acidity of the proton $\alpha$-to the carbonyl group.
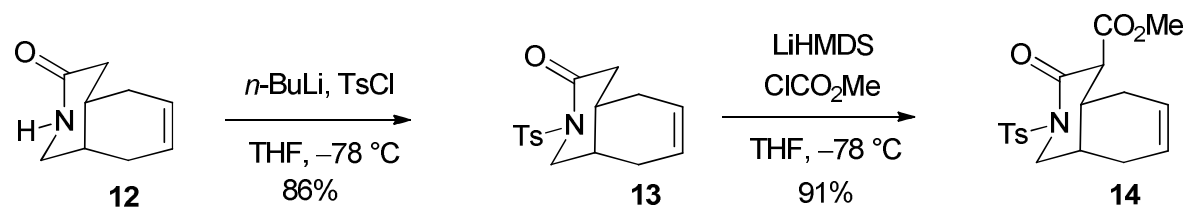

Scheme 2.13 Functionalization of BC system.

With compound $\mathbf{1 4}$ in hand we had to choose an alkylating agent which had to bear an appropriate functionality that could allow, in a later stage, the construction of the macrocyclic $D$ ring of madangamine. Considering that the presence of a double bond is incompatible with our synthetic plan, which involves an aminohydroxylation reaction which proceeds via epoxidation of the ciclohexene ring, a suitable chain bearing an acetal function at the terminal position was installed. The easy hydrolysis of the dioxolane moiety affords an aldehyde that would allow access of a wide range of unsaturated compounds including: terminal olefins, $Z$ iodoalkenes or acetylenes. The resulting C-9 functionalized diazatricyclic compounds would represent the ideal platform to perform different type of macrocyclization reactions, some of them discussed in Chapter 3. 


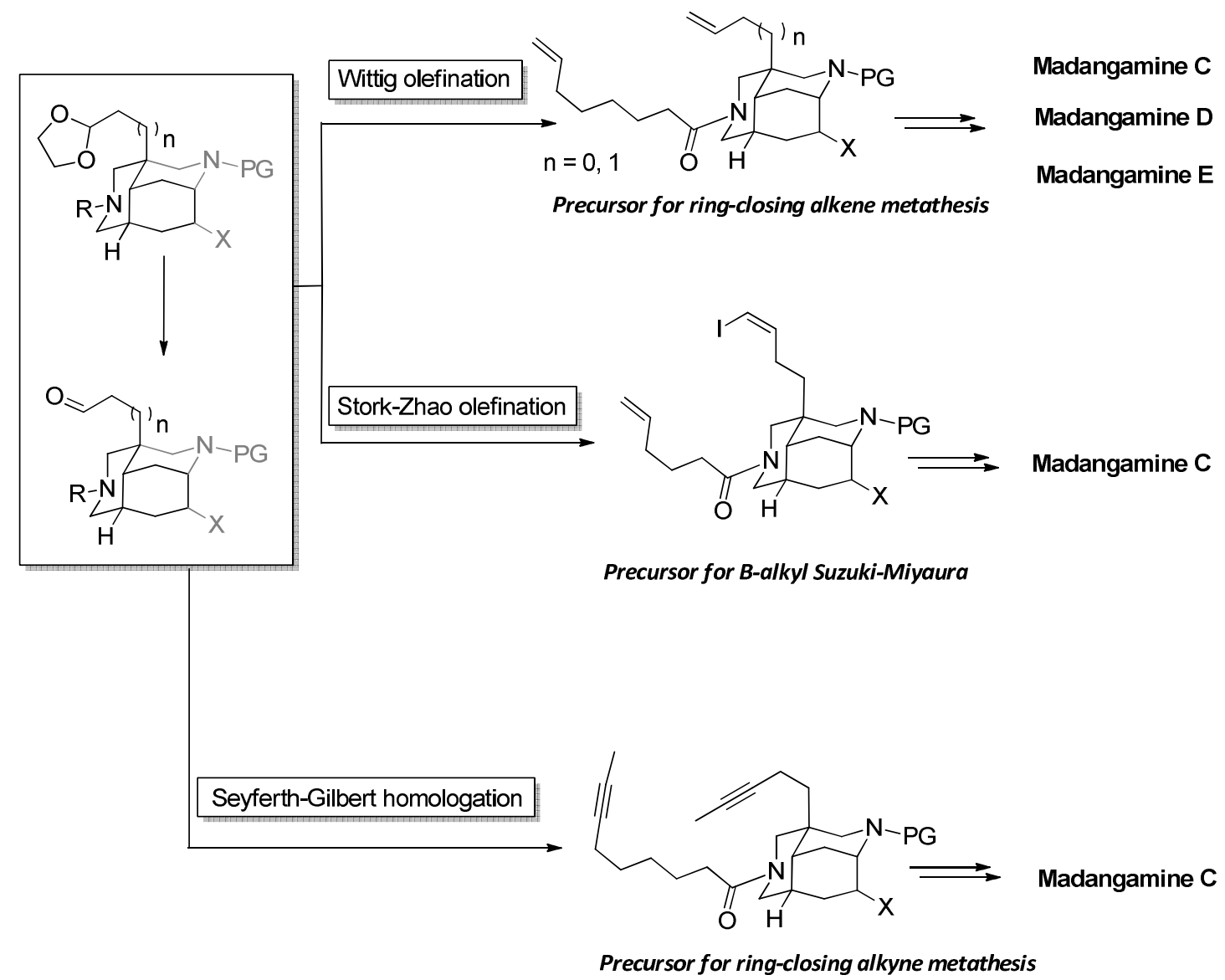

Figure 2.6 $\mathrm{D}$ rings annulation strategies.

The quaternary C-9 stereocenter of madangamines was installed using $\mathrm{NaH}$ as base and 3-(3iodopropyl)-1,3-dioxolane as alkylating agent. The reaction proceeded from compound $\mathbf{1 4}$ with an excellent facial stereoselectivity due to the compact convex geometry of the bicyclic system that allowed that the alkylation took place preferentially at the most accessible exo face. This strategy permitted the installation of the quaternary stereocenter at C-9 position and compound 15 was generated with an excellent, but not complete, diastereselectivity.
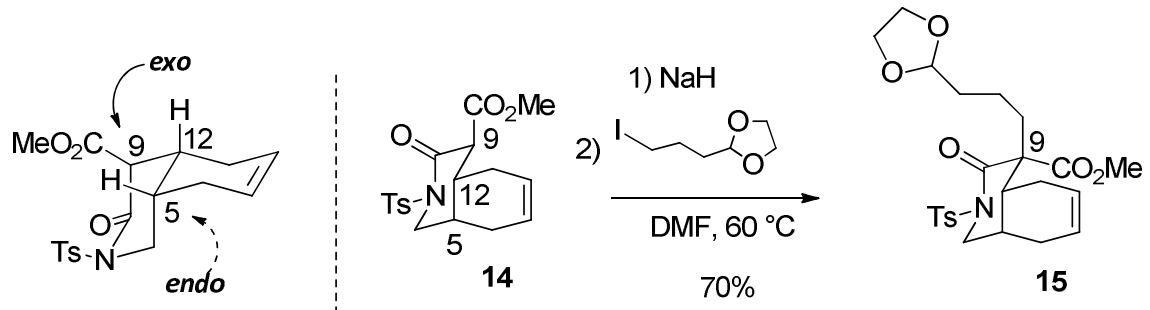

d. $r>96: 4$

Scheme 2.14 Stereoselective alkylation. 
The achievement of our first goal, represented by the construction of the functionalized BC azabicyclic system of madangamines, would be reached simply treating compound $\mathbf{1 5}$ with $\mathrm{LiAlH}_{4}$ which would provoke the reduction of both carbonyl functions leading directly to compound 18. Unfortunately, as already described in Section 2.2, the electron withdrawing nature of the $N$-tosyl protecting group would induce the opening reaction of the lactam ring $B$ ring affording diol XIV. ${ }^{60}$

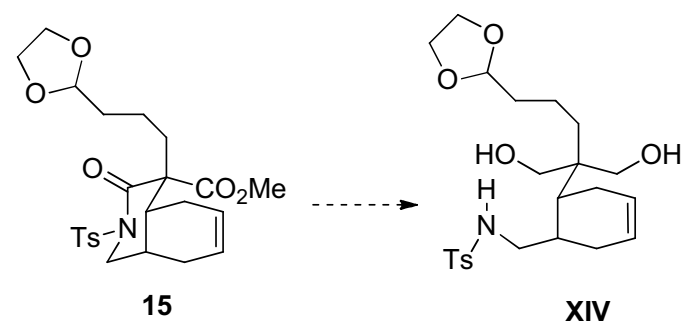

Figure 2.7 A ring opening reaction.

These observations led us to overcome this drawback extending the synthetic sequence with a three steps procedure in which the reduction reaction was performed into $N$-unprotected amide 16. Thus, after the deprotection of the $\mathbf{N}$-sulfonamide $\mathbf{1 5}$ with a sodium naphtalenide solution both carbonylic functions were reduced with $\mathrm{LiAlH}_{4}$. The resulting desired aminoalcohol $\mathbf{1 7}$ was finally converted to the $\mathrm{N}$-Boc derivative $\mathbf{1 8}$ with excellent yields.

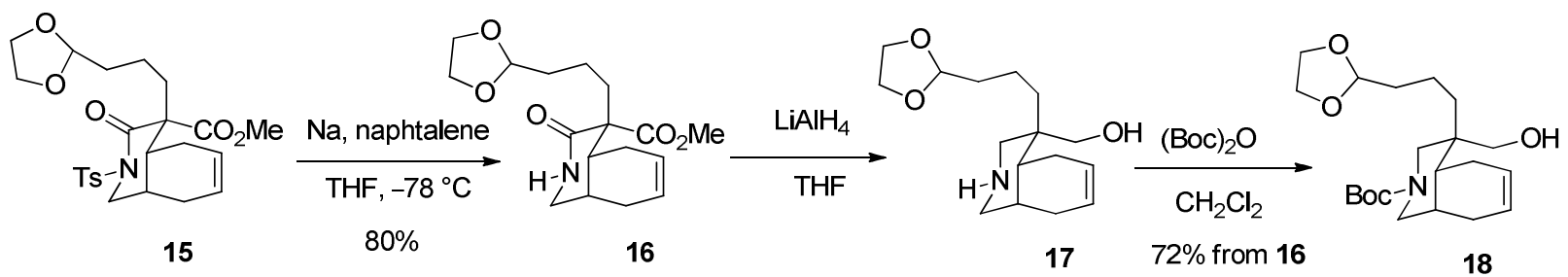

Scheme 2.15 Synthesis of BC core of madangamine.

The route described herein allows the access to the enantiopure azabicyclic compound $\mathbf{1 8}$ holding the three contiguous stereocenters, including the quaternary one, and with the correct functionalizations needed to perform the closure of the A ring and the formation of macrocylic $D$ ring of madangamines.

${ }^{60}$ (a) Hosaka, T.; Torisawa, Y.; Nakagawa, M. Tetrahedron Lett. 1997, 38, 3535-3538. 


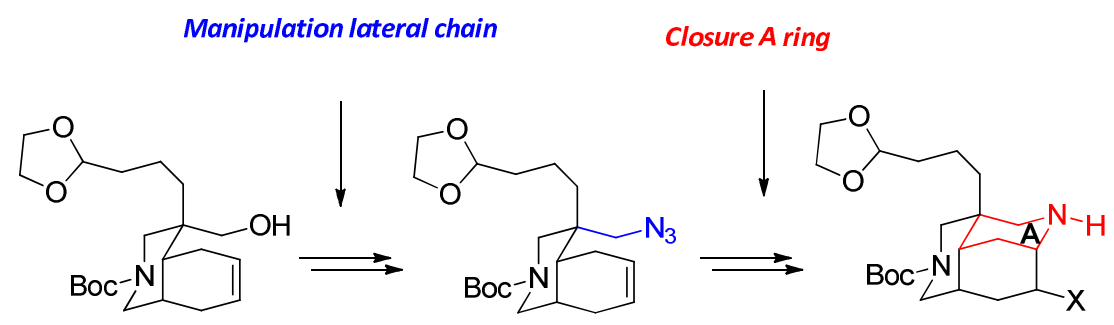

Figure 2.8 Synthetic plan toward the tricyclic core.

\subsection{AN ALTERNATIVE ROUTE TO THE BC SYSTEM}

Even if the synthetic strategy reported above permitted to reach the construction of the $B C$ heart of madangamines, we were obliged to pay an expensive price, from a synthetic point view.

In fact, starting from the elaborated diallyl compound 7, a total of eleven steps were needed to achieve the synthesis of the bicyclic compound 18. Analyzing the Scheme 2.16 it is possible to notice that, practically a half of the synthetic steps of this sequence, are redundant and include the installation/cleavage and re-installation of the ester function at the C-9 position and the change of two different nitrogen protecting groups to finally generate the aminoalcohol 18.

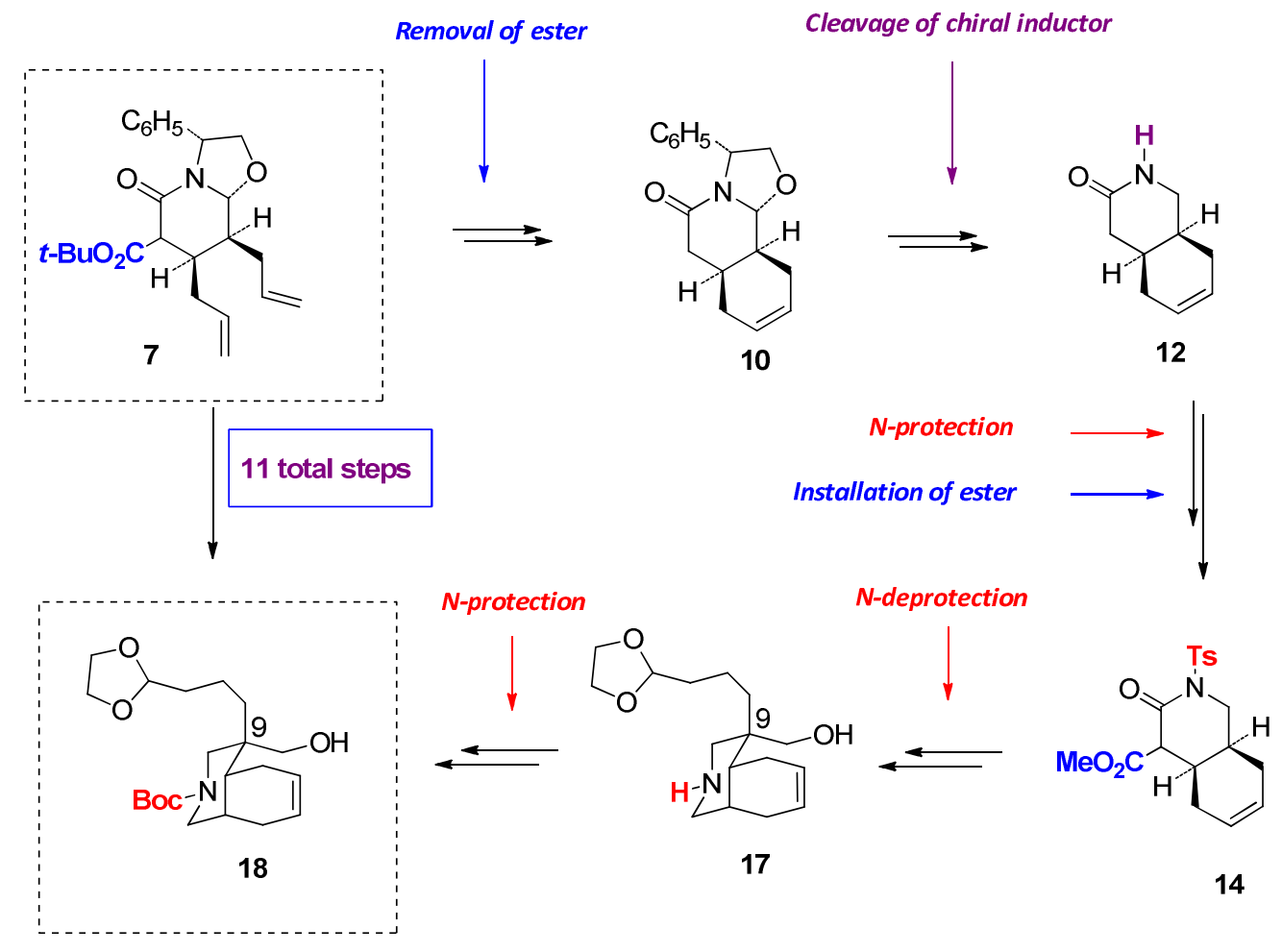

Scheme 2.16 Synthesis of BC system. 
Furthermore there are another two observations which are worth to remember:

- Part of the precious starting material is lost during the removal of the activating ester moiety, due the acid-promoted epimerization of the C-5 position.

- The impossibility to scale (over $300 \mathrm{mg}$ ) the debenzylation reaction difficulty the production of starting material in a very early stage of the synthesis (see Section 2.3).

As we mentioned in Section 2.2 the synthetic approach presented until now is a modification of an "original plan" which was designed to overcome the phenyl ethanol removal troubles, attributed to the interfering presence of the methoxycarbonyl function at the C-9 position. The synthetic consequences caused by this single failure were severe and the synthetic tricks necessary to reach our goal complicated the whole process.

Considering that we are still in an early stage of the process and we need to dispose of enough quantity of starting material to achieve the synthesis of the diazatricyclic system and the subsequent annulation of the macrocyclcic rings we decided to tackle our synthetic problems.

\subsubsection{Formulation of the Alternative Strategy}

We always have been conscious of the existence of two different ways to avoid this unwanted pathway and consequentially reduce significantly the numbers of synthetic steps. Both processes would start with the synthesis of tricyclic lactam $\mathbf{X V}$ which already incorporates all the above key features, including the methoxycarbonyl function that dually enhances the reactivity of the double bond towards the conjugate addition, and simultaneously acts as a masked aminomethyl moiety, required to construct the piperidine ring $\mathrm{A}$.

Tricyclic system $\mathbf{X V}$ can be prepared with (1) a stereoselective conjugate addition followed by (2) a ring-closing metathesis, whereas the crucial quaternary stereocenter at C-9 would be installed by (3) a stereoselective alkylation reaction. As mention before in a previous work the synthesis of some alkylated compounds constructed with this three step sequence was studied. 


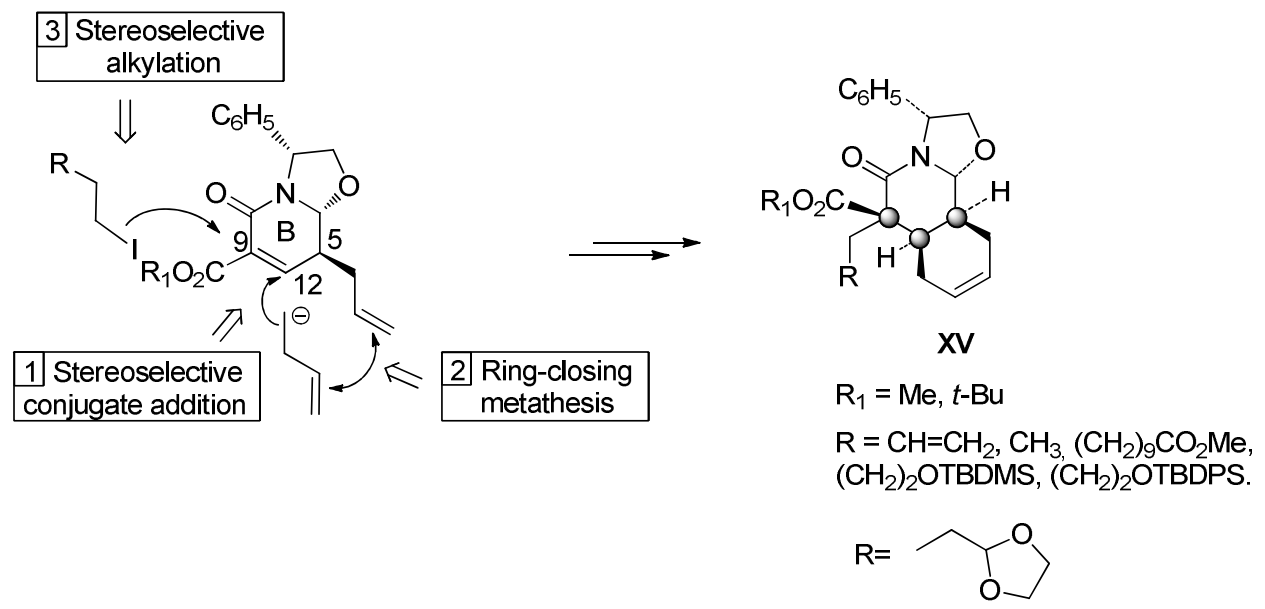

Figure 2.9 Preparation of functionalized tricyclic lactam.

With these arguments in mind we thought it was worth stopping our journey toward the synthesis of madangamine, come back to our original synthetic plan and concentrate our efforts to achieve the construction of the azabicyclic compound $\mathbf{1 8}$ starting from the fully substituted tricyclic lactam $\mathbf{X V}$ since this process would allow to reach the goal, halving the synthetic sequence with the consequent big save in terms of time and synthetic steps.

To this end, we plan to explore a methodology for the removal of the phenylethanol moiety from the amino alcohol resulting from the $\mathrm{LiAlH}_{4}$-reduction of $\mathbf{X V}$ while, in an alternative route, we will study the problematic $\mathrm{C}-\mathrm{O}$ oxazolidine opening reaction from tricyclic lactam $\mathbf{X V}$.

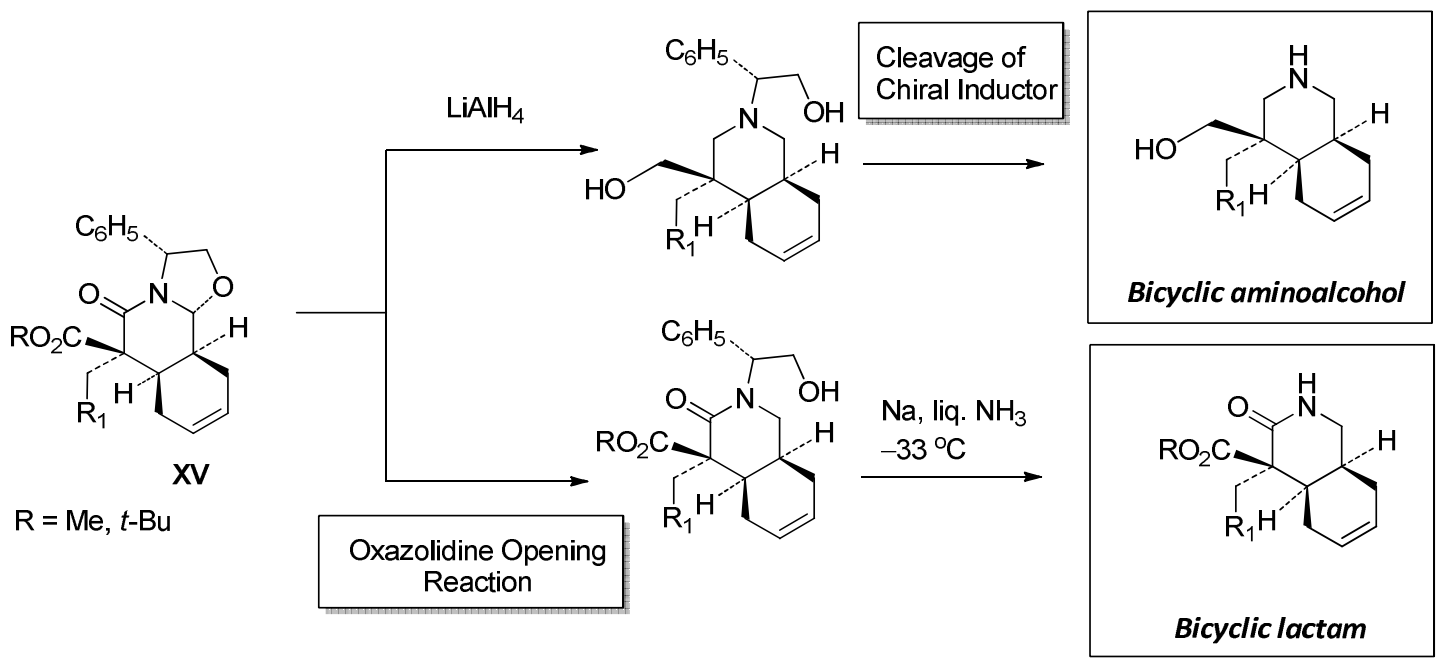

Figure 2.10 Different strategies for the cleavage of the chiral inductor

The synthesis of the functionalized tricyclic compounds started with the ring-closing metathesis of the diallyl compounds $\mathbf{7}$ and $\mathbf{8}$ which were converted to the tricyclic lactams $\mathbf{1 9}$ 
and 20 using a 2nd generation Grubbs catalyst in dichloromethane. In this case, the alcoxycarbonyl group present in the C-9 position not only increase the acidity of the $\mathrm{C}=\mathrm{O} \alpha$ position, permitting the subsequent alkylation reaction, but acts as a precursor of the aminomethyl chain required for the synthesis of $A$ ring of the $A B C$ diazatricyclic core of madangamine. Then, the crucial quaternary C-9 stereocenter of madangamines was generated with complete facial selectivity and excellent chemical yields by alkylation of the epimeric mixture of 19 and 20 to give the lactams 21 and 22 as single isomers. The compactly architecture of the starting tricyclic compound clearly forced alkylation to occur from the more accessible exo-face.

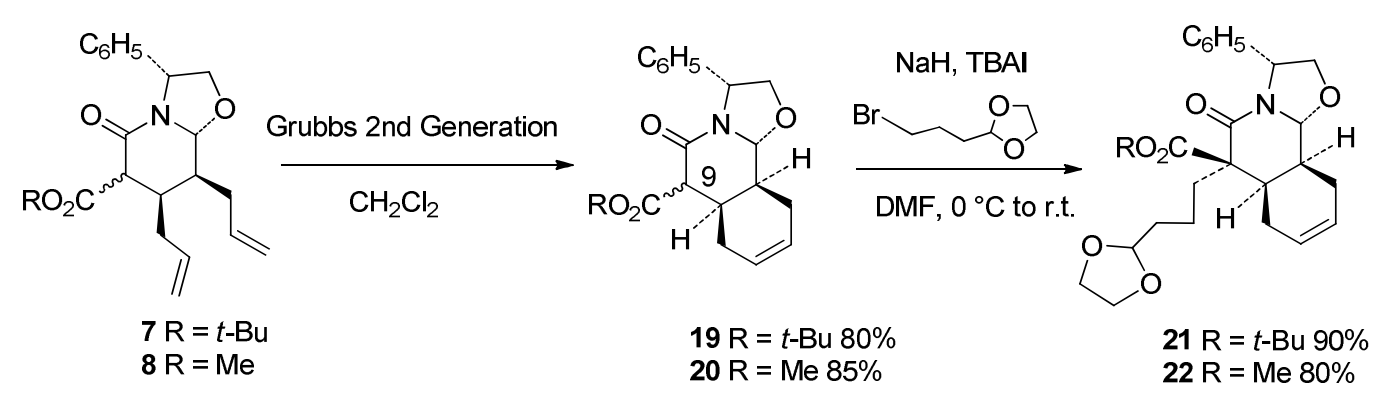

Scheme 2.17 Synthesis of tricyclic lactam 21 and 22.

\subsubsection{Cleavage of Phenylethanol Moiety from Amine Derivatives}

$\mathrm{LiAlH}_{4}$ reduction of the alkylated compound $\mathbf{2 1}$ caused the cleavage of the $\mathrm{C}-\mathrm{O}$ bond of the oxazolidine ring and the simultaneous reduction of the ester and amide carbonyl groups to give the respective alcohol $\mathbf{2 3}$ in excellent yield.
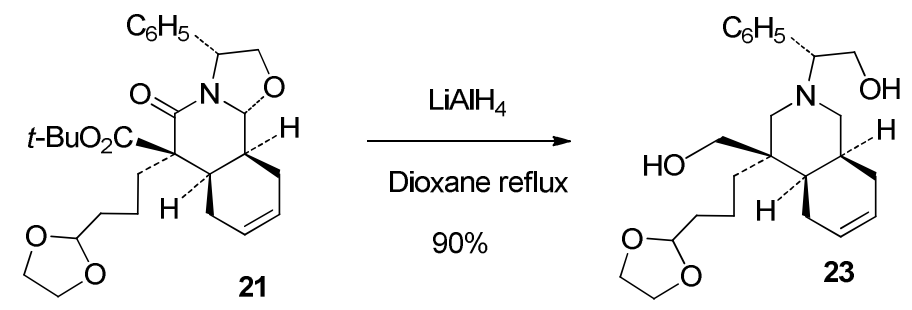

Scheme 2.18 Reduction of tricyclic lactam.

Since the removal of the chiral inductor using a catalytic hydrogenation is incompatible due to the presence of the endocyclic double bond in the $C$ ring, needful to generate the piperidine $A$ ring in a later stage, we decided, as first attempt, to cleavage the phenyl ethanol moiety applying the typical $N$-dealkyl or $N$-debenzylation reactions normally used in tertiary amines. 


\subsubsection{Oxidative Ceavage with Lead Tetraacetate and CAN}

We assume that the first reagent had to be tested on our compound was lead tetraacetate since has been successfully used in transformations of 1,2-aminoalcohols to the corresponding amines. The methodology appeared promising and its ability and selectivity in the removal of phenylethanol moieties from both secondary and tertiary amines, has been largely demonstrated in several papers ${ }^{61}$. The reaction would involve the oxidative cleavage of the aminoalcohol while an acidic hydrolysis of the resulting imine would afford the corresponding secondary amine.

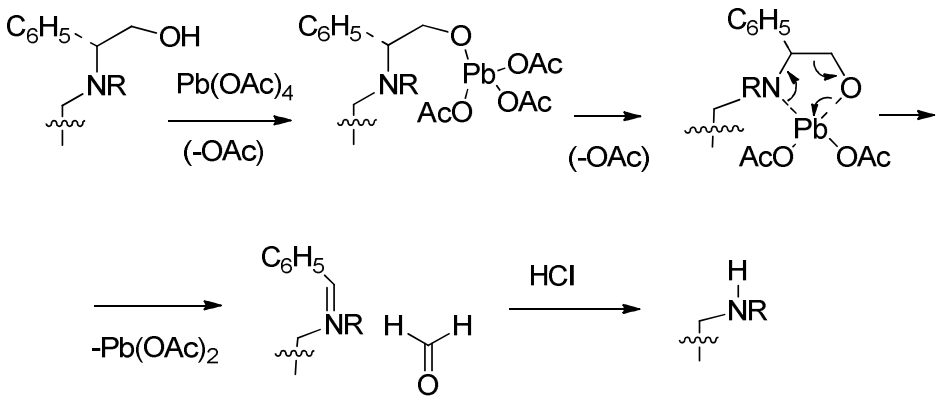

Scheme 2.19 Mechanism of reaction.

In order to test its efficacy we treat aminoalcohol XVII with lead tetraacetate in a 2:1 solution of dichloromethane and methanol at low temperature. Unfortunately the use of this procedure resulted unsuccessful and, despite the use of a several different experimental protocols, the expected secondary amine was obtained with poor yield. The only significant result was represented by the aisolation of a small quantity of dimeric compound XVIII, formed as a resulting of a side reaction of the secondary amine with the formaldehyde generated during the process.

${ }^{61}$ (a) Poerwono, H.; Higashiyama, K.; Yamauchi, T.; Takahashi, H. Heterocycle 1997, 46, 385-400. (b) Chakraborty, T. K.; Reddy, G. V. J.Org. Chem. 1992, 57, 5462-5469. (c) Williams, R. M.; Zhai, W.; Aldous, D. J.; Aldous, S. C. J. Org. Chem. 1992, 57, 6527-6532. (d) Mokhallalati, K.; Pridgen, L. N. Synth. Commun 1993, 14, 2055-2064. (e) Higashiyama, K.; Inoue, T.; Yamacuchi, T.; Takahashi, H. J. Chem. Soc. Perkin Trans. 1 1995, 111-115. (f) Mokhallalati, K.; Wu M. J.; Pridgen, L. N. Tetrahedron Lett. 1993, .34, 47-50. (g) Wu M. J.; Pridgen, L. N. J.Org. Chem. 1991, 56, 1340-1344. 


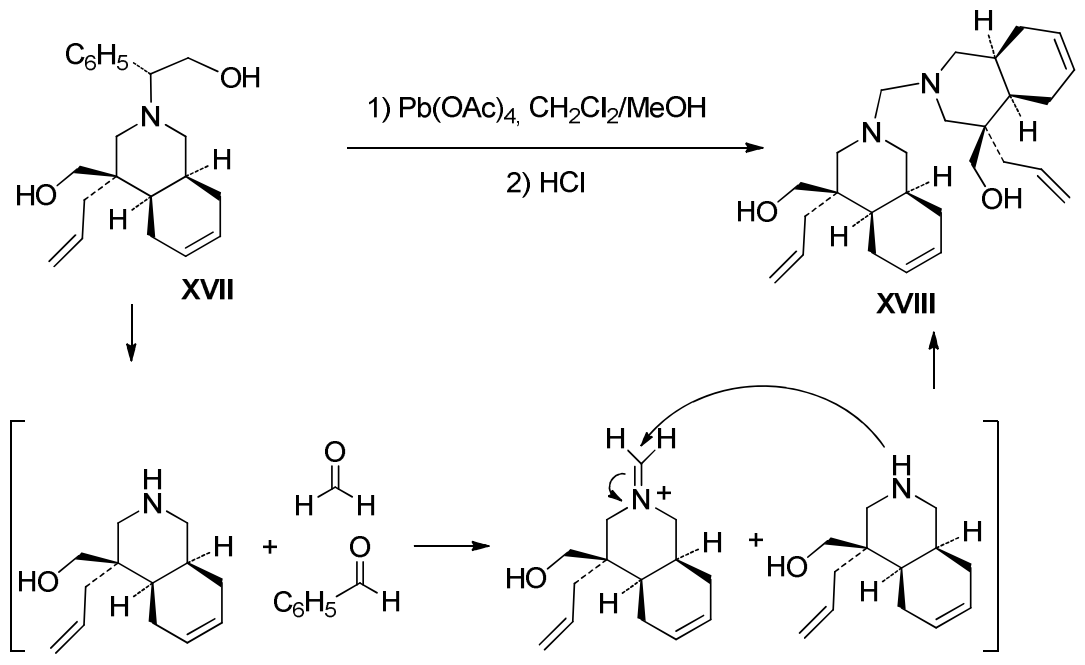

Scheme 2.20 Formation of dimer.

In view of these disappointing results we moved our attention to CAN (cerium ammonium nitrate) which represents another oxidative reagent with similar characteristics. ${ }^{62}$ Furthermore the reaction conditions, which are normally used, are very mild and mostly functional groups remain unaffected during the reaction.

The mechanism, shown in Scheme 2.21, would suggest that the reaction between the alcohol unit of the chiral inductor and CAN would produce a radical cleavage of the $\mathrm{C}-\mathrm{C}$ bond which would afford the benzyl radical species and formaldehyde. The radical has to be converted to a cation species by a one-electron transfer oxidation of one more CAN while the addition of water would form a hemiacetal, easily converted to the final compound by the removal of the benzaldehyde.

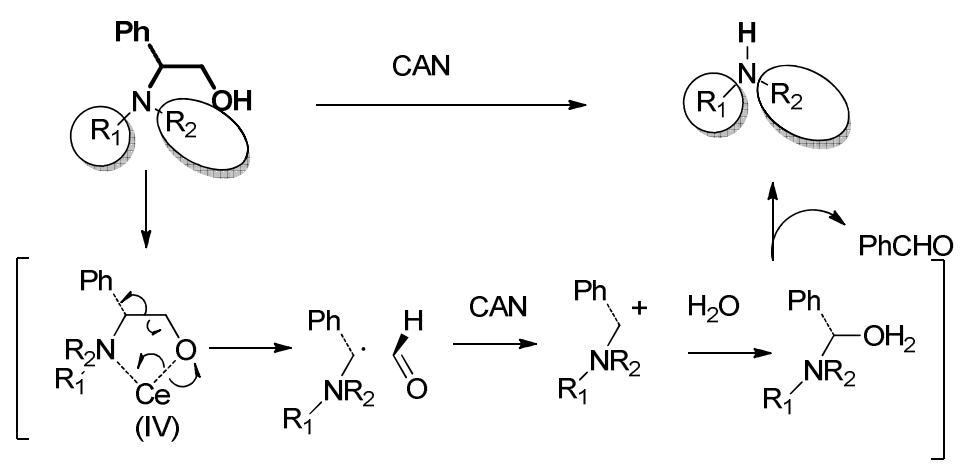

Scheme 2.21 Mechanism of reaction.

\footnotetext{
${ }^{62}$ (a) Bull, S. D.; Davies, S. G.; Fenton, G.; Mulvaney, A. W.; Prasad, R. S.; Smith, A. D. J. Chem. Soc. Perkin Trans. 1 2000, 3765-3774. (b) Fujioka, H.; Hirose, H.; Ohba, Y.; Murai, K.; Nakahara, K.; Kita, Y. Tetrahedron 2007, 63, 625-637. (c) Lan, H. Q.; Ye, J. L.; Wang, A. E.; Ruan, Y. P.; Huang, P. Q. Chem. Eur. 2011, 17, 958-968.
} 
Although CAN is recognized as a good reagent for the selective deprotection hydroxyethyl units from 2-hydroxyethyl amines, no report has described its ability with cyclic amines. Indeed treatment of aminoalcohol $\mathbf{2 3}$ with aqueous CAN gave unsatisfactory results.
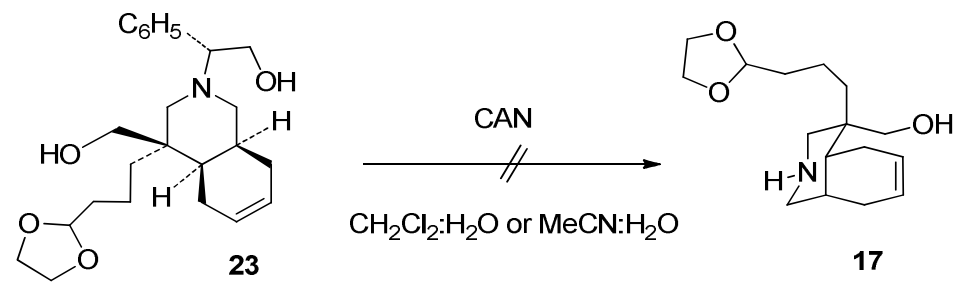

Scheme 2.22 Reaction with CAN.

\subsubsection{Acid Mediated Elimination of the Chiral Inductor}

Firmly motivated to overcome the problem and inspired by some recent few works concerning the synthesis of fluorinated amino acids ${ }^{63}$ we decided to undertake another methodology which would involve the removal of the phenylethanol moiety simply by treatment of the corresponding tertiary amine with acid. Scheme 2.23 illustrates two examples in which a clean removal of the phenylethanol side chain was easily achieved in excellent yield by reaction of the aminoalcohols with a concentrated solution of refluxing $\mathrm{HCl}$.

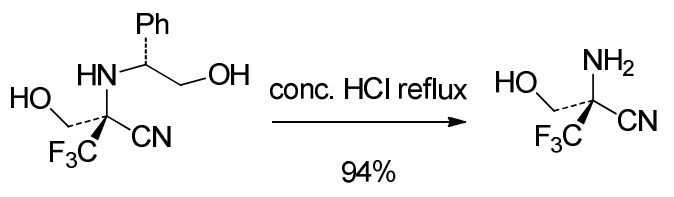

$94 \%$<smiles>CC(=O)OCC1CC=CC(N(Cc2ccccc2)c2ccccc2)(C(O)(F)F)C1(O)O</smiles><smiles>OCC1C=CCC(CO)N1</smiles>

Scheme 2.23 Removal of chiral inductor with $\mathrm{HCl}$.

Unfortunately the application of this procedure on our compound did not afford the desired secondary amine and, treatment of compound 23 with $\mathrm{HCl}$ under several experimental procedures, gave only mixtures of undesired byproducts. This result demonstrate that probably the outcome of the reaction is strictly dependent by the presence of the electron

\footnotetext{
${ }^{63}$ Fustero, S.; Albert, L.; Mateu, N.; Chiva, G.; Miró, J.; González, J.; Aceña, J. L. Chem. Eur. J. 2012, 18, 3753-3764. (b) Simon, J.; Nguyen, T.; Chelain, E.; Lensen, N.; Pytkowicz, J.; Chaume, G.; Brigaud, T. Tetrahedron: Assimetry 2011, 22, 309314.
} 
withdrawing trifluoromethyl group at the $\mathrm{C}-2$ position that weakens the benzylic $\mathrm{C}-\mathrm{N}$ bond promoting the removal of the chiral inductor.

\subsubsection{3.. Trans-Acylating Reagents}

We next turned our attention to another classic methodology which involves the use of transacylating reagents which have been largely used to perform the selective $N$-dealkylation of tertiary amines in high yields. ${ }^{64}$

The most popular reactive of this family is 1-chloroethyl chloroformate (ACE-Cl) which has proven to be effective in the removal of the phenylglycinol chiral inductor from tertiary cyclic amines. ${ }^{65}$ The reaction proceeds via a nucleophilic attack of the chloride ion at the benzylic position, leading to the formation of the carbamate ester. This intermediate is deACEylated by evaporating the reaction mixture in vacuo and then heating the residue in $\mathrm{MeOH}$ (see Scheme 2.24).

Its compatibility with our compound and the easiness to be removed make ACE-Cl the perfect candidate for our cleavage studies on the piperidinic model compound $\mathbf{2 4}$, generated by reduction and TBDMS protection of lactam compound $2 .{ }^{66}$ Subsequent treatment of compound $\mathbf{2 4}$ with ACE-Cl in a refluxing solution of 1,2-dichloroethane, followed by heating of the residue in $\mathrm{MeOH}$, afforded allyl piperidine $\mathbf{2 5}$ with modest yield.

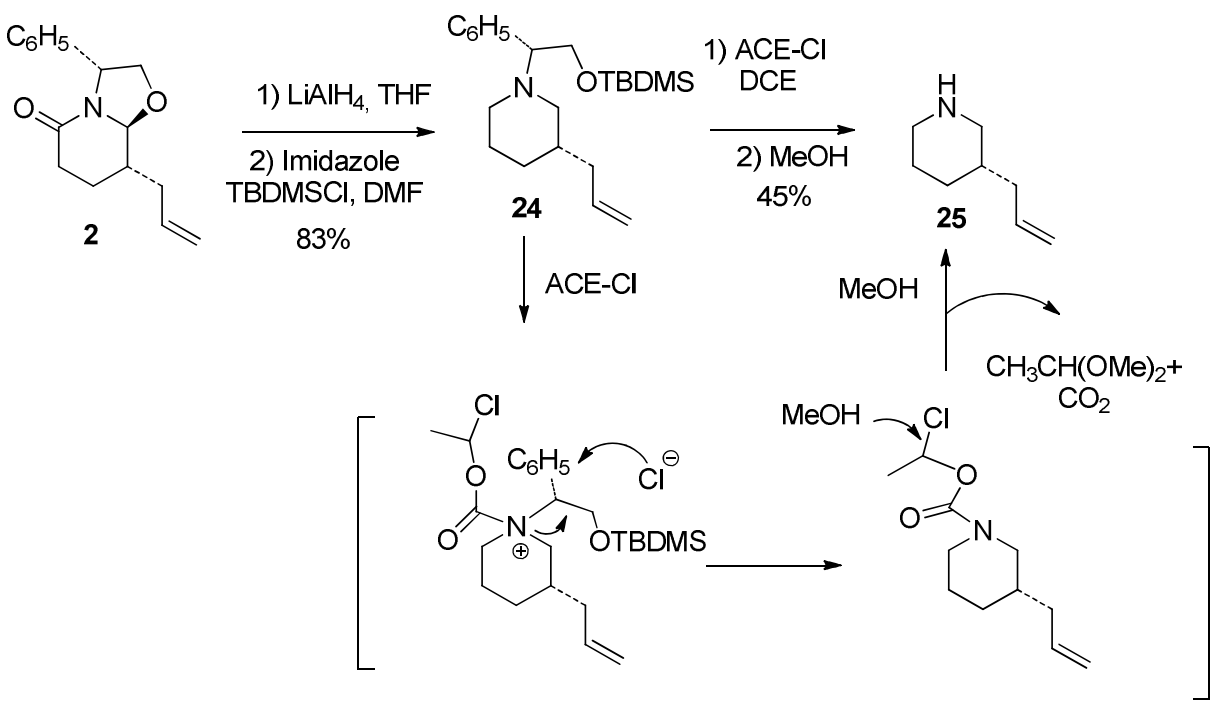

Scheme 2.24 Debenzylation reaction with ACE-CI.

\footnotetext{
${ }^{64}$ (a) Olofson, R. A.; Abbott, D. E. J. Org. Chem. 1984, 49, 2795-2799. (b) Olofson, R. A.; Martz, J. T.; Senet, J. P.; Piteau, M.; Malfroof, T. J. Org. Chem. 1984, 49, 2081-2082. (c) Bhat, R. G.; Ghosh, Y.; Chandrasekaran, S. Tetrahedron Lett. 2004, 45, 7983-7985.

65 (a) Ennis, M. D.; Hoffman, R. L.; Ghazal, N.B.; Old, D. W.; Mooney, P. A. J. Org. Chem. 1996, 61, 58135817. (b) Agami, C.; Bihan, D.; Hamon, L.; Kadouri-Puchot, C.; Lusinchi, M. Eur. J. Org. Chem. 1998, 2461-2465.

${ }^{66}$ Lactam 2 is the minor isomer synthesized during the cyclocondensation reaction.
} 
The same methodology was therefore applied on our compound using both unprotected aminoalcohol 23 or TBDMS protected compound $\mathbf{2 6}$ but, despite our efforts, the reaction didn't take place and in all cases it was only possible to recover starting or decomposed material. Several attempts were tried to promote the reaction including: (i) the screening of various solvents (ii) the use of a proton sponge ${ }^{67}$ or hydrogen carbonate, ${ }^{68}$ in order to elude the protonation of the starting amine by the chloridric acid formed in the reaction, or (iii) the introduction of the iodine ion to accelerate the nucleophilic pathway ${ }^{69}$ and avoid the formation of byproducts. Under no circumstances were possible to reproduce the results obtained on the model compound and achieve the synthesis of the desired secondary amine. The use of Troc- $\mathrm{Cl}$ instead of ACE-Cl gave identical results. ${ }^{18}$

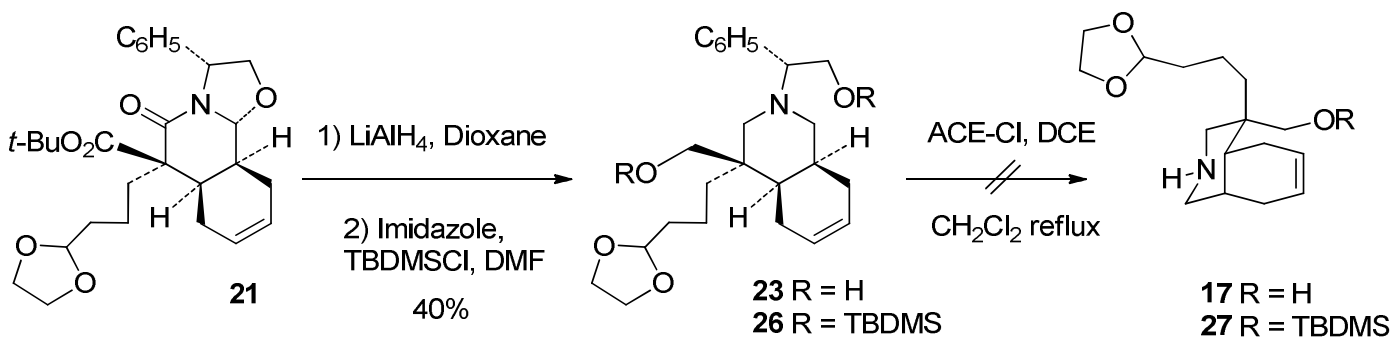

Scheme 2.25 Reaction with ACE-Cl.

\subsubsection{Dissolving-Metal Reductions}

Once we discarded the use of oxidatives and trans-acylative methodologies we decided to start the exploration of dissolving-metal reductions.

Reductions of organic compounds using a "solution of electrons" in ammonia are known as dissolving-metal reductions. When alkali metals (lithium, sodium, potassium and calcium) are dissolved in liquid ammonia, metal atoms loose electrons and their solvation gives the typical blue color of the solution. The efficiency of these reactions for the selective cleavage of C-N benzylic compounds has already been proven in the removal of the chiral phenylethanol moiety from our isoquinolinic bicyclic lactam 12 (See Section 2.3).

Therefore the application of the conventional procedure with sodium or lithium in liquid ammonia, on our amino alcohol compound 23, gave the desired secondary amine $\mathbf{1 7}$ with poor yield $(<15 \%)$ together with a complex mixture of byproducts, some of them derived from a dearomatization of the phenyl group.

\footnotetext{
${ }^{67}$ Harmann, B. C.; Hartwig, J. F. J. Am. Chem. Soc. 1997, 119, 7236-7239.

${ }^{68}$ Yamada, K.; Kurokawa, T.; Tokuyama, H.; Fukuyama, T. J. Am. Chem. Soc. 2003, 125, 12382-12383.

${ }^{69}$ Coe, J. W.; Brooks, P. R.; Vetelino, M. G.; Bashore, C. G.; Bianco, K.; Flick, A. C. Tetrahedron Lett. 2011, $52,953-954$.
} 

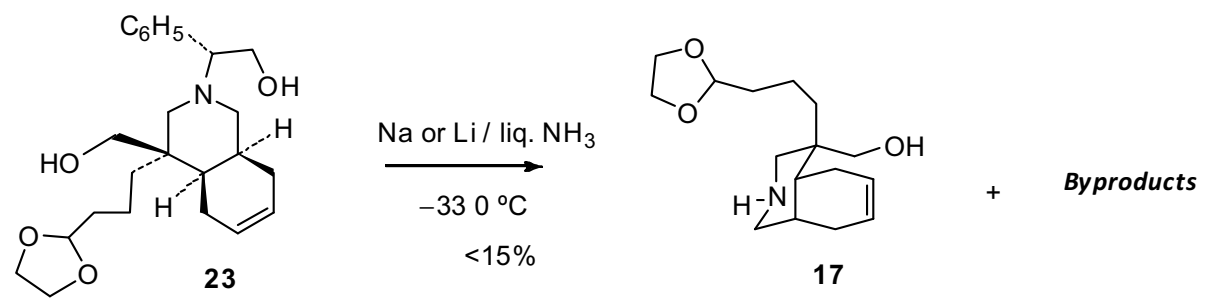

Scheme 2.26 Dissolving metal methodology.

Fortunately, the application of the Benkeser protocol, described by Angle in his synthesis of palaustrine, ${ }^{70}$ which employs lithium in a mixture of triethylamine/THF/ethylenediamine instead liquid ammonia, ${ }^{71}$ worked. Treatment of both TBDMS and unprotected 23 and 26 compounds with lithium using the Angle conditions gave aminoalcohol $\mathbf{1 7}$ that was immediately converted without any purification to the $\mathrm{N}$-Boc derivatives $\mathbf{1 8}$ or $\mathbf{2 8 .}$

Disappointingly, due to the complicated operational procedure, the reaction was sparsely reproducible and sometimes afforded a complex mixture of unwanted compounds. In order to control the course of the reaction avoiding, or at least reducing, the formation of byproducts we tried the use of different solvents, the substitution of lithium with sodium but all our efforts result unsuccessful.

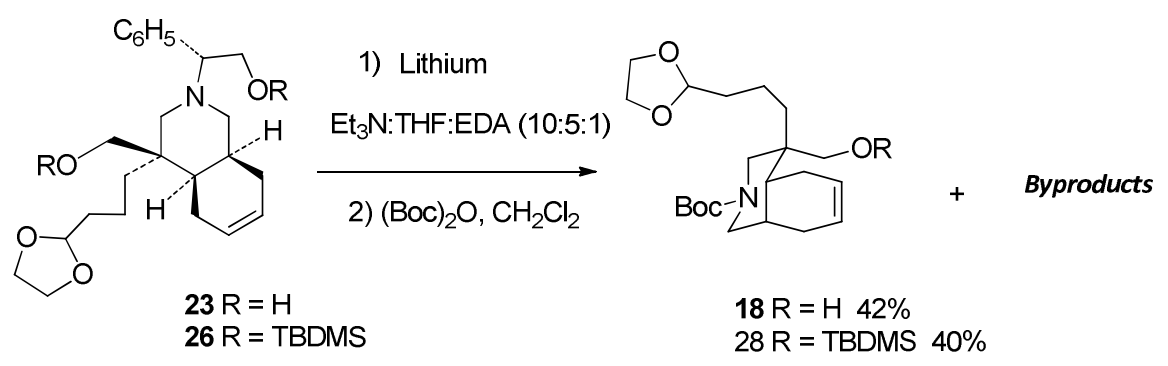

Scheme 2.27 Removal of the chiral inductor with lithium.

We thought that the reason of our failures was the impossibility to dosage the exact quantity of lithium, considering that this metal is sold in wire and has to be cut with a scissor with the consequent different shape and dimensions of the resulting pieces.

Based on these experimental observations we started again to search for solutions. SiGNa Chemistry has developed a technology for encapsulating alkali metals into nano-structured porous oxides, such as silica gel and alumina. The encapsulation facilitates the manipulation and reduces the dangers associated with the handling of alkali metals while retains its reducing

\footnotetext{
${ }^{70}$ (a) Angle, S. R.; Henry, R. M. J. Org. Chem. 1998, 63, 7490-7497. (b) Passarella, D.; Angoli, M.; Giardini, A.; Lesma, G.; Silvani, A.; Danieli, B. Org. Lett. 2002, 4, 2925-2928.

${ }^{71}$ Garst, E. M.; Dolby, J. L.; Esfandiari, S.; Fedoruk, N. A.; Chamberlain, N. C.; Avey, A. A. J. Org. Chem. 2000, 65, 7098-7104.
} 
power. The company has developed three categories of alkali metal nanostructured silica materials (M-SG) which differ in the reductive reactivity compared with neat alkali metals.

The literature described a large number of applications of the reagents in organic chemistry including cleavage of sulfonamides, ${ }^{72}$ ester reductions, Birch reactions and debenzylations. ${ }^{73}$ The examples shown in Scheme 2.28 were conducted in ethereal solvents, typically in THF with 2.5-5 equivalents of $\mathrm{M}-\mathrm{SG}(\mathrm{I})$ at room temperature and demonstrate the efficacy of this class of reagents in debenzylation reactions.
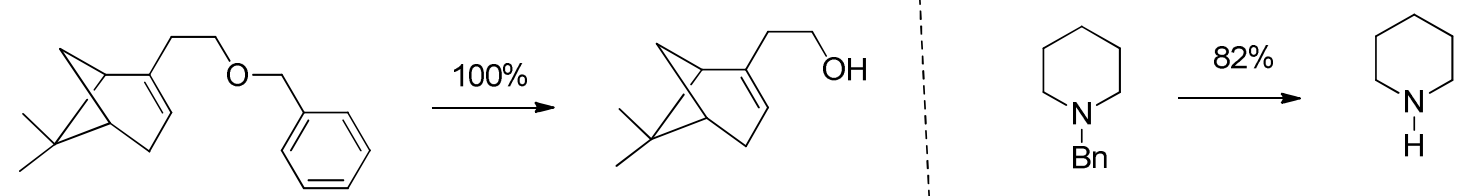

Scheme 2.28 Applications of M-SG.

Even if the only reagent available in the market is formed by a mixture of sodium and potassium ${ }^{74}$ we decided to test its ability to cleavage the $\mathrm{C}-\mathrm{N}$ benzylic bond on our compound. We thought it could replace the lithium metal avoiding at the same time the typical problems related to its manipulation and, as a consequence, the formation of the by-products.

Thus, a solution of compound $\mathbf{2 3}$ in THF was treated with Na-SG(I) at room temperature and, after 12 hours the reaction showed the formation of the desired amino product with modest yield. Indeed the best results were observed when a sub-stoichiometric amount of ethylenediamine (EDA) was added. From compound $\mathbf{2 3}$ and using the reaction conditions described above, we obtained the $N$-protected amino alcohol 18 in low overall yield, which were not able to improve.
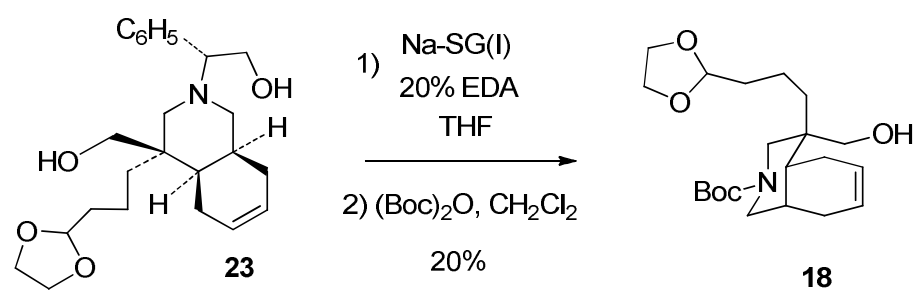

+ starting material

Scheme 2.29 Reaction with Na-SG(I).

\footnotetext{
${ }^{72}$ Nandi, P.; Redko, M. Y.; Petersen, K.; Dye, J. L.; Lefenfeld, M.; Vogt, P. F.; Jackson, J. E. Org. Lett. 2008, 10, 5441-5444.

${ }^{73}$ Nandi, P.; Dye, J. L.; Jackson, J. E. Tetrahedron Lett. 2009, 50, 3864-3866.

${ }^{74}$ Stabilized lithium-alumina gel materials (M-AG) has been recently developed by the same company but it isn't available in the market yet.
} 


\subsubsection{Cleavage of Chiral Inductor by Mesylation/Elimination}

It is worth mentioning others attempts of removal the phenylglycinol chiral auxiliary from amino-substrates which are reported in the literature and contemplate a two steps sequences. $^{75}$

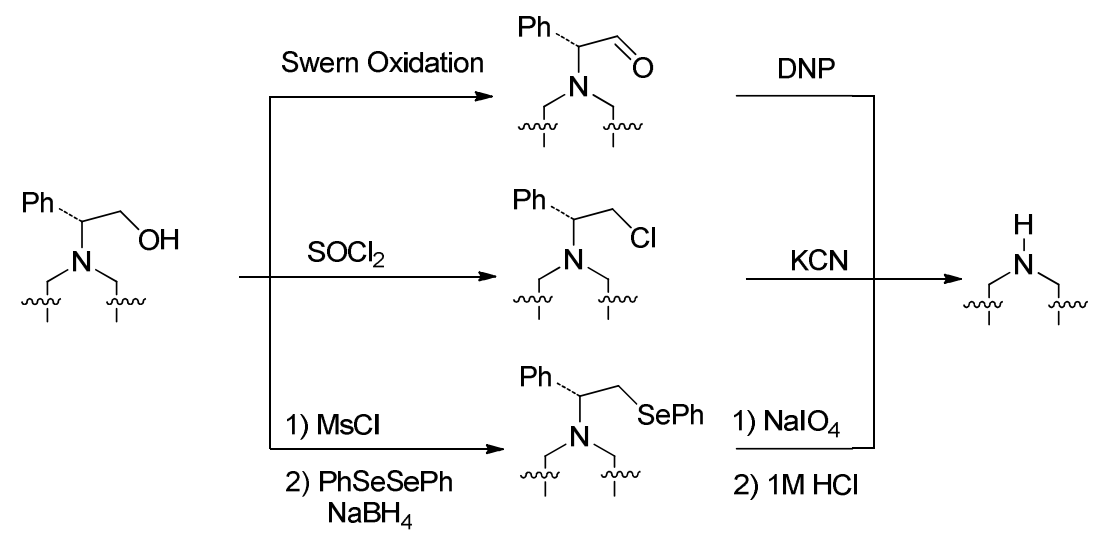

Figure 2.11 Miscellaneous methodologies.

None of this methodology is viable for our purpose, due to the presence of the hydroxymethyl chain at the C-9 position of our compound $\mathbf{2 3}$, which would have to be selectively reduced and protected in an early stage of the synthesis. Nevertheless we focused our attention on the formation of the mesyl and chloride intermediates of the last two entries (see Figure 2.12).

Theoretically a mesylation of both primary alcohols would lead to compound 29, while, a subsequent elimination reaction, would take place only in the hydroxymethyl chain of the phenylethanol fragment affording 30. In summary a two steps process would generate an enamine which can be hydrolyze with $\mathrm{HCl}$ leading to the free amine compound 31: an advanced intermediate of our synthetic sequence.

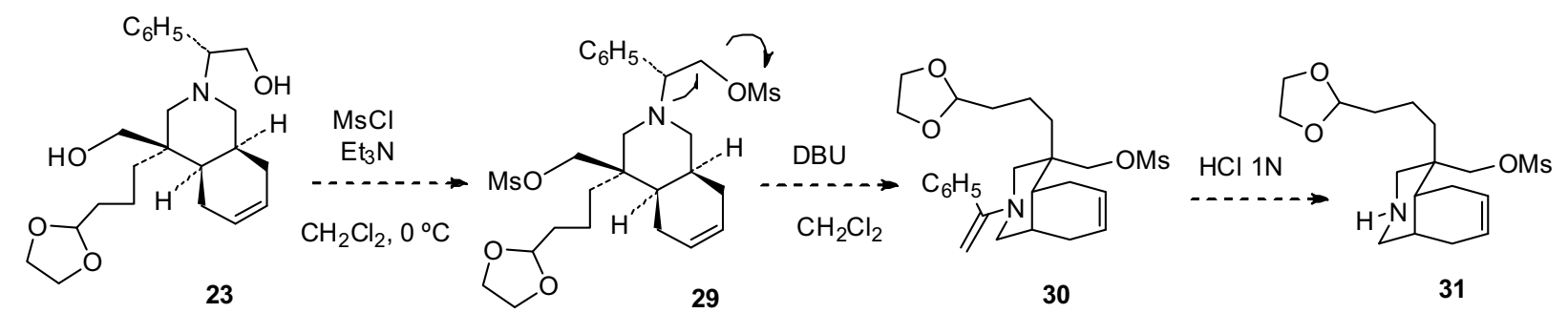

Scheme 2.30 Mesylation/elimination strategy.

\footnotetext{
${ }^{75}$ (a) Lebeuf, R.; Robert, F.; Schenk, K.; Landais, Y. Org. Lett. 2006, 8, 4755-4758. (b) Andrés, J. A.; Herráiz-Sierra, I.; Pedrosa, R.; Pérez-Encabo, A. Eur. J. Org. Chem. 2000, 1719-1726. (c) Agami, C.; Couty, F.; Evano, G. Tetrahedron: Assimetry 2000, 11, 4639-4643.
} 
Unfortunately due the nucleophilicity of our tertiary amine we were not able to obtain the double mesylated compound $\mathbf{2 9}$ and the reaction led only to the formation of the aziridinium ion 32 which forced us to leave this route.
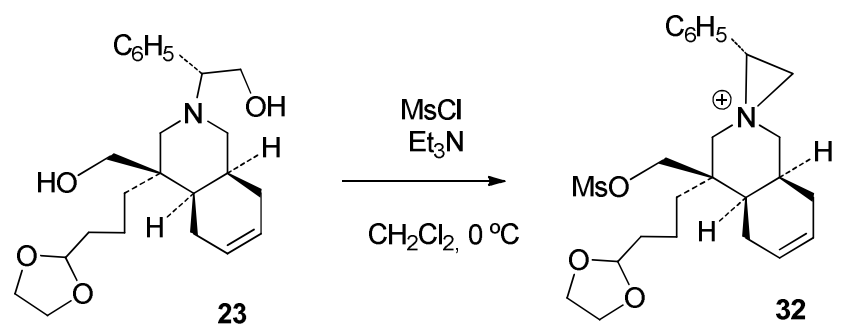

Scheme 2.31 Formation of aziridinium compound.

Unsatisfied of all these results, but still motivated to find a way to reduce the number of synthetic steps, we decided to change our strategy and concentrate our efforts exploring alternative methodologies to perform the cleavage of the $\mathrm{C}-\mathrm{O}$ bond of the oxazolidine.

\subsubsection{Removal of the Chiral Inductor from Tricyclic Lactams}

\subsubsection{C-O Bond Cleavage Studies}

As already disclosed in the previous sections treatment of tricyclic lactam II under classical $\mathrm{TiCl}_{4}$ and triethylsilane conditions did not afford the expected reduced compound IV. ${ }^{76}$ In view of the difficulty to perform the chemoselective reduction of the oxazolidine $\mathrm{C}-\mathrm{O}$ bond it was decided to try various combinations of reducing agents and Lewis acids.

Despite the exploration of several reaction conditions and reagents we couldn't achieve the desired compound confirming what was previously mentioned about the influence of the substituent at the C-9 position of lactam.

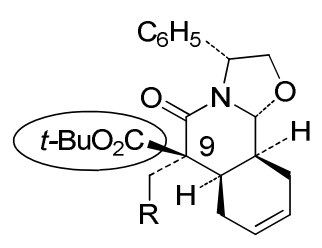

II

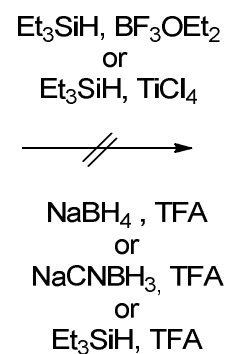

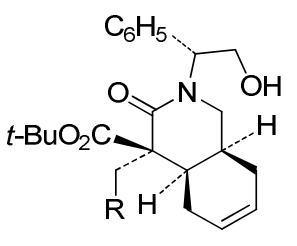

IV

$\mathrm{Et}_{3} \mathrm{SiH}, \mathrm{TFA}$

Scheme 2.32 Reductive ring opening reaction.

\footnotetext{
${ }^{76}$ Snyder, L.; Meyers, A.I. J. Org. Chem. 1993, 58, 36-42.
} 
With the aim of find out a solution and establish if the behavior of these compounds depends on the presence in the piperidine ring of a specific substituent or on the inductive proprieties of the ester group at C-9 position, we decided to use lactams 33-36 as model compounds (Scheme 2.33).

Starting compound $\mathbf{3 3}$ was prepared with excellent yield treating lactam $\mathbf{2}$ with a solution of LiHMDS and the appropriate chloroformiate. As predictable, compound $\mathbf{3 3}$ was not reduced under $\mathrm{TiCl}_{4}$ and triethylsilane conditions. Thus the ester moiety was transformed into primary alcohol and $\mathbf{3 4}$ subjected to reduction with a combination of $\mathrm{Et}{ }_{3} \mathrm{SiH} / \mathrm{TiCl}_{4}$ or $\mathrm{Et}_{3} \mathrm{SiH}_{3} \mathrm{BF}_{3} \mathrm{OEt}_{2}$, but in all cases the oxazolidine ring opening reaction didn't take place. The same conditions were then used with the corresponding mesylate $\mathbf{3 5}$ and azide $\mathbf{3 6}$ derivatives but again the results were unsatisfactory.

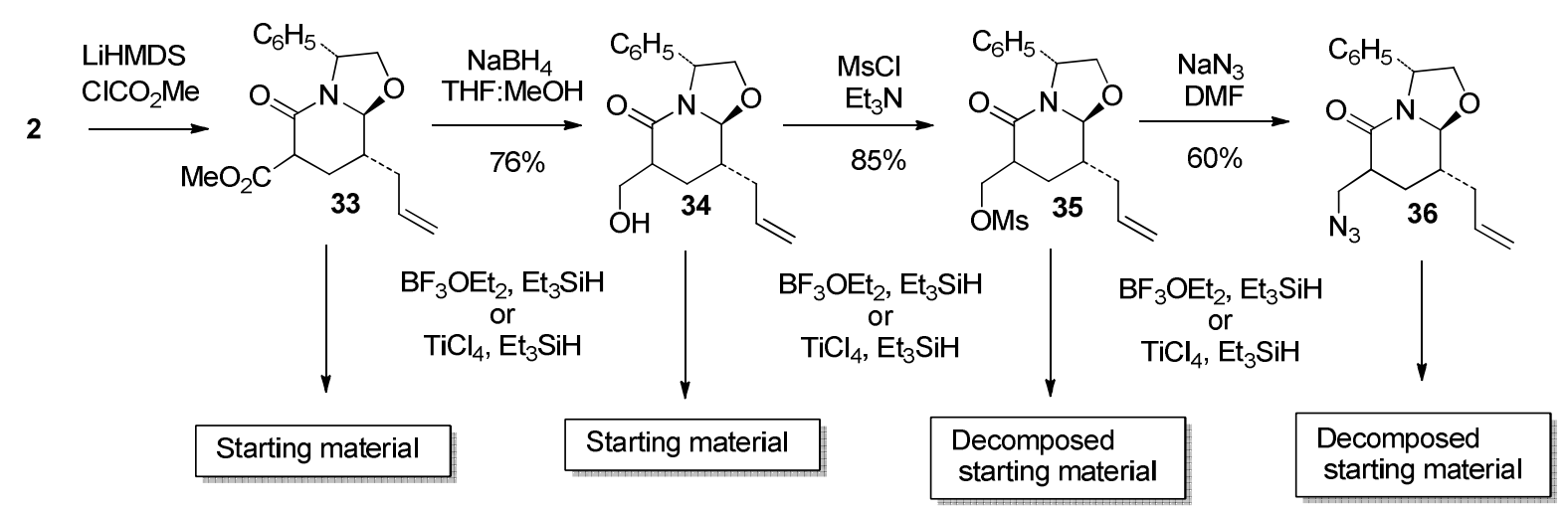

Scheme 2.33 Attempts on a model lactam.

The results presented above point out the interference of some substituents at the C-9 position which dramatically hinder the oxazolidine reduction and suggest the use of a different methodology to overcome the problem.

\subsubsection{C-N bond Cleavage Studies}

In view of these considerations we decide to overturn the process cleaving in the first place the $\mathrm{C}-\mathrm{N}$ benzylic bond. The $\mathrm{C}-\mathrm{O}$ bond of resulting hydroxyl- or alkoxy-lactam could be easily reduced in a later stage with conventional methods.

As mentioned above the methodology that involves the use of sodium metal in liquid ammonia is able to cleave the $\mathrm{C}-\mathrm{N}$ benzylic bond, with excellent yields, in amides but poorly in amines. With these observations in mind our plan to remove the chiral inductor would involve an inversion of the order of the classical two step methodology (see Section 2.3). Hence the C-N reductive opening oxazolidine reaction followed by a Lewis acid-promoted reduction of the 
resulting hidroxy lactam would led to the free amide compound $\mathbf{X I X}$. Subsequent reductions of carbonylic functions with $\mathrm{LiAlH}_{4}$ followed by $\mathrm{N}$-Boc protection of the resulting secondary amine would finally afford compound 18.

Unfortunately, as already argue, dissolving-metal reductions are very effective reactions but, due its complicated experimental protocol, lack in selectivity. Thus, as predictable, the reaction was able to perform the oxazolidine opening reaction and afforded the corresponding alkoxyand hydroxyl- lactams together with products derived by the reduction of the ester group. Afterwards the complex mixture of unstable compounds was treated with $\mathrm{Et}_{3} \mathrm{SiH}$ or $\mathrm{NaCNBH}_{3}$ and alternatively $\mathrm{TiCl}_{4}$ or TFA but, the conversion into the desired free amide compounds $\mathbf{3 7}$ and $\mathbf{3 8}$, was obtained with low unacceptable yields $(<25 \%)$.

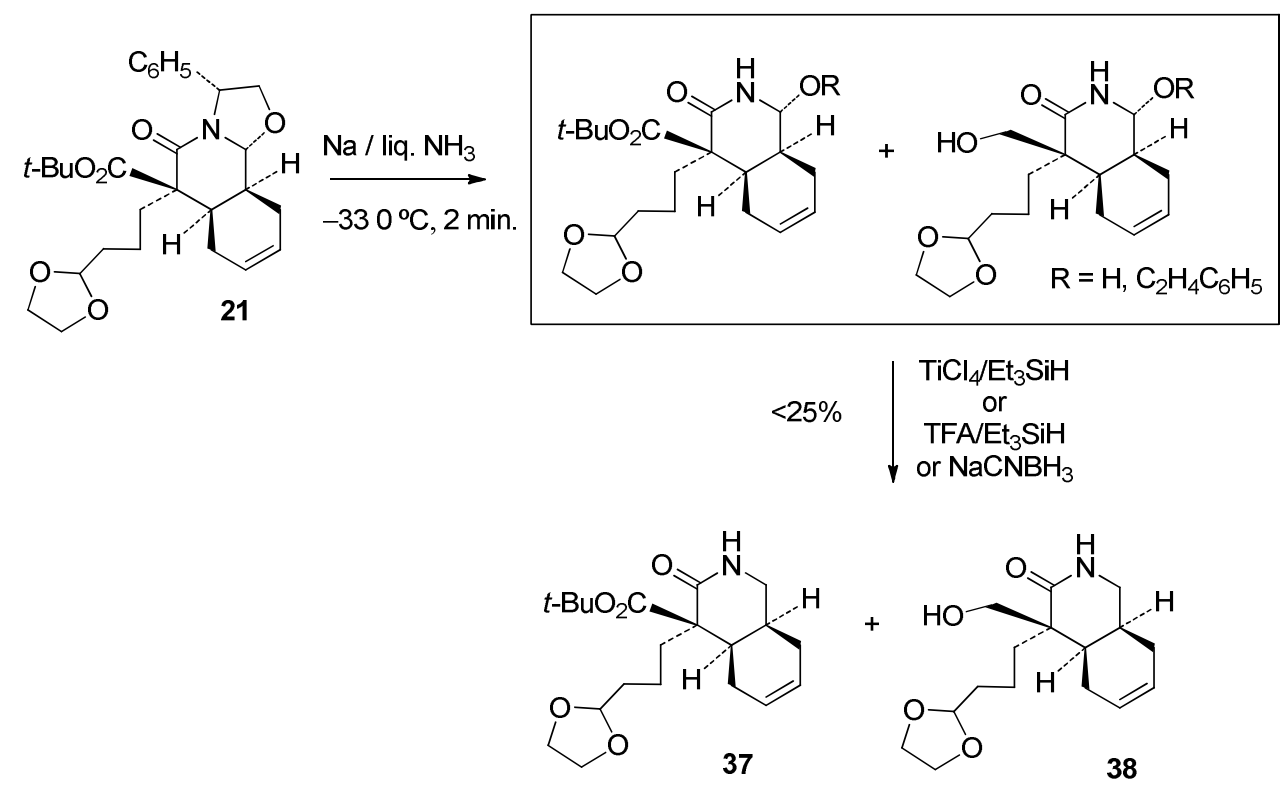

Scheme 2.34 C-N bond cleavage methodologies.

Interestingly, treatment of the crude resulting unstable products with a high excess of $\mathrm{LiAlH}_{4}$ (15 equiv.) in refluxing dioxane during a period of 48 hours provoked the contemporary reduction of the hemiaminal moieties and both carbonylic groups affording, as only product, the aminoalcohol $\mathbf{1 7}$. The protection of the secondary amine with di-tert-butyl-dicarbonate finally gave the desired $\mathrm{N}$-Boc derivate $\mathbf{1 8}$ with a considerable overall yield of $45 \%$.

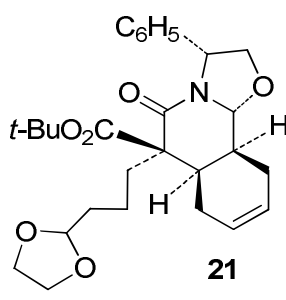

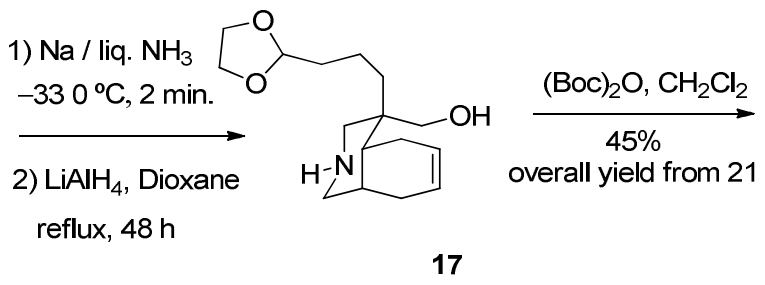<smiles>O=C(O)N1CCCCC1(O)CCC1OCCO1</smiles>

Scheme 2.35 Cleavage of phenyl ethanol moiety 
This methodology represents an important breakthrough in the synthesis of the azabicyclic intermediate 18. We have definitely found a reliable way to cleave the phenylglicynol chiral inductor from our tricyclic compound and consequently fix our main synthetic hindrance. The advantages resulting from this improvement are outstanding and the return to our "original synthetic plan" permitted the synthesis of the fully-functionalized azabicyclic compound $\mathbf{1 8}$ in a faster and scalable easier way.

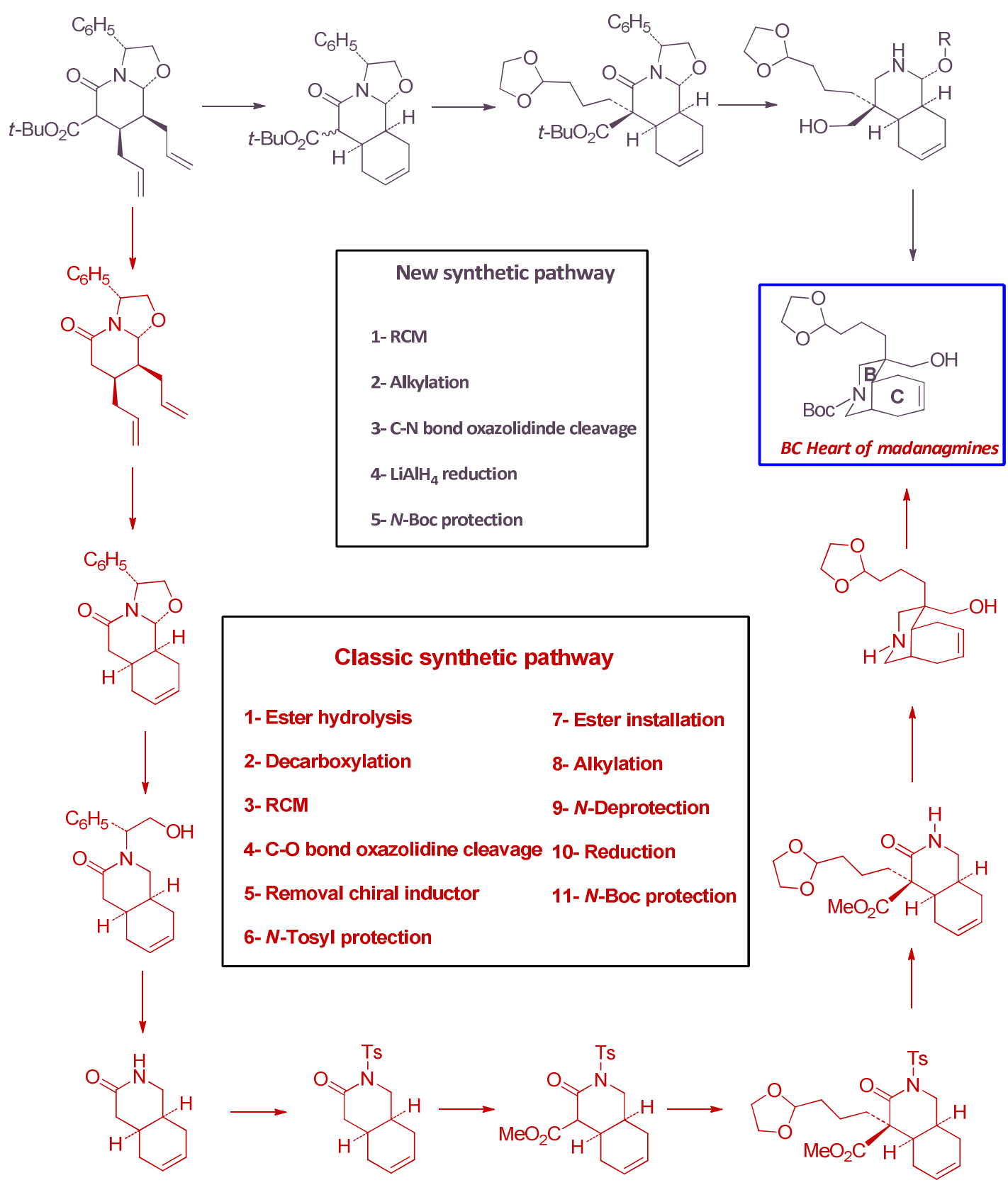

Figure 2.12 Synthetic routes toward BC system.

The new synthetic sequence, represented below, excludes the two main drawbacks of the previous strategy represented by the removal and re-introduction of the alkoxycarbonate in 
the $\mathrm{C}-9$ position and the nitrogen protecting group changes. This route allows the production of an important scaffold, direct precursor of the $A B C$ central core of madangamine, in only five scalable steps, in contrast to the eleven steps of the previous sequence, permitting to save a lot of time and precious starting material during the process.

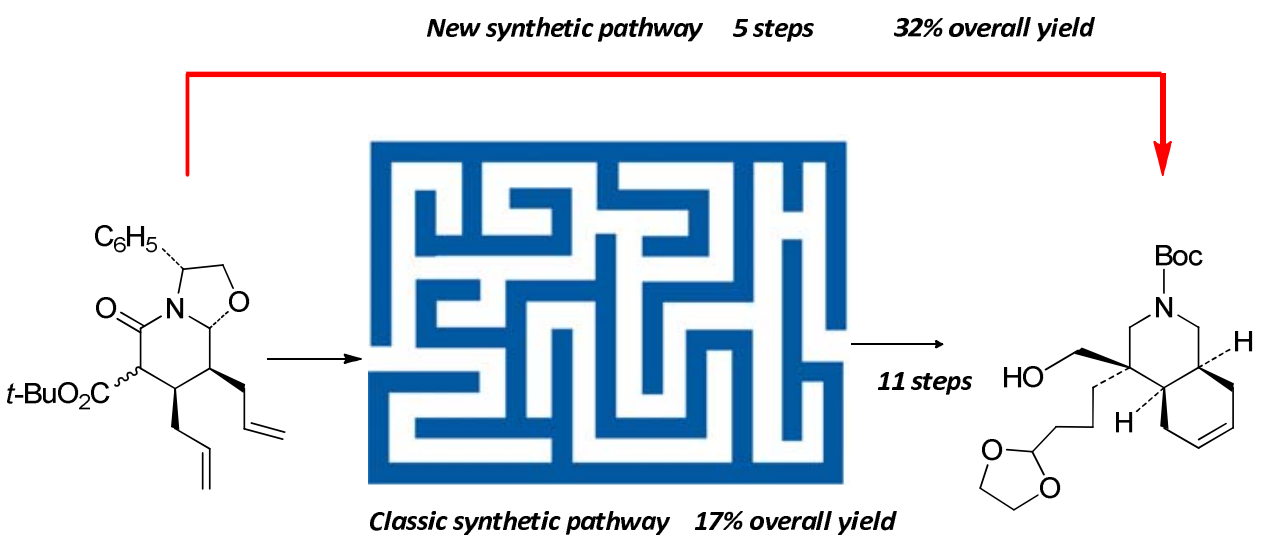

Figure 2.13 Improvements of the new strategy.

\subsection{SYNTHESIS OF ABC TRICYCLIC CORE OF MADANGAMINE}

With a solid and scalable synthetic sequence, which allows the access to the bicyclic heart of madangamine, the next challenge has been represented by the construction of the central diazatricyclic ring system common to madangamines A-E.

The azabicyclic compound $\mathbf{1 8}$ possesses two main features we can use to assemble the $A B C$ system:

- The primary alcohol at the C-9 position, with an appropriate spatial arrangement, which can be easily converted into an aminomethyl moiety.

- The endocyclic double bond in the $C$ ring which would take part in the aminohydroxylation process leading contemporary to the construction of the tricyclic system, the formation of the C-2 stereocenter and the C-3 functionality, essential for the construction of the macrocyclic $\mathrm{E}$ ring.

In summary our basic idea is to assemble the piperidine A ring performing an intramolecular aminohydroxylation reaction between the amino moiety, easily obtainable from the primary alcohol, and the unsaturation among the ciclohexene $\mathrm{C}$ ring. 


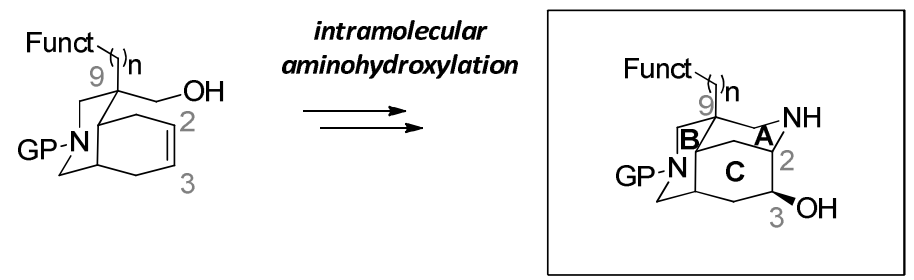

Figure 2.14 Assembly of the diazatricyclcic core.

The synthetic sequence started with transformation of the hydroxyl functional group of $\mathbf{1 8}$ into the azide compound which would serve as masked amino functionality. Azide $\mathbf{4 0}$ was first tried to introduce under Mitsunobu conditions but the result of the process was unsatisfactory and a two steps sequence synthesis via mesylate was found to be necessary. Even under these conditions the transformation of mesylated compound $\mathbf{3 9}$ into the required azide $\mathbf{4 0}$ proceeded slowly and required strong reaction conditions to reach a satisfactory conversion.

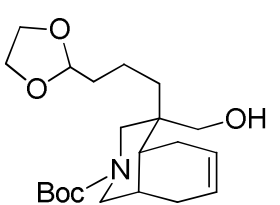

18

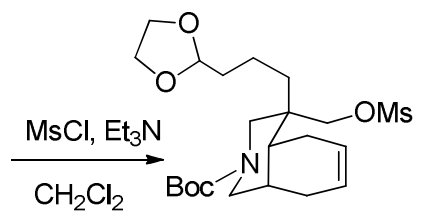

39

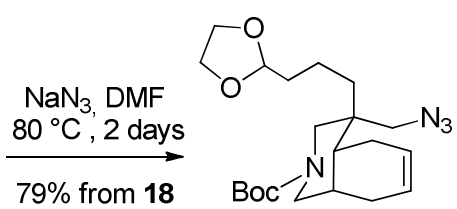

40

Scheme 2.36 Preparation of azide compounds.

With the bicyclic azide $\mathbf{4 0}$ in hands we started our aminohydroxylation studies that would lead to the construction of the diazatricyclic system.

In a first generation synthesis of the $A B C$ system, ${ }^{51}$ we were inspired by the construction of the tricyclic core described by Weinreb, ${ }^{37}$ which involves an aminomercuriation reaction followed by oxidation. ${ }^{77}$ The application of this strategy started with the reduction of the azide XVII into the amine XVIII and culminated with the first enantiopure synthesis of the diazatricylic system of madangamines XIX which confirm the great synthetic potentiality of our retrosynthetic analysis.

\footnotetext{
${ }^{77}$ For further applications of the aminomercuration reaction in total synthesis, see: (a) Saitoh, Y; Moriyama, Y.; Takahashi, T.; Khuong-Huu, Q. Tetrahedron Lett. 1980, 21, 75-78. (b) Kametani, T.; Suzuki, Y.; Honda, T. J. Chem. Soc., Perkin Trans.1, 1986, 1373-1377. (c) Kalinin, V. N.; Moiseev, S. K.; Bakhmutov, V. I.; Chrepanov, I. A. J. Organom. Chem. 1990, 383, 85-91. (d) Bai, X.; Mascarella, W.; Bowen, W. D.; Carroll, F. I. J. Chem. Soc., Chem. Commun. 1994, 2401-2402.
} 


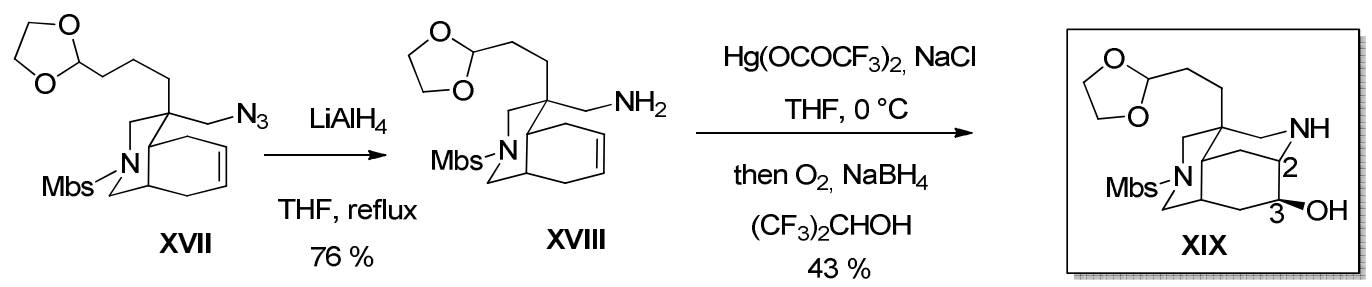

Scheme 2.37 Aminomercuriation methodology.

Unfortunately the process was tedious and a large and complicated experimental protocol was required. Furthermore the low yield associated to the process and the use of some toxic or expensive reagents limit a large scale synthesis of the core, essential condition to reach our final target. Thus we decided to replace this procedure with a more convenient one.

Hence the new methodology, design for the construction of the diazatricyclcic core of madangamine, was centered on the use of an alternative aminohydroxylation reaction. This strategy would avoid the use uncomfortable operational procedure preserving the capability to assemble the piperidinic $A$ ring and installing the C-2 and C-3 stereocenters in an efficient and simple procedure which provided the proper 1,2-amino alcohol.

Our attention was then turned to the possibility to reach our target molecule using, as a key step, a nucleophilic epoxide opening reaction as alternative of the aminomercuriation procedure. The outcome of the process depends on the stereoselechemical course of the epoxidation of the endocyclic double bond which has to be exo-selective. The stereospecific endo-face nature of the nucleophilic attack at the epoxide ring with an appropriate amine function would guarantee the formation of the 1,2 aminoalcohol with an anti-selectivity. Thus, the azide $\mathbf{4 0}$ was treated with meta-chloroperbenzoic acid to provoke the cyclohexene double bond epoxidation. As expected the reaction proceeded with a perfect exo-selectivity affording the azido-epoxide compound $\mathbf{4 1}$ as only isomer. This stereochemical result can be explained taking into account that the epoxidation reaction occurs from the less hindered exo-face of the compact convex architecture of our bicyclic system.

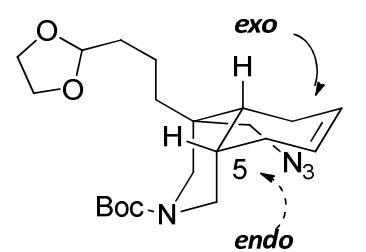

40

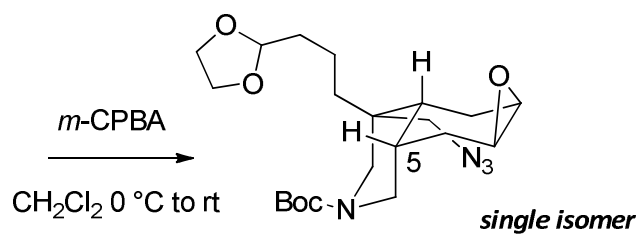

41

Scheme 2.38 Stereoselective epoxidation.

The resulting epoxide compound $\mathbf{4 1}$ was subjected to a selective reduction of the azide function through a mild Staundinger reaction allowing the formation of the resulting amine 
which underwent a smooth in situ cyclization leading to the closure of the piperidine ring $A$ and the formation of the resulting enantiopure diazatricyclic alcohol 42. In view of these observations appear evident that the stereochemical course of the reaction has been directed by the geometrical disposition of the aminomethyl chain and its endo-nucleophilic attack to the epoxide ring. ${ }^{51}$

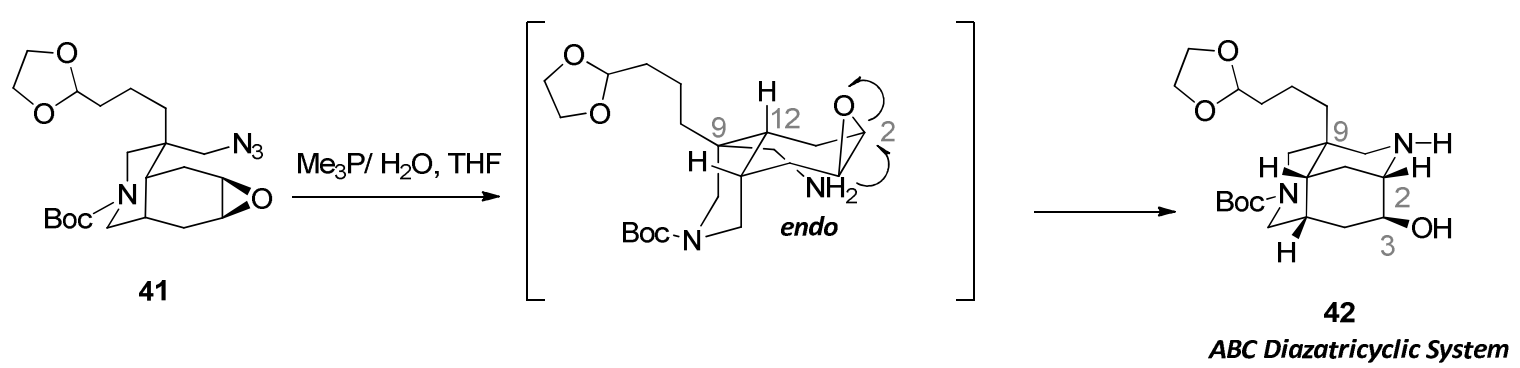

Scheme 2.39 Closure of A ring.

The resulting tricyclic system $\mathbf{4 2}$ was immediately protected as $\mathbf{N}$-tosyl derivatives $\mathbf{4 3}$ while the tertiary alcohol at $\mathrm{C}-3$ position was converted in the benzylic ether affording the fullyfunctionalized $A B C$ diazatricyclic system $\mathbf{4 4}$.

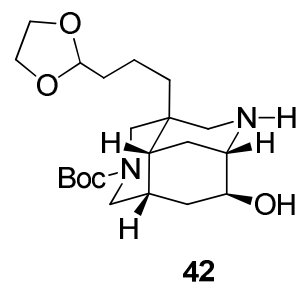

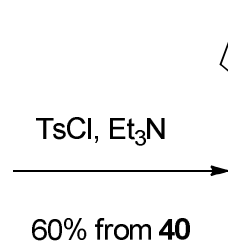

$60 \%$ from 40

42

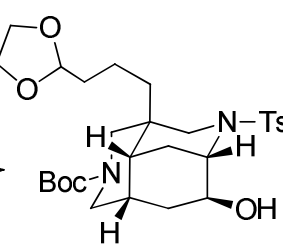

43

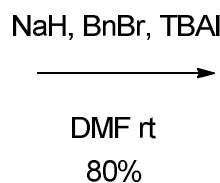

$80 \%$

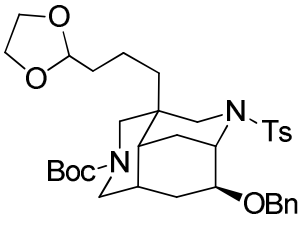

44

The methodology described set a big improvement in term of yields and simplicity: the aminohydroxilation reaction was carried out without the use of uncomfortable experimental processes and dangerous reagents like mercury or oxygen. The excellent yields of every step permitted to avoid unnecessary chromatographic purifications and $\mathrm{N}$-tosyl, $\mathrm{O}$-benzyl protected tricylic compound $\mathbf{4 4}$ was obtained with excellent yield.

In summary the diazatricyclic core, which would virtually permit the access to madangamine A$E$, has been enantioselectively assembled in a fast and scalable way, with the suitable functionality at the C-3 and C-9 positions which would permit to address the construction of both western and eastern macrocyclic $\mathrm{D}$ and $\mathrm{E}$ rings. 


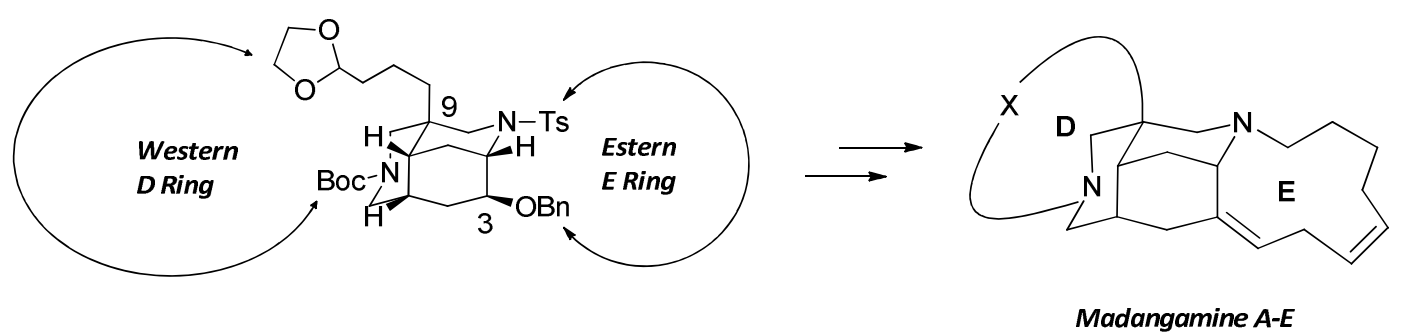

Figure 2.15 Fully-functionalized diazatricyclic ring. 

ㄴ...... 


\subsection{INTRODUCTION}

With a robust and convenient synthesis of the tricyclic system in hand we focused our attention on the second part of our synthetic plan which involves the construction of the macrocyclic rings of madangamine. Our studies started with the assembly of the western macrocyclic $D$ ring and then proceeded with the construction of the common unsaturated eastern E ring which would permit to complete the first total synthesis of the alkaloid.

One of the most evident advantages of our synthetic approach is that, starting from a common simple building block, we can virtually accede to all type of madangamine. Indeed we can hypothetically introduce any type of carbon chain at the C-9 position, at an early stage of synthesis, allowing the entry to different functionalized diazatricyclic precursors. What we need now is to develop effective strategies for the construction of the macrocyclic rings $D$ from these functionalized-central cores.

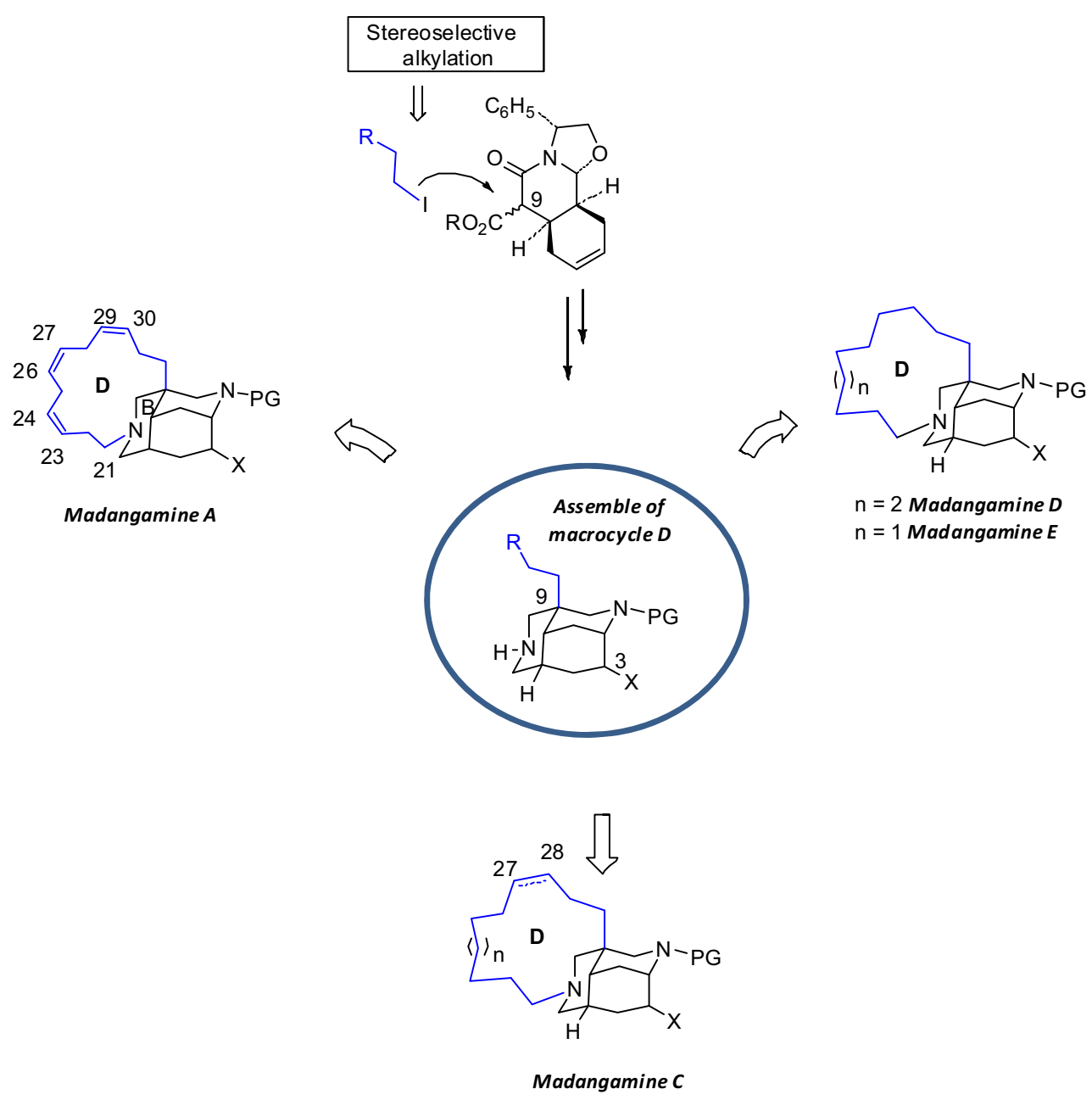

Figure 3.1 Synthetic plan for the annulation of macrocyclic $D$ rings. 
In the present section will be presented different approaches to assemble macrocyclic rings of various dimensions, completely unsaturated, presenting a single double bond or a Z-skipped system of olefins. The annulation reactions will be studied and optimized on simple piperidinic model compounds and the most promising methodologies will be applied on the diazatricyclic system.
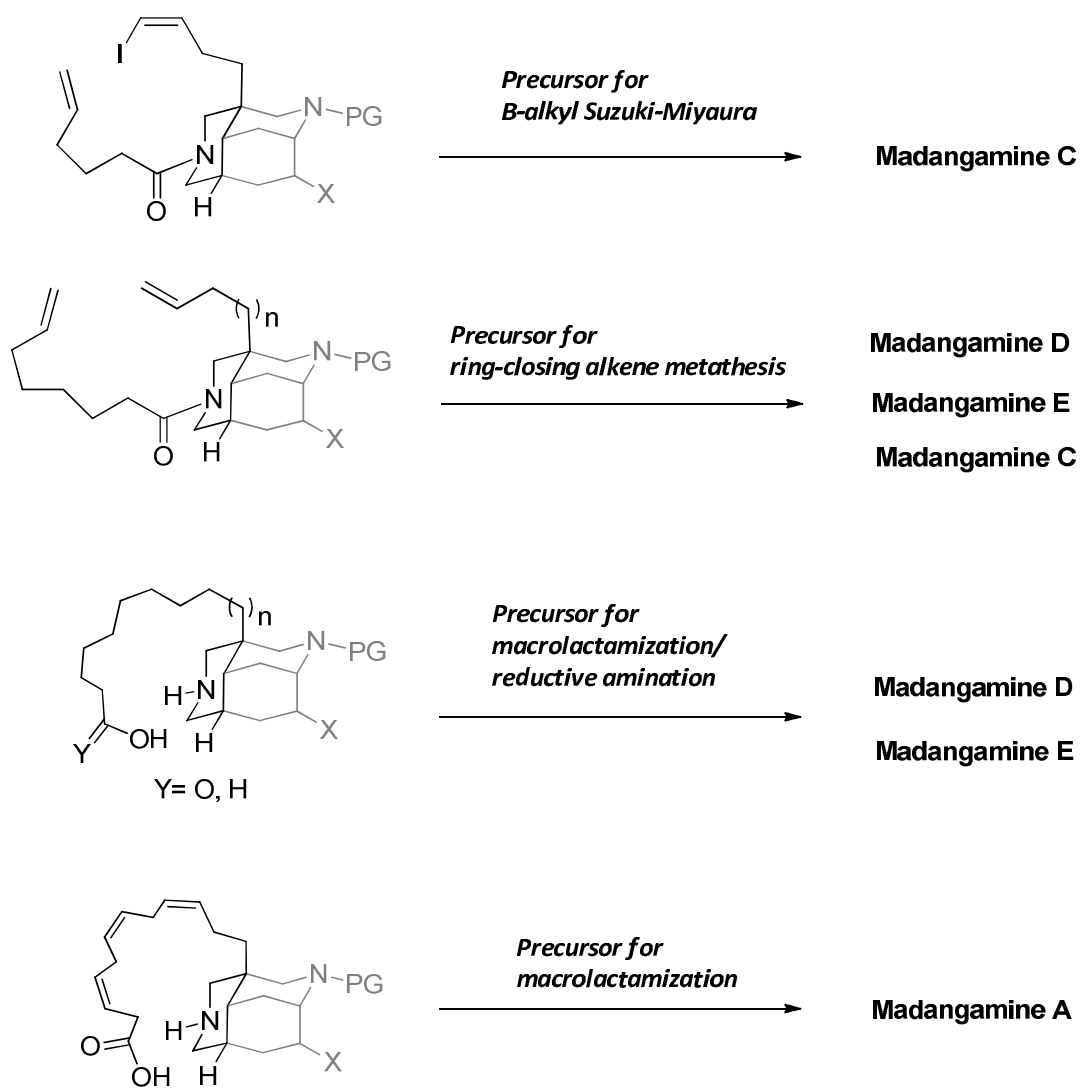

Precursor for

macrolactamization

Madangamine A

Figure 3.2 Possible synthetic pathways.

The use of a cheap and easy-to-prepare appropriate simplified model derivatives offers the possibility to work with structures which chemical behavior is, or would expected to be, similar to our diazatricylic compound. In most cases they have permitted to find the best experimental conditions or, at least, to predict the course of the reaction saving a lot of time and precious starting material. ${ }^{78}$.

\footnotetext{
${ }^{78}$ Proto, S.; Amat, M.; Pérez, M.; Ballette, R.; Romagnoli, F.; Mancinelli, A.; Bosch, J. Org. Lett. 2012, 14,
} 3916-3919. 
Once we will found an appropriate and viable methodology for the closure of the western $D$ ring, we will apply it in order to reach the construction the tetracyclic $A B C D$ system. Finally, the assembly of the eastern 11-membered $E$ ring we will be studied to provide first total synthesis of an alkaloid of the madangamine group.

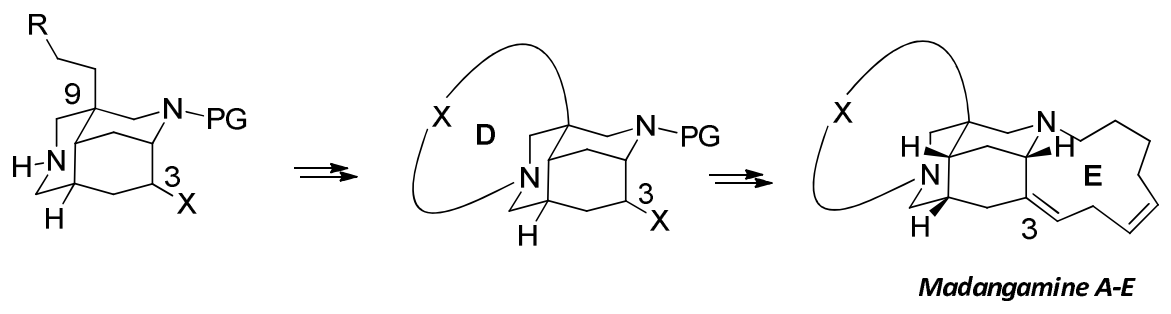

Figure 3.3 Annulation of E ring.

\subsection{CONTRIBUTION FROM PREVIOUS WORK}

As already mentioned in the previous section, part of the work presented herein has been made in conjunction with Dr. Stefano Proto with whom I share the first two years of my experimental studies. The outcome of this project has been possible thanks to this collaboration and the chemical heritage he left. Indeed I had the possibility to follow his footprints among the synthetic pathway together with the chance to learn from his errors. His experimental studies ended with the assembly of the tetracyclic $A B C D$ and $A B C E$ systems of madangamine $D$ but, due some complications during the process, he wasn't able to combine the two macrocyclization strategies to finally achieve the goal.

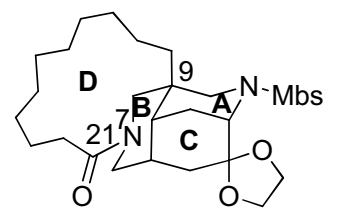

$A B C D$ System

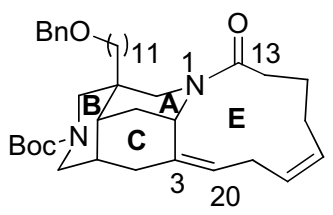

ABCE System

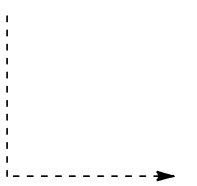

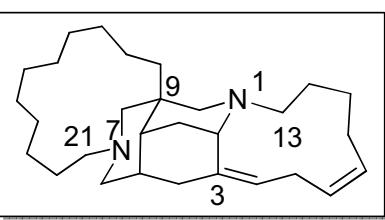

Madangamine D

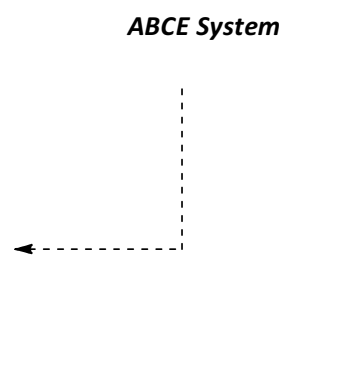

Figure 3.4 Previous work. 


\subsubsection{Macroclactamization Approach to the ABCD Tetracyclic System}

The studies concerning the elaboration of the western $D$ ring of madangamine $D$ started with the exploration of intramolecular macrolactamization and reductive amination reactions from diazatricyclic compound $\mathbf{X X}$ which incorporates a saturated 11-carbon chain at the C-9 quaternary carbon.

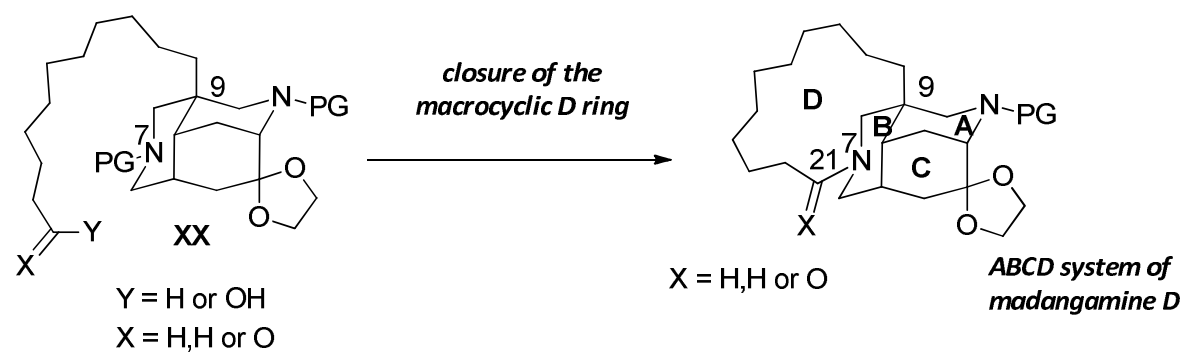

Figure 3.5 Macrolactamization and reductive amination reaction approach.

As already mentioned the use of a model compound played a guiding role in all our investigations representing a useful platform we can use to predict the viability of our plans.

Thus, $\delta$-valeroctam was used as starting material for the synthesis of the piperidinic model compound and, once protected as $\mathrm{N}$-Boc carbamate 45 , was acylated at the $\alpha \mathrm{C}=\mathrm{O}$ position with methylchloroformiate. This group increased the acidity of the proton at $\mathrm{C}-3$ permitting the subsequent alkylation with the 11-membered iodoalkyl chain. Alkylated compound $\mathbf{4 6}$ was then $N$-Boc deprotected and reduced with $\mathrm{LiAlH}_{4}$. Once re-protected as $N$-Boc carbamate the resulting hydroxymethyl chain was converted into the benzyl ester $\mathbf{4 7}$ which was finally treated with TBAF to give free alcohol 48.

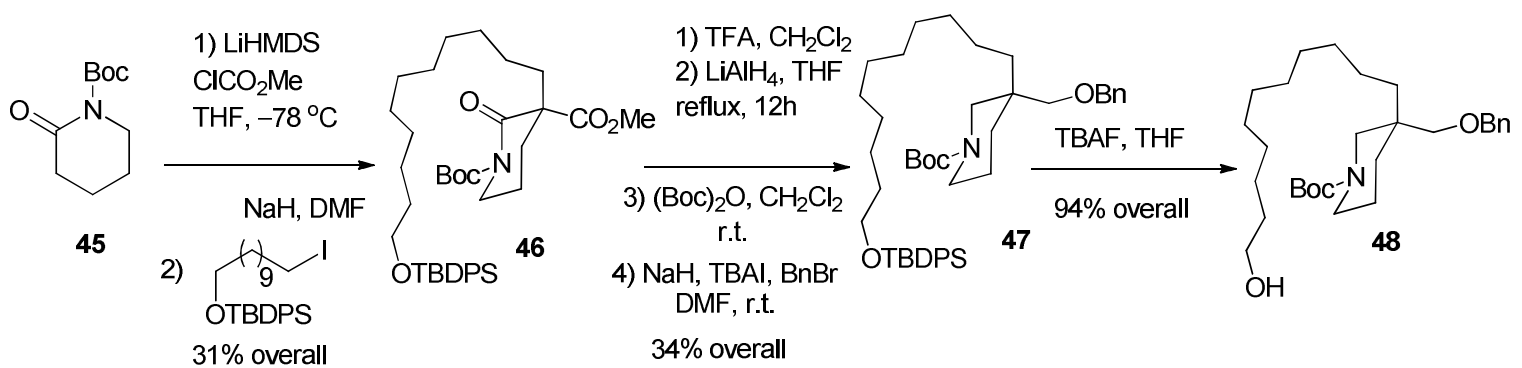

Scheme 3.1 Synthesis of model compound. 
Compound 48 was then subjected to oxidation alternatively with Dess-Martin periodinane to aldehide $\mathbf{4 9}$ or with PDC to carboxylic acid 51. The attempts to perform the macrocyclization reaction using a reductive amination approach, starting from the amino-aldehyde compound, allowed to assembly the macrocyclic D ring $\mathbf{5 0}$ with $40 \%$ yields while, starting from the corresponding aminoacid, the macrolactamization approach gave the D ring $\mathbf{5 2}$ with a $32 \%$ of yields.

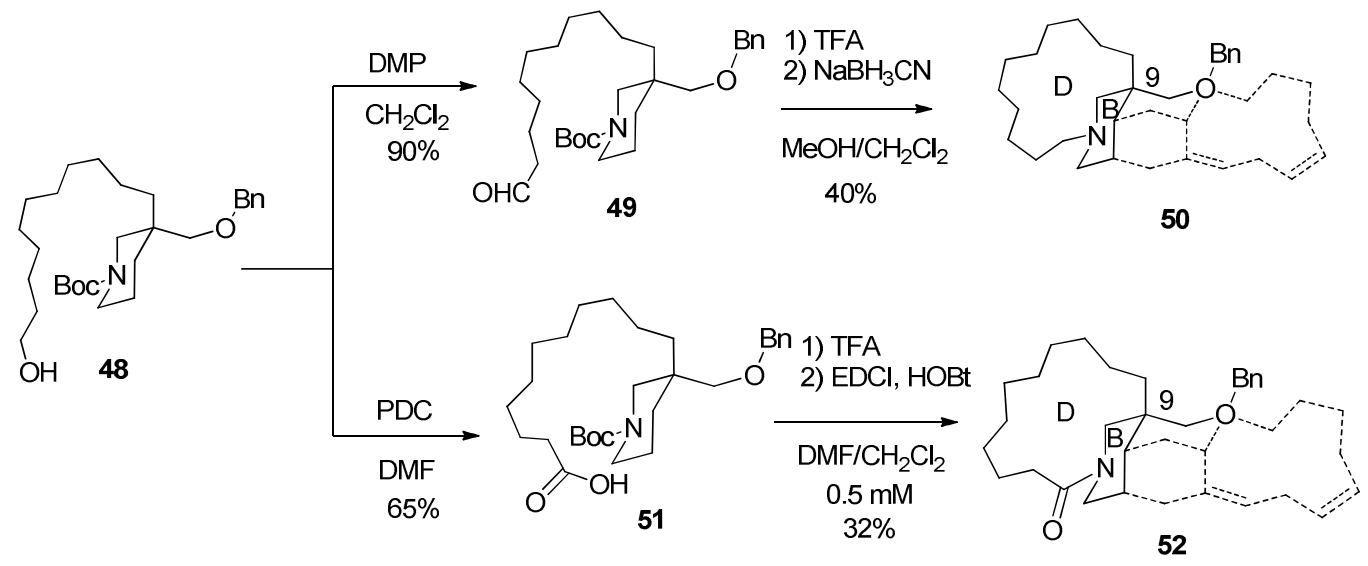

Scheme 3.2 Macrocyclization reactions.

Having achieved these results, and once we found the best experimental conditions, the same methodologies were then applied on the diazatricylcic system 53. The more promising reductive-amination reaction led only to the formation of oligomeric products while, with the macrolactamization methodology, it was possible to achieve the first synthesis of the $A B C D$ tetracyclic system of madangamine D $54 .^{47}$
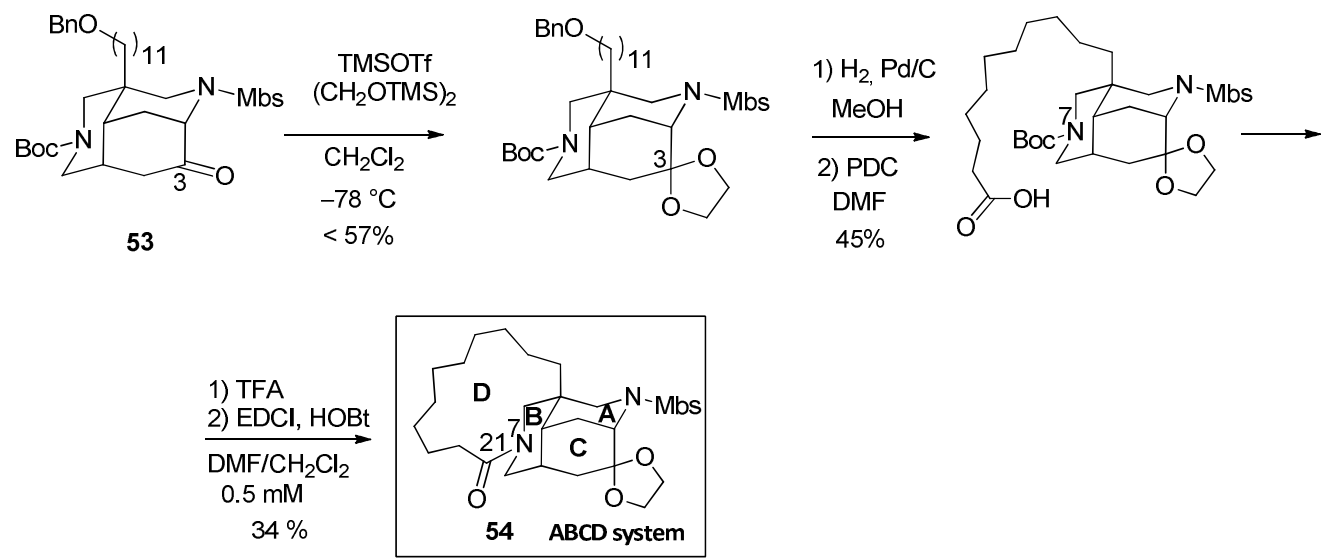

Scheme 3.3 Intramolecular macrolactamization. 
However intramolecular macrolactamization has proven to be a viable process to construct large membered macrocyclic rings, the results herein reported point out some limitations. The elevated number of degrees of freedom of the 11-membered carbon chain, which lack unsaturations, favored the formation of oligomeric products provoking a ruinous loss of material at this stage of the synthesis. Moreover the preceding synthetic steps, especially the protection of the ketone at C-3 and the oxidation of the primary alcohol to carboxylic acid, proceeded with moderate to low yields causing an important drop of the overall yield $(<9 \%$ from tricyclic compound XXI).

\subsection{ASSEMBLING OF THE D RING: ANNULATION STUDIES}

Thus, with these precious informations in hands, we decided to search for more convenient methodologies, able to assemble the $A B C D$ tetracyclic system of different type of madangamines and capable to provide enough material to complete the first total synthesis of the alkaloid.

\subsubsection{Construction of Macrocyclic D Ring of Madangamine C}

The western $D$ ring of madangamine $C$ possesses a single isolated Z-unsaturation among the macrocycle between positions 27 and 28 . This structure represents a very common motif found in different marine natural products like manzamine ${ }^{79}$ or nakadomarine ${ }^{80}$ which share the same biogenetic origins of our alkaloid.

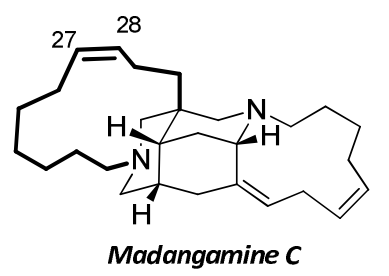

Figure 3.6 Structure of madangamine $C$.

The study of a methodology that could afford Z-isolated double bonds in "madangamine type" alkaloids was explored by Winkler ${ }^{81}$ in 1998 and later by Fukuyama ${ }^{82}$ in 2010. In their works

\footnotetext{
${ }^{79}$ Sakai, R.; Higa, T.; Jefford C.W.; Bernardinelli G.; J. Am. Chem. Soc. 1986, 108, 6404-6405.

${ }^{80}$ (a) Kobayashi, J.; Watanabe, D.; Kawasaki, N.; Tsuda, M. J. Org. Chem. 1997, 62, 9236-9239. (b) Kobayashi, J.; Tsuda, M.; Ishibashi, M. Pure Appl. Chem. 1999, 71, 1123-1126.

${ }^{81}$ Winkler, J. D.; Axten, J. M. J. Am. Chem. Soc. 1998, 120, 6425-6426.
} 
both achieved the formation of the unsaturated ring of nakadomarine $A$ and manzamine $A$, respectively, via macrocyclization reactions followed by a Lindlar-catalyzed partial hydrogenation of the alkyne.
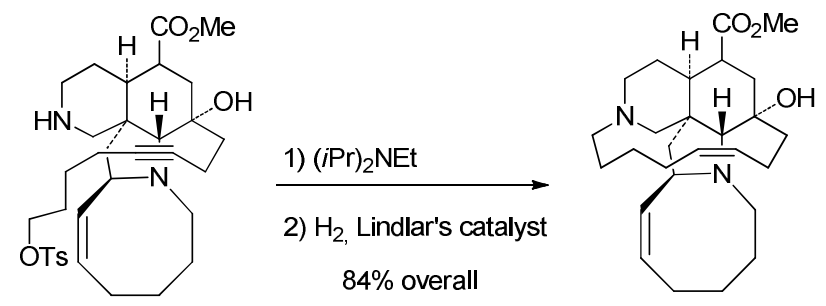

Figure 3.7 Annulation of macrocyclic ring of manzamine.

During the successive years the general strategy used to assemble these types of rings included ring-closing metathesis reactions which always produced a mixture of $Z / E$ isomers ${ }^{83}$ in which an HPLC separation to afford the pure $Z$ compounds is needed in many cases.<smiles>C=CCCCN1CCC2C(C(OC)OC)=CC3(CCC=C)OC(=O)N4C(C=C)CCC2C43C1</smiles>

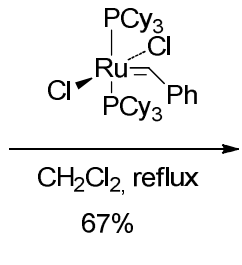<smiles>C=CC1CC23CN4CCCCC(CC4)C(C(OC)OC)=CC2C3OC(=O)N1</smiles>

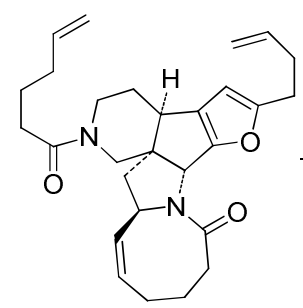
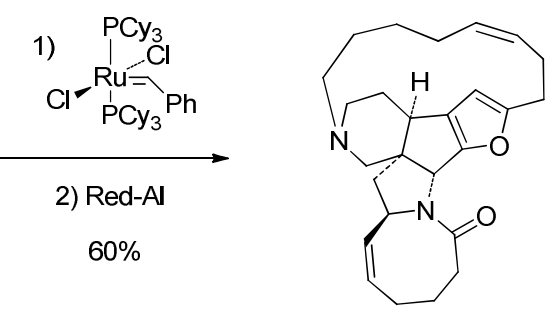

Figure 3.8 RCM macrocyclization strategies.

An interesting improvement addressed to overcome the lack of stereocontrol for the alkene geometry in RCM macrocyclizations, was introduced by Funk ${ }^{84}$ and Fürstner ${ }^{85}$. These authors

\footnotetext{
82 Toma, T.; Kita, Y.; Fukuyama, T. J. Am. Chem. Soc. 2010, 132, 10233-10235.

${ }^{83}$ (a) Ono, K.; Nakagawa, M.; Nishida, A. Angew. Chem. Int. Ed. 2004, 43, 2020-2023. (b) Young, I. S.; Kerr, M. A. J. Am. Chem. Soc. 2007, 129, 1465-1469. (c) Jakubec, P.; Cockfield, D. M.; Dixon, D. J. J. Am. Chem. Soc. 2009, 131, 16632-16633. (d) Cheng, B.; Wu, F.; Yang, X.; Zhou, Y.; Wan, X.; Zhai, H. Chem. Eur. J. 2011, 17, 12569-12572. (e) Martin, S. F.; Humphrey, J. M.; Ali, A.; Hillier, M. C. J. Am. Chem. Soc. 1999, 121, 866-867.
} 
accomplished the synthesis of the cis-unsaturated macrocyclic ring of nakadomarine $A$ through a ring-closing alkyne metathesis followed by Lindlar-catalyzed Z-stereoselective hydrogenation.
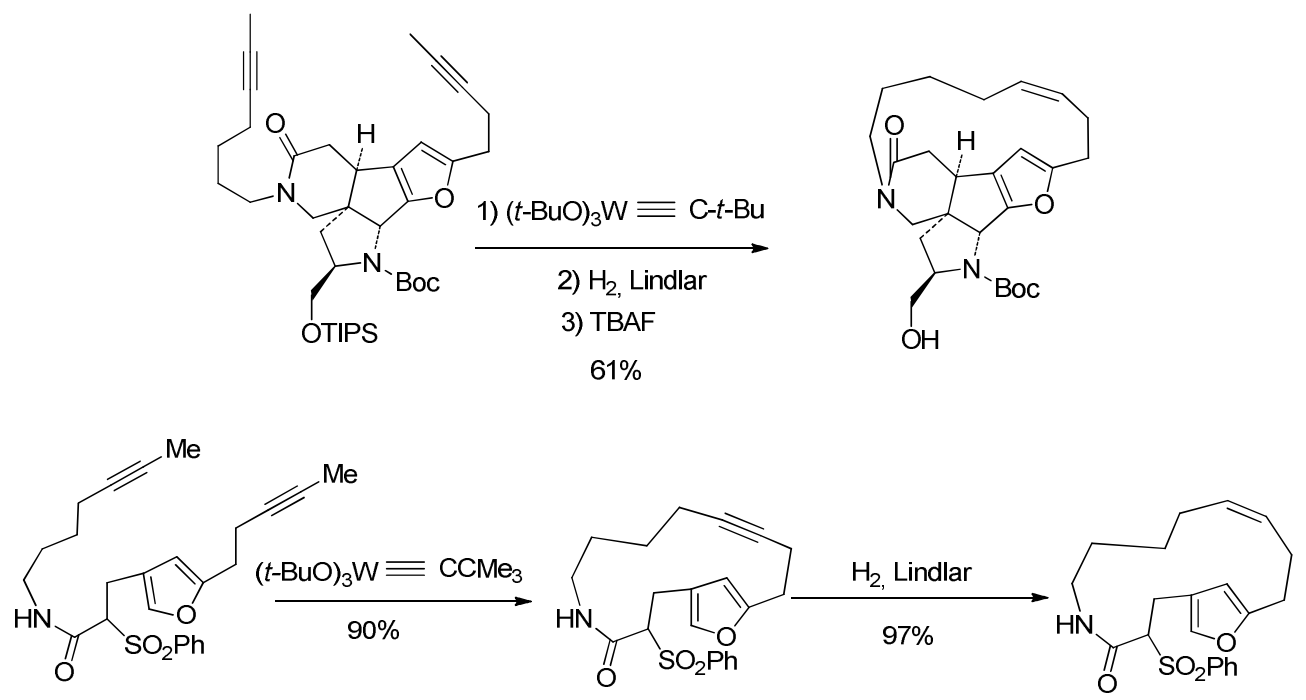

Figure 3.9 Ring-closing enyne metathesis strategies.

\subsubsection{Intramolecular B-Alkyl Suzuky-Miyaura Approach: Model Studies}

With the aim to analyze different methodologies able to generate large-dimensioned rings with control on the stereochemical outcome, we decided to explore the use of intramolecular $B$-Suzuki-Miyaura reactions. ${ }^{86}$ These cross coupling palladium-catalyzed reactions represent a powerful tool to form C (sp3)-C (sp2) bonds under mild conditions, in good yields and providing access to either $E$ - or Z-alkenes. In general only few methods have been reported for the highly selective access to Z-alkenes, presumably because of the thermodynamic control favors the lower-in-energy $E$-alkenes The cross coupling step in B-Suzuki-Miyaura reactions is stereospecific and results in the retention of the stereochemistry of the starting alkenyl halides: the control of the alkene isomer, therefore, has to be established before the cross coupling step.

Hence, starting from an aldehyde derivative bearing a terminal double bond, the Suzuki annulation process for the assembly of a macrocyclic ring involves three steps: the stereoselective formation of a Z-iodoalkene, the hydroboration reaction of the terminal olefin

\footnotetext{
${ }^{84}$ Nilson, M. G.; Funk, R. L. Org. Lett., 2010, 12, 4912-4915.

${ }^{85}$ (a) Fürstner, A.; Grela, K. Angew. Chem., Int. Ed. 2000, 39, 1234-1236. (b) Fürstner, A.; Guth, O.; Rumbo, A.; Seidel, G. J. Am. Chem. Soc. 1999, 121, 11108-11113.

${ }^{86}$ (a) Miyaura, N.; Ishiyama, T.; Ishikawa, M.; Suzuki, A. Tetrahedron Lett. 1986, 27, 6369-6321. (b) Miyaura, N.; Ishiyama, T.; Sasaki, H.; Ishikawa, M.; Satoh, M.; Suzuki, A. J. Am. Chem. Soc. 1989, 111, 314-321. (c) Oh-e, T.; Miyayra, N.; Suzuki, A. J. Org. Chem. 1993, 58, 2201-2208. (d) Sato, M.; Miyaura, N.; Suzuki, A. Chem. Lett. 1989, 1405-1408.
} 
with 9-BBN, ${ }^{87}$ and finally the palladium-catalyzed coupling reaction of the alkyl borane moiety with the Z-iodoalkene.

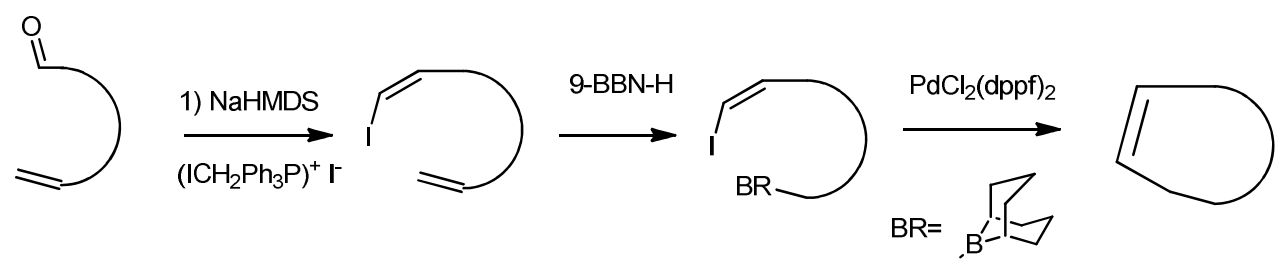

Figure 3.10 Intramolecular Suzuki annulation.

As already mentioned the reaction will be studied on a piperidinic model compound, synthesized from from commercially available $\delta$-valerolactam, which would allow the entry to diolefin compound $\mathbf{5 9}$ in only five steps.

The process started with the direct alkylation of the $\delta$-valerolactam with $\mathbf{5 5 ^ { 8 8 }}$ which afforded, with quantitative yield, functionalized compound 56. The reduction of the amide, and the subsequent acylation of the resulting amine $\mathbf{5 7}$ with $\mathbf{5}$-hexenoic acid, was followed by the hydrolysis of the acetal which led to the formation of aldehyde $\mathbf{5 8}$.

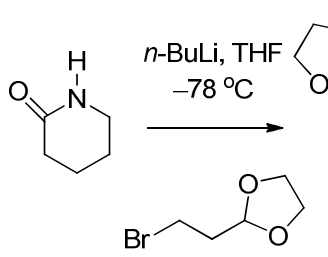

55

quant.
1)

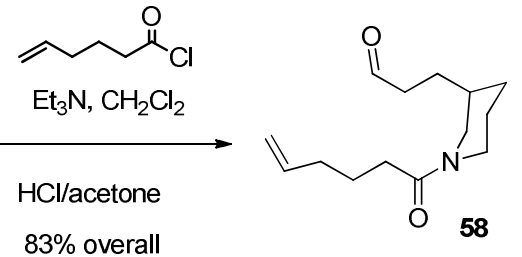

Scheme 3.4 Construction of model compound.

The synthesis of the Z-iodoalkene was achieved using the Stork-Zhao Wittig olefination. ${ }^{89}$ Thus, the protocol would involve the deprotonation of the iodoalkyl phosphonium salt with sodium hexamethyldisilazane to yield the ylide that would react with aldehydes to generate the Z-alkenyl halides in good to excellent selectivity. Not only disubstituted Z-alkenyl halides,

\footnotetext{
${ }^{87}$ (a) Burgess, K.; Van der Donk, W. A.; Jarstfer, M. B.; Ohlmeyer, M. J. J. Am. Chem. Soc. 1991, 113, 6139-6144.

88 (a) Potts, K. T.; Rochanapruk, T.; Padwa, A.; Coats, S. J.; Hadjiarapoglou, L. J. Org. Chem. 1995, 60, 3795-3805. (b) Magnus, P.; Rainey, T. Tetrahedron 2001, 57, 8647-8651.

${ }^{89}$ (a) Stork, G.; Zhao, K. Tetrahedron Lett. 1989, 30, 2173-2174. (b) Stork, G.; Zhao, K. J. Am. Chem. Soc. 1990, 112, 5875-5876.
} 
but also trisubstituted ones can be prepared using this method. ${ }^{90}$ These products are useful synthons in organic synthesis, especially in cross coupling reactions.

$$
\begin{aligned}
& \left(\mathrm{ICH}_{2} \mathrm{Ph}_{3} \mathrm{P}\right)^{+} \mathrm{I}^{-} \stackrel{\text { NaHMDS, THF, }-23{ }^{\circ} \mathrm{C}}{\text { then } \mathrm{RCHO},-78{ }^{\circ} \mathrm{C} \text { to } 22{ }^{\circ} \mathrm{C}} \underset{\mathrm{R}}{\stackrel{\mathrm{R}=\text { alkyl, }}{\text { aryl }}} \\
& 60-90 \% \text { yield } \\
& \text { Z:E 9:1 to } 98: 2
\end{aligned}
$$

Scheme 3.5 Synthesis of Z-iodoalkenes.

Unfortunately the application of this protocol, between aldehyde $\mathbf{5 8}$ and the required ylide, gave an inseparable mixture of the Z-vinyliodide $\mathbf{5 9}$ together with the undesired geminal diiodide 60 .

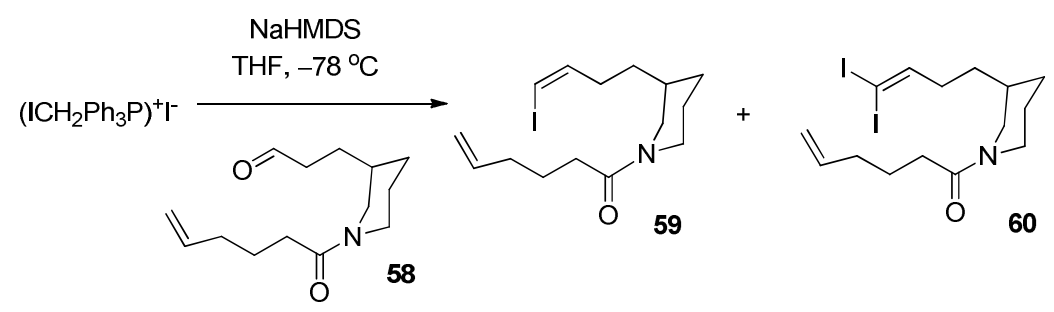

Scheme 3.6 Stork-Zhao Wittig reaction.

Similar results were obtained by the groups of Bestmann ${ }^{91}$ and Menche ${ }^{92}$ which tried to avoid the formation of byproducts by simply carrying out the reaction through an "inverted addition" of the phosphonium salt to the base.

As shown in Scheme 3.7 the formation of the undesired geminal iodide 60 can be explained considering an $\mathrm{I}-\mathrm{H}$ exchange caused by the slow addition of the base. This process could promote the nucleophilic attack of the ylide $\boldsymbol{a}$ to the phosphonium salt $\boldsymbol{b}$ which would bring to the formation of dimeric species $c$. The compound could undergo to another nucleophilic substitution, mediated by a iodide, that would finally lead to the formation of $\boldsymbol{d}$ and $\boldsymbol{e}$. The inverse addition of the base should avoid the first nucleophilic attack and consequentially the formation of the dimer $c$.

Following this procedure we were able to obtain the Z-iodoalkene $\mathbf{5 9}$ with a moderate $46 \%$ yield in a pure form.

\footnotetext{
${ }^{90}$ Chen, J.; Wang, T.; Zaho, K. Tetrahedron Lett. 1994, 35, 2827-2828.

91 Bestmann, H. J.; Rippel, H. C.; Dostalek, R. Tetrahedron Lett. 1989, 30, 5261-5262.

92 Li, P.; Li, J.; Arikan, F.; Ahlbrecht, W.; Dieckmann, M.; Menche, D. J. Org. Chem. 2010, 75, 2429-2444.
} 


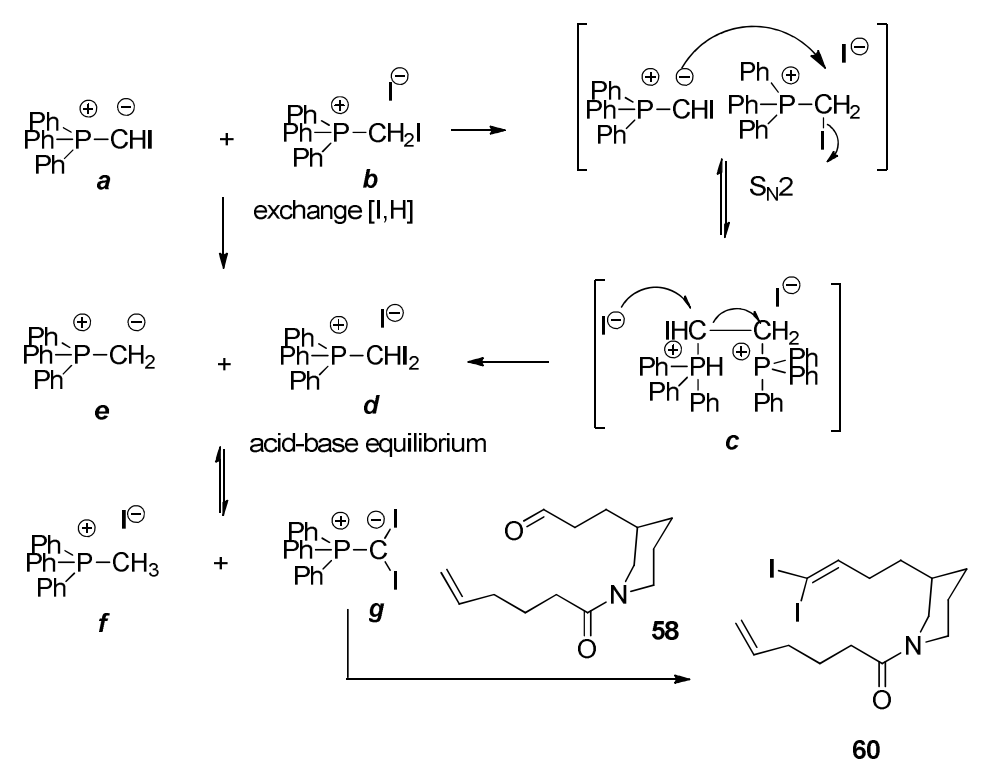

Scheme 3.7 Proposed mechanism of geminal diiodide.

With compound $\mathbf{5 9}$ in hand we started to study the intramolecular B-Suzuky-Miyaura coupling reaction that would give the unsaturated macrocyclic $D$ ring of madangamine $C$. In the quest of using these cross coupling reactions, the nature of bases, $\mathrm{Pd}$ catalysts, and solvents must be taken into consideration.

Thus, in a first attempt, we decided to use a protocol described by Danishefsky ${ }^{93}$ which performed the regioselective terminal hydroboration of the double bond with 9-BBN and the coupling reaction with $\mathrm{Pd}(0)$ with a bidentate bis(diphenylphosphino)ferrocene ligand in presence of a thallium base in a very diluted conditions.

The selection of the thallium(I)ethoxyde as base was justified considering its proprieties in increasing the rate of the coupling, probably due to acceleration of the hydroxyl-halogen exchange at palladium. ${ }^{94,95}$

$$
\mathrm{L}_{2} \mathrm{Pd}_{-}^{\mathrm{R}_{1}} \underset{-\mathrm{MX}}{\stackrel{\mathrm{MOH}}{\longrightarrow}}-\mathrm{L}_{2} \mathrm{Pdd}^{\mathrm{R}_{\mathrm{OH}}}
$$

Scheme 3.8 Hydroxyl-halogen exchange.

A $\beta$-hydride elimination can be occurs instead of the reductive elimination when the substrate possess hydrogens in the $\beta$-position. For this reason a bidentate palladium catalyst was used. The bis(diphenylphosphino)ferrocene ligand helps to avoid the elimination by enforcing a cisgeometry between the substituents and facilitating the reductive elimination.

\footnotetext{
${ }^{93}$ Chemler, R.; Danishefsky, S. J. Org. Lett. 2000, 2, 2695-2698.

${ }^{94}$ Frank, S. A.; Chen, H.; Kunz, R. K.; Schnaderbeck, M. J.; Roush, W. R. Org.Lett. 2000, 2, 2691-2694.

${ }^{95}$ Uenishi, J.; Beau, J.; Armstrong, R. W.; Kishi, Y. J. Am. Chem. Soc. 1987, 109, 4756-4658.
} 


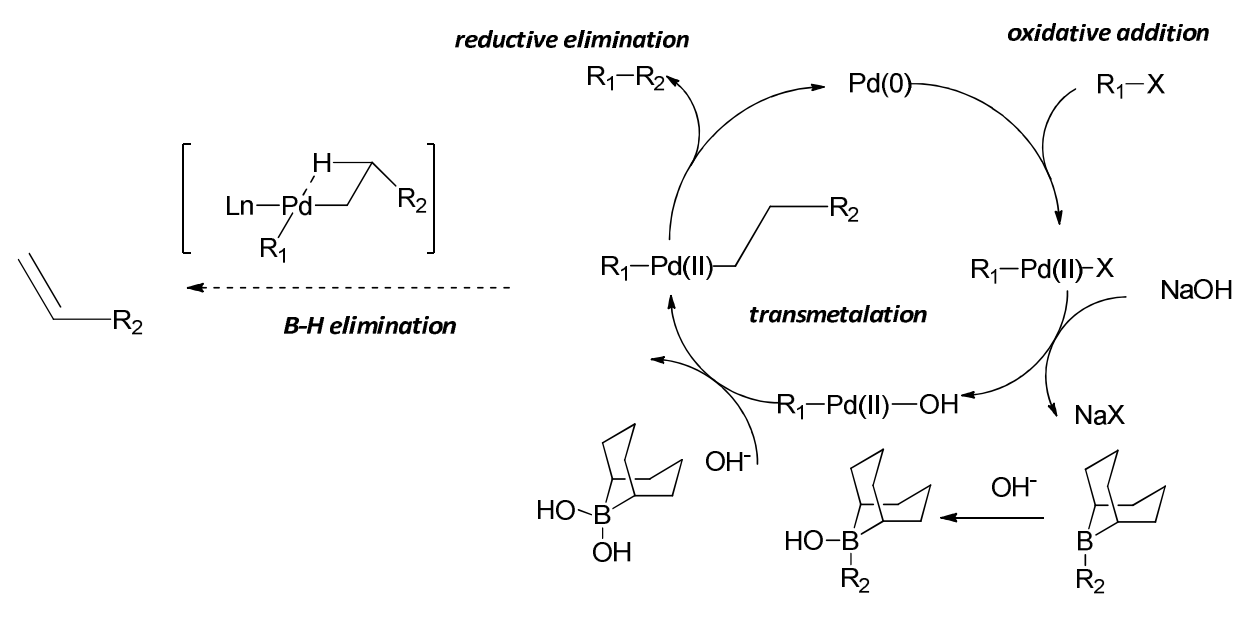

Figure 3.11 Catalytic cycle.

With these consideration in mind we carried out the reaction adding the solution of hydroborated compound $\mathbf{5 9}$ and the base to the solution of catalyst $\left[\mathrm{PdCl}_{2}(\mathrm{dppf})\right]$ and $\mathrm{AsPh}_{3}$ via a syringe pump over a period of 5 hours. A high dilution of $0.003 \mathrm{M}$ was used to avoid the formation of the oligomeric undesired products.

Unfortunately our boronic acid-TIOEt adduct was not soluble not even using of water as a cosolvent. The substitution of the thallium base for cesium carbonate ${ }^{96}$ gave only trace of the desired compound 61, which presence was revealed by a LC-MS analysis, together with oligomeric byproducts.

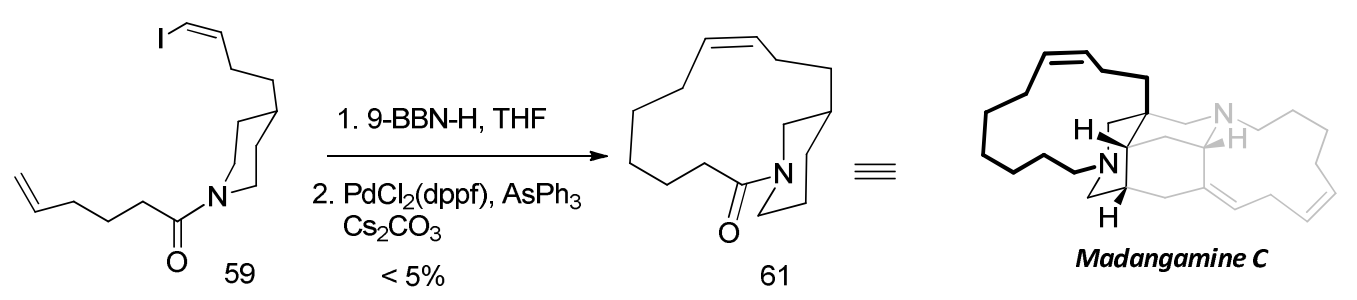

Scheme 3.9 Intramolecular Suzuki-Miyaura reaction.

These results underline the difficulty of this task and point out the entropically favorable formation of oligomeric byproducts which was probably promoted by the elevated number of degrees of freedom of both unsaturated chains and the elevated strain energy of the ring. The use of the piperidinic model compound provided important informations regarding the construction of the unsaturated macrocyclic ring, allowing the evaluation of the efficacy of the strategy and avoiding useless waste of valuable material.

${ }^{96}$ Trost, B. M.; Lee, C. B. J. Am. Chem. Soc. 1998, 120, 6818-6819. 
Due to the poor results obtained in the cyclization step, and the difficulties that involved the construction of the required $Z$-iodoalkene, we decided for the moment to consider alternative approaches and center our attention on other methodologies for the generation of the 13-and 14- membered $D$ rings of madangamine $D$ and $E$.

\subsubsection{Ring-closing Metathesis Strategy: Construction of D Ring of Madangamine D and E}

Catalytic ring-closing metathesis is one of the most powerful tools for the construction of carbon-carbon bonds; the market availability of a wide range of catalysts makes these reactions indispensable methodologies to prepare cyclic and acyclic organic compounds. The stability, easiness to handle and the tolerance to a huge variety of functional groups make ruthenium catalysts the most used catalytic systems in metathesis reactions including: ringclosing metathesis (RCM), cross-metathesis (CM), ring-opening metathesis (ROMP) and enyne metathesis (RCEYM). ${ }^{97}$
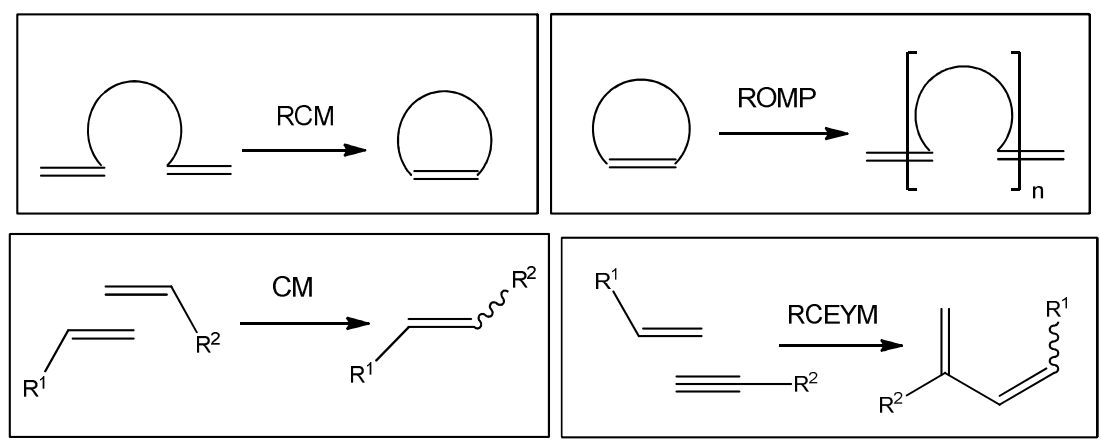

Figure 3.12 Metathesis reactions.

The validity and importance of these processes have been largely demonstrated by hundreds of publications regarding theoretical study and applications but, in this paragraph, we will try to focus our attention on the RCM and, in particularly, on its application in the synthesis of large rings. ${ }^{98}$ Our objective is to explore the formation of 13 - and 14-member saturated macrocyclic rings ${ }^{99}$ of madangamine $\mathrm{D}$ and $\mathrm{E}$ via ring-closing metathesis reactions followed by hydrogenation of the resulting double bonds.

\footnotetext{
${ }^{97}$ Nicolaou, K. C.; Bulger, P. G.; Sarlah, D. Angew. Chem. Int. Ed. 2005, 44, 4490- 4527.

98 (a) Gradillas, A.; Pérez-Castells, J. Angew. Chem. Int. Ed. 2006, 45, 6086-6101. (b) Fürstner, A. Chem. Commun. 2011, 47, 6505-6511.

${ }^{99}$ Rao, H. S. P.; Rafi, S.; Ratish Kumar, P.; Guravaiah, C.; Muthanna, N. Tetrahedron Lett. 2012, 53, 68776880.
} 
This procedure has been validating during the last years in the synthesis of different largedimensioned rings of alkaloids such as pinnatoxin $A^{100}$, isooncinotine ${ }^{101}$ and saraine $A .{ }^{102}$

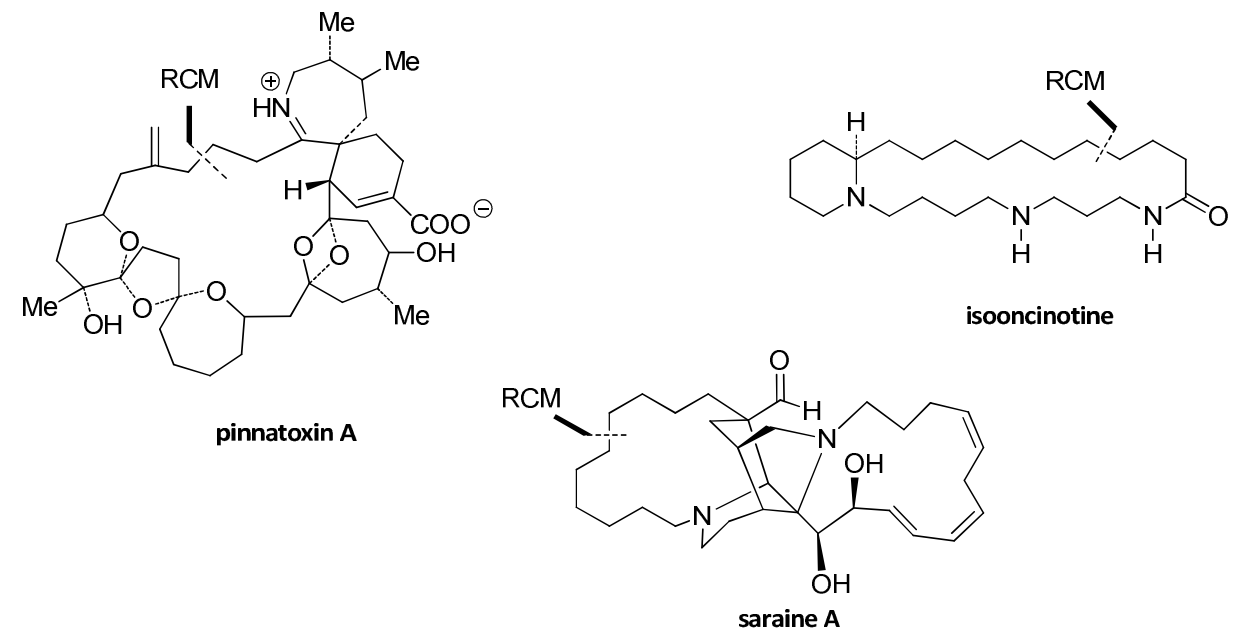

Figure 3.13 Alkaloids containing macrocyclic rings.

Inspired by these recent works we decided to undertake this route and to study the assembly of the macrocyclic $D$ ring of madangamine $D$ and $E$ starting from simple model compounds. Our synthetic plan involves the synthesis of piperidinic dienes bearing unsaturated chains of different lengths, required for the macroannulations of different $D$ rings. Our intention is to validate this methodology and finally to find the best reaction conditions that will be applied in the synthesis of the madangamine alkaloids.

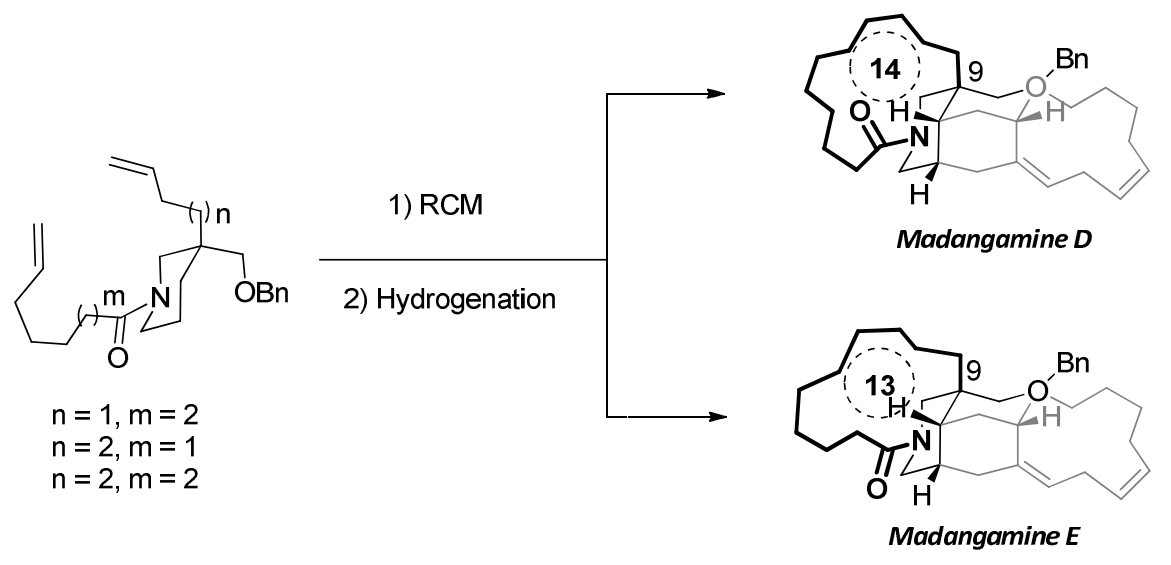

Figure 3.14 RCM annulation strategy.

\footnotetext{
${ }^{100}$ Sakamoto, S.; Sakazaki, H.; Hagiwara, K.; Kamada, K.; Ishii, K.; Noda, T.; Inoue, M.; Hirama, M. Angew. Chem. 2004, 116, 2200-2204; Angew. Chem. Int. Ed. 2004, 43, 6505-6510.

${ }^{101}$ Scheiper, B.; Glorius, F.; Leitner, A.; Fürstner, A. Proc. Natl. Acad. Sci. USA 2004, 101, 11960-11965.

${ }^{102}$ Garg, K. N.; Hiebert, S.; Overman, L. E. Angew. Chem. Int. Ed. 2006, 45, 2912-2915.
} 
The main problem related to the construction of large rings is the formation of byproducts, derived by a competitive cross-metathesis reaction, which would provoke the formation of oligomeric compounds. The parameters to control the course of the reaction are: (a) the catalysts, (b) the length of the chains and the site of ring close, (c) the dimension of the macrocyclic rings, (d) the dilution factor, (e) and some parameters such as time, solvent and temperature. ${ }^{103,}$

\subsubsection{Ring-closing Metathesis Approach: Model Studies}

The synthesis of our dienes model compounds started from $N$-Boc-protected derivative 62 . The alkylation step was carried out from $\mathrm{N}$-unprotected 3-methoxycarbonyl-2-piperidone $\mathbf{6 3}$ by treatment with $\mathrm{NaH}$ and the corresponding four or five membered bromo-alkene derivatives.

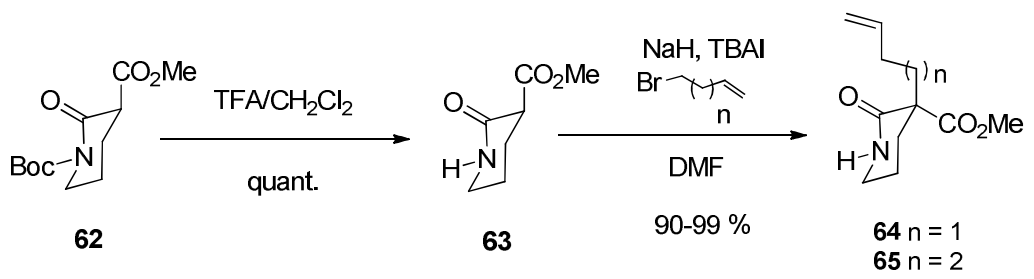

Scheme 3.10 Synthesis of piperidinic model system.

The reduction of both carbonylic moieties of compounds 64 and 65 with $\mathrm{LiAlH}_{4}$ afforded amines $\mathbf{6 6}$ and $\mathbf{6 7}$ while, after the protection of the resulting primary alcohols as benzyl derivates the compounds were acylated with terminal unsaturated chains affording the required dienes 68,69 and 70 .

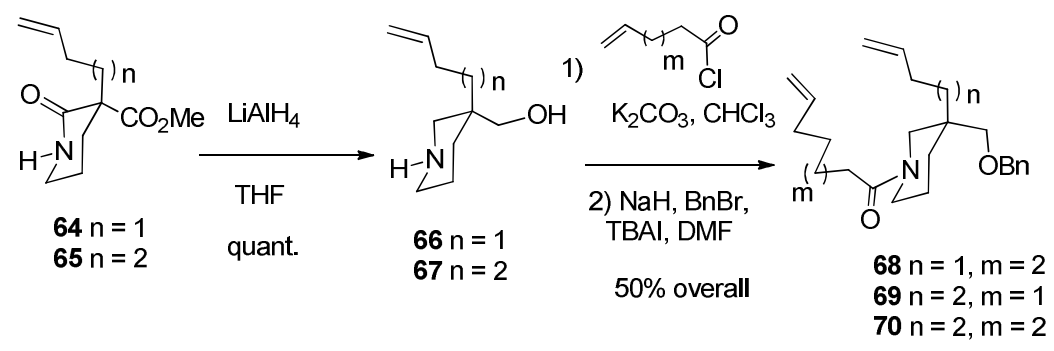

Scheme 3.11 Synthesis of the diene.

${ }^{103}$ (a) Clavier, H.; Nolan, S. P. Chem. Eur. J. 2007, 13, 8029-8036. (b) Yamamoto, K.; Biswas, K.; Gaul, C.; Danishefsky, S. J. Tetrahedron Lett. 2003, 44, 3297-3299. (c) Conrad, J. C.; Fogg, D. E. Curr. Org. Chem., 2006, 10, 185-202. 
In our first attempt we decided to study the assembly of the 13-membered $D$ ring of madangamine $E$ starting from compound 68 embodying a four membered carbon chain at the C-3 position and acylated with an eight membered terminal olefin (see Scheme 3.12).

In order to avoid the formation of dimeric products the reaction was carried out in a very high diluted condition $(0.4 \mathrm{mM})$ and diene 68 were slowly added via syringe pump to a refluxing solution of the catalyst in dichloromethane. When a 1st generation Grubbs catalyst was used, the desired 13-membered macrocyclic ring $\mathbf{7 1}$ was isolated with $40 \%$ of yield in an inseparable mixture of $E / Z$ isomers. In order to increase the yield we next tried the use the 2 nd generation Grubbs catalyst and, applying the same synthetic protocol described above, the reaction led to the formation of the desired cycloolefin with $38 \%$ of yield.

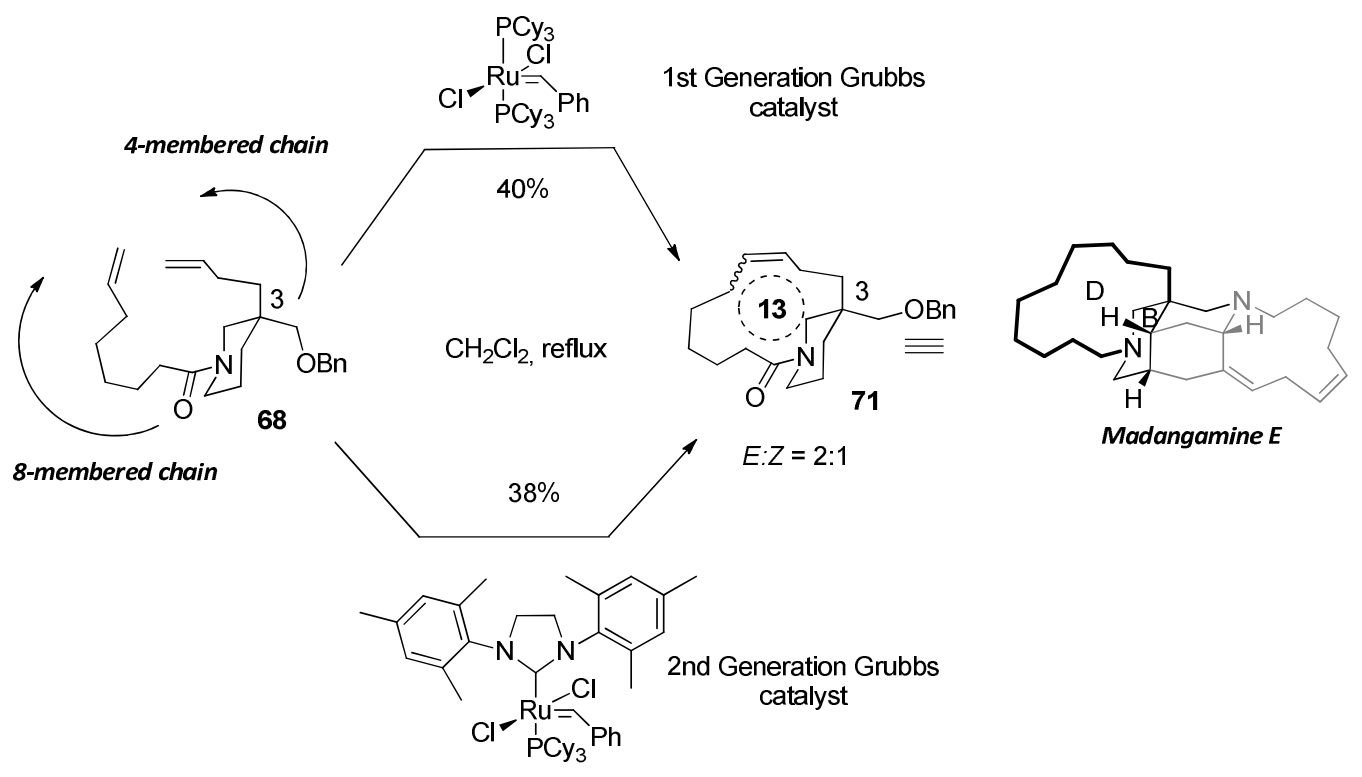

Scheme 3.12 13-memebered RCM reactions.

Considering that the rate, products and selectivity of RCM processes are determined by both catalyst and substrate and, taking into account that the use of the 2nd generation Grubbs catalyst didn't improve the yields of the macrocyclization reaction, we decided to change the chains length of diene. Instead of compound $\mathbf{6 8}$ we decided to use the diene $\mathbf{6 9}$ bearing a five membered carbon chain at C-3 position and a seven membered carbon chain on the nitrogen $(n=2, m=1)$. Our intention is to estabilish if these modifications could make more favorable the end to end encounters probabilities of the olefins and increase the effective molarity (EM), defined as the ratio between the rate constant of RCM macrocyclization and the oligomeric reactions.

Thanks to these modifications the reaction showed a little improvement, in terms of yield, and the use of a 2 nd generation Grubbs catalyst with diene 69 gave the 13-membered macrocyclic ring $\mathbf{7 2}$ with $53 \%$ yield together with $25 \%$ of dimeric compound. 


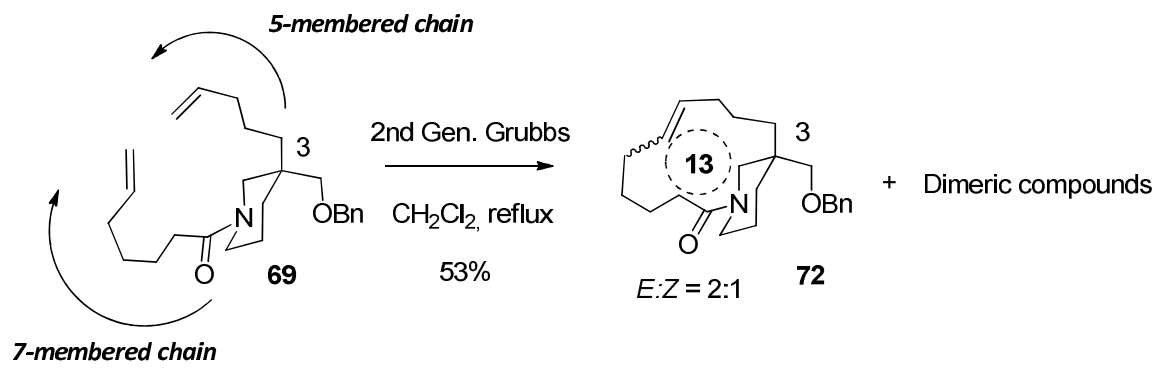

Scheme 3.13 13-membered RCM reaction.

In view of these results we decided to assemble the 14-membered $D$ ring of madangamine $D$ applying the same protocol described above with piperidinic diene 70. Thus, reaction of compound $\mathbf{7 0}$ with a 2nd generation Grubbs catalyst gave surprisingly the unsaturated macrocyclic ring $\mathbf{7 3}$ with $84 \%$ yields and a complete $E$-stereoselectivity.

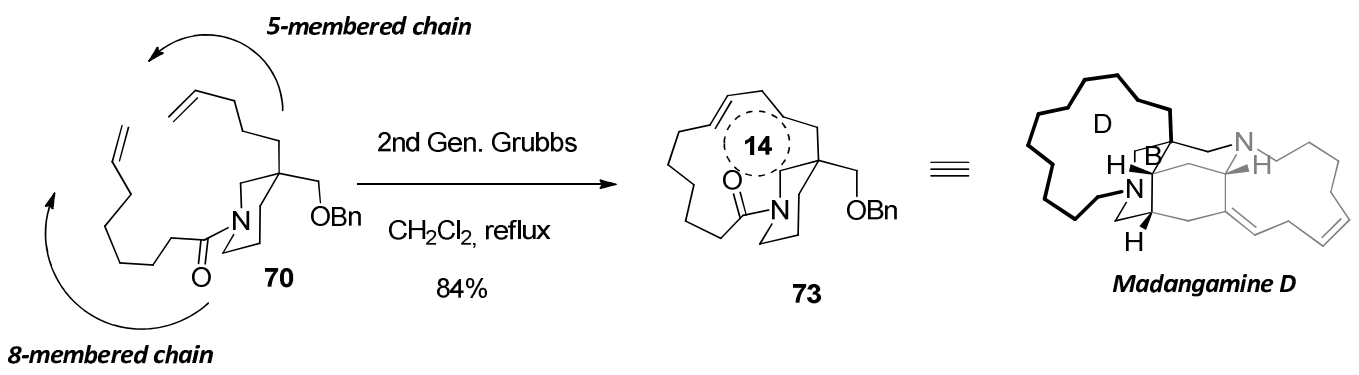

Scheme 3.14 14-memebered RCM reaction.

The results obtained showed a significant difference in the assembly of the 13- and 14membered macrocyclic rings. We thought we could force the formation of the macrocyclic $D$ ring of madangamine $\mathrm{E}$, and overcame some of the problems related to the high dilution, modifying some of the parameters of the reaction by using a flow chemistry approach.

\subsubsection{Ring-closing Metathesis Strategy: Flow Chemistry Approach}

The low effective molarity (EM) of the dienes 68 and 69 , precursors of macrocyclic $D$ ring of madangamine $E$, resulted in a competition between ring-closing and cross metathesis reactions. 

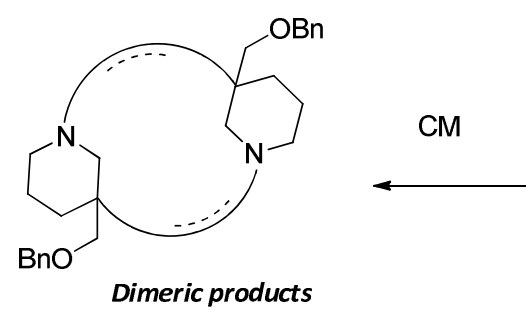

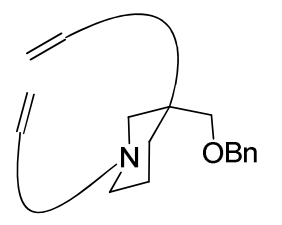

68 or 69

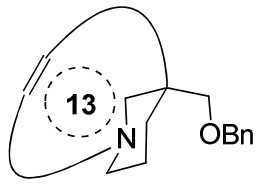

71 or 72

Figure 3.15 RCM versus CM.

To improve the EM values of our problematic diolefinics compounds 68 and $\mathbf{6 9}$ and facilitate the synthesis of macrocylic compounds are required high dilutions that, consequently, reduce the kinetics of the reaction. The high temperature often used to overcome this effect has a detrimental impact on the catalysts activity and lifetime.

Flow chemistry represents an attractive solution not only for the opportunity to improve the synthesis of the 13-membered ring but also open the possibility to carry out one of the crucial step of the synthesis of madangamine in a continuous-flow system. We thought we could exploit this technique to avoid the use of extremely diluted condition and the formation of byproducts. ${ }^{104}$

\subsubsection{Flow Reactors Structure and Main Principles}

In flow chemistry, ${ }^{105}$ a chemical reaction is performed in a continuously flowing stream in a network of interconnecting channels: where they join one another, the fluids come into contact and the reaction takes place.

In terms of equipment, a typical flow chemistry apparatus is composed by two or more pumps connected with an appropriate reactor. The liquid reagents are collected in tanks, or simply in flasks, and pumped, with independents flow rates through a T-junction, inside the reactor where the reaction takes place. The whole process can be monitored at the end of this circuit, in real time, by diode array UV detectors or mass spectrometry techniques.

\footnotetext{
${ }^{104}$ Monfette, S.; Eyholzer, M.; Roberge, D. M.; Fogg, D. E. Chem. Eur. J. 2010, 16, 11720-11725.

${ }^{105}$ For reviews of flow chemistry see: (a) Wegner, J.; Ceylan, S.; Kirschning, A. Chem. Commun. 2011, 47, 4583-4592. (b) Pastre, J. C.; Browne, D. L.; Ley, S. V. Chem. Soc. Rev. 2013, 42, 8849-8869. (c) Jas, G.; Kirschning, A. Chem. Eur. J. 2003, 9, 5708-5723.
} 


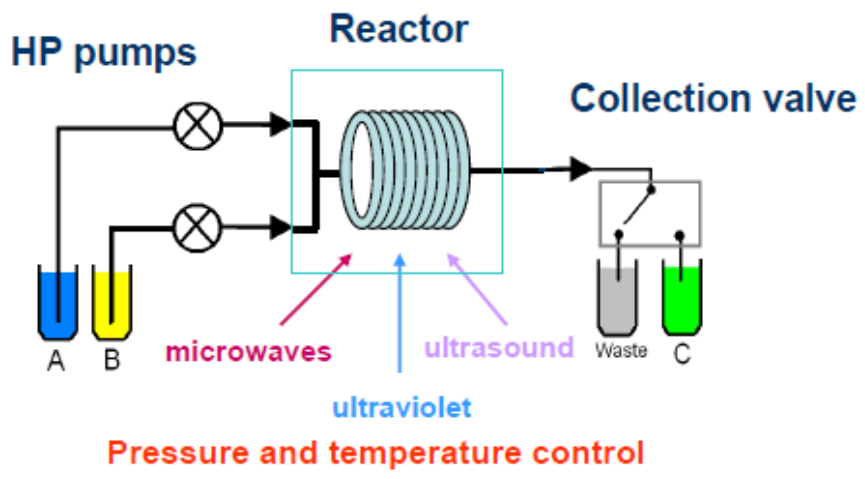

Figure 3.16 Flow reactors structure.

The core of the equipment is represented by the reactor: laboratory scale flow reactors can be generally divided into two classes on the basis of channels size and volume: micro- and mesoflow reactors. ${ }^{106}$ Micro-flow reactors present channels having diameter from 10 to $1000 \mu \mathrm{m}$, whereas meso-flow reactors are characterized by larger channels with diameter up to 1000 $\mu \mathrm{m}$.

The main advantages associated with the flow processes can be comprised in two classes: the first one is associated with the small dimensions of the reactor channels and includes the precise control of the reaction conditions, the efficient mass and heat transfer and the possibility of working under superheating conditions. The second aspect is related to the continuous nature of the process and includes the simplicity in reaction, scale-up, the possibility of performing sequential synthetic steps with independent control of reaction conditions and the opportunity to introduce in-line purification by means of supported scavengers.

The main principles of the flow chemistry are:

- The reaction stoichiometry depends not only on the concentration of the reagent but also on the flow rate which can be controlled and changed during the reaction.

- The reaction time is determined by the ratio between the reactor volume and the flow rate.

$$
\mathrm{RT}(\mathrm{min})=\text { Reactor Volume }(\mathrm{mL}) / \text { Total Flow Rate }(\mathrm{mL} / \mathrm{min})
$$

${ }^{106}$ Geyer, K.; Codee, J. D. C.; Seeberger, P. H. Chem. Eur. J. 2006, 12, 8434-8442. 


\subsubsection{Continuous Flow RCM Macrocyclization: Model Studies}

Keeping in mind the limitations of our RCM-batch transannulation reactions, and fascinated by the promises of this recent methodology, we decided to explore the efficiency of macrocyclizations performed in a flow system.

Our attempts began with the annulation reaction of compound 70.with the aim to find the best reaction conditions to carry out the process. Hence, the reaction was performed using a flow apparatus, equipped with a $10 \mathrm{~mL}$ reactor, with two constant flow streams of 0.120 $\mathrm{mL} / \mathrm{min}$ each. ${ }^{107}$ The first one was formed by a $0.01 \mathrm{M}$ solution of diene $\mathbf{7 0}$ in toluene and the second by a solution of a 2nd generation Grubbs catalyst in the same solvent. The reaction took place at $150{ }^{\circ} \mathrm{C}$ leading to a complete conversion of the starting material into compound 73 and confirming the efficiency of the flow technique.

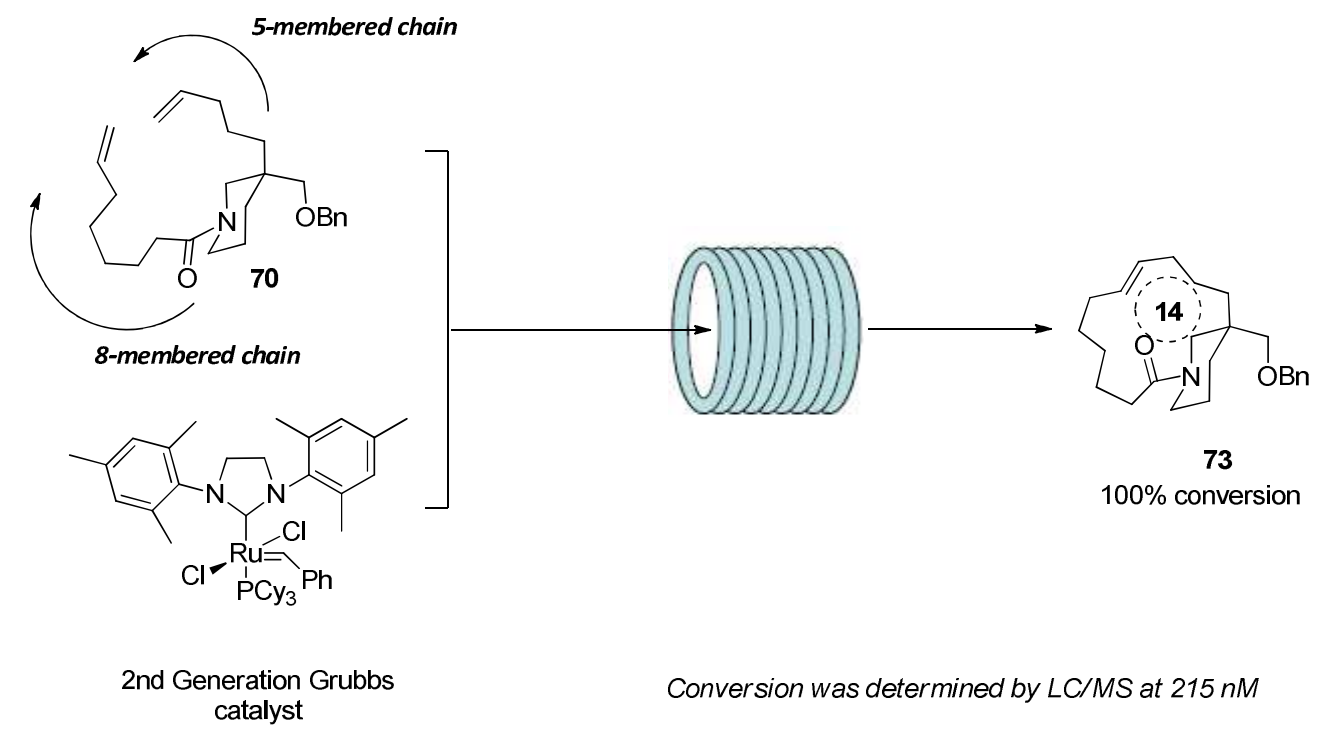

Scheme 3.15 14-memebered RCM-flow reaction.

In view of these promising results we next tested the use of a PEG-supported Hoveyda-Grubbs catalyst under continuous flow chemistry conditions.

The immobilizations of ruthenium catalysts in polymers have been largely exploited during the last decade ${ }^{108}$ for the ease of catalysts separation and recycling purposes. The PEG-supported

${ }^{107} 0.120 \mathrm{~mL} / \mathrm{min}$ was the minimum flow rate permitted by the apparatus.

108 (a) Buchmeiser, M. R. Chem. Rev. 2009, 109, 303-321. (b) Ahmed, M.; Barrett, A. G. M.; Braddock, D. C.; Cramp, S. M.; Procopiou, P. A. Tetrahedron Lett. 1999, 40, 8657-8662. (c) Halbach, T. S.; Mix, S.; Fischer, D.; Maechling, S.; Krause, J. O.; Sievers, C.; Blechert, S.; Nuyken, O.; Buchmeiser, M. R. J. Org. Chem. 2005, 70, 4687-4694. 
catalyst, synthesized by the group of Yao, ${ }^{109}$ was find to be very effective, easy to recover by precipitation with ethylic ether and once recycled could be reused up to six cycles without a substantial decrease in activity.

In the macrocyclization reaction of diene $\mathbf{7 0}$ with the PEG-supported catalyst was observed $86 \%$ of conversion of the starting material and the final product $\mathbf{7 3}$ could be easily purified by removal of the organic solvent, treatment of the residue with ethylic ether and filtration.

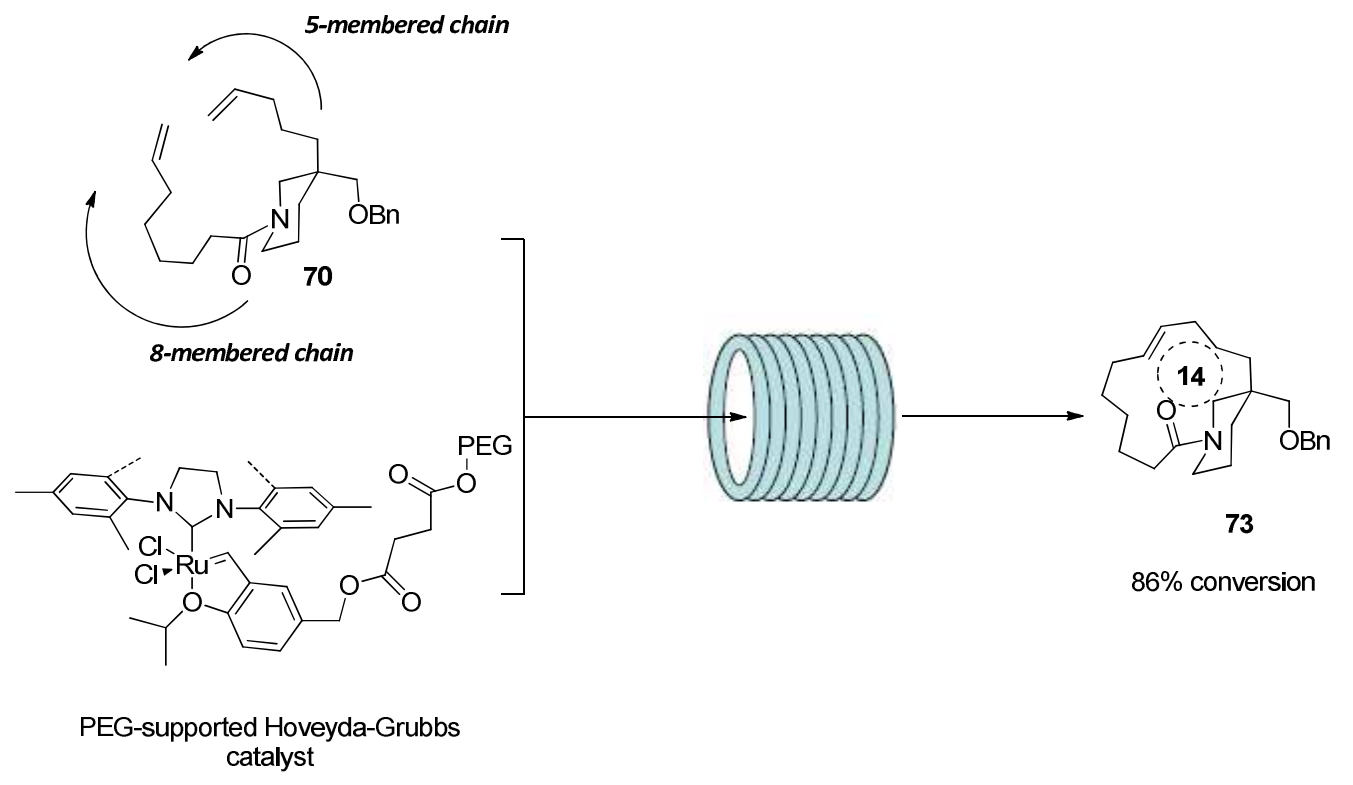

Scheme 3.16 RCM-flow reaction.

Once we established the best RCM-flow conditions we started to exploit the problematic formation of the 13-membered macrocyclic ring of madangamine E. The same protocol described above was applied in the reactions between diene 68 and the 2nd generation Grubbs catalyst but the results were unsatisfactory: the LC-MS reveled only $50 \%$ of conversion and the presence of unreacted starting material and oligomeric byproducts. When compound 69 was used, which differs in the length of both chains, the reaction gave identical results.

${ }^{109}$ (a) Yao, Q. Angew. Chem., Int. Ed. 2000, 39, 3896-3898. (b) Yao, Q.; Motta, A. R. Tetrahedron Lett. 2004, 45, 2447-2451. 


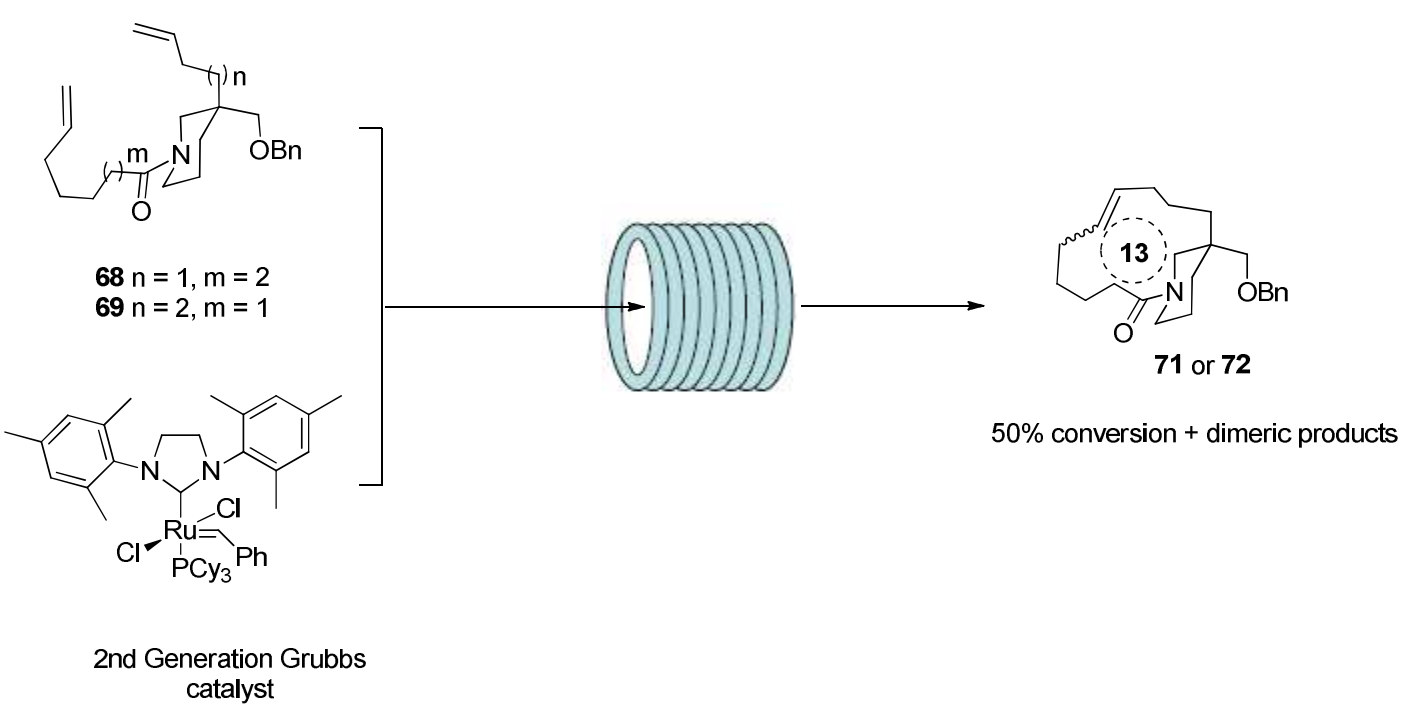

Scheme 3.17 13-membered RCM-flow reaction.

The results of these experiments pointed out two important facts: the first one is that the low yields related to the formation of the 13-membered cycle can be attributed to the slow reaction rate that didn't permit to reach a complete conversion. ${ }^{110}$ The second observation is that both batch and flow reactions demonstrated that an important parameter, in ring-closing macrocyclization reactions, is the size of the ring, no matter which is the position of the unsaturation among the cycle.

The substantial difference, in terms of yields, between the 13- and the 14-membered rings could be explained considering that the success of ring closing reactions is determined largely by strain in the nascent ring system, and the probability of end-to-end encounter between the chains. Figure 3.17 represents the behavior of model lactones ${ }^{111}$ and shows how the strain energy increases or decreases depending on the size of the ring due to the transannular interactions. As we can see in the graphic, the ring strain for a 14-membered ring is quite lower than the corresponding 13-membered.

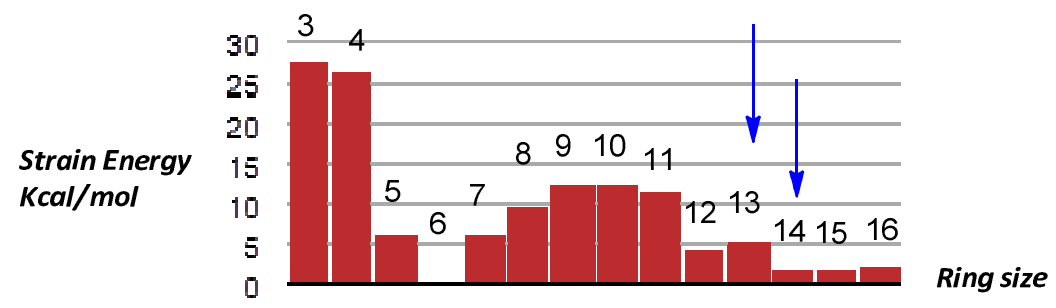

Figure 3.17 Strain energy variations.

\footnotetext{
${ }^{110}$ A $10 \mathrm{~mL}$ reactor was used with the minimum permitted flow rate, to increase the reaction time we should use a bigger reactor.

${ }^{111}$ Conrad, J. C.; Eelman, M. D.; Duarte Silva, J. A.; Monfette, S.; Parnas, H. H.; Snelgrove, J. L.; Fogg, D. E. J. Am. Chem. Soc. 2007, 129, 1024-1025.
} 
In summary, the RCM strategy for the synthesis of the macrocyclic $D$ ring of madangamine $D$ and $E$ has been examined including the use of three different ruthenium catalysts. Some of the efforts were directed towards the annulation of the 13-membered ring, which resulted in moderate yields, while the assembly of the larger 14-membered ring was achieved with excellent results. Further studies of RCM reactions resulted in the exploration of flow chemistry techniques however this methodology wasn't able to improve the yields of the problematic 13-membered ring construction of madangamine $\mathrm{E}$.

\subsubsection{Construction of $D$ Ring of Madangamine A}

Madangamine $\mathrm{A}$ is probably the most important alkaloid of the family due to its structural complexity and its pharmacological proprieties. The main feature is to possess a polyunsaturated $\mathrm{D}$ ring composed by 15 carbons with a Z,Z,Z-skipped system of olefins.

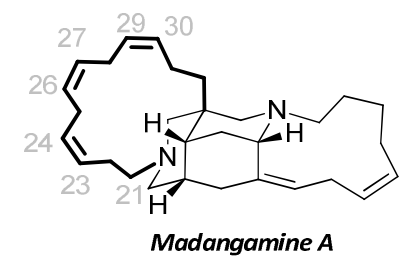

Figure 3.18 Structure of madangamine A.

This architectural characteristic is a common motif which can be found in many biologically active compounds such as marine alkaloids, pheromones, and its construction represents a synthetic intriguing challenge for chemists. ${ }^{112}$<smiles>CCC1(C)CC[C@H](/C(C)=C/C(C)/C=C/[C@@H]2CC(C)=C(C)C(=O)O2)O[C@H]1C</smiles>

Jerangolid $D$

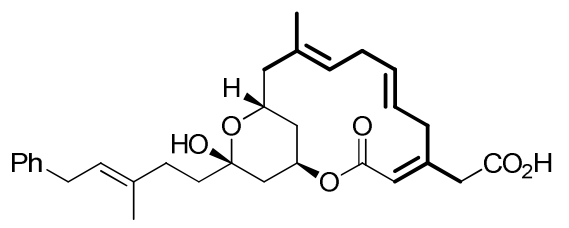

Ripostatin A<smiles>CCCCC/C=C\C/C=C\C/C=C\C/C=C\CCC(=O)O</smiles>

Arachidonic acid<smiles>C/C=C(/C)C/C=C/CCc1nc(-c2nc(C(C)C[C@H]3CC(O)C[C@](C)(O)[C@H]3C)co2)co1</smiles>

Hennoxazole A

Figure 3.19 Alkaloids containing skipped olefins.

112 (a) Tang, W.; Prusov, E. V. Org. Lett. 2012, 17, 4690-4693. (b) Pospısil, J.; Markó, I. E. J. Am. Chem. Soc. 2007, 129, 3516-3517. (c) Yokokawa, F.; Asano, T.; Shioiri, T. Org. Lett. 2000, 2, 4169-4172. 
Our purpose is to set up a protocol for the construction of this complex macrocyclic ring using a piperidine model and eventually apply the methodology on the synthesis of madangamine $A$.

The general strategy would involve first the synthesis of a piperidinic ring, substituted at C-3 position with a 12-membered carbon chain bearing the Z-skipped system of olefins and an appropriate terminal functionalization (74 or $\mathbf{7 5}$ ). In a second stage of the synthesis we will explore the annulation of the macrocyclic ring through intramolecular macrolactamization or reductive amination reactions. Following this synthetic plan the construction of the $D$ ring of madangamine A $\mathbf{7 6}$ and $\mathbf{7 7}$ could be achieved, for the first time, in a fast and convergent way.

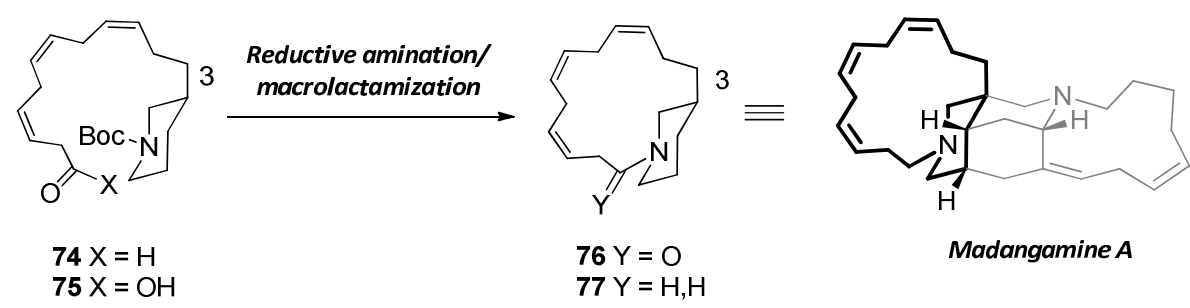

Scheme 3.18 D ring annulation strategy.

\subsubsection{Synthesis of Z-Skipped D Ring of Madangamine A: First Synthetic Approach}

In our first synthetic strategy we planned to incorporate the carbon chain $\mathbf{7 9}$, bearing two skipped system of olefins and a terminal alkyne, on the piperidine compound 78 . The stereoselective partial cis-hydrogenation of the resulting piperidine-derivate $\mathbf{8 0}$ would afford compound $\mathbf{8 1}$ which represents our starting point for the macrocylization studies.

79

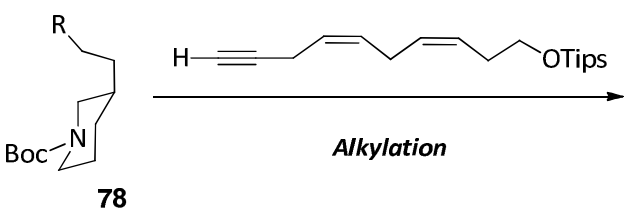

78
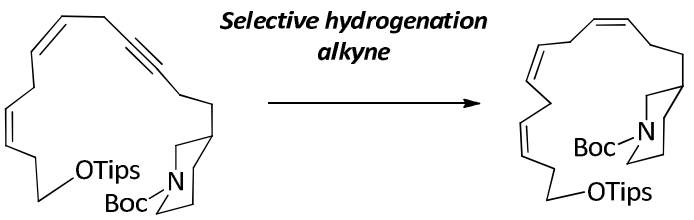

$\mathrm{R}=$ Leaving group

80

81

Scheme 3.19 First synthetic approach.

Keeping in mind this synthetic plan, our first challenge was represented by the synthesis of the $(Z, Z)$ 1,4-dienic systems, a common feature found in many organic compounds such as polyunsaturated fatty acids (PUFA). The methodologies generally used to build these structures can be divided in two big groups: 
- A preparation using sequential Wittig reactions, which would bring directly to the construction of the Z-olefin system.

- The synthesis of a poly-alkynil chain and the subsequent selective cis-hydrogenation of the resulting triple bonds.

The Wittig reaction is a useful methodology for the generation of olefins and most of its attractiveness is due to the possibility to control the $E / Z$ geometry of the double bond. The $Z$ skipped system can be achieved through this procedure using the classical cis-Wittig reaction conditions that include the use of unstabilized ylides, free-lithium bases and the control of some parameters like dilution and temperature.

The synthesis of arachidonic acid reported by Santelli ${ }^{113}$ represents an example of the versatility of this methodology for the synthesis of these Z-skipped olefins.

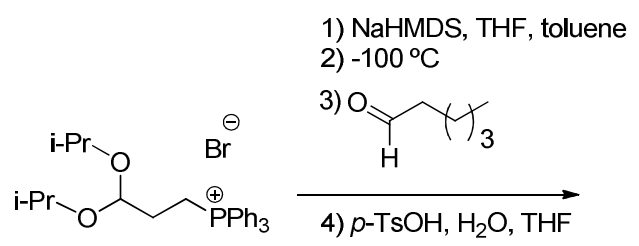

A $96 \%$

1) NaHMDS, THF, toluene 2) $-100^{\circ} \mathrm{C}$

A

$$
\begin{aligned}
& \text { 3) } \mathrm{i} \\
& \begin{array}{l}
\text { 4) } \mathrm{p}-\mathrm{TsOH}, \mathrm{H}_{2} \mathrm{O}, \mathrm{THF} \\
\quad 87 \%
\end{array}
\end{aligned}
$$

1) NaHMDS, THF, toluene 2) $-100^{\circ} \mathrm{C}$

A

$$
\begin{gathered}
\text { 4) } p-\mathrm{TsOH}, \mathrm{H}_{2} \mathrm{O}, \mathrm{THF} \\
88 \%
\end{gathered}
$$

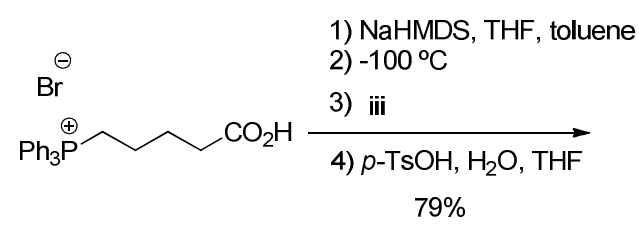

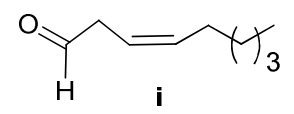
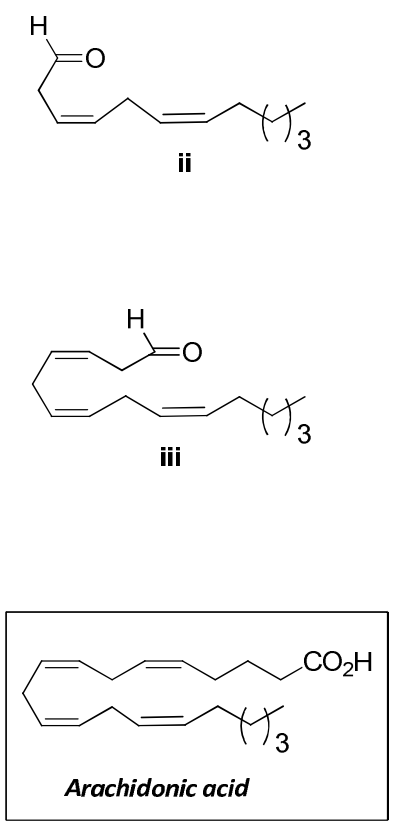

Scheme 3.20 Synthesis. of arachidonic acid.

${ }^{113}$ Viala, J.; Santelli, M. J. Org. Chem. 1988, 53, 6121-6123. 
As already mentioned the second strategy involves the preparation of a poli-alkynil system by a cross-coupling reaction between a terminal alkyne and propargylic halides. The subsequent semi-hydrogenation of the corresponding acetylenic moiety could be achieved using different methodologies described in literature.

The most classical ones include the hydrogenation with Lindlar catalyst (palladium oxide deposited on calcium carbonate and poisoned with lead acetate and quinolone) or with the nickel based Brown catalyst, ${ }^{114}$ while other methodologies like the hydroboration followed by protonolysis or the use of titanium isopropoxide-isopropylmagnesium chloride system ${ }^{115}$ seem to be also efficient methods to obtain Z-olefins.

An application of this methodology was reported by Chaik of in the synthesis of $18 R$-HETE. ${ }^{116}$ As shown below the synthesis of the TMS-protected acetylenic triene $\mathbf{X X I}$ was achieved by using a $\mathrm{Cu}$ (I) coupling reactions and a selective hydrogenation with a Brown catalyst P-2 as a key steps. This catalytic system permits the Z-selective hydrogenation of the alkyne without affecting the terminal TMS-acetylenic moiety.

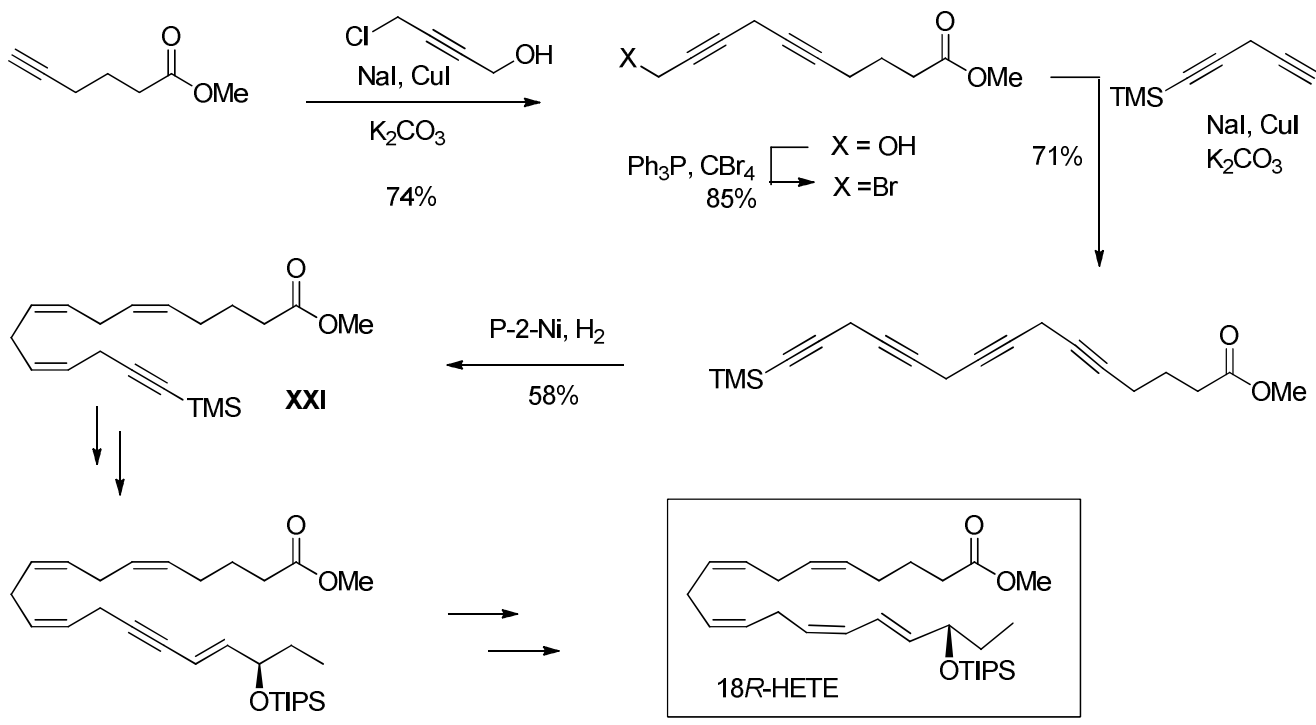

Scheme 3.21 Synthesis of 18R-HETE.

The catalyst was obtained through a reaction between sodium borohydride and a solution of nickel salts, when the reaction takes place in aqueous media a black precipitate is formed (P-1$\mathrm{Ni)} .{ }^{117}$ This material showed an activity comparable with nickel Raney but is non-magnetic and non-pyrophoric. When the same catalytic system was synthesized using a suspension of the

\footnotetext{
${ }^{114}$ (a) Brown, C. A.; Ahuja, V. K.; J. Chem. Soc. Chem. Commun. 1973, 553-554. (b) Brown, C. A.; Ahuja, V. K. J. Org. Chem. 1973, 38, 2226-2230.

${ }^{115}$ (a) Hungerford, N. L.; Kitching, W. Chem. Commun. 1996, 1697-1698. (b) Harada, K.; Urabe, H.; Sato, F. Tetrahedron Lett. 1995, 36, 3203-3206

${ }^{116}$ Krishnamurthy, V. R.; Dougherty, A.; Haller, C. A.; Chaikof, E. L. J. Org. Chem. 2011, 76, 5433-5437.

${ }^{117}$ Brown, C. A. J. Org. Chem. 1970, 35, 1900-1904.
} 
nickel salt in ethanol ${ }^{118}$ a colloidal black suspension was produced, the material called $\mathrm{P}-2-\mathrm{Ni}$ showed different characteristics compare to the other. This catalytic system is less effective than the P-1 type but more selective in the hydrogenation of conjugate dienes and in the partial hydrogenation of alkynes in cis-olefins while has no activity in the hydrogenation of the terminal TMS-protected alkyne. ${ }^{119,111}$ Furthermore the nickel catalyst can be generated in situ through the reduction of nickel acetate by $\mathrm{NaBH}_{4}$, which makes it straightforward to control the exact catalyst loading for the reaction.

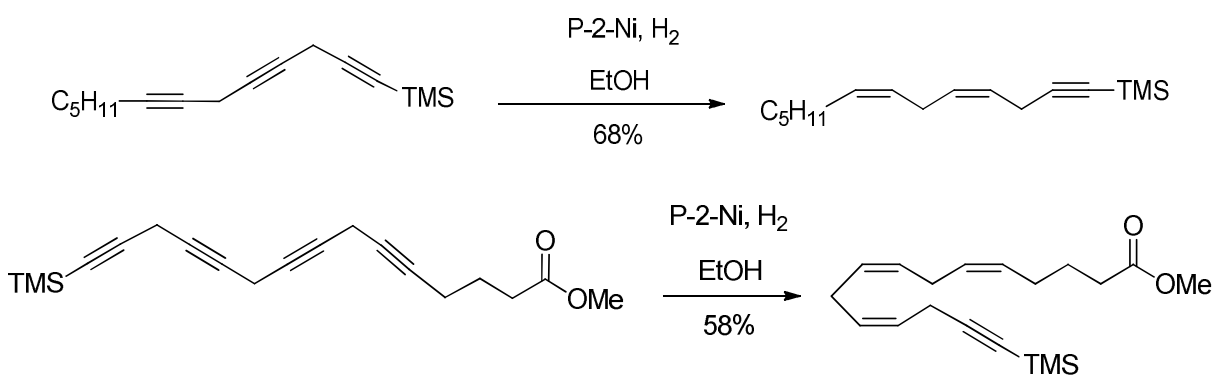

Scheme 3.22 Hydrogenations with P-2-Ni.

\subsubsection{Construction of Unsaturated Chain}

The assembly of our unsaturated chain $\mathbf{7 9}$ could be achieved using a Brown hydrogenation of the two central triple bonds of tryine $\mathbf{8 5}$ which was obtained after two cross coupling reactions between terminal alkynes and propargylic halides.

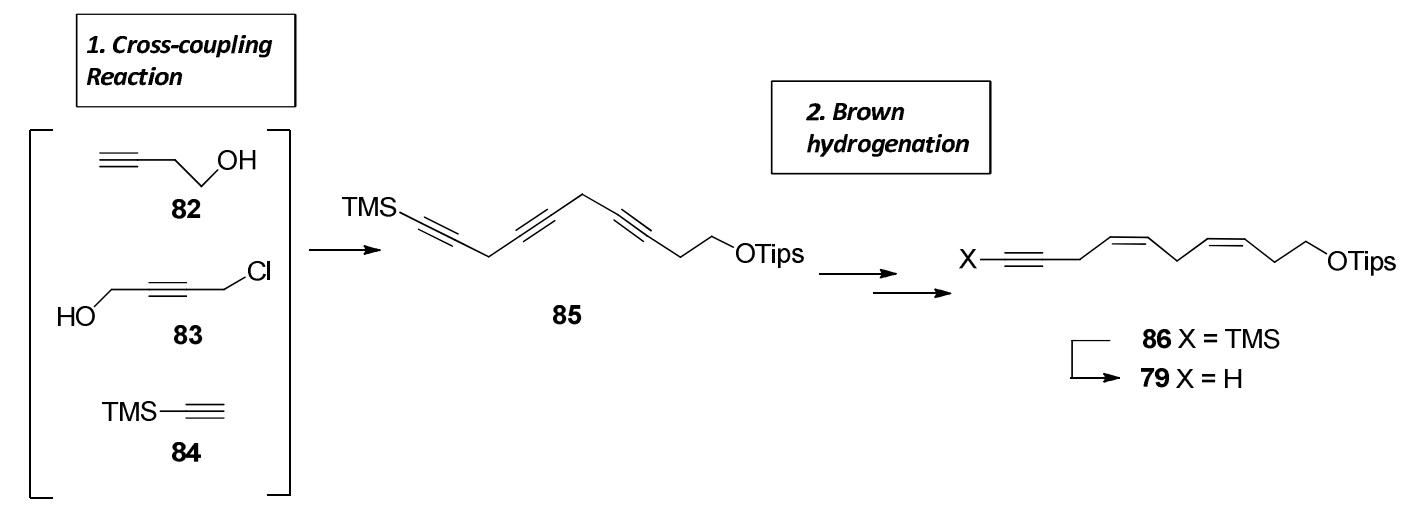

Scheme 3.23 Construction of unsaturated chain.

\footnotetext{
${ }^{118}$ (a) Brown, H. C.; Brown, C. A. Tetrahedron, 1966, 22, Suppl. 8 Part I, 149-164. (b) Brown, C. A.; Brown, H. C. J. Org. Chem. 1966, 31, 3989-3995.

${ }^{119}$ Gueugnot, S.; Alami, M.; Linstrumelle, G., Mambu, L.; Petit, Y.; Larchevêque, M. Tetrahedron 1996, $52,6635-6646$.
} 
The synthetic sequence started with the 4-chloro butynol $83,{ }^{120}$ which was prepared by slowly addition of thionyl chloride to a solution of diol 87 via syringe pump. After the purification by distillation, compound $\mathbf{8 3}$ was obtained with moderate yield.

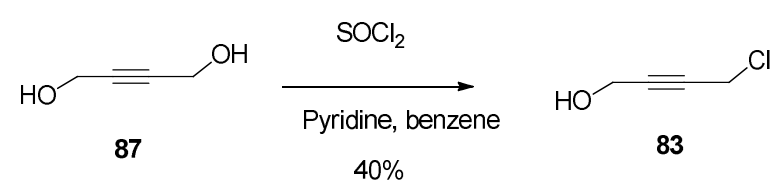

Scheme 3.24 Synthesis of 4-chloro butynol.

The subsequent reaction between the Tips-protected terminal alkyne 88, prepared from compound $\mathbf{8 2}$, and the propargylic halide $\mathbf{8 3}$ proceeded smoothly, when classical copper crosscoupling conditions were used ( $\mathrm{Cul}, \mathrm{Nal}$ and potassium carbonate as base), affording dyine $\mathbf{8 9}$ with excellent yield.

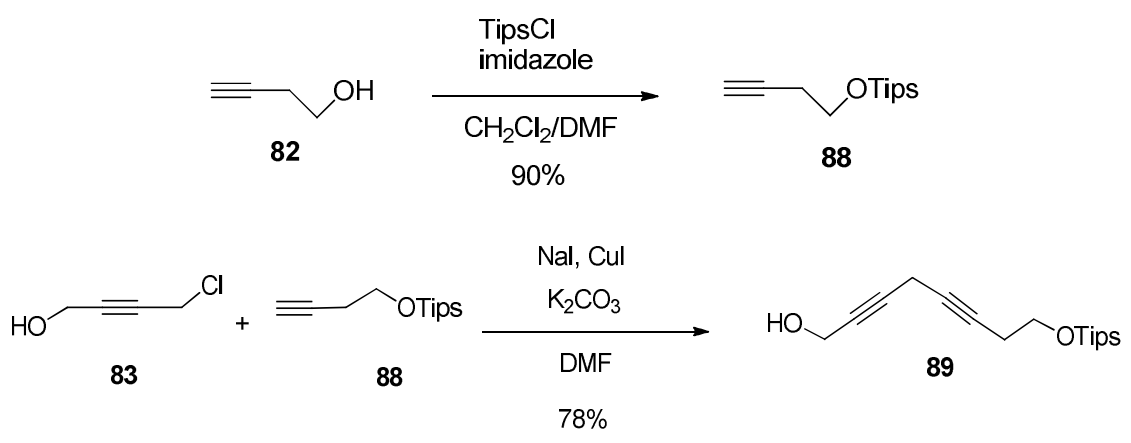

Scheme 3.25 Cross coupling reaction.

Transformation of alcohol $\mathbf{8 9}$ into the corresponding bromo-derivative, using the classical Appel reaction, proceeded with very low yield and a two-step synthesis was necessary to synthesize the required propargylic halide 90 . The resulting bromo derivative was then subjected to the second cross-coupling reaction with a terminal TMS-protected alkyne affording the desired tryine 85 .

${ }^{120}$ The compound is commercial available but expensive. 


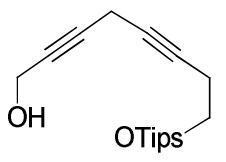

89
1) $\mathrm{Et}_{3} \mathrm{~N}, \mathrm{MsCl}$

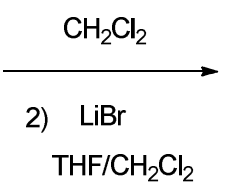

$73 \%$ overall<smiles>[18OH]CCCC#CCBr</smiles>

90

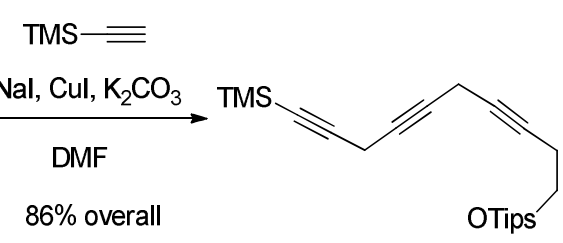

85

Scheme 3.26 Synthesis of tryine.

The next challenging issue was represented by the regio- and stereoselective cis-reduction of the central alkyne functionalities of $\mathbf{8 5}$ to give the desired $Z-Z$ diene $\mathbf{8 6}$ with the terminal TMSalkyne function (see scheme 3.27).

Our approach was based on the use of the Brown catalyst which was prepared in situ by treating a vigorously stirred solution of nickel acetate in $95 \%$ ethanol with a solution of sodium borohydride under hydrogen atmosphere. The presence of ethylendiamine was found to increase the selectivity in the formation of the Z-olefins and to avoid the over reduction of the substrates into alkane.

To our delight, a clean conversion of the skipped triyne 85 into the corresponding $Z$ - $Z$ dienealkyne system $\mathbf{8 6}$ was obtained in $75 \%$ yield. The subsequent deprotection of the triple bond with potassium carbonate afforded unsaturated compound 79.

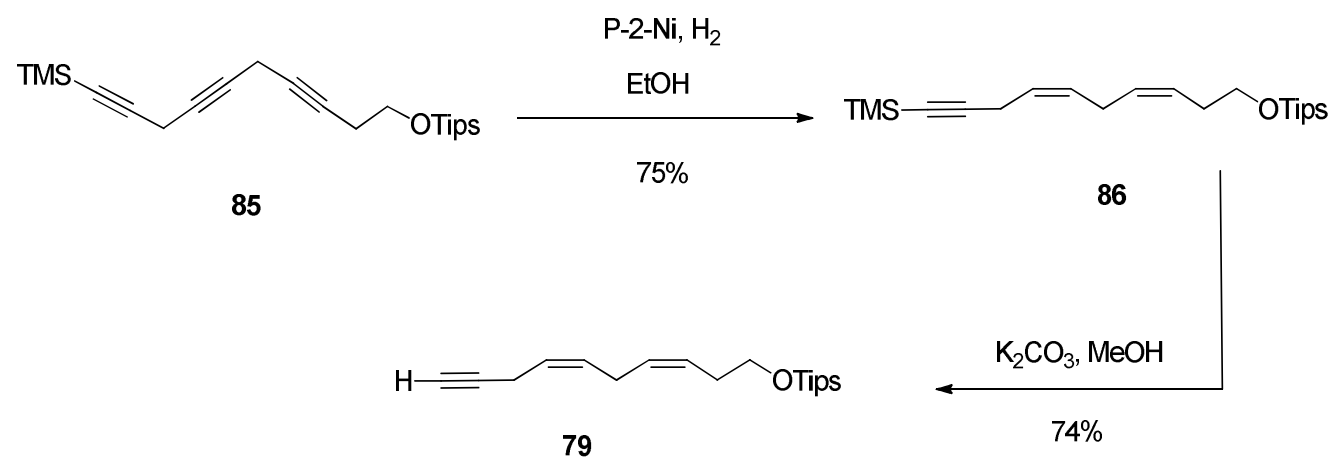

Scheme 3.27 Synthesis of unsaturated chain.

Following this simple protocol we were able to obtain in few steps the nucleophilic unsaturated chain $\mathbf{7 9}$ ready to be incorporated into the piperidine ring. 


\subsubsection{Incorporation of the Unsaturated Chain into the Piperidinic Model Compound}

The preparation of the required mesyl-substituted piperidine was achieved using the commercial 3-ethanol piperidine $\mathbf{9 1}$ as starting material. Unfortunately the reaction of the piperidine 92 with our unsaturated carbon chain 79 gave unsatisfactory results. When the reaction was conducted using buthyllithium ${ }^{121}$ at room temperature, it was possible to observe the formation of a small quantity of product and unreacted starting material, while an increase of the temperature or the reactions time led to the degradation of the chain.
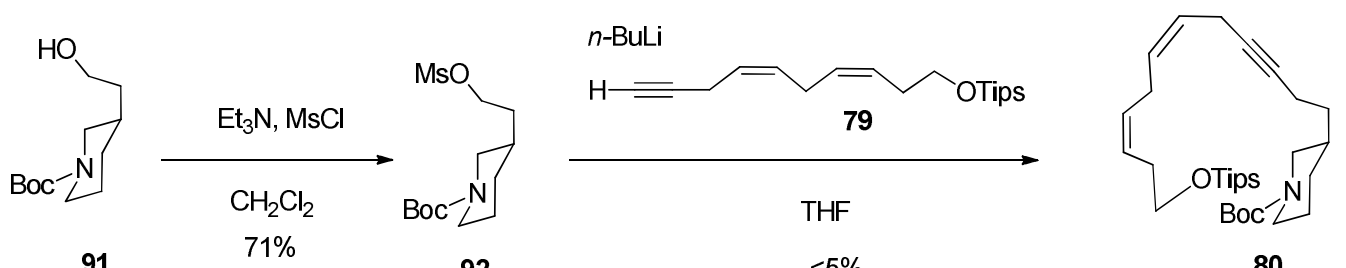

$<5 \%$

80

Scheme 3.28 Installation of carbon chain 79.

We thought that the problem could be found in the poor stability of the unsaturated chain in a basic media which probably caused the isomerization of the double bonds system and didn't allow the formation of the desired anion necessary to perform the alkylation reaction.

\subsubsection{Synthesis of Z-Skipped D Ring of Madangamine A: Second Synthetic Approach}

In order to overcome the alkylation problems we decided to change our strategy using piperidine 93 bearing a side chain at C-3 position with an acetylenic moiety. The new synthetic plan would involve an alkylation reaction between 93 and the unsaturated chain 95 . The subsequent selective reduction of the triple bond of compound $\mathbf{8 0}$ would afford the $Z$-triene 81 required in our macrocyclization studies (Path $A$ ).

Another alternative approach would contemplate a copper cross-coupling reaction between the piperidine $\mathbf{9 3}$ and skipped diyne $\mathbf{9 0}$. The simultaneously hydrogenation of the triyne $\mathbf{9 4}$ would lead to the synthesis of the required Z-skipped double bond system $\mathbf{8 1}$ (Path B).

${ }^{121}$ (a) Du, Y.; Zheng, J.-F.; Wang, Z.-G.; Jiang, L.-J.; Ruan, Y.-P.; Huang, P.-Q. J. Org. Chem. 2010, 75, 46194622. (b) Schumacher, R. F.; Rosário, A. R.; de Souza, A. C. G.; Menezes, P. H.; Zeni, G. Org. Lett. 2010, 12, 1952-1955. (c) Davison, E. C.; Fox, M. E.; Holmes, A. B.; Roughley, S. D.; Smith, C. J.; Williams, G. M.; Davies, J. E.; Raithby P. R.; Adams, J. P.; Forbes, I. T.; Press, N. J.; Thompson, M. J. J. Chem. Soc., Perkin Trans 1, 2002, 1494-1514. 


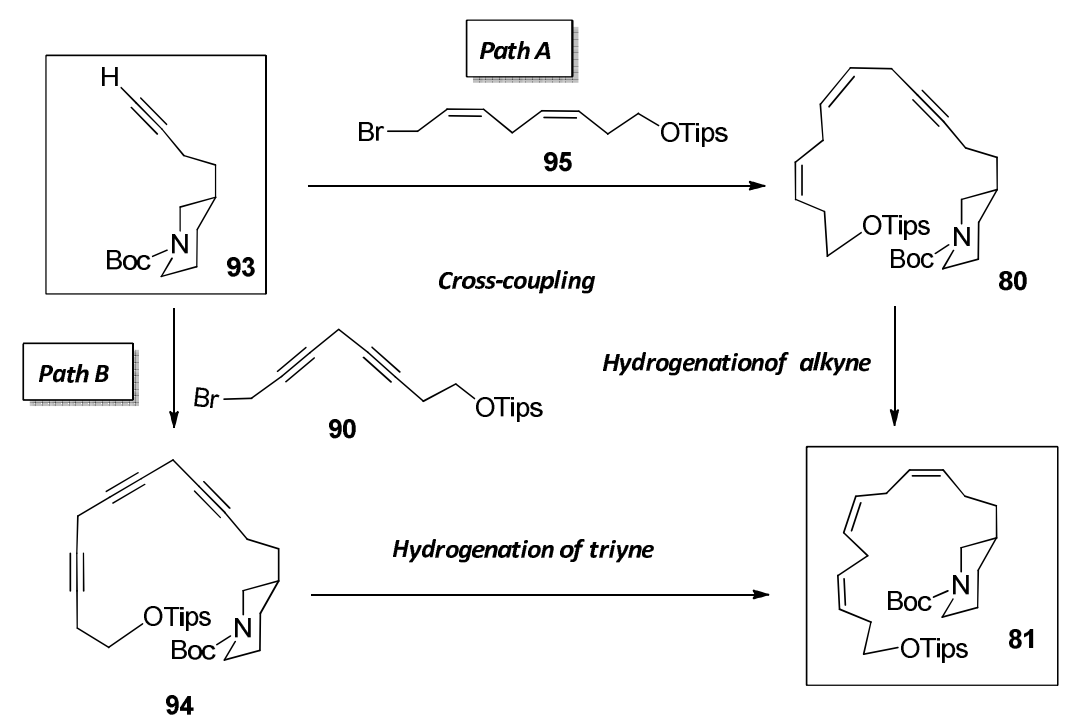

Scheme 3.29 Alternative approaches.

The starting piperidinic material 93 was readily obtained through an alkylation at $\alpha \mathrm{C}=\mathrm{O}$ position of the $\delta$-valerolactam with the appropriate carbon chain. The iodo-derivatives was prepared from TMS-protected 3-butynol and introduced at the C-3 position of the valerolactam giving compound 96. The subsequent reduction of the amide with $\mathrm{LiAlH}_{4}$, followed by the formation of $\mathrm{N}$-Boc carbamate and the deprotection of the triple bond afforded compound 93 .

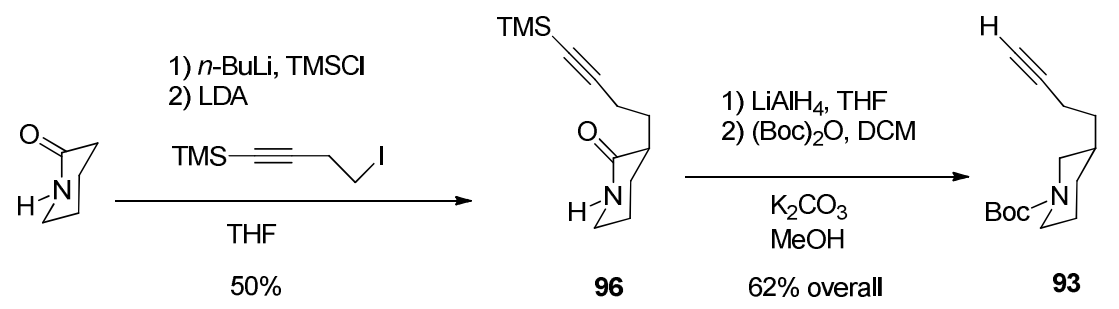

Scheme 3.30 Construction piperidine model. 


\subsubsection{Model Studies Toward the Construction of the D Ring of Madangamine A: Path A}

The required unsaturated carbon chain was readily available starting from the previously synthesized compound 89. Thus, the dyine was transformed in the corresponding olefin, through a selective Brown hydrogenation, and subsequently converted into the bromoderivative 95 .

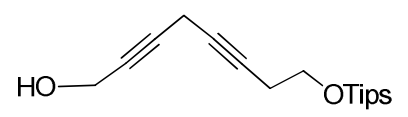

89

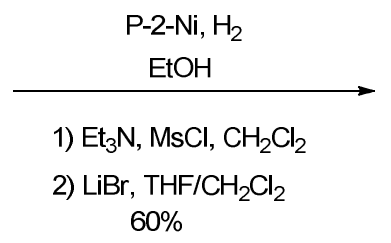

$\mathrm{Br}$

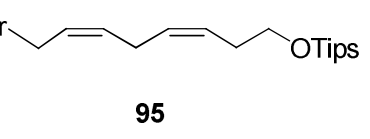

Scheme 3.31 Synthesis of unsaturated carbon chain 95.

Finally the cross-coupling reaction between bromo-derivative chain $\mathbf{9 5}$ and piperidine $\mathbf{9 3}$ took place giving piperidinic compound $\mathbf{8 0}$ with excellent yields.

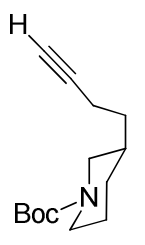

93

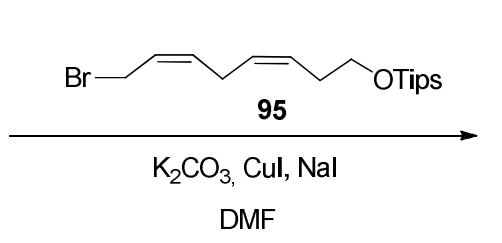

$86 \%$

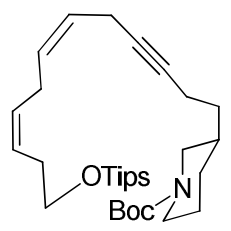

80

Scheme 3.32 Cross coupling reaction.

The crucial hydrogenation step of the triple bond of compound $\mathbf{8 0}$ presented some problems. The use of the Brown's P-2-Ni catalyst resulted unsuccessful and when was substituted with the classical Lindlar's catalyst no conversion to piperidine $\mathbf{8 1}$ were observed. A third approach was based on the use of $\mathrm{Zn}(\mathrm{Cu} / \mathrm{Ag})$ reagent as catalytic system ${ }^{122}$ but neither this methodology was able to perform the expected hydrogenation of the triple bond.

\footnotetext{
122 (a) Näf, F.; Decorzant, R.; Thommen, W.; Willhalm, B.; Ohloff, G. Helv. Chim. Acta 1975, 58, 10161037. (b) Aerssens, M. H. P. J.; Brandsma, L. J. Chem. Soc., Chem. Commun. 1984, 735-736. (c) Boland, W.; Schroer, N.; Sieler, C.; Feigel, M. Helv. Chim. Acta 1987, 70, 1025-1040.
} 


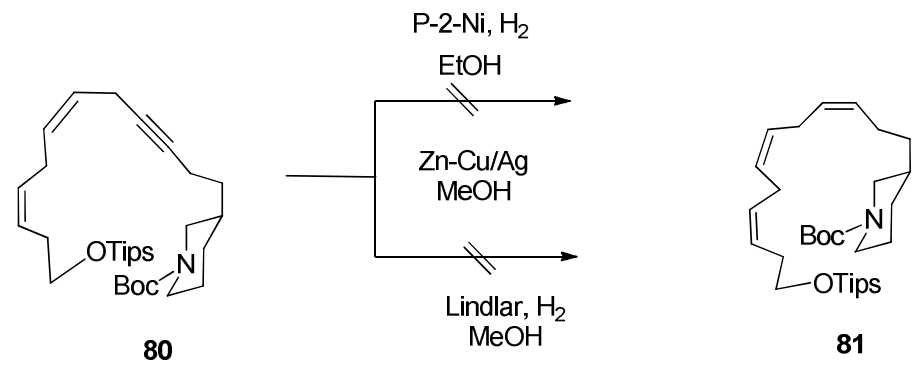

Scheme 3.33 Catalytic hydrogenation.

The difficulties we found in this step made us leave this synthetic plan and undertake the pathway B.

\subsubsection{Model Studies Toward the Construction of the D Ring of Madangamine A: Path B}

Our new challenge was then represented by the synthesis of the piperidinic model compound 94 embodying the 12-membered carbon chain bearing the tryine system. Selective hydrogenation of the alkyne moieties would lead to the formation of piperidine XXIII which represents the platform for our macrocyclization studies.

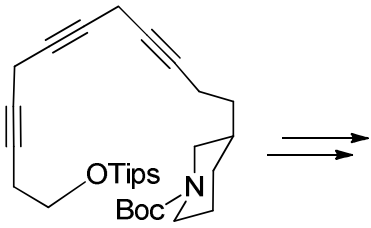

94

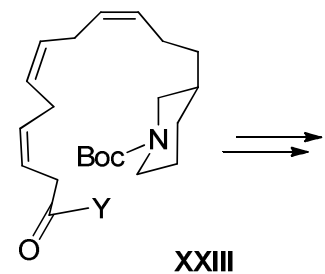

$\mathrm{Y}=\mathrm{OH}, \mathrm{H}$

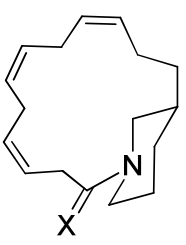

$\mathrm{X}=\mathrm{H}, \mathrm{H} ; \mathrm{O}$

Figure 3.20 Second pathway.

Thus, compound 94 was prepared using a classical copper cross-coupling reaction between the previously synthesized diyne $\mathbf{9 0}$ (see Scheme 3.26) with piperidine 93.

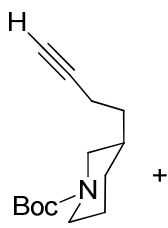

93

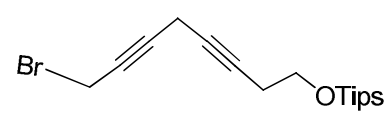

90

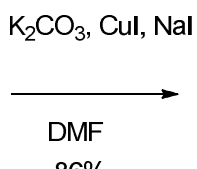

$86 \%$

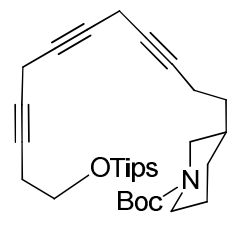

94

Scheme 3.34. Construction of 94 . 
With compound $\mathbf{9 4}$ in hand we next tried to perform the selective hydrogenation reaction. In our first attempt we performed the reaction with the Lindlar's catalyst but the reaction gave a mixture of over-reduced product together with unreacted starting material. The use of Brown's catalyst or titanium isopropoxide-isopropylmagnesium chloride system ${ }^{115}$ afforded required compound $\mathbf{8 1}$ with poor yields and an increase of the load of catalysts lead to the degradation of the compound, probably due to the isomerization of the unsaturated system.

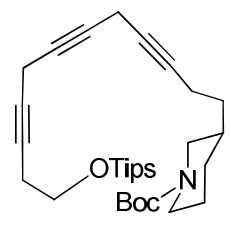

94

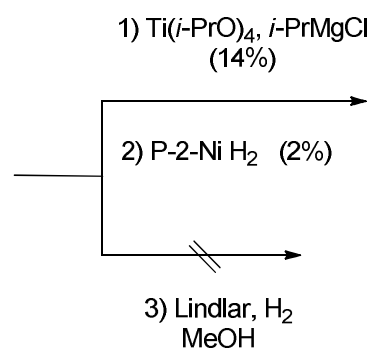

Lindlar, $\mathrm{H}_{2}$ MeH

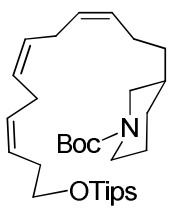

81

Scheme 3.35 Hydrogenation of alkynes.

Therefore, in our last attempt, we decided to apply another well-known methodology used for the selective cis-reduction of the alkyne that involves a hydroboration reaction of the alkyne followed by protonolysis. Thus the use of dicyclohexylborane followed by treatment with acetic acid ${ }^{123}$ was finally able to generate our compound $\mathbf{8 1}$ bearing the desired Z,Z,Z-skipped system of olefins with excellent yields.

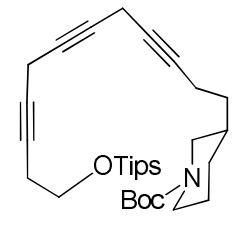

94

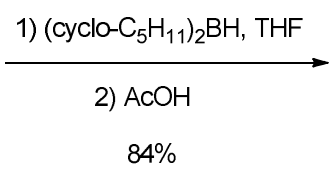

$84 \%$

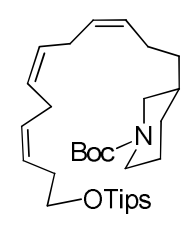

81

Scheme 3.36 Hydroboration/protonolysis reaction.

\subsubsection{Closure of the Macrocyclic D Ring of Madangamine A}

With compound $\mathbf{8 1}$ in hands we started to study the macrocyclization reactions that, as planned before, included an intramolecular aminoreduction or macrolactamization reactions.

${ }^{123}$ Brudermüller, M.; Musso, H. Angew. Chem., Int. Ed. Engl. 1988, 27, 298-299. 
The deprotection of the primary alcohol from compound $\mathbf{8 1}$ was achieved using hydrogen chloride ${ }^{124}$ in methanol solution since the classical use of TBAF resulted in an isomerization of the lateral chain. The resulting alcohol 97 was then oxidized with a Dess-Martin reagent to give aldehide $\mathbf{7 4}$ that was used to study the intramolecular aminoreduction macrocyclization. Unfortunately the reaction didn't take place and was observed only the formation of complex products probably derived by a degradation of the starting material.
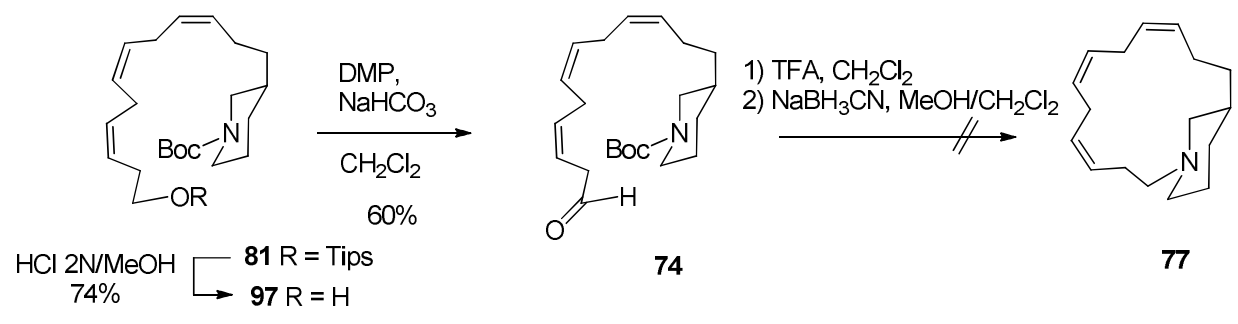

Scheme 3.37 Reductive amination.

The further oxidation of the aldehyde $\mathbf{7 4}$ to carboxylic acid $\mathbf{7 5}$ was performed using sodium chlorite. The following deprotection reaction gave the resulting aminoacid that was immediately treated with a combination of EDCl and $\mathrm{HOBt}$ which finally afforded compound $\mathbf{7 6}$ bearing the desired unsaturated $D$ ring of madangamine $A$.

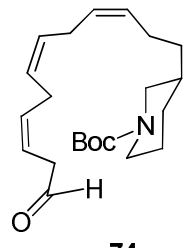

74

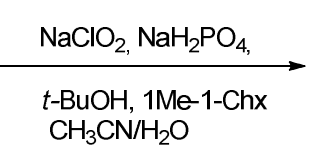

$\mathrm{CH}_{3} \mathrm{CN} / \mathrm{H}_{2} \mathrm{O}$

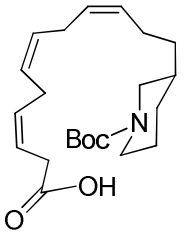

75

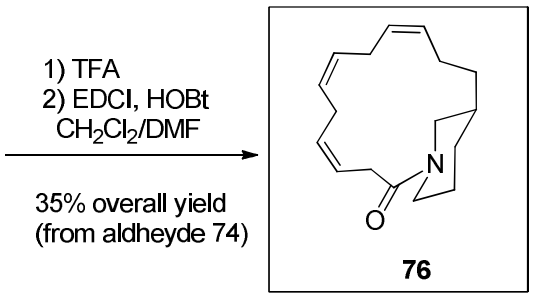

Scheme 3.38 Closure of A ring.

In summary the synthesis of the 15 -membered $D$ ring was achieved in a convergent way. The key steps included (i) a cross coupling reaction between the piperidine and the diyne chain (ii) a hydroboration followed by protonolysis and (iii) an intramolecular macrolactamization reaction. ${ }^{125}$

\footnotetext{
${ }^{124}$ (a) Ogilvie, K. K.; Thompson, E. A.; Quilliam, M. A.; Westmore, J. B. Tetrahedron Lett. 1974, 15, 28652868. (b) Magnus, P.; Giles, M.; Bonnert, R.; Johnson, G.; McQuire, L.; Deluca, M.; Merritt, A.; Kim, C. S.; Vicker, N. J. Am. Chem. Soc. 1993, 115, 8116-8129.

${ }^{125}$ Campbell, J. E.; Englund, E. E.; Burke, S. D. Org. Lett. 2002, 4, 2273-2275.
} 


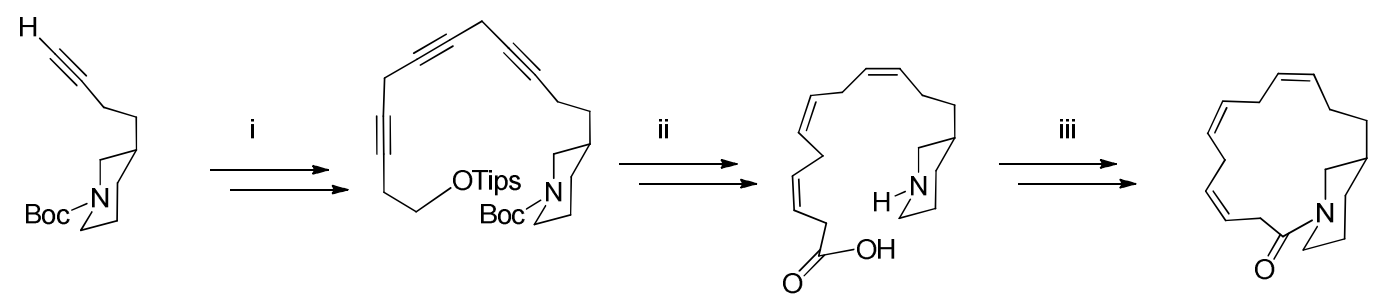

Scheme 3.39 Synthesis of the A ring.

The methodology described could be applied for the synthesis of the $D$ ring of madangamine $A$ using an advanced tricyclic precursor bearing a cyclic acetal, precursor of an aldehyde, at C-9 position. A Seyfert-Gilbert homologation ${ }^{126}$ reaction could convert the carbonylic group into a terminal alkyne which could react, through a cross-coupling reaction, with $\mathbf{9 0}$. The result tryine would be converted to the Z-skipped system of olefins and cyclized to finally give the tetracyclic $A B C D$ system of madangamine $A$.

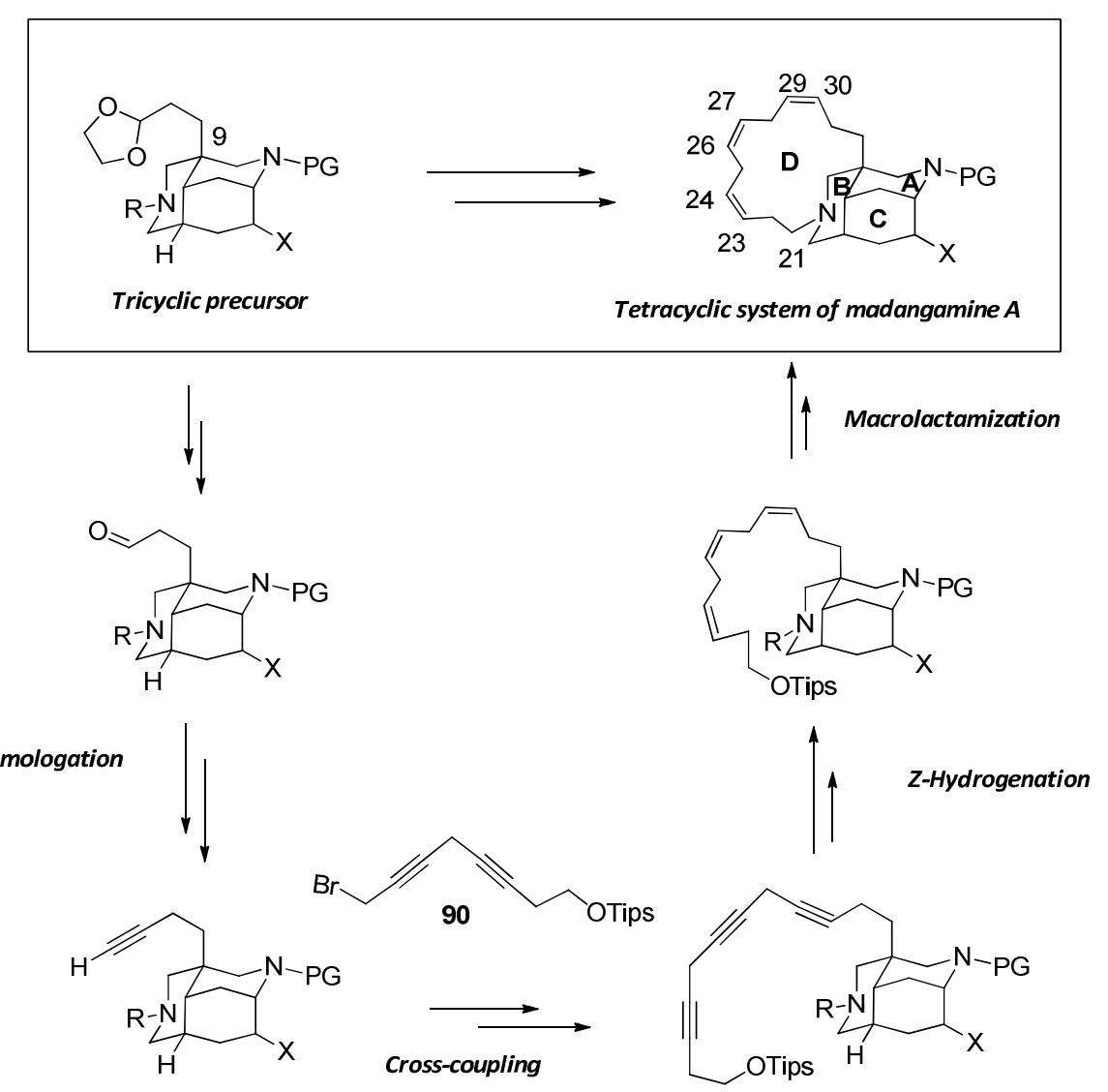

Scheme 3.40 Application of the methodology.

${ }^{126}$ (a) Gilbert, J. C.; Weerasooriya, U. J. Org. Chem. 1982, 47, 1837-1845. (b) Müller, S.; Liepold, B.; Roth, G. J.; Bestmann, H. J. Synlett 1996, 521-522. 
In summary, using appropriate simplified piperidine model compounds, we have explored different synthetic routes to construct the 13 - and 14 -membered $D$ rings of madangamines C-E as well as the all-cis-triunsaturated 15 -membered $D$ ring of madangamine $A$.

\subsection{CONSTRUCTION OF TETRACYCLIC ABCD SYSTEM}

The results obtained in the model compounds pointed out the efficiency of the RCM reactions for the synthesis of large rings. The surprising excellent yields obtained in the construction of the 14-membered $D$ ring, led us to madangamine $D$ as the target molecule for our synthetic endeavor.

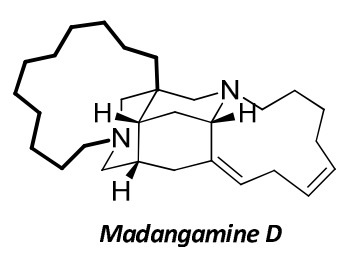

Figure 3.21 Structure of madangamine $D$.

According to our investigation, the construction of the completely saturated 14-membered D ring could be reached applying the RCM protocol, previously set up on the model compound, followed by hydrogenation reaction of the resulting double bond. Thus, the synthesis of the $A B C D$ tetracyclic system of madangamine $D$ from an advanced tricyclic compound would involve: (i) the incorporation at N-7 position of a carbon chain with a terminal double bond and the generation of the other terminal olefin from the dioxolane; (ii) the macrocyclization by a ring-closing metathesis reaction. We hoped that the chemical behavior of the piperidine model compound could be extrapolated to our complex tricyclic diene.

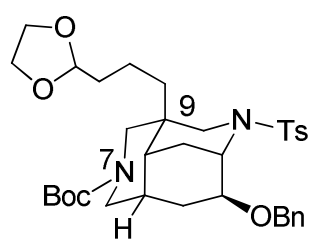

Tricyclic precursor

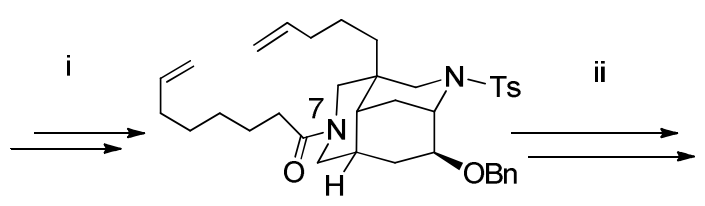

Diene precursor of RCM

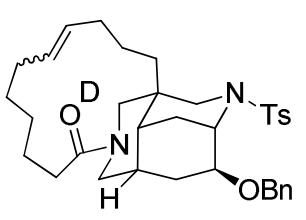

ABCD tetracyclic system

Figure 3.22 RCM strategy. 


\subsubsection{Assembly of the 14-Membered Ring of Madangamine D}

Our purpose by now is the application of the most promising RCM strategy that involve the formation of the required diene bearing a 5-membered carbon chain at C-9 position and a 8membered chain on the nitrogen of the $B$ ring.

The synthesis of the di-olefin started with the functionalized tricyclic compound $\mathbf{4 4}$ which was treated with a mixture of TFA $/ \mathrm{CH}_{2} \mathrm{Cl}_{2}$ in anhydrous conditions which were necessary to perform the Boc-deprotection of the piperidinic $\mathrm{B}$ ring without affecting the dioxolane group. The excess of TFA was co-evaporated with toluene and the resulting amine $\mathbf{1 0 0}$ was then acylated with octenoyl chloride 101, previously prepared from the commercial acid, affording compound 102 with excellent yields.

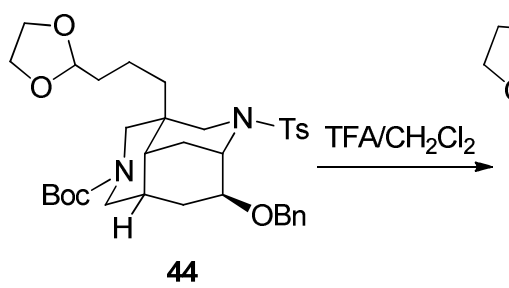

44

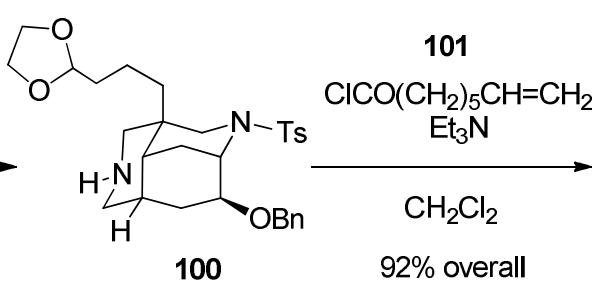

100

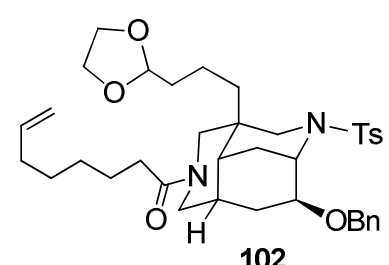

102

Scheme 3.41 Preparation of the olefin.

The aldehyde $\mathbf{1 0 3}$ was readily obtained after the acidic hydrolyisis of the dioxolane $\mathbf{1 0 2}$ which was immediately used in the subsequent Wittig methylenation reaction which afforded the desired di-olefin 98.

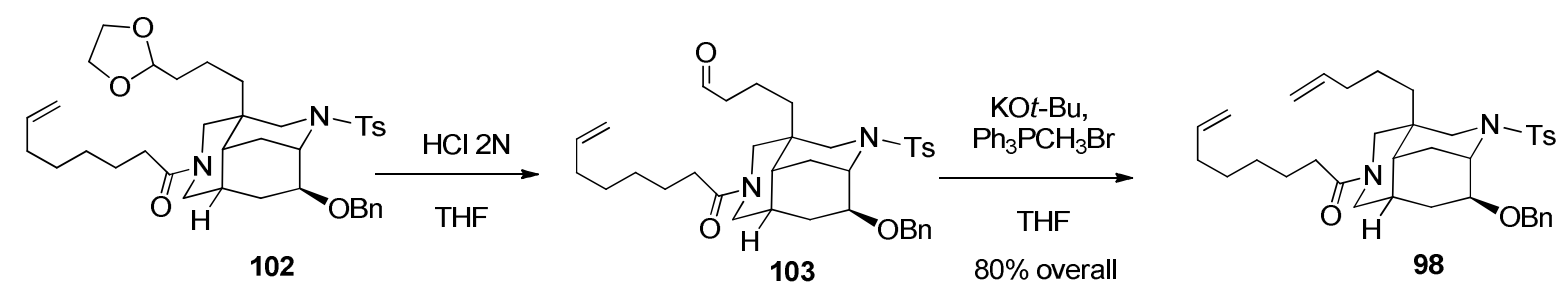

Scheme 3.42 Synthesis of the diene 98.

Starting from diene 98 we tried to apply the RCM protocol, described in Section 3.3.2, which involves a slowly addition of the olefin via syringe pump in a $0.2 \mathrm{mM}$ refluxing solution of a 2 nd generation Grubbs catalyst in dichloromethane. As we hoped the reaction underwent a macrocyclization and afforded the unsaturated tetracyclic system 99 in $54 \%$ of yield in a 2:1 
mixture of $E: Z$ isomers. No cross-metathesis products were observed and the reaction permitted the recovery of unreacted starting material. ${ }^{47}$

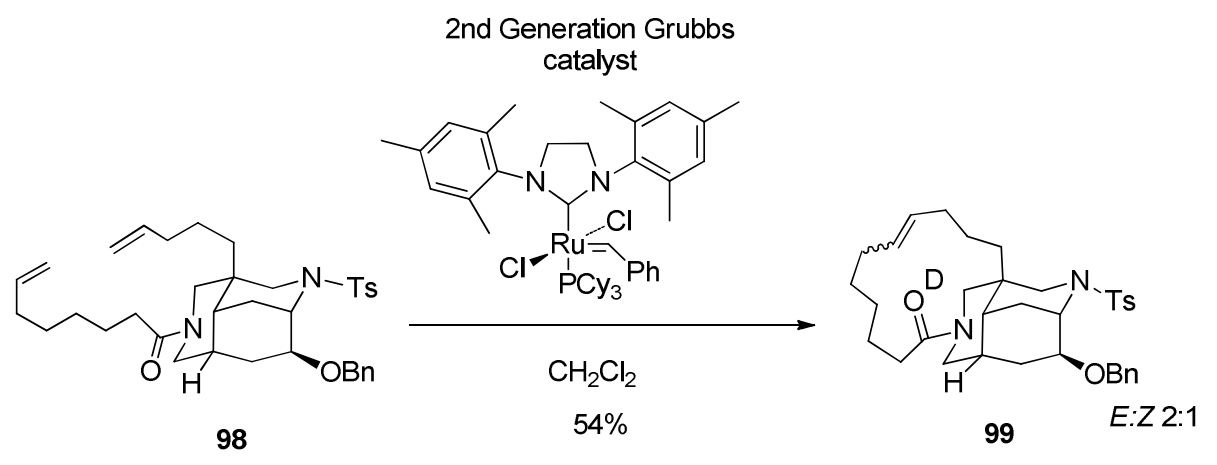

Scheme 3.43 RCM reaction with 2nd generation Grubbs catalyst.

The results obtained in the RCM macrocyclization reaction were satisfactory but not as excellent as the ones observed in the piperidine model. In our attempts to improve the yield of the reaction we first tried to carry out the reaction avoiding the slow addition of the diene via syringe pump, since we thought that the high temperature could inactivate the Grubbs catalyst. We next tried to work in a more concentrated solution but, in both cases, we were not able to improve the yields. Taking into account that our efforts for optimize the metathesis reaction were unsuccessful (change of temperature, catalyst's loading, solvent and time of reaction) and, considering that we were far from the end and a consistent amount of tetracycle compund $\mathbf{9 9}$ were needed to complete the synthesis, we decided to carry out the reaction using other ruthenium catalysts. Thus, when the reaction was conducted with a 2 nd generation Hoveyda-Grubbs catalyst, we observed a little yield improvement (64\%) and a substantial difference in the $E: Z$ ratio (1:1). To our delight, the reaction of diene 98 with the 1st generation Grubbs catalyst afforded the desired unsaturated tetracyclic system $\mathbf{9 9}$ with $90 \%$ yield and a complete inversion of the $E: Z$ ratio selectivity ( $E: Z$ ratio $1: 2$ ), compared with the use of the 2 nd generation Grubbs catalyst. 


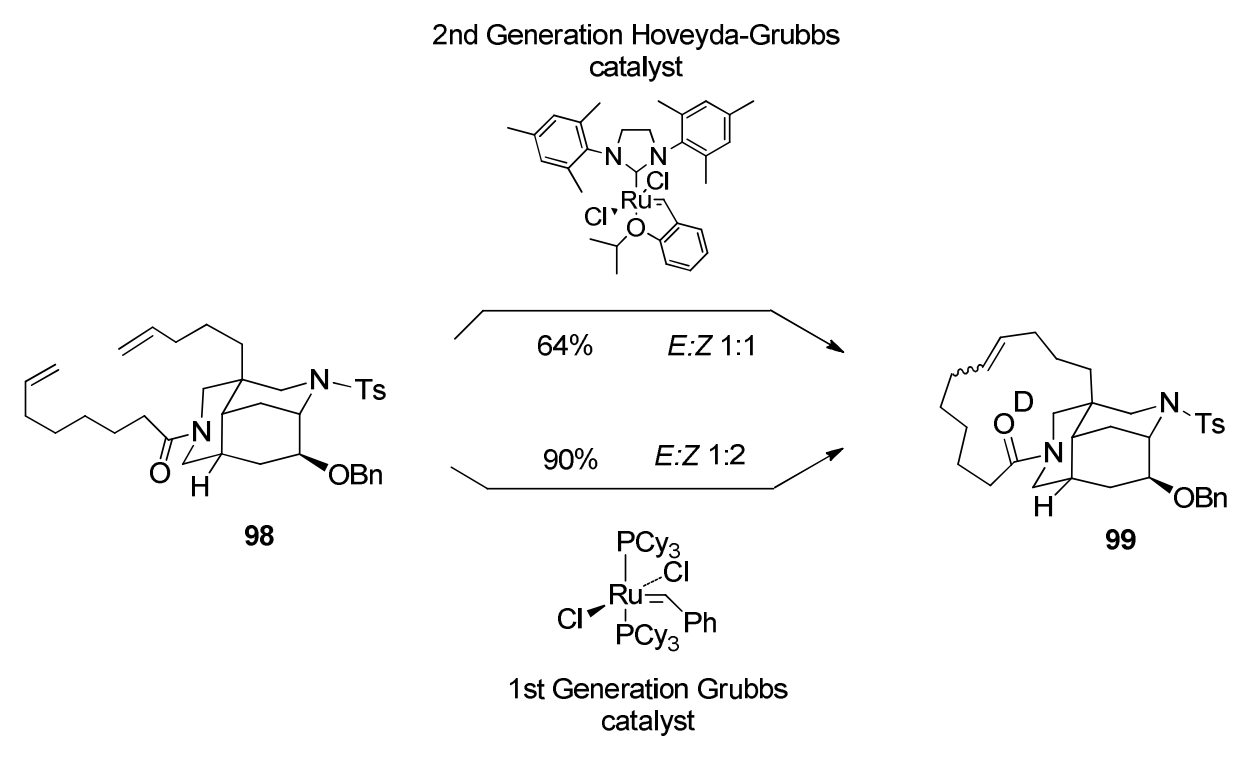

Scheme 3.44 Annulation of macrocyclic ring D.

Grubbs and coworkers, in a recent study ${ }^{127}$ toward the RCM reaction of a 14-membered lactone, have showed the existence of a significant difference of stereoselectivity in the use of the first or second generation Grubbs catalysts. The graphic below shows the sterechemical $E / Z$ depedence of the reaction on the the type of catalysts used in the RCM of a 14-membered ring. In addition, the high trans-preference observed in the reaction with the 2 nd generation catalyst seems to be due to secondary isomerization of the ring-closed product.

These results can be extrapolated to our substrate to explain the observed $E / Z$ stereoselectivity.

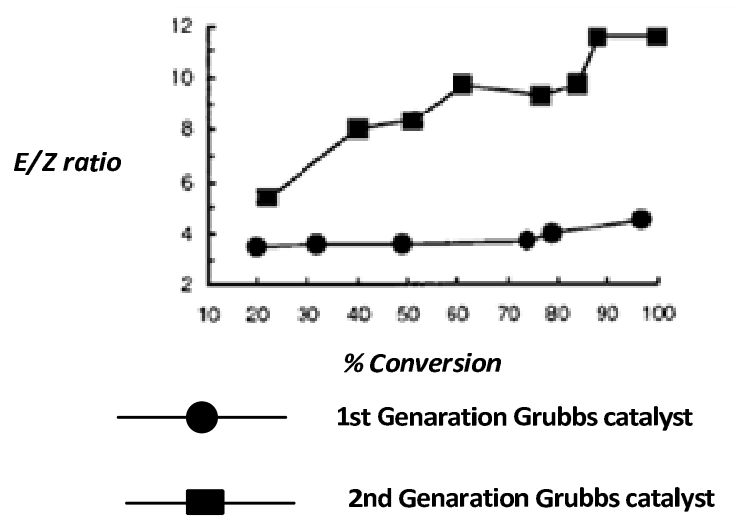

Figure 3.23 Stereoselectivity of Grubbs catalysts.

${ }^{127}$ (a) Lee, C. W.; Grubbs, R. H. Org. Lett. 2000, 2, 2145-2147. (b) For reviews of the alkene-metathesis reaction, see reference 58 . 
The subsequent hydrogenation at atmospheric pressure of compound 99, with conventional palladium catalyst $\left[\mathrm{Pd} / \mathrm{C}\right.$ or $\left.\mathrm{Pd}(\mathrm{OH})_{2}\right]$ in common solvents, such as methanol, ethanol and THF, led to the formation of tertracyclic system 104 but wasn't able to remove the benzyl group from the tertiary alcohol.

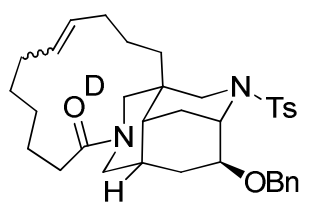

99

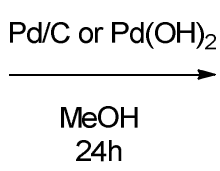

$24 \mathrm{~h}$

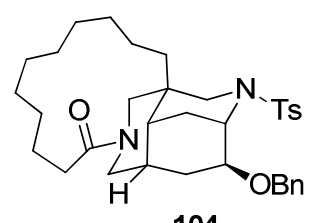

104

Scheme 3.45 Hydrogenation reactions.

We next tried the use of platinum oxide in methanol at room temperature and atmospheric pressure which tragically caused the hydrogenation of the benzene ring leading to the formation of compound 105.

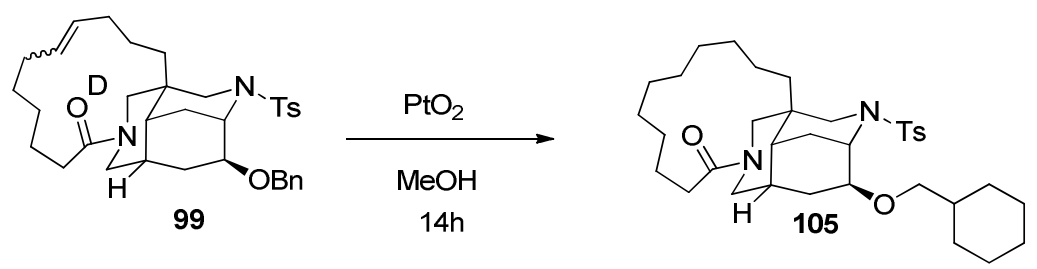

Scheme 3.46 Hydrogenation with $\mathrm{PtO}_{2}$.

The problem was finally solved changing the solvent. The use of $\mathrm{Pd} / \mathrm{C}$ catalyst in a 9:1 solution of ethanol and water at last permitted to afford tertiary alcohol which was immediately oxidized to ketone 106 with Dess-Martin periodinane.

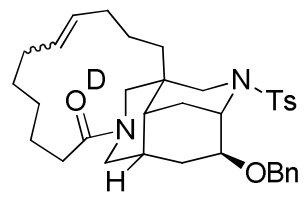

99

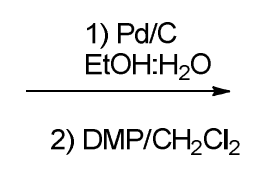

$75 \%$ overall from 98

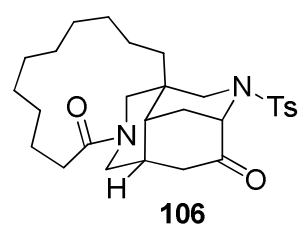

106

Scheme 3.47 Synthesis of the tetracyclic ketone. 
Tetracyclic compound $\mathbf{1 0 6}$ represents an important intermediate in our synthesis since can be produced in high yields with only few steps starting from the functionalized diazatricyclic core. Therefore the presence of the ketone moiety, at C-3 position, makes this compound the perfect candidate to reach the final stage of the synthesis: the elaboration of the last $\mathrm{E}$ ring.

In summary we have validated and optimized the RCM methodology previously studied on the piperidinic model compound and we have set up a protocol that permitted to achieve the synthesis of the functionalized tetracyclic $A B C D$ system of madangamine $D$ from diene $\mathbf{9 8}$ with an almost quantitative yield and using a very efficient synthetic procedure.

The next phase of our work will include the construction of the eastern E ring of the alkaloid which would lead to complete the first total synthesis of madangamine D.

\subsection{ANNULATION OF MACROCYCLIC RING E}

The 11- membered $E$ ring common of madangamine A-E possess a peculiar motif formed by a $Z, Z$ skipped system of olefins. In Section 3.3.3 we have already presented the features of this structure and point out its complexity from a synthetically point of view. The construction of the $E$ ring of madangamines is even more synthetically intricate since one of the Z-olefin, which forms the skipped system, is directly connected with the $C$ ring of the alkaloid.

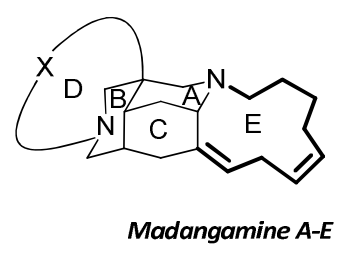

Figure 3.24 General structure of madangamines.

With the tetracyclic $A B C D$ ketone 106 in hands our synthetic plan for the assembly of the eastern $\mathrm{E}$ ring of madangamine will involve two different strategies:

- A stepwise pathway described by Kibayashi which would start with a Still-Gennari modification of the Horner-Wadsworth-Emmons olefination ${ }^{128}$ to install the C-3/C-20 $Z$-double bond. The remaining six carbon of the lateral chain would be inserted through a palladium-catalyzed coupling reaction with the appropriate (Z)-

\footnotetext{
${ }^{128}$ Still, W. C.; Gennari, C. Tetrahedron Lett. 1983, 24, 4405-4408.
} 
vinylstannane while the annulation of the $\mathrm{E}$ ring could be performed with a reductive amination.

- A convergent strategy in which a Wittig reaction between the tetracyclic ketone and a suitable phosphonium salt would incorporate the required 8-membered $Z, Z$ skipped chain in a single step. The subsequent intramolecular macrolactamization reaction would afford the desired pentacyclic system.

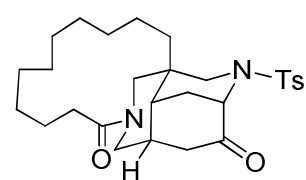

ABCD Tetracyclic System
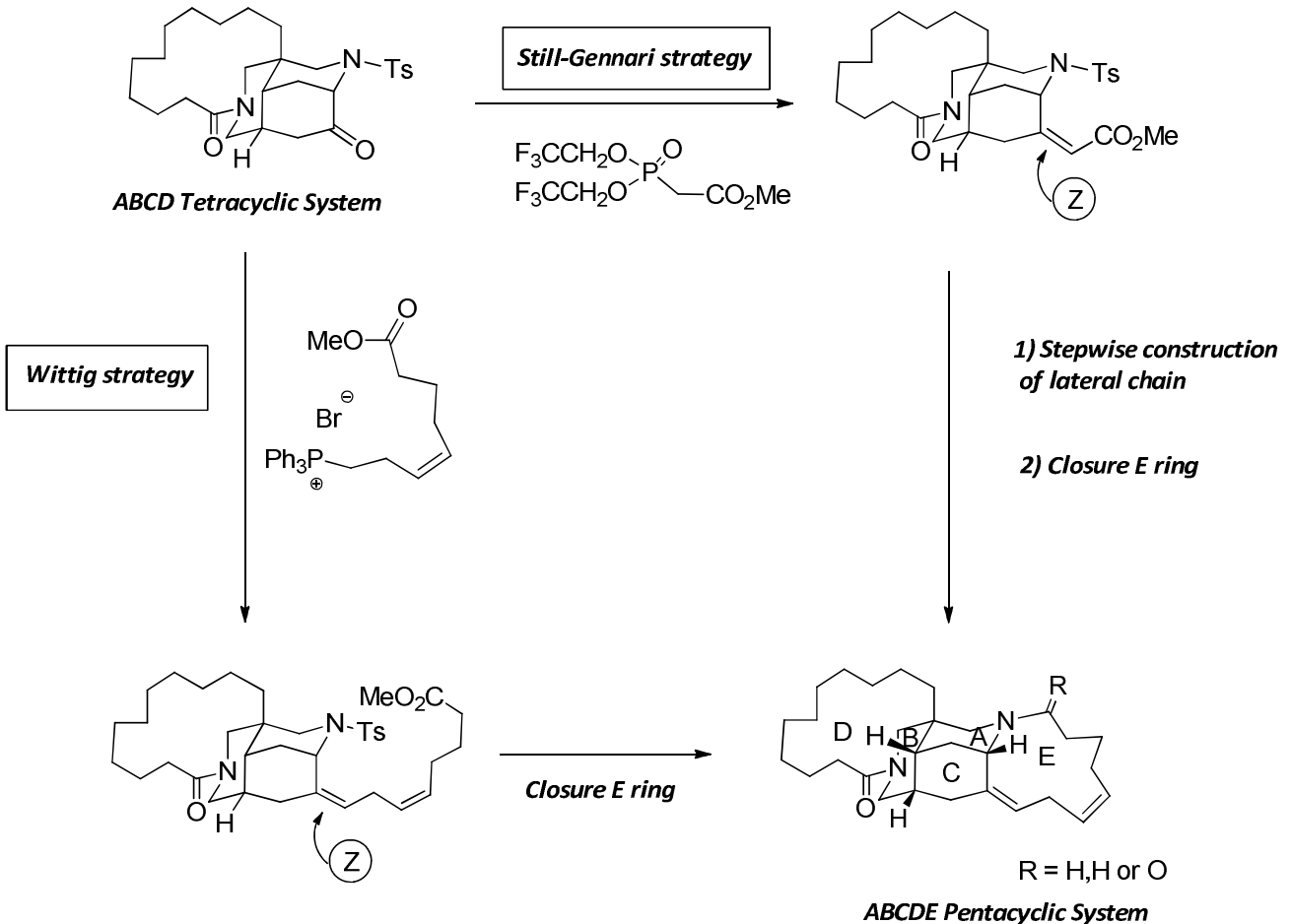

Scheme 3.48 Synthetic strategies.

The synthetic plan for the annulation of the E ring will follow the trend used until now, consisting on the exploration of different methodologies first on simple model compounds and the application of the more promising protocol on our tetracyclic system.

\subsubsection{Synthesis of Model Compounds}

The morphan model was synthesized thanks to the important collaboration of Federica Romagnoli who, despite the short time spent in our laboratories, was able to give a priceless contribution in the project.

Thus, the AC model compound was constructed, in very few steps, starting from the commercially available 4-vinylcyclohexene and using, as a key step, the intramolecular 
aminohydroxidation methodology previously described in the synthesis of the $A B C$ core of madangamine.

The hydroboration of the exocyclic double bond of 107 afforded the primary alcohol 108 which was easily converted, through a two steps sequence, to the corresponding azide 109.

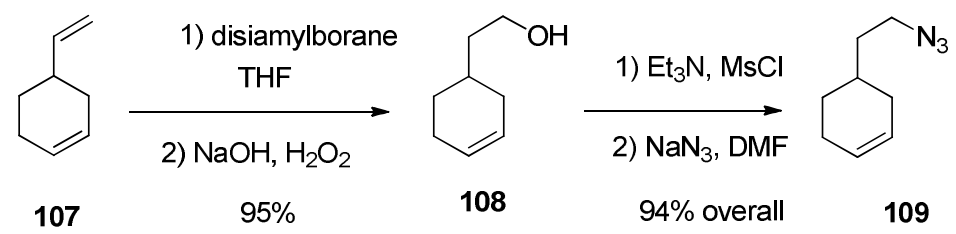

Scheme 3.49 Synthesis of azide 109.

The construction of the A ring was achieved via epoxidation of the cyclohexene double bond of 109 followed by a Staudinger reduction of the azide moiety. The initially formed amino epoxide underwent a smooth in situ cyclization, directly leading to the corresponding amino alcohols (110, 111 and 112), which were then $\mathrm{N}$-protected and immediately oxidized to ketones 113, 114 and 115.

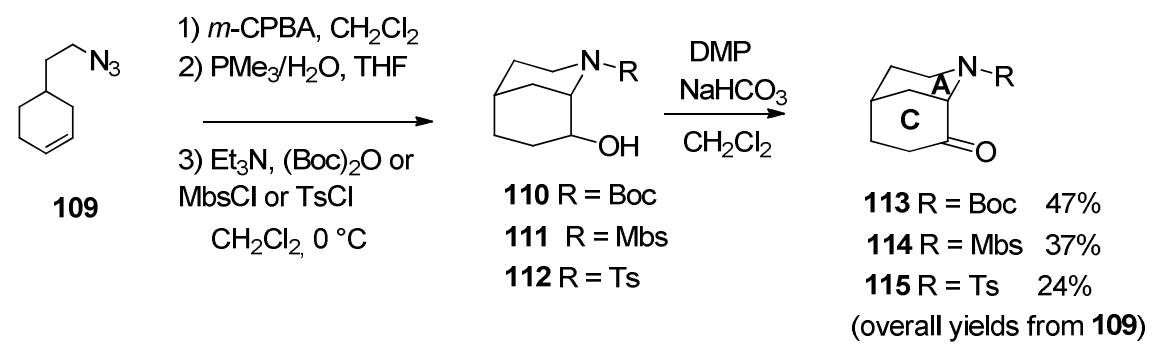

Scheme 3.50 Construction of AC morphan models.

This synthetic plan allowed to access to a azabicyclic system which represents not only the AC fragment of madangamine but a model compound, similar to the Kibayashi's one, we can use to test different methodologies to access to the 11-membered $Z, Z$ unsaturated $E$ ring.

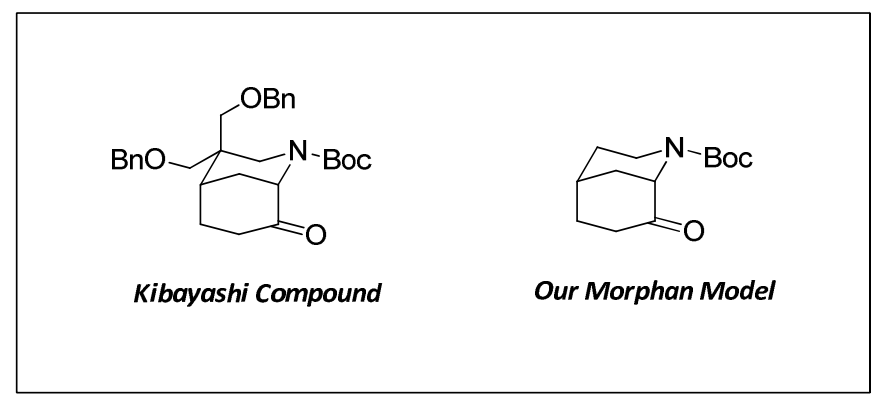

Figure 3.25 Model compounds. 


\subsubsection{Annulation of E Ring on Model Compounds}

\subsubsection{Still-Gennary Strategy}

The construction of the 11-membered $\mathrm{E}$ ring of madangamine was successful achieved by Kibayashi and coworkers in $2006^{40}$ starting from the morphan system 57a. ${ }^{129}$ The methodology used to install the crucial Z-unsaturation at the C-3 position involved a Still-Gennari (Z)selective Wittig-Horner olefination, which afforded the resulting ester 58a with an incredible high E:Z ratio of 11:1.

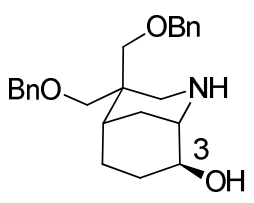

$57 a$

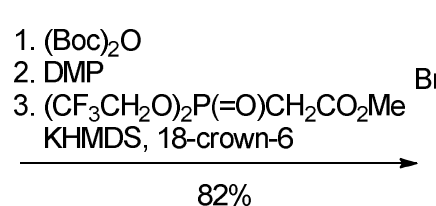

$82 \%$

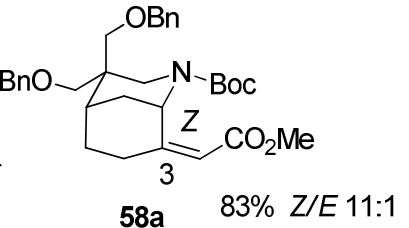

58a $\quad 83 \%$ Z/E 11:1

Scheme 3.51 Still-Gennari reaction.

Taking into account that, apparently, this strategy has demonstrated to be particularly effective in the installation of the Z-isomer of the related ester, we decided to test the Kibayashi's methodology in our model compounds $\mathbf{1 1 3}$ and 114. Unfortunately the application of the described Still-Gennari olefination under either the original ${ }^{119}$ or slightly modified ${ }^{130}$ reaction conditions resulted in the generation of $Z / E$ mixtures of alkenes, in which the undesired $E$-isomer was predominant (35:65 ratio).

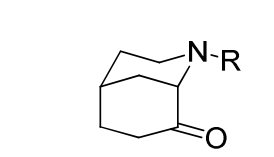

$113 \mathrm{R}=\mathrm{Boc}$

$114 \mathrm{R}=\mathrm{Mbs}$

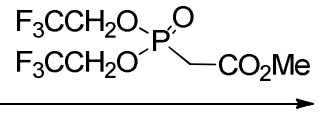

$$
\text { a. or b. }
$$

$10-50 \%$

Z/E 35:65

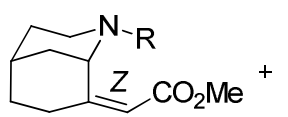

$116 \mathrm{R}=\mathrm{Boc}$

$117 \mathrm{R}=\mathrm{Mbs}$

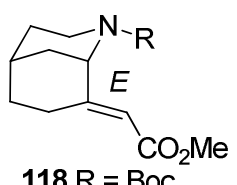

$118 \mathrm{R}=\mathrm{Boc}$

$119 \mathrm{R}=\mathrm{Mbs}$

Conditions: a. KHMDS, $18-$ crown- $6, \mathrm{THF},-78^{\circ} \mathrm{C}$

b. $\mathrm{LiCl}, \mathrm{DBU}, \mathrm{THF},-78^{\circ} \mathrm{C}$

Scheme 3.52 Application of Kibayashi protocol.

\footnotetext{
${ }^{129}$ See section 1.5.2

${ }^{130}$ Blanchette, M. A.; Choy, W.; Davis, J. T.; Essenfeld, A. P.; Masamune, S.; Roush, W. R.; Sakai, T. Tetrahedron Lett. 1984, 25, 2183-2186.
} 


\subsubsection{Wittig Strategy}

In view of these disappointing results we decided to put aside this stepwise strategy and undertake another synthetic route that would involve (i) the direct installation of the eightmembered carbon chain at C-3 position, and (ii) the subsequent annulation of the E ring through an intramolecular macrolactamization. We were convinced that the required $Z, Z$ poliunsaturated system could be directly installed to our azabicycle model using a $Z$-selective Wittig reaction with an appropriate phosphonium salt. The control over the selectivity of the reaction could be achieved using a suitable "nonstabilized" phosphonium salt. This ideal ylide already contains the central cis $\mathrm{C}-17 / \mathrm{C}-18$ double bond present in the $\mathrm{E}$ ring of madangamines, and possesses the ester functionality required for the final macrolactamization.

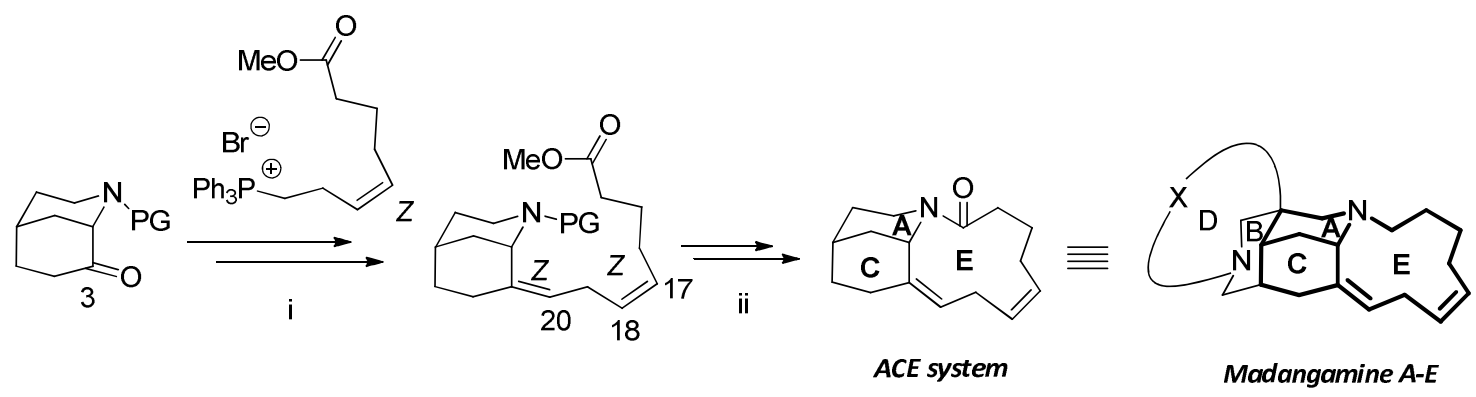

Scheme 3.53 Wittig strategy.

The desired unsaturated chain 124 was prepared, as a single cis-isomer, through a Wittig reaction between the phosphonium bromide 122, prepared starting from 3-bromopropanol, and methyl-5-oxopentanoate 123.

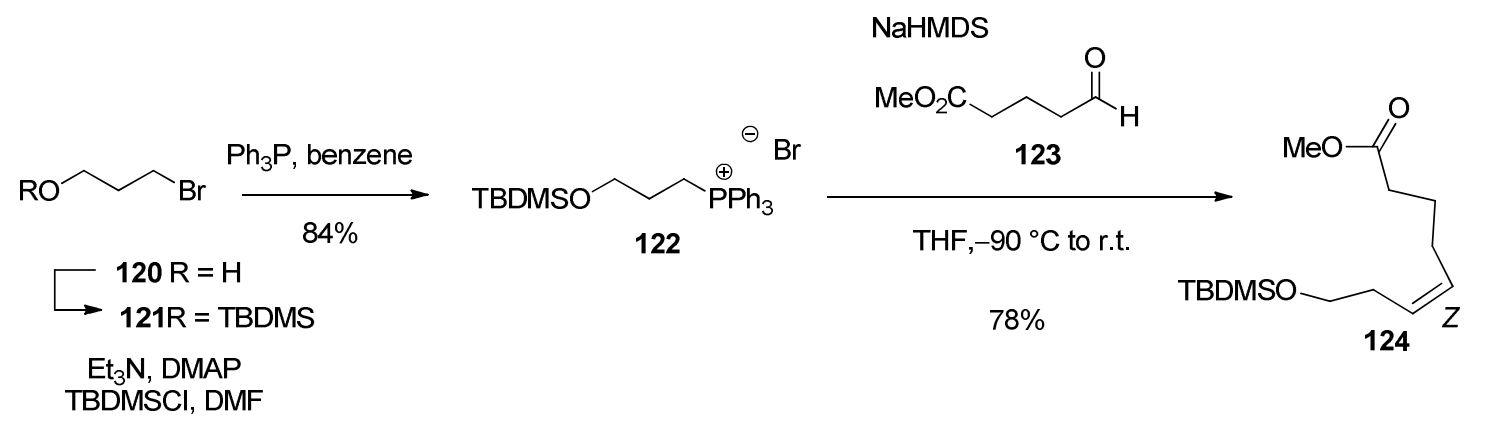

Scheme 3.54 Construction of unsaturated chain 124.

The required Wittig reactive was readily obtained after the deprotection of the silyl ether $\mathbf{1 2 4}$ and the subsequent transformation of the resulting alcohol $\mathbf{1 2 5}$ into the phosphonium salt 126. ${ }^{131}$

${ }^{131}$ The synthesis of the phosphonium salt 126 was accomplished by modification of known procedures: (a) Zamboni, R.; Milette, S.; Rokach, J. Tetrahedron Lett. 1983, 24, 4899-4902. (b) Wang, S. S.; Rokach, J.; 


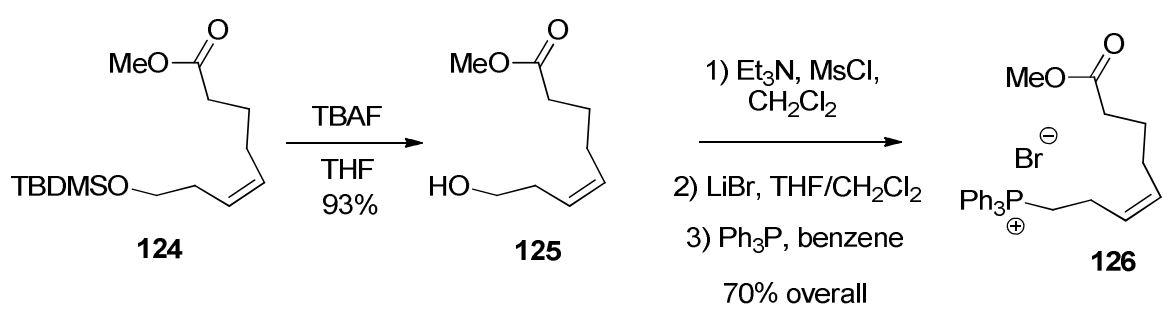

Scheme 3.55 Synthesis of phosphonium salt 126.

Compound 126 belongs to the nonstabilized class of ylides, which doesn't possess conjugating substituents (e.g., COOMe, $\mathrm{CN}$ or $\mathrm{SO}_{2} \mathrm{Ar}$ ) on the ylidic carbon, and that generally reacts in Wittig reactions under kinetic control giving preferentially Z-olefins.

The carbonyl compound and the ylide approach at right angles and large substituents are kept away from each other due to the steric effects. The first step of the Wittig reaction is represented by the formation of the oxaphosphetane in which the steric 1,2 or 1,3 repulsions lead to the distortion of the cycle and the formation of a syn-puckered-oxaphosphetane which can undergo in a syn-elimination to give preferentially the corrisponding Z-alkene.

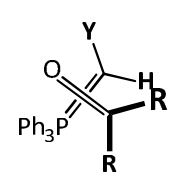

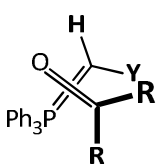<smiles>CCCCCCCC</smiles>

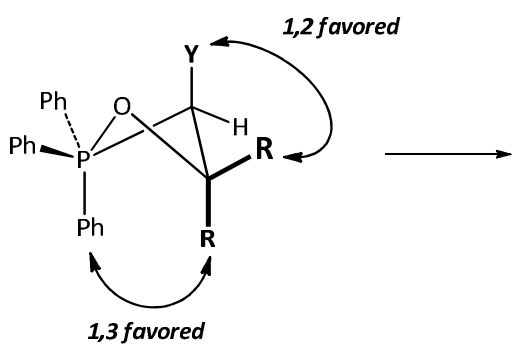

Syn-Oxaphosphetane

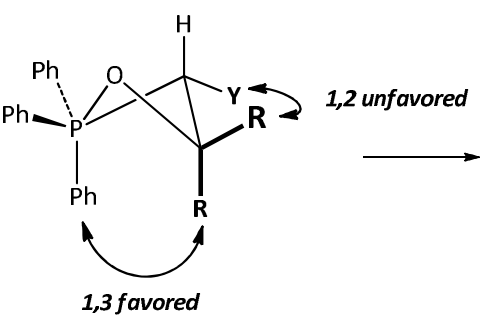

Anti-Oxaphosphetane

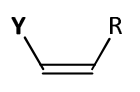

(Z)-alkenes

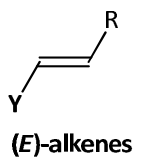

(E)-alkenes

Figure 3.26 Stereoselectivity in Wittig reactions.

The critical coupling reaction between phosphonium salt 126 and the morphan model compound $\mathbf{1 1 5}$ conducted to the formation of desired compounds $\mathbf{1 2 7}$ and $\mathbf{1 2 8}$ with an

Powell, W. S.; Dekle, C.; Feinmark, S. J. Tetrahedron Lett. 1994, 35, 4051-4054. (c) Sandri, J.; Viala, J. J. Org. Chem. 1995, 60, 6627-6630. 
excellent $E: Z$ ratio of $1: 10$, confirmed by $2 D$ NOESY experiment, which clearly showed the presence of a NOE effects between $\mathrm{H}-4 / \mathrm{H}-20$ and $\mathrm{H}-2 / \mathrm{H}-19$.

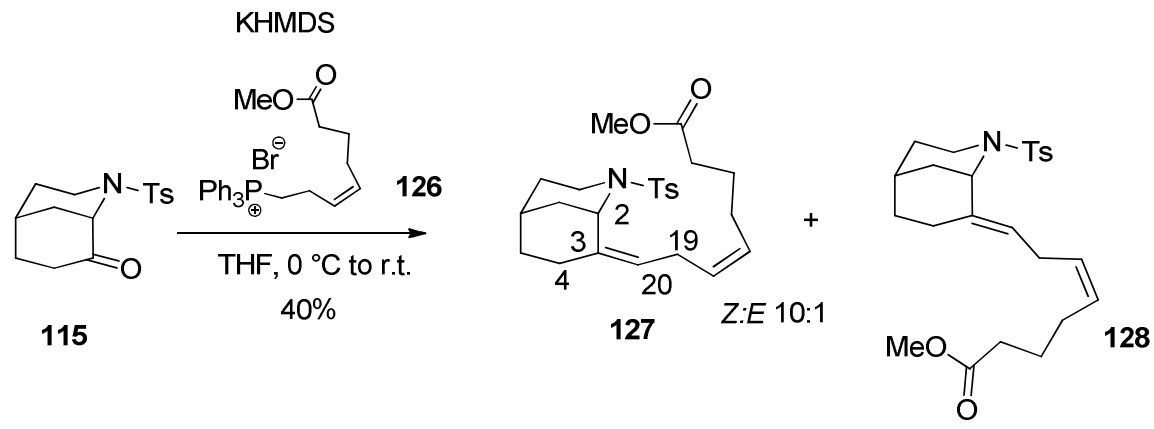

Scheme 3.56 Wittig reaction.

Once we installed the fragment $\mathrm{C}-13 / \mathrm{C}-20$ on the azabicyclic morphan we applied the macrolactamization protocol that would led to the synthesis of the ACE ring system of madangamine. The synthetic plan involved the removal of the sulfonamide protecting group from 127 and 128, with a sodium naphtalenide solution, followed by hydrolysis of the ester moiety which provided the resulting amino acids. The crude compounds were finally treated with a HOBt/EDCl mixture of coupling agents that afforded tricyclic lactam 131, bearing the $(Z, Z)$-unsaturated 11 -membered ring of the target alkaloids.

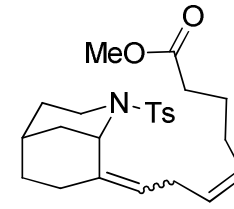

127-128

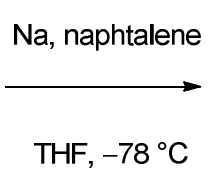

$95 \%$

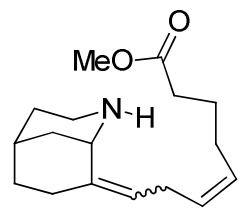

129-130

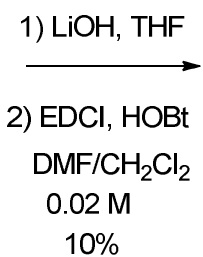

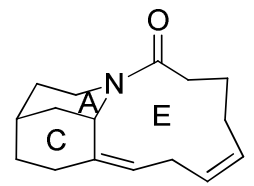

131

ACE System

Scheme 3.57 Closure of E ring.

Completely satisfied with this results we decided to go a little further and apply the same methodology described for the morphan using the fully-functionalized tricyclic compound $\mathbf{1 3 2}$.

Thus, the required Z-selective Wittig reaction, conducted with potassium hexamethyldisilazide, wasn't able to afford the desired compound and it has been necessary a substitution with sodium hexamethyldisilazide and a slight warming of the solution to accelerate the reaction. After one hour at $60{ }^{\circ} \mathrm{C}$ the desired Z-exo-olefin was obtained as a mayor product ( $E: Z$ ratio of 7:1) with $45 \%$ of yield. The inseparable mixture of diastereomeric compounds 133 and 134 were transformed into amino acids, and finally subjected to the described intramolecular macrolactamization reaction which provided the ABCE tetracyclic system 135. 


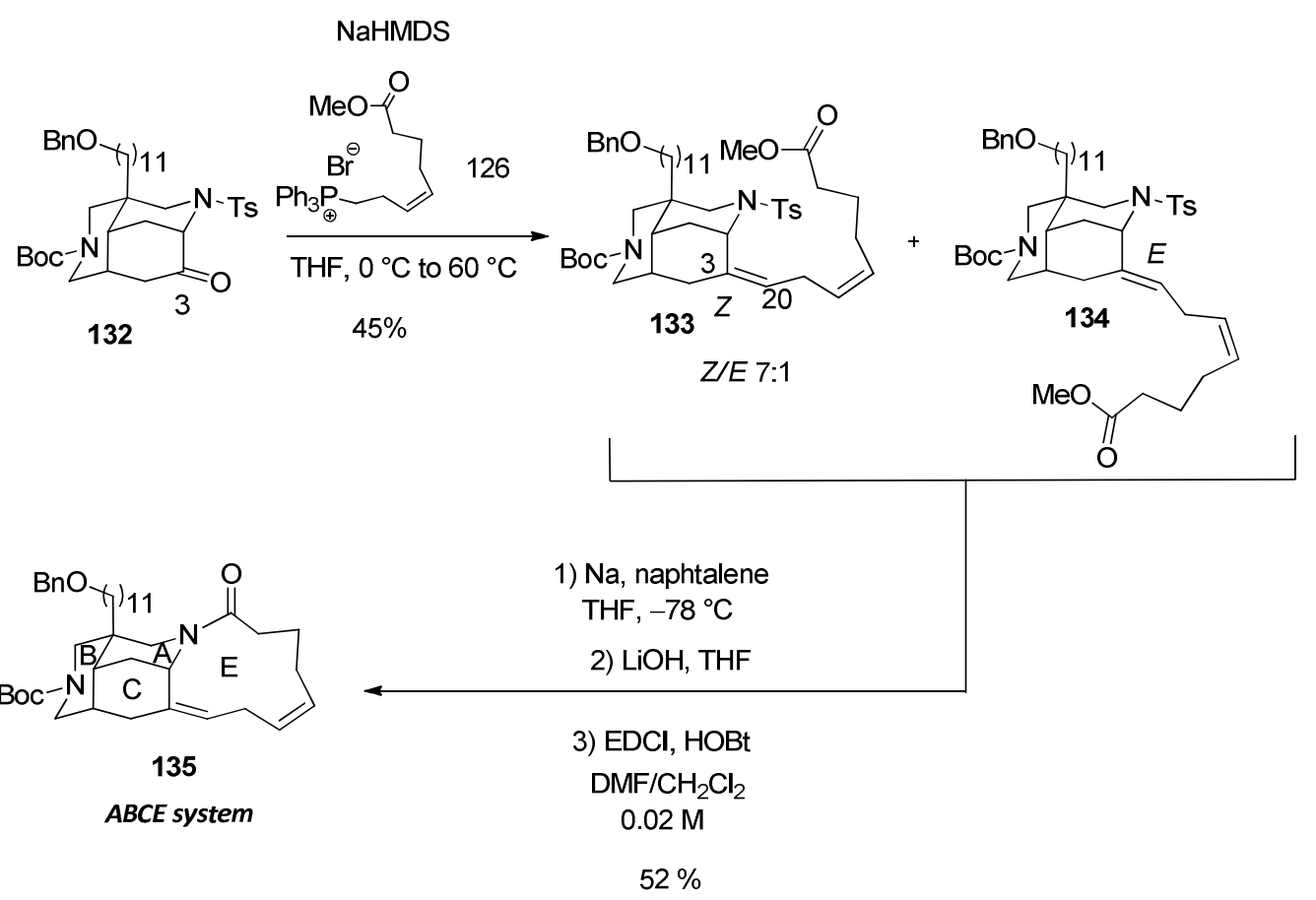

Scheme 3.58 Synthesis ABCE system.

These results confirm the efficiency of this procedure for the assembly of the eastern E ring. The synthetic plan for the stereoselective installation of the C-13/C-20 fragment was first achieved using the $A C$ system and then validated on a complex functionalized diazatricyclic ring, while the efficiency of the intramolecular macrolactamization for the construction of the 11-membered ring was confirmed in both models.

\subsubsection{Synthesis ABCDE Pentacyclic System of Madangamine D}

At this stage the next last challenge was represented by the annulation of the last eastern $\mathrm{E}$ ring, which access could be achieved applying the described methodology on the tetracyclic $A B C D$ system of madangamine $D$.

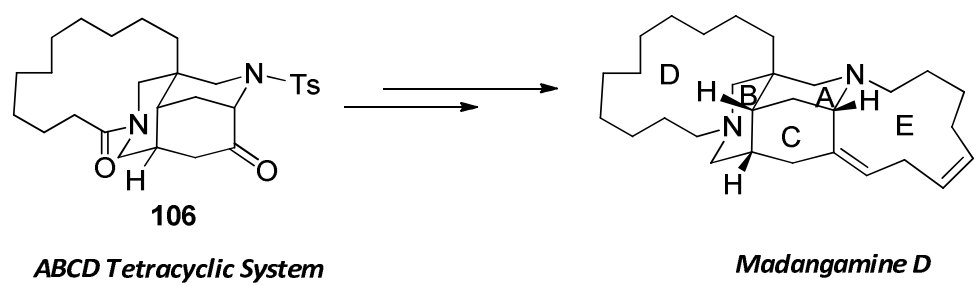

Scheme 3.59 From tetracycle to madangamine D. 
The incorporation of the $(Z, Z)$-unsaturated eight-carbon fragment, required to complete the synthesis of madangamine $D$, was carry out, as previously described for the models, using the phosphonium salt 126 and the tetracyclic ketone 106. In our first attempts the reaction gave a mixture of complex products and no traces of the desired compounds were observed. The problem was found in the extremely hygroscopicity of the phosphonium salt which impeded the formation of the ylide. ${ }^{132}$ In fact only when 126 was dried by repeated dilution/reconcentration in dry 1:1 THF: toluene, followed by overnight subjection in vacuo with inline phosphorous pentoxide, was able to react with sodium hexamethyldisilazide affording the typical orange color of the ylide.

Apparently this problem was not observed in the previously assays withy the bi- or tricyclic model compounds because of the larger-scale of the reactions. In our case the process was conducted with only few milligrams of products and the presence of a minimum amount of moisture seemed to be detrimental for the outcome of the reaction.

Thus, the reaction between the tetracyclic ketone 106 and the anhydrous Wittig reactive 126 afforded the desired diastereoisomeric mixture of alkenes 136 and $\mathbf{1 3 7}$ with excellent yields but with a loss in stereoselectivity (E:Z ratio 1:2.2).

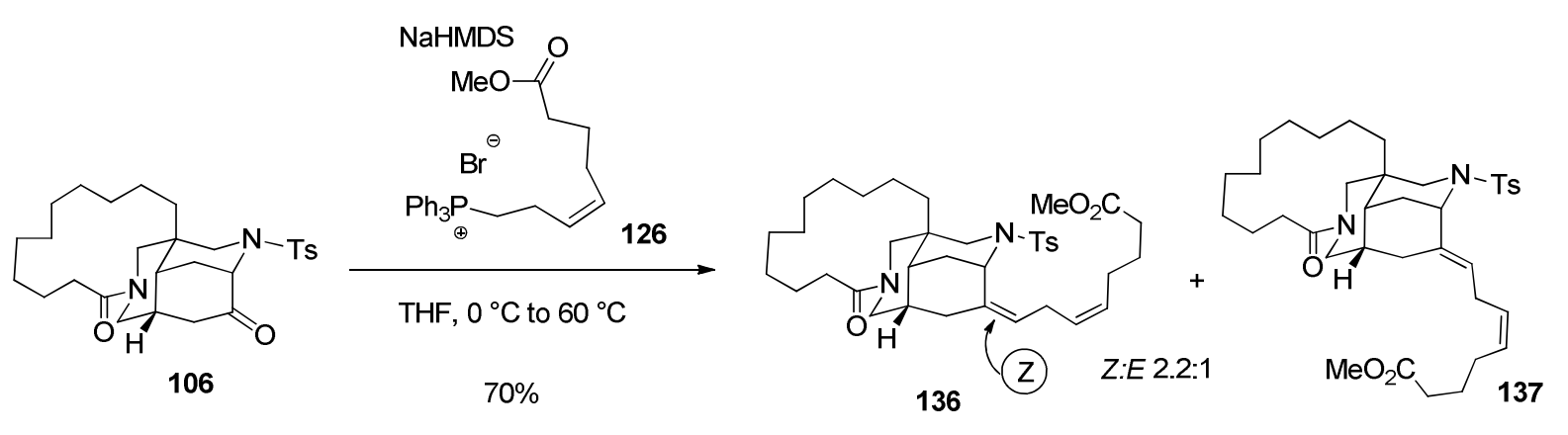

Scheme 3.60 Stereoselective Wittig reaction.

The removal of the tosyl group from compounds $\mathbf{1 3 6}$ and $\mathbf{1 3 7}$ permitted an easy separation of the diastereomeric mixture of the amines while the hydrolysis of the ester function afforded the desired carboxylic compound, which was immediately cyclized through an intramolecular macrolactamization reaction to give 138. The annulation reaction was performed in diluted conditions and, the aminoacid compound, was slowly added via syringe pump over a period of six hours to a solution containing the coupling agents.

${ }^{132}$ Christiansen, M. A.; Andrus, M. B. Tetrahedron Lett. 2012, 53, 4805-4808. 


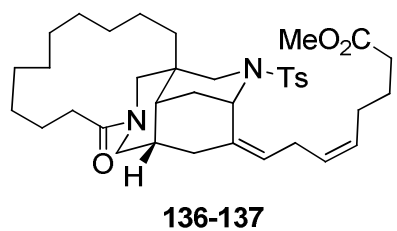

136-137
1) $\mathrm{Na}$, naphtalene, $\mathrm{THF},-78^{\circ} \mathrm{C}$ 2) $\mathrm{LiOH}, \mathrm{THF}$

3) $\mathrm{EDCl}, \mathrm{HOBt}$, $\mathrm{CH}_{2} \mathrm{Cl}_{2} / \mathrm{DMF}$

$75 \%$ overall

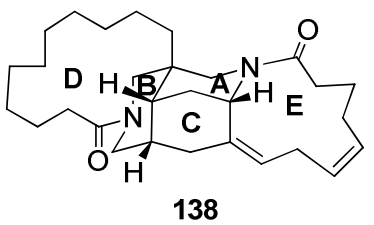

ABCDE Pentacyclic System

Scheme 3.61 Synthesis of the pentacyclic system.

The methodology described allowed the formation of pentacyclic dilactam compound $\mathbf{1 3 8}$ with excellent yields and the final reduction of both amide with $\mathrm{LiAlH}_{4}$ permitted to access to pure madangamine $\mathrm{D}$. The ${ }^{1} \mathrm{H}$ and ${ }^{13} \mathrm{C}$ NMR data of our synthetic madangamine were coincident with those reported for the natural product.

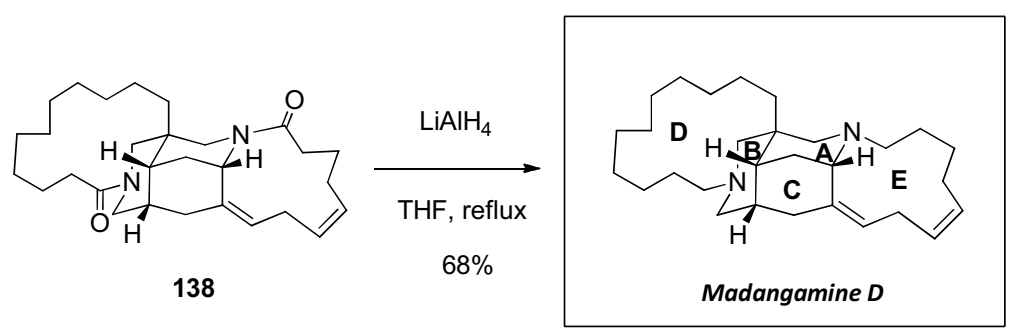

Scheme 3.62 Synthesis of madangamine D.

We have reported the first total synthesis of an alkaloid of the madangamine group, ${ }^{48}$ and confirmed their absolute configuration which, until now, has only been inferred by correlation with that of their presumed biosynthetic precursors, ingenamines. Synthetic madangamine $D$ possess unambiguous $2 S, 5 S, 9 R, 12 R$ absolute configuration and a specific rotation $\{[\alpha]=+$ 101.3 (c $\left.\left.0.29, \mathrm{CHCl}_{3}\right)\right\}$ with the same sign as in the closely related madangamines A-C. According to these data we can confirms the absolute configuration of this alkaloid family assigned by Andersen. ${ }^{19}$

As already discuss in the previous section no biological activity data has been reported to date for madangamine $D$, since was isolated as inseparable mixture with madangamine $E$ and further pharmacological research has been impeded by the low quantity of alkaloid available.

Thanks to collaboration with Pharmamar Company we could test the biological activity of madangamine $D$. The alkaloid showed significant in vitro cytotoxic activity against human colon $\mathrm{HT} 29\left(\mathrm{Gl}_{50} 4.4 \mu \mathrm{g} / \mathrm{mL}\right)$ and pancreas PSN1 $\left(\mathrm{Gl}_{50} 7.4 \mu \mathrm{g} / \mathrm{mL}\right)$ cancer cell lines, but was inactive against lung NSCLC A549 and breast MDA-MB-231 cancer cell lines at the highest assayed concentration $(>10 \mu \mathrm{g} / \mathrm{mL})$. 


\subsection{PRIMERA SÍNTESIS TOTAL DE LA (+)-MADANGAMINA D}

La madangamina $\mathrm{D}$ es un complejo alcaloide pentacíclico aislado de Xestospongia ingens, una esponja de la familia Haplosclerida que se encuentra en las aguas de la costa de la isla de Madang en Papúa Nueva Guinea.

Estructuralmente este alcaloide está constituido por un núcleo diazatricíclico central (anillos $A B C$ ) y dos puentes carbonados que forman dos macrociclos. El anillo $D$ está formado por una cadena completamente saturada de 11 carbonos que conecta la posición C-9 con el nitrógeno $\mathrm{N}-7$, mientras que el anillo $\mathrm{E}$, de 11 miembros, posee un sistema insaturado de tipo "skypped" con dos Z-olefinas, una de ellas directamente conectada a la posición C-3 del sistema tricíclico central.

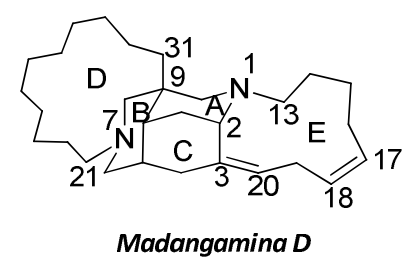

Figura 3.27 Madangamina D.

Hasta el presente ninguna síntesis total de un alcaloide perteneciente a la familia de las madangaminas ha sido descrita y la actividad biológica de la madangamina $D$ no ha sido estudiada debido a su limitada disponibilidad. En la presente Tesis Doctoral hemos querido sintetizar la madangamina D para confirmar su estructura, incluyendo su configuración absoluta, y preparar suficiente material para un eventual estudio biológico.

Nuestra estrategia sintética implica la utilización de una lactama bicíclica quiral, derivada del fenilglicinol, y a partir de esta, se pretende generar del núcleo diazatricíclico $A B C$ con la funcionalización y la substitución requerida para la posterior construcción de los macrocíclos $D$ y E de la madangamina $D$.

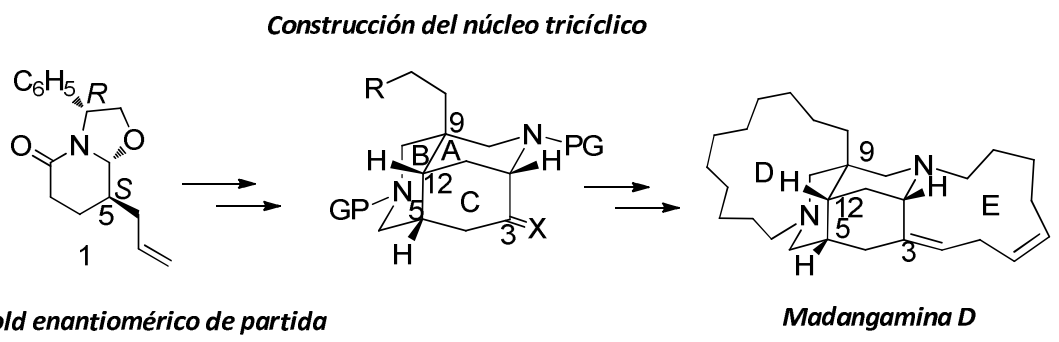

Figura 3.28 Estrategia sintética.

La lactama bicíclica 1 se preparó mediante una reacción de ciclocondensación entre el $(R)$ fenilglicinol y el 4-formil-6-heptonato, obtenido a su vez por adición conjugada del acrilato de metilo al 4-pentenal. La reacción de ciclodeshidratación conduce preferentemente a un solo estereoisómero de los cuatro posibles, debido al proceso de resolución cinética dinámica que tiene lugar por la presencia de un centro estereogénico configuracionalmente lábil en el substrato racémico. En esta etapa no solamente se generó el anillo piperidínico B de las 
madangaminas sino que además se instauró el primer estereocentro del alcaloide (posición 5 de las madangaminas).

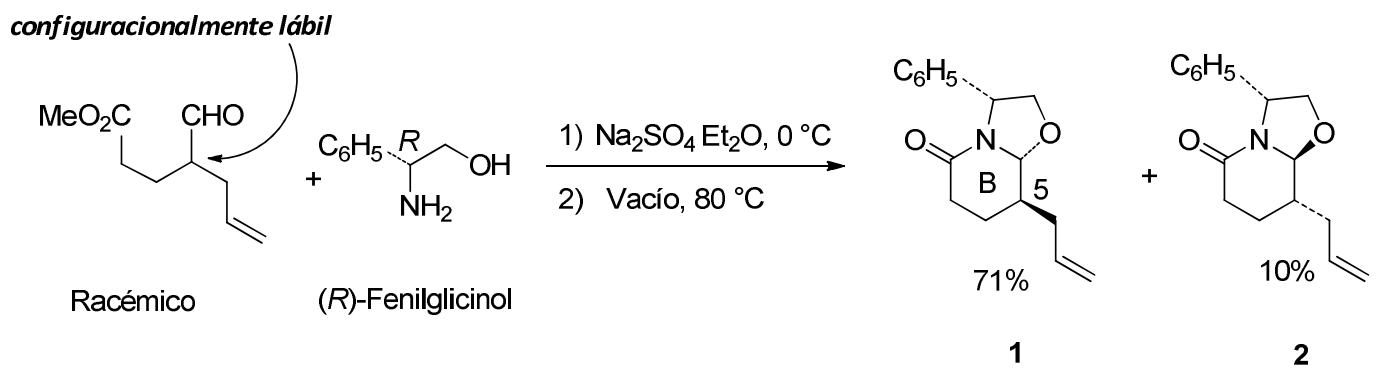

Esquema 3.63 Obtención lactama bicíclica inicial 1. Generación primer estereocentro.

La lactama insaturada $\mathbf{5}$ fue preparada en dos etapas a partir del compuesto 1. La introducción de tert-butoxicarbonilo y fenilselenio en posición $\alpha$ respecto al grupo carbonilo de la lactama proporcionó los seleno derivados 3, como mezcla de isómeros en la posición C-9. La oxidación posterior del selenio con peróxido de hidrógeno en presencia de piridina, proporcionó la lactama insaturada 5, la cual fue inmediatamente utilizada en la etapa siguiente sin previa purificación. Cabe comentar que la activación del doble enlace mediante la presencia del grupo activante alcoxicarbonilo es imprescindible para que la posterior reacción de adición conjugada tenga lugar.

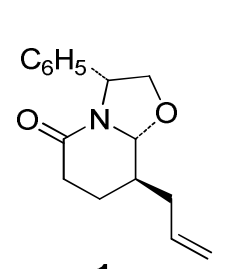

1
1) LiHMDS

2) $(\mathrm{Boc})_{2} \mathrm{O}$

3) $\mathrm{C}_{6} \mathrm{H}_{5} \mathrm{SeCl}$

$\mathrm{THF},-78^{\circ} \mathrm{C}$ $93 \%$

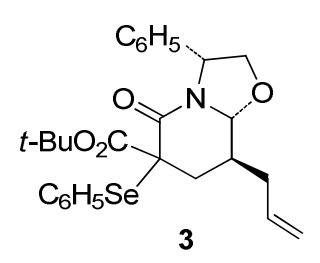

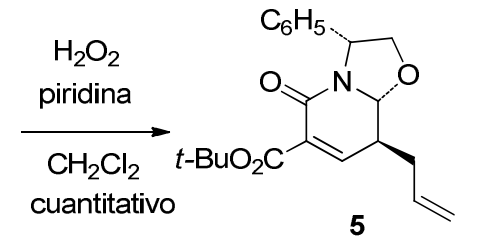

5

Esquema 3.64 Preparación de la lactama insaturada 5.

La adicción conjugada del alil cuprato a lactama $\alpha, \beta$-insaturada activada 5 , tuvo lugar de forma completamente estereoselectiva en la generación del nuevo enlace, proporcionando la diallyl lactama 7 con excelente rendimiento como mezcla de epímeros en posición 9. La reacción de adición nos permitió instalar el segundo estereocentro de la madangamina en la posición C-12 y obtener la relación cis entre los sustituyentes alilo, relación necesaria para la posterior construcción del anillo $\mathrm{C}$.

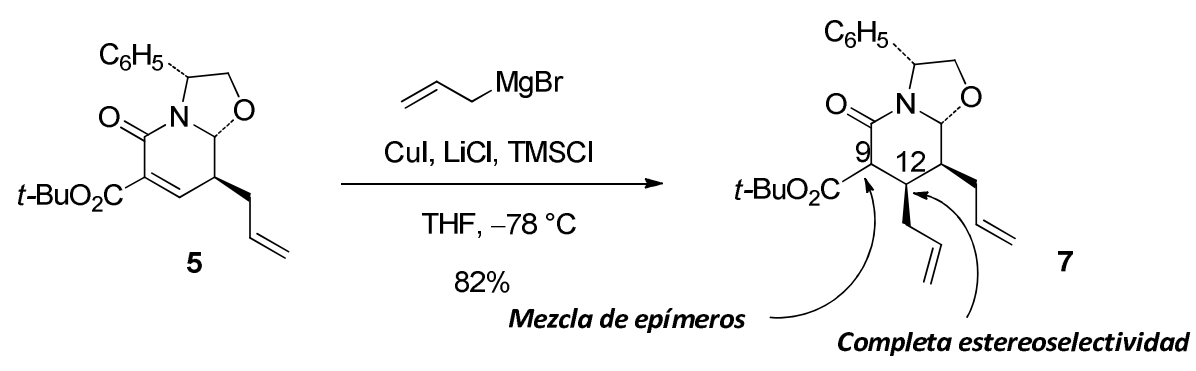

Esquema 3.65 Adición conjugada. Generación segundo estereocentro. 
El cierre del anillo carbocíclico tuvo lugar mediante tratamiento de los dienos $\mathbf{7}$ con el reactivo de Grubbs de segunda generación que, a través de una reacción de ring-closing metátesis, generó la lactama tricíclica $\mathbf{1 9}$ con excelente rendimiento. El anillo $\mathrm{C}$, formado a través de esta reacción, posee la instauración requerida para la construcción posterior del anillo piperidínico A necesaria para completar la síntesis del núcleo diazatríciclico de las madangaminas, que queremos construir mediante de reacción de amino-hidroxilación intramolecular.

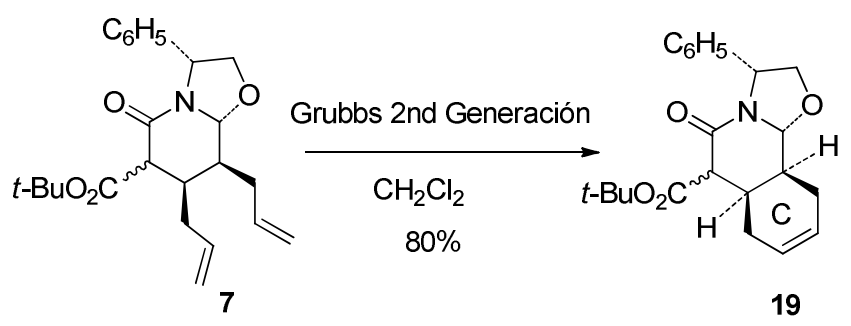

Esquema 3.66 Cierre del anillo carbocíclico C.

El centro cuaternario presente en la posición 9 de las madangaminas, se instaló aprovechando de la acidez de los protones metilénicos contiguos a los carbonilos del compuesto 19, mediante de una reacción de alquilación con el agente alquilante 3-(3-bromopropil)-1,3dioxolano. La reacción tuvo lugar por la cara más accesible del sistema isoquinolínico, proporcionado el compuesto $\mathbf{2 1}$ como único isómero, instalando de esta manera el tercer estereocentro del alcaloide.

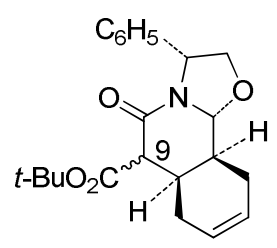

19

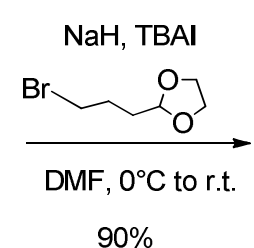

$90 \%$

Esquema 3.67 Incorporación del centro cuaternario.

Para la eliminación del inductor quiral empleamos un procedimiento en tres etapas que implica: rotura del enlace $\mathrm{C}-\mathrm{N}$ bencílico del compuesto $\mathbf{2 0}$ mediante tratamiento con una disolución de sodio en amoniaco líquido, posterior reducción de la mezcla compleja de las alcoxi- y oxilactamas resultantes con $\mathrm{LiAlH}_{4} \mathrm{y}$, finalmente, protección del grupo amino presente en 17 en forma de $\mathbf{N}$-Boc derivado 18.
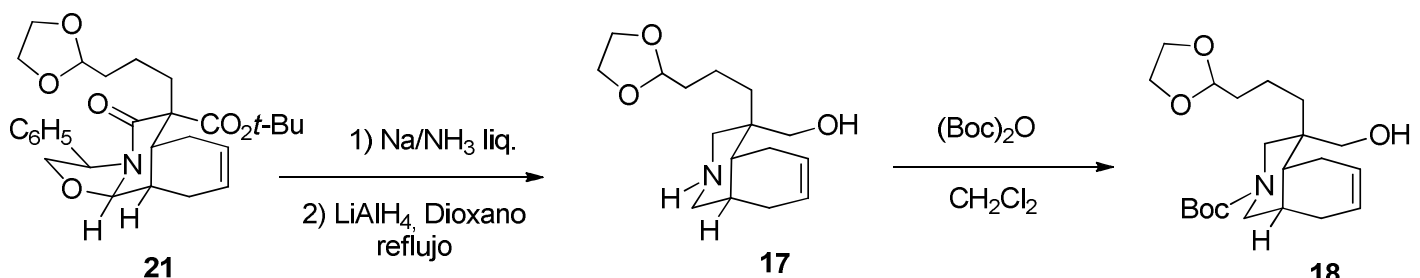

45\% rendiemiento global desde 21

Esquema 3.68 Eliminación del inductor quiral. 
La mesilación del alcohol primario del compuesto 18, seguido de una reacción de sustitución nucleófila con azida sódica permitió la obtención de la azida 40. La reacción de este compuesto con $m$-CPBA llevó a la epoxidación del doble enlace presente en el anillo ciclohexénico $C$ en una reacción que, debido a la estructura convexa del sistema bicíclico, proporcionó como único isómero el compuesto $4 \mathbf{4}$.

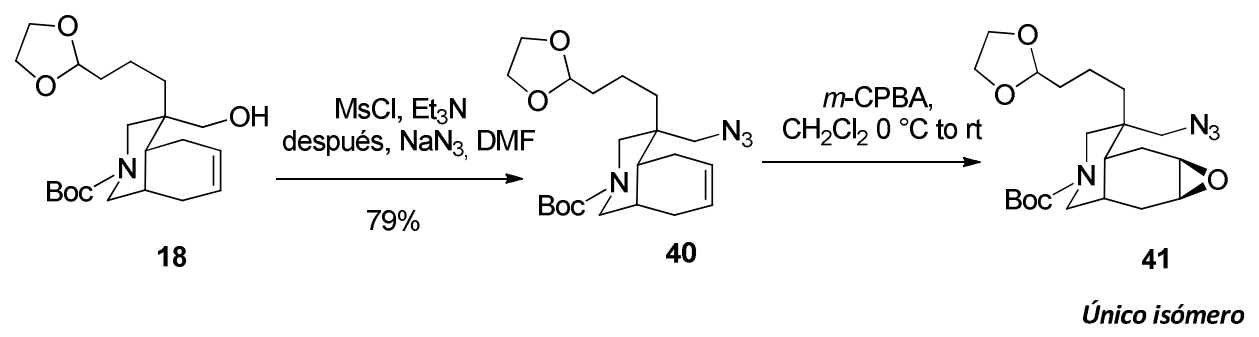

Esquema 3.69 Preparación del precursor para la cierre del anillo piperidínico A.

La síntesis del núcleo diazatricíclico $A B C$, común a las madangaminas $A-E$, se efectuó a través de una reacción de aminohidroxilación intramolecular en la cual, una reducción de Staundinger de la azida 41, llevó a la formación del intermedio amino-epóxido que experimentó una espontánea ciclación formando el compuesto tricíclico $\mathbf{4 2}$.

De esta manera se pudo instalar el último estereocentro de las madangaminas (posición C-2) y obtener el sistema tricíclico con la funcionalización y la substitución adecuada en las posiciones C-3 y C-9 necesarias para la posterior construcción de los anillos macrocíclicos D y E.

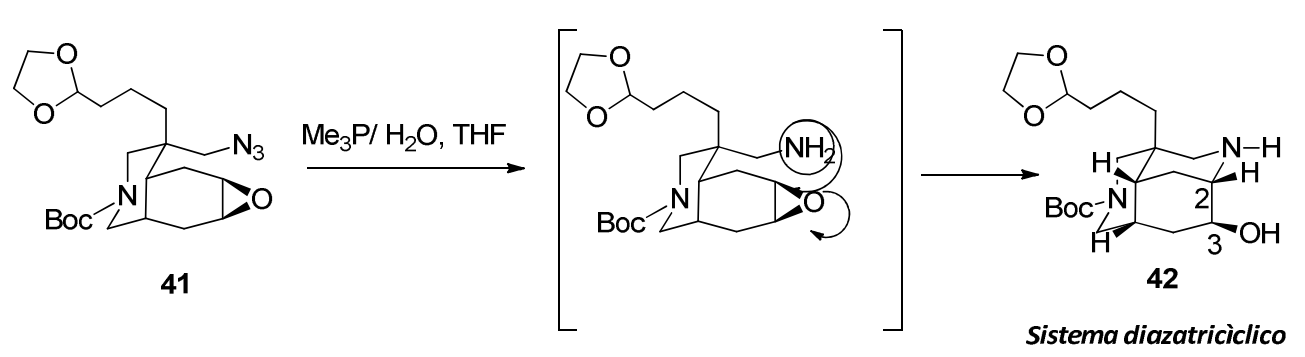

Esquema 3.70 Síntesis del núcleo diazatricíclico $A B C$.

Una vez sintetizado en núcleo central de las madangaminas, la siguiente fase de la síntesis implicó la construcción del anillo $D$ de la madangamina $D$, completamente saturado y que presenta 14 miembros, macrociclación que queremos realizar mediante una reacción de ringclosing metátesis.

Para ello, en primer lugar a partir del compuesto tricíclico $\mathbf{4 2}$ se protegieron las funciones de amina en forma de tosil derivado y de alcohol en forma de benzilo, proporcionando el triciclo 44 de forma satisfactoria. 


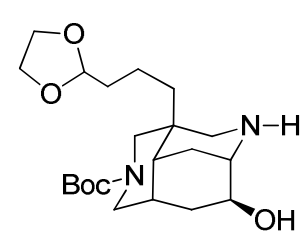

42

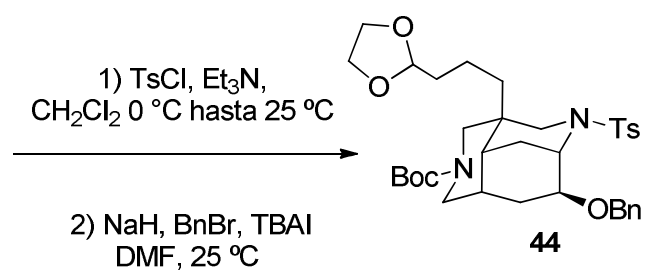

$48 \%$ desde 40

Sistema diazatriciclico

Esquema 3.71 Protección de las funciones de amina y alcohol.

El triciclo 44 fue posteriormente tratado con una disolución de TFA en diclorometano obteniendo la amina segundaria $\mathbf{1 0 0}$, la cual se aciló inmediatamente con cloruro de octenoilo, previamente preparado a partir del correspondiente ácido, obteniendo el compuesto 102.

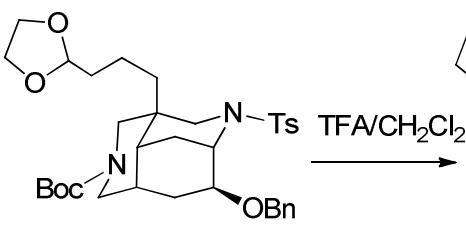

44<smiles>CN1C2CNCC(CCC3OCCO3)(CC2Br)C1Br</smiles>

100

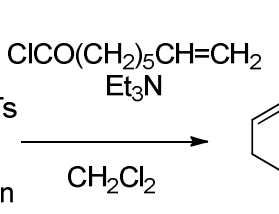

$92 \%$ global

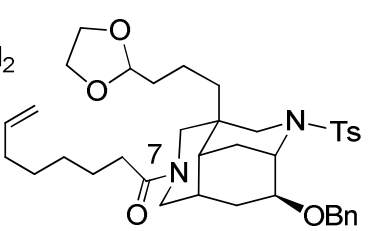

102

Esquema 3.72 Incorporación de la cadena carbonada en el nitrógeno 7.

El dieno 98, necesario para la construcción del anillo $D$, se generó finalmente a partir del compuesto 102 a través de una reacción de olefinación de tipo Wittig entre el aldehído 103, obtenido a través de una hidrólisis acida del dioxolano, y la correspondiente sal de fosfonio.

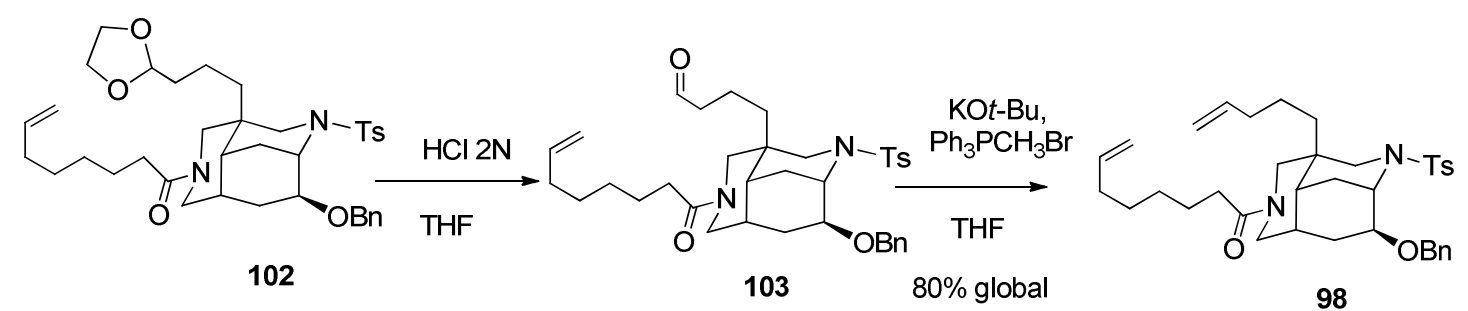

Esquema 3.73 Obtención del dieno precursor del anillo D de la madangamina D.

La cierre del anillo $D$ fue realizada mediante ring-closing metátesis utilizando el catalizador de Grubbs de primera generación, que mostró una actividad superior respecto al de segunda, y que condujo con excelentes rendimientos al sistema tetracíclico $A B C D 99$ de la madangamina D. 


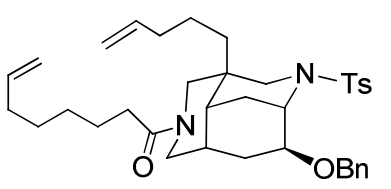

98

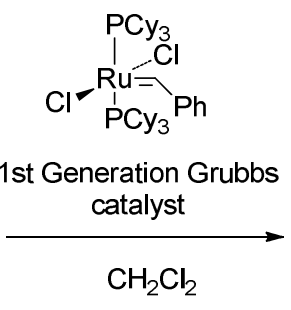

$90 \%$

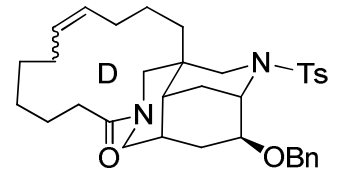

99

$E: Z 1: 2$

Esquema 3.74 Síntesis del sistema tetracíclico $A B C D$ de la madangamina $D$

La posterior hidrogenación catalítica, realizada con Pd/C en una disolución de 9:1 de etanol y agua, provocó la reducción del doble enlace del macrociclo con simultánea desbencilación del alcohol terciario 99, mientras que la oxidación del alcohol resultante con el reactivo de DessMartin proporcionó la cetona $\mathbf{1 0 6}$ con excelente rendimiento.

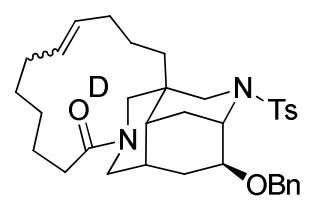

99

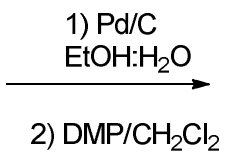

$75 \%$ rendimiento global desde 98

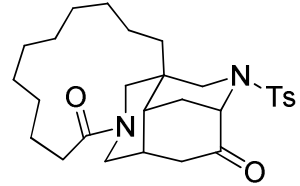

106

Esquema 3.75 Obtención de la cetona tetracíclica 106.

El anillo macrocíclico E, mucho más complejo por presentar una doble insaturación, se construyó de manera convergente, mediante una reacción Z-estereoselectiva de Wittig entre la cetona 106 y una sal de fosfonio apropiada, previamente sintetizada y caracterizada por la presencia de una $Z$-instauración y de un grupo éster terminal.

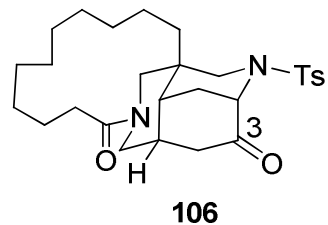

106

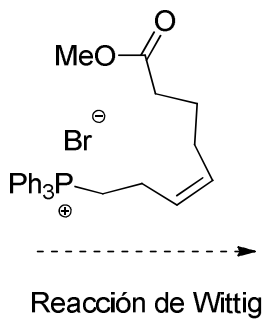

Reacción de Wittig

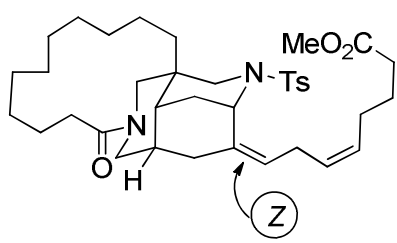

Esquema 3.76 Estrategia para la incorporación cadena en C-3 para la construcción anillo E.

La sal de fosfonio 126 fue sintetizada a partir del ester 124 preprarado mediante una reacción estereoselectiva de Wittig entre el aldehído 123 y el la sal de fosfonio 122. Tras desprotección del grupo silil éter con TBAF, el alcohol $\mathbf{1 2 5}$ fue finalmente convertido al correspondiente bromuro y, a partir de este, se generó la sal de fosfonio 126. 


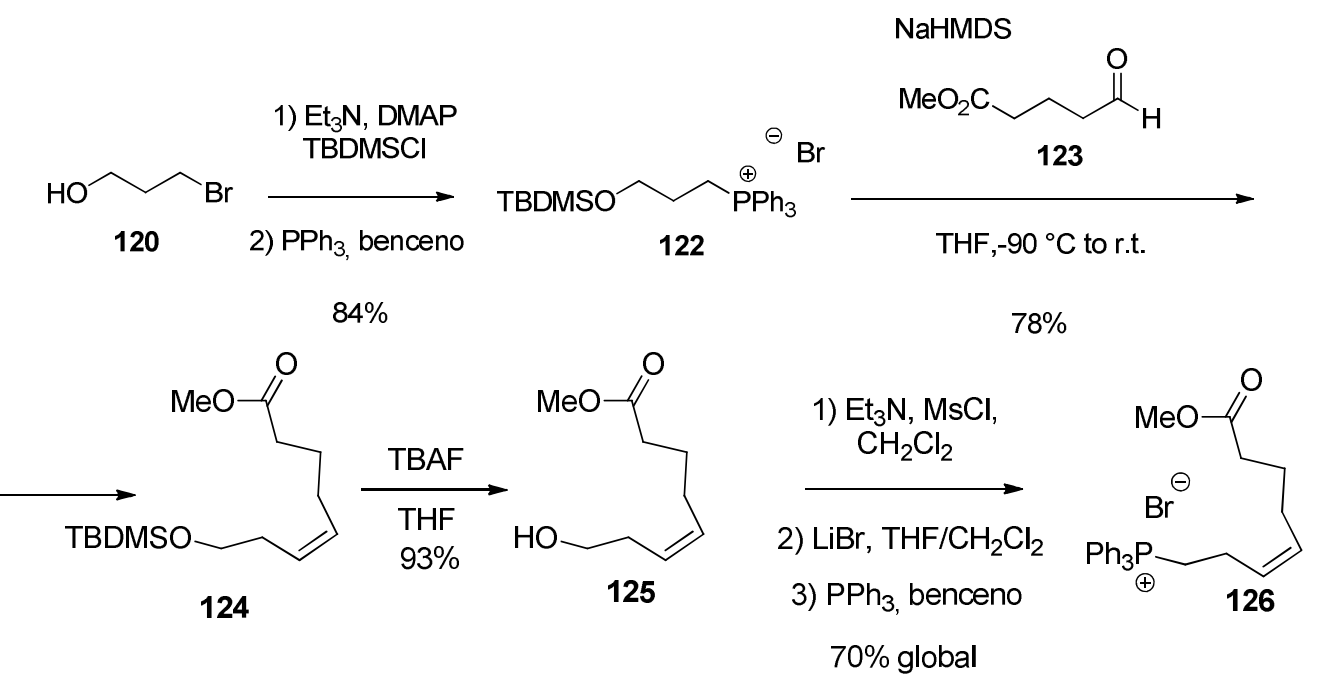

Esquema 3.77 Preparación de la sal de fosfonio 126.

La posterior reacción de Z-olefinacíon selectiva de Wittig entre la cetona 106 y la sal de fosfonio 126 nos permitió incorporar el fragmento to C-3/C-20 al sistema tetracíclico conduciendo a la formación del compuesto 137 con excelentes rendimientos y como mezcla de los isómeros $E$ y $Z$ (E:Z ratio 1:2.2).

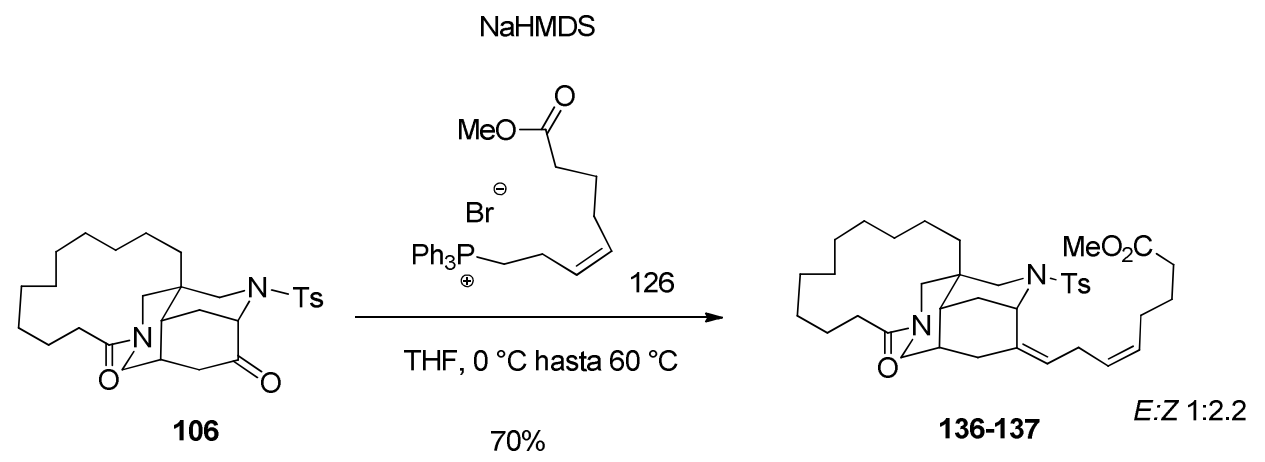

Esquema 3.78 Reacción de Wittig, generación de la Z-olefina.

La desprotección del nitrógeno piperidínico del anillo A con una disolución de sodio y naftaleno en THF a baja temperatura y la siguiente hidrólisis del éster con hidróxido de litio condujo a la formación del aminoácido 139.
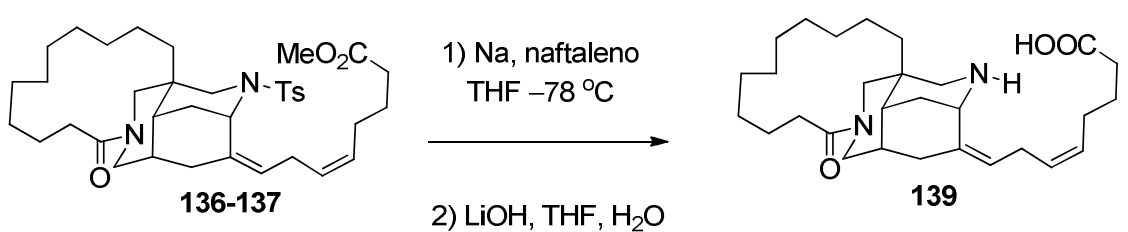

Esquema 3.79 Obtención del precursor de macrolactamización.

El sistema pentacíclico $A B C D E$ de la madangamina $D$ fue obtenido con excelentes resultados mediante una reacción de macrolactamización intramolecular, en condiciones diluidas, 
adicionando el aminoácido $\mathbf{1 3 9}$ a través de una syringe pump a una disolución de DMF y diclorometano contente los agentes acoplantes EDCl, HOBt.

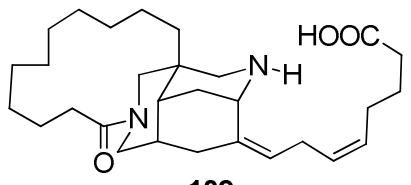

139

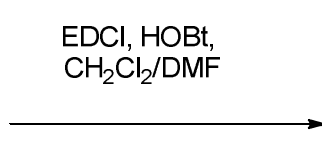

$75 \%$

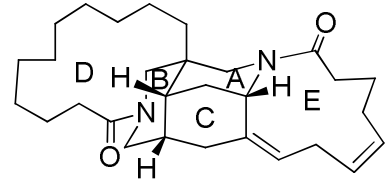

138

Sistema pentacíclico $A B C D E$

Esquema 3.80 Obtención sistema pentacíclico.

Finalmente la reducción de los dos carbonilos de lactama del sistema pentacíclico obtenido proporcionó la madangamina $\mathrm{D}$ como único producto.

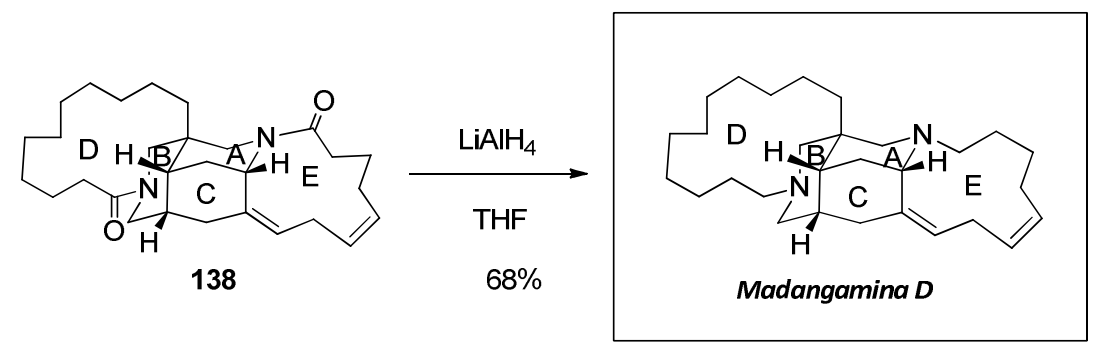

Esquema 3.81 Obtención de la madagamina D.

La presente estrategia sintética permitió obtener por primera vez la madangamina $D$ en forma enantiopura y establecer su configuración absoluta. La madangamina $D$ sintética posee sin ambigüedades la configuración absoluta $2 S, 5 S, 9 R, 12 R$ y una rotación específica $\{[\alpha]=+$ 101.3 ( $c$ 0,29, $\mathrm{CHCl}_{3}$ ) \} con el mismo signo que en las madangamines A-C. De acuerdo con estos datos podemos confirmar la configuración absoluta de esta familia de alcaloides asignado por Andersen.

La actividad biológica la madangamina $\mathrm{D}$, hasta ahora totalmente desconocida debido a su aislamiento en forma de mezcla inseparable con la madangamina $\mathrm{E}$ y por la baja cantidad de muestra disponible, fue ensayada en los laboratorios de Pharmamar. El alcaloide mostró significativa actividad citotóxica in vitro comparable a la de la madangamina $A$, siendo activa contra líneas celulares de cáncer HT29 de colon humano $\left(\mathrm{Gl}_{50} 4,4 \mu \mathrm{g} / \mathrm{mL}\right)$ y PSN1 páncreas $\left(\mathrm{GI}_{50}\right.$ $7,4 \mu \mathrm{g} / \mathrm{mL}$ ), pero fue inactiva contra las líneas celulares de cáncer pulmonar NSCLC A549 y de mama MDA-MB-231 en la concentración más alta ensayada $(>10 \mu \mathrm{g} / \mathrm{mL}) .{ }^{48}$ 
Chapter 4 

1. Se ha ratificado el potencial sintético de las lactamas bicíclicas quirales con estructura de oxazolopiperidona derivadas del $(R)$-fenilglicinol para la síntesis enantioselectivas de productos naturales complejos que contienen una subunidad de piperidina.

2. Se ha mejorado la metodología sintética anteriormente desarrollada en nuestro grupo de investigación permitiendo acceder al corazón $\mathrm{BC}$ de las madangaminas de manera escalable y rápida. La nueva ruta sintética prevé la eliminación del inductor quiral a través de una metodología compatible con la presencia del doble enlace endocíclico en una fase inicial de la síntesis. El compuesto obtenido ya presenta todas las funcionalizaciones necesarias para completar la síntesis del núcleo diazatriciclico común a las madangaminas A-E.

3. En la nueva secuencia sintética se ha conseguido eliminar algunas etapas redundantes incluyendo la eliminación de la función ester y su posterior reintroducción o los cambios de grupos protectores del nitrógeno piperidínico. De esta manera se podido reducir sensiblemente el número de etapas sintéticas y duplicar los rendimientos globales que llevan a la síntesis enantioselectiva del azabicílico correspondiente a los anillos $\mathrm{B}$ y $\mathrm{C}$ de las madangaminas.

4. Se ha puesto de manifiesto la eficiencia de la reacción de aminohydroxilacción intramolecular capaz de acceder al núcleo diazatricíclico $A B C$, común a las madangaminas $A-E$, de forma rápida y económica excluyendo la manipulación de reactivos altamente tóxicos o la utilización de procedimientos experimentales complejos e incómodos.

5. Se han estudiado reacciones de macrociclación de anillos de diferente tamaño pertenecientes a diferentes madangaminas. Las metodologías han sido aplicadas en un modelo piperidínico sencillo con el objetivo de encontrar las mejores condiciones de reacción y poder evaluar su eficacia. Como resultados de estos estudios exploratorios se identificó la reacción de cierre de anillo por metátesis como la más prometedora para acceder al sistema tetracíclico $A B C D$ de la madangamina $\mathrm{D}$.

6. El potencial sintético de las reacciones de ring-closing metátesis como procedimiento para la anulación de anillos macrocíclicos ha sido demostrada con la construcción del anillo de 14 eslabones, permitiendo el acceso manera eficaz al sistema $A B C D$ de la madangamina $D$.

7. Se ha aplicado con éxito la metodología sintética que presenta una reacción de tipo Wittig, descrita anteriormente en el grupo de investigación, para la introducción de la cadena carbonada instaurada en la posición C-3 del sistema tetracíclico, con una estereoselectividad preferentemente $Z$. Esta ruta, desarrollada precedentemente en un modelo morfánico, permite la formación del 
sistema insaturado de tipo Z-skipped, mientras que, la siguiente reacción de macrolactamizacción, permite la construcción del anillo E de la madangamina D.

8. La reducción final del compuesto diaminico, permite la obtención del la madangamna D. Por primera vez se ha llevado a cabo la síntesis total de un alcaloide perteneciente a la familia de las madangaminas. La (+)-madangamina $D$ sintetizada permitió confirmar la configuración absoluta asignada por Andersen basada en la correlación con un precursor biogenético común.

9. Gracias a la obtención del compuesto en estado puro se ha podido ensayar la actividad biológica de este alcaloide y confirmar sus propiedades citotóxicas hacia diferentes líneas celulares, ensayos que hasta el presente no habían podido efectuarse al aislarse conjuntamente con la madangamina $E$ y en poca cantidad de su fuente natural. La madangamina $D$ sintética, así pues, muestra citotoxicidad in vitro contra las líneas celulares de cáncer HT29 de colon humano y PSN1 páncreas, pero es inactivo contra el NSCLC de pulmón A549 y el cáncer de mama MDA-MB231 líneas de células a la concentración más alta ensayada $(10 \mu \mathrm{g} / \mathrm{ml})$. 
Chapter 5 



\section{Experimental procedures and spectroscopic data}

General Procedures: All air sensitive manipulations were carried out under a dry argon or nitrogen atmosphere. THF and toluene were carefully dried and distilled from sodium/benzophenone prior to use. $\mathrm{CH}_{2} \mathrm{Cl}_{2}$ was dried and distilled from $\mathrm{CaH}_{2}$. Other solvents and all standard reagents were purchased from Aldrich, Fluka or Alfa Aesar and were used without further purification.

Analytical thin-layer chromatography was performed on $\mathrm{SiO}_{2}$ (Merck silica gel $60 \mathrm{~F}_{254}$ ), and the spots were located with $1 \%$ aqueous $\mathrm{KMnO}_{4}$. Chromatography refers to flash chromatography and was carried out on $\mathrm{SiO}_{2}$ (SDS silica gel 60 ACC, 35-75 mm, 230-240 mesh ASTM). NMR spectra were recorded at 300 or $400 \mathrm{MHz}\left({ }^{1} \mathrm{H}\right)$ and 75.4 or $100.6 \mathrm{MHz}\left({ }^{13} \mathrm{C}\right)$, and chemical shifts are reported in $\delta$ values downfield from TMS or relative to residual chloroform (7.26 ppm, 77.0 ppm) as an internal standard. Data are reported in the following manner: chemical shift, integrated intensity, multiplicity, coupling constant $(J)$ in hertz $(\mathrm{Hz})$, and assignment (when possible). Assignments and stereochemical determinations are given only when they are derived from definitive two-dimensional NMR experiments (HSQC-COSY). IR spectra were performed in a spectrophotometer Nicolet Avantar 320 FT-IR and only noteworthy IR absorptions $\left(\mathrm{cm}^{-1}\right)$ are listed. Optical rotations were measured on Perkin-Elmer 241 polarimeter. $[\alpha]_{D}$ values are given in $10^{-1} \mathrm{deg} \mathrm{cm}^{2} \mathrm{~g}^{-1}$. High resolution mass spectra (HMRS; LC/MSD TOF Agilent Technologies) were performed by Centres Científics i Tecnològics de la Universitat de Barcelona. 
<smiles>C=CCC(C=O)CCC(C)=O</smiles>

\section{Methyl-4-formyl-6-heptenoate.}

4-Pentenal $(4.7 \mathrm{ml}, 0.047 \mathrm{~mol}$ ) was added dropwise during $0.5 \mathrm{~h}$ to a stirred, ice-cold suspension of anhydrous potassium carbonate $(2.0 \mathrm{~g}, 0.16 \mathrm{~mol})$ in piperidine $(12 \mathrm{ml}, 0.1 \mathrm{~mol})$. Stirring was continued at room temperature for 2 days and the mixture filtered through sintered glass. The residue was washed thoroughly with ether which was subsequently removed under reduced pressure affording the piperidine enamine of 4-pentenal, as a pale yellow oil. To a solution of the above enamine in dry acetonitrile $(75 \mathrm{ml})$, cooled below $5{ }^{\circ} \mathrm{C}$, was added methyl acrylate $(7.5 \mathrm{ml}, 0.03 \mathrm{~mol})$ dissolved in $19 \mathrm{ml}$ of dry acetonitrile during 30 $\min$. The mixture was stirred at room temperature for $5 \mathrm{~h}$ and then refluxed for $72 \mathrm{~h}$. Glacial acetic acid $(10.5 \mathrm{ml})$ in water $(75 \mathrm{ml})$ was added and the mixture refluxed for further $8 \mathrm{~h}$. Ether $(250 \mathrm{ml})$ was added to the residue and the organic layer was washed with $3 \mathrm{~N} \mathrm{HCl}(100 \mathrm{ml})$, saturated $\mathrm{NaHCO}_{3}(100 \mathrm{ml})$, brine $(100 \mathrm{ml})$, and finally dried with $\mathrm{MgSO}_{4}$ and filtered. Removal of the organic solvents left methyl-4-formyl-6-heptenoate $(5.10 \mathrm{~g}, 64 \%)$ as a brown oil which was used in the next reaction without further purification.

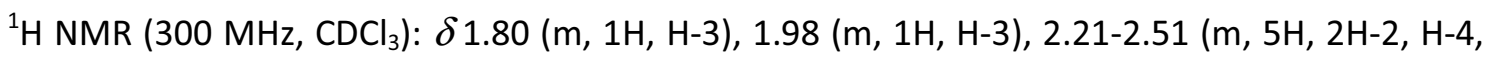
$2 \mathrm{H}-5), 3.67\left(\mathrm{~s}, 3 \mathrm{H}, \mathrm{CH}_{3}\right), 5.09(\mathrm{~m}, 2 \mathrm{H}, \mathrm{H}-7), 5.76(\mathrm{~m}, 1 \mathrm{H}, \mathrm{H}-6), 9.63(\mathrm{~d}, \mathrm{~J}=2.1 \mathrm{~Hz}, 1 \mathrm{H}, \mathrm{CHO})$.

${ }^{13} \mathrm{C} \mathrm{NMR}\left(100.6 \mathrm{MHz}, \mathrm{CDCl}_{3}\right): \delta 23.2$ (C-3), $31.2(\mathrm{C}-5), 32.9$ (C-2), $50.3\left(\mathrm{CH}_{3}\right), 51.7$ (C-4), 117.6 (C7), 134.3 (C-6), 173.4 (C-1), 203.7 (CHO). 

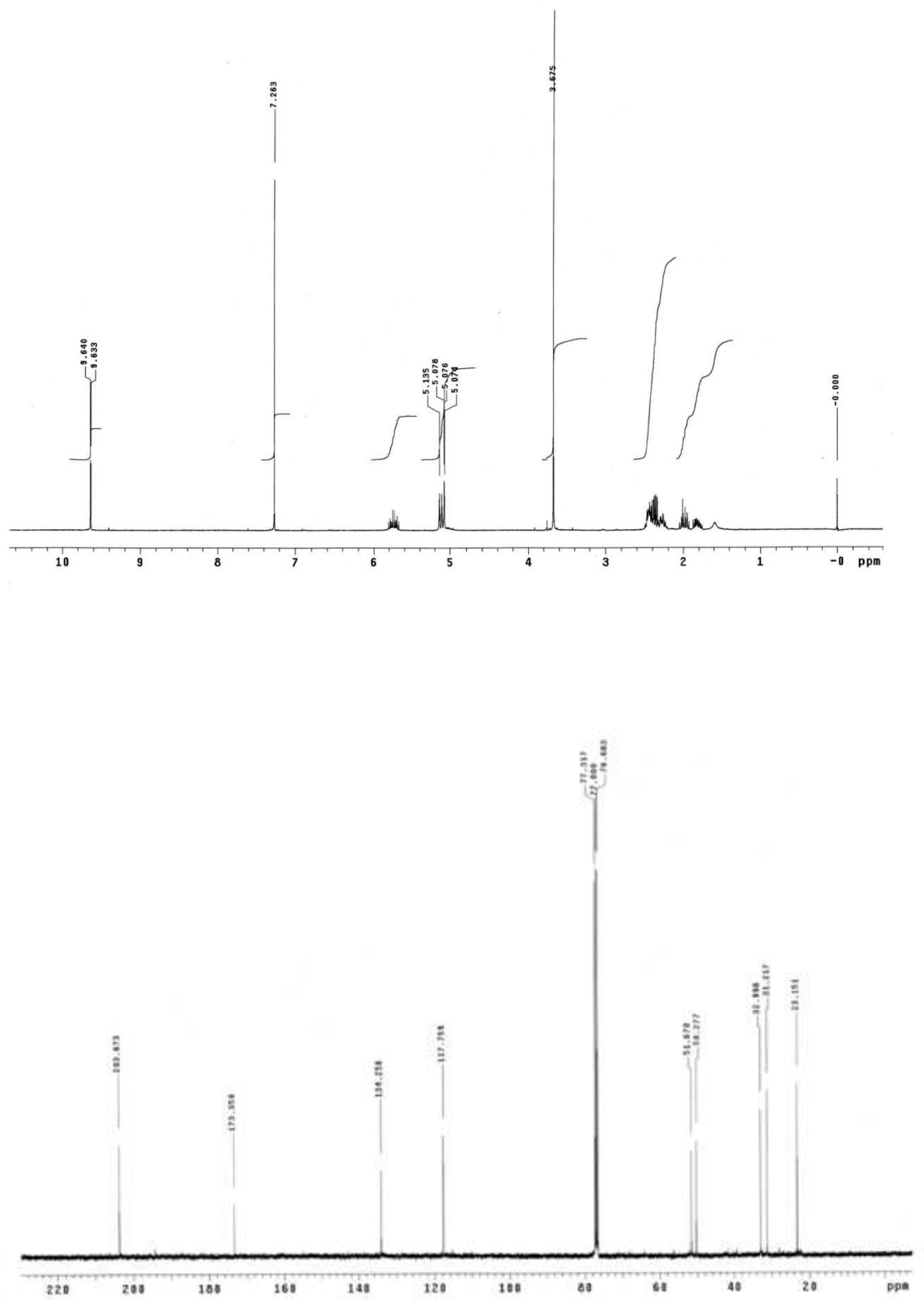
<smiles>C=CC[C@H]1CCC(=O)N2C(C)COC12</smiles>

$(3 R, 8 R, 8 \mathrm{a} R)$-8-Allyl-5-oxo-3-phenyl-2,3,6,7,8,8a-hexahydro-5H-oxazolo[3,2-a]pyridine (1).

A mixture of racemic methyl 4-formyl-6-heptenoate $(4.6 \mathrm{~g}, 29 \mathrm{mmol}),(R)$-phenylglycinol (3.97 g, $29 \mathrm{mmol})$, and anhydrous $\mathrm{Na}_{2} \mathrm{SO}_{4}(17.0 \mathrm{~g}, 12 \mathrm{mmol})$ in $\mathrm{Et}_{2} \mathrm{O}(115 \mathrm{~mL})$ was stirred at $0{ }^{\circ} \mathrm{C}$ for 2 h. The resulting suspension was filtered through Celite ${ }^{\circledR}$ and concentrated under reduced pressure. The residue was heated at $80{ }^{\circ} \mathrm{C}$ for $18 \mathrm{~h}$ under vacuum $(10-15 \mathrm{mmHg})$. Flash chromatography (8:2 hexane-EtOAc to EtOAc) of the residue afforded oxazolopiperidone 1 (5.3 g, 71\%) and its (8S,8aS)-diastereoisomer 2 (753 mg, 10\%).

Compound $\mathbf{1}$ (major isomer, lower $\mathrm{R}_{f}$ ):

$[\alpha]_{D}^{22}=-32.8(c 1.0, \mathrm{EtOH})$.

IR (film): 1655 (NCO) $\mathrm{cm}^{-1}$.

${ }^{1} \mathrm{H}$ NMR (400 MHz, CDCl 3 , COSY, gHSQC): $\delta 1.45$ (dddd, $J=13.8,13.8,12.0,7.2 \mathrm{~Hz}, 1 \mathrm{H}, \mathrm{H}-7$ ), $2.02\left(\mathrm{~m}, 3 \mathrm{H}, \mathrm{H}-7, \mathrm{H}-8, \mathrm{CH}_{2}\right.$ allyl), 2.30 (ddd, $J=18.0,12.0,6.6 \mathrm{~Hz}, 1 \mathrm{H}, \mathrm{H}-6$ ), 2.42 (ddd, $J=18.0$, 7.2, $1.8 \mathrm{~Hz}, 1 \mathrm{H}, \mathrm{H}-6), 2.62\left(\mathrm{~m}, 1 \mathrm{H}, \mathrm{CH}_{2}\right.$ allyl), 4.01 (dd, J = 9.0, $\left.1.2 \mathrm{~Hz}, 1 \mathrm{H}, \mathrm{H}-2\right), 4.11$ (dd, J = 9.0, $6.9 \mathrm{~Hz}, 1 \mathrm{H}, \mathrm{H}-2), 4.53(\mathrm{~d}, J=8.7 \mathrm{~Hz}, 1 \mathrm{H}, \mathrm{H}-8 \mathrm{a}), 4.92(\mathrm{~d}, J=6.6 \mathrm{~Hz}, 1 \mathrm{H}, \mathrm{H}-3), 5.12\left(\mathrm{~m}, 2 \mathrm{H}, \mathrm{CH}_{2}=\right)$, 5.86 (dddd, $J=16.5,10.2,7.8,6.0 \mathrm{~Hz}, 1 \mathrm{H}, \mathrm{CH}=)$, 7.20-7.30 (m, $\left.5 \mathrm{H}, \mathrm{C}_{6} \mathrm{H}_{5}\right)$.

${ }^{13} \mathrm{C} \mathrm{NMR}\left(100.6 \mathrm{MHz}, \mathrm{CDCl}_{3}\right): \delta 23.6$ (C-7), 31.2 (C-6), $35.4\left(\mathrm{CH}_{2}\right.$ allyl), 39.1 (C-8), 58.9 (C-3), 73.7 (C-2), 91.9 (C-8a), $117.4\left(\mathrm{CH}_{2}=\right), 126.2,128.4\left(\mathrm{C}-0, m \mathrm{C}_{6} \mathrm{H}_{5}\right), 127.4\left(\mathrm{C}-p \mathrm{C}_{6} \mathrm{H}_{5}\right), 134.6(\mathrm{CH}=), 141.4$ (C-i $\left.\mathrm{C}_{6} \mathrm{H}_{5}\right), 167.1$ (NCO).

Elemental analysis calcd (\%) for $\mathrm{C}_{16} \mathrm{H}_{19} \mathrm{NO}_{2} \cdot 1 / 4 \mathrm{H}_{2} \mathrm{O}: \mathrm{C} 73.40, \mathrm{H}$ 7.51, N 5.35; found: $\mathrm{C} 73.71, \mathrm{H}$ 7.25, N 5.41. 

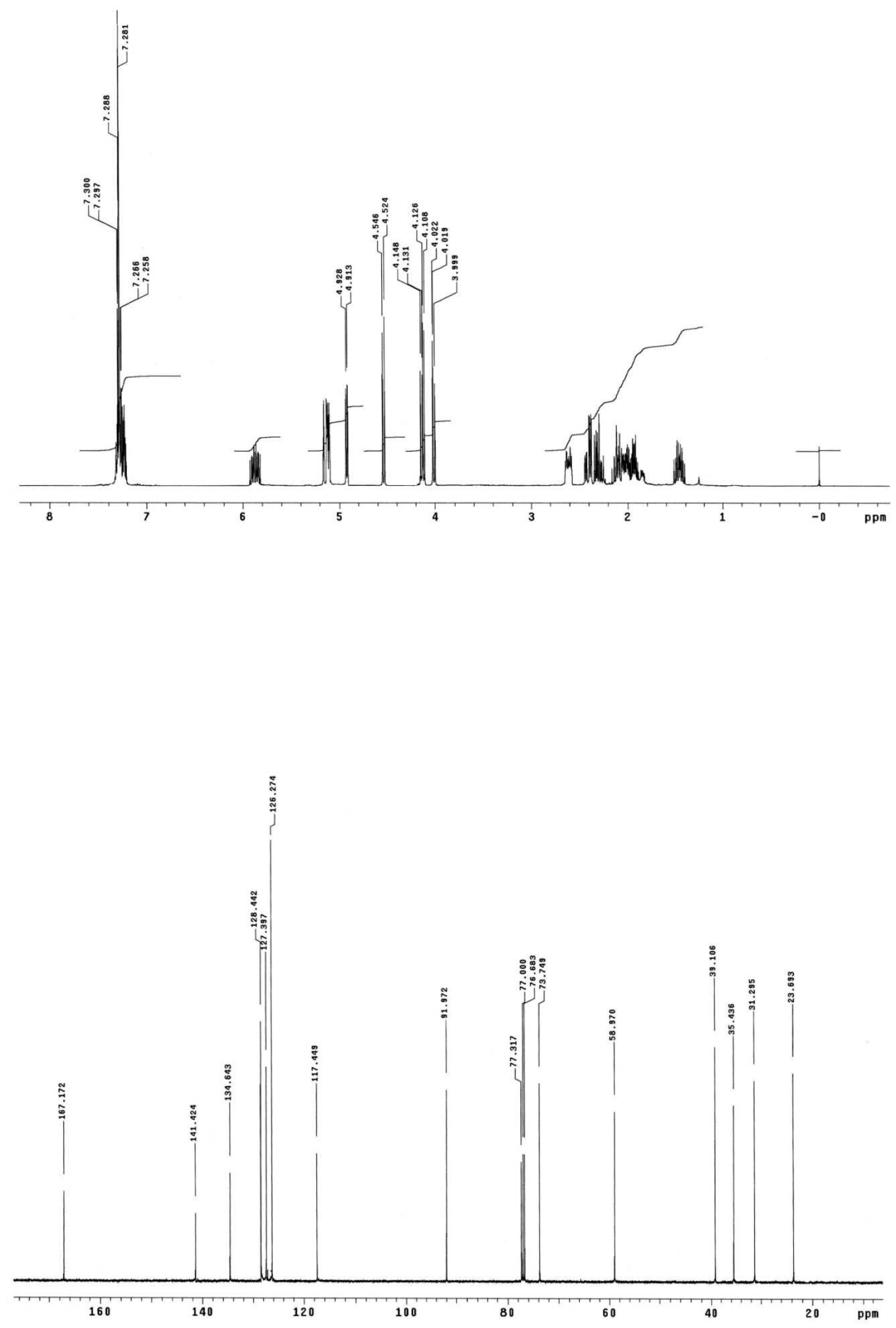
<smiles>C=CCC1CCC(=O)N2C(C)COC12</smiles>

Compound $\mathbf{2}$ (minor isomer, higher $\mathrm{R}_{f}$ ):

$[\alpha]_{D}^{22}=-59.9(c$ 1.0, EtOH).

IR (film): 1658 (NCO) $\mathrm{cm}^{-1}$.

${ }^{1} \mathrm{H} \mathrm{NMR}\left(400 \mathrm{MHz}, \mathrm{CDCl}_{3}\right.$, COSY, gHSQC): $\delta 1.53(\mathrm{~m}, 1 \mathrm{H}, \mathrm{H}-7), 1.66(\mathrm{~m}, 1 \mathrm{H}, \mathrm{H}-8), 1.96(\mathrm{~m}, 1 \mathrm{H}, \mathrm{H}-$ 7), 2.07 (dt, $J=16.5,8.4,8.4 \mathrm{~Hz}, 1 \mathrm{H}, \mathrm{CH}_{2}$ allyl), 2.35 (ddd, $\left.J=18.6,12.0,6.6 \mathrm{~Hz}, 1 \mathrm{H}, \mathrm{H}-6\right), 2.56$ ( $m, 2 \mathrm{H}, \mathrm{H}-6, \mathrm{CH}_{2}$ allyl), 3.75 (dd, J = 9.0, 7.8 Hz, 1H, H-2), 4.47 (dd, J = 9.0, 8.1 Hz, 1H, H-2), 4.69 (d, $J=8.4 \mathrm{~Hz}, 1 \mathrm{H}, \mathrm{H}-8 \mathrm{a}), 5.13\left(\mathrm{~m}, 2 \mathrm{H}, \mathrm{CH}_{2}=\right), 5.25$ (t, $J=7.8 \mathrm{~Hz}, 1 \mathrm{H}, \mathrm{H}-3$ ), 5.83 (dddd, $J=16.5$, $10.2,8.1,6.0 \mathrm{~Hz}, 1 \mathrm{H}, \mathrm{CH}=)$, 7.25-7.34 (m, $\left.5 \mathrm{H}, \mathrm{C}_{6} \mathrm{H}_{5}\right)$.

${ }^{13} \mathrm{C} \mathrm{NMR}\left(100.6 \mathrm{MHz}, \mathrm{CDCl}_{3}\right.$ ): $\delta 22.7$ (C-7), 31.3 (C-6), $35.8\left(\mathrm{CH}_{2}\right.$ allyl), 39.5 (C-8), 58.3 (C-3), 72.3 (C-2), 92.0 (C-8a), $117.4\left(\mathrm{CH}_{2}=\right), 126.0,128.7\left(\mathrm{C}-0, m \mathrm{C}_{6} \mathrm{H}_{5}\right), 127.5\left(\mathrm{C}-p \mathrm{C}_{6} \mathrm{H}_{5}\right), 134.6(\mathrm{CH}=), 139.4$ $\left(\mathrm{C}-i \mathrm{C}_{6} \mathrm{H}_{5}\right), 168.7$ (NCO).

Elemental analysis calcd for $\mathrm{C}_{16} \mathrm{H}_{19} \mathrm{NO}_{2} \cdot 1 / 4 \mathrm{H}_{2} \mathrm{O}: \mathrm{C}, 73.40 ; \mathrm{H}, 7.51 ; \mathrm{N}, 5.35$. Found: $\mathrm{C}, 73.27 ; \mathrm{H}$, $7.25 ; \mathrm{N}, 5.51$. 

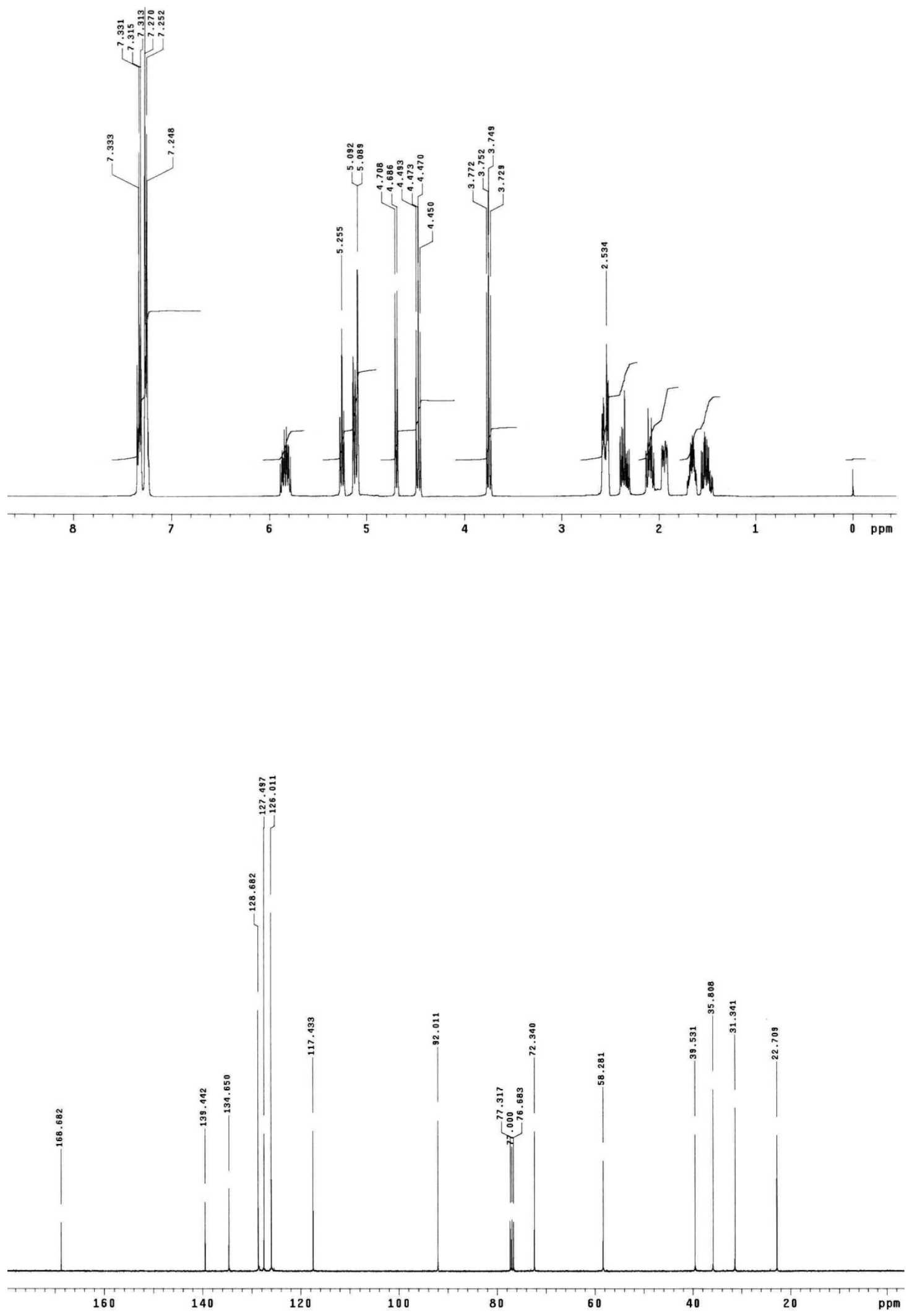


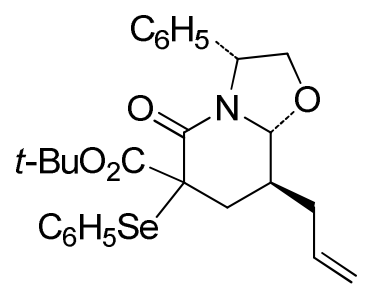

(3R,8S,8aR)-8-Allyl-6-(tert-butoxycarbonyl)-5-oxo-3-phenyl-6-(phenylselenyl)-2,3,6,7,8,8ahexahydro-5H-oxazolo-[3,2-a]pyridine (3).

Lithium bis(trimethylsilyl)amide $(3.85 \mathrm{~mL}$ of a $1 \mathrm{M}$ solution in THF, $3.85 \mathrm{mmol}$ ) was slowly added at $-78{ }^{\circ} \mathrm{C}$ to a solution of lactam $1(450 \mathrm{mg}, 1.75 \mathrm{mmol})$ in anhydrous THF (30 mL), and the resulting mixture was stirred for $90 \mathrm{~min}$. Then, di-tert-butyl dicarbonate $(420 \mathrm{mg}, 1.92$ $\mathrm{mmol})$ in THF (2 mL) and, after 90 min of continuous stirring at $-78^{\circ} \mathrm{C}$, phenylselenyl chloride $(503 \mathrm{mg}, 2.62 \mathrm{mmol})$ in THF $(4 \mathrm{~mL})$ were added to the solution. The resulting mixture was stirred for a further $1 \mathrm{~h}$ and poured into saturated aqueous $\mathrm{NH}_{4} \mathrm{Cl}$. The aqueous layer was extracted with EtOAc, and the combined organic extracts were dried over anhydrous $\mathrm{MgSO}_{4}$, filtered and concentrated under reduced pressure. Flash chromatography (9:1 to 8:2 hexaneEtOAc) of the resulting oil afforded the C-6 epimers a $(520 \mathrm{mg})$ and $\mathbf{b}(320 \mathrm{mg})$ of the seleno derivatives $\mathbf{3}$ as brown oils (93\% overall yield).

Epimer a (major, higher $\mathrm{R}_{f}$ ):

IR (film): 1665 (NCO), 1726 (COO) $\mathrm{cm}^{-1}$.

${ }^{1} \mathrm{H} \mathrm{NMR}\left(400 \mathrm{MHz}, \mathrm{CDCl}_{3}, \mathrm{gHSQC}\right): \delta 1.29\left[\mathrm{~s}, 9 \mathrm{H},\left(\mathrm{CH}_{3}\right)_{3} \mathrm{C}\right], 1.79(\mathrm{dd}, J=14.4,12.3 \mathrm{~Hz}, 1 \mathrm{H}, \mathrm{H}-7)$, $1.93\left(\mathrm{~m}, 1 \mathrm{H}, \mathrm{CH}_{2}\right.$ allyl), 1.98 (m, 1H, H-8), 2.36 (dd, J = 14.4, 3.0 Hz, 1H, H-7), $2.43\left(\mathrm{~m}, 1 \mathrm{H}, \mathrm{CH}_{2}\right.$ allyl), 3.87 (d, $J=9.0 \mathrm{~Hz}, 1 \mathrm{H}, \mathrm{H}-8 \mathrm{a}$ ), 3.95 (dd, $J=9.0,6.0 \mathrm{~Hz}, 1 \mathrm{H}, \mathrm{H}-2$ ), 3.99 (dd, $J=9.0,2.1 \mathrm{~Hz}$, $1 \mathrm{H}, \mathrm{H}-2$ ), $4.82\left(\mathrm{dd}, J=6.0,2.1 \mathrm{~Hz}, 1 \mathrm{H}, \mathrm{H}-3\right.$ ), $5.03\left(3 \mathrm{~m}, 2 \mathrm{H}, \mathrm{CH}_{2}=\right.$ ), 5.70 (dddd, $J=16.5,10.8,8.1$, $6.6 \mathrm{~Hz}, 1 \mathrm{H}, \mathrm{CH}=), 7.26-7.68\left(\mathrm{~m}, 10 \mathrm{H}, \mathrm{C}_{6} \mathrm{H}_{5}\right)$.

${ }^{13} \mathrm{C} \mathrm{NMR}\left(100.6 \mathrm{MHz}, \mathrm{CDCl}_{3}\right): \delta 27.5\left[\left(\mathrm{CH}_{3}\right)_{3} \mathrm{C}\right], 34.5\left(\mathrm{CH}_{2}\right.$ allyl), 36.7 (C-7), 37.9 (C-8), 55.8 (C-6), 59.3 (C-3), 74.0 (C-2), $82.8\left[\left(\mathrm{CH}_{3}\right)_{3} \mathrm{C}\right], 91.5$ (C-8a), $117.6\left(\mathrm{CH}_{2}=\right), 126.7-128.6,138.3$ (C-o, $m, p$ $\left.\mathrm{C}_{6} \mathrm{H}_{5}\right), 129.5\left(\mathrm{C}-i \mathrm{C}_{6} \mathrm{H}_{5}\right), 133.9(\mathrm{CH}=), 140.7\left(\mathrm{C}-i \mathrm{C}_{6} \mathrm{H}_{5}\right), 163.6(\mathrm{C}=\mathrm{O}), 168.7(\mathrm{C}=\mathrm{O})$.

HRMS calcld for $\left[\mathrm{C}_{27} \mathrm{H}_{31} \mathrm{NO}_{4} \mathrm{Se}+\mathrm{H}\right]^{+}: 514.1491$, found: 514.1479 . 

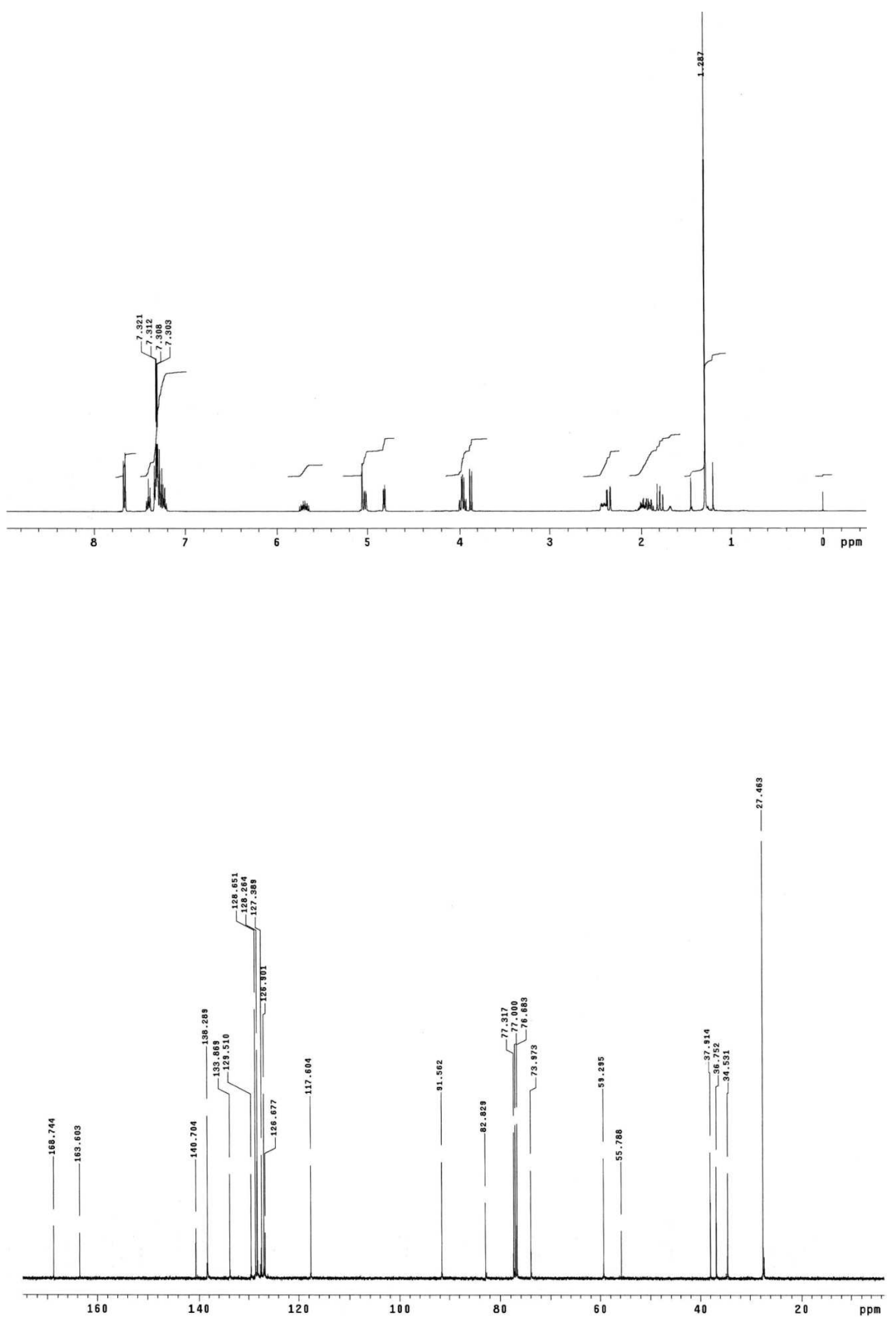


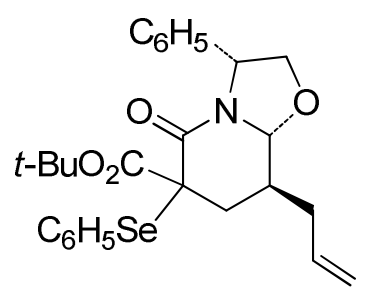

Epimer b (minor, lower $R_{f}$ ):

IR (film): 1661 (NCO), 1732 (COO) $\mathrm{cm}^{-1}$.

${ }^{1} \mathrm{H}$ NMR (400 MHz, CDCl 3 , gHSQC): $\delta 1.45\left[\mathrm{~s}, 9 \mathrm{H},\left(\mathrm{CH}_{3}\right)_{3} \mathrm{C}\right], 1.95\left(\mathrm{~m}, 1 \mathrm{H}, \mathrm{CH}_{2}\right.$ allyl), 1.99 (dd, $J=$ 15.0, $10.8 \mathrm{~Hz}, 1 \mathrm{H}, \mathrm{H}-7$ ), 2.10 (dd, J = 15.0, $4.5 \mathrm{~Hz}, 1 \mathrm{H}, \mathrm{H}-7$ ), 2.20 (m, $1 \mathrm{H}, \mathrm{H}-8), 2.48\left(\mathrm{~m}, 1 \mathrm{H}, \mathrm{CH}_{2}\right.$ allyl), 4.08 (dd, $J=9.0,1.2 \mathrm{~Hz}, 1 \mathrm{H}, \mathrm{H}-2), 4.17(\mathrm{dd}, J=9.0,6.6 \mathrm{~Hz}, 1 \mathrm{H}, \mathrm{H}-2), 4.61(\mathrm{~d}, J=8.7 \mathrm{~Hz}, 1 \mathrm{H}$, $\mathrm{H}-8 \mathrm{a}$ ), 4.95 (dd, $J=6.6,1.2 \mathrm{~Hz}, 1 \mathrm{H}, \mathrm{H}-3$ ), $4.98\left(3 \mathrm{~m}, 2 \mathrm{H}, \mathrm{CH}_{2}=\right), 5.56$ (dddd, $J=16.5,10.2,8.4,6.0$ $\mathrm{Hz}, 1 \mathrm{H}, \mathrm{CH}=)$, 7.22-7.52 (m, 10H, $\left.\mathrm{C}_{6} \mathrm{H}_{5}\right)$.

${ }^{13} \mathrm{C} \mathrm{NMR}\left(100.6 \mathrm{MHz}, \mathrm{CDCl}_{3}\right): \delta 27.8\left[\left(\mathrm{CH}_{3}\right)_{3} \mathrm{C}\right], 34.2$ (C-7), $35.2\left(\mathrm{CH}_{2}\right.$ allyl), 36.9 (C-8), 56.8 (C-6), 59.4 (C-3), 73.9 (C-2), $82.9\left[\left(\mathrm{CH}_{3}\right)_{3} \mathrm{C}\right], 91.6$ (C-8a), $117.5\left(\mathrm{CH}_{2}=\right), 126.9-128.6,138.1$ (C-o, $m, p$ $\left.\mathrm{C}_{6} \mathrm{H}_{5}\right), 129.3\left(\mathrm{C}-i \mathrm{C}_{6} \mathrm{H}_{5}\right), 134.2(\mathrm{CH}=), 140.6\left(\mathrm{C}-i \mathrm{C}_{6} \mathrm{H}_{5}\right), 163.5(\mathrm{C}=\mathrm{O}), 169.5(\mathrm{C}=\mathrm{O})$.

HRMS calcd for $\left[\mathrm{C}_{27} \mathrm{H}_{31} \mathrm{NO}_{4} \mathrm{Se}+\mathrm{H}\right]^{+}: 514.1491$, found: 514.1472 . 

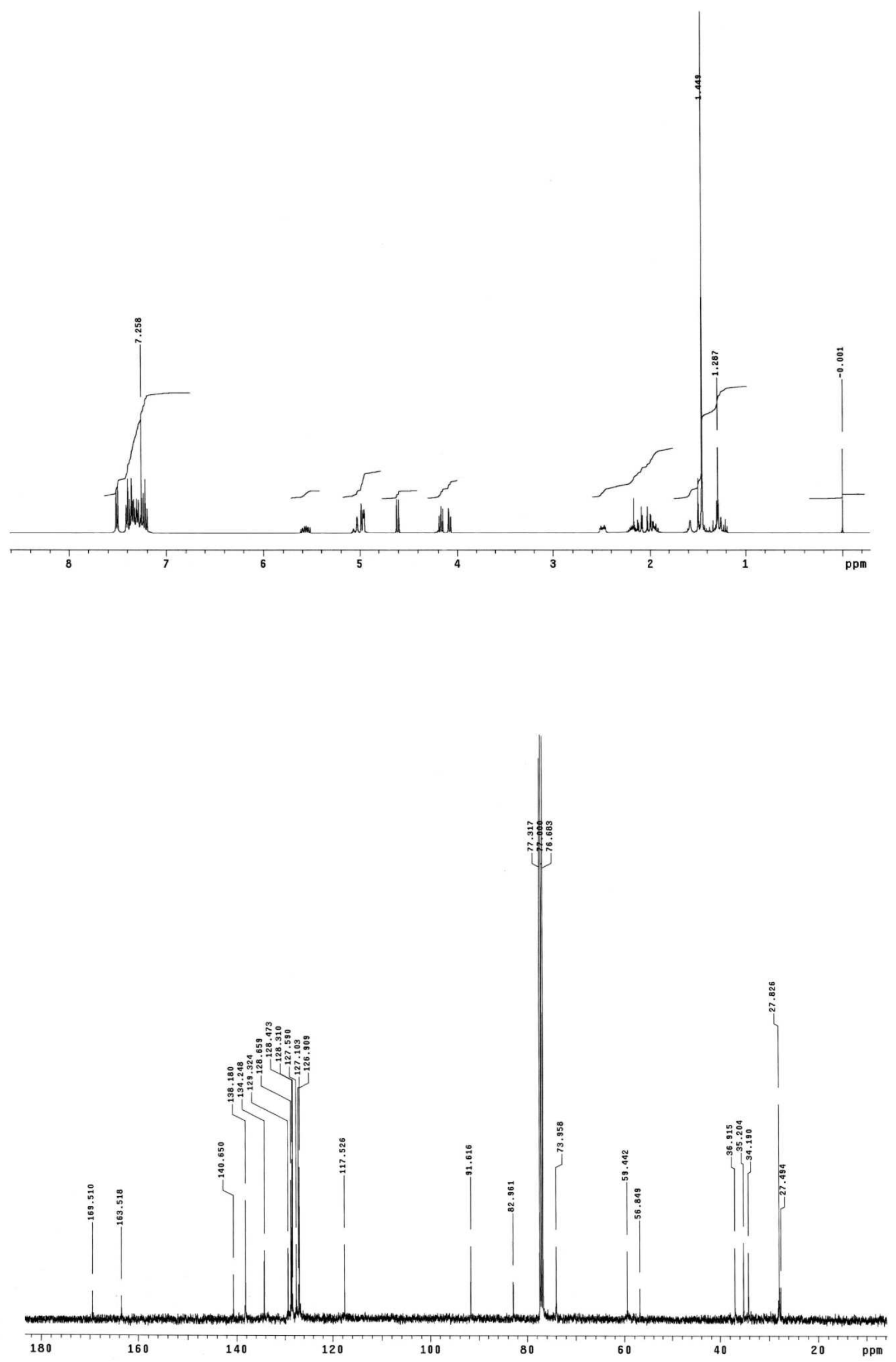


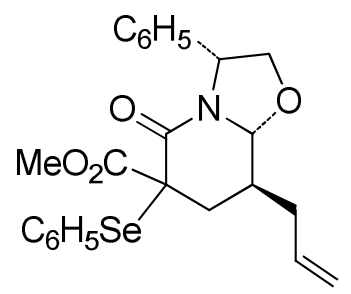

(3R,8S,8aR)-8-Allyl-6-(methoxycarbonyl)-5-oxo-3-phenyl-6-(phenylselenyl)-2,3,6,7,8,8ahexahydro-5H-oxazolo[3,2-a]pyridine (4).

Lithium bis(trimethylsilyl) amide (1 M in THF, $10 \mathrm{~mL}$ ) was slowly added at $-78 \stackrel{\circ}{\circ}$ to a solution of lactam $1(2.0 \mathrm{~g}, 1.07 \mathrm{mmol})$ in anhydrous THF $(120 \mathrm{ml})$, and the resulting mixture was stirred for $90 \mathrm{~min}$. Then, methyl chloroformate $(0.6 \mathrm{~mL}, 7.78 \mathrm{mmol})$ and, after $90 \mathrm{~min}$ of continuous stirring at $-78^{\circ} \mathrm{C}, \mathrm{PhSeCl}(2.08 \mathrm{~g}, 10.8 \mathrm{mmol})$ were added to the solution. The resulting mixture was stirred for $1 \mathrm{~h}$ and poured into saturated aqueous $\mathrm{NH}_{4} \mathrm{Cl}$. The aqueous layer was extracted with EtOAc, and the combined organic extracts were dried and concentrated. Flash chromatography (9:1 hexane-EtOAc to $1: 1$ hexane-EtOAc) of the resulting oil afforded $\mathbf{4}$ as a mixture of C- 6 epimers ( $3.14 \mathrm{~g}, 86 \%$ overall yield).

Epimer a (higher $\left.R_{f}\right)$ :

IR (NaCl) $1667,1725 \mathrm{~cm}^{-1}$.

${ }^{1} \mathrm{H}$ NMR (400 MHz, CDCl 3 , COSY, gHSQC): $\delta 1.82$ (dd, $J=14.0,12.4 \mathrm{~Hz}, 1 \mathrm{H}, \mathrm{CH}_{2}$ allyl), $1.98(\mathrm{~m}$, $2 \mathrm{H}, \mathrm{H}-7, \mathrm{H}-8), 2.33$ (dd, J = 14.0, $2.8 \mathrm{~Hz}, 1 \mathrm{H}, \mathrm{CH}_{2}$ allyl), 2.41 (m, 1H, H-7), 3.58 (s, 3H, $\mathrm{CH}_{3}$ ), 4.00 (dd, $J=9.2,2.0 \mathrm{~Hz}, 1 \mathrm{H}, \mathrm{H}-2$ ), 4.05 (dd, $J=9.2,6.8 \mathrm{~Hz}, 1 \mathrm{H}, \mathrm{H}-2$ ), 4.15 (d, $J=8.8 \mathrm{~Hz}, 1 \mathrm{H}, \mathrm{H}-8 \mathrm{a}$ ), 4.87 (dd, $J=6.8,2.0 \mathrm{~Hz}, 1 \mathrm{H}, \mathrm{H}-3$ ), $5.03\left(\mathrm{~m}, 2 \mathrm{H}, \mathrm{CH}_{2}=\right), 5.71$ (dddd, $J=16.8,10.4,7.6,6.4 \mathrm{~Hz}, 1 \mathrm{H}$, $\mathrm{CH}=)$, 7.26-7.66 (m, 10H, ArH).

${ }^{13} \mathrm{C} \mathrm{NMR}\left(100.6 \mathrm{MHz}, \mathrm{CDCl}_{3}\right): \delta 34.8(\mathrm{C}-7), 36.6\left(\mathrm{CH}_{2}\right), 37.7$ (C-8), $53.0\left(\mathrm{CH}_{3}\right), 54.0$ (C-6), 59.4 (C3), 74.0 (C-2), 91.8 (C-8a), $117.7\left(\mathrm{CH}_{2}=\right), 126.4-138.2$ (C-o, $\left.m, p\right), 133.7$ ( $\left.\mathrm{CH}=\right), 140.5$ (C-i), 163.3 (NCO), 170.8 (COO).

$[\alpha]_{D}^{22}=-99.3\left(c 0.7, \mathrm{CHCl}_{3}\right)$.

HRMS calcd for $\mathrm{C}_{24} \mathrm{H}_{25} \mathrm{NO}_{4} \mathrm{Se}: 471.0948$, found: 471.0955 . 

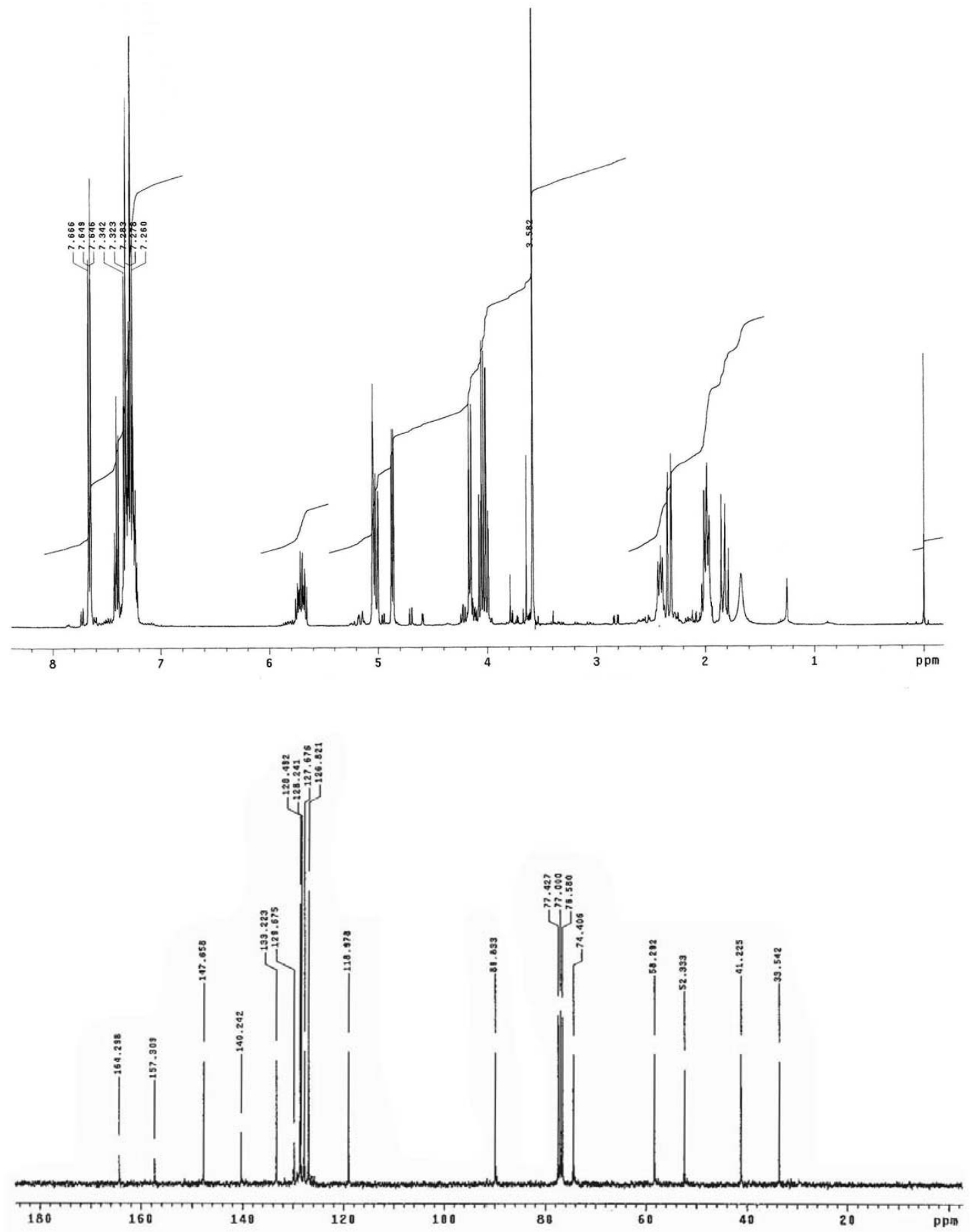
Epimer b (lower $R_{f}$ ):

${ }^{1} \mathrm{H}$ NMR (400 MHz, CDCl 3 , COSY, gHSQC) $\delta 1.98\left(\mathrm{~m}, 1 \mathrm{H}, \mathrm{CH}_{2}\right.$ allyl), 2.00 (dd, $J=15.2,11.6 \mathrm{~Hz}, 1 \mathrm{H}$, $\mathrm{H}-7$ ), 2.08 (dd, $J=15.2,4.0 \mathrm{~Hz}, 1 \mathrm{H}, \mathrm{H}-7), 2.32(\mathrm{~m}, 1 \mathrm{H}, \mathrm{H}-8), 2.52\left(\mathrm{~m}, 1 \mathrm{H}, \mathrm{CH}_{2}\right.$ allyl), 3.73 (s, 3H, $\mathrm{CH}_{3}$ ), $4.10(\mathrm{dd}, J=9.2,1.2 \mathrm{~Hz}, 1 \mathrm{H}, \mathrm{H}-2$ ), 4.17 (dd, $J=9.2,6.8 \mathrm{~Hz}, 1 \mathrm{H}, \mathrm{H}-2$ ), $4.63(\mathrm{~d}, J=9.2 \mathrm{~Hz}, 1 \mathrm{H}$, $\mathrm{H}-8 \mathrm{a}), 4.97\left(\mathrm{~m}, 3 \mathrm{H}, \mathrm{H}-3, \mathrm{CH}_{2}=\right), 5.59$ (dddd, $\left.J=16.0,10.0,8.0,6.0 \mathrm{~Hz}, 1 \mathrm{H}, \mathrm{CH}=\right)$, 7.23-7.47 (m, $10 \mathrm{H}, \mathrm{ArH})$.

${ }^{13} \mathrm{C} \mathrm{NMR}\left(100.6 \mathrm{MHz}, \mathrm{CDCl}_{3}\right) \delta 33.6(\mathrm{C}-7), 35.0\left(\mathrm{CH}_{2}\right), 36.5$ (C-8), $53.3\left(\mathrm{CH}_{3}\right), 55.6$ (C-6), 59.6 (C3), 73.8 (C-2), 91.6 (C-8a), $117.8\left(\mathrm{CH}_{2}=\right), 126.8-138.3$ (C-o, $\left.m, p\right), 134.0(\mathrm{CH}=), 140.5$ (C-i), 162.8 (NCO), 171.0 (COO).

$[\alpha]_{D}^{22}=+18.46\left(c 0.5, \mathrm{CHCl}_{3}\right)$.

HRMS calcd for $\mathrm{C}_{24} \mathrm{H}_{25} \mathrm{NO}_{4} \mathrm{Se}: 471.0948$, found: 471.0946 . 

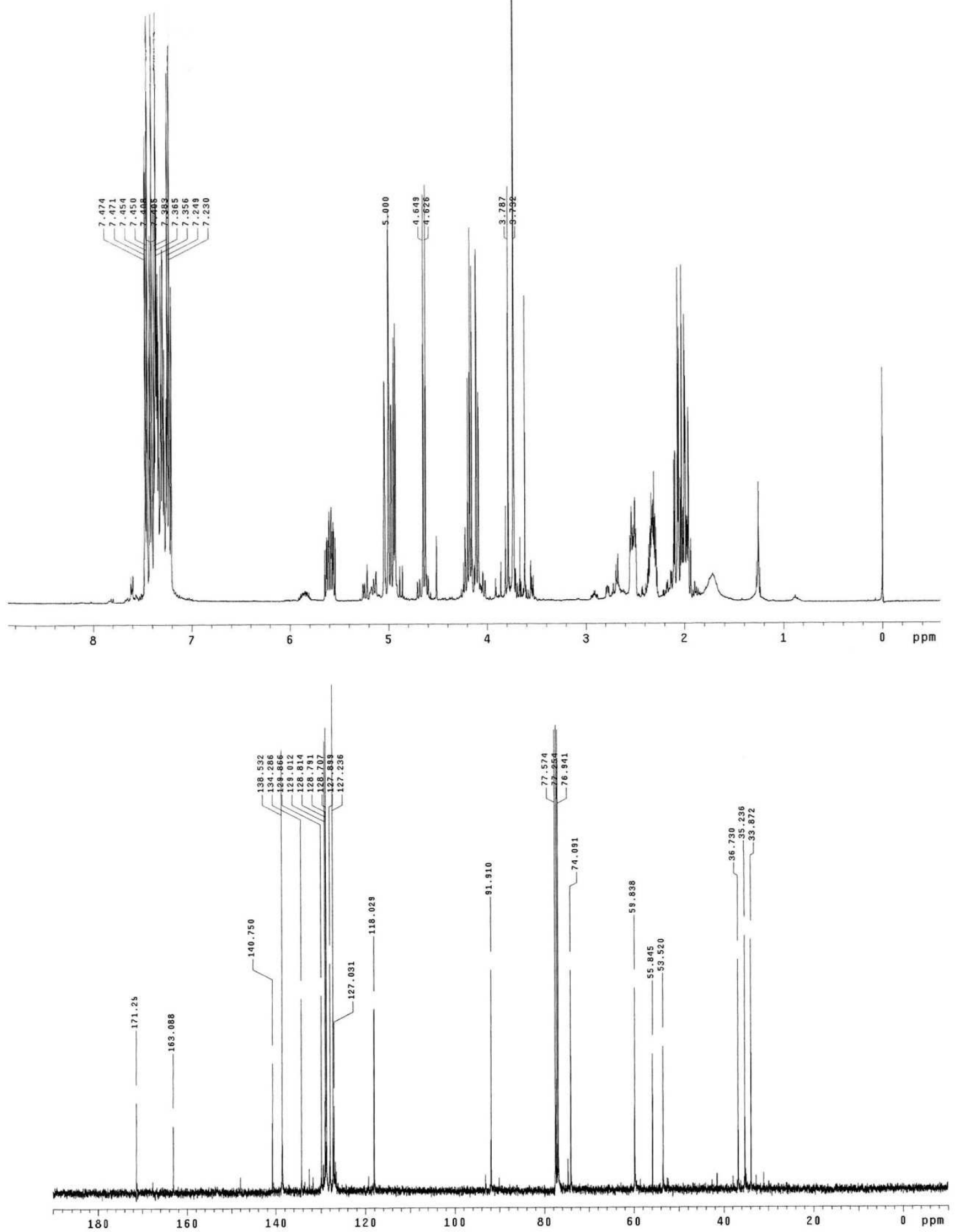
<smiles>C=CC[C@H]1C=C(C(=O)OC(C)(C)C)C(=O)N2C(C)COC12</smiles>

(3R,8S,8aR)-8-Allyl-6-(tert-butoxycarbonyl)-5-oxo-3-phenyl-2,3,8,8a-tetrahydro-5Hoxazolo[3,2-a]pyridine (5).

Aqueous $\mathrm{H}_{2} \mathrm{O}_{2}(30 \%, 0.37 \mathrm{~mL}, 12.4 \mathrm{mmol})$ and pyridine $(0.17 \mathrm{~mL}, 2.01 \mathrm{mmol})$ were added to a solution of the selenides $3(0.92 \mathrm{~g}, 1.77 \mathrm{mmol})$ in $\mathrm{CH}_{2} \mathrm{Cl}_{2}(159 \mathrm{~mL})$. The resulting mixture was stirred at room temperature for $2 \mathrm{~h}$ and poured into distilled water. The two phases were separated, and the organic layer was washed with water, dried, filtered and concentrated under reduced pressure to give crude unsaturated lactam 5, which was used in the next reaction without further purification.

Compound $\mathbf{5}$ (spectroscopic data from the crude):

IR (film): 1675 (NCO), 1731 (COO) $\mathrm{cm}^{-1}$.

${ }^{1} \mathrm{H}$ NMR $\left(300 \mathrm{MHz}, \mathrm{CDCl}_{3}\right): \delta 1.49\left[\mathrm{~s}, 9 \mathrm{H},\left(\mathrm{CH}_{3}\right)_{3} \mathrm{C}\right], 2.31$ (dt, $J=14.4,9.3,9.3 \mathrm{~Hz}, 1 \mathrm{H}, \mathrm{CH}_{2}$ allyl), $2.68\left(\mathrm{ddm}, J=14.4,4.8 \mathrm{~Hz}, 1 \mathrm{H}, \mathrm{CH}_{2}\right.$ allyl), 2.89 (dddd, $\left.J=10.5,9.3,4.8,1.8 \mathrm{~Hz}, 1 \mathrm{H}, \mathrm{H}-8\right), 4.15$ (dd, $J=9.0,1.2 \mathrm{~Hz}, 1 \mathrm{H}, \mathrm{H}-2$ ), 4.22 (dd, J = 9.0, $6.6 \mathrm{~Hz}, 1 \mathrm{H}, \mathrm{H}-2$ ), 4.87 (d, $J=10.5 \mathrm{~Hz}, 1 \mathrm{H}, \mathrm{H}-8 \mathrm{a}$ ), 5.05 (dd, $J=6.6,1.2 \mathrm{~Hz}, 1 \mathrm{H}, \mathrm{H}-3$ ), $5.24\left(\mathrm{~m}, 2 \mathrm{H}, \mathrm{CH}_{2}=\right), 5.86$ (dddd, $J=16.5,10.2,8.7,5.7 \mathrm{~Hz}, 1 \mathrm{H}$, $\mathrm{CH}=)$, 7.10-7.20 (m, 6H, H-7, $\left.\mathrm{C}_{6} \mathrm{H}_{5}\right)$.

${ }^{13} \mathrm{C} \mathrm{NMR}\left(75.4 \mathrm{MHz}, \mathrm{CDCl}_{3}\right): \delta 28.0\left[\left(\mathrm{CH}_{3}\right)_{3} \mathrm{C}\right], 33.7\left(\mathrm{CH}_{2}\right.$ allyl), 41.0 (C-8), 58.2 (C-3), 74.5 (C-2), $82.1\left[\left(\mathrm{CH}_{3}\right)_{3} \mathrm{C}\right], 90.0$ (C-8a), $118.7\left(\mathrm{CH}_{2}=\right), 126.6,128.6\left(\mathrm{C}-0, m \mathrm{C}_{6} \mathrm{H}_{5}\right), 127.6\left(\mathrm{C}-p \mathrm{C}_{6} \mathrm{H}_{5}\right), 131.6$ (C6), $133.5(\mathrm{CH}=), 140.5\left(\mathrm{C}-i \mathrm{C}_{6} \mathrm{H}_{5}\right), 145.2(\mathrm{C}-7), 157.6(\mathrm{C}=\mathrm{O}), 163.2(\mathrm{C}=\mathrm{O})$. 

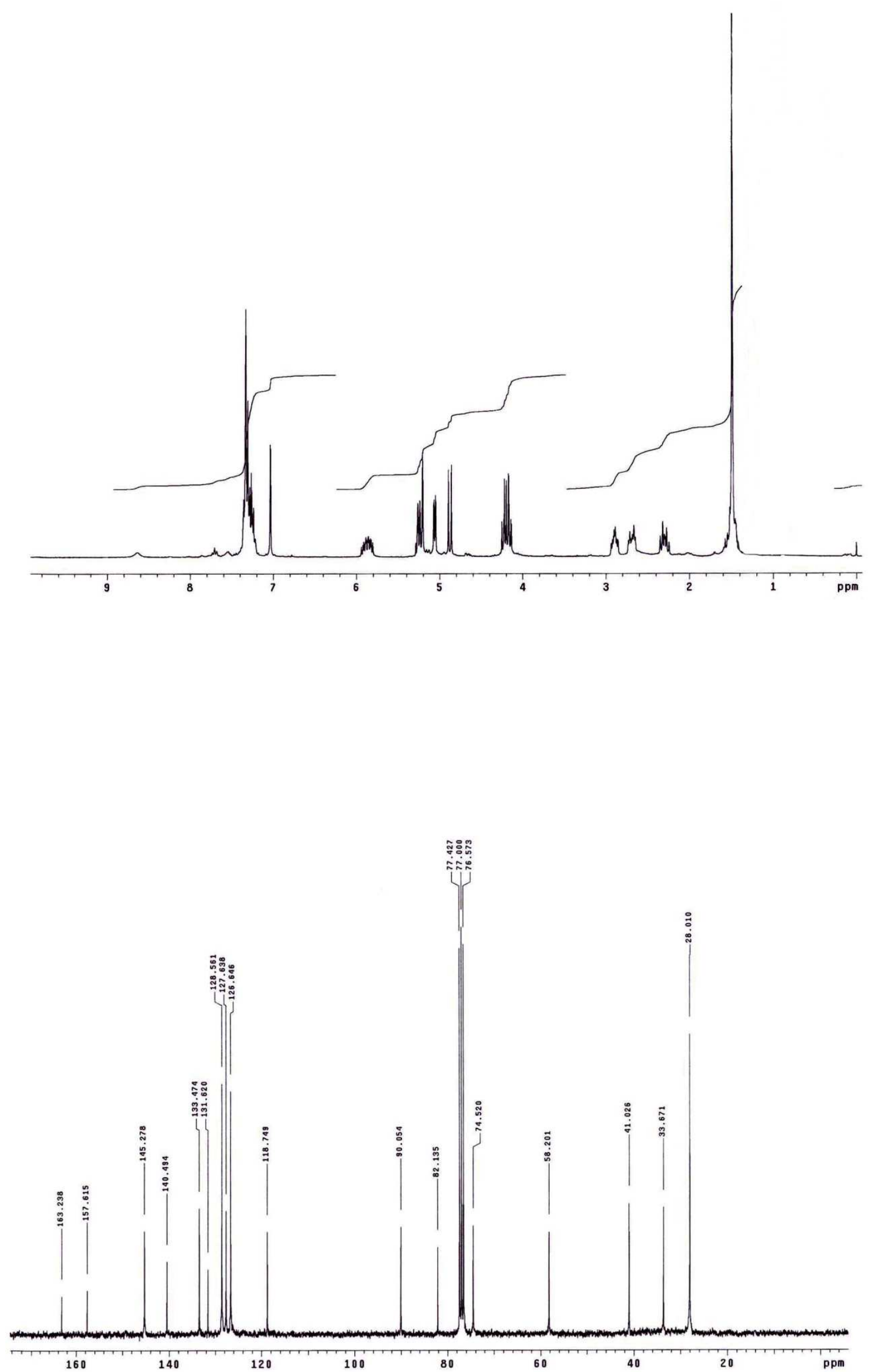
<smiles>C=CC[C@H]1C=C(C(C)=O)C(=O)N2C(C)COC12</smiles>

(3R,8S,8aR)-8-Allyl-6-(methoxycarbonyl)-5-oxo-3-phenyl-2,3,8,8a-tetrahydro-5H-oxazolo[3,2a]pyridine (6).

$30 \%$ aqueous $\mathrm{H}_{2} \mathrm{O}_{2}(0.73 \mathrm{~mL}, 23.8 \mathrm{mmol})$ and pyridine $(0.36 \mathrm{~mL}, 4.4 \mathrm{mmol})$ were added to a solution of selenides $4(1.6 \mathrm{~g}, 2.8 \mathrm{mmol})$ in $\mathrm{CH}_{2} \mathrm{Cl}_{2}(231 \mathrm{~mL})$, and the resulting mixture was stirred at $\mathrm{rt}$ for $2 \mathrm{~h}$. The two phases were separated, and the organic layer was washed with water $(10 \times 20 \mathrm{~mL})$, dried, and concentrated to give crude $6(1.65 \mathrm{~g})$ as an oil, which was kept at $-30{ }^{\circ} \mathrm{C}$ and used in the next reaction without further purification.

IR (NaCl) $1673,1741 \mathrm{~cm}^{-1}$.

${ }^{1} \mathrm{H}$ NMR $\left(300 \mathrm{MHz}, \mathrm{CDCl}_{3}\right): \delta 2.31\left(\mathrm{dt}, J=14.4,8.7 \mathrm{~Hz}, 1 \mathrm{H}, \mathrm{CH}_{2}\right.$ allyl), $2.70(\mathrm{dm}, J=14.4 \mathrm{~Hz}, 1 \mathrm{H}$, $\mathrm{CH}_{2}$ allyl), $2.91(\mathrm{~m}, 1 \mathrm{H}, \mathrm{H}-8), 3.78\left(\mathrm{~s}, 3 \mathrm{H}, \mathrm{CH}_{3}\right), 4.20$ (dd, $\left.J=9.0,2.4 \mathrm{~Hz}, 1 \mathrm{H}, \mathrm{H}-2\right), 4.24$ (dd, $J=$ 9.0, 6.0 Hz, 1H, H-2), 4.87 (d, J = 10.5 Hz, 1H, H-8a), 5.03 (dd, J = 6.0, 2.4 Hz, 1H, H-3), 5.27 (m, $2 \mathrm{H}, \mathrm{CH}_{2}=$ ), 5.86 (dddd, $\left.J=15.3,10.8,8.7,5.7 \mathrm{~Hz}, 1 \mathrm{H}, \mathrm{CH}=\right), 7.20-7.36(\mathrm{~m}, 5 \mathrm{H}, \mathrm{ArH}$ ).

${ }^{13} \mathrm{C} \mathrm{NMR}\left(75.4 \mathrm{MHz}, \mathrm{CDCl}_{3}\right): \delta 33.5\left(\mathrm{CH}_{2}\right), 41.2(\mathrm{C}-8), 52.3\left(\mathrm{CH}_{3}\right), 58.3(\mathrm{C}-3), 74.4(\mathrm{C}-2), 89.8$ (C8a), $119.0\left(\mathrm{CH}_{2}=\right), 126.8,128.5(\mathrm{C}-o, m), 127.7$ (C-p), 129.7 (C-6), $133.3(\mathrm{CH}=), 140.2(\mathrm{C}-i), 147.6$ (C-7), 157.3 (COO), 164.3 (NCO). 

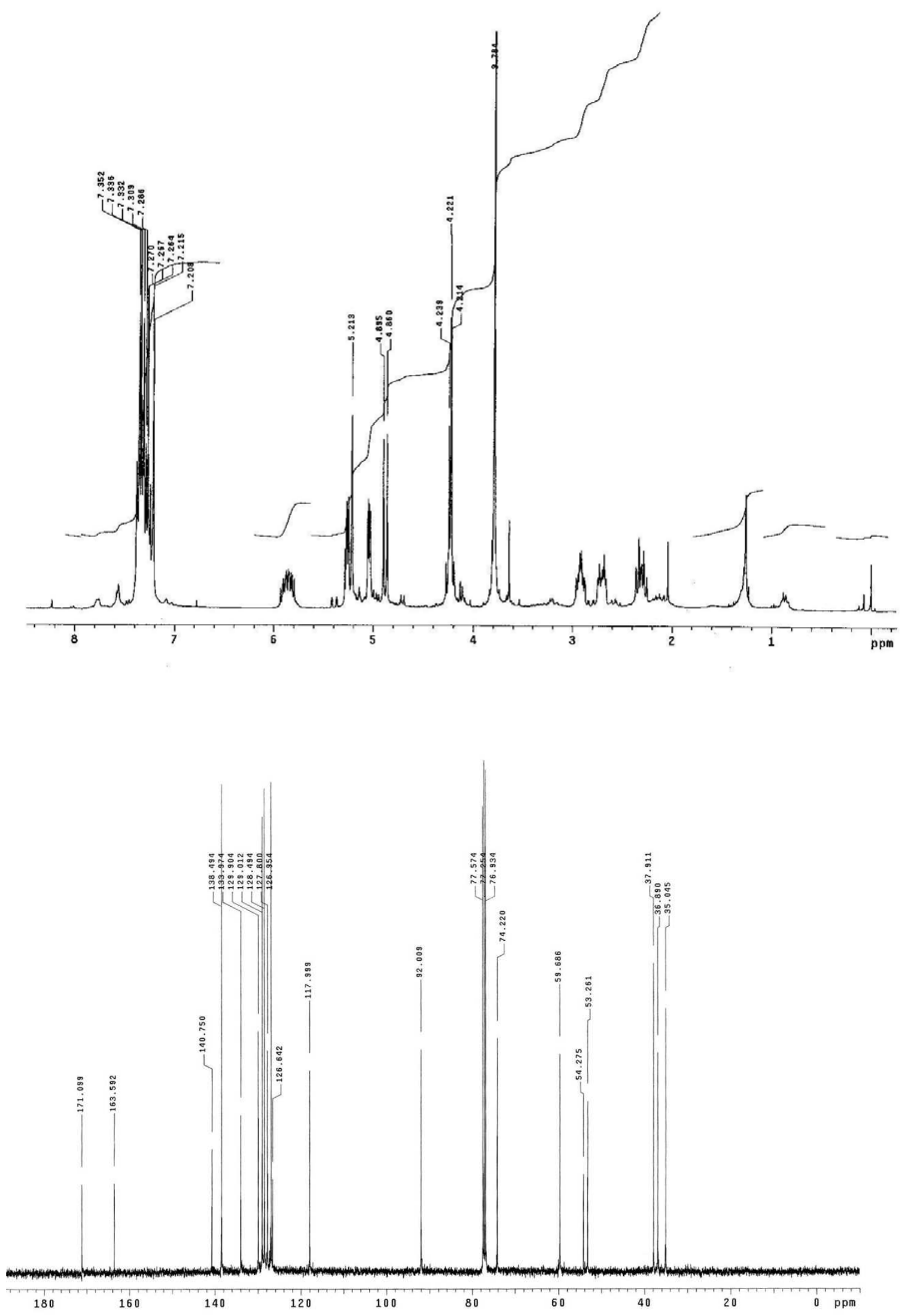
<smiles>C=CCC1C(C(=O)OC(C)(C)C)C(=O)N2C(C)COC2C1CC=C</smiles>

$(3 R, 7 R, 8 S, 8 \mathrm{a} R)$-6-(tert-Butoxycarbonyl)-7,8-diallyl-5-oxo-3-phenyl-2,3,6,7,8,8a-hexahydro$5 H$-oxazolo[3,2-a]pyridine (7).

$\mathrm{LiCl}(301 \mathrm{mg}, 7.11 \mathrm{mmol})$ was dried at $80^{\circ} \mathrm{C}$ for $1 \mathrm{~h}$ under vacuum $(10-15 \mathrm{mmHg}$ ) in a twonecked, $100 \mathrm{~mL}$ round-bottomed flask. Then, Cul $(1.35 \mathrm{~g}, 7.11 \mathrm{mmol})$ and THF $(20 \mathrm{~mL})$ were added under inert atmosphere, and the mixture was stirred at room temperature for $5 \mathrm{~min}$. The suspension was cooled at $-78^{\circ} \mathrm{C}$, and allylmagnesium bromide $(7.11 \mathrm{~mL}$ of a $1 \mathrm{M}$ solution in $\left.\mathrm{Et}_{2} \mathrm{O}, 7.11 \mathrm{mmol}\right), \mathrm{TMSCl}(0.9 \mathrm{~mL}, 7.11 \mathrm{mmol})$, and the crude unsaturated lactam 5 (1.77 $\mathrm{mmol})$ in THF $(20 \mathrm{~mL})$ were successively added. The resulting mixture was stirred at $-78^{\circ} \mathrm{C}$ for $18 \mathrm{~h}$. The reaction was quenched with saturated aqueous $\mathrm{NH}_{4} \mathrm{Cl}$, and filtered through Celite ${ }^{\circledR}$. The aqueous layer was extracted with EtOAc, and the combined organic extracts were dried over anhydrous $\mathrm{MgSO}_{4}$, filtered and concentrated under reduced pressure. Flash chromatography (9:1 to 7:3 hexane-EtOAc) gave the C-6 epimers a (major, $494 \mathrm{mg}, 71 \%$ from selenide derivative) and $\mathbf{b}$ (minor, $77 \mathrm{mg}, 11 \%$ from selenide derivative) of the diallyl derivative 7 as colourless oils ( $82 \%$ overall yield).

Epimer 7a (major, lower $\mathrm{R}_{f}$ ) (6S):

$[\alpha]_{D}^{22}=-70.7\left(c 0.5, \mathrm{CHCl}_{3}\right)$.

IR (film): 1664 (NCO), 1729 (COO) $\mathrm{cm}^{-1}$.

${ }^{1} \mathrm{H}$ NMR $\left(400 \mathrm{MHz}, \mathrm{CDCl}_{3}, \mathrm{COSY}, \mathrm{gHSQC}\right): \delta 1.35\left[\mathrm{~s}, 9 \mathrm{H},\left(\mathrm{CH}_{3}\right)_{3} \mathrm{C}\right], 1.80(\mathrm{dt}, J=12.3,9.0,9.0 \mathrm{~Hz}$, $1 \mathrm{H}, \mathrm{CH}_{2}$ allyl), 2.20 (dt, $J=13.8,10.2,10.2 \mathrm{~Hz}, 1 \mathrm{H}, \mathrm{CH}_{2}$ allyl), $2.33(\mathrm{dm}, J=11.4 \mathrm{~Hz}, 1 \mathrm{H}, \mathrm{H}-7)$, $2.45\left(\mathrm{dm}, J=13.8 \mathrm{~Hz}, 1 \mathrm{H}, \mathrm{CH}_{2}\right.$ allyl), $2.63\left(\mathrm{~m}, 2 \mathrm{H}, \mathrm{H}-8, \mathrm{CH}_{2}\right.$ allyl), 3.30 (s, $\left.1 \mathrm{H}, \mathrm{H}-6\right), 4.00$ (dd, $J=$ 9.0, $1.2 \mathrm{~Hz}, 1 \mathrm{H}, \mathrm{H}-2$ ), 4.14 (dd, J = 9.0, $6.9 \mathrm{~Hz}, 1 \mathrm{H}, \mathrm{H}-2$ ), 4.60 (d, J = 9.6 Hz, 1H, H-8a), 4.92 (dd, J $=6.9,1.2 \mathrm{~Hz}, 1 \mathrm{H}, \mathrm{H}-3$ ), 5.08-5.21 (m, 4H, $\left.\mathrm{CH}_{2}=\right), 5.68$ (dddd, $J=15.0,10.5,8.4,4.8 \mathrm{~Hz}, 1 \mathrm{H}, \mathrm{CH}=$ ), 5.84 (dddd, $J=15.3,9.9,8.4,4.8 \mathrm{~Hz}, 1 \mathrm{H}, \mathrm{CH}=)$, 7.20-7.35 (m, $5 \mathrm{H}, \mathrm{C}_{6} \mathrm{H}_{5}$ ).

${ }^{13} \mathrm{C} \mathrm{NMR}\left(100.6 \mathrm{MHz}, \mathrm{CDCl}_{3}\right): \delta 27.8\left[\left(\mathrm{CH}_{3}\right)_{3} \mathrm{C}\right], 31.6,31.7\left(\mathrm{CH}_{2}\right.$ allyl), 36.5 (C-7), 38.7 (C-8), 52.8 (C-6), 59.6 (C-3), 74.1 (C-2), 81.8 [( $\left.\left.\mathrm{CH}_{3}\right)_{3} \mathrm{C}\right], 89.4$ (C-8a), 117.4, $118.3\left(\mathrm{CH}_{2}=\right), 126.4,128.4$ (C-o, $\left.m \mathrm{C}_{6} \mathrm{H}_{5}\right), 127.4\left(\mathrm{C}-p \mathrm{C}_{6} \mathrm{H}_{5}\right), 134.7,135.1(\mathrm{CH}=), 140.8\left(\mathrm{C}-i \mathrm{C}_{6} \mathrm{H}_{5}\right), 162.7(\mathrm{C}=\mathrm{O}), 168.9(\mathrm{C}=\mathrm{O})$. 
Elemental analysis calcd (\%) for $\mathrm{C}_{24} \mathrm{H}_{31} \mathrm{NO}_{4}$ : C 72.52, $\mathrm{H} 7.86, \mathrm{~N} 3.52$; found: $\mathrm{C} 72.49, \mathrm{H} 7.94, \mathrm{~N}$ 3.40 .
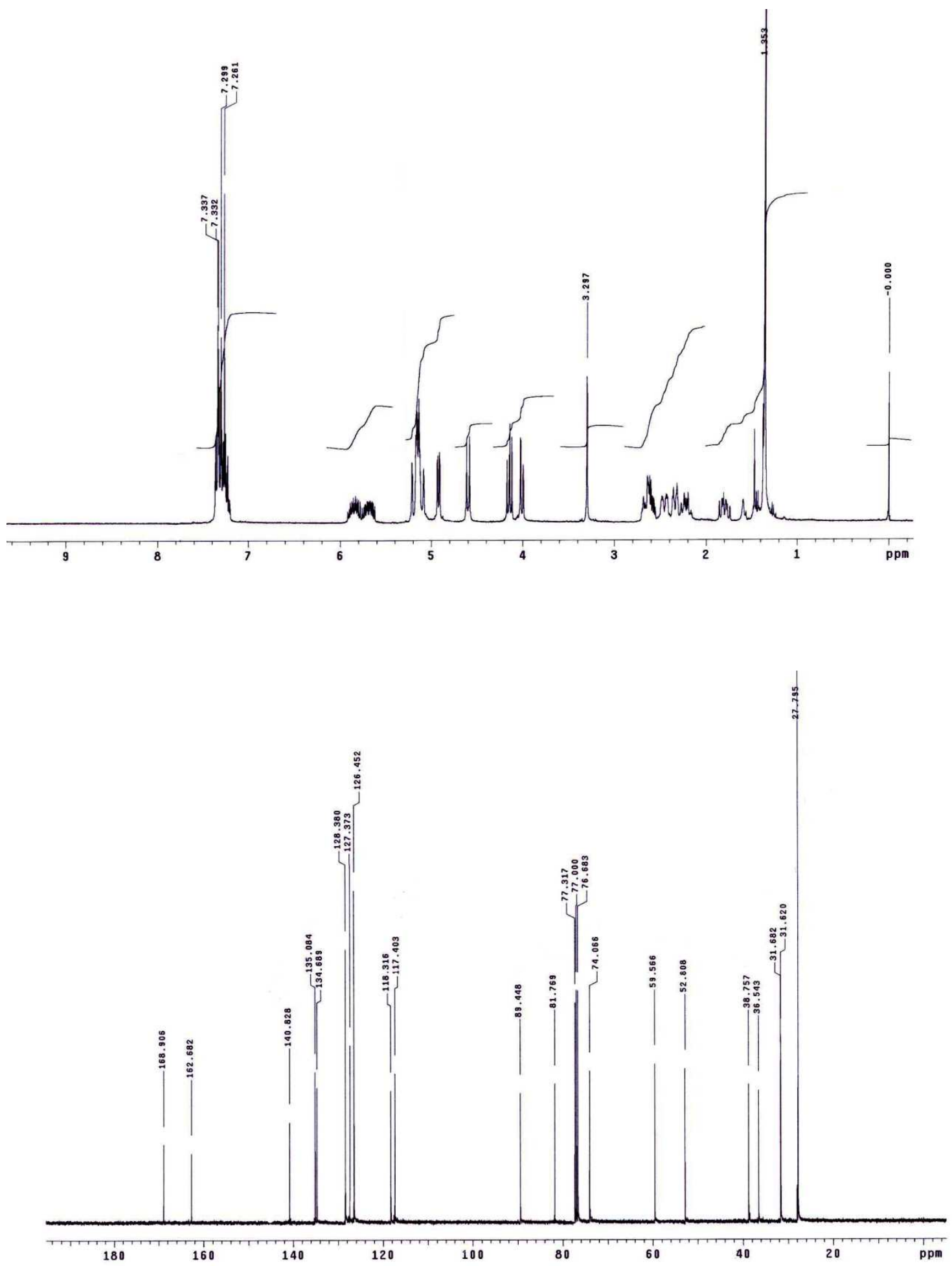
<smiles>C=CCC1[C@H](C(=O)OC(C)(C)C)C(=O)N2C([13CH3])CO[C@@H]2[C@H]1CC=C</smiles>

Epimer $\mathbf{7 b}$ (minor, higher $\left.\mathrm{R}_{f}\right)(6 R)$ :

IR (film): 1669 (NCO), 1727 (COO) $\mathrm{cm}^{-1}$.

${ }^{1} \mathrm{H}$ NMR $\left(300 \mathrm{MHz}, \mathrm{CDCl}_{3}\right): \delta 1.47\left[\mathrm{~s}, 9 \mathrm{H},\left(\mathrm{CH}_{3}\right)_{3}\right], 1.86\left(\mathrm{~m}, 1 \mathrm{H}, \mathrm{CH}_{2}\right.$ allyl), $2.18\left(\mathrm{~m}, 1 \mathrm{H}, \mathrm{CH}_{2}\right.$ allyl), $2.33\left(\mathrm{~m}, 2 \mathrm{H}, \mathrm{H}-7, \mathrm{CH}_{2}\right.$ allyl), $2.51\left(\mathrm{~m}, 2 \mathrm{H}, \mathrm{H}-8, \mathrm{CH}_{2}\right.$ allyl), 3.21 (d, J = 8.1 Hz, 1H, H-6), 4.05 (dd, J = 9.0, $1.2 \mathrm{~Hz}, 1 \mathrm{H}, \mathrm{H}-2$ ), 4.18 (dd, J = 9.0, $6.6 \mathrm{~Hz}, 1 \mathrm{H}, \mathrm{H}-2$ ), 4.77 (d, J = 9.0 Hz, 1H, H-8a), 4.94 (dd, J $=6.6,1.2 \mathrm{~Hz}, 1 \mathrm{H}, \mathrm{H}-3), 5.10-5.17\left(\mathrm{~m}, 4 \mathrm{H}, \mathrm{CH}_{2}=\right), 5.73(\mathrm{~m}, 1 \mathrm{H}, \mathrm{CH}=), 5.89(\mathrm{~m}, 1 \mathrm{H}, \mathrm{CH}=), 7.26-7.35$ $\left(m, 5 \mathrm{H}, \mathrm{C}_{6} \mathrm{H}_{5}\right)$.

${ }^{13} \mathrm{C} \mathrm{NMR}\left(100.6 \mathrm{MHz}, \mathrm{CDCl}_{3}\right): \delta 27.8\left[\left(\mathrm{CH}_{3}\right)_{3} \mathrm{C}\right], 32.7,35.5\left(\mathrm{CH}_{2}\right.$ allyl), 36.7 (C-7), 41.6 (C-8), 54.4 (C-6), 59.0 (C-3), 73.8 (C-2), 81.9 [( $\left.\left.\mathrm{CH}_{3}\right)_{3} \mathrm{C}\right], 89.8$ (C-8a), 118.2, $119.4\left(\mathrm{CH}_{2}=\right), 126.4,128.4$ (C-o, $\left.m \mathrm{C}_{6} \mathrm{H}_{5}\right), 127.5\left(\mathrm{C}-p \mathrm{C}_{6} \mathrm{H}_{5}\right), 132.7,133.4(\mathrm{CH}=), 140.8\left(\mathrm{C}-i \mathrm{C}_{6} \mathrm{H}_{5}\right), 163.2(\mathrm{C}=\mathrm{O}), 169.7(\mathrm{C}=\mathrm{O})$.

MS m/z (\%): 397 (2) [M+], 381 (5), 342 (8), 341 (34), 340 (21), 324 (17), 300 (12), 296 (27), 256 (15), 254 (38), 214 (16), 146 (26), 128 (12), 120 (28), 117 (15), 104 (100).

HRMS calcld for $\left[\mathrm{C}_{24} \mathrm{H}_{32} \mathrm{NO}_{4}+\mathrm{H}\right]^{+}: 398.2326$, found: 398.2320 . 

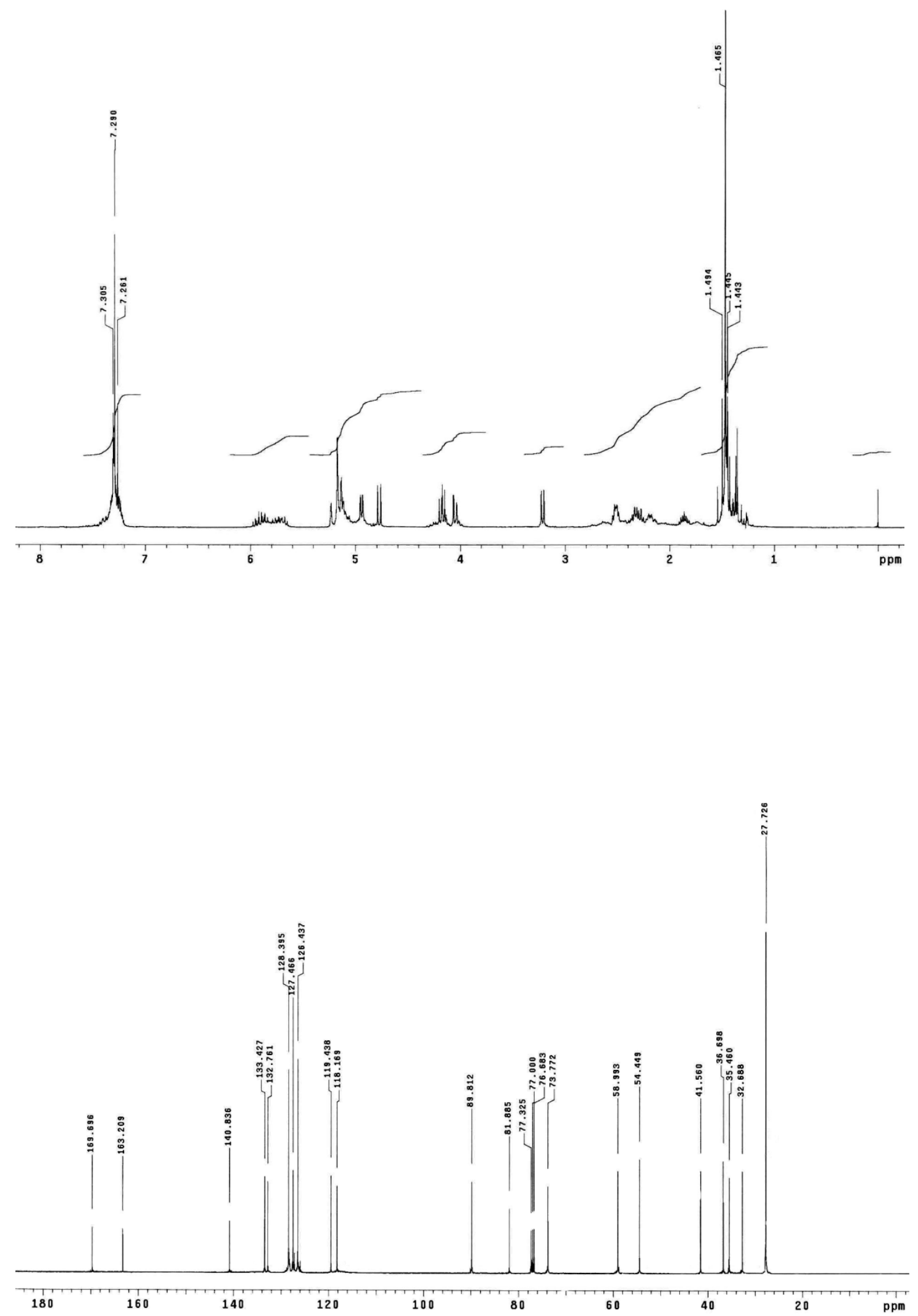
<smiles>C=CCC1C2OCC(CC)N2C(=O)C[C@H]1CC=C</smiles>

$(3 R, 7 S, 8 S, 8 \mathrm{a} R)-7,8$-Diallyl-5-oxo-3-phenyl-2,3,6,7,8,8a-hexahydro-5H-oxazolo[3,2-a]pyridine (9a).

TFA (1.5 mL, $0.02 \mathrm{mmol}$ ) was added to a solution of the diallyl derivative 7 (406 mg, 1.02 mmol) in anhydrous $\mathrm{CH}_{2} \mathrm{Cl}_{2}(4 \mathrm{~mL})$ at room temperature under inert atmosphere, and the resulting mixture was stirred for 30 minutes. The reaction was quenched with saturated aqueous $\mathrm{Na}_{2} \mathrm{CO}_{3}$. The aqueous phase was washed with $\mathrm{CH}_{2} \mathrm{Cl}_{2}(2 \times 5 \mathrm{~mL})$, and the combined organic extracts were dried over anhydrous $\mathrm{Na}_{2} \mathrm{SO}_{4}$, filtered, and concentrated. The crude was then redissolved in anhydrous toluene $(15 \mathrm{~mL})$, and the resulting solution was heated at reflux for $18 \mathrm{~h}$. The resulting solution was cooled and poured into brine, the aqueous phase was extracted with EtOAc, and the combined organic extracts were dried over anhydrous $\mathrm{Na}_{2} \mathrm{SO}_{4}$, filtered, and concentrated under reduced pressure. Flash chromatography (7:3 to 6:4 hexaneEtOAc) afforded compound 9 a ( $233 \mathrm{mg}, 77 \%)$ as a colourless oil.

$[\alpha]_{D}^{22}=-17.0\left(c 0.2, \mathrm{CHCl}_{3}\right)$.

IR (film): 1661 (COO) $\mathrm{cm}^{-1}$.

${ }^{1} \mathrm{H}$ NMR (400 MHz, $\mathrm{CDCl}_{3}, \mathrm{COSY}, \mathrm{gHSQC}$ ): $\delta 1.81$ (ddd, $J=13.6,10.8,8.8 \mathrm{~Hz}, 1 \mathrm{H}, \mathrm{CH}_{2}$ allyl), 2.13 (m, 2H, H-7, H-8), 2.23 (m, 1H, $\mathrm{CH}_{2}$ allyl), 2.28 (dd, J = 18.0, $\left.6.0 \mathrm{~Hz}, 1 \mathrm{H}, \mathrm{H}-6\right), 2.38\left(\mathrm{~m}, 1 \mathrm{H}, \mathrm{CH}_{2}\right.$ allyl), 2.44 (dd, $J=18.0,1.6 \mathrm{~Hz}, 1 \mathrm{H}, \mathrm{H}-6), 2.64$ (dt, $J=14.0,5.2,5.2 \mathrm{~Hz}, 1 \mathrm{H}, \mathrm{CH}_{2}$ allyl), 4.00 (dd, $J$ $=8.8,0.8 \mathrm{~Hz}, 1 \mathrm{H}, \mathrm{H}-2$ ) , 4.13 (dd, J = 8.8, $6.8 \mathrm{~Hz}, 1 \mathrm{H}, \mathrm{H}-2$ ), 4.63 (d, J = 9.2 Hz, 1H, H-8a), 4.89 (dd, $J=6.8,0.8 \mathrm{~Hz}, 1 \mathrm{H}, \mathrm{H}-3), 5.05-5.20\left(\mathrm{~m}, 4 \mathrm{H}, \mathrm{CH}_{2}=\right), 5.69(\mathrm{~m}, 1 \mathrm{H}, \mathrm{CH}=), 5.86$ (dddd, $J=16.0,10.4$, 8.8, 6.0 Hz, $1 \mathrm{H}, \mathrm{CH}=), 7.26-7.30\left(\mathrm{~m}, 5 \mathrm{H}, \mathrm{C}_{6} \mathrm{H}_{5}\right)$.

${ }^{13} \mathrm{C} \mathrm{NMR}\left(100.6 \mathrm{MHz}, \mathrm{CDCl}_{3}\right): \delta 32.2\left(2 \mathrm{CH}_{2}\right.$ allyl), 32.5 (C-7), 36.3 (C-6), 42.2 (C-8), 59.4 (C-3), 73.8 (C-2), 89.6 (C-8a), $117.3\left(\mathrm{CH}_{2}=\right), 117.6\left(\mathrm{CH}_{2}=\right), 126.3,128.5\left(\mathrm{C}-0, m \mathrm{C}_{6} \mathrm{H}_{5}\right), 127.4\left(\mathrm{C}-p \mathrm{C}_{6} \mathrm{H}_{5}\right)$, $135.0(\mathrm{CH}=), 135.9(\mathrm{CH}=), 141.5\left(\mathrm{C}-i \mathrm{C}_{6} \mathrm{H}_{5}\right), 166.9$ (NCO).

Elemental analysis calcd (\%) for $\mathrm{C}_{19} \mathrm{H}_{23} \mathrm{NO}_{2} \cdot 1 / 44 \mathrm{H}_{2} \mathrm{O}$ : C 75.59, $\mathrm{H}$ 7.85, $\mathrm{N}$ 4.64; found: $\mathrm{C} 75.49, \mathrm{H}$ $7.76, \mathrm{~N} 4.45$. 

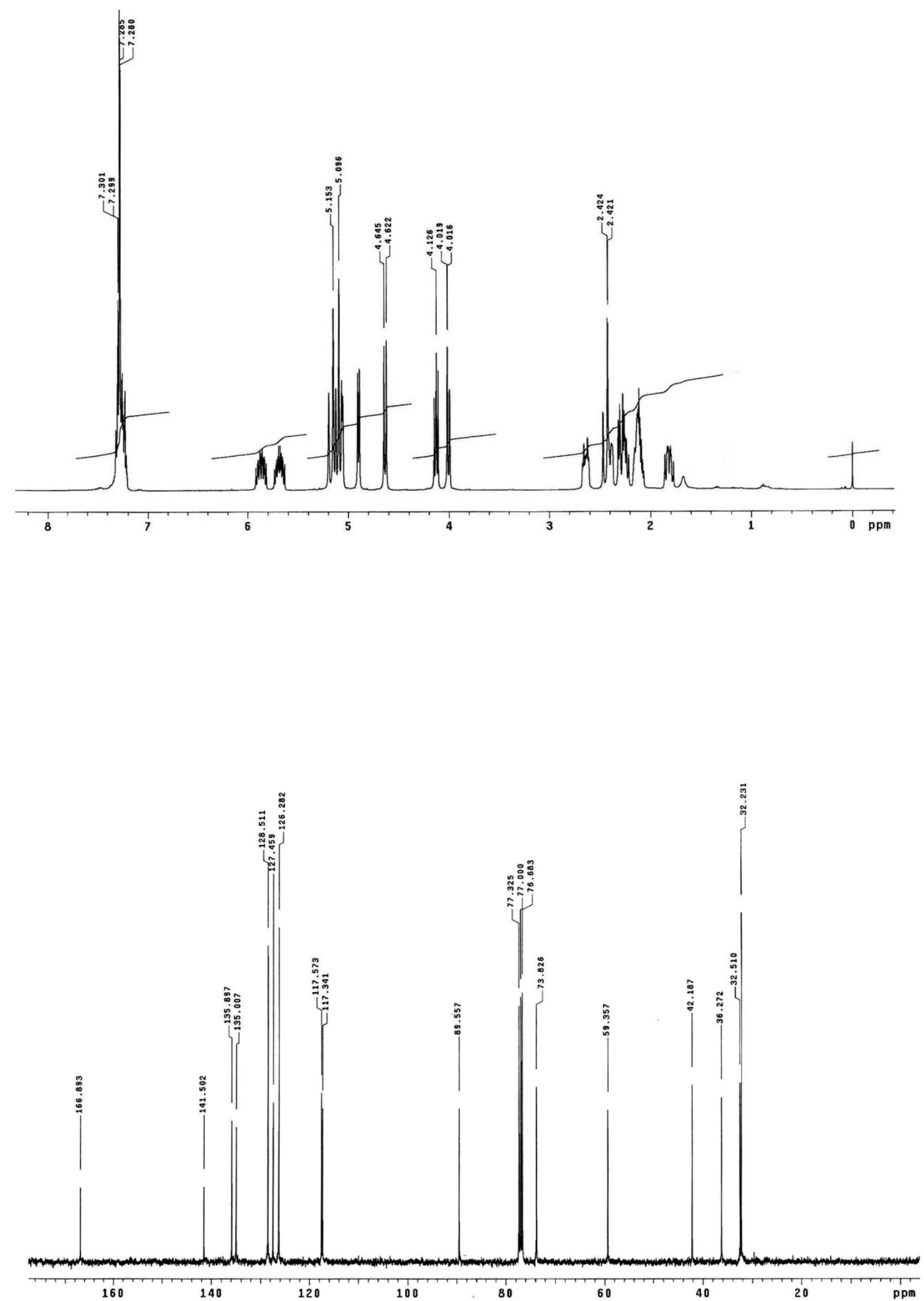


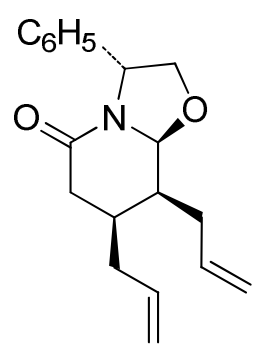

Compound 9b:

IR (film): 1643 (NCO) $\mathrm{cm}^{-1}$.

${ }^{1} \mathrm{H}-\mathrm{NMR}\left(400 \mathrm{MHz}, \mathrm{CDCl}_{3}\right.$, COSY, gHSQC): $\delta 2.01$ (m, $1 \mathrm{H}, \mathrm{CH}_{2}$ allyl), 2.08-2.23 (m, 5H, H-6, H-7, $3 \mathrm{H} \mathrm{CH}_{2}$ allyl), $2.37(\mathrm{~m}, 1 \mathrm{H}, \mathrm{H}-8), 2.49(\mathrm{~m}, 1 \mathrm{H}, \mathrm{H}-6), 3.78(\mathrm{dd}, J=9.2,7.6 \mathrm{~Hz}, 1 \mathrm{H}, \mathrm{H}-2), 4.46(\mathrm{dd}, J$ $=9.2,8.0 \mathrm{~Hz}, 1 \mathrm{H}, \mathrm{H}-2), 4.89-5.11\left(\mathrm{~m}, 4 \mathrm{H}, \mathrm{CH}_{2}=\right), 5.14(\mathrm{~d}, J=4.4 \mathrm{~Hz}, 1 \mathrm{H}, \mathrm{H}-8 \mathrm{a}), 5.20(\mathrm{t}, J=7.6 \mathrm{~Hz}$, $1 \mathrm{H}, \mathrm{H}-3), 5.73(\mathrm{~m}, 1 \mathrm{H}, \mathrm{CH}=), 5.91$ (dddd, $J=17.6,10.0,8.0,6.0 \mathrm{~Hz}, 1 \mathrm{H}, \mathrm{CH}=), 7.25-7.40(\mathrm{~m}, 5 \mathrm{H}$, $\left.\mathrm{C}_{6} \mathrm{H}_{5}\right)$.

${ }^{13} \mathrm{C}-\mathrm{NMR}\left(100.6 \mathrm{MHz}, \mathrm{CDCl}_{3}\right): \delta 25.9\left(\mathrm{CH}_{2}\right.$ allyl), 33.8 (C-7), 34.3 (C-6), $36.5\left(\mathrm{CH}_{2}\right.$ allyl), 39.0 (C-8), 58.1 (C-3), 72.6 (C-2), 91.0 (C-8a), $115.4\left(\mathrm{CH}_{2}=\right), 117.3\left(\mathrm{CH}_{2}=\right), 126.1,128.7\left(\mathrm{C}-0, m \mathrm{C}_{6} \mathrm{H}_{5}\right), 127.5$ (C-p $\left.\mathrm{C}_{6} \mathrm{H}_{5}\right), 135.3(\mathrm{CH}=), 137.9(\mathrm{CH}=), 139.3\left(\mathrm{C}-i \mathrm{C}_{6} \mathrm{H}_{5}\right), 168.1$ (NCO).

$[\alpha]_{D}^{22}=-94.6\left(c 2.1, \mathrm{CHCl}_{3}\right)$.

m.p. $91-93^{\circ} \mathrm{C}$

HRMS calcld for $\left[\mathrm{C}_{19} \mathrm{H}_{23} \mathrm{NO}_{2}+\mathrm{Na}\right]^{+}: 320.1621$, found: 320.1626 . 

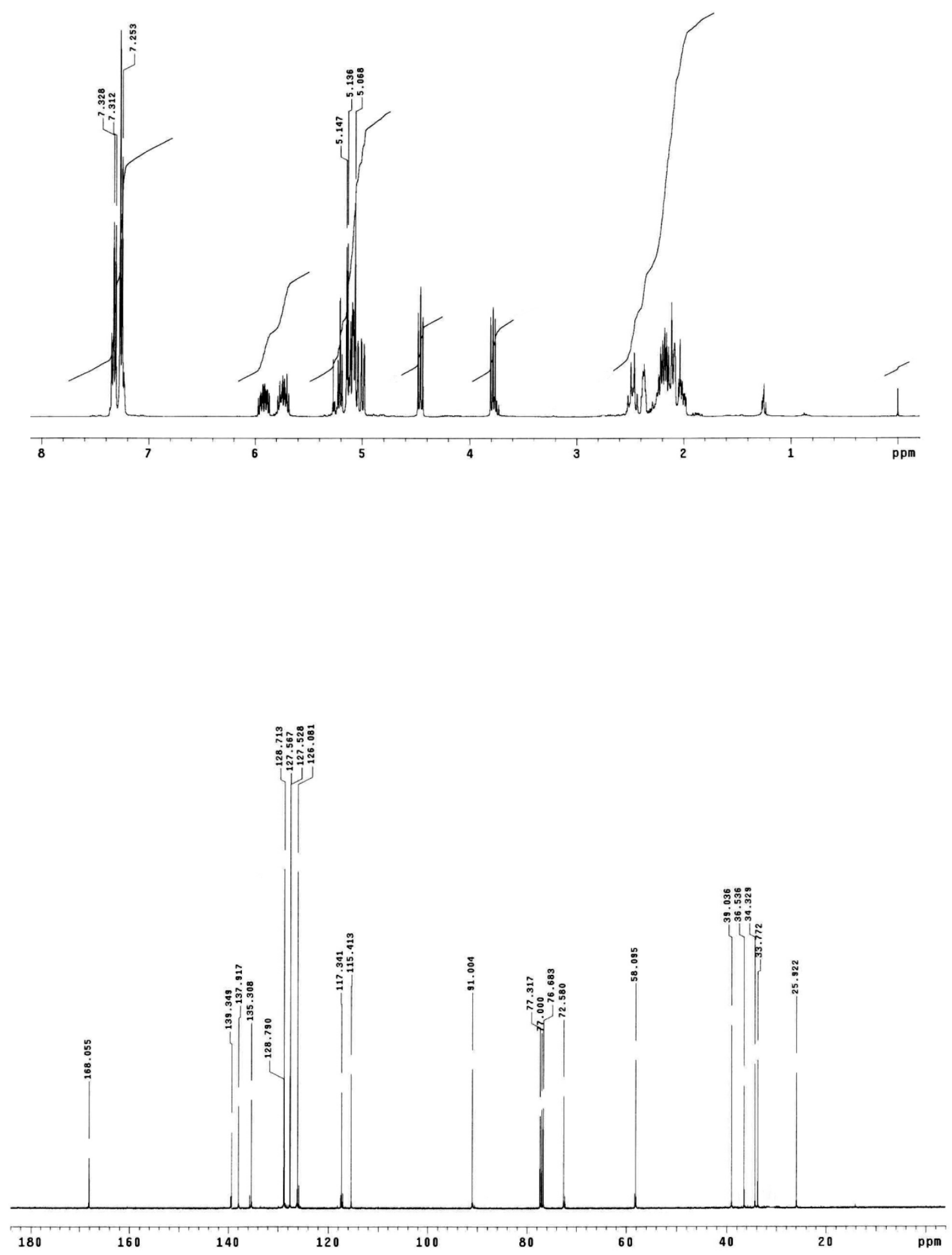


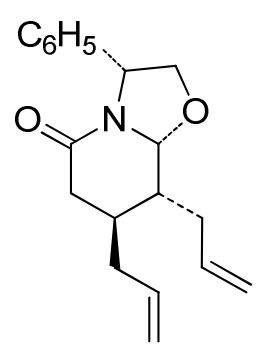

Compound 9c:

IR (film): 1661 (NCO) $\mathrm{cm}^{-1}$.

${ }^{1} \mathrm{H}-\mathrm{NMR}\left(400 \mathrm{MHz}, \mathrm{CDCl}_{3}\right.$, COSY, gHSQC): $\delta 1.58(\mathrm{~m}, 1 \mathrm{H}, \mathrm{H}-8), 1.94\left(\mathrm{~m}, 1 \mathrm{H}, \mathrm{CH}_{2}\right.$ allyl), 1.98 (m, $1 \mathrm{H}, \mathrm{H}-7$ ), 2.10 (dd, $J=18.4,10.8 \mathrm{~Hz}, 1 \mathrm{H}, \mathrm{H}-6), 2.47$ (m, 3H, $\mathrm{CH}_{2}$ allyl), 2.58 (dd, $J=18.4,5.6 \mathrm{~Hz}$, $1 \mathrm{H}, \mathrm{H}-6), 3.74(\mathrm{t}, J=8.4 \mathrm{~Hz}, 1 \mathrm{H}, \mathrm{H}-2), 4.46(\mathrm{t}, J=8.4 \mathrm{~Hz}, 1 \mathrm{H}, \mathrm{H}-2), 4.81(\mathrm{~d}, J=8.4 \mathrm{~Hz}, 1 \mathrm{H}, \mathrm{H}-8 \mathrm{a})$, 5.05-5.20 (m, 4H, $\left.\mathrm{CH}_{2}=\right), 5.25$ (t, J = 8.4 Hz, 1H, H-3), 5.72 (dddd, J = 16.9, 9.6, 7.2, 7.2 Hz, $1 \mathrm{H}$, $\mathrm{CH}=), 5.88$ (dddd, $J=16.8,10.0,7.6,6.8 \mathrm{~Hz}, 1 \mathrm{H}, \mathrm{CH}=), 7.23-7.32\left(\mathrm{~m}, 5 \mathrm{H}, \mathrm{C}_{6} \mathrm{H}_{5}\right)$.

${ }^{13} \mathrm{C}-\mathrm{NMR}\left(100.6 \mathrm{MHz}, \mathrm{CDCl}_{3}\right): \delta 31.7$ (C-7), $31.8\left(\mathrm{CH}_{2}\right.$ allyl), $35.7\left(\mathrm{CH}_{2}\right.$ allyl), 36.9 (C-6), 42.5 (C8), 58.2 (C-3), 72.3 (C-2), 90.5 (C-8a), $118.1\left(\mathrm{CH}_{2}=\right), 118.2\left(\mathrm{CH}_{2}=\right), 125.9,128.6\left(\mathrm{C}-0, m \mathrm{C}_{6} \mathrm{H}_{5}\right)$, $127.5\left(\mathrm{C}-p \mathrm{C}_{6} \mathrm{H}_{5}\right), 133.5(\mathrm{CH}=), 134.0(\mathrm{CH}=), 139.3\left(\mathrm{C}-i \mathrm{C}_{6} \mathrm{H}_{5}\right), 168.3$ (NCO).

HRMS calcld for $\left[\mathrm{C}_{19} \mathrm{H}_{23} \mathrm{NO}_{2}+\mathrm{H}\right]^{+}:$:298.1801, found: 298.1801 . 

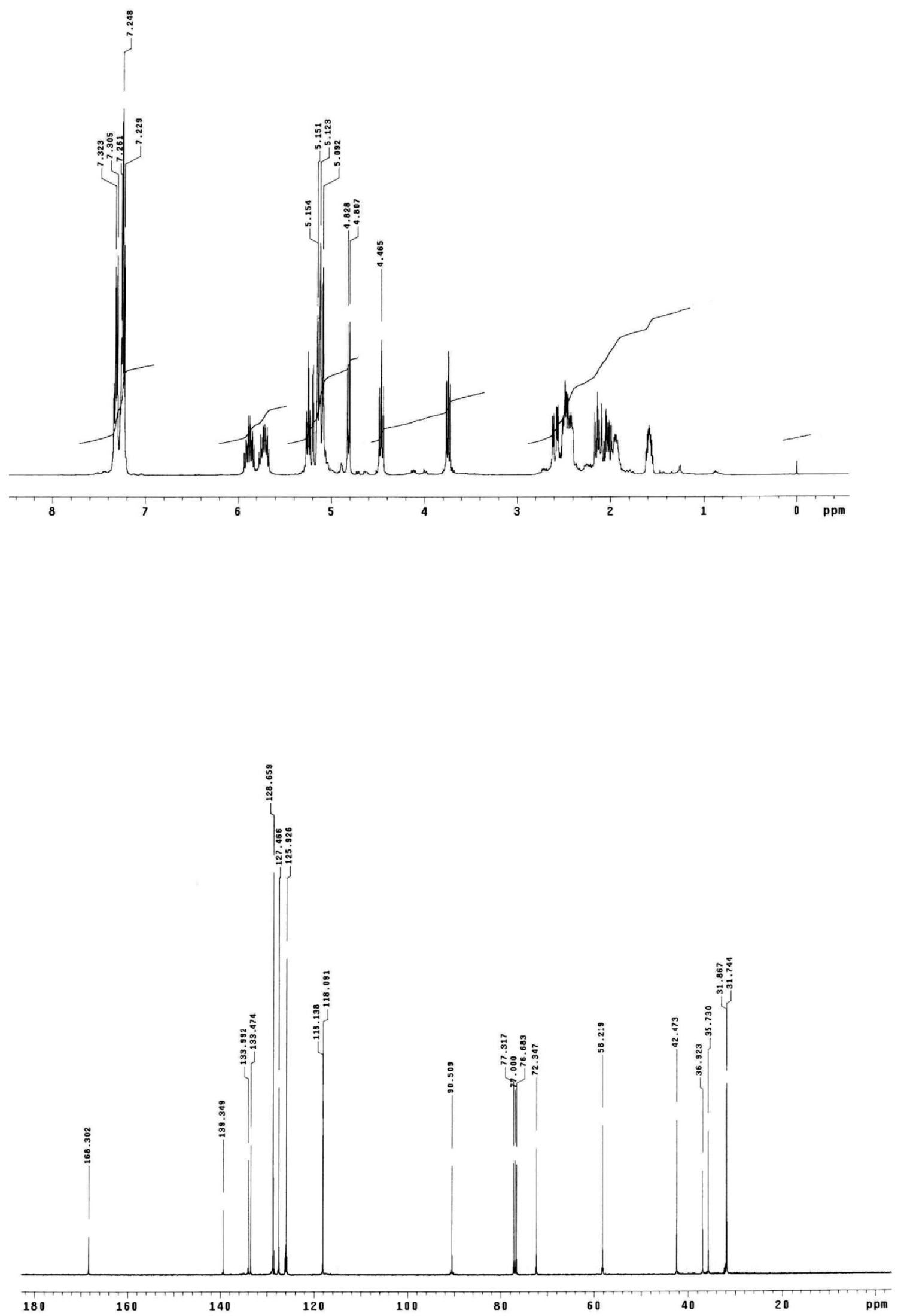


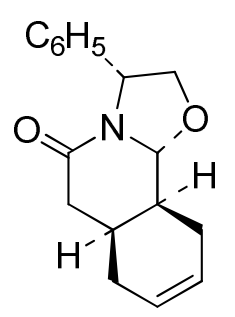

(3R,6aS,10aS,10bR)-5-0xo-3-phenyl-2,3,6,6a,7,10,10a,10b-octahydro-5H-oxazolo[3,2a]isoquinoline (10).

Second generation Grubbs catalyst ( $340 \mathrm{mg}, 0.4 \mathrm{mmol}$ ) was added to a solution of lactam 9 (1.59 g, $5.3 \mathrm{mmol})$ in $\mathrm{CH}_{2} \mathrm{Cl}_{2}(756 \mathrm{~mL})$. The mixture was stirred at room temperature for $4 \mathrm{~h}$ and concentrated under reduced pressure. Flash chromatography (9:1 to 7:3 hexane-EtOAc) afforded tricyclic lactam 10 (1.18 g, 83\%) as a brown solid.

$[\alpha]_{D}^{22}=+68.6\left(c 1.0, \mathrm{CHCl}_{3}\right)$.

IR (film): 1658 (COO) $\mathrm{cm}^{-1}$.

${ }^{1} \mathrm{H}$ NMR (400 MHz, CDCl 3 , COSY, gHSQC): $\delta 1.93-2.03(\mathrm{~m}, 1 \mathrm{H}, \mathrm{H}-7), 2.13$ (dd, $J=16.4,5.6 \mathrm{~Hz}, 1 \mathrm{H}$, $\mathrm{H}-7$ ), 2.23 (d, J = $18.0 \mathrm{~Hz}, 1 \mathrm{H}, \mathrm{H}-6), 2.30$ (m, 2H, H-10a, H-6a), 2.45 (s, 2H, H-10), 2.54 (dd, J = 18.0, 6.4 Hz, 1H, H-6), 3.98 (d, J = 9.2 Hz, 1H, H-2), 4.11 (dd, J = 9.2, $6.8 \mathrm{~Hz}, 1 \mathrm{H}, \mathrm{H}-2$ ), 4.86 (d, J = $9.6 \mathrm{~Hz}, 1 \mathrm{H}, \mathrm{H}-10 \mathrm{~b}), 4.91(\mathrm{~d}, J=6.8 \mathrm{~Hz}, 1 \mathrm{H}, \mathrm{H}-3), 5.70(\mathrm{~m}, 2 \mathrm{H}, \mathrm{H}-8, \mathrm{H}-9), 7.20-7.30\left(\mathrm{~m}, 5 \mathrm{H}, \mathrm{C}_{6} \mathrm{H}_{5}\right)$.

${ }^{13} \mathrm{C} \mathrm{NMR}\left(100.6 \mathrm{MHz}, \mathrm{CDCl}_{3}\right.$ ): $\delta 25.6$ (C-10), 28.5 (C-7), 29.1 (C-6a), 36.0 (C-10a), 38.3 (C-6), 59.4 (C-3), 73.6 (C-2), 87.3 (C-10b), 124.6, 125.1 (C-8, C-9), 127.3, 128.5 (C-o, $m \mathrm{C}_{6} \mathrm{H}_{5}$ ), 127.4 (C-p $\left.\mathrm{C}_{6} \mathrm{H}_{5}\right), 141.6\left(\mathrm{C}-i \mathrm{C}_{6} \mathrm{H}_{5}\right), 166.7$ (NCO).

m.p. $138-140{ }^{\circ} \mathrm{C}$.

Elemental analysis calcd (\%) for $\mathrm{C}_{17} \mathrm{H}_{19} \mathrm{NO}_{2} \cdot{ }^{1} /{ }_{4} \mathrm{H}_{2} \mathrm{O}$ : C 74.56, $\mathrm{H}$ 7.18, N 5.11; found: $\mathrm{C} 74.67, \mathrm{H}$ 7.09, N 4.99 . 
Experimental data and spectra
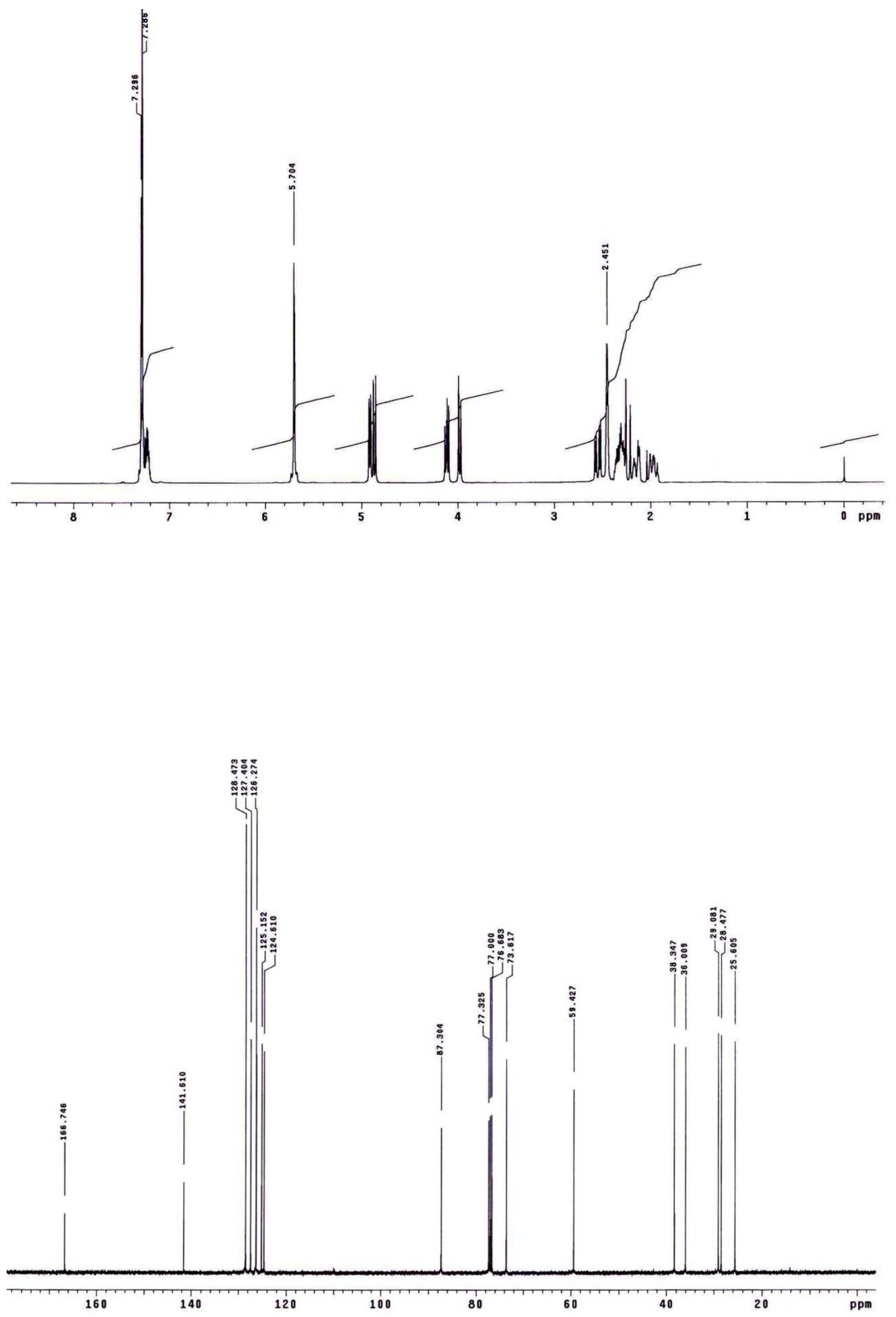

169 
<smiles>CC(CO)N1C[C@H]2CC=CC[C@H]2CC1=O</smiles>

(4aS,8aS)-2-[(1R)-2-Hydroxy-1-phenylethyl]-3-oxo-1,2,3,4,4a,5,8,8a-octahydroisoquinoline (11).

$\mathrm{Et}_{3} \mathrm{SiH}(155 \mu \mathrm{L}, 0.97 \mathrm{mmol})$ and $\mathrm{TiCl}_{4}(171 \mu \mathrm{L}, 1.56 \mathrm{mmol})$ were added under inert atmosphere to a solution of lactam 10 (105 mg, $0.39 \mathrm{mmol})$ in anhydrous $\mathrm{CH}_{2} \mathrm{Cl}_{2}(10 \mathrm{~mL})$, and the resulting dark solution was heated at reflux for $24 \mathrm{~h}$. The mixture was poured into saturated aqueous $\mathrm{NaHCO}_{3}(10 \mathrm{~mL})$, and the resulting solution was extracted with $\mathrm{CH}_{2} \mathrm{Cl}_{2}$. The combined organic extracts were dried over anhydrous $\mathrm{Na}_{2} \mathrm{SO}_{4}$, filtered, and concentrated under reduced pressure. Flash chromatography (9:1 hexane-EtOAc to only EtOAc) gave isoquinoline 11 (98 $\mathrm{mg}, 92 \%)$ as a white solid.

$[\alpha]_{D}^{22}=-20.5\left(c 0.4, \mathrm{CHCl}_{3}\right)$.

IR (film): $3375(\mathrm{OH}), 1614(\mathrm{COO}) \mathrm{cm}^{-1}$.

${ }^{1} \mathrm{H}$ NMR $\left(400 \mathrm{MHz}, \mathrm{CDCl}_{3}\right.$, COSY, gHSQC): $\delta 1.93(\mathrm{~m}, 2 \mathrm{H}, \mathrm{H}-5, \mathrm{H}-8), 2.15(\mathrm{~m}, 4 \mathrm{H}, \mathrm{H}-5, \mathrm{H}-8, \mathrm{H}-4 \mathrm{a}$, H-8a), 2.40 (dd, $J=18.0,6.0 \mathrm{~Hz}, 1 \mathrm{H}, \mathrm{H}-4$ ), 2.54 (dd, $J=18.0,5.6 \mathrm{~Hz}, 1 \mathrm{H}, \mathrm{H}-4$ ), 2.88 (dd, $J=12.0$, $4.0 \mathrm{~Hz}, 1 \mathrm{H}, \mathrm{H}-1$ ), 3.16 (dd, J = 12.0, $7.2 \mathrm{~Hz}, 1 \mathrm{H}, \mathrm{H}-1$ ), 3.64 (br. s, 1H, OH), $4.08\left(\mathrm{~m}, 1 \mathrm{H}, \mathrm{H}-2^{\prime}\right), 4.14$ (dd, $\left.J=11.2,5.2 \mathrm{~Hz}, 1 \mathrm{H}, \mathrm{H}-2^{\prime}\right), 5.60(\mathrm{~m}, 2 \mathrm{H}, \mathrm{H}-6, \mathrm{H}-7), 5.89$ (dd, J = 9.2, $\left.5.2 \mathrm{~Hz}, 1 \mathrm{H}, \mathrm{H}-1^{\prime}\right), 7.10-$ $7.20\left(\mathrm{~m}, 5 \mathrm{H}, \mathrm{C}_{6} \mathrm{H}_{5}\right)$.

${ }^{13} \mathrm{C} \mathrm{NMR}\left(100.6 \mathrm{MHz}, \mathrm{CDCl}_{3}\right.$ ): $\delta 26.2,27.6$ (C-5, C-8), 28.7 (C-4a), 29.7 (C-8a), 36.0 (C-4), 44.7 (C1), 57.1 (C-1'), 60.4 (C-2'), 124.3, 124.5 (C-6, C-7), 127.3 (C-p $\left.\mathrm{C}_{6} \mathrm{H}_{5}\right), 127.5,128.3\left(\mathrm{C}-0, m \mathrm{C}_{6} \mathrm{H}_{5}\right.$ ), $136.9\left(\mathrm{C}-i \mathrm{C}_{6} \mathrm{H}_{5}\right), 170.6$ (NCO).

m.p. $84-86^{\circ} \mathrm{C}$

Elemental analysis calcd (\%) for $\mathrm{C}_{17} \mathrm{H}_{21} \mathrm{NO}_{2}$ : C 75.25, H 7.80, N 5.16; found: C 74.96, H 7.78, N 5.07 . 

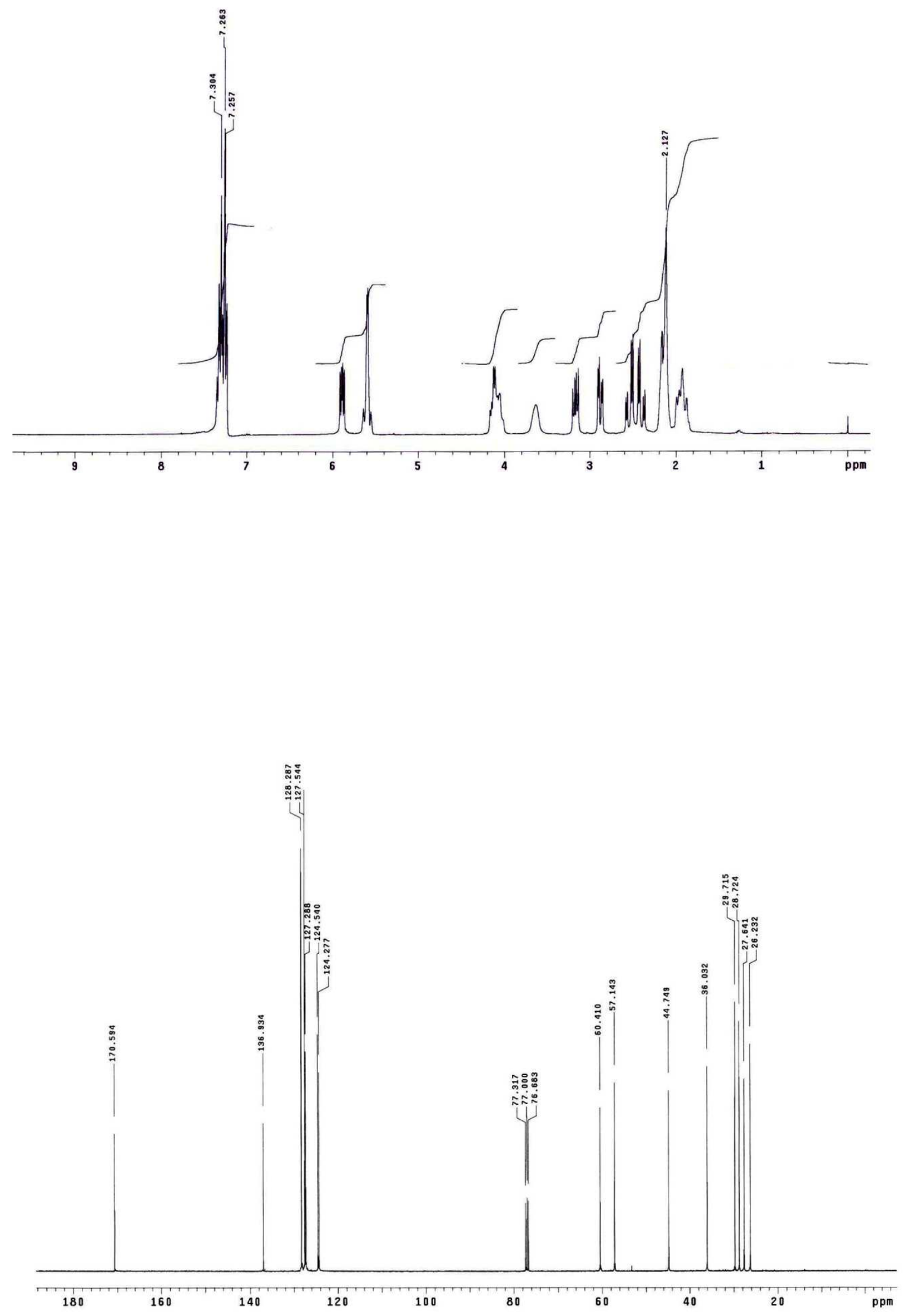


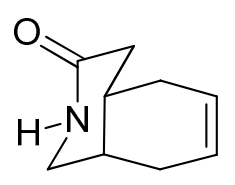

(4aS,8aS)-3-0xo-1,2,3,4,4a,5,8,8a-octahydroisoquinoline (12).

Into a three-necked, $100 \mathrm{~mL}$ round-bottomed flask equipped with a coldfinger condenser charged with dry ice-acetone were condensed $15 \mathrm{~mL}$ of $\mathrm{NH}_{3}$ at $-78{ }^{\circ} \mathrm{C}$, and then a solution of lactam $11(190 \mathrm{mg}, 0.70 \mathrm{mmol})$ in THF $(10 \mathrm{~mL})$ was added. The temperature was raised to -33 ${ }^{\circ} \mathrm{C}$ and sodium metal was added in small portions until the blue colour persisted. The mixture was stirred at $-33^{\circ} \mathrm{C}$ for $1 \mathrm{~min}$. The reaction was quenched by the addition of solid $\mathrm{NH}_{4} \mathrm{Cl}$ until the blue colour disappeared, and then the mixture was stirred at room temperature for $4 \mathrm{~h}$. The resulting residue was digested at room temperature with $\mathrm{CH}_{2} \mathrm{Cl}_{2}$, and the suspension was filtered through Celite ${ }^{\circledast}$. The solution was concentrated under reduced pressure. Flash chromatography of the resulting oil (2:1 hexane- EtOAc to 60:1 EtOAc-MeOH) afforded bicyclic lactam 12 (98 mg, 93\%) as a white solid.

$[\alpha]_{D}^{22}=-30.2(c 1.0, \mathrm{MeOH})$.

IR (film): $3300(\mathrm{NH}), 1665$ (COO) $\mathrm{cm}^{-1}$.

${ }^{1} \mathrm{H}$ NMR (400 MHz, CDCl 3 , COSY, gHSQC): $\delta 1.88-2.04(\mathrm{~m}, 2 \mathrm{H}, \mathrm{H}-8, \mathrm{H}-5), 2.15-2.30(\mathrm{~m}, 4 \mathrm{H}, \mathrm{H}-8$, H-5, H-4a, H-8a), 2.30 (dd, J = 16.8, $6.4 \mathrm{~Hz}, 1 \mathrm{H}, \mathrm{H}-4$ ), 2.38 (dd, $J=16.8,4.4 \mathrm{~Hz}, 1 \mathrm{H}, \mathrm{H}-4$ ), 3.23 (ddd, $J=12.0,6.0,2.0 \mathrm{~Hz}, 1 \mathrm{H}, \mathrm{H}-1$ ), 3.31 (ddd, $J=12.0,4.8,1.6 \mathrm{~Hz}, 1 \mathrm{H}, \mathrm{H}-1$ ), $5.63(\mathrm{~s}, 2 \mathrm{H}, \mathrm{H}-6, \mathrm{H}-$ 7), 6.73 (br. s, 1H, NH).

${ }^{13} \mathrm{C} \mathrm{NMR}\left(100.6 \mathrm{MHz}, \mathrm{CDCl}_{3}\right.$ ): $\delta 26.0$ (C-8), 28.3 (C-5), 29.1, 29.2 (C-4a, C-8a), 34.8 (C-4), 44.6 (C1), $124.5,124.6$ (C-6, C-7), 172.2 (NCO).

m.p. $65-67^{\circ} \mathrm{C}$

Elemental analysis calcd (\%) for $\mathrm{C}_{9} \mathrm{H}_{13} \mathrm{NO}$ : C 71.49, H 8.67, N 9.26; found: C 71.42, H 8.58, N 9.16. 

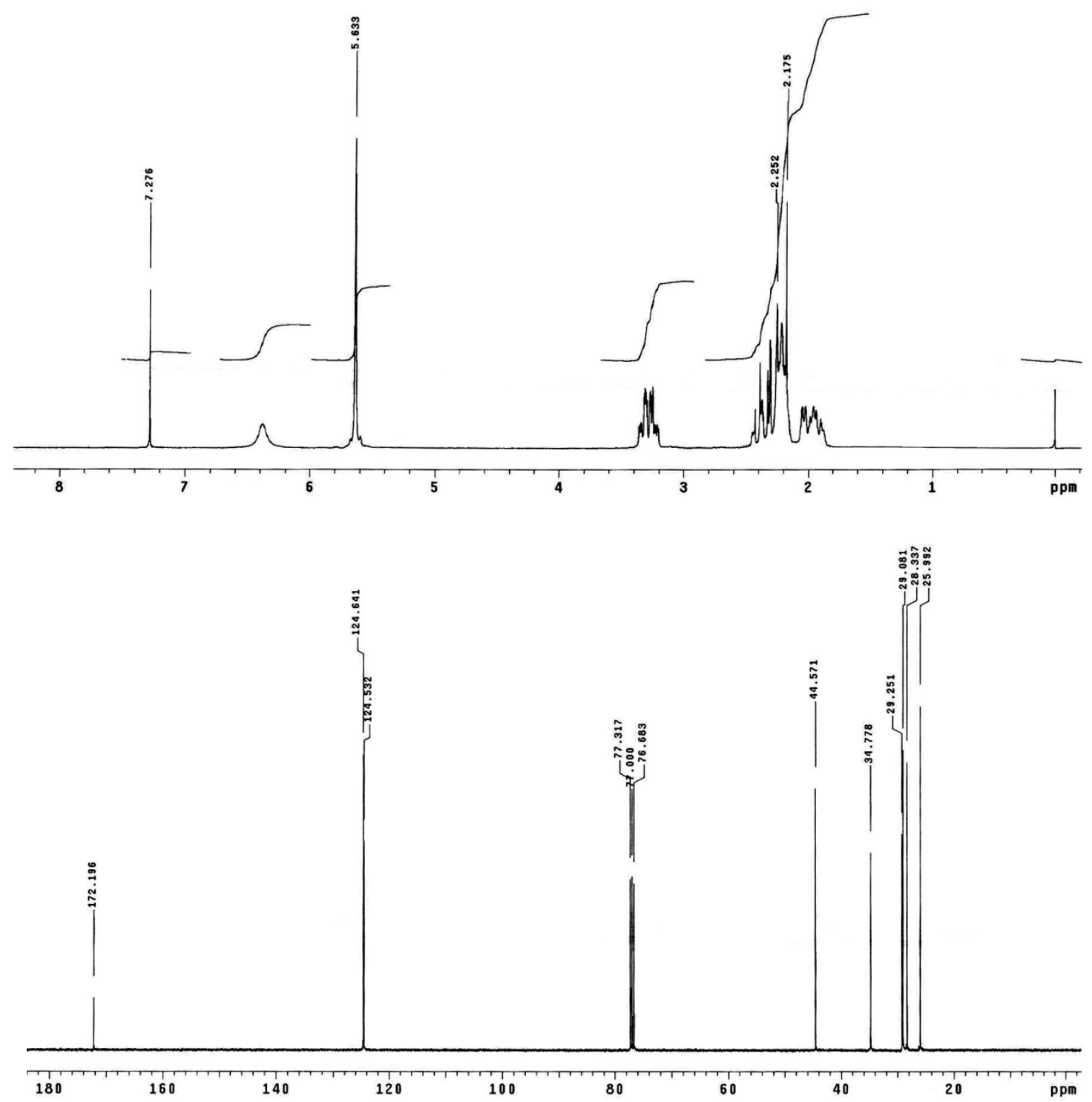


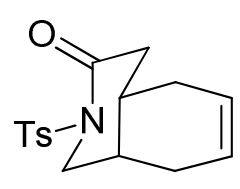

(4aS,8aS)-3-0xo-2-(p-toluenesulfonyl)-1,2,3,4,4a,5,8,8a-octahydroisoquinoline (13).

$n$-BuLi (1.24 mL of a solution 1.6 $\mathrm{M}$ in hexane, $1.98 \mathrm{mmol}$ ) was added dropwise at $-78{ }^{\circ} \mathrm{C}$ to a stirred solution of bicyclic lactam $12(250 \mathrm{mg}, 1.65 \mathrm{mmol})$ in anhydrous THF (10 mL) under inert atmosphere. After stirring for $45 \mathrm{~min}$ at $-78^{\circ} \mathrm{C}$, a solution of $p$-toluenesulfonyl chloride (347 $\mathrm{mg}, 1.82 \mathrm{mmol}$ ) in anhydrous THF ( $2 \mathrm{~mL}$ ) was added via cannula, and the stirring was continued for $4 \mathrm{~h}$ at $-78^{\circ} \mathrm{C}$. The reaction was quenched by the addition of saturated aqueous $\mathrm{NH}_{4} \mathrm{Cl}$ solution, and the resulting solution was extracted with EtOAc. The combined organic extracts were dried over anhydrous $\mathrm{Na}_{2} \mathrm{SO}_{4}$, filtered, and concentrated under reduced pressure. Flash chromatography (8:2 hexane-EtOAc) afforded lactam 13 (432 mg, 86\% yield) as a white solid.

$[\alpha]_{D}^{22}=-17.5\left(c 0.14, \mathrm{CHCl}_{3}\right)$.

IR (film): 1690 (NCO), $1165\left(\mathrm{SO}_{2}-\mathrm{O}\right) \mathrm{cm}^{-1}$.

${ }^{1} \mathrm{H}$ NMR (400 MHz, CDCl 3 , COSY, gHSQC): $\delta 1.74(\mathrm{dm}, J=17.2 \mathrm{~Hz}, 1 \mathrm{H}, \mathrm{H}-5), 1.96(\mathrm{dm}, J=17.2$ $\mathrm{Hz}, 1 \mathrm{H}, \mathrm{H}-8$ ), 2.19 (m, 2H, H-5, H-4a), 2.31 (m, 2H, H-8, H-8a), 2.33 (dd, J = 18.0, 7.2 Hz, 1H, H4), $2.42\left(\mathrm{~s}, 3 \mathrm{H}, \mathrm{CH}_{3} \mathrm{Ts}\right), 2.43(\mathrm{dd}, J=18.0,5.1 \mathrm{~Hz}, 1 \mathrm{H}, \mathrm{H}-4), 3.85(\mathrm{dd}, J=12.0,5.1 \mathrm{~Hz}, 1 \mathrm{H}, \mathrm{H}-1$ ), 3.89 (dd, $J=12.0,6.0 \mathrm{~Hz}, 1 \mathrm{H}, \mathrm{H}-1$ ), 5.61 (s, 2H, H-6, H-7), 7.30 (d, J = $8.8 \mathrm{~Hz}, 2 \mathrm{H}, \mathrm{H}-m \mathrm{Ts}$ ), 7.89 (d, $J=8.8 \mathrm{~Hz}, 2 \mathrm{H}, \mathrm{H}-\mathrm{o} \mathrm{Ts})$.

${ }^{13} \mathrm{C}$ NMR (100.6 MHz, CDCl $)$ ): $\delta 21.6\left(\mathrm{CH}_{3} \mathrm{Ts}\right), 25.5$ (C-8), 27.9 (C-5), 28.9 (C-4a), 30.2 (C-8a), 37.1 (C-4), 49.3 (C-1), 124.1, 124.4 (C-6, C-7), 128.6 (C-o), 129.2 (C-m Ts), 136.0 (C-i Ts), 144.6 (C-p Ts), 169.8 (NCO).

m.p. $70-72^{\circ} \mathrm{C}$.

HRMS (ESI) calcd for $\left[\mathrm{C}_{16} \mathrm{H}_{19} \mathrm{NO}_{3} \mathrm{~S}+\mathrm{H}\right]^{+}: 306.1158$, found: 306.1155 .

Elemental analysis calcd (\%) for $\mathrm{C}_{16} \mathrm{H}_{19} \mathrm{NO}_{3} \mathrm{~S}$ : C 62.93, H 6.27, N 4.59; found: C 62.93, H 6.36, N 4.48 . 

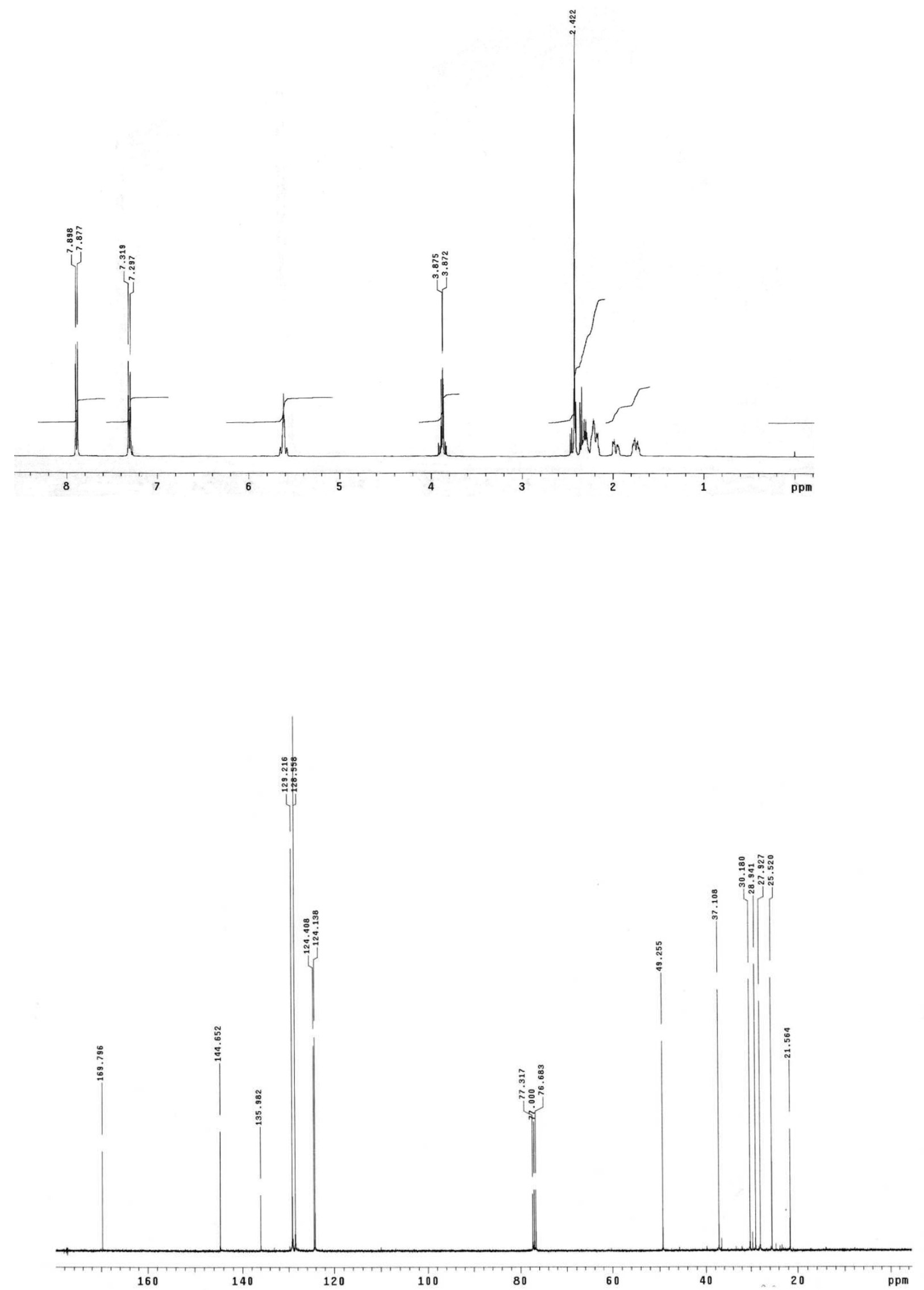
<smiles>COC(=O)C1CC=CCC2CCCN2C1=O</smiles>

(4aR,8aS)-4-Methoxycarbonyl-3-oxo-2-( $p$-toluenesulfonyl)-1,2,3,4,4a,5,8,8aoctahydroisoquinoline (14).

Lithium bis(trimethylsilyl)amide $(528 \mu \mathrm{l}$ of a $1 \mathrm{M}$ solution in hexane, $0.53 \mathrm{mmol}$ ) was added at $-78{ }^{\circ} \mathrm{C}$ to a stirred solution of lactam $13(124 \mathrm{mg}, 0.41 \mathrm{mmol})$ in anhydrous THF $(5 \mathrm{~mL})$ under inert atmosphere. After stirring for $1 \mathrm{~h}$ at $-78^{\circ} \mathrm{C}$, methyl chloroformate $(31 \mu \mathrm{l}, 0.41 \mathrm{mmol})$ was added and stirring was continued for $3 \mathrm{~h}$ at $-78^{\circ} \mathrm{C}$. The reaction was quenched by the addition of saturated aqueous $\mathrm{NH}_{4} \mathrm{Cl}$, and the resulting solution was extracted with EtOAc. The combined organic extracts were dried over anhydrous $\mathrm{Na}_{2} \mathrm{SO}_{4}$, filtered, and concentrated under reduced pressure. Flash chromatography (9:1 to 7:3 hexane-EtOAc) afforded the $N$-tosyl derivative 14 (136 mg, 91\%).

IR (film): 1686 (NCO), 1740 (COO) $\mathrm{cm}^{-1}$.

${ }^{1} \mathrm{H}$ NMR (400 MHz, CDCl, COSY, gHSQC): $\delta 1.77(\mathrm{dm}, J=18.0 \mathrm{~Hz}, 1 \mathrm{H}, \mathrm{H}-8), 1.93(\mathrm{~m}, 1 \mathrm{H}, \mathrm{H}-5)$, 2.25 (m, 2H, H-5, H-8), 2.43 (s, 3H, CH $\left.\mathrm{CH}_{3} \mathrm{Ts}\right), 2.45$ (m, 1H, H-8a), 2.55 (m, 1H, H-4a), 3.33 (d, J = $8.0 \mathrm{~Hz}, 1 \mathrm{H}, \mathrm{H}-4$ ), 3.68 (s, 3H, $\mathrm{CH}_{3} \mathrm{O}$ ), 3.89 (dd, $J=12.0,6.0 \mathrm{~Hz}, 1 \mathrm{H}, \mathrm{H}-1$ ), 3.95 (dd, $J=12.0,4.4$ $\mathrm{Hz}, 1 \mathrm{H}, \mathrm{H}-1), 5.63(\mathrm{~m}, 2 \mathrm{H}, \mathrm{H}-6, \mathrm{H}-7), 7.31$ (d, J = 8.4 Hz, 2H, H-m Ts), 7.88 (d, J = 8.4 Hz, 2H, H-O Ts).

${ }^{13} \mathrm{C}$ NMR (100.6 MHz, CDCl $): \delta 21.6\left(\mathrm{CH}_{3} \mathrm{Ts}\right), 25.2$ (C-5), 27.3 (C-8), 28.8 (C-8a), 33.2 (C-4a), 49.3 (C-1), 52.7 ( $\mathrm{CH}_{3} \mathrm{O}$ ), 53.2 (C-4), 123.8, 124.6 (C-6, C-7), 128.6 (C-o Ts), 129.3 (C-m Ts), 135.4 (C-i Ts), 144.9 (C-p Ts), 166.0 (NCO), 169.5 (COO).

HRMS (ESI) calcd for $\left[\mathrm{C}_{18} \mathrm{H}_{21} \mathrm{NO}_{5} \mathrm{~S}+\mathrm{H}\right]^{+}: 364.1213$, found: 364.1214 .

Elemental analysis calcd (\%) for $\mathrm{C}_{18} \mathrm{H}_{21} \mathrm{NO}_{5} \mathrm{~S}$ : C 59.49, H 5.82, N 3.85; found: C 59.45, H 5.84, N 3.83. 

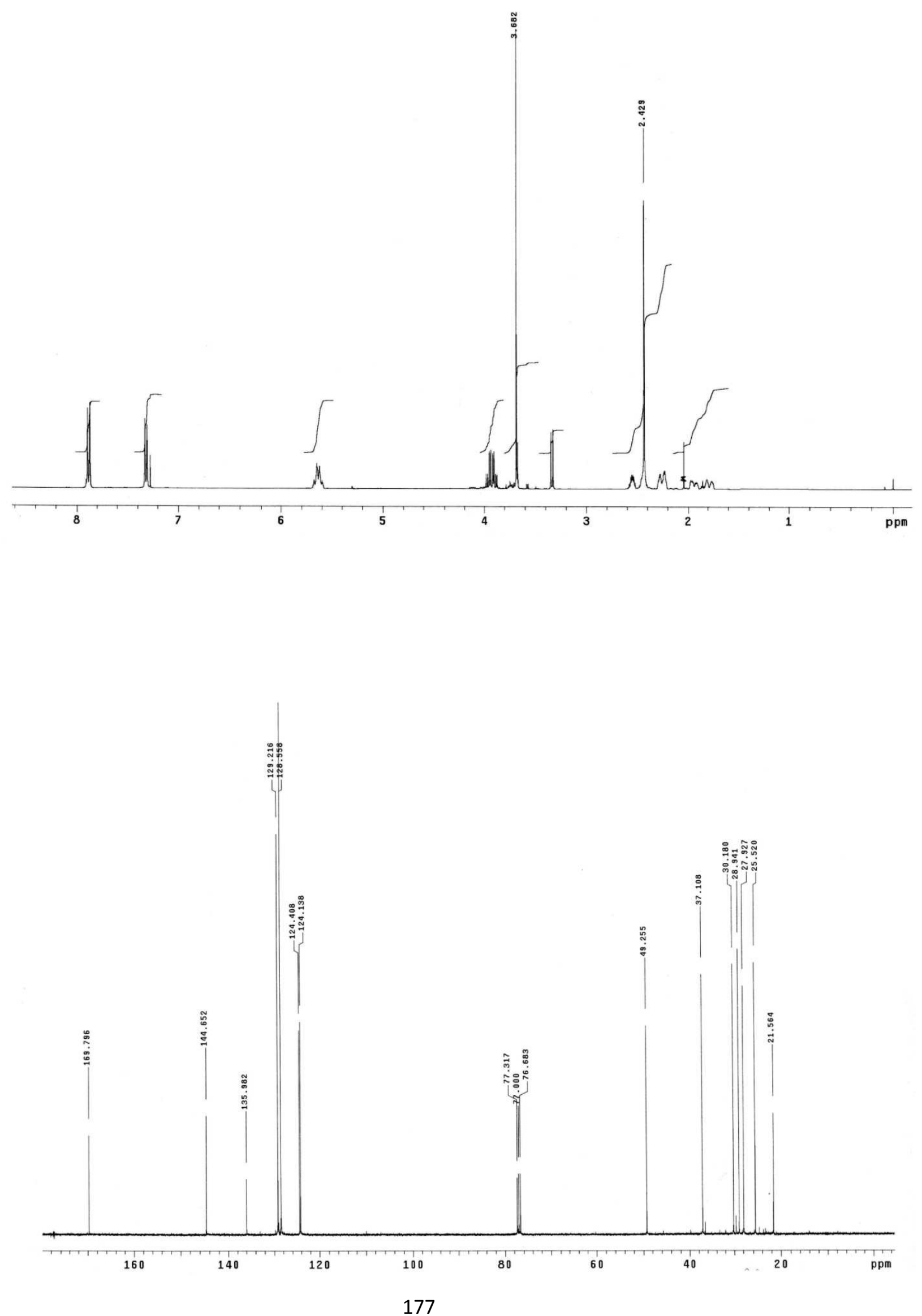


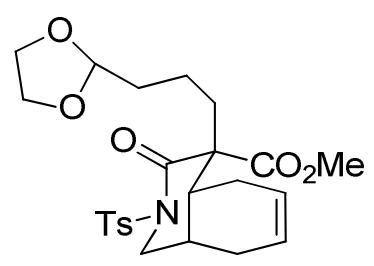

(4R,4aR,8aS)-4-[3-(1,3-Dioxolan-2-yl)propyl]-4-(methoxycarbonyl)-3-oxo-2-(ptoluensulfonyl)-1,2,3,4,4a,5,8,8a-octahydroisoquinoline (15).

A mixture of lactam 14 (85 mg, $0.24 \mathrm{mmol}$ ) and $\mathrm{NaH}$ (12 mg of a $60 \%$ dispersion in mineral oil, $0.28 \mathrm{mmol})$ in anhydrous DMF $(1 \mathrm{~mL})$ was stirred at $0{ }^{\circ} \mathrm{C}$ under argon atmosphere for $1 \mathrm{~h}$. Then, 2-(2-iodoprophyl)-1,3-dioxolane $(226 \mathrm{mg}, 0.95 \mathrm{mmol}$ ) was added, and the solution was stirred at $60{ }^{\circ} \mathrm{C}$ for $20 \mathrm{~h}$. After cooling to room temperature, saturated aqueous $\mathrm{NH}_{4} \mathrm{Cl}$ was added and was extracted with ethyl acetate. The combined organic extracts were dried, filtered and concentrated under reduced pressure. Flash chromatography (95:5 to 7:3 hexaneEtOAc) afforded compound 15 (113 mg, 70\%).

$[\alpha]_{D}^{22}=-28.28\left(c 0.99, \mathrm{CHCl}_{3}\right)$.

${ }^{1} \mathrm{H}$ NMR $\left(400 \mathrm{MHz}, \mathrm{CDCl}_{3}, \mathrm{COSY}, \mathrm{gHSQC}\right): \delta 1.25\left(\mathrm{~m}, 1 \mathrm{H}, \mathrm{H}-2^{\prime}\right), 1.42\left(\mathrm{~m}, 1 \mathrm{H}, \mathrm{H}-2^{\prime}\right), 1.51(\mathrm{~m}, 2 \mathrm{H}$, H-3'), $1.83(\mathrm{dm}, J=17.4 \mathrm{~Hz}, 1 \mathrm{H}, \mathrm{H}-5), 1.93\left(\mathrm{~m}, 2 \mathrm{H}, \mathrm{H}-1^{\prime}\right), 2.00(\mathrm{dm}, J=17.4 \mathrm{~Hz}, 1 \mathrm{H}, \mathrm{H}-5), 2.03$ $(\mathrm{dm}, J=16.4 \mathrm{~Hz}, 1 \mathrm{H}, \mathrm{H}-8), 2.17$ (ddd, $J=9.6,5.6,3.2 \mathrm{~Hz}, 1 \mathrm{H}, \mathrm{H}-4 \mathrm{a}), 2.41(\mathrm{dm}, J=16.4 \mathrm{~Hz}, 1 \mathrm{H}, \mathrm{H}-$ 8), $2.43\left(\mathrm{~s}, 3 \mathrm{H}, \mathrm{CH}_{3} \mathrm{Ts}\right), 2.55(\mathrm{~m}, 1 \mathrm{H}, \mathrm{H}-8 \mathrm{a}), 3.54\left(\mathrm{~s}, 3 \mathrm{H}, \mathrm{CH}_{3} \mathrm{O}\right), 3.76(\mathrm{t}, J=12.0 \mathrm{~Hz}, 1 \mathrm{H}, \mathrm{H}-1 \mathrm{ax})$, $3.80\left(\mathrm{~m}, 2 \mathrm{H}, \mathrm{CH}_{2} \mathrm{O}\right), 3.90\left(\mathrm{~m}, 2 \mathrm{H}, \mathrm{CH}_{2} \mathrm{O}\right), 3.96(\mathrm{dd}, \mathrm{J}=12.0,4.8 \mathrm{~Hz}, 1 \mathrm{H}, \mathrm{H}-1 \mathrm{eq}), 4.71(\mathrm{t}, J=4.8 \mathrm{~Hz}$, $\left.1 \mathrm{H}, \mathrm{CHO}_{2}\right), 5.61(\mathrm{~m}, 2 \mathrm{H}, \mathrm{H}-6, \mathrm{H}-7), 7.30(\mathrm{~d}, \mathrm{~J}=8.0 \mathrm{~Hz}, 2 \mathrm{H}, \mathrm{H}-\mathrm{m}), 7.88(\mathrm{~d}, J=8.4 \mathrm{~Hz}, 2 \mathrm{H}, \mathrm{H}-\mathrm{o})$.

${ }^{13} \mathrm{C} \mathrm{NMR}\left(100.6 \mathrm{MHz}, \mathrm{CDCl}_{3}\right): \delta 19.6\left(\mathrm{C}-2{ }^{\prime}\right), 21.6\left(\mathrm{CH}_{3} \mathrm{Ts}\right), 24.5$ (C-5), 27.8 (C-8), 28.0 (C-8a), 33.9 (C-3'), 37.0 (C-1'), 38.2 (C-4a), 46.9 (C-1), $51.9\left(\mathrm{CH}_{3} \mathrm{O}\right), 59.7$ (C-4), $64.7\left(2 \mathrm{CH}_{2} \mathrm{O}\right), 104.1$ (C-4'), 124.2, 124.4 (C-6, C-7), 128.6, 129.1 (C-o, m), 135.7 (C-i), 144.6 (C-p), 168.4 (NCO), 171.0 (CCO). HRMS calcd for $\left[\mathrm{C}_{24} \mathrm{H}_{31} \mathrm{NO}_{7} \mathrm{~S}+\mathrm{H}\right]^{+}: 478.18$, found: 478.1888 . 

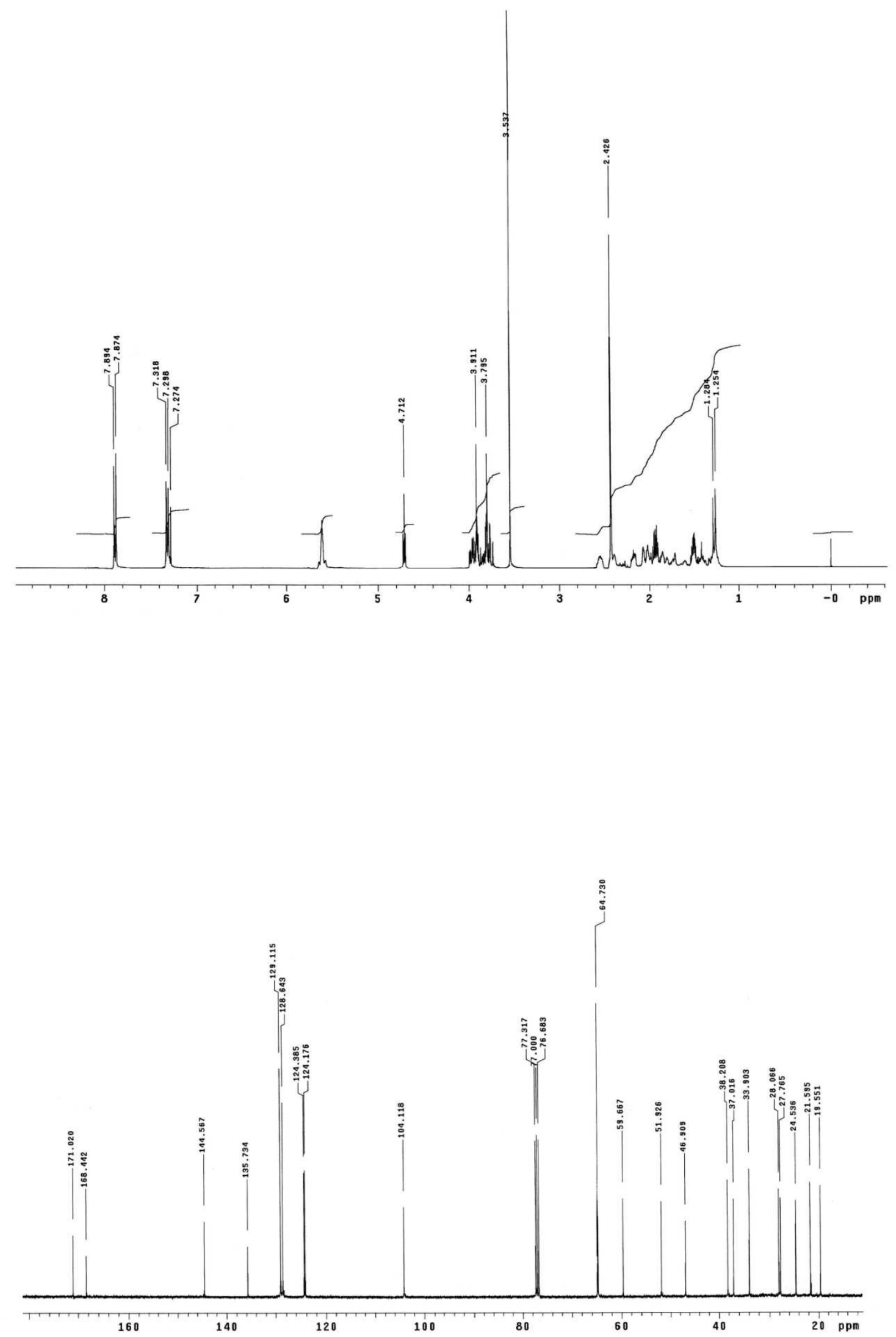


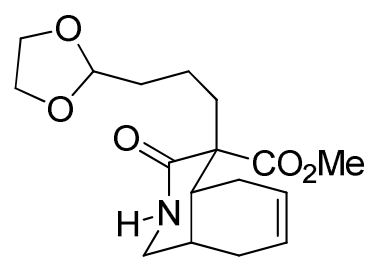

(4R,4aR,8aS)-4-[3-(1,3-Dioxolan-2-yl)propyl]-4-(methoxycarbonyl)-3-oxo-1,2,3,4,4a,5,8,8aoctahydroisoquinoline (16).

Sodium metal $(320 \mathrm{mg}, 13.3 \mathrm{mmol}$ ) was added at room temperature to a solution of naphthalene (890 mg, $13.3 \mathrm{mmol})$ in THF $(20 \mathrm{~mL}$ ). After stirring for $3 \mathrm{~h}$, part of the mixture (4.55 mL) was added to a solution of sulfonamide 15 (162 mg, $0.35 \mathrm{mmol})$ in THF ( $9 \mathrm{~mL})$ at -78 ${ }^{\circ} \mathrm{C}$. After $15 \mathrm{~min}$, saturated aqueous $\mathrm{NH}_{4} \mathrm{Cl}$ was carefully added, and the resulting solution was extracted with ethyl acetate. The combined extracts were washed with brine, dried $\left(\mathrm{Na}_{2} \mathrm{SO}_{4}\right)$, and concentrated under reduced pressure. Flash chromatography (9:1 to 1:1 hexane-EtOAc) afforded amide 16 (85 mg, 75\%).

${ }^{1} \mathrm{H}$ NMR $\left(400 \mathrm{MHz}^{\mathrm{C} C D C l}{ }_{3}, \mathrm{COSY}, \mathrm{gHSQC}\right): \delta 1.51\left(\mathrm{~m}, 1 \mathrm{H}, \mathrm{H}-2^{\prime}\right), 1.65\left(\mathrm{~m}, 2 \mathrm{H}, \mathrm{H}-3^{\prime}\right), 1.79(\mathrm{~m}, 2 \mathrm{H}$, H-2', H-5), $1.93(\mathrm{dm}, J=18.0 \mathrm{~Hz}, 1 \mathrm{H}, \mathrm{H}-8), 2.03\left(\mathrm{~m}, 2 \mathrm{H}, \mathrm{H}-1^{\prime}\right), 2.19(\mathrm{~m}, 2 \mathrm{H}, \mathrm{H}-4 \mathrm{a}, \mathrm{H}-5), 2.35$ (dm, $J=18.0 \mathrm{~Hz}, 1 \mathrm{H}, \mathrm{H}-8), 2.51(\mathrm{~m}, 1 \mathrm{H}, \mathrm{H}-8 \mathrm{a}), 3.12(\mathrm{dt}, J=12.0,4.8 \mathrm{~Hz}, 1 \mathrm{H}, \mathrm{H}-1), 3.36(\mathrm{t}, J=12.0 \mathrm{~Hz}$, $1 \mathrm{H}, \mathrm{H}-1), 3.75\left(\mathrm{~s}, 3 \mathrm{H}, \mathrm{CH}_{3} \mathrm{O}\right), 3.82\left(\mathrm{~m}, 2 \mathrm{H}, \mathrm{CH}_{2} \mathrm{O}\right), 3.94\left(\mathrm{~m}, 2 \mathrm{H}, \mathrm{CH}_{2} \mathrm{O}\right), 4.84(\mathrm{t}, J=4.8 \mathrm{~Hz}, 1 \mathrm{H}$, $\left.\mathrm{CHO}_{2}\right), 5.59(\mathrm{~m}, 2 \mathrm{H}, \mathrm{H}-6, \mathrm{H}-7), 6.84$ (br. s, $\left.1 \mathrm{H}, \mathrm{NH}\right)$.

${ }^{13} \mathrm{C}$ NMR (100.6 MHz, CDCl $)$ ): $\delta 19.9$ (C-2'), 24.5 (C-5), 26.9 (C-8a), 27.8 (C-8), 34.1 (C-3'), 37.1 (C-1'), 38.6 (C-4a), 42.6 (C-1), $51.7\left(\mathrm{CH}_{3} \mathrm{O}\right), 57.2(\mathrm{C}-4), 64.6\left(2 \mathrm{CH}_{2} \mathrm{O}\right), 104.2\left(\mathrm{C}-4^{\prime}\right), 124.1,124.7$ (C-6, C-7), 170.4 (NCO), 172.2 (CCO). 

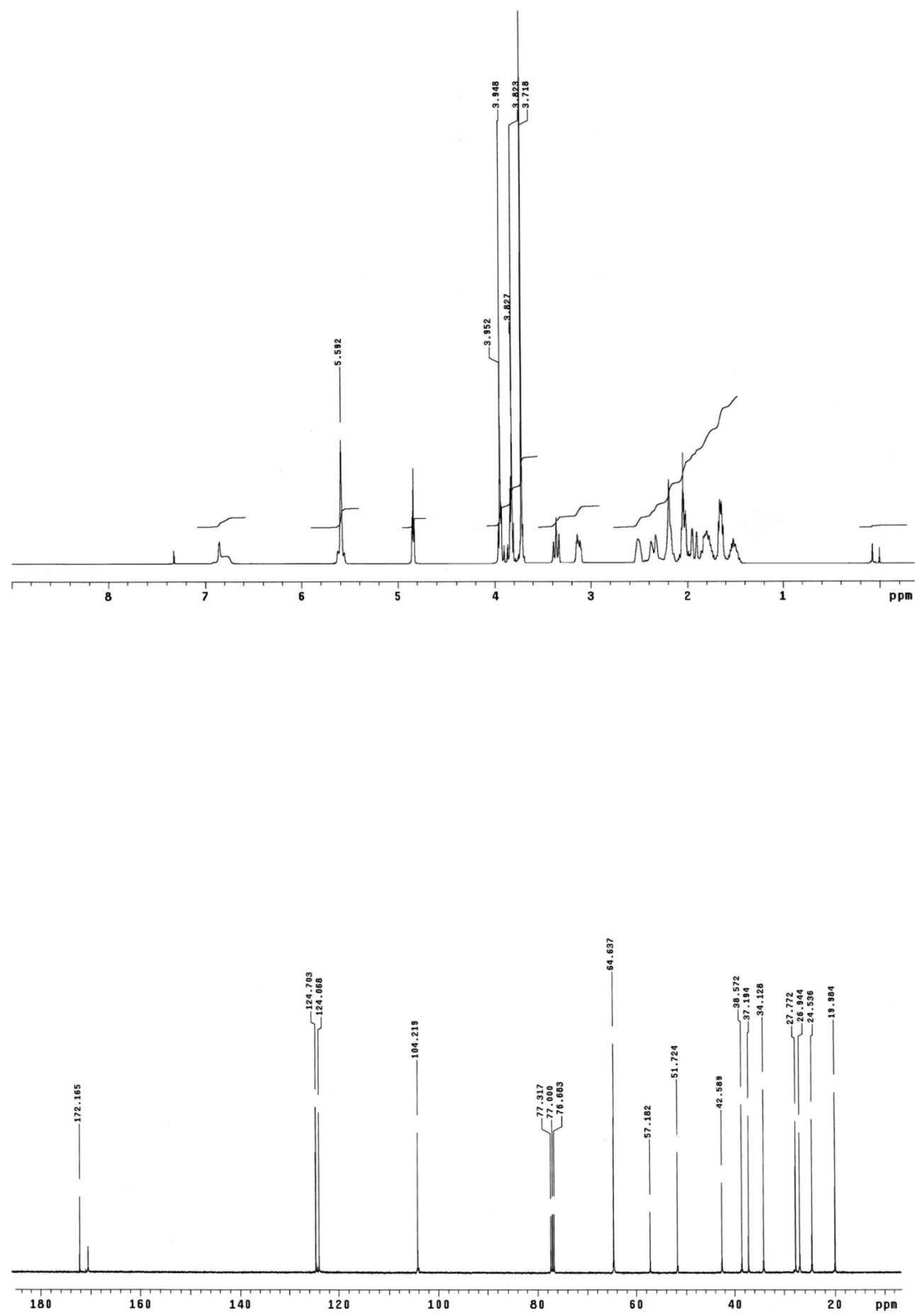


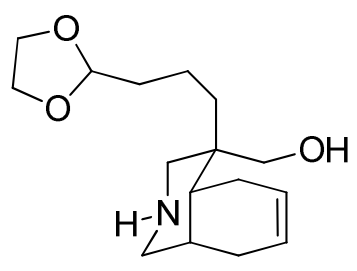

(4R,4aR,8aS)-4-[3-(1,3-Dioxolan-2-yl)propyl]-4-(methoxycarbonyl)-1,2,3,4,4a,5,8,8aoctahydroisoquinoline (17).

Lithium aluminum hydride ( $237 \mathrm{mg}, 6.25 \mathrm{mmol}$ ) was added to a solution of compound 16 (404 $\mathrm{mg}, 1.25 \mathrm{mmol})$ in anhydrous THF $(81 \mathrm{~mL})$ under argon atmosphere at $0{ }^{\circ} \mathrm{C}$ and the mixture was stirred at reflux for $5 \mathrm{~h}$. The resulting suspension was cooled to $0{ }^{\circ} \mathrm{C}$, and the reaction was quenched by slowly addition of distilled water. The aqueous layer was extracted with EtOAc, and the combined organic extracts were dried and concentrated to afford aminoalcohol derivate 17 (381 $\mathrm{mg}$ ) as a yellow oil which was used in the next step without purification.

${ }^{1} \mathrm{H}$ NMR (400 MHz, CDCl 3 , COSY, gHSQC): $\delta 1.43\left(\mathrm{~m}, 2 \mathrm{H}, \mathrm{H}-2^{\prime}\right), 1.69\left(\mathrm{~m}, 4 \mathrm{H}, \mathrm{H}-1^{\prime}, \mathrm{H}-3^{\prime}\right), 1.73$ $(\mathrm{dm}, J=18.4 \mathrm{~Hz}, 1 \mathrm{H}, \mathrm{H}-8), 1.87(\mathrm{~m}, 1 \mathrm{H}, \mathrm{H}-4 \mathrm{a}), 1.96(\mathrm{dm}, J=15.6 \mathrm{~Hz}, 1 \mathrm{H}, \mathrm{H}-5), 2.03-2.19(\mathrm{~m}, 2 \mathrm{H}$, $\mathrm{H}-8 \mathrm{H}-5), 2.23(\mathrm{~m}, 1 \mathrm{H}, \mathrm{H}-8 \mathrm{a}), 2.58(\mathrm{~m}, 4 \mathrm{H}, \mathrm{H}-1, \mathrm{H}-3), 3.36\left(\mathrm{~m}, 2 \mathrm{H}, \mathrm{CH}_{2} \mathrm{OH}\right), 3.84,3.95(2 \mathrm{~m}, 2 \mathrm{H}$, $\left.\mathrm{CH}_{2} \mathrm{O}\right), 4.88\left(\mathrm{t}, \mathrm{J}=4.8 \mathrm{~Hz}, 1 \mathrm{H}, \mathrm{CHO}_{2}\right), 5.58(\mathrm{~m}, 2 \mathrm{H}, \mathrm{H}-6, \mathrm{H}-7)$.

${ }^{13} \mathrm{C}$ NMR (100.6 MHz, CDCl $): \delta 17.2$ (C-2'), 21.9 (C-5), 29.0 (C-8a), 29.3 (C-8), 30.6 (C-1'), 34.0 (C-3'), 34.2 (C-4a), 39.6 (C-4), 46.9, 47.1 (C-1, C-3), $64.4\left(\mathrm{CH}_{2} \mathrm{OH}\right), 64.7,64.8\left(2 \mathrm{CH}_{2} \mathrm{O}\right), 104.5$ (C4'), 124.5, 124.7 (C-6, C-7). 

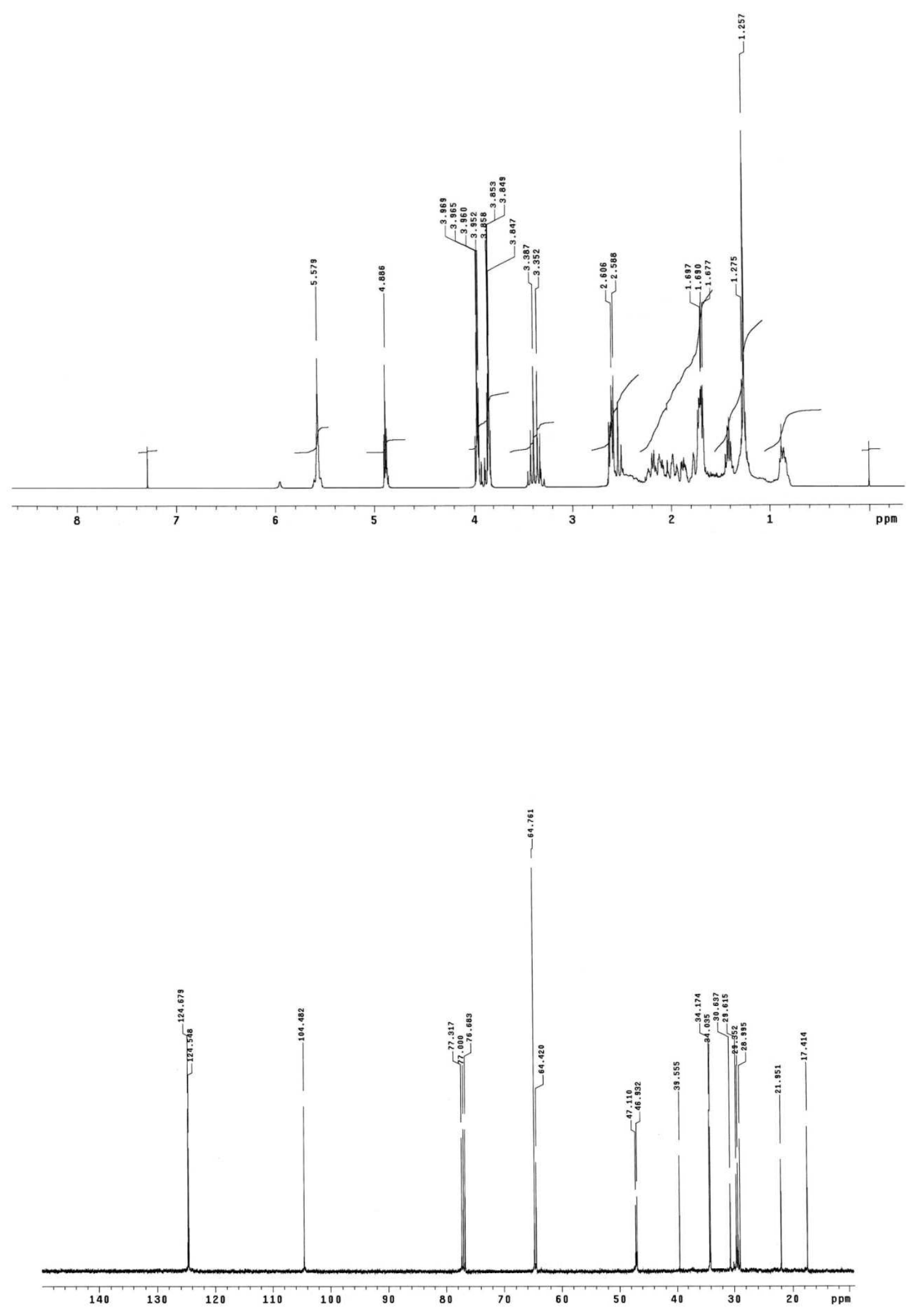
<smiles>CCCCCCCCCCCCCCCCCC(C)(C)C</smiles>

(3R,6aR,10aS,10bR)-6-(tert-Butotxycarbonyl)-5-oxo-3-phenyl-2,3,6,6a,7,10,10a,10b- octahydro$5 H$-oxazolo[3,2-a]isoquinoline (19).

Second generation Grubbs catalyst ( $200 \mathrm{mg}, 5 \% \mathrm{~mol}$ ) was added to a stirred solution of diallyllactams 7 (1.87 g, $4.71 \mathrm{mmol}$ ) in $\mathrm{CH}_{2} \mathrm{Cl}_{2}$. The resulting mixture was stirred at room temperature for $18 \mathrm{~h}$ and then concentrated under reduced pressure. Flash chromatography (hexane to 8:2 hexane-EtOAc) gave the C-6 epimers of lactam 19 (1.38 g, 80\%).

${ }^{1} \mathrm{H}$ NMR (400 MHz, CDCl 3 , COSY, gHSQC): $\delta 1.35\left[\mathrm{~s}, 9 \mathrm{H},\left(\mathrm{CH}_{3}\right)_{3} \mathrm{C}\right], 1.96(\mathrm{tm}, J=16.0 \mathrm{~Hz}, 1 \mathrm{H}, \mathrm{H}-7)$, $2.19(\mathrm{~m}, 1 \mathrm{H}, \mathrm{H}-7), 2.45(\mathrm{~m}, 2 \mathrm{H}, \mathrm{H}-10), 2.50(\mathrm{~m}, 1 \mathrm{H}, \mathrm{H}-6 \mathrm{a}), 2.80$ (m, 1H, H-10a), $3.06(\mathrm{~s}, 1 \mathrm{H}, \mathrm{H}-6)$, 3.99 (dd, $J=8.8,1.6 \mathrm{~Hz}, 1 \mathrm{H}, \mathrm{H}-2$ ), 4.13 (dd, $J=8.8,7.2 \mathrm{~Hz}, 1 \mathrm{H}, \mathrm{H}-2$ ), 4.84 (d, J=10.0 Hz, $1 \mathrm{H}, \mathrm{H}-$ 10b), 4.94 (dd, J = 7.2, 1.6 Hz, 1H, H-3), $5.70(\mathrm{~m}, 2 \mathrm{H}, \mathrm{H}-8, \mathrm{H}-9), 7.20-7.36\left(\mathrm{~m}, 5 \mathrm{H}, \mathrm{C}_{6} \mathrm{H}_{5}\right)$.

${ }^{13} \mathrm{C} \mathrm{NMR}\left(100.6 \mathrm{MHz}, \mathrm{CDCl}_{3}\right): \delta 25.2(\mathrm{C}-10), 27.8$ [( $\left.\left.\mathrm{CH}_{3}\right) \mathrm{C}\right], 27.9$ (C-7), 33.1 (C-10a), 33.4 (C-6a), 53.4 (C-6), 59.5 (C-3), 73.8 (C-2), 81.8 [( $\left.\left.\mathrm{CH}_{3}\right) \mathrm{C}\right], 87.1$ (C-10b), 124.7, 125.1 (C-8, C-9), 126.4, $128.3\left(\mathrm{C}-0, m \mathrm{C}_{6} \mathrm{H}_{5}\right), 127.4\left(\mathrm{C}-p \mathrm{C}_{6} \mathrm{H}_{5}\right), 141.1\left(\mathrm{C}-i \mathrm{C}_{6} \mathrm{H}_{5}\right), 163.5,168.2$ (NCO, COO).

HRMS (ESI) calcd for $\left[\mathrm{C}_{22} \mathrm{H}_{27} \mathrm{NO}_{4}+\mathrm{H}\right]^{+}: 370.2013$, found: 370.2011 . 

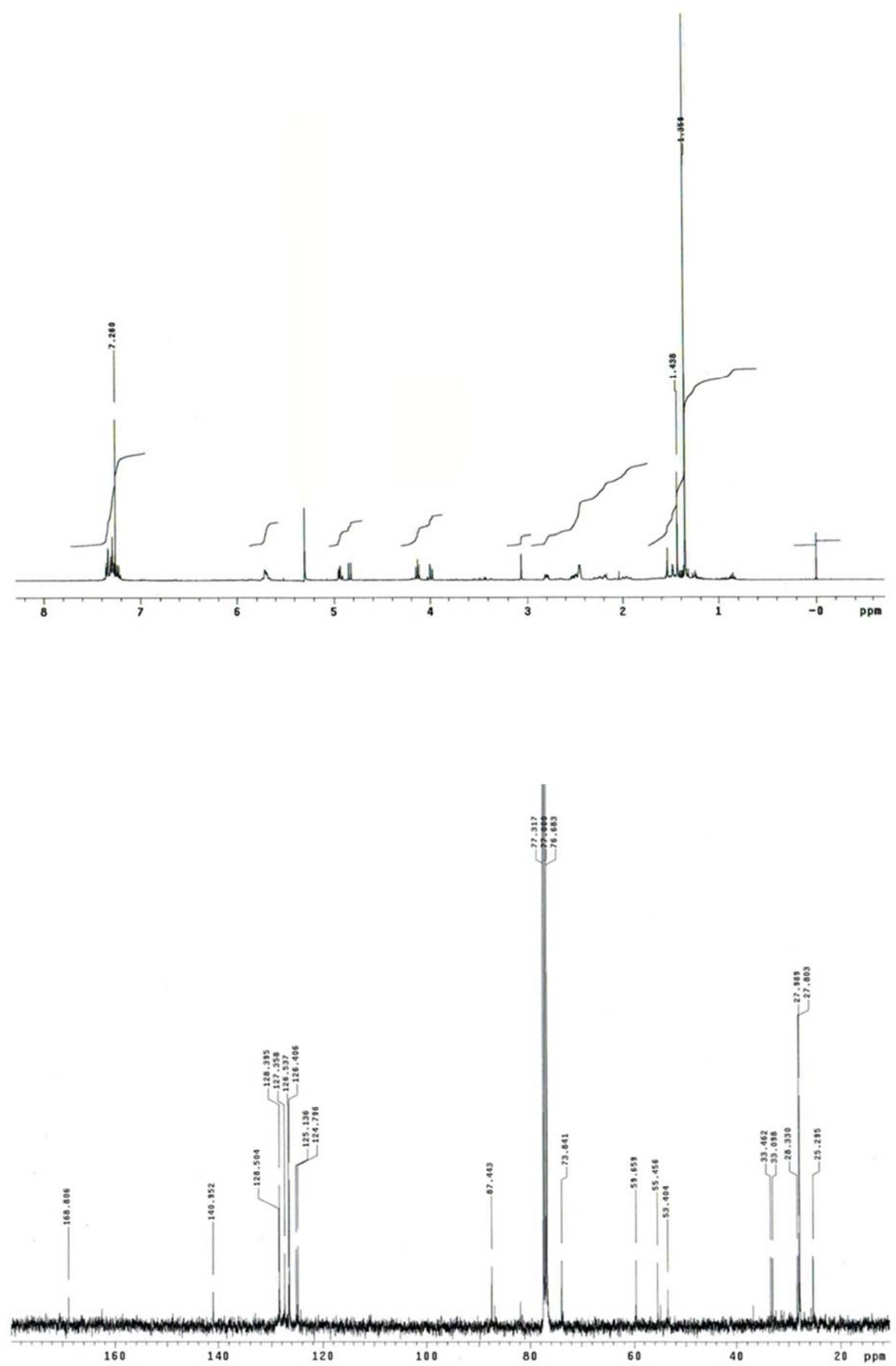


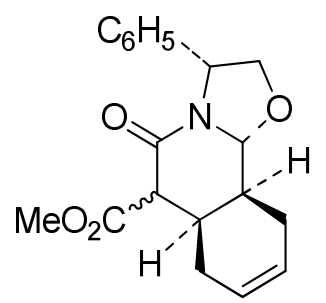

(3R,6aR,10aS,10bR)-6-(Methoxycarbonyl)-5-oxo-3-phenyl-2,3,6,6a,7,10,10a,10b-octahydro $5 \mathrm{H}$-oxazolo[2,3-a]isoquinoline (20).

Second generation Grubbs catalyst (642 mg) was added to a solution of lactam 8 (3.58 g, 10.1 $\mathrm{mmol})$ in $\mathrm{CH}_{2} \mathrm{Cl}_{2}(1.44 \mathrm{~L})$. The mixture was stirred for $18 \mathrm{~h}$ at room temperature, and the resulting suspension was concentrated. Flash chromatography (4:1 to 3:2 hexane-EtOAc) of the residue gave tricyclic lactam 20 as a mixture of C- 6 epimers $(2.8 \mathrm{~g}, 85 \%$ yield).

IR (film): 1667, 1738 (CO) $\mathrm{cm}^{-1}$.

Compound (6R)-20 (major):

${ }^{1} \mathrm{H} \mathrm{NMR}\left(400 \mathrm{MHz}, \mathrm{CDCl}_{3}, \mathrm{COSY}, \mathrm{gHSQC}\right): \delta 2.00(\mathrm{~m}, 1 \mathrm{H}, \mathrm{H}-7), 2.20(\mathrm{~m}, 1 \mathrm{H}, \mathrm{H}-7), 2.43(\mathrm{~m}, 2 \mathrm{H}, \mathrm{H}-$ 10), $2.50(\mathrm{~m}, 1 \mathrm{H}, \mathrm{H}-6 \mathrm{a}), 2.70(\mathrm{~m}, 1 \mathrm{H}, \mathrm{H}-10 \mathrm{a}), 3.18(\mathrm{~s}, 1 \mathrm{H}, \mathrm{H}-6), 3.60\left(\mathrm{~s}, 3 \mathrm{H}, \mathrm{CH}_{3} \mathrm{O}\right), 3.96(\mathrm{dd}, J=$ 9.0, 1.2 Hz, 1H, H-2), 4.12 (dd, J = 9.0, 6.9 Hz, 1H, H-2), 4.85 (d, J = 9.9 Hz, 1H, H-10b), 4.92 (dd, $J=6.9,1.2 \mathrm{~Hz}, 1 \mathrm{H}, \mathrm{H}-3), 5.69(\mathrm{~m}, 2 \mathrm{H}, \mathrm{H}-8, \mathrm{H}-9), 7.22-7.35\left(\mathrm{~m}, 5 \mathrm{H}, \mathrm{C}_{6} \mathrm{H}_{5}\right)$.

${ }^{13} \mathrm{C}$ NMR (100.6 MHz, CDCl $): \delta 25.1$ (C-10), 28.0 (C-7), 32.6 (C-10a), 33.5 (C-6a), $52.2\left(\mathrm{CH}_{3} \mathrm{O}\right)$, 53.9 (C-6), 59.4 (C-3), 73.6 (C-2), 87.1 (C-10b), 124.4, 124.8 (C-8, C-9), 126.8, 128.0 (C-o, m $\left.\mathrm{C}_{6} \mathrm{H}_{5}\right), 127.2\left(\mathrm{C}-p \mathrm{C}_{6} \mathrm{H}_{5}\right), 140.6\left(\mathrm{C}-i \mathrm{C}_{6} \mathrm{H}_{5}\right), 162.0$ (NCO), 170.2 (COO).

Compound (6S)-3 (minor):

${ }^{13} \mathrm{C} \mathrm{NMR}\left(100.6 \mathrm{MHz}, \mathrm{CDCl}_{3}\right.$, selected resonances): $\delta 24.7$ (C-10), 32.6 (C-10a), 36.7 (C-6a), 51.8 $\left(\mathrm{CH}_{3}\right), 53.7$ (C-6), 59.6 (C-3), 73.3 (C-2), 86.6 (C-10b), 140.9 (C-i), 162.4 (NCO), 169.1 (COO).

Anal. Calcd for $\mathrm{C}_{19} \mathrm{H}_{21} \mathrm{O}_{4} \mathrm{~N} \cdot{ }^{1} /{ }_{4} \mathrm{H}_{2} \mathrm{O}: \mathrm{C}, 68.76 ; \mathrm{H}, 6.53 ; \mathrm{N}, 4.22$. Found: $\mathrm{C}, 68.82 ; \mathrm{H}, 6.90 ; \mathrm{N}, 4.20$. 

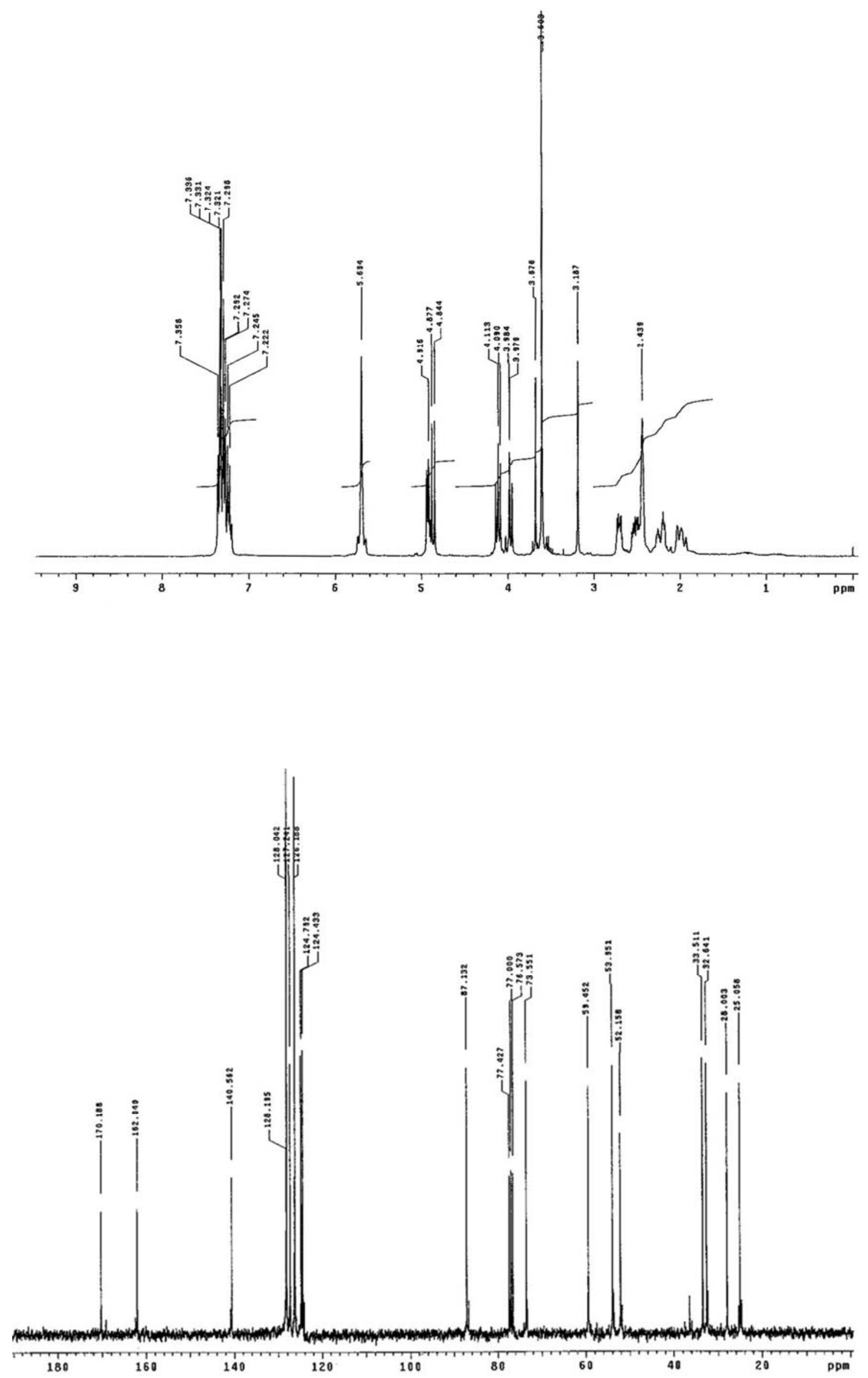


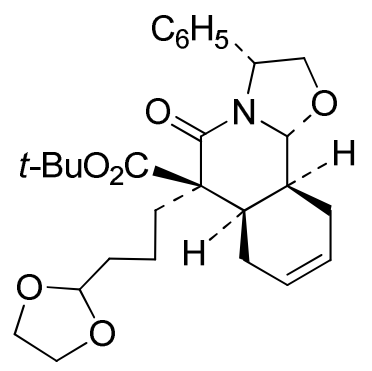

(3R,6R,6aR,10aS,10bR)-6-(tert-Butotxycarbonyl)-6-[3-(1,3-dioxolan-2-yl)propyl]-5-oxo $-3-$ phenyl-2,3,6,6a,7,10,10a,10b-octahydro-5H-oxazolo[3,2-a]isoquinoline (21).

Lactam 19 (2.0 g, $5.4 \mathrm{mmol}$ ) was added to a suspension of $\mathrm{NaH}$ ( $396 \mathrm{mg}$ of a $60 \%$ dispersion in mineral oil, $5.9 \mathrm{mmol})$ in anhydrous $\mathrm{DMF}(10 \mathrm{~mL})$ at $0{ }^{\circ} \mathrm{C}$ under an inert atmosphere, and the resulting mixture was stirred at this temperature for 1 h. 2-(3-Bromopropyl)-1,3-dioxolane $\mathrm{e}^{133}$ $(3.87 \mathrm{~g}, 27.0 \mathrm{mmol})$ and TBAI $(398 \mathrm{mg}, 1.08 \mathrm{mmol})$ were then added at $0{ }^{\circ} \mathrm{C}$, and the mixture was stirred overnight at room temperature. Saturated aqueous $\mathrm{NH}_{4} \mathrm{Cl}$ was added, and the mixture was extracted with $\mathrm{Et}_{2} \mathrm{O}$ and then with $\mathrm{CH}_{2} \mathrm{Cl}_{2}$. The combined organic extracts were dried, filtered, and concentrated under reduced pressure. Flash chromatography (hexane to $8: 2$ hexane-EtOAC) of the resulting residue afforded lactam 21 (2.34 g, 90\%).

$[\alpha]_{D}^{22}=-51.7\left(c 0.63, \mathrm{CHCl}_{3}\right)$.

IR (KBr): 1728, $1659(\mathrm{C}=\mathrm{O}) \mathrm{cm}^{-1}$.

${ }^{1} \mathrm{H}$ NMR (400 MHz, CDCl 3 , COSY, gHSQC): $\delta 1.22\left(\mathrm{~m}, 2 \mathrm{H}, \mathrm{H}-2^{\prime}\right), 1.39\left(\mathrm{~m}, 2 \mathrm{H}, \mathrm{H}-3^{\prime}\right), 1.45[\mathrm{~s}, 9 \mathrm{H}$, $\left.\left(\mathrm{CH}_{3}\right)_{3} \mathrm{C}\right], 1.81\left(\mathrm{dd}, J=6.0,2.0 \mathrm{~Hz}, 1 \mathrm{H}, \mathrm{H}-1^{\prime}\right), 1.84\left(\mathrm{dd}, J=6.0,2.4 \mathrm{~Hz}, 1 \mathrm{H}, \mathrm{H}-1^{\prime}\right), 2.00(\mathrm{dm}, J=$ 12.4 Hz, 1H, H-7), 2.17 (dm, J = 12.4 Hz, 1H, H-7), 2.23 (m, 1H, H-10), 2.29 (ddd, J = 11.6, 5.6, $3.6 \mathrm{~Hz}, 1 \mathrm{H}, \mathrm{H}-6 \mathrm{a}), 2.44(\mathrm{~m}, 1 \mathrm{H}, \mathrm{H}-10), 2.51(\mathrm{~m}, 1 \mathrm{H}, \mathrm{H}-10 \mathrm{a}), 3.78,3.88\left(2 \mathrm{~m}, 4 \mathrm{H}, 2 \mathrm{CH}_{2} \mathrm{O}\right), 4.04$ (dd, $J=8.8,1.2 \mathrm{~Hz}, 1 \mathrm{H}, \mathrm{H}-2), 4.13(\mathrm{dd}, J=8.8 \mathrm{~Hz}, 1 \mathrm{H}, \mathrm{H}-2), 4.63\left(\mathrm{t}, J=4.8 \mathrm{~Hz}, 1 \mathrm{H}, \mathrm{H}-4^{\prime}\right), 4.89(\mathrm{~m}, 2 \mathrm{H}$, $\mathrm{H}-3, \mathrm{H}-10 \mathrm{~b}), 5.69$ (m, 2H, H-8, H-9), 7.20-7.35 (m, 5H, $\left.\mathrm{C}_{6} \mathrm{H}_{5}\right)$.

${ }^{13} \mathrm{C}$ NMR $(100.6 \mathrm{MHz}, \mathrm{CDCl}$ ) $): \delta 20.0$ (C-2'), 25.7, 25.8 (C-5, C-8), $28.0\left[\left(\mathrm{CH}_{3}\right)_{3} \mathrm{C}\right], 33.6$ (C-10a), 34.2 (C-3'), 37.3 (C-1'), 38.9 (C-6a), 46.9 (C-1), 59.1 (C-4), 59.7 (C-3), 64.6, $64.7\left(2 \mathrm{CH}_{2} \mathrm{O}\right), 73.5$ (C-2), $81.6\left[\left(\mathrm{CH}_{3}\right)_{3} \mathrm{C}\right], 87.0$ (C-10b), 104.4 (C-4'), 124.1, 125.1 (C-8, C-9), 126.7, 128.3 (C-o, $m$ $\left.\mathrm{C}_{6} \mathrm{H}_{5}\right), 127.4\left(\mathrm{C}-p \mathrm{C}_{6} \mathrm{H}_{5}\right), 142.2\left(\mathrm{C}-i \mathrm{C}_{6} \mathrm{H}_{5}\right), 165.5$ (NCO), 170.0 (CCO).

HRMS (ESI) calcd for $\left[\mathrm{C}_{28} \mathrm{H}_{37} \mathrm{NO}_{6}+\mathrm{H}\right]^{+}: 484.2694$, found: 484.2685 .

${ }^{133}$ Varseev, G. N.; Maier, M. E. Org. Lett. 2005, 7, 3881-3884. 

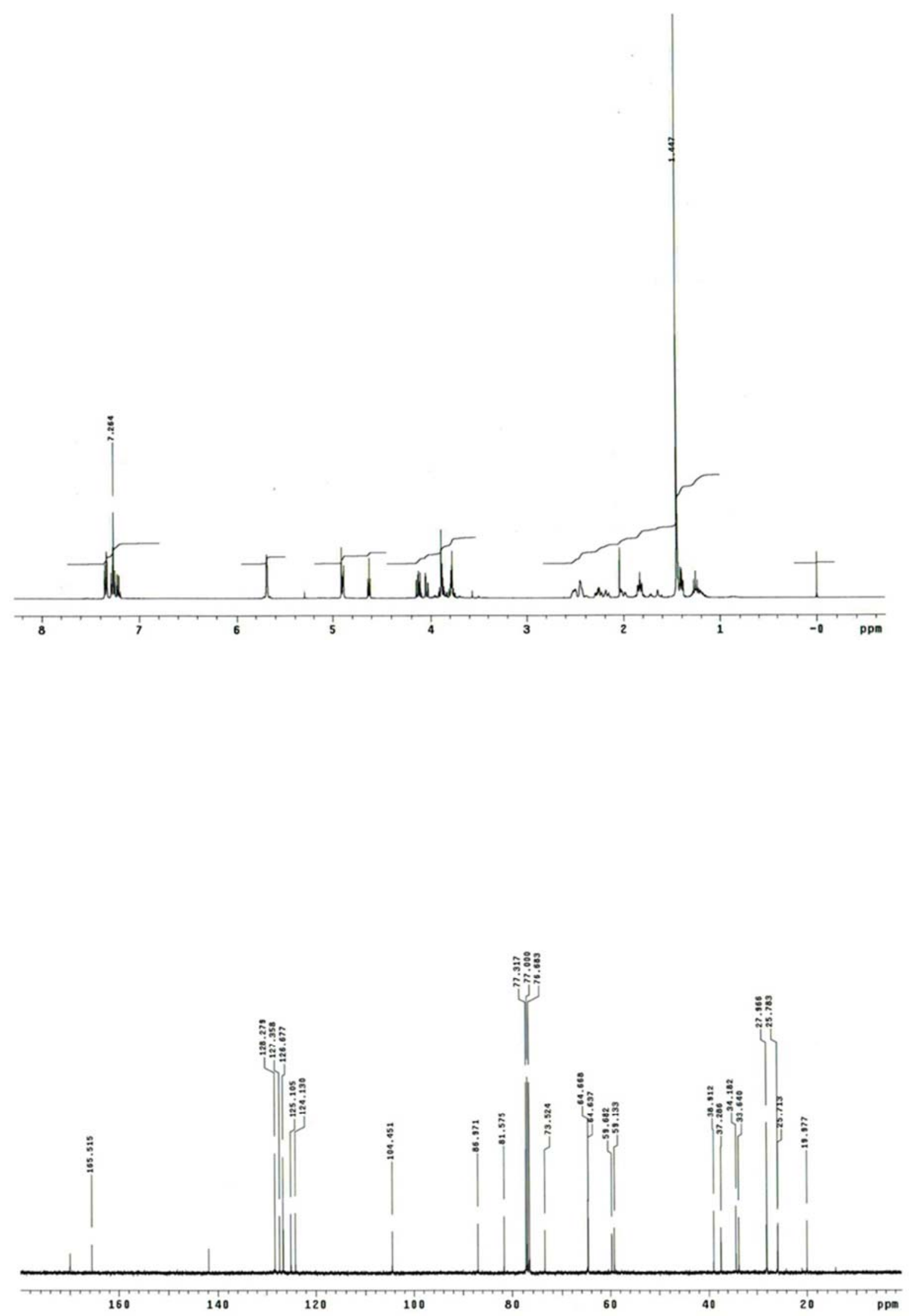
<smiles>COC(=O)[C@]1(CCCC2OCCO2)C(=O)N2C(C)COC2[C@H]2CC=CC[C@H]21</smiles>

(3R,6R,6aR,10aS,10bR)-6-[3-(1,3-Dioxolan-2-yl)propyl]-6-(methoxycarbonyl)-5-oxo-3-phenyl2,3,6,6a,7,10,10a,10b-octahydro-5H-oxazolo[3,2-a]isoquinoline (22).

A solution of isoquinoline $20(880 \mathrm{mg}, 2.691 \mathrm{mmol})$ in DMF $(2 \mathrm{~mL})$ was added to a cooled $\left(0^{\circ} \mathrm{C}\right)$ suspension of $\mathrm{NaH}$ (161 $\mathrm{mg}$ of a $60 \%$ dispersion in mineral oil, $4.031 \mathrm{mmol}$ ) in anhydrous DMF $(20 \mathrm{~mL})$ under an inert atmosphere, and the resulting mixture was stirred at $0{ }^{\circ} \mathrm{C}$ for 1 h. 2 - $(3-$ Bromopropyl)-1,3-dioxolane (1.94 g, $13.4 \mathrm{mmol}$ ) and TBAI (198 mg, $0.538 \mathrm{mmol})$ were added at $0{ }^{\circ} \mathrm{C}$, and the mixture was stirred overnight at room temperature. Saturated aqueous $\mathrm{NH}_{4} \mathrm{Cl}$ was added, and the mixture was extracted with diethyl ether and then with $\mathrm{CH}_{2} \mathrm{Cl}_{2}$. The combined organic extracts were dried, filtered, and concentrated under reduced pressure. Flash chromatography (hexane to 6:4 hexane-EtOAc) of the resulting oil afforded lactam 22 (905 mg, 80\%).

$[\alpha]_{D}^{22}=-29.7$ (c 2.1, $\left.\mathrm{CHCl}_{3}\right)$.

IR (film): 1668, 1737 (CO) $\mathrm{cm}^{-1}$.

${ }^{1} \mathrm{H}$ NMR (400 MHz, CDCl 3 , COSY, gHSQC): $\delta 1.13-1.23\left(\mathrm{~m}, 2 \mathrm{H}, \mathrm{H}-2^{\prime}\right), 1.40\left(\mathrm{~m}, 2 \mathrm{H}, \mathrm{H}-3^{\prime}\right), 1.81-$ $1.97\left(\mathrm{~m}, 3 \mathrm{H}, 2 \mathrm{H}-1^{\prime}, \mathrm{H}-7\right), 2.11-2.20(3 \mathrm{~m}, 2 \mathrm{H}, \mathrm{H}-7, \mathrm{H}-10), 2.28$ (ddd, $J=12.4,6.0,4.0 \mathrm{~Hz}, 1 \mathrm{H}, \mathrm{H}-$ 6a), $2.45(\mathrm{~m}, 1 \mathrm{H}, \mathrm{H}-10), 2.54(\mathrm{~m}, 1 \mathrm{H}, \mathrm{H}-10 \mathrm{a}), 3.70\left(\mathrm{~s}, 3 \mathrm{H}, \mathrm{CH}_{3} \mathrm{O}\right), 3.77,3.89\left(2 \mathrm{~m}, 4 \mathrm{H}, \mathrm{CH}_{2} \mathrm{O}\right), 4.07$ $(\mathrm{dd}, J=9.2,1.6 \mathrm{~Hz}, 1 \mathrm{H}, \mathrm{H}-2), 4.15(\mathrm{dd}, J=9.2 \mathrm{~Hz}, 1 \mathrm{H}, \mathrm{H}-2), 4.61\left(\mathrm{t}, J=4.8 \mathrm{~Hz}, 1 \mathrm{H}, \mathrm{H}-4^{\prime}\right), 4.91(\mathrm{~m}$, $2 \mathrm{H}, \mathrm{H}-3, \mathrm{H}-10 \mathrm{~b}), 5.67(\mathrm{~m}, 2 \mathrm{H}, \mathrm{H}-8, \mathrm{H}-9), 7.20-7.39\left(\mathrm{~m}, 5 \mathrm{H}, \mathrm{C}_{6} \mathrm{H}_{5}\right)$.

${ }^{13} \mathrm{C}$ NMR (100.6 MHz, CDCl $): \delta 19.9$ (C-2'), 25.6, 25.7 (C-7, C-10), 33.6 (C-10a), 34.1 (C-3'), 37.4 (C-1'), 39.5 (C-6a), $51.9\left(\mathrm{CH}_{3} \mathrm{O}\right), 58.9$ (C-6), 59.8 (C-3), 64.6, $64.7\left(2 \mathrm{CH}_{2} \mathrm{O}\right), 73.5$ (C-2), 87.5 (C10b), 104.4 (C-4'), 124.3, 124.7 (C-8, C-9), 126.8, 128.3 (C-o, $m \mathrm{C}_{6} \mathrm{H}_{5}$ ), 127.5 (C-p $\mathrm{C}_{6} \mathrm{H}_{5}$ ), 141.6 (C$\left.i \mathrm{C}_{6} \mathrm{H}_{5}\right), 165.4$ (NCO), 172.1 (CCO).

HRMS calcd for $\left[\mathrm{C}_{25} \mathrm{H}_{31} \mathrm{NO}_{6}+\mathrm{H}\right]^{+}: 442.2224$, found: 442.2227 . 

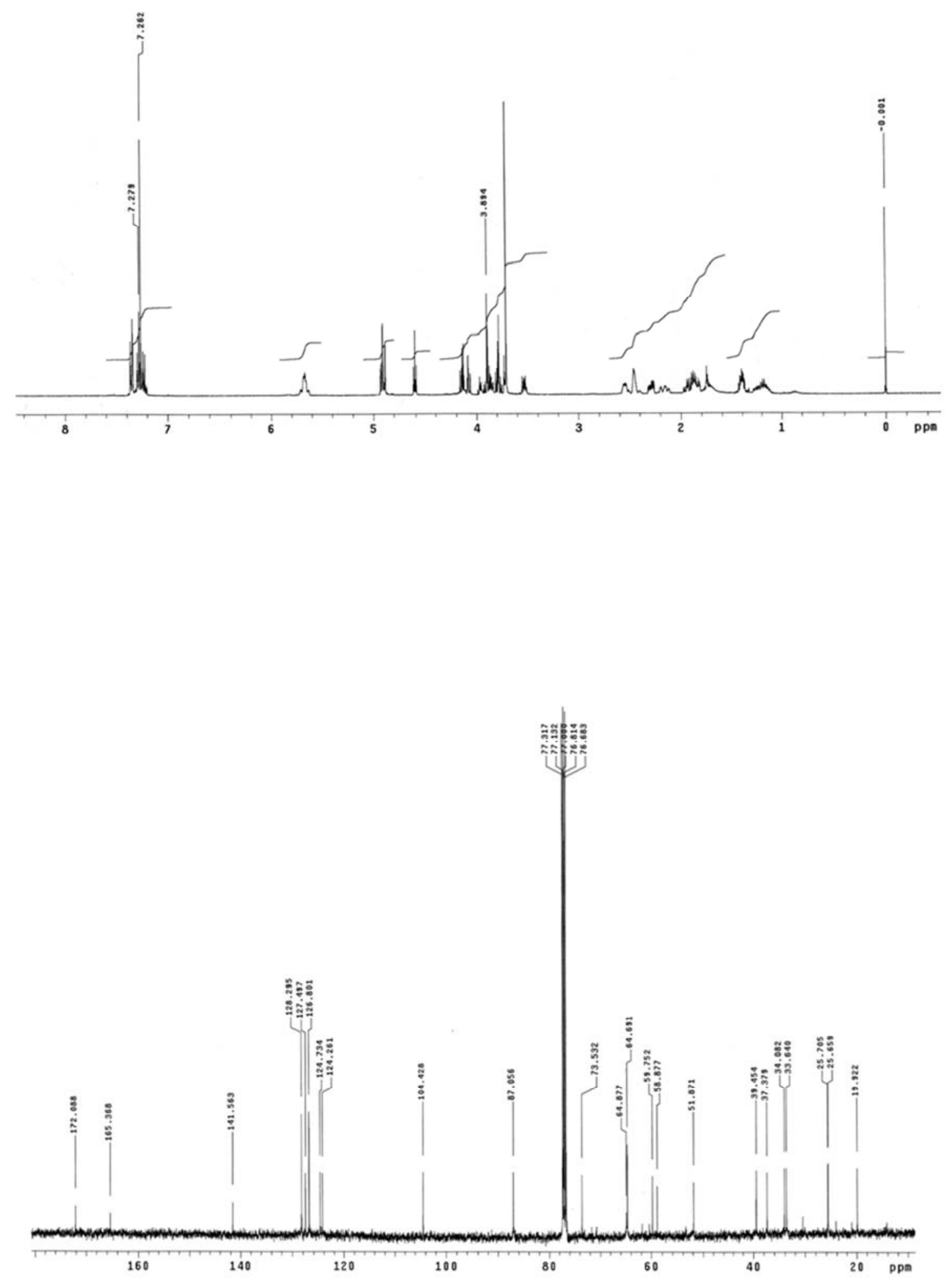
<smiles>C=CC[C@H]1C(C(=O)OC)C(=O)N2C(CCCCC)COC12</smiles>

$(3 R, 7 R, 8 S, 8 \mathrm{a} R)$-7,8-Diallyl-6-(methoxycarbonyl)-5-oxo-3-phenyl-2,3,6,7,8,8a-hexahydro-5Hoxazolo[3,2-a]pyridine (8).

$\mathrm{LiCl}(1.2 \mathrm{~g}, 28.4 \mathrm{mmol})$ was dried at $80^{\circ} \mathrm{C}$ for $1 \mathrm{~h}$ under vacuum $(10-15 \mathrm{mmHg})$ in a threenecked, $250 \mathrm{~mL}$ round-bottomed flask. Then, Cul $(5.4 \mathrm{~g}, 28.4 \mathrm{mmol})$ and THF (150 mL) were added under an inert atmosphere, and the mixture was stirred at room temperature for $5 \mathrm{~min}$. The suspension was cooled to $-78^{\circ} \mathrm{C}$, and allylmagnesium bromide $(28.4 \mathrm{~mL}$ of a $1 \mathrm{M}$ solution in $\left.\mathrm{Et}_{2} \mathrm{O}, 28.4 \mathrm{mmol}\right), \mathrm{TMSCl}(3.6 \mathrm{~mL}, 28.4 \mathrm{mmol})$, and unsaturated lactam 6 (7.11 mmol) in THF $(5 \mathrm{~mL})$ were successively added. The resulting mixture was stirred at $-78{ }^{\circ} \mathrm{C}$ for $18 \mathrm{~h}$. The reaction was quenched with saturated aqueous $\mathrm{NH}_{4} \mathrm{Cl}$, and the resulting mixture was filtered through Celite ${ }^{\circledast}$. The aqueous layer was extracted with EtOAc, and the combined organic extracts were dried over anhydrous $\mathrm{MgSO}_{4}$, filtered, and concentrated under reduced pressure. Flash chromatography (9:1 to 7:3 hexane-EtOAc) of the resulting oil gave 8 ( $2.05 \mathrm{~g}$, $81 \%$ yield) as a mixture of C-6 epimers (ratio 2:1).

Epimer 8a (major) (6S):

IR (film): 1665, 1736 (CO) $\mathrm{cm}^{-1}$.

${ }^{1} \mathrm{H} \mathrm{NMR} \mathrm{(300} \mathrm{MHz,} \mathrm{CDCl} 3$, COSY, gHSQC): $\delta 1.80$ (ddd, $J=14.1,12.0,9.0 \mathrm{~Hz}, 1 \mathrm{H}, \mathrm{CH}_{2}$ allyl), 2.16 (dt, $J=14.1,9.3,9.3 \mathrm{~Hz}, 1 \mathrm{H}, \mathrm{CH}_{2}$ allyl), $2.34(\mathrm{dm}, J=12.0 \mathrm{~Hz}, 1 \mathrm{H}, \mathrm{H}-7), 2.44-2.70(\mathrm{~m}, 3 \mathrm{H}, \mathrm{H}-8$, $\mathrm{CH}_{2}$ allyl), $3.43(\mathrm{~d}, J=1.5 \mathrm{~Hz}, 1 \mathrm{H}, \mathrm{H}-6), 3.60\left(\mathrm{~s}, 3 \mathrm{H}, \mathrm{CH}_{3} \mathrm{O}\right), 4.02(\mathrm{dd}, J=9.3,1.8 \mathrm{~Hz}, 1 \mathrm{H}, \mathrm{H}-2), 4.15$ (dd, $J=9.3,7.2 \mathrm{~Hz}, 1 \mathrm{H}, \mathrm{H}-2$ ), 4.62 (d, $J=9.6 \mathrm{~Hz}, 1 \mathrm{H}, \mathrm{H}-8 \mathrm{a}$ ), 4.91 (dd, $J=7.2,1.8 \mathrm{~Hz}, 1 \mathrm{H}, \mathrm{H}-3$ ), $5.14\left(\mathrm{~m}, 4 \mathrm{H}, \mathrm{CH}_{2}=\right.$ ), 5.68 (dddd, $J=15.0,10.2,9.0,4.8 \mathrm{~Hz}, 1 \mathrm{H}, \mathrm{CH}=$ ), 5.84 (dddd, $J=15.3,9.9$, 8.7, $5.1 \mathrm{~Hz}, 1 \mathrm{H}, \mathrm{CH}=)$, 7.26-7.33 (m, $\left.5 \mathrm{H}, \mathrm{C}_{6} \mathrm{H}_{5}\right)$.

${ }^{13} \mathrm{C} \mathrm{NMR}\left(75.4 \mathrm{MHz}, \mathrm{CDCl}_{3}\right): \delta 31.6,31.8\left(\mathrm{CH}_{2}\right), 36.9$ (C-7), 38.5 (C-8), $51.5(\mathrm{C}-6), 52.3\left(\mathrm{CH}_{3} \mathrm{O}\right)$, 59.6 (C-3), 73.9 (C-2), 89.4 (C-8a), 117.4, $118.5\left(\mathrm{CH}_{2}=\right)$, 126.4, 128.2 (C-o, $m$ ), 127.4 (C-p), 134.4, $134.8(\mathrm{CH}=), 140.5(\mathrm{C}-\mathrm{i}), 162.3$ (NCO), 170.6 (COO). 
Epimer $\mathbf{8 b}$ (minor) $(6 R)$ :

${ }^{13} \mathrm{C} \mathrm{NMR}\left(75.4 \mathrm{MHz}, \mathrm{CDCl}_{3}\right): \delta 32.8,35.9\left(\mathrm{CH}_{2}\right), 36.5(\mathrm{C}-7), 41.9(\mathrm{C}-8), 52.5\left(\mathrm{CH}_{3} \mathrm{O}\right), 53.7(\mathrm{C}-6)$, 59.2 (C-3), 73.8 (C-2), 89.8 (C-8a), 118.4, $119.6\left(\mathrm{CH}_{2}=\right), 126.7-128.5$ (C-o, $\left.m, p\right), 132.8,133.4$ $(\mathrm{CH}=), 140.6(\mathrm{C}-\mathrm{i}), 162.5$ (NCO), 170.8 (COO).

HRMS (ESI) calcd for $\left[\mathrm{C}_{21} \mathrm{H}_{25} \mathrm{NO}_{4}+\mathrm{H}\right]^{+}: 356.1783$, found: 356.1779 .

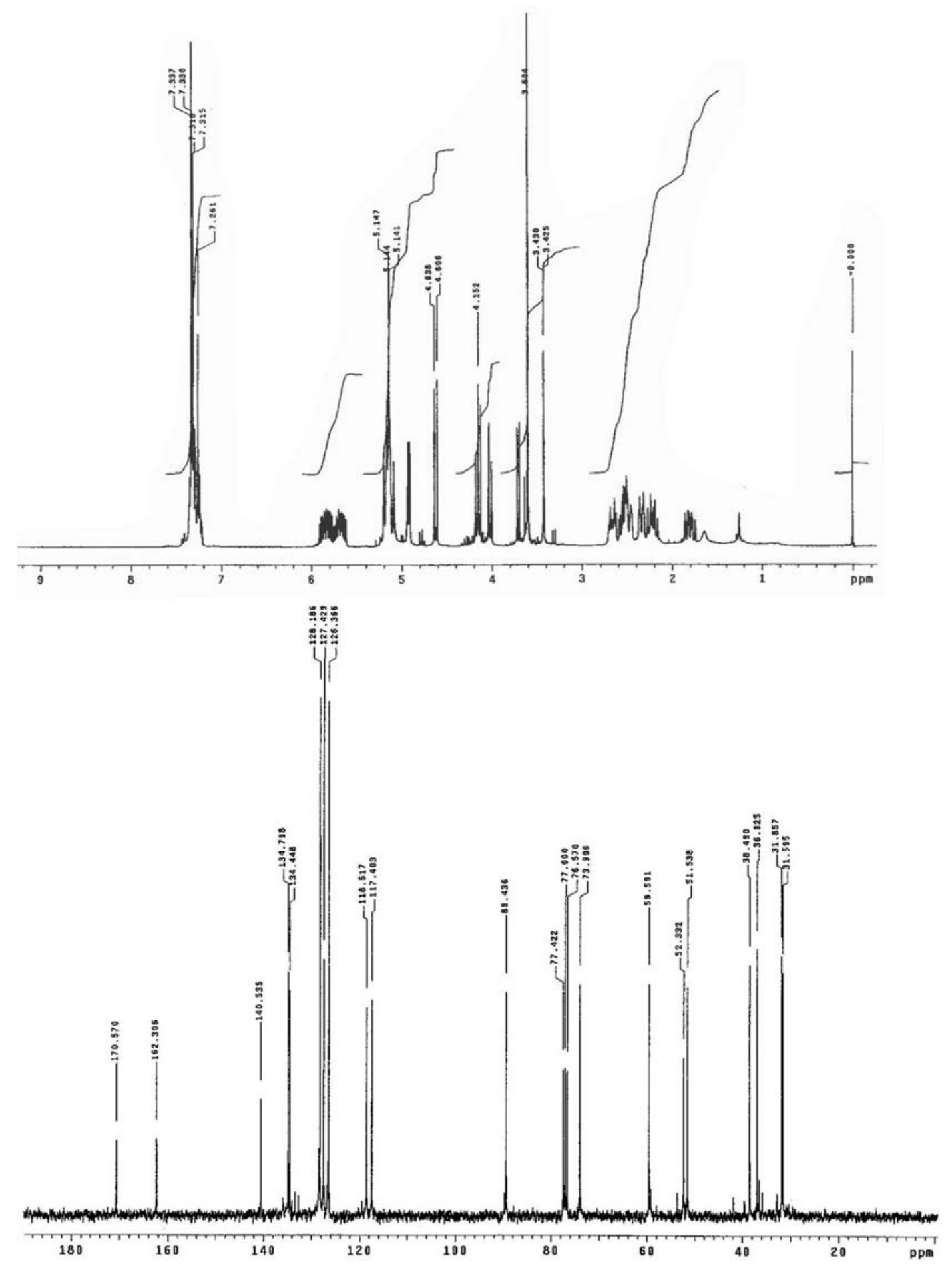




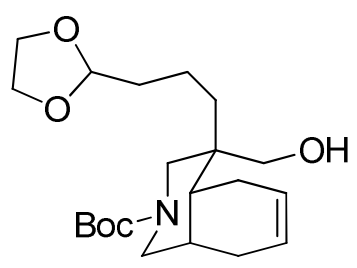

(4R,4aR,8aS)-2-(tert-Butoxycarbonyl)-4-[3-(1,3-dioxolan-2-yl)propyl]-4-(hydroxymethyl)-

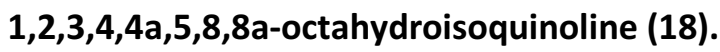

First step: Liquid ammonia $(15 \mathrm{~mL})$ was condensed at $-78^{\circ} \mathrm{C}$ in a three-necked, $100 \mathrm{~mL}$ roundbottomed flask equipped with a coldfinger condenser charged with dry ice-acetone, and then a solution of lactam 21 (200 mg, $0.414 \mathrm{mmol})$ in THF (10 mL) was added. The temperature was raised to $-33{ }^{\circ} \mathrm{C}$ and sodium metal was added in small portions until the blue color persisted. The mixture was stirred at $-33^{\circ} \mathrm{C}$ for $2 \mathrm{~min}$. The reaction was quenched by the addition of solid $\mathrm{NH}_{4} \mathrm{Cl}$ until the blue colour disappeared, and the mixture was stirred at room temperature for $4 \mathrm{~h}$. The residue was digested at room temperature with $\mathrm{CH}_{2} \mathrm{Cl}_{2}$, and the resulting suspension was filtered through Celite ${ }^{\circledR}$. The solution was concentrated under reduced pressure.

Second step: Lithium aluminum hydride $(235 \mathrm{mg}, 6.21 \mathrm{mmol})$ was added under an argon atmosphere to a solution of the above residue in anhydrous dioxane $(14 \mathrm{~mL})$ and the mixture was stirred at reflux overnight. The resulting suspension was cooled to $0{ }^{\circ} \mathrm{C}$, and the reaction was quenched with distilled water. The aqueous layer was extracted with $\operatorname{Et}_{2} \mathrm{O}$, and the combined organic extracts were dried, filtered, and concentrated to afford the corresponding amino alcohol $\mathbf{1 7}$ as a yellow oil, which was used in the next step without purification.

Third step: Di-tert-butyl dicarbonate $(98 \mathrm{mg}, 0.455 \mathrm{mmol}$ ) was added dropwise to a solution of the above amino 17 alcohol in anhydrous $\mathrm{CH}_{2} \mathrm{Cl}_{2}(7 \mathrm{~mL})$ at room temperature under an inert atmosphere, and the resulting mixture was stirred for $20 \mathrm{~h}$. The solution was then poured into saturated aqueous $\mathrm{NH}_{4} \mathrm{Cl}$ and extracted with $\mathrm{CH}_{2} \mathrm{Cl}_{2}$. The combined organic extracts were washed with brine, dried over anhydrous $\mathrm{MgSO}_{4}$, filtered, and concentrated under reduced pressure. Flash chromatography (9:1 to 1:1 hexane-EtOAc) of the residue gave carbamate 18 (71 mg, 45\% overall yield from 21).

$[\alpha]_{D}^{22}=-14.4\left(\right.$ c $\left.2.6, \mathrm{CHCl}_{3}\right)$.

IR (KBr): $3480(\mathrm{OH}), 1688(\mathrm{C}=\mathrm{O}) \mathrm{cm}^{-1}$.

${ }^{1} \mathrm{H}$ NMR (400 MHz, CDCl 3 , COSY, gHSQC): $\delta 1.45$ [s, 11H, (CH3)3C), H-2'], $1.50\left(\mathrm{~m}, 2 \mathrm{H}, \mathrm{H}-1^{\prime}\right)$, $1.63\left(\mathrm{~m}, 2 \mathrm{H}, \mathrm{H}-3^{\prime}\right), 1.81$ (d, J = $\left.18.0 \mathrm{~Hz}, 1 \mathrm{H}, \mathrm{H}-8\right), 1.90(\mathrm{~m}, 1 \mathrm{H}, \mathrm{H}-4 \mathrm{a}), 1.99(\mathrm{dm}, J=18.8 \mathrm{~Hz}, 1 \mathrm{H}$, 
$\mathrm{H}-5), 2.09(\mathrm{dm}, J=18.8 \mathrm{~Hz}, 1 \mathrm{H}, \mathrm{H}-5), 2.14(\mathrm{~m}, 1 \mathrm{H}, \mathrm{H}-8 \mathrm{a}), 2.23(\mathrm{~d}, J=18.0 \mathrm{~Hz}, 1 \mathrm{H}, \mathrm{H}-8), 2.70-2.97$ ( $m, 2 \mathrm{H}, \mathrm{H}-1, \mathrm{H}-3), 3.42\left(\mathrm{~s}, 2 \mathrm{H}, \mathrm{CH}_{2} \mathrm{OH}\right), 3.52-3.63(\mathrm{~m}, 2 \mathrm{H}, \mathrm{H}-1, \mathrm{H}-3), 3.85,3.95\left(2 \mathrm{~m}, 4 \mathrm{H}, 2 \mathrm{CH}_{2} \mathrm{O}\right)$, $4.87\left(\mathrm{t}, J=4.8 \mathrm{~Hz}, 1 \mathrm{H}, \mathrm{H}-4^{\prime}\right), 5.59(\mathrm{~m}, 2 \mathrm{H}, \mathrm{H}-6, \mathrm{H}-7)$.

${ }^{13} \mathrm{C}$ NMR (100.6 MHz, CDCl3): $\delta 17.6$ (C-2'), 21.4 (C-5), 27.8 (C-8a), $\left.28.3\left[\left(\mathrm{CH}_{3}\right)_{3} \mathrm{C}\right)\right], 28.5(\mathrm{C}-8)$, 30.7 (C-1'), 34.2 (C-3', C-4a), 40.8 (C-4), 43.7-45.7 (C-1, C-3), 63.6, 64.2 ( $\left.\mathrm{CH}_{2} \mathrm{OH}\right), 64.7,64.8$ $\left.\left(2 \mathrm{CH}_{2} \mathrm{O}\right), 79.4\left[\left(\mathrm{CH}_{3}\right)_{3} \mathrm{C}\right)\right], 104.4\left(\mathrm{C}-4^{\prime}\right), 124.5(\mathrm{C}-6, \mathrm{C}-7), 155.0$ (NCOO).

HRMS (ESI) calcd for $\left[\mathrm{C}_{22} \mathrm{H}_{35} \mathrm{NO}_{5}+\mathrm{H}\right]^{+}: 382.2588$, found: 382.2585 .
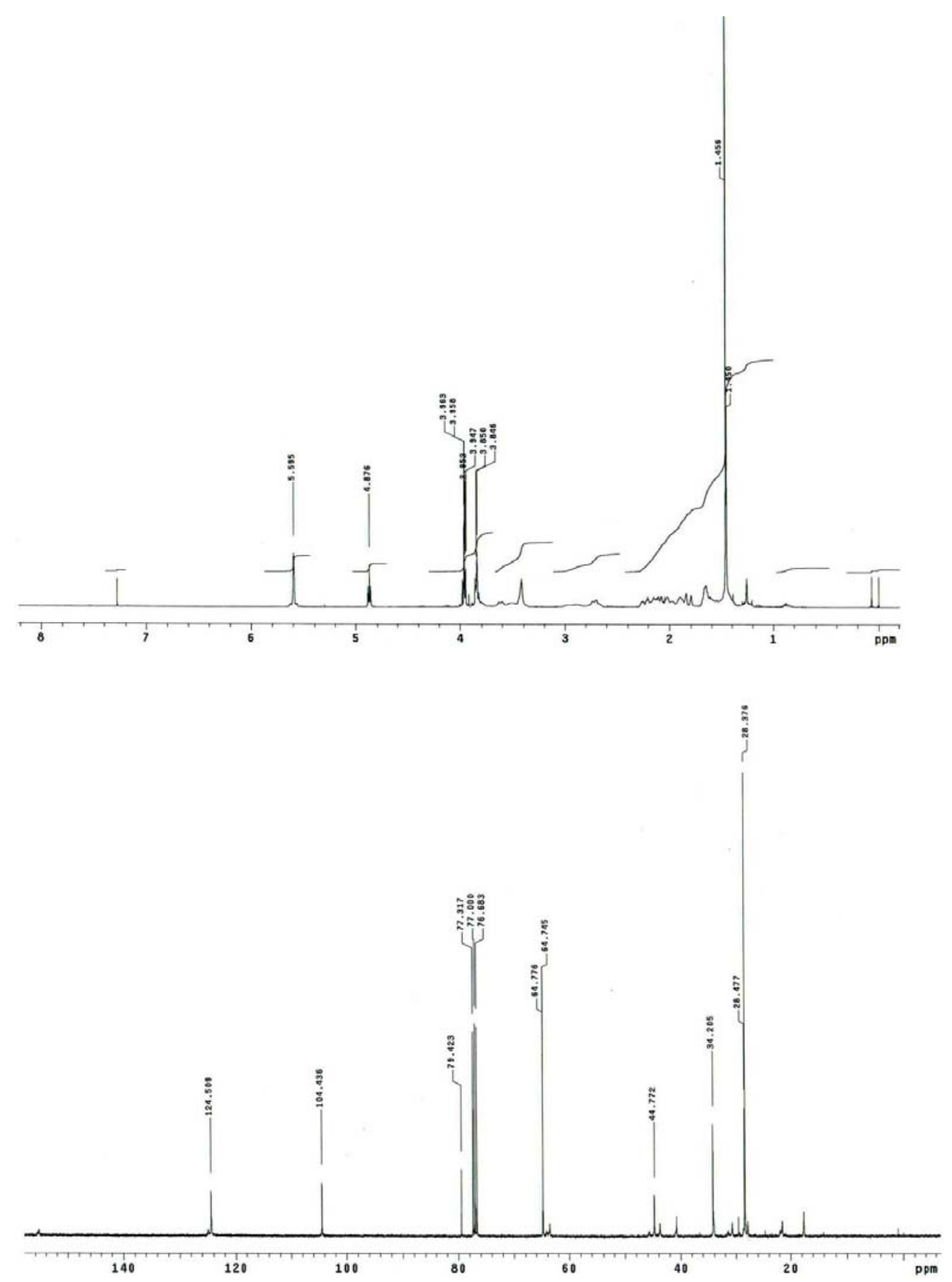


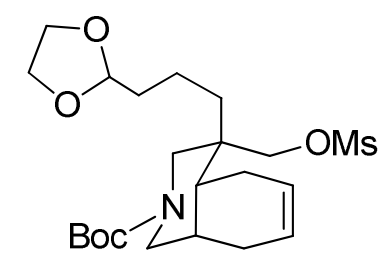

(4R,4aR,8aS)-2-(tert-Butoxycarbonyl)-4-[3-(1,3-dioxolan-2-yl)propyl]-4(methansulfonyloximethyl)-1,2,3,4,4a,5,8,8a-octahydroisoquinoline (39).

Anhydrous $\mathrm{Et}_{3} \mathrm{~N}(2.18 \mathrm{~mL}, 15.6 \mathrm{mmol})$ and methanesulfonyl chloride $(1.21 \mathrm{~mL}, 15.6 \mathrm{mmol})$ were added at $0{ }^{\circ} \mathrm{C}$ under an inert atmosphere to a stirred solution of alcohol 18 (1.98 g, 5.21 mmol) in anhydrous $\mathrm{CH}_{2} \mathrm{Cl}_{2}(86 \mathrm{~mL})$, and the resulting mixture was stirred at room temperature for $4 \mathrm{~h}$. The reaction was quenched with a saturated aqueous $\mathrm{NH}_{4} \mathrm{Cl}$ solution, and the mixture was extracted with $\mathrm{CH}_{2} \mathrm{Cl}_{2}$. The combined organic extracts were dried over anhydrous $\mathrm{Na}_{2} \mathrm{SO}_{4}$, filtered, and concentrated under reduced pressure. The resulting mesylate $\mathbf{3 9}$ was obtained as a yellow oil, which was used in the next step without purification.

${ }^{1} \mathrm{H}$ NMR (400 MHz, CDCl 3 , COSY, gHSQC): $\left.\delta 1.42\left(\mathrm{~m}, 2 \mathrm{H}, \mathrm{H}-2^{\prime}\right), 1.45,1.46\left[\mathrm{~s}, 9 \mathrm{H},\left(\mathrm{CH}_{3}\right)_{3} \mathrm{C}\right)\right], 1.64$ $\left(\mathrm{m}, 4 \mathrm{H}, \mathrm{H}-\mathrm{1}^{\prime}, \mathrm{H}-3^{\prime}\right), 1.83$ (d, J = $\left.17.6 \mathrm{~Hz}, 1 \mathrm{H}, \mathrm{H}-8\right), 1.94(\mathrm{~m}, 1 \mathrm{H}, \mathrm{H}-4 \mathrm{a}), 1.99$ (dm, J = $17.2 \mathrm{~Hz}, 1 \mathrm{H}$, $\mathrm{H}-5), 2.10(\mathrm{dm}, J=17.2 \mathrm{~Hz}, 1 \mathrm{H}, \mathrm{H}-5), 2.16(\mathrm{~m}, 1 \mathrm{H}, \mathrm{H}-8 \mathrm{a}), 2.25(\mathrm{dm}, J=17.6 \mathrm{~Hz}, 1 \mathrm{H}, \mathrm{H}-8), 2.78-$ $2.81(2 \mathrm{~s}, 2 \mathrm{H}, \mathrm{H}-1, \mathrm{H}-3), 3.03\left(\mathrm{~s}, 3 \mathrm{H}, \mathrm{CH}_{3} \mathrm{~S}\right), 3.66-3.73(2 \mathrm{~m}, 2 \mathrm{H}, \mathrm{H}-1, \mathrm{H}-3), 3.84,3.95(2 \mathrm{~m}, 4 \mathrm{H}$, $\left.2 \mathrm{CH}_{2} \mathrm{O}\right), 4.00\left(\mathrm{~s}, 2 \mathrm{H}, \mathrm{CH}_{2} \mathrm{OS}\right), 4.86\left(\mathrm{t}, J=4.4 \mathrm{~Hz}, 1 \mathrm{H}, \mathrm{H}-4^{\prime}\right), 5.60(\mathrm{~m}, 2 \mathrm{H}, \mathrm{H}-6, \mathrm{H}-7)$.

$\left.\left.{ }^{13} \mathrm{C} \mathrm{NMR}(100.6 \mathrm{MHz}, \mathrm{CDCl})^{3}\right): \delta 17.2\left(\mathrm{C}-2^{\prime}\right), 21.4(\mathrm{C}-5), 27.8(\mathrm{C}-8 \mathrm{a}), 28.3\left[\left(\mathrm{CH}_{3}\right)_{3} \mathrm{C}\right), \mathrm{C}-8\right], 31.0(\mathrm{C}-$ 1'), 33.5 (C-4a), 34.3 (C-3'), $37.1\left(\mathrm{CH}_{3} \mathrm{SO}\right), 39.8$ (C-4), 43.4-44.6 (C-1, C-3), 64.7, $64.8\left(2 \mathrm{CH}_{2} \mathrm{O}\right)$, $\left.70.2\left(\mathrm{CH}_{2} \mathrm{OS}\right), 79.8\left[\left(\mathrm{CH}_{3}\right)_{3} \mathrm{C}\right)\right], 104.1\left(\mathrm{C}-4^{\prime}\right), 123.8,124.5$ (C-6, C-7), 155.0 (NCOO).

HRMS (ESI) calcd for $\left[\mathrm{C}_{22} \mathrm{H}_{37} \mathrm{NO}_{7} \mathrm{~S}+\mathrm{NH}_{4}\right]^{+}:$477.2629, found: 477.2621 . 

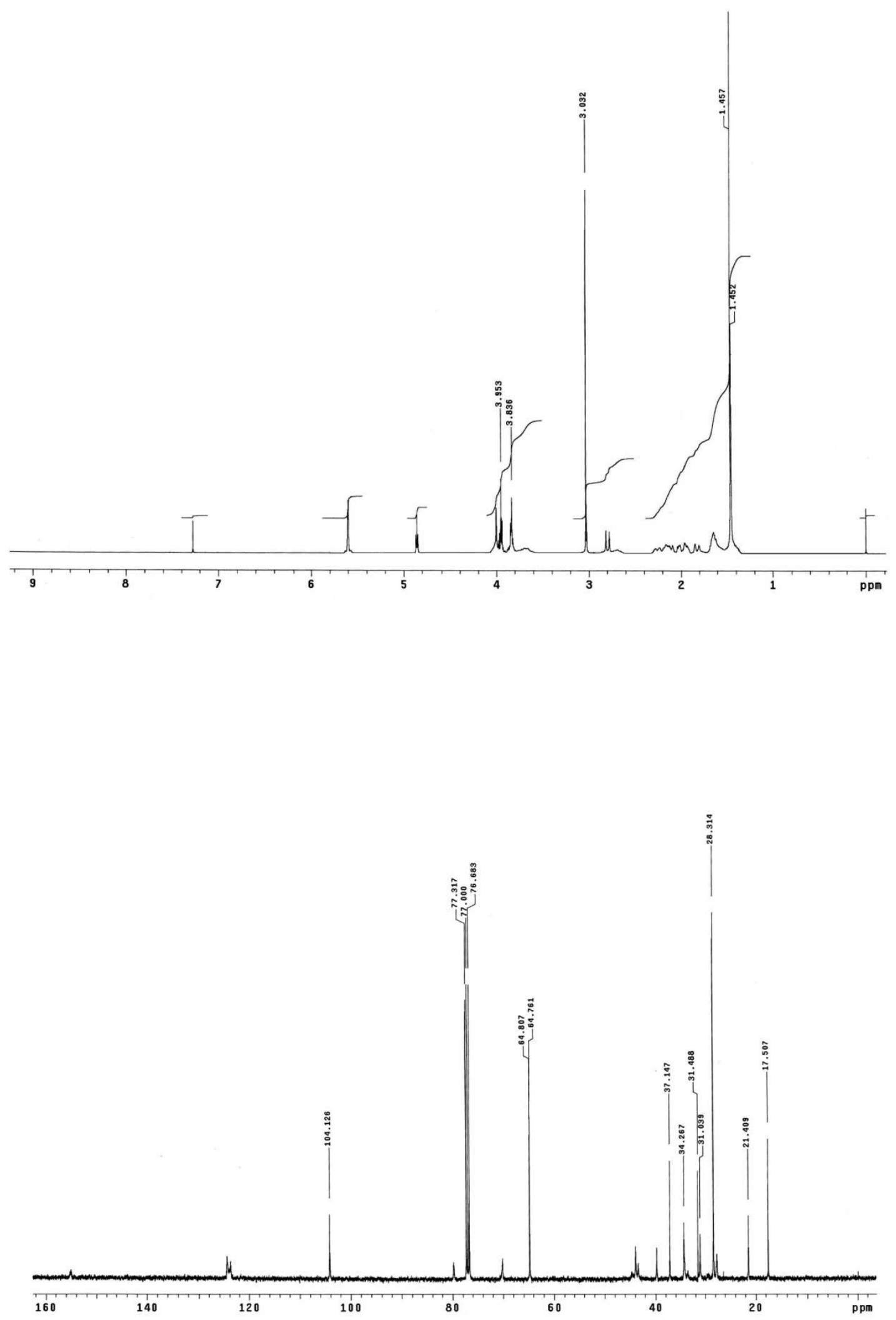


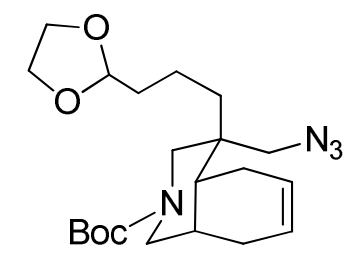

(4R,4aR,8aS)-4-(Azidomethyl)-2-(tert-butoxycarbonyl)-4-[3-(1,3-dioxolan-2-yl)propyl]$1,2,3,4,4 a, 5,8,8 a-o c t a h y d r o i s o q u i n o l i n e ~(40)$.

$\mathrm{NaN}_{3}(2.03 \mathrm{~g}, 31.2 \mathrm{mmol})$ was added to a solution of the above mesylate $39(5.21 \mathrm{mmol})$ in anhydrous DMF (15 mL), and the mixture was heated to $90^{\circ} \mathrm{C}$. After $48 \mathrm{~h}$, more $\mathrm{NaN}_{3}(2.03 \mathrm{~g}$, $31.2 \mathrm{mmol}$ ) was added, and the resulting mixture was stirred at $90{ }^{\circ} \mathrm{C}$ for an additional $24 \mathrm{~h}$ and quenched with distilled water. The aqueous layer was extracted with $\mathrm{CH}_{2} \mathrm{Cl}_{2}$, and the combined organic extracts were dried, filtered, and concentrated under reduced pressure to give an oil. Flash chromatography (9:1 hexane-EtOAc) afforded azide $\mathbf{4 0}$ (1.68 g, 79\%).

$[\alpha]_{D}^{22}=-45.3\left(\mathrm{c} 0.9, \mathrm{CHCl}_{3}\right)$.

IR (film): $2098\left(\mathrm{~N}_{3}\right), 1688(\mathrm{C}=\mathrm{O}) \mathrm{cm}^{-1}$.

$\left.\left.{ }^{1} \mathrm{H} \mathrm{NMR} \mathrm{(400} \mathrm{MHz,} \mathrm{CDCl}{ }_{3}, \mathrm{COSY}, \mathrm{gHSQC}\right): \delta 1.38\left(\mathrm{~m}, 2 \mathrm{H}, \mathrm{H}-2^{\prime}\right), 1.42\left[\mathrm{~s}, 10 \mathrm{H},\left(\mathrm{CH}_{3}\right)_{3} \mathrm{C}\right), \mathrm{H}-1^{\prime}\right], 1.60$ (m, 1H, H-1') $1.65\left(m, 2 \mathrm{H}, \mathrm{H}-3^{\prime}\right), 1.81(\mathrm{~d}, J=18.4 \mathrm{~Hz}, 1 \mathrm{H}, \mathrm{H}-8), 1.86(\mathrm{~m}, 1 \mathrm{H}, \mathrm{H}-4 \mathrm{a}), 1.95(\mathrm{dm}, J=$ $17.6 \mathrm{~Hz}, 1 \mathrm{H}, \mathrm{H}-5), 2.08(\mathrm{dm}, J=17.6 \mathrm{~Hz}, 1 \mathrm{H}, \mathrm{H}-5), 2.16(\mathrm{~m}, 1 \mathrm{H}, \mathrm{H}-8 \mathrm{a}), 2.24(\mathrm{~m}, 1 \mathrm{H}, \mathrm{H}-8), 2.61-$ $2.74(\mathrm{~m}, 2 \mathrm{H}, \mathrm{H}-1, \mathrm{H}-3), 3.22\left(\mathrm{~s}, 2 \mathrm{H}, \mathrm{CH}_{2} \mathrm{~N}_{3}\right), 3.62-3.73(2 \mathrm{~m}, 2 \mathrm{H}, \mathrm{H}-1, \mathrm{H}-3), 3.85,3.95(2 \mathrm{~m}, 4 \mathrm{H}$, $\left.2 \mathrm{CH}_{2} \mathrm{O}\right), 4.82\left(\mathrm{t}, J=4.4 \mathrm{~Hz}, 1 \mathrm{H}, \mathrm{H}-4^{\prime}\right), 5.60(\mathrm{~m}, 2 \mathrm{H}, \mathrm{H}-6, \mathrm{H}-7)$.

${ }^{13} \mathrm{C} \mathrm{NMR}\left(100.6 \mathrm{MHz}, \mathrm{CDCl}_{3}\right): \delta 17.7$ (C-2'), 21.5 (C-5), 27.8 (C-8a), 28.3 [(CH3)3C), C-8], 31.7 (C1'), 34.5 (C-4a), 34.8 (C-3'), 42.2 (C-4), 43.5-45.0 (C-1, C-3), $54.0\left(\mathrm{CH}_{2} \mathrm{~N}_{3}\right), 64.8\left(2 \mathrm{CH}_{2} \mathrm{O}\right), 79.7$ $\left.\left[\left(\mathrm{CH}_{3}\right)_{3} \mathrm{C}\right)\right], 104.3\left(\mathrm{C}-4^{\prime}\right), 123.9,124.6$ (C-6, C-7), 155.0 (NCOO).

HRMS (ESI) calcd for $\left[\mathrm{C}_{21} \mathrm{H}_{34} \mathrm{~N}_{4} \mathrm{O}_{4}+\mathrm{H}\right]^{+}:$: 407.2653, found: 407.2645 . 

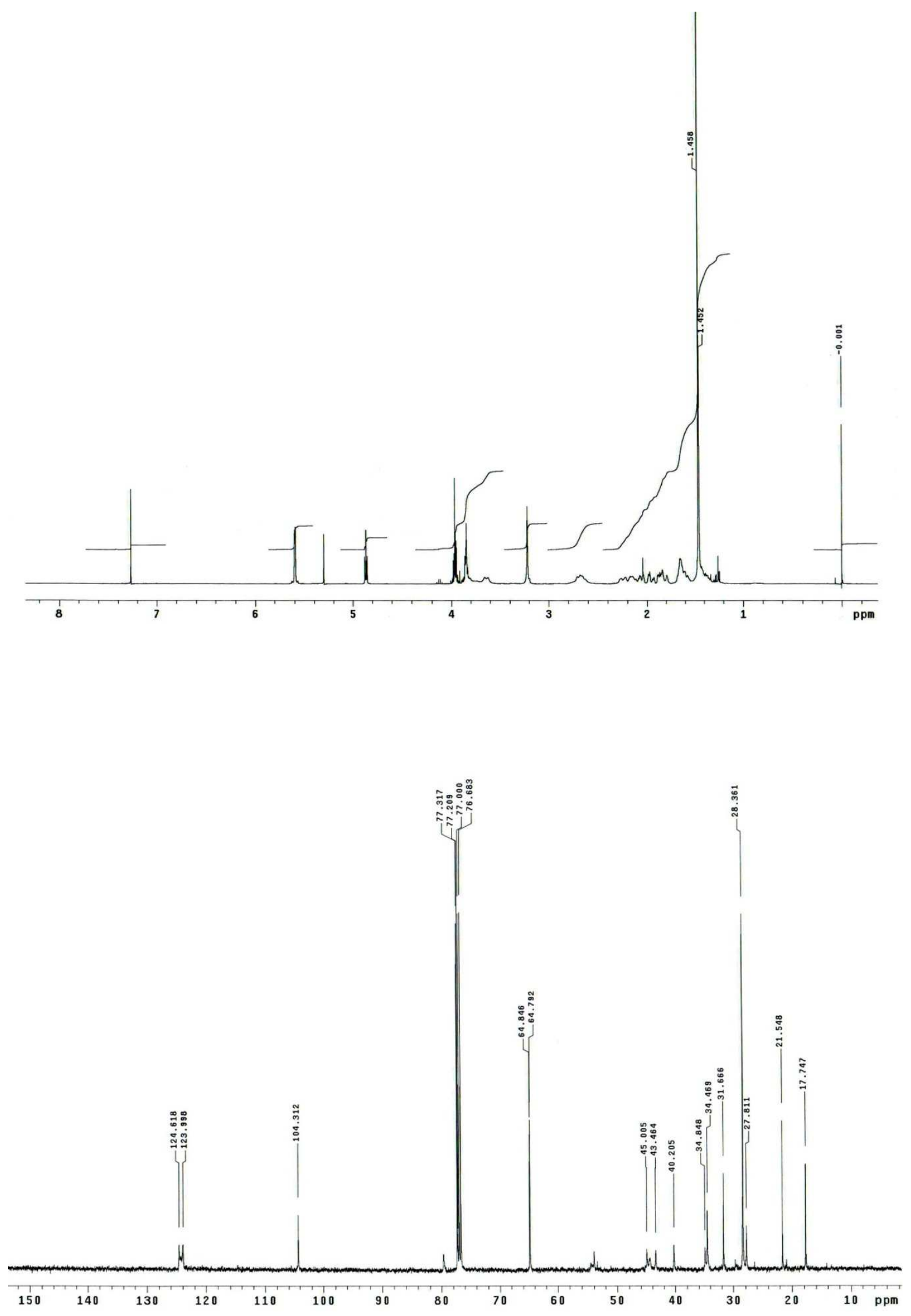


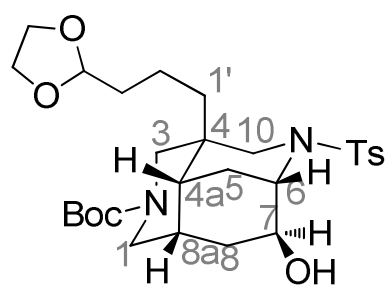

(IUPAC numbering)

(4R,4aR,6S,7S, 8aS)-2-(tert-Butoxycarbonyl)-4-[3-(1,3-dioxolan-2-yl)propyl]-7hydroxy-6,4-(iminomethano)-9-(p-toluenesulfonyl)perhydroisoquinoline (43).

First step: $m$-Chloroperoxybenzoic acid (1.48 g, $6.61 \mathrm{mmol}, \leq 77 \%$ of purity) was added to a cold $\left(0{ }^{\circ} \mathrm{C}\right)$ solution of azide $40(1.22 \mathrm{~g}, 3.00 \mathrm{mmol})$ in $\mathrm{CH}_{2} \mathrm{Cl}_{2}(43 \mathrm{~mL})$, and the mixture was allowed to warm slowly to room temperature. After $5 \mathrm{~h}$, the reaction was quenched with saturated aqueous $\mathrm{NaHCO}_{3}$, and the mixture was extracted with $\mathrm{CH}_{2} \mathrm{Cl}_{2}$. The organic extracts were dried (anhydrous $\mathrm{MgSO}_{4}$ ), filtered, and concentrated under reduced pressure to give azido epoxide 41, which was used in the next step without purification.

Second step: $\mathrm{Me}_{3} \mathrm{P}(4.8 \mathrm{~mL}$ of a $1 \mathrm{M}$ solution in $\mathrm{DMF}, 4.80 \mathrm{mmol})$ was added to a solution of the above azido epoxide $41(3.00 \mathrm{mmol})$ in THF $(60 \mathrm{~mL})$, and the mixture was stirred at room temperature for 2 hours. Water $(6 \mathrm{~mL})$ was added, and the resulting mixture was stirred overnight at room temperature and concentrated under reduced pressure to afford a diazatricyclic alcohol 42.

Third step: $\mathrm{Et}_{3} \mathrm{~N}(410 \mu \mathrm{L}, 3.00 \mathrm{mmol})$ was added dropwise to a stirring solution of the aminoalcohol 42 in anhydrous $\mathrm{CH}_{2} \mathrm{Cl}_{2}(50 \mathrm{~mL})$ at $0{ }^{\circ} \mathrm{C}$. A solution of $p$-toluenesulfonyl chloride (572 $\mathrm{mg}, 3.00 \mathrm{mmol}$ ) in anhydrous $\mathrm{CH}_{2} \mathrm{Cl}_{2}(5 \mathrm{~mL}$ ) was transferred via a cannula, and the stirring was continued at $0{ }^{\circ} \mathrm{C}$ for $2.5 \mathrm{~h}$. The reaction was then quenched with saturated aqueous $\mathrm{NH}_{4} \mathrm{Cl}$, and the aqueous layer was extracted with $\mathrm{CH}_{2} \mathrm{Cl}_{2}$. The combined organic extracts were dried over anhydrous $\mathrm{Na}_{2} \mathrm{SO}_{4}$, filtered, and concentrated under reduced pressure to afford the protected tricyclic compound $\mathbf{4 3}$ (998 $\mathrm{mg}, 60 \%$ overall yield from the azide 41) after flash chromatography (9:1 to 1:1 hexane-EtOAc).

$[\alpha]_{D}^{22}=+36.1\left(c 3.1, \mathrm{CHCl}_{3}\right)$.

IR (film): $3500(\mathrm{OH}), 1689(\mathrm{C}=0) \mathrm{cm}^{-1}$.

${ }^{1} \mathrm{H}$ NMR (400 MHz, CDCl $\left.{ }_{3}, \mathrm{COSY}, \mathrm{gHSQC}\right): \delta 1.19\left(\mathrm{~m}, 4 \mathrm{H}, \mathrm{H}-1^{\prime}, \mathrm{H}-2^{\prime}\right), 1.43\left[\mathrm{~s}, 11 \mathrm{H},\left(\mathrm{CH}_{3}\right)_{3} \mathrm{C}, \mathrm{H}-3^{\prime}\right]$, $1.52(\mathrm{~m}, 1 \mathrm{H}, \mathrm{H}-4 \mathrm{a}), 1.60-1.71(\mathrm{~m}, 3 \mathrm{H}, \mathrm{H}-5,2 \mathrm{H}-8), 1.94(\mathrm{~d}, J=14.4 \mathrm{~Hz}, 1 \mathrm{H}, \mathrm{H}-5), 2.04(\mathrm{~m}, 1 \mathrm{H}, \mathrm{H}-$ 8a), $2.43\left(\mathrm{~s}, 3 \mathrm{H}, \mathrm{CH}_{3} \mathrm{Ts}\right), 2.60-2.78(\mathrm{~m}, 2 \mathrm{H}, \mathrm{H}-1, \mathrm{H}-3), 3.12(\mathrm{~m}, 1 \mathrm{H}, \mathrm{H}-10), 3.28(\mathrm{~d}, \mathrm{~J}=13.2 \mathrm{~Hz}, 1 \mathrm{H}$, 
$\mathrm{H}-10), 3.84\left(\mathrm{~m}, 2 \mathrm{H}, \mathrm{CH}_{2} \mathrm{O}\right), 3.87(\mathrm{~m}, 1 \mathrm{H}, \mathrm{H}-7), 3.90$ (masked, $\left.2 \mathrm{H}, \mathrm{H}-3, \mathrm{H}-1\right), 3.95\left(\mathrm{~m}, 2 \mathrm{H}, \mathrm{CH}_{2} \mathrm{O}\right)$, 4.00 (s, 1H, H-6), $4.76\left(\mathrm{~m}, 1 \mathrm{H}, \mathrm{H}-4^{\prime}\right), 7.27(\mathrm{~d}, J=8.0 \mathrm{~Hz}, 2 \mathrm{H}, \mathrm{H}-m \mathrm{Ts}), 7.69$ (d, J = 8.0 Hz, 2H, H-O Ts).

${ }^{13} \mathrm{C}$ NMR (100.6 MHz, CDCl $): \delta 17.3\left(\mathrm{C}-2^{\prime}\right), 21.4\left(\mathrm{CH}_{3} \mathrm{Ts}\right), 21.9$ (C-5), $28.3\left[\left(\mathrm{CH}_{3}\right)_{3} \mathrm{C}\right], 30.3(\mathrm{C}-8 \mathrm{a})$, 32.5 (C-8), 32.8 (C-4), 34.0 (C-3'), 34.9 (C-4a), 36.1 (C-1'), 46.0-50.0 (C-10, C-1, C-3), 50.8 (C-6), 64.7, $64.8\left(2 \mathrm{CH}_{2} \mathrm{O}\right), 67.7$ (C-7), $79.8\left[\left(\mathrm{CH}_{3}\right)_{3} \mathrm{C}\right], 104.0$ (C-4'), 126.8 (C-o Ts), 129.6 (C-m Ts), 136.5 (C-i Ts), 143.1 (C-p Ts), 155.5 (NCOO).

HRMS (ESI) calcd for $\left[\mathrm{C}_{28} \mathrm{H}_{42} \mathrm{~N}_{2} \mathrm{O}_{7} \mathrm{~S}+\mathrm{H}\right]^{+}: 551.2785$, found: 551.2788 .
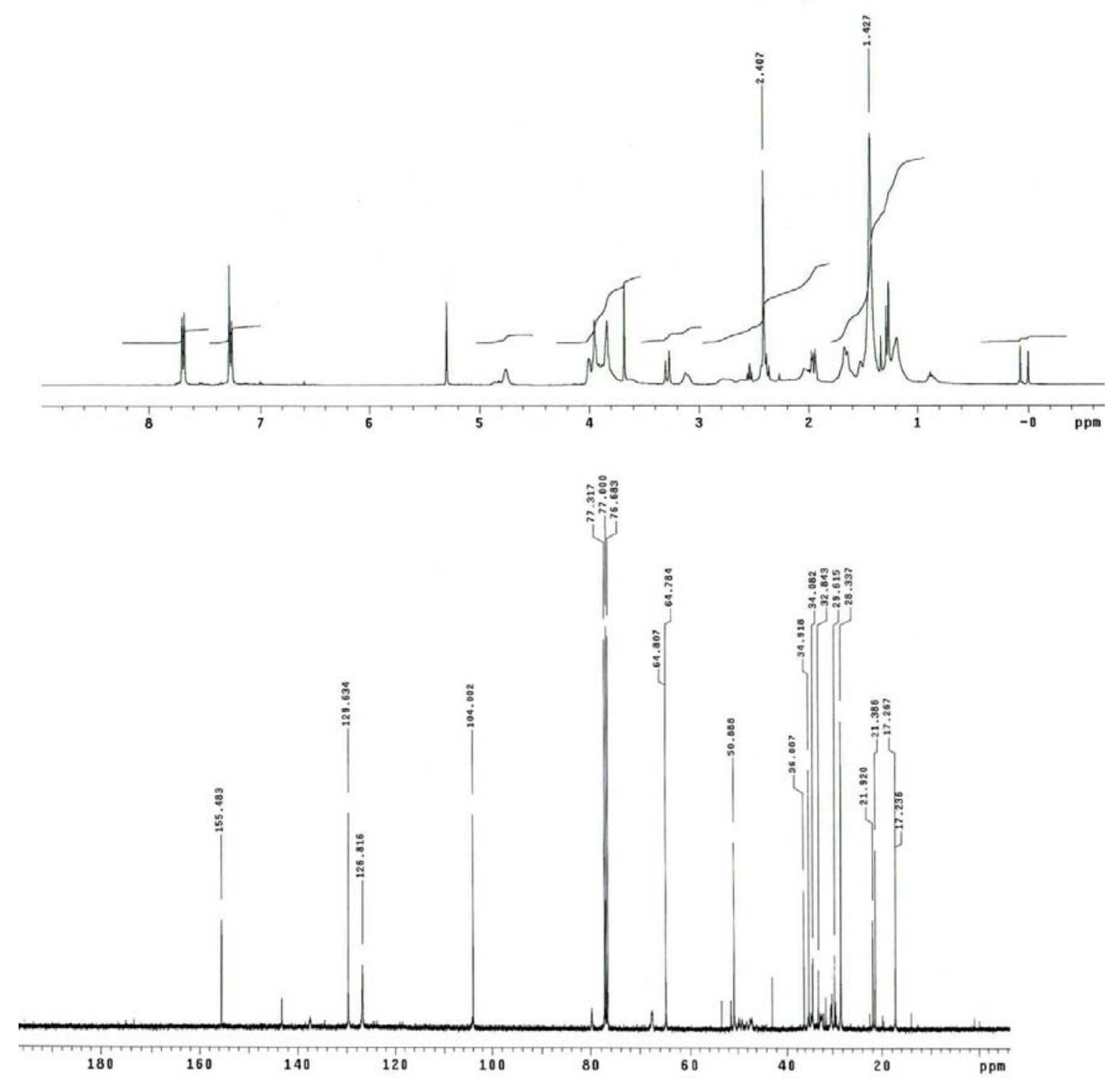


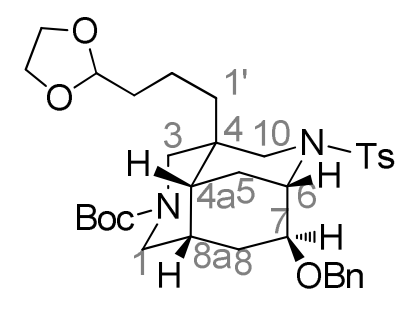

(IUPAC numbering)

(4R,4aR,6S,7S, 8aS)-7-(Benzyloxy)-2-(tert-butoxycarbonyl)-4-[3-(1,3-dioxolan-2-

yl)propyl]-6,4-(iminomethano)-9-(p-toluenesulfonyl)perhydroisoquinoline (44).

$\mathrm{NaH}$ (180 mg of a $60 \%$ dispersion in mineral oil, $4.53 \mathrm{mmol}$ ) was added to a solution of tricyclic compound 43 (1.66 g, $3.02 \mathrm{mmol})$ in anhydrous DMF (40 mL), and the mixture was stirred at 0 ${ }^{\circ} \mathrm{C}$ for $1 \mathrm{~h}$. Then, benzyl bromide $(2.58 \mathrm{~g}, 15.12 \mathrm{mmol}$, previously filtered over a neutral alumina pad) and TBAI (223 $\mathrm{mg}, 0.60 \mathrm{mmol}$ ) were added at room temperature, and the resulting suspension was stirred overnight. The reaction was quenched by the addition of distilled water, and the aqueous layer was extracted with $\mathrm{Et}_{2} \mathrm{O}$. The combined organic extracts were dried over anhydrous $\mathrm{Na}_{2} \mathrm{SO}_{4}$, filtered, and concentrated under reduced pressure. Flash chromatography (9:1 to 1:1 hexane-EtOAc) of the residue gave the benzyloxy derivative $\mathbf{4 4}$ $(1.55 \mathrm{~g}, 80 \%)$ as a colourless oil.

$[\alpha]_{D}^{22}=+38.8\left(c 0.75, \mathrm{CHCl}_{3}\right)$.

IR (film): $1689(\mathrm{C}=0) \mathrm{cm}^{-1}$.

${ }^{1} \mathrm{H}$ NMR $\left(400 \mathrm{MHz}, \mathrm{CDCl}_{3}, \mathrm{COSY}, \mathrm{gHSQC}\right): \delta 1.25$ (s, 4H, H-1', H-2'), 1.42 [s, $\left.11 \mathrm{H},\left(\mathrm{CH}_{3}\right)_{3} \mathrm{C}, \mathrm{H}-3^{\prime}\right]$, $1.50(\mathrm{~m}, 2 \mathrm{H}, \mathrm{H}-4 \mathrm{a}, \mathrm{H}-8), 1.70(\mathrm{dm}, J=13.6 \mathrm{~Hz}, 1 \mathrm{H}, \mathrm{H}-5), 1.75$ (m, 1H, H-8), 1.92 (dt, J = 13.6, 2.4 $\mathrm{Hz}, 1 \mathrm{H}, \mathrm{H}-5), 1.99$ (m, 1H, H-8a), 2.37 (s, 3H, $\left.\mathrm{CH}_{3} \mathrm{Ts}\right), 2.62-2.70$ (2m, 2H, H-1, H-3), 3.04 (d, J = $13.2 \mathrm{~Hz}, 1 \mathrm{H}, \mathrm{H}-10), 3.32$ (d, J = 13.2 Hz, 1H, H-10), 3.40 (br. s, 1H, H-7), 3.52-3.62 (2m, 2H, H-1, $\mathrm{H}-3), 3.84,3.95\left(2 \mathrm{~m}, 4 \mathrm{H}, 2 \mathrm{CH}_{2} \mathrm{O}\right), 4.20(\mathrm{~m}, 1 \mathrm{H}, \mathrm{H}-6), 4.51\left(\mathrm{~m}, 2 \mathrm{H}, \mathrm{CH}_{2} \mathrm{Bn}\right), 4.78\left(\mathrm{~m}, 1 \mathrm{H}, \mathrm{H}-4^{\prime}\right)$, 7.10-7.60 (m, $\left.9 \mathrm{H}, \mathrm{C}_{6} \mathrm{H}_{5}, \mathrm{Ts}\right)$.

${ }^{13} \mathrm{C} \mathrm{NMR}\left(100.6 \mathrm{MHz}, \mathrm{CDCl}_{3}\right): \delta 17.3\left(\mathrm{C}-2^{\prime}\right), 21.4\left(\mathrm{CH}_{3} \mathrm{Ts}\right), 22.6(\mathrm{C}-5), 28.4\left[\left(\mathrm{CH}_{3}\right)_{3} \mathrm{C}\right], 29.9(\mathrm{C}-8)$, 30.8 (C-8a), 31.9 (C-4), 34.2 (C-3'), 35.0 (C-4a), 36.1 (C-1'), 43.9 (C-3'), 47.0-49.9 (C-1, C-3), 48.2 (C-10), 51.0 (C-6), $64.8\left(2 \mathrm{CH}_{2} \mathrm{O}\right), 70.5\left(\mathrm{CH}_{2} \mathrm{Bn}\right), 73.4$ (C-7), $79.8\left[\left(\mathrm{CH}_{3}\right)_{3} \mathrm{C}\right], 104.1\left(\mathrm{C}-4^{\prime}\right), 126.7-$ 129.6 (C-o, $m$ Ts, C-o, $m, p \mathrm{C}_{6} \mathrm{H}_{5}$ ), 137.4 (C-i Ts), 138.5 (C-i $\mathrm{C}_{6} \mathrm{H}_{5}$ ), 143.0 (C-p Ts), 155.5 (NCOO). HRMS (ESI) calcd for $\left[\mathrm{C}_{35} \mathrm{H}_{48} \mathrm{~N}_{2} \mathrm{O}_{7} \mathrm{~S}+\mathrm{H}\right]^{+}: 641.3255$, found: 641.3255 . 

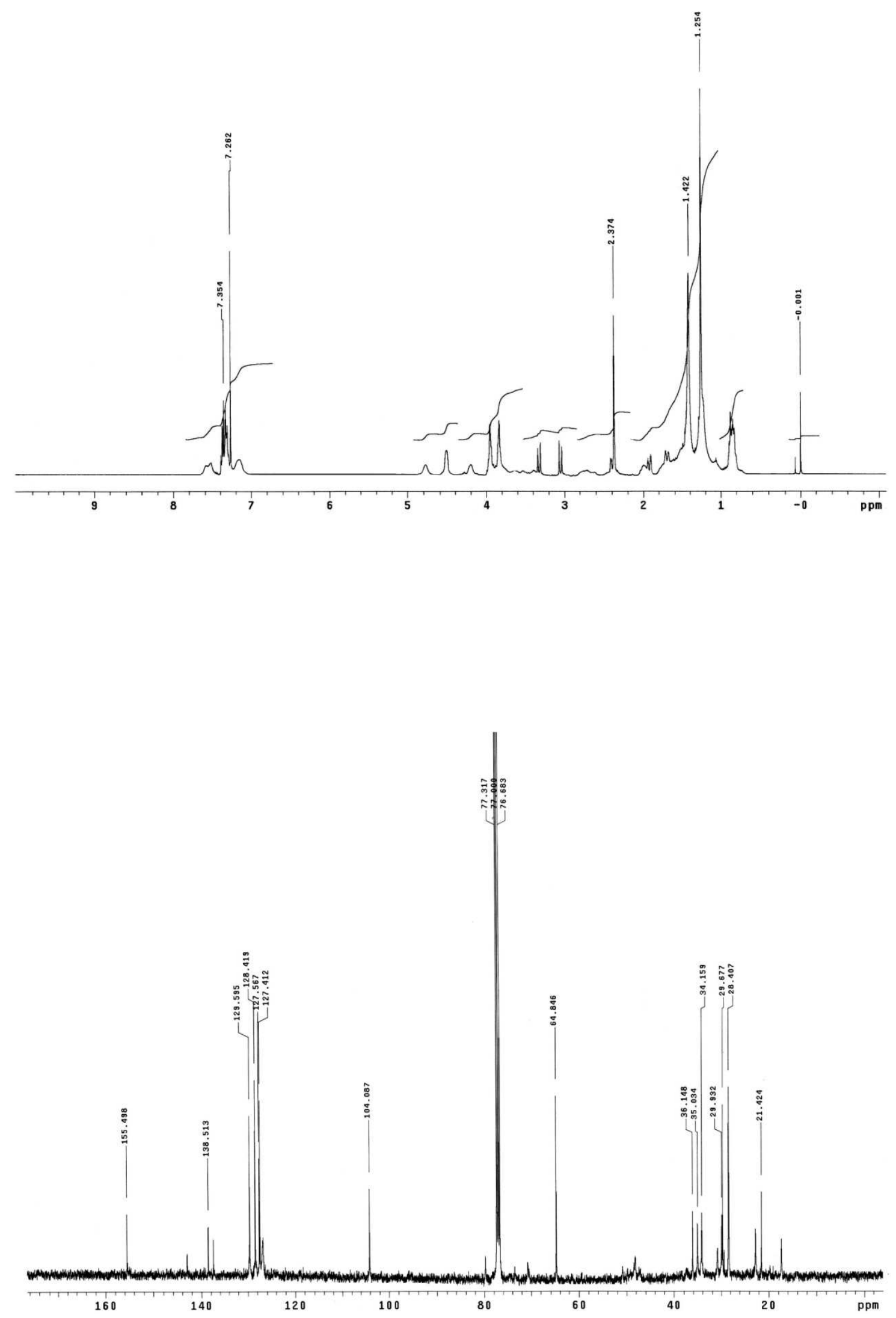


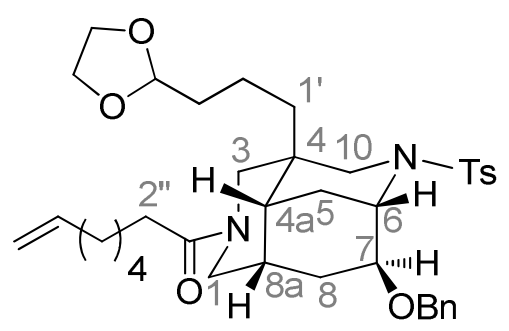

(IUPAC numbering)

(4R,4aR,6S,7S,8aS)-7-(Benzyloxy)-4-[3-(1,3-dioxolan-2-yl)propyl]-6,4-(iminomethano)-2-(7octenoyl)-9-(p-toluenesulfonyl)perhydroisoquinoline (102).

First step: TFA $(7 \mathrm{~mL})$ was added to a solution of the above tricyclic compound $(1.15 \mathrm{~g}, 1.79$ mmol) in anhydrous $\mathrm{CH}_{2} \mathrm{Cl}_{2}(21 \mathrm{~mL})$, and the mixture was stirred at room temperature for 30 minutes. Toluene $(2 \times 20 \mathrm{~mL})$ was added, and the resulting solution was concentrated under reduced pressure to give the deprotected derivative 100 , which was used in the next step without purification.

${ }^{1} \mathrm{H}$ NMR (400 MHz, CDCl 3 , COSY, gHSQC): $\delta 1.22\left(\mathrm{~m}, 2 \mathrm{H}, \mathrm{H}-2^{\prime}\right), 1.36\left(\mathrm{~m}, 1 \mathrm{H}, \mathrm{H}-1^{\prime}\right), 1.52(\mathrm{~m}, 3 \mathrm{H}$, $\left.2 \mathrm{H}-3^{\prime}, \mathrm{H}-1^{\prime}\right), 1.65(\mathrm{~m}, 1 \mathrm{H}, \mathrm{H}-4 \mathrm{a}), 1.78(\mathrm{dm}, J=13.6 \mathrm{~Hz}, 2 \mathrm{H}, \mathrm{H}-4 \mathrm{a}, \mathrm{H}-8), 1.90(\mathrm{dm}, J=13.6 \mathrm{~Hz}, 1 \mathrm{H}$, $\mathrm{H}-8), 1.98(\mathrm{dm}, J=13.6 \mathrm{~Hz}, 1 \mathrm{H}, \mathrm{H}-5), 2.25(\mathrm{~m}, 1 \mathrm{H}, \mathrm{H}-8 \mathrm{a}), 2.37$ (s, 3H, $\left.\mathrm{CH}_{3} \mathrm{Ts}\right), 2.78,2.98-3.05$ $(2 \mathrm{~m}, 4 \mathrm{H}, \mathrm{H}-1, \mathrm{H}-3), 3.31$ (br. s, $1 \mathrm{H}, \mathrm{H}-7$ ), 3.48 (d, J = 13.2 Hz, 1H, H-10), 3.53 (d, J=13.2 Hz, 1H, $\mathrm{H}-10), 3.82,3.94\left(2 \mathrm{~m}, 4 \mathrm{H}, 2 \mathrm{CH}_{2} \mathrm{O}\right), 4.18$ (br. $\left.\mathrm{s}, 1 \mathrm{H}, \mathrm{H}-6\right), 4.43\left(\mathrm{~m}, 2 \mathrm{H}, \mathrm{CH}_{2} \mathrm{Bn}\right), 4.79(\mathrm{t}, J=4.4 \mathrm{~Hz}$, $\left.1 \mathrm{H}, \mathrm{H}-4^{\prime}\right)$, 7.15-7.57 (m, 9H, $\left.\mathrm{C}_{6} \mathrm{H}_{5}, \mathrm{Ts}\right)$.

${ }^{13} \mathrm{C} \mathrm{NMR}\left(100.6 \mathrm{MHz}, \mathrm{CDCl}_{3}\right): \delta 17.0\left(\mathrm{C}-2{ }^{\prime}\right), 21.4\left(\mathrm{CH}_{3} \mathrm{Ts}\right), 22.5$ (C-5), 27.7 (C-8a), 28.9 (C-8), 32.3 (C-4a), 33.1 (C-4), 33.6 (C-3'), 36.0 (C-1'), 46.2 (C-10), 47.6, 48.7 (C-1, C-3), 47.8 (C-6), 64.8 (2 $\left.\mathrm{CH}_{2} \mathrm{O}\right), 70.7\left(\mathrm{CH}_{2} \mathrm{Bn}\right.$ ), 72.8 (C-7), 103.7 (C-4'), 126.6-129.8 (C-o, $m$ Ts, C-o, $m, p \mathrm{C}_{6} \mathrm{H}_{5}$ ), 137.2 (C-i Ts), $138.0\left(\mathrm{C}-i \mathrm{C}_{6} \mathrm{H}_{5}\right), 143.4$ (C-p Ts).

HRMS (ESI) calcd for $\left[\mathrm{C}_{30} \mathrm{H}_{40} \mathrm{~N}_{2} \mathrm{O}_{5} \mathrm{~S}+\mathrm{H}\right]^{+}: 541.2731$, found: 541.2736 . 

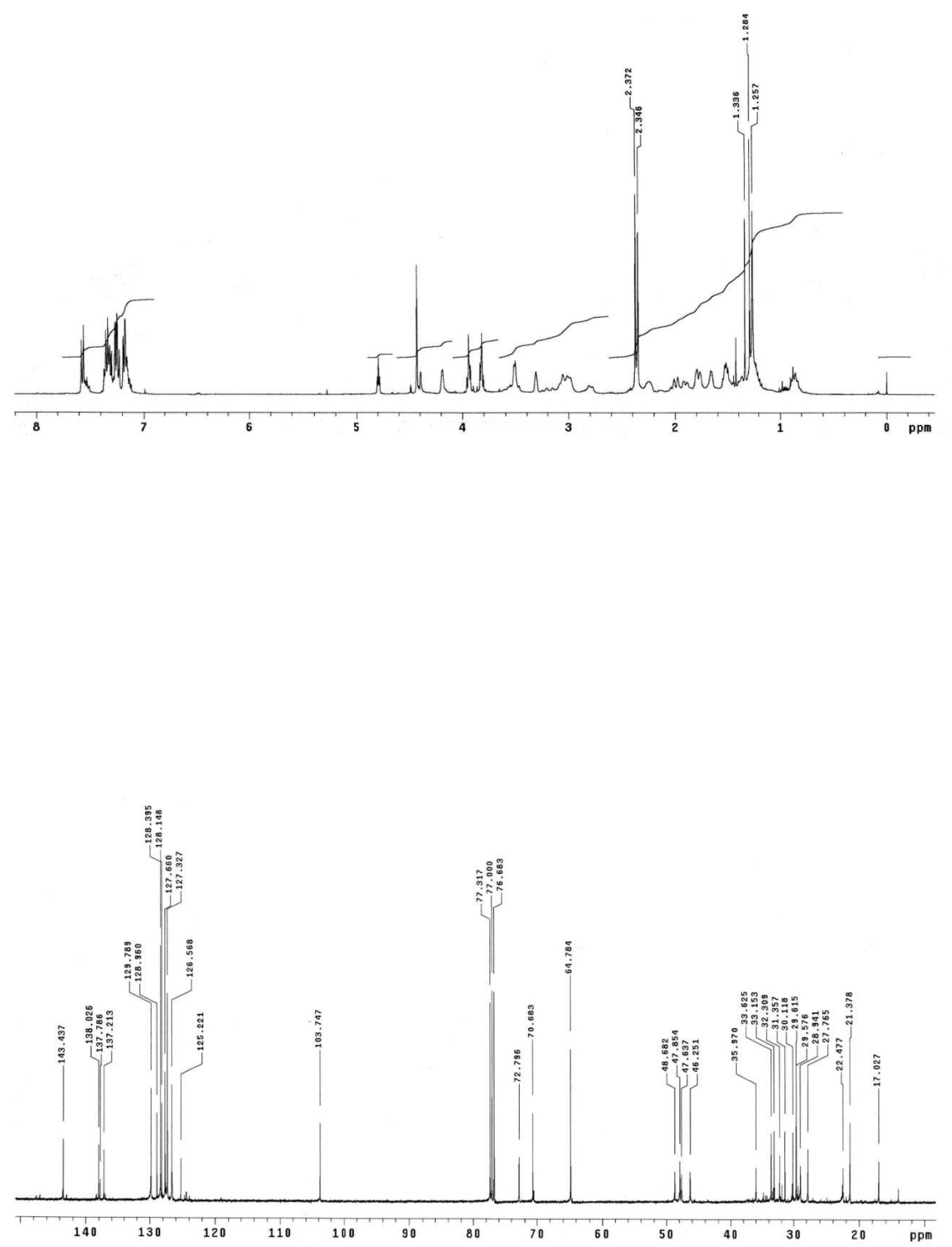
Preparation of 7-octenoyl chloride: $\mathrm{A} \mathrm{CH}_{2} \mathrm{Cl}_{2}$ solution of oxalyl chloride $(2 \mathrm{M}, 5.8 \mathrm{~mL}, 11.7 \mathrm{mmol})$ was added to a mixture of 7-octenoic acid $(1.37 \mathrm{~mL}, 8.97 \mathrm{mmol})$ and 2 drops of $\mathrm{DMF}$, and the resulting mixture was stirred at room temperature for $15 \mathrm{~min}$. $\mathrm{Et}_{2} \mathrm{O}$ was added, and the resulting mixture was filtered and concentrated under reduced pressure to give crude 7octenoyl chloride.

Second step: $\mathrm{Et}_{3} \mathrm{~N}(1.30 \mathrm{~mL}, 9.33 \mathrm{mmol})$ was added at $0{ }^{\circ} \mathrm{C}$ to a solution of the above secondary amine 100 (1.79 mmol) in anhydrous $\mathrm{CH}_{2} \mathrm{Cl}_{2}(85 \mathrm{~mL})$, and the resulting mixture was stirred at 0 ${ }^{\circ} \mathrm{C}$ for $10 \mathrm{~min}$. A solution of the freshly prepared $101(8.97 \mathrm{mmol})$ in anhydrous $\mathrm{CH}_{2} \mathrm{Cl}_{2}(4 \mathrm{~mL})$ was added at $0{ }^{\circ} \mathrm{C}$, and the mixture was stirred at $0{ }^{\circ} \mathrm{C}$ for $3 \mathrm{~h}$ and at room temperature overnight. Distilled water was added, and the resulting mixture was stirred for 20 minutes. The aqueous layer was extracted with $\mathrm{CH}_{2} \mathrm{Cl}_{2}$, and the combined organic extracts were dried over anhydrous $\mathrm{Na}_{2} \mathrm{SO}_{4}$, filtered, and concentrated under reduced pressure. Flash chromatography (hexane to 8:2 hexane-EtOAc) of the residue afforded amide 102 (1.09 g, $92 \%$, overall yield from the Boc derivative 44).

${ }^{1} \mathrm{H}$ NMR (400 MHz, CDCl 3 , COSY, gHSQC): $\delta 1.20-1.50$ (m, 10H, H-1', H-2', H-3', H-4", H-5") 1.59 (m, 3H, H-4a, 2H-3") $, 1.64(\mathrm{~m}, 1 \mathrm{H}, \mathrm{H}-8), 1.69(\mathrm{dm}, J=13.2 \mathrm{~Hz}, 1 \mathrm{H}, \mathrm{H}-5), 1.78(\mathrm{td}, J=14.8,5.6$ $\mathrm{Hz}, 1 \mathrm{H}, \mathrm{H}-8), 1.93(\mathrm{dt}, J=13.2,2.8 \mathrm{~Hz}, 1 \mathrm{H}, \mathrm{H}-5), 2.06$ (m, 3H, H-8a, 2H-6"), 2.25 (m, 2H, H-2")), 2.29, 2.86 (d, $J=14.0 \mathrm{~Hz}, 1 \mathrm{H}, \mathrm{H}-3 \mathrm{ax}$ ), 2.39 (s, 3H, $\mathrm{CH}_{3} \mathrm{Ts}$ ), 2.53, 3.06 (2dd, $J=13.2,2.8 \mathrm{~Hz}, 1 \mathrm{H}$, $\mathrm{H}-1), 3.00,3.27(2 \mathrm{~d}, J=13.6 \mathrm{~Hz}, 1 \mathrm{H}, \mathrm{H}-10), 3.05,3.36(2 \mathrm{~d}, J=13.6 \mathrm{~Hz}, 1 \mathrm{H}, \mathrm{H}-10), 3.37,4.30(2 \mathrm{~d}$, $J=13.2 \mathrm{~Hz}, 1 \mathrm{H}, \mathrm{H}-3), 3.40,3.56(2 \mathrm{br} . \mathrm{s}, 1 \mathrm{H}, \mathrm{H}-7), 3.54,4.43(2 \mathrm{~d}, J=13.2 \mathrm{~Hz}, 1 \mathrm{H}, \mathrm{H}-1), 3.82,3.93$ $\left(2 \mathrm{~m}, 4 \mathrm{H}, 2 \mathrm{CH}_{2} \mathrm{O}\right), 4.14,4.22(2 \mathrm{br} . \mathrm{s}, 1 \mathrm{H}, \mathrm{H}-6), 4.50$ (d, J = $12.4 \mathrm{~Hz}, 1 \mathrm{H}, \mathrm{CH}_{2} \mathrm{Bn}$ ), 4.56 (d, $J=12.0$ $\left.\mathrm{Hz}, 1 \mathrm{H}, \mathrm{CH}_{2} \mathrm{Bn}\right), 4.73,4.79\left(2 \mathrm{t}, J=4.8 \mathrm{~Hz}, 1 \mathrm{H}, \mathrm{H}-4^{\prime}\right), 4.93-5.04\left(\mathrm{~m}, 2 \mathrm{H}, \mathrm{CH}_{2}=\right), 5.80(\mathrm{tdt}, J=13.2$, $13.2,10.0,6.8,6.8 \mathrm{~Hz}, 1 \mathrm{H}, \mathrm{CH}=)$, 7.05-7.60 (m, $\left.9 \mathrm{H}, \mathrm{Ts}, \mathrm{C}_{6} \mathrm{H}_{5}\right)$.

${ }^{13} \mathrm{C}$ NMR (100.6 MHz, $\left.\mathrm{CDCl}_{3}\right): \delta$ 17.3, 17.4 (C-2'), $21.4\left(\mathrm{CH}_{3} \mathrm{Ts}\right), 22.3,22.6$ (C-5), 24.9 (C-3"), 28.6, 28.7, 28.8, 28.9 (C-4", C-5"), 29.7, 30.0 (C-8), 30.4, 31.0 (C-8a), 33.1, 33.2 (C-2"), 33.5 (C6"), 34.4 (C-3'), 34.6, 34.8 (C-4a), 34.9, 35.3 (C-4), 36.1, 36.2 (C-1'), 43.9, 50.5 (C-1), 46.5, 46.8 (C-10), 47.8, 48.4 (C-6), 48.0, 52.2 (C-3), 64.7, $64.8\left(2 \mathrm{CH}_{2} \mathrm{O}\right), 70.5,70.8\left(\mathrm{CH}_{2} \mathrm{Bn}\right), 73.7,74.7$ (C7), 103.9, 104.0 (C-4'), 114.3, $144.4\left(\mathrm{CH}_{2}=\right)$, 126.6-129.6 (C-o, $m$ Ts, C-o, $\left.m, p \mathrm{C}_{6} \mathrm{H}_{5}\right), 137.4-138.8$ (C-i Ts, $\mathrm{C}_{6} \mathrm{H}_{5}, \mathrm{CH}=$ ), 143.0, 143.2 (C- $p$ Ts), 172.6, 172.7 (NCO).

HRMS (ESI) calcd for $\left[\mathrm{C}_{38} \mathrm{H}_{52} \mathrm{~N}_{2} \mathrm{O}_{6} \mathrm{~S}+\mathrm{H}\right]^{+}: 665.3619$, found: 665.3608 . 

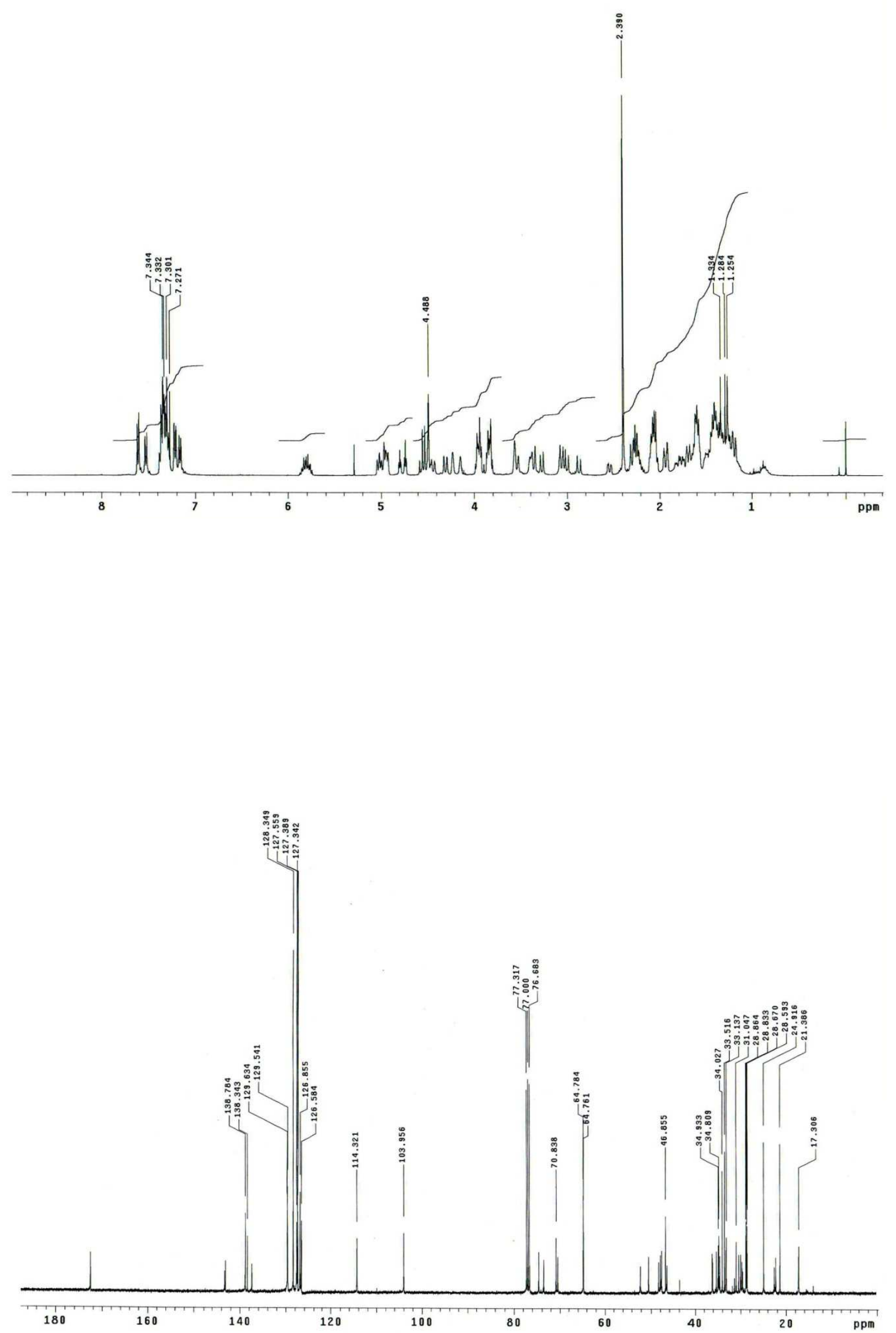


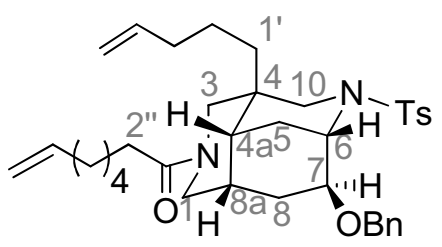

(IUPAC numbering)

(4R,4aR,6S,7S,8aS)-7-(Benzyloxy)-6,4-(iminomethano)-2-(7-octenoyl)-4-(4-pentenyl)

$-2-(p-$ toluenesulfonyl)perhydroisoquinoline (98).

First step: Aqueous $\mathrm{HCl}(3.0 \mathrm{~mL}$ of a $1 \mathrm{M}$ solution, $3.0 \mathrm{mmol})$ was added to a solution of acetal $102(100 \mathrm{mg}, 0.15 \mathrm{mmol})$ in THF $(1.0 \mathrm{~mL})$, and the resulting mixture was stirred at room temperature for $2 \mathrm{~h}$. The reaction was quenched by addition of solid $\mathrm{K}_{2} \mathrm{CO}_{3}$, and the mixture was basified to $\mathrm{pH}$ 8. The resulting mixture was extracted with $\mathrm{CH}_{2} \mathrm{Cl}_{2}$, and the combined organic extracts were washed with brine, dried over anhydrous $\mathrm{Na}_{2} \mathrm{SO}_{4}$, filtered, and concentrated under reduced pressure to give the corresponding aldehyde $\mathbf{1 0 3}$ as a yellow oil, which was used in the next step without purification.

${ }^{1} \mathrm{H}$ NMR (400 MHz, CDCl 3 , COSY, gHSQC): $\delta 1.25-1.49\left(\mathrm{~m}, 8 \mathrm{H}, \mathrm{H}-1^{\prime}, \mathrm{H}-2^{\prime}, \mathrm{H}-4^{\prime \prime}, \mathrm{H}-5^{\prime \prime}\right), 1.58-1.65$ (m, 4H, H-8, H-4a, 2H-3"), 1.74 (dm, J = $14.0 \mathrm{~Hz}, 1 \mathrm{H}, \mathrm{H}-5$ ), 1.79 (dd, J = 14.0, 6.4 Hz, 1H, H-8), $1.96(\mathrm{dm}, J=14.0 \mathrm{~Hz}, 1 \mathrm{H}, \mathrm{H}-5), 2.07\left(\mathrm{~m}, 3 \mathrm{H}, \mathrm{H}-8 \mathrm{a}, 2 \mathrm{H}-6^{\prime \prime}\right), 2.26\left(\mathrm{t}, J=7.6 \mathrm{~Hz}, 4 \mathrm{H}, \mathrm{H}-2^{\prime \prime}, \mathrm{H}-3^{\prime}\right)$, 2.35, $2.90(\mathrm{~d}, J=13.2 \mathrm{~Hz}, 1 \mathrm{H}, \mathrm{H}-3 \mathrm{ax}), 2.39$ (s, 3H, $\mathrm{CH}_{3} \mathrm{Ts}$ ), 2.55, 3.08 (dd, J = 13.2, 3.2 Hz, $1 \mathrm{H}, \mathrm{H}-$ 1ax), 2.98, $3.03(2 \mathrm{~d}, J=13.6 \mathrm{~Hz}, 1 \mathrm{H}, \mathrm{H}-10 \mathrm{ax}), 3.29,3.38$ (2d, $J=13.6 \mathrm{~Hz}, 1 \mathrm{H}, \mathrm{H}-10 \mathrm{eq}), 3.33,3.50$ (2br. s, 1H, H-7), 3.41, 4.34 (2d, J = 13.2 Hz, 1H, H-3eq), 3.55, 4.45 (2d, J = 13.2 Hz, 1H, H-1eq), 4.14, 4.22 (2br. s, $1 \mathrm{H}, \mathrm{H}-6$ ), 4.48 (d, $J=12.0 \mathrm{~Hz}, 1 \mathrm{H}, \mathrm{CH}_{2} \mathrm{Bn}$ ), 4.55 (d, $J=12.0 \mathrm{~Hz}, 1 \mathrm{H}, \mathrm{CH}_{2} \mathrm{Bn}$ ), $4.97\left(\mathrm{~m}, 2 \mathrm{H}, \mathrm{CH}_{2}=\right), 5.81(\mathrm{~m}, 1 \mathrm{H}, \mathrm{CH}=)$, 7.13-7.60 (m, 9H, Ts, $\left.\mathrm{C}_{6} \mathrm{H}_{5}\right), 9.67,9.75(2 \mathrm{~s}, 1 \mathrm{H}, \mathrm{CHO})$.

${ }^{13} \mathrm{C}$ NMR $\left(100.6 \mathrm{MHz}, \mathrm{CDCl}_{3}\right): \delta 15.3,15.5\left(\mathrm{C}-2^{\prime}\right), 21.0,21.4\left(\mathrm{CH}_{3} \mathrm{Ts}\right), 22.4,22.5$ (C-5), 24.9 (C3"), 28,6, 28.7, 28.8, 28.9 (C-4", C-5"), 29.7, 30.0 (C-8), 30.5, 31.0 (C-8a), 31.5 (C-4), 33.1, 33.2 (C-2") 33.5 (C-6") , 34.6, 34.9 (C-4a), 35.4, 35.5 (C-1'), 43.9 (C-3'), 46.5, 50.1 (C-1), 46.6 (C-10), 47.8, 52.1 (C-3), 47.9, 48.4 (C-6), 70.4, $70.7\left(\mathrm{CH}_{2} \mathrm{Bn}\right), 73.2,74.2$ (C-7), 114.3, $114.4\left(\mathrm{CH}_{2}=\right)$, 126.6-129.6 (C-o, $m$ Ts, C-o, $m, p \mathrm{C}_{6} \mathrm{H}_{5}$ ), 137.3-138.9 (C-i Ts, C-i $\mathrm{C}_{6} \mathrm{H}_{5}, \mathrm{CH}=$ ), 143.0 (C-p Ts), 172.5, 172.7 (NCO), 201.4, 201.5 (CHO).

HRMS (maldi) calcd for $\left[\mathrm{C}_{36} \mathrm{H}_{48} \mathrm{~N}_{2} \mathrm{O}_{5} \mathrm{~S}+\mathrm{Na}\right]^{+}: 643.32$, found: 643.30 . 

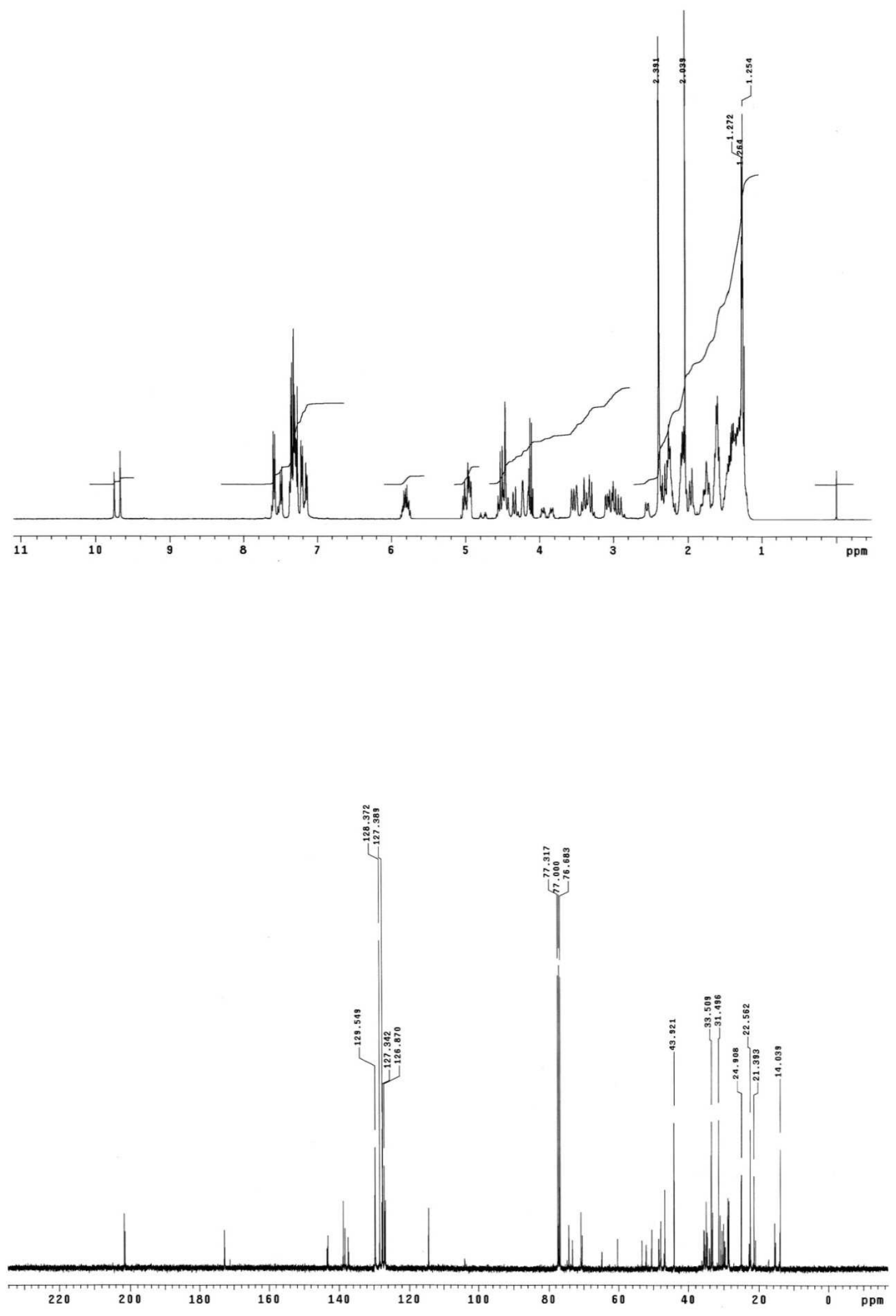
Second step: KOtBu $(750 \mu \mathrm{L}$ of a $1 \mathrm{M}$ solution in THF, $0.75 \mathrm{mmol}$ ) was added dropwise to a solution of methyltriphenylphosphonium bromide ( $375 \mathrm{mg}, 1.05 \mathrm{mmol}$ ) in THF (4 mL), and the solution was stirred at room temperature for $1 \mathrm{~h}$. The resulting yellow suspension was added to a solution of the above aldehyde $103(0.15 \mathrm{mmol})$ in THF $(4 \mathrm{~mL})$ and the mixture was stirred at room temperature overnight. The reaction was quenched by addition of saturated aqueous $\mathrm{NH}_{4} \mathrm{Cl}$, and the aqueous layer was extracted with $\mathrm{Et}_{2} \mathrm{O}$. The combined organic extracts were dried over anhydrous $\mathrm{Na}_{2} \mathrm{SO}_{4}$, filtered, and concentrated under reduced pressure. Flash chromatography (hexane to 6:4 hexane-EtOAc) of the resulting oil afforded diene $98 \mathbf{8}(73 \mathrm{mg}$, $80 \%)$.

$[\alpha]_{D}^{22}=+38.5\left(c 1.5, \mathrm{CHCl}_{3}\right)$.

IR (film): $1641(\mathrm{C}=0) \mathrm{cm}^{-1}$.

${ }^{1} \mathrm{H}$ NMR (400 MHz, CDCl, COSY, gHSQC): $\delta 1.08-1.43\left(\mathrm{~m}, 8 \mathrm{H}, 2 \mathrm{H}-1^{\prime}, 2 \mathrm{H}-2^{\prime}, 2 \mathrm{H}-4{ }^{\prime \prime}, 2 \mathrm{H}-5^{\prime \prime}\right), 1.55$ (m, 1H, H-4a), $1.61\left(\mathrm{~m}, 3 \mathrm{H}, 2 \mathrm{H}-3^{\prime \prime}, \mathrm{H}-8\right), 1.71(\mathrm{dm}, \mathrm{J}=14.8 \mathrm{~Hz}, 1 \mathrm{H}, \mathrm{H}-5), 1.80\left(\mathrm{~m}, 2 \mathrm{H}, \mathrm{H}-3^{\prime}, \mathrm{H}-8\right)$, $1.91\left(\mathrm{~m}, 1 \mathrm{H}, \mathrm{H}-3^{\prime}\right), 1.94(\mathrm{dm}, J=14.8 \mathrm{~Hz}, 1 \mathrm{H}, \mathrm{H}-5), 2.06\left(\mathrm{~m}, 3 \mathrm{H}, \mathrm{H}-6^{\prime \prime}, \mathrm{H}-8 \mathrm{a}\right), 2.20,2.84(2 \mathrm{~d}, J=$ $14.0 \mathrm{~Hz}, 1 \mathrm{H}, \mathrm{H}-3 \mathrm{ax}$ ), 2.26 (t, J = 8.0 Hz, 2H, H-2"), 2.40 (s, 3H, CH $\mathrm{CH}_{3} \mathrm{Ts}$ ), 2.54, 3.07 (2dd, J = 12.8, $2.8 \mathrm{~Hz}, 1 \mathrm{H}, \mathrm{H}-1 \mathrm{ax}), 2.98,3.04(2 \mathrm{~d}, J=13.6 \mathrm{~Hz}, 1 \mathrm{H}, \mathrm{H}-10 \mathrm{ax}), 3.26,3.36(2 \mathrm{~d}, J=13.6 \mathrm{~Hz}, 1 \mathrm{H}, \mathrm{H}-$ 10eq), 3.36, $4.31(2 \mathrm{~d}, J=14.0 \mathrm{~Hz}, 1 \mathrm{H}, \mathrm{H}-3 \mathrm{eq}), 3.40,3.57$ (2br. $\mathrm{s}, 1 \mathrm{H}, \mathrm{H}-7), 3.55,4.43(2 \mathrm{~d}, J=$ $12.8 \mathrm{~Hz}, 1 \mathrm{H}, \mathrm{H}-1 \mathrm{eq}), 4.14,4.23$ (2br. s, $1 \mathrm{H}, \mathrm{H}-6), 4.51$ (d, $J=12.0 \mathrm{~Hz}, 1 \mathrm{H}, \mathrm{CH}_{2} \mathrm{Bn}$ ), 4.56 (d, $J=$ $\left.12.0 \mathrm{~Hz}, 1 \mathrm{H}, \mathrm{CH}_{2} \mathrm{Bn}\right), 4.95\left(\mathrm{~m}, 4 \mathrm{H}, \mathrm{CH}_{2}=\right)$, 5.61-5.90 (m, 2H, CH=), 7.08-7.60 (m, 9H, Ts, $\left.\mathrm{C}_{6} \mathrm{H}_{5}\right)$.

${ }^{13} \mathrm{C} \mathrm{NMR}\left(100.6 \mathrm{MHz}, \mathrm{CDCl}_{3}\right): \delta 21.0,21.4\left(\mathrm{CH}_{3} \mathrm{Ts}\right), 22.2,22.3,22.4,22.6$ (C-2', C-5), 24.7, 25.0 (C-3") , 28.7, 28.8, 28.9 (C-4", C-5"), 30.1, 30.6 (C-8), 31.1, 31.5 (C-8a), 33.2, 33.3 (C-2"), 33.6 (C-6") 34.0 (C-3'), 34.8, 35.3 (C-4a), 35.7, 35.8 (C-1'), 36.6 (C-4), 46.5, 50.6 (C-1), 46.7, 46.8 (C10), 47.9, 48.4 (C-6), 48.2, 52.3 (C-3), 70.5, 70.9 ( $\mathrm{CH}_{2} \mathrm{Bn}$ ), 73.7, 74.7 (C-7), 114.3, 114.4, 114.8, $115.1\left(2 \mathrm{CH}_{2}=\right), 126.7-129.6\left(\mathrm{C}-0, m\right.$ Ts, $\left.\mathrm{C}-0, m, p \mathrm{C}_{6} \mathrm{H}_{5}\right), 137.4,138.0,138.1,138.4,138.5(2 \mathrm{CH}=$, C-i Ts, C- $\left.i \mathrm{C}_{6} \mathrm{H}_{5}\right), 143.1,143.3$ (C-p Ts), 172.6, 172.7 (NCO). 

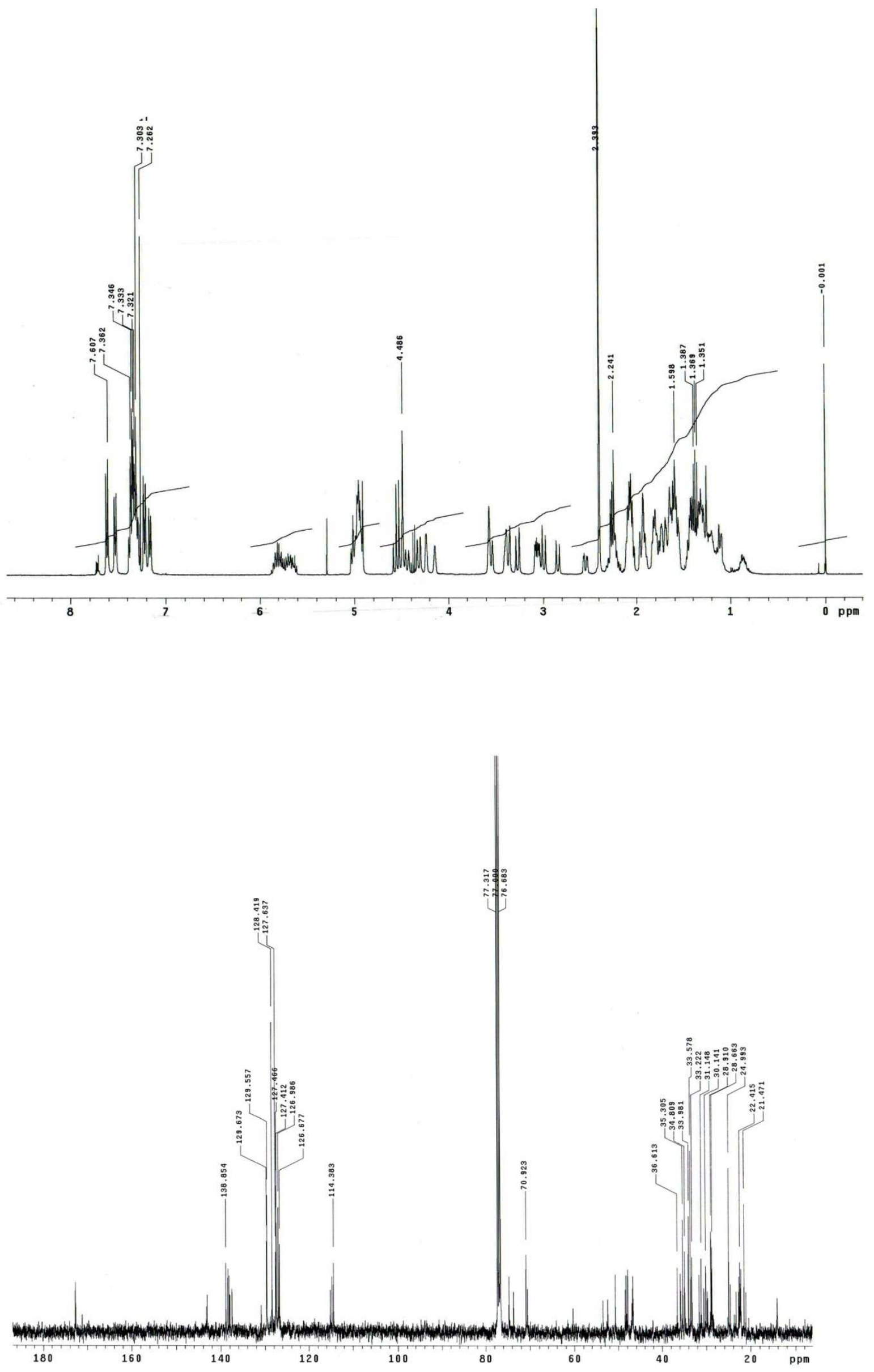


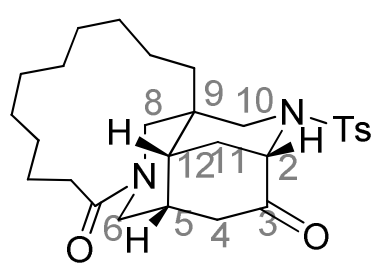

(madangamine numbering)

\section{(2S,5S,9R,12R) Tetracyclic keto lactam 106.}

Ring-closing metathesis reaction: A solution of compound 98 (202 $\mathrm{mg}, 0.326 \mathrm{mmol}$ ) in $\mathrm{CH}_{2} \mathrm{Cl}_{2}$ $(1.3 \mathrm{~L})$ was sparged with a flow of argon through a gas dispersion tube for $1 \mathrm{~h}$. The solution was heated to reflux and then a solution of 1st generation Grubbs catalyst ( $27 \mathrm{mg}, 0.0326$ $\mathrm{mmol})$ in $\mathrm{CH}_{2} \mathrm{Cl}_{2}(10 \mathrm{~mL})$ was added in one portion through the septum. The solution was heated to reflux for $8 \mathrm{~h}$ under a flow of argon. DMSO $(0.3 \mathrm{~mL})$ was added, and the resulting solution was allowed to cool to room temperature over $12 \mathrm{~h}$. The solvent was evaporated under reduced pressure. Flash chromatography filtration (8:2 to 1:1 hexane-EtOAc) of the residue afforded the unsaturated tetracyclic lactam 99 as a 2:1 mixture of $Z / E$ isomers.

${ }^{1} \mathrm{H}$ NMR (400 MHz, CDCl 3 , COSY, gHSQC, selected resonances, spectral data of the major isomer (Z) from a mixture of isomers): $\delta 1.36(\mathrm{~m}, 1 \mathrm{H}, \mathrm{H}-12), 1.46(\mathrm{dm}, J=13.6 \mathrm{~Hz}, 1 \mathrm{H}, \mathrm{H}-11)$, $1.78(\mathrm{dm}, J=10.4 \mathrm{~Hz}, 1 \mathrm{H}, \mathrm{H}-4), 1.84-2.22(\mathrm{~m}, 10 \mathrm{H}, \mathrm{H}-11, \mathrm{H}-5, \mathrm{H}-4, \mathrm{H}-22), 2.44\left(\mathrm{~s}, 3 \mathrm{H}, \mathrm{CH}_{3} \mathrm{Ts}\right)$, $2.53(\mathrm{~m}, 1 \mathrm{H}, \mathrm{H}-22), 2.59$ (d, J = 11.6 Hz, 1H, H-10ax), 2.99 (d, J = $11.6 \mathrm{~Hz}, 1 \mathrm{H}, \mathrm{H}-10 \mathrm{eq}), 3.04$ (d, J $=14.0 \mathrm{~Hz}, 1 \mathrm{H}, \mathrm{H}-6 \mathrm{ax}), 3.20$ (d, J = $11.6 \mathrm{~Hz}, 1 \mathrm{H}, \mathrm{H}-8 \mathrm{ax}), 3.63$ (m, 2H, H-2, H-8eq), 4.22 (m, 1H, H3), 4.31 (d, $J=14.0 \mathrm{~Hz}, 1 \mathrm{H}, \mathrm{H}-6 \mathrm{eq}), 4.57$ (d, $J=11.6 \mathrm{~Hz}, 1 \mathrm{H}, \mathrm{CH}_{2} \mathrm{Bn}$ ), 4.64 (d, J = 11.6 Hz, $1 \mathrm{H}, \mathrm{CH}_{2}$ $\mathrm{Bn}), 5.13(\mathrm{~m}, 2 \mathrm{H}, \mathrm{CH}=), 7.26-7.36\left(\mathrm{~m}, 7 \mathrm{H}, \mathrm{C}_{6} \mathrm{H}_{5}, \mathrm{H}-m \mathrm{Ts}\right), 7.60$ (d, J = 8.4 Hz, 2H, H-o Ts).

${ }^{13} \mathrm{C} \mathrm{NMR}\left(100.6 \mathrm{MHz}, \mathrm{CDCl}_{3}\right): \delta 21.0\left(\mathrm{CH}_{3} \mathrm{Ts}\right), 21.5(\mathrm{C}-11), 22.6\left(\mathrm{CH}_{2}\right), 25.6\left(\mathrm{CH}_{2}\right), 25.7\left(\mathrm{CH}_{2}\right), 27.6$ $\left(\mathrm{CH}_{2}\right), 28.8\left(\mathrm{CH}_{2}\right), 29.0$ (C-5), 30.5 (C-4), $30.9\left(\mathrm{CH}_{2}\right), 31.5\left(\mathrm{CH}_{2}\right), 31.9$ (C-22), 36.0 (C-9), 38.4 $\left(\mathrm{CH}_{2}\right), 40.8$ (C-6), 47.6, 49.4 (C-10, C-8), 50.6 (C-2), $71.6\left(\mathrm{CH}_{2} \mathrm{Bn}\right), 77.1$ (C-3), 127.1 (C-o, m, $p$ $\mathrm{C}_{6} \mathrm{H}_{5}, \mathrm{C}-0, m \mathrm{Ts}$ ), 131.6, 132.0 (C=), 133.6 (C-p Ts), $138.8\left(\mathrm{C}-i \mathrm{C}_{6} \mathrm{H}_{5}\right.$ ), 143.6 (C-i Ts), 174.5 (NCO). HRMS (maldi) calcd for $\left[\mathrm{C}_{35} \mathrm{H}_{46} \mathrm{~N}_{2} \mathrm{O}_{4} \mathrm{~S}+\mathrm{Na}\right]^{+}: 613.3$, found: 613.3 . 
Hydrogenation step: $\mathrm{Pd} / \mathrm{C}$ (100 mg, 50\% in weight) was added to a solution of the above unsaturated lactam $99(0.326 \mathrm{mmol})$ in a 9:1 $\mathrm{EtOH}-\mathrm{H}_{2} \mathrm{O}$ mixture $(20 \mathrm{~mL})$, and the resulting mixture was stirred under a hydrogen atmosphere at room temperature overnight to afford a saturated alcohol as a single product, which was immediately used in the subsequent step.

${ }^{1} \mathrm{H}$ NMR (400 MHz, $\mathrm{CDCl}_{3}$, selected resonances): $\delta 1.38(\mathrm{~m}, 1 \mathrm{H}, \mathrm{H}-12), 1.63-1.79(\mathrm{~m}, 3 \mathrm{H}, 2 \mathrm{H}-4$, $\mathrm{H}-11), 1.86(\mathrm{dm}, J=13.2 \mathrm{~Hz}, 1 \mathrm{H}, \mathrm{H}-11), 2.04(\mathrm{~m}, 1 \mathrm{H}, \mathrm{H}-5), 2.13\left(\mathrm{~m}, 2 \mathrm{H}, \mathrm{CH}_{2}\right), 2.43\left(\mathrm{~s}, 3 \mathrm{H}, \mathrm{CH}_{3}\right.$ Ts), 2.79 (dd, $J=113.6,4.4 \mathrm{~Hz}, 1 \mathrm{H}, \mathrm{H}-6 \mathrm{ax}), 2.98$ (d, J = 12.8 Hz, 1H, H-8ax), 3.13 (d, J = $124 \mathrm{~Hz}$, 1H, H-10ax), 3.33 (d, J = 12.4 Hz, 1H, H-10eq), 3.59 (d, J = 12.8 Hz, 1H, H-8eq), 3.82(br. s, 1H, H2), 4.02 (br. s, $1 \mathrm{H}, \mathrm{H}-3$ ), 4.10 (d, J = $13.6 \mathrm{~Hz}, 1 \mathrm{H}, \mathrm{H}-6 \mathrm{eq}$ ), 7.31 (d, J = $8.4 \mathrm{~Hz}, 2 \mathrm{H}, \mathrm{H}-\mathrm{m}$ Ts), 7.68 (d, $J=8.4 \mathrm{~Hz}, 2 \mathrm{H}, \mathrm{H}-\mathrm{O} \mathrm{Ts})$.

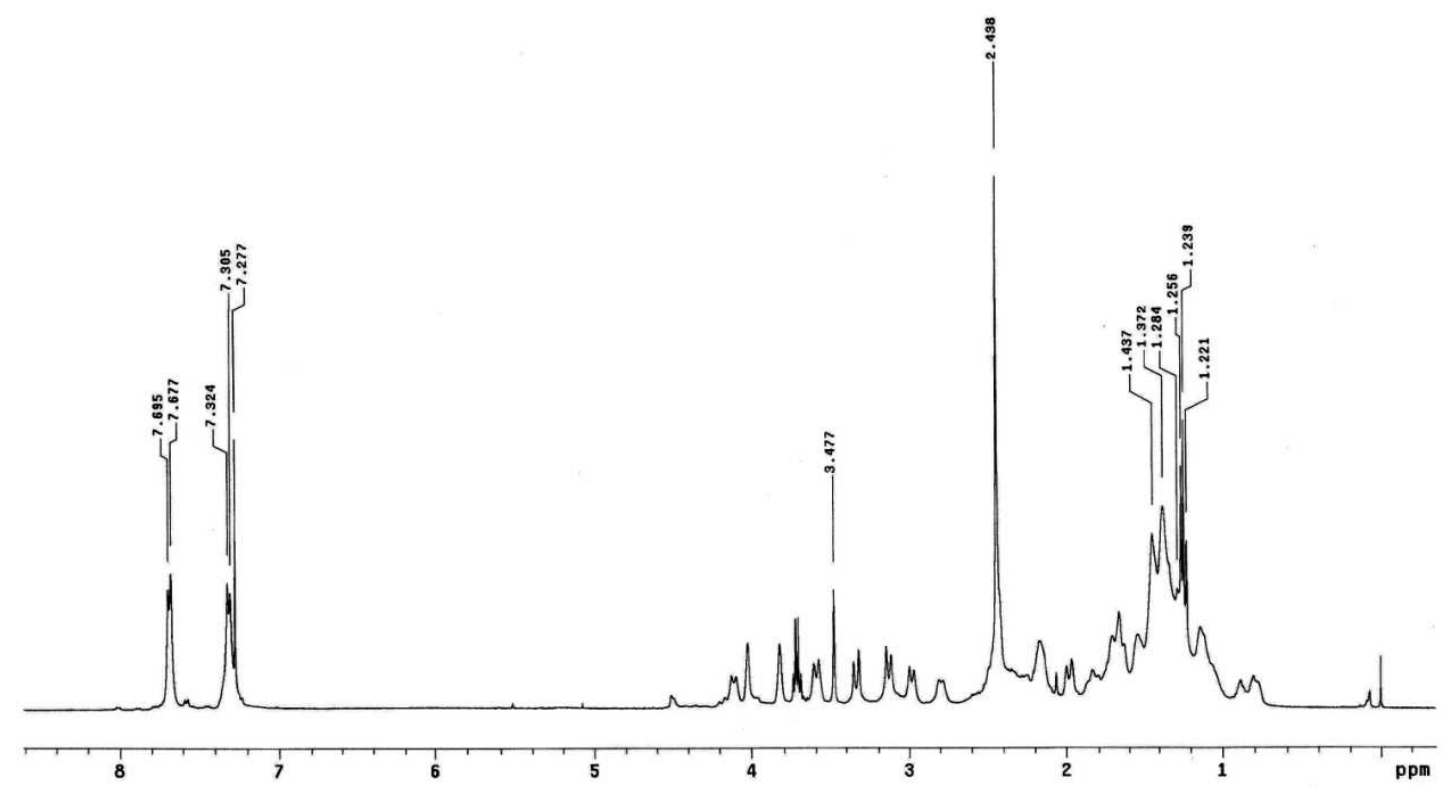


Oxidation step: Dess-Martin periodinane $(414 \mathrm{mg}, 0.97 \mathrm{mmol}$ ) was added to an ice-cold solution of the above alcohol in $\mathrm{CH}_{2} \mathrm{Cl}_{2}(20 \mathrm{~mL})$, and the resulting mixture was stirred at room temperature for $4 \mathrm{~h}$. The solution was poured into a saturated aqueous solution of $\mathrm{NaHCO}_{3}$ and $\mathrm{Na}_{2} \mathrm{~S}_{2} \mathrm{O}_{3}$, and the mixture was stirred for $1 \mathrm{~h}$ and extracted with $\mathrm{CH}_{2} \mathrm{Cl}_{2}$. The combined organic extracts were dried over anhydrous $\mathrm{Na}_{2} \mathrm{SO}_{4}$, filtered, and concentrated under reduced pressure. Flash chromatography (7:3 to 1:1 hexane-EtOAc) of the residue afforded ketone 106 (122 mg, 75\% from diene 98).

$[\alpha]_{D}^{22}=+33.8\left(c 1.4, \mathrm{CHCl}_{3}\right)$.

IR (film): 1721, $1636(C=0) \mathrm{cm}^{-1}$.

${ }^{1} \mathrm{H}$ NMR (400 MHz, CDCl 3 , COSY, gHSQC): $\delta 1.00(\mathrm{~m}, 1 \mathrm{H}, \mathrm{H}-31), 1.24-1.42\left(\mathrm{~m}, 16 \mathrm{H}, \mathrm{CH}_{2}\right), 1.50-$ $1.65(m, 2 \mathrm{H}, \mathrm{H}-11, \mathrm{H}-12)$, 2.10-2.22 (m, 4H, H-22, H-5, H-4, H-31), 2.35-2.50 (m, 3H, H-22, H-11, H-4), 2.42 (s, 3H, CH $\mathrm{CH}_{3}$ ), 2.91 (dd, J = 12.8, 1.2 Hz, 1H, H-6ax), 2.98 (d, J=13.2 Hz, 1H, H-10ax), $3.16(\mathrm{~d}, J=14.0 \mathrm{~Hz}, 1 \mathrm{H}, \mathrm{H}-8 \mathrm{ax}$ ), 3.54 (d, $J=13.2 \mathrm{~Hz}, 1 \mathrm{H}, \mathrm{H}-10 \mathrm{eq}), 3.75$ (d, J=14.0, 1H, H-8eq), $4.08(\mathrm{~d}, J=12.8 \mathrm{~Hz}, 1 \mathrm{H}, \mathrm{H}-6 \mathrm{eq}), 4.13(\mathrm{~m}, 1 \mathrm{H}, \mathrm{H}-2), 7.29$ (d, J = $7.6 \mathrm{~Hz}, 2 \mathrm{H}, \mathrm{H}-m \mathrm{Ts}), 7.62(\mathrm{~d}, J=$ $7.6 \mathrm{~Hz}, 2 \mathrm{H}, \mathrm{H}-\mathrm{O} \mathrm{Ts})$.

${ }^{13} \mathrm{C} \mathrm{NMR}\left(100.6 \mathrm{MHz}, \mathrm{CDCl}_{3}\right): \delta 20.7\left(\mathrm{CH}_{2}\right), 21.5\left(\mathrm{CH}_{3} \mathrm{Ts}\right), 22.7\left(\mathrm{CH}_{2}\right), 23.3\left(\mathrm{CH}_{2}\right), 24.1\left(\mathrm{CH}_{2}\right), 25.6$ $\left(\mathrm{CH}_{2}\right), 26.3\left(\mathrm{CH}_{2}\right), 26.4\left(\mathrm{CH}_{2}\right), 27.3\left(\mathrm{CH}_{2}\right), 29.0(\mathrm{C}-11), 33.7(\mathrm{C}-22), 34.8\left(\mathrm{CH}_{2}\right), 35.8(\mathrm{C}-5), 36.6(\mathrm{C}-$ 12), 38.0 (C-9), 43.2 (C-4), 45.2 (C-10), 45.8 (C-6), 50.9 (C-8), 57.4 (C-2), 127.8 (C-o Ts), 129.4 (C$m$ Ts), 134.6 (C-p Ts), 144.0 (C-i Ts), 174.0 (NCO), 205.4 (C=O).

HRMS (maldi) calcd for $\left[\mathrm{C}_{28} \mathrm{H}_{40} \mathrm{~N}_{2} \mathrm{O}_{4} \mathrm{~S}+\mathrm{Na}\right]^{+}: 523.26$, found: 523.30 . 

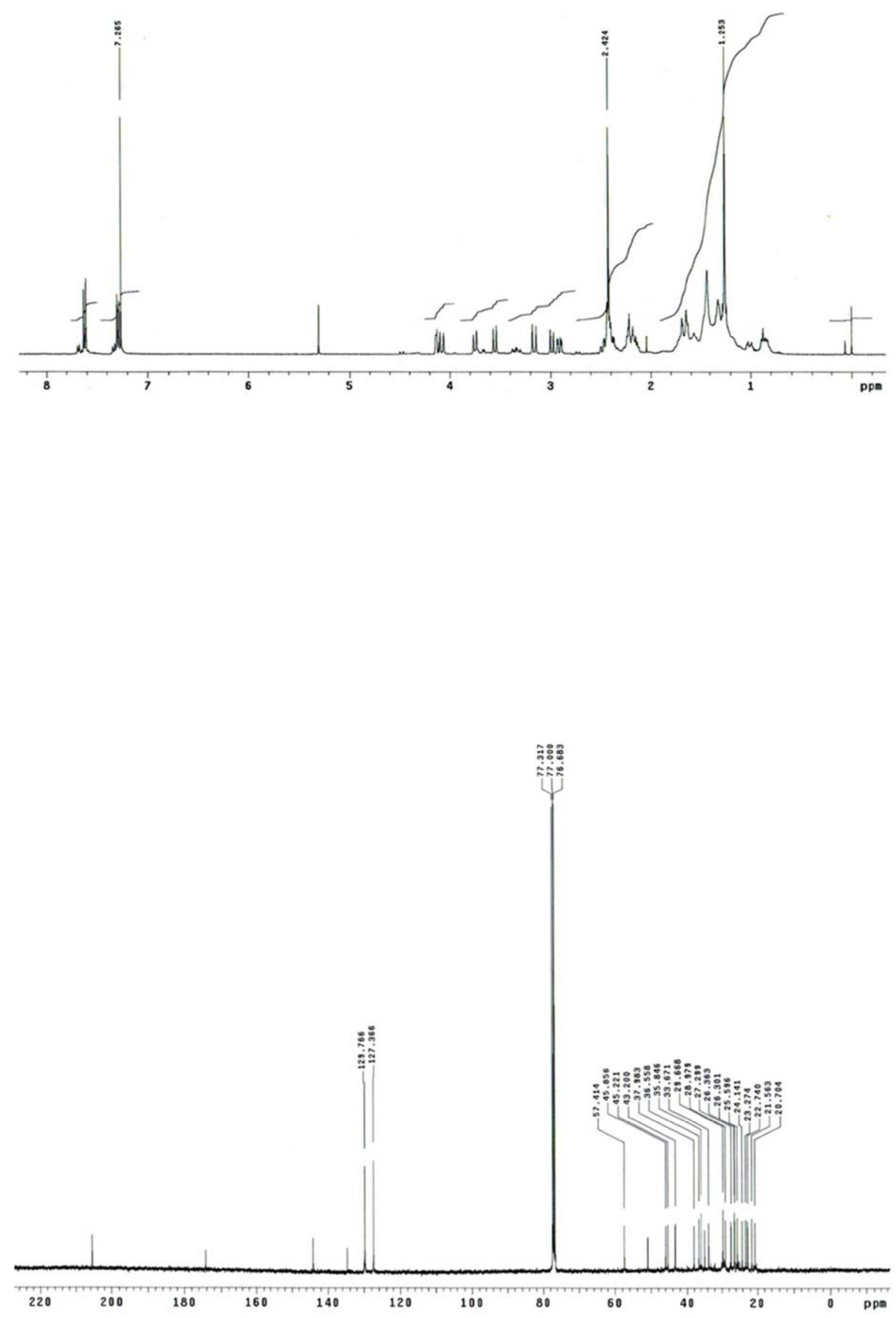


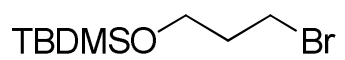

\section{3-Bromo-1-(tert-butyldimethylsililoxy)propane (121).}

Triethylamine $(2.0 \mathrm{~mL}, 14.2 \mathrm{mmol})$, dimethylaminopiridine $(67 \mathrm{mg}, 0.52 \mathrm{mmol})$ and tertbutyldimethylsilane chloride $(2.0 \mathrm{~g}, 13.2 \mathrm{mmol})$ were added to a solution of 3-bromopropanol (1.0 mL, $11 \mathrm{mmol})$ in anhydrous THF $(16 \mathrm{~mL})$ under inert atmosphere at room temperature. After $18 \mathrm{~h}$ stirring at room temperature, saturated $\mathrm{NH}_{4} \mathrm{Cl}$ was added and the resulting mixture was extracted with $\mathrm{CH}_{2} \mathrm{Cl}_{2}$. The combined organic extracts were dried over anhydrous $\mathrm{Na}_{2} \mathrm{SO}_{4}$, and concentrated under reduced pressure. Flash chromatography (hexane) gave protected alcohol 121 (253 mg, 99\%) as colourless oil.

${ }^{1} \mathrm{H}$ RMN $\left(400 \mathrm{MHz}, \mathrm{CDCl}_{3}\right): \delta 0.07\left(\mathrm{~s}, 6 \mathrm{H}, \mathrm{CH}_{3} \mathrm{Si}\right), 0.90\left[\mathrm{~s}, 9 \mathrm{H},\left(\mathrm{CH}_{3}\right)_{3} \mathrm{C}\right], 2.03(\mathrm{~m}, 2 \mathrm{H}, \mathrm{H}-2), 3.51(\mathrm{t}$, $J=6.6 \mathrm{~Hz}, 2 \mathrm{H}, \mathrm{H}-1), 3.73(\mathrm{t}, J=5.4 \mathrm{~Hz}, 2 \mathrm{H}, \mathrm{H}-3)$.

${ }^{13} \mathrm{C} \mathrm{NMR}\left(100.6 \mathrm{MHz}, \mathrm{CDCl}_{3}\right): \delta-5.4\left(2 \mathrm{CH}_{3} \mathrm{Si}\right), 18.3\left[\left(\mathrm{CH}_{3}\right)_{3} \mathrm{CSi}\right], 25.9\left[\left(\mathrm{CH}_{3}\right)_{3} \mathrm{CSi}\right], 30.7\left(\mathrm{CH}_{2} \mathrm{Br}\right)$, $35.5\left(\mathrm{CH}_{2}\right), 60.4\left(\mathrm{CH}_{2} \mathrm{O}\right)$. 

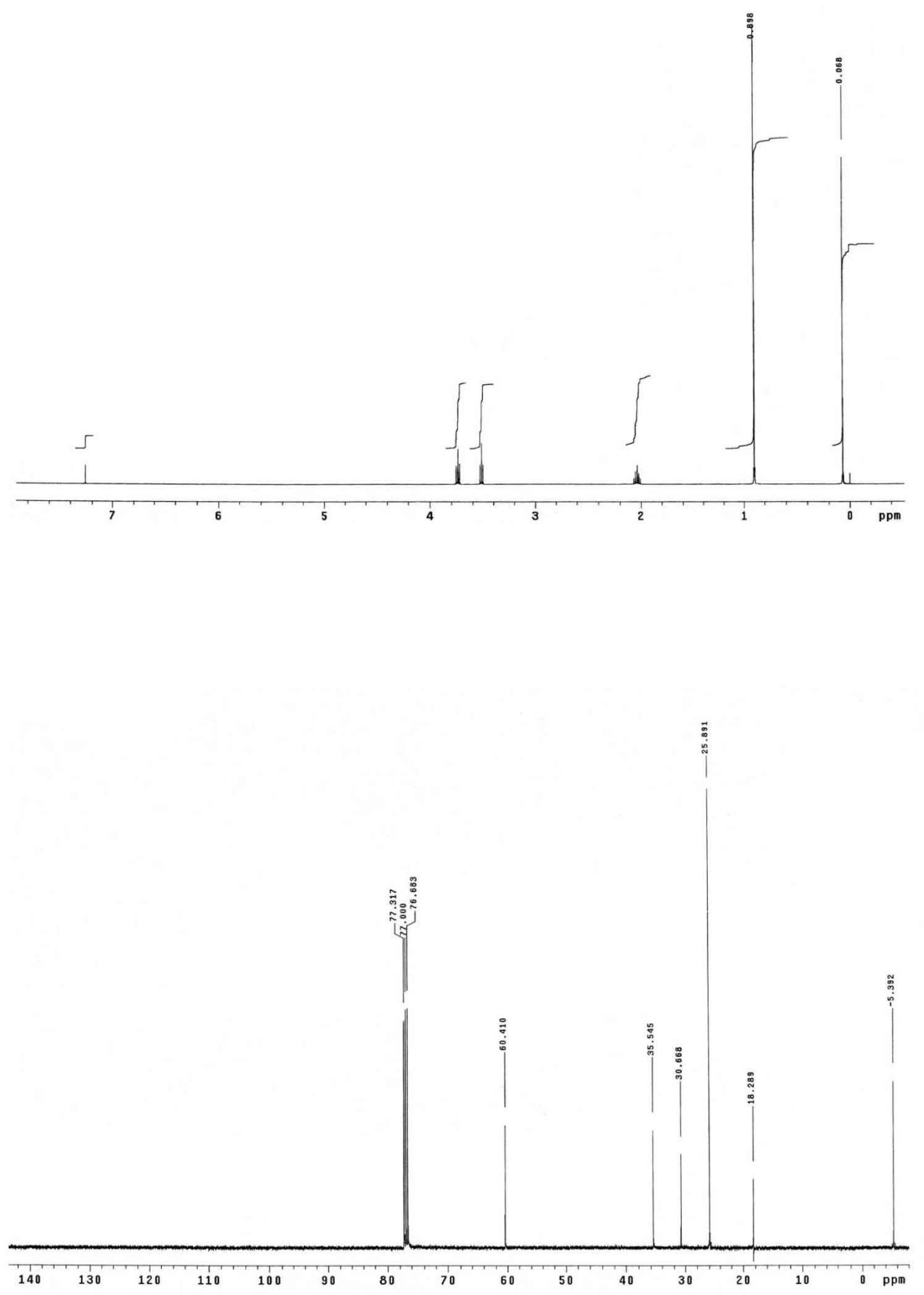


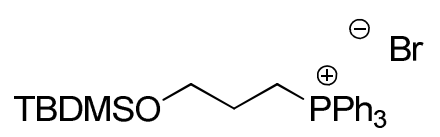

3-(tert-Butyldimethylsilyloxy)propyltriphenylphosphonium bromide (122).

Triphenylphosphine $(8.7 \mathrm{~g}, 33 \mathrm{mmol})$ was added to a solution of compound 121 (6.9 g, 28 $\mathrm{mmol}$ ) in anhydrous benzene $(5 \mathrm{~mL})$ under argon atmosphere. The mixture was heated at 85 ${ }^{\circ} \mathrm{C}$ for $18 \mathrm{~h}$ and then was cooled to room temperature. Ether was added to the resulting solution and a white solid was formed. The residue was filtered and washed several times with ether. Flash chromatography of the resulting solid $\left(\mathrm{CH}_{2} \mathrm{Cl}_{2}\right.$ to $\left.96: 4 \mathrm{CH}_{2} \mathrm{Cl}_{2} / \mathrm{MeOH}\right)$ afforded phosphonium salt 122 (12 g, 84\%).

$\left.{ }^{1} \mathrm{H} \mathrm{RMN} \mathrm{(300} \mathrm{MHz,} \mathrm{CDCl}\right): \delta 0.04\left(\mathrm{~s}, 6 \mathrm{H}, \mathrm{CH}_{3} \mathrm{Si}\right), 0.85\left[\mathrm{~s}, 9 \mathrm{H},\left(\mathrm{CH}_{3}\right)_{3} \mathrm{C}\right], 1.95(\mathrm{~m}, 2 \mathrm{H}, \mathrm{H}-2), 3.98$ $(\mathrm{m}, 4 \mathrm{H}, \mathrm{H}-1, \mathrm{H}-3), 7.69-7.90\left(\mathrm{~m}, 15 \mathrm{H}, \mathrm{C}_{6} \mathrm{H}_{5}\right)$.

${ }^{13} \mathrm{C} \mathrm{NMR}(100.6 \mathrm{MHz}, \mathrm{CDCl})$ ): $\delta$-5.3 $\left(2 \mathrm{CH}_{3} \mathrm{Si}\right), 18.2\left[\left(\mathrm{CH}_{3}\right)_{3} \mathrm{CSi}\right], 18.7,19.2\left(\mathrm{CH}_{2} \mathrm{P}\right), 25.9\left[\left(\mathrm{CH}_{3}\right)_{3} \mathrm{CSi}\right]$, 26.0, $26.1\left(\mathrm{CH}_{2}\right), 61.6,61.8\left(\mathrm{CH}_{2} \mathrm{O}\right), 118.0,118.8\left(\mathrm{C}-i \mathrm{C}_{6} \mathrm{H}_{5}\right), 130.3-133.7\left(\mathrm{C}-0, m \mathrm{C}_{6} \mathrm{H}_{5}\right), 134.9(\mathrm{C}-$ $\left.p \mathrm{C}_{6} \mathrm{H}_{5}\right)$.

m.p. $216^{\circ} \mathrm{C}$

HRMS (ESI) calcd for $\left[\mathrm{C}_{21} \mathrm{H}_{22} \mathrm{OP}-\mathrm{TBDMSi}+\mathrm{H}\right]^{+}:$321.1403, found: 321.1398. 

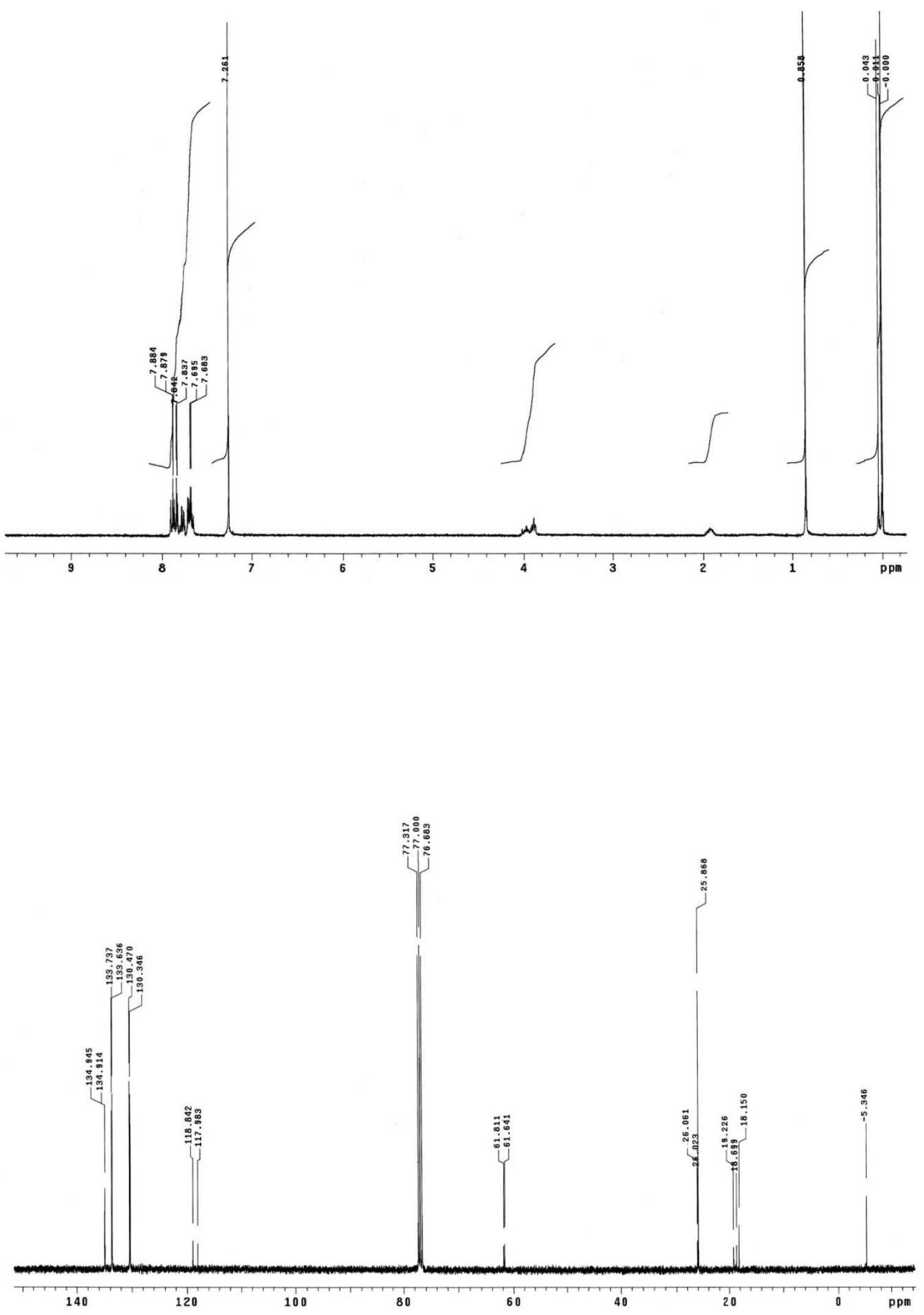
<smiles>COC(=O)CCC/C=C\CCOC(C)(C)C</smiles>

\section{(Z)-Methyl 8-(tert-butyldimethylsilyloxy)-5-octenoate (124).}

Sodium bis(trimethylsilyl)amide $(8.75 \mathrm{~mL}$ of a solution $1 \mathrm{M}$ in THF, $8.75 \mathrm{mmol}$ ) was added dropwise to a solution of compound $122(4.5 \mathrm{~g}, 8.75 \mathrm{mmol})$ in a mixture of solvents formed by THF $(99 \mathrm{~mL})$ and toluene $(22 \mathrm{~mL})$ at $-30{ }^{\circ} \mathrm{C}$ under inert atmosphere and the resulting mixture was stirred at room temperature for $4 \mathrm{~h}$. A solution of methyl 4-formylpentanoate $123(2.2 \mathrm{~g}$, $17.5 \mathrm{mmol})$ in $\mathrm{THF}(15 \mathrm{~mL})$ was then added at $-78^{\circ} \mathrm{C}$ and the mixture was stirred at the same temperature overnight. The reaction was quenched with saturated aqueous $\mathrm{NH}_{4} \mathrm{Cl}$, and the resulting solution was extracted with EtOAc. The combined organic extracts were dried over anhydrous $\mathrm{Na}_{2} \mathrm{SO}_{4}$, filtered, and concentrated under reduced pressure. Flash chromatography (hexane to 8:2 hexane-EtOAc) afforded compound 124 (1.94 g, 78\%).

IR (film): 1742 (COO) $\mathrm{cm}^{-1}$.

${ }^{1} \mathrm{H}$ NMR (400 MHz, CDCl 3 , COSY, gHSQC): $\delta 0.05\left(\mathrm{~s}, 6 \mathrm{H}, \mathrm{CH}_{3} \mathrm{Si}\right), 0.89\left[\mathrm{~s}, 9 \mathrm{H},\left(\mathrm{CH}_{3}\right)_{3} \mathrm{CSi}\right], 1.69(\mathrm{~m}$, $2 \mathrm{H}, 2 \mathrm{H}-3), 2.08(\mathrm{~m}, 2 \mathrm{H}, 2 \mathrm{H}-4), 2.25(\mathrm{~m}, 2 \mathrm{H}, 2 \mathrm{H}-7), 2.31(\mathrm{t}, J=8.0 \mathrm{~Hz}, 2 \mathrm{H}, 2 \mathrm{H}-2), 3.60(\mathrm{t}, J=6.4$ $\left.\mathrm{Hz}, 2 \mathrm{H}, \mathrm{CH}_{2} \mathrm{OSi}\right), 3.67\left(\mathrm{~s}, 3 \mathrm{H}, \mathrm{CH}_{3} \mathrm{O}\right), 5.40(\mathrm{~m}, 2 \mathrm{H}, \mathrm{H}-5, \mathrm{H}-6)$.

${ }^{13} \mathrm{C} \mathrm{NMR}\left(75.4 \mathrm{MHz}, \mathrm{CDCl}_{3}\right): \delta-5.3\left(\mathrm{CH}_{3} \mathrm{Si}\right), 18.3\left[\left(\mathrm{CH}_{3}\right)_{3} \mathrm{CSi}\right], 24.8(\mathrm{C}-3), 25.9$ [( $\left.\left.\mathrm{CH}_{3}\right)_{3} \mathrm{CSi}\right], 26.7$ (C4), 31.1 (C-7), 33.5 (C-2), $51.5\left(\mathrm{CH}_{3} \mathrm{O}\right), 62.9$ (C-8), 126.9, 130.3 (C-5, C-6), 174.1 (NCO).

HRMS (ESI) calcd for $\left[\mathrm{C}_{15} \mathrm{H}_{30} \mathrm{O}_{3} \mathrm{Si}+\mathrm{H}\right]^{+}: 287.2000$, found: 287.2042 . 

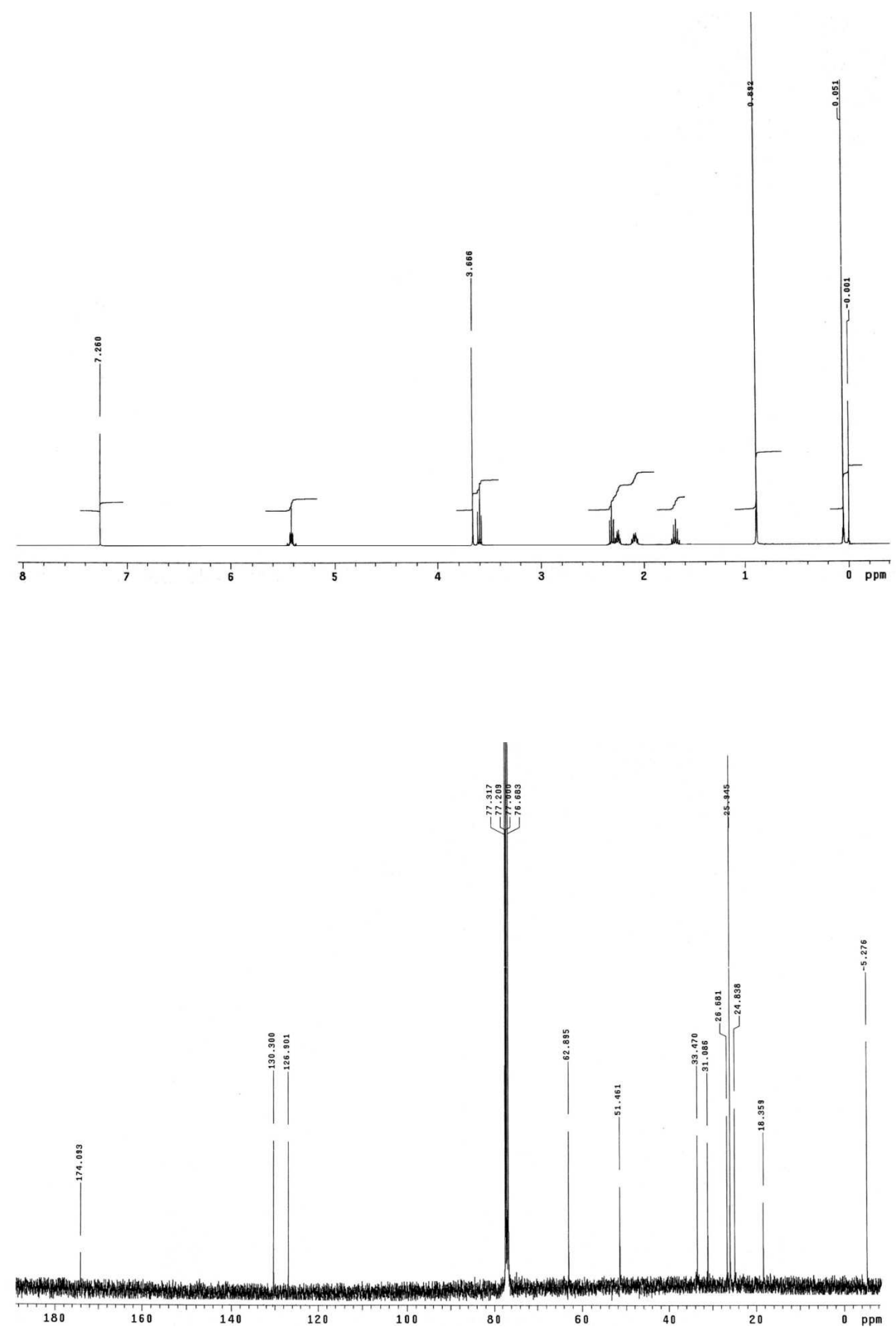


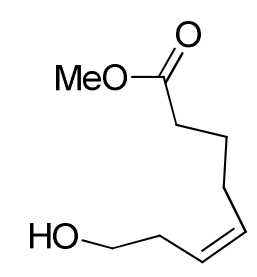

(Z)-Methyl 8-hydroxy-5-octenoate (125).

TBAF (1.94 mL of a solution $1 \mathrm{M}$ in THF, $1.94 \mathrm{mmol}$ ) was added at $0{ }^{\circ} \mathrm{C}$ to a solution of silyl derivative 124 (275 mg, $0.97 \mathrm{mmol}$ ) in THF (15 mL) under inert atmosphere and the resulting mixture was stirred overnight at room temperature. The reaction was quenched with saturated aqueous $\mathrm{NaHCO}_{3}$, and the resulting mixture was extracted with EtOAc. The combined organic extracts were dried over anhydrous $\mathrm{Na}_{2} \mathrm{SO}_{4}$, filtered, and concentrated under reduced pressure. Flash chromatography (8:2 to 7:3 hexane-EtOAc) afforded compound 125 (153 mg, 93\%).

IR (film): $3465(\mathrm{OH}), 1732(\mathrm{COO}) \mathrm{cm}^{-1}$.

${ }^{1} \mathrm{H} \mathrm{NMR}\left(400 \mathrm{MHz}, \mathrm{CDCl}_{3}, \mathrm{COSY}, \mathrm{gHSQC}\right): \delta 1.71(\mathrm{~m}, 2 \mathrm{H}, \mathrm{H}-3), 2.11(\mathrm{~m}, 2 \mathrm{H}, \mathrm{H}-4), 2.30(\mathrm{t}, J=6.0$ $\mathrm{Hz}, 2 \mathrm{H}, \mathrm{H}-7), 2.33(\mathrm{t}, J=7.2 \mathrm{~Hz}, 2 \mathrm{H}, \mathrm{H}-2), 2.65\left(\mathrm{t}, J=6.4 \mathrm{~Hz}, 2 \mathrm{H}, \mathrm{CH}_{2} \mathrm{OH}\right), 3.67\left(\mathrm{~s}, 3 \mathrm{H}, \mathrm{CH}_{3} \mathrm{O}\right), 5.39-$ $5.55(2 \mathrm{~m}, 2 \mathrm{H}, \mathrm{H}-5, \mathrm{H}-6)$.

${ }^{13} \mathrm{C}-\mathrm{NMR}\left(100.6 \mathrm{MHz}, \mathrm{CDCl}_{3}\right): \delta 24.8(\mathrm{C}-3), 28.6(\mathrm{C}-4), 30.8$ (C-7), $33.4(\mathrm{C}-2), 51.5\left(\mathrm{CH}_{3} \mathrm{O}\right), 62.2$ $\left(\mathrm{CH}_{2} \mathrm{OH}\right), 126.5(\mathrm{C}-6), 131.8$ (C-5), 174.1 (NCO). 

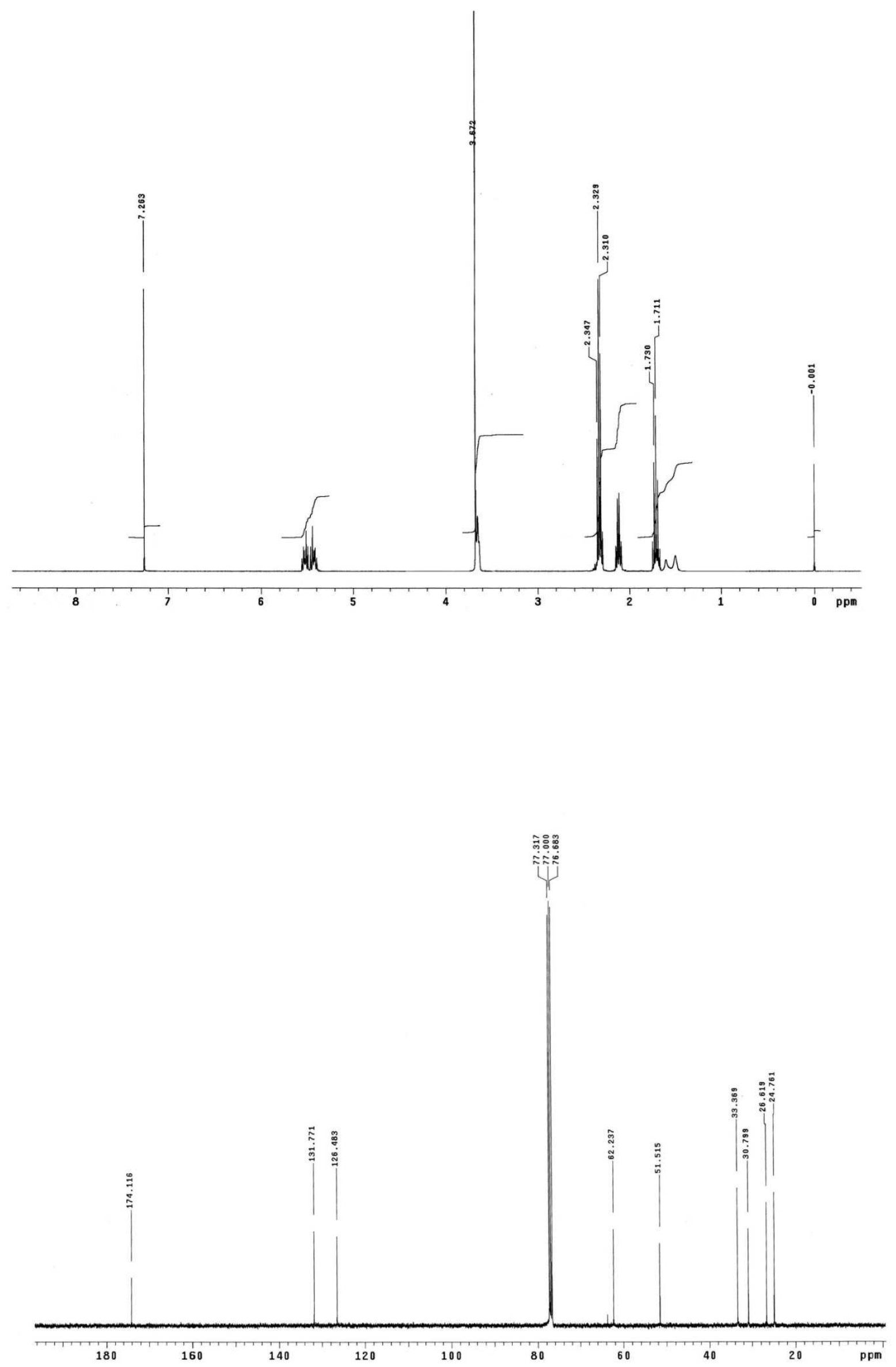


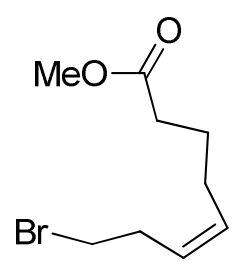

(Z)-Methyl 8-(tert-butyldimethylsilyloxy)-5-octenoate.

Mesylation step: Triethylamine (190 $\mu \mathrm{L}, 1.35 \mathrm{mmol})$ and mesyl chloride (130 $\mu \mathrm{L}, 1.8 \mathrm{mmol})$ were added dropwise to a solution of alcohol 125 (153 mg, $0.9 \mathrm{mmol}$ ) in anhydrous $\mathrm{CH}_{2} \mathrm{Cl}_{2}(12$ $\mathrm{mL}$ ) cooled to $0{ }^{\circ} \mathrm{C}$. After the end of addition, the mixture was allowed to heat slowly to room temperature and was stirred for additional $2 \mathrm{~h}$. Saturated aqueous $\mathrm{NH}_{4} \mathrm{Cl}$ was added, and the mixture was extracted with $\mathrm{CH}_{2} \mathrm{Cl}_{2}$. The combined organic layers were dried over anhydrous $\mathrm{Na}_{2} \mathrm{SO}_{4}$, filtered, and the solvent was removed under reduced pressure. Flash chromatography (6:4 to $1: 1$ hexane-EtOAc) afforded the mesyl derivative ( $239 \mathrm{mg}, 99 \%$ yield).

Bromination step: A solution of $\operatorname{LiBr}(2.9 \mathrm{~g}, 33.3 \mathrm{mmol})$ in THF $(13 \mathrm{~mL})$ was added to a solution of the above crude $(818 \mathrm{mg}, 3.33 \mathrm{mmol})$ in $\mathrm{CH}_{2} \mathrm{Cl}_{2}(9.3 \mathrm{~mL})$ at $0{ }^{\circ} \mathrm{C}$ under inert atmosphere. The resulting mixture was stirred overnight at room temperature. Distilled water was added, and the mixture was extracted with $\mathrm{CH}_{2} \mathrm{Cl}_{2}$. The combined organic layers were dried over anhydrous $\mathrm{Na}_{2} \mathrm{SO}_{4}$, filtered, and the solvent was removed under reduced pressure. Flash chromatography (hexane to 3:7 hexane-EtOAc) afforded bromo derivative ( $374 \mathrm{mg}, 75 \%$ yield).

IR (film): 1730 (COO) $\mathrm{cm}^{-1}$.

${ }^{1} \mathrm{H}$ NMR $\left(300 \mathrm{MHz}, \mathrm{CDCl}_{3}\right): \delta 1.71(\mathrm{~m}, 2 \mathrm{H}, 2 \mathrm{H}-3), 2.14(\mathrm{~m}, 2 \mathrm{H}, 2 \mathrm{H}-4), 2.36(\mathrm{t}, J=7.2 \mathrm{~Hz}, 2 \mathrm{H}, 2 \mathrm{H}-$ 2), $2.55(\mathrm{qd}, J=7.2,1.2 \mathrm{~Hz}, 2 \mathrm{H}, 2 \mathrm{H}-7), 3.01\left(\mathrm{~s}, 3 \mathrm{H}, \mathrm{CH}_{3} \mathrm{SO}_{2}\right), 3.67\left(\mathrm{~s}, 3 \mathrm{H}, \mathrm{CH}_{3} \mathrm{O}\right), 4.21(\mathrm{t}, J=6.8$ $\mathrm{Hz}, 2 \mathrm{H}, 2 \mathrm{H}-8), 5.41(\mathrm{~m}, 1 \mathrm{H}, \mathrm{CH}=), 5.56(\mathrm{~m}, 1 \mathrm{H}, \mathrm{CH}=)$.

${ }^{13} \mathrm{C} \mathrm{NMR}\left(100.6 \mathrm{MHz}, \mathrm{CDCl}_{3}\right): \delta 24.5$ (C-3), 26.6, 27.3 (C-4, C-7), 32.2 (C-8), $37.4\left(\mathrm{CH}_{3} \mathrm{SO}_{2}\right), 51.5$ $\left(\mathrm{CH}_{3} \mathrm{O}\right), 69.0$ (C-2), 123.9 (C-6), 132.6 (C-5), 173.8 (NCO). 

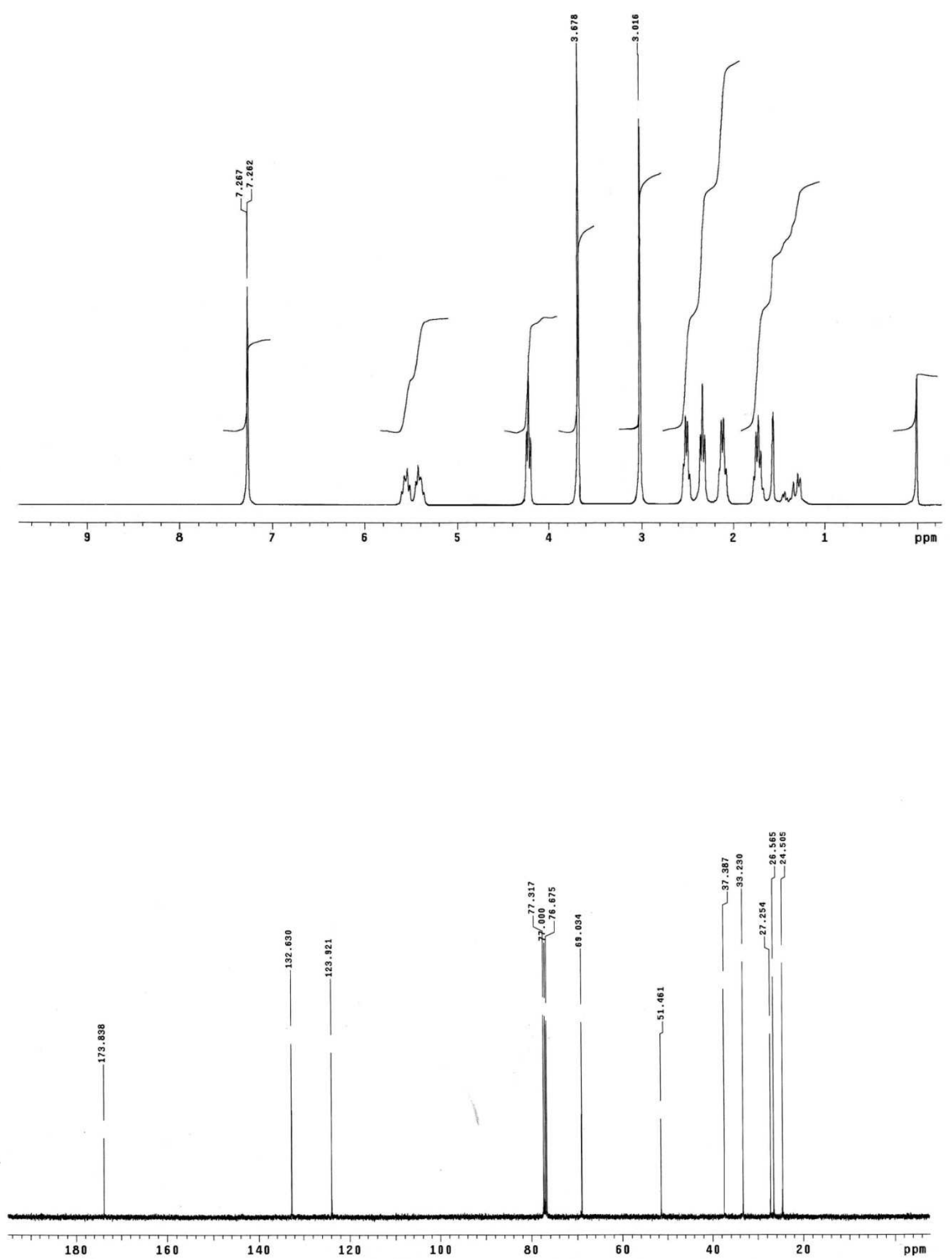
<smiles>COC(=O)CC/C=C\CCPc1ccccc1</smiles>

(Z)[-8-(Methoxycarbonyl)-3-octen-1-yl]triphenylphosphonium bromide (126).

Triphenylphosphine $(113 \mathrm{mg}, 0.43 \mathrm{mmol}$ ) was added to a solution of the above bromo derivative (100 $\mathrm{mg}, 0.43 \mathrm{mmol})$ in anhydrous benzene $(1.2 \mathrm{~mL})$ under argon atmosphere. The mixture was heated at $85^{\circ} \mathrm{C}$ for $18 \mathrm{~h}$, then was cooled to room temperature and concentrated under reduced pressure. Flash chromatography (hexane to 1:9 hexane- $\mathrm{CH}_{2} \mathrm{Cl}_{2}$ and then $\mathrm{CH}_{2} \mathrm{Cl}_{2}$ to $96: 4 \mathrm{CH}_{2} \mathrm{Cl}_{2} / \mathrm{MeOH}$ ) afforded phosphonium salt 126 (200 mg, 94\%).

${ }^{1} \mathrm{H} \mathrm{NMR}\left(400 \mathrm{MHz}, \mathrm{CDCl}_{3}\right): \delta 1.57(\mathrm{~m}, 2 \mathrm{H}, 2 \mathrm{H}-3), 1.85(\mathrm{q}, J=7.6 \mathrm{~Hz}, 2 \mathrm{H}, 2 \mathrm{H}-4), 2.21(\mathrm{t}, J=7.2 \mathrm{~Hz}$, $2 \mathrm{H}, 2 \mathrm{H}-2), 2.43(\mathrm{~m}, 2 \mathrm{H}, 2 \mathrm{H}-7), 3.60\left(\mathrm{~s}, 3 \mathrm{H}, \mathrm{CH}_{3} \mathrm{O}\right), 4.00(\mathrm{~m}, 2 \mathrm{H}, 2 \mathrm{H}-8), 5.38(\mathrm{~m}, 1 \mathrm{H}, \mathrm{CH}=), 5.65(\mathrm{~m}$, $1 \mathrm{H}, \mathrm{CH}=), 7.67-7.92\left(\mathrm{~m}, 15 \mathrm{H}, \mathrm{C}_{6} \mathrm{H}_{5}\right)$.

${ }^{13} \mathrm{C}$ NMR (100.6 MHz, CDCl $)$ ): $\delta 20.3$ (C-2), 23.2 (C-1), 24.4 (C-6), 26.5 (C-5), 33.2 (C-7), 51.4 $\left(\mathrm{CH}_{3} \mathrm{O}\right), 117.9,118.8\left(\mathrm{C}-i \mathrm{C}_{6} \mathrm{H}_{5}\right), 127.2,127.4(\mathrm{CH}=), 130.4,130.5,133.7,133.8\left(\mathrm{C}-\mathrm{o}, \mathrm{m} \mathrm{C}_{6} \mathrm{H}_{5}\right)$, $131.0(\mathrm{CH}=), 134.9,135.0\left(\mathrm{C}-p \mathrm{C}_{6} \mathrm{H}_{5}\right), 173.9$ (NCO). 

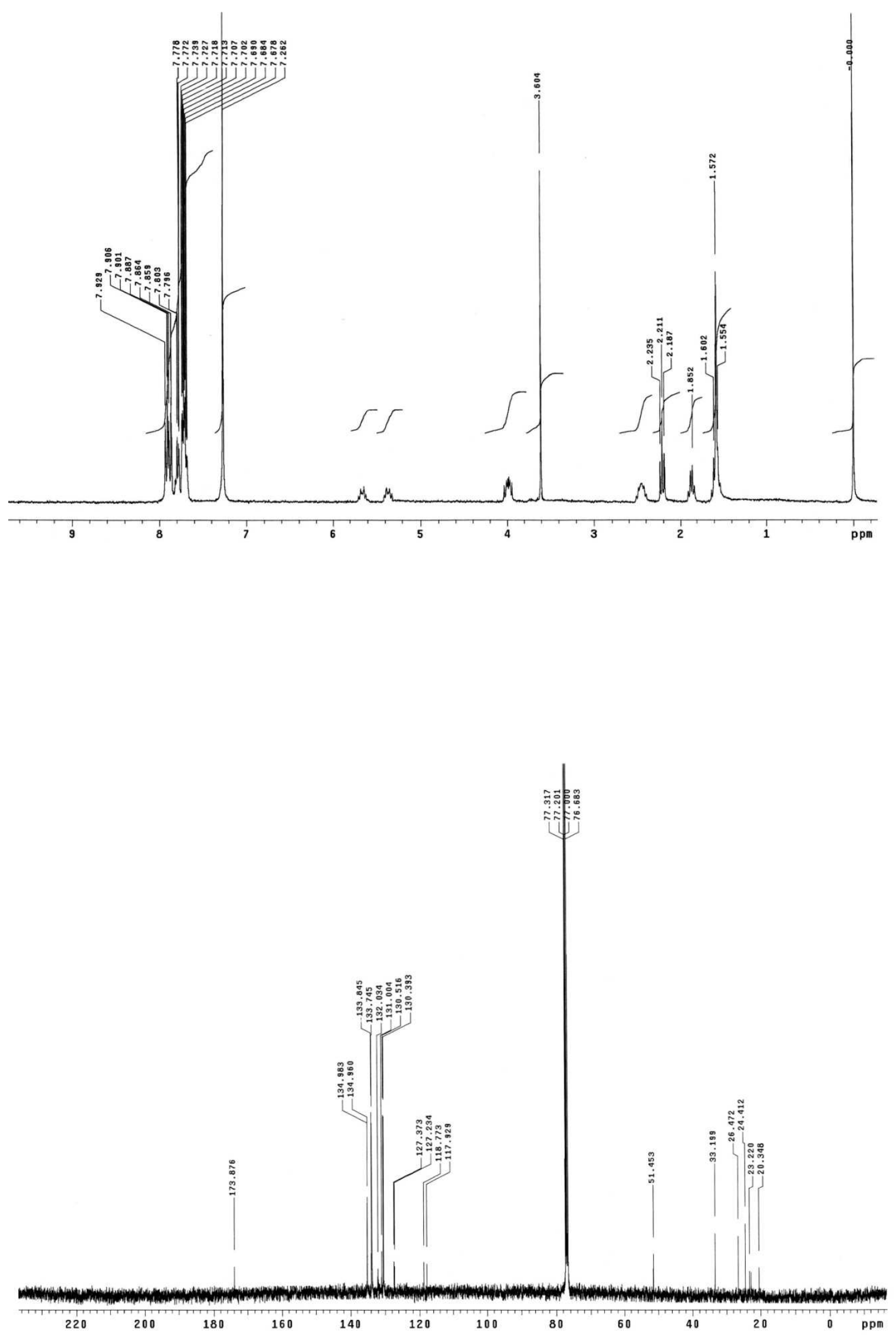

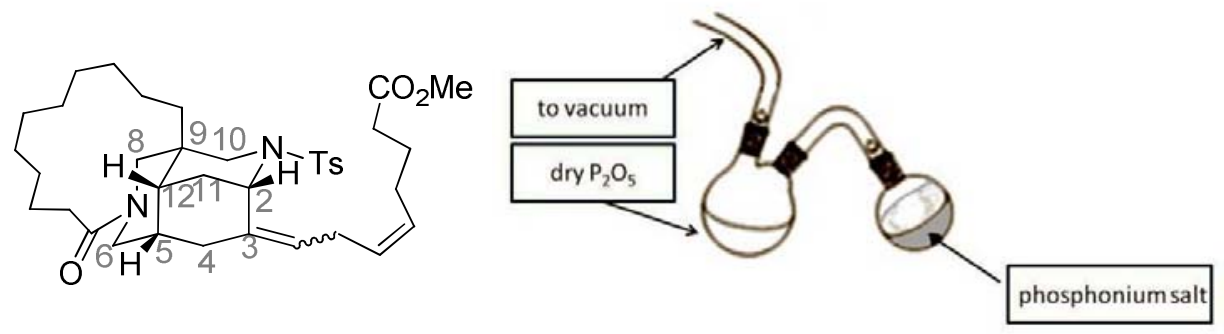

(madangamine numbering)

(2S,5S,9R,12R) Tetracyclic derivative 136-137.

(Z)-(8-Methoxy-8-oxo-3-octenyl)triphenylphosphonium bromide (126) ${ }^{134}$ was dried by repeated dilution with anhydrous 1:1 THF-toluene and concentration under reduced pressure, followed by overnight storing under vacuum over a phosphorus pentoxide system $^{135}$ (see illustration). Sodium bis(trimethylsilyl)amide (1.03 mL of a $1 \mathrm{M}$ solution in THF, $1.03 \mathrm{mmol}$ ) was added to a solution of this phosphonium salt $(566 \mathrm{mg}, 1.14 \mathrm{mmol})$ in anhydrous THF $(2.0 \mathrm{~mL})$ under an inert atmosphere at $0{ }^{\circ} \mathrm{C}$. After $1 \mathrm{~h}$ of stirring at this temperature, a solution of tetracyclic ketone 106 (173 mg, $0.346 \mathrm{mmol})$ in anhydrous THF $(1.0 \mathrm{~mL})$ was added, and the resulting mixture was stirred at $0{ }^{\circ} \mathrm{C}$ for $1 \mathrm{~h}$, at room temperature for $90 \mathrm{~min}$, and at $60^{\circ} \mathrm{C}$ for 2 h. The reaction was quenched with a saturated aqueous $\mathrm{NH}_{4} \mathrm{Cl}$. The mixture was extracted with EtOAc, and the combined organic extracts were dried over anhydrous $\mathrm{Na}_{2} \mathrm{SO}_{4}$, filtered, and concentrated under reduced pressure. Flash chromatography (hexane to 7:3 hexaneEtOAc) of the residue gave an inseparable mixture of isomers of compounds 136-137 (155 mg, $E / Z 2.2: 1$ ratio, $70 \%)$.

Major isomer (spectral data from a mixture of isomers):

IR (film): 1733, $1638(\mathrm{C}=0) \mathrm{cm}^{-1}$.

${ }^{1} \mathrm{H}$ NMR (400 MHz, CDCl 3 , COSY, gHSQC): $\delta 0.80(\mathrm{~m}, 1 \mathrm{H}, \mathrm{H}-31), 1.10-1.39\left(\mathrm{~m}, 14 \mathrm{H}, \mathrm{H}-11, \mathrm{CH}_{2}\right)$, $1.49\left(\mathrm{~m}, 3 \mathrm{H}, \mathrm{H}-12, \mathrm{CH}_{2}\right), 1.62\left(\mathrm{~m}, 3 \mathrm{H}, \mathrm{H}-15, \mathrm{CH}_{2}\right), 1.88(\mathrm{~m}, 1 \mathrm{H}, \mathrm{H}-5), 2.01-2.10(\mathrm{~m}, 5 \mathrm{H}, 2 \mathrm{H}-22$, 2H-16, H-4), $2.13(\mathrm{~m}, 1 \mathrm{H}, \mathrm{H}-31), 2.21(\mathrm{~m}, 1 \mathrm{H}, \mathrm{H}-11), 2.25$ (t, J = $7.6 \mathrm{~Hz}, 2 \mathrm{H}, \mathrm{H}-14), 2.34(\mathrm{~s}, 3 \mathrm{H}$, $\left.\mathrm{CH}_{3} \mathrm{Ts}\right), 2.35(\mathrm{~m}, 1 \mathrm{H}, \mathrm{H}-4), 2.61$ (dd, J = 13.6, 3.6 Hz, 1H, H-6ax), 2.67 (m, 1H, H-19), 2.86 (m, $1 \mathrm{H}, \mathrm{H}-19$ ), 2.87 (d, J = 13.6 Hz, 1H, H-8ax), 2.98 (d, J = 12.4 Hz, 1H, H-10ax), 3.45 (d, J = $12.4 \mathrm{~Hz}$, $1 \mathrm{H}, \mathrm{H}-10 \mathrm{eq}$ ), 3.55 (d, J = $13.6 \mathrm{~Hz}, 1 \mathrm{H}, \mathrm{H}-8 \mathrm{eq}), 3.60$ (s, 3H, $\mathrm{CH}_{3} \mathrm{O}$ ), 4.17 (d, J = 13.6 Hz, $\left.1 \mathrm{H}, \mathrm{H}-6\right)$,

\footnotetext{
${ }^{134}$ Sandri, J.; Viala, J. J. Org. Chem. 1996, 60, 6627-6630.

${ }^{135}$ Christiansen, M. A.; Andrus, M. B. Tetrahedron Lett. 2012, 53, 4805-4808.
} 
$4.76(\mathrm{~m}, 1 \mathrm{H}, \mathrm{H}-2), 5.00(\mathrm{tm}, J=7.6 \mathrm{~Hz}, 1 \mathrm{H}, \mathrm{H}-20), 5.21-5.32(\mathrm{~m}, 2 \mathrm{H}, \mathrm{H}-17, \mathrm{H}-18), 7.17(\mathrm{~d}, J=8.4$ $\mathrm{Hz}, 2 \mathrm{H}, \mathrm{H}-m \mathrm{Ts}), 7.53$ (d, J=8.4 Hz, 2H, H-o Ts).

${ }^{13} \mathrm{CNMR}(100.6 \mathrm{MHz}, \mathrm{CDCl}): \delta 20.8\left(\mathrm{CH}_{2}\right), 21.5\left(\mathrm{CH}_{3} \mathrm{Ts}\right), 22.5\left(\mathrm{CH}_{2}\right), 23.5\left(\mathrm{CH}_{2}\right), 24.6\left(\mathrm{CH}_{2}\right), 24.8$ $\left(\mathrm{CH}_{2}\right), 25.5$ (C-19), $25.7\left(\mathrm{CH}_{2}\right), 26.4\left(\mathrm{CH}_{2}\right), 26.6\left(\mathrm{C}-16, \mathrm{CH}_{2}\right), 27.5\left(\mathrm{CH}_{2}\right), 30.6(\mathrm{C}-11), 33.5(\mathrm{C}-14)$, 33.6 (C-4), 34.1 (C-31), 35.6 (C-5), 36.1 (C-22), 37.3 (C-9), 38.1 (C-12), 45.7 (C-10), 46.5 (C-6), 46.9 (C-2), 51.5 ( $\mathrm{CH}_{3} \mathrm{O}$ ), 52.6 (C-8), 124.8 (C-20), 127.1 (C-o Ts), 128.5, 129.1 (C-17, C-18), 129.4 (C-m Ts), 135.4, 136.3 (C-p Ts, C-3), 143.2 (C-i Ts), 173.8 (NCO, COO).

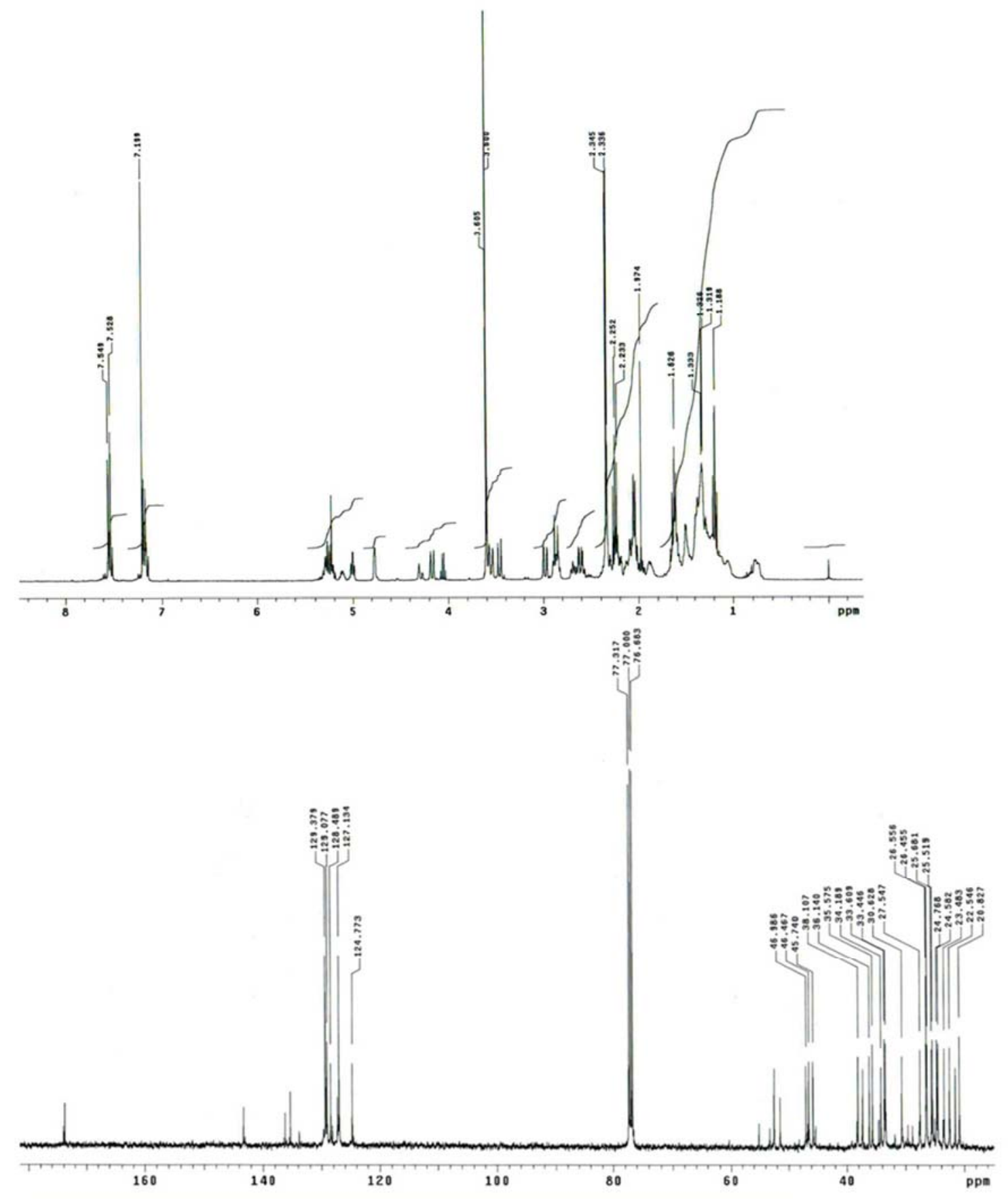




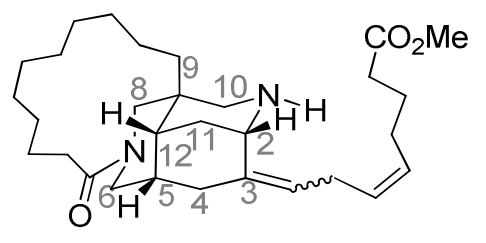

(madangamine numbering)

$(2 S, 5 S, 9 S, 12 R)$ Tetracyclic secondary amine.

Sodium metal (192 mg, $8.02 \mathrm{mmol}$ ) was added at room temperature to a solution of naphthalene (534 mg, $4.16 \mathrm{mmol})$ in THF $(12 \mathrm{~mL})$. After stirring for $2 \mathrm{~h}$, part of the mixture (2.44 mL) was added at $-78{ }^{\circ} \mathrm{C}$ to a solution of the above tosyl derivatives $136-137$ (120 mg, $0.188 \mathrm{mmol})$ in THF $(4.5 \mathrm{~mL})$. After $10 \mathrm{~min}$ at $-78^{\circ} \mathrm{C}$, a few drops of saturated aqueous $\mathrm{NH}_{4} \mathrm{Cl}$ were carefully added, and the resulting solution was dried over anhydrous $\mathrm{MgSO}_{4}$, filtered, and concentrated under reduced pressure. Flash chromatography (1:1 hexane-EtOAc to EtOAc) of the residue afforded the secondary amine $(62 \mathrm{mg}, 68 \%)$.

${ }^{1} \mathrm{H}$ NMR (400 MHz, $\mathrm{CDCl}_{3}$, selected resonances): $\delta 0.85(\mathrm{~m}, 1 \mathrm{H}, \mathrm{H}-31), 1.68(\mathrm{t}, J=7.6 \mathrm{~Hz}, 2 \mathrm{H}, \mathrm{H}-$ 15), 2.31 (t, J = 7.6 Hz, 2H, H-14), 2.64 (dd, J = 13.2, $2.8 \mathrm{~Hz}, \mathrm{H}, \mathrm{H}-6 \mathrm{ax}), 2.77$ (m, 1H, H-19), 2.84 (d, $J=12.4 \mathrm{~Hz}, 1 \mathrm{H}, \mathrm{H}-10 \mathrm{ax}$ ), 2.86 (d, $J=13.6 \mathrm{~Hz}, 1 \mathrm{H}, \mathrm{H}-8 \mathrm{ax}$ ), 3.08 (d, $J=12.4 \mathrm{~Hz}, 1 \mathrm{H}, \mathrm{H}-10 \mathrm{eq}$ ), 3.65 (d, J = $13.6 \mathrm{~Hz}, 1 \mathrm{H}, \mathrm{H}-8 \mathrm{eq}), 3.66$ (s, $3 \mathrm{H}, \mathrm{CH}_{3} \mathrm{O}$ ), 3.81 (br. s, $1 \mathrm{H}, \mathrm{H}-2$ ), 4.40 (d, J = $13.2 \mathrm{~Hz}, 1 \mathrm{H}$, $\mathrm{H}-6 \mathrm{eq}$ ), 5.20 (br. $\mathrm{t}, \mathrm{J}=6.4 \mathrm{~Hz}, 1 \mathrm{H}, \mathrm{H}-20), 5.35$ ( $\mathrm{m}, 2 \mathrm{H}, \mathrm{H}-17, \mathrm{H}-18$ ). 


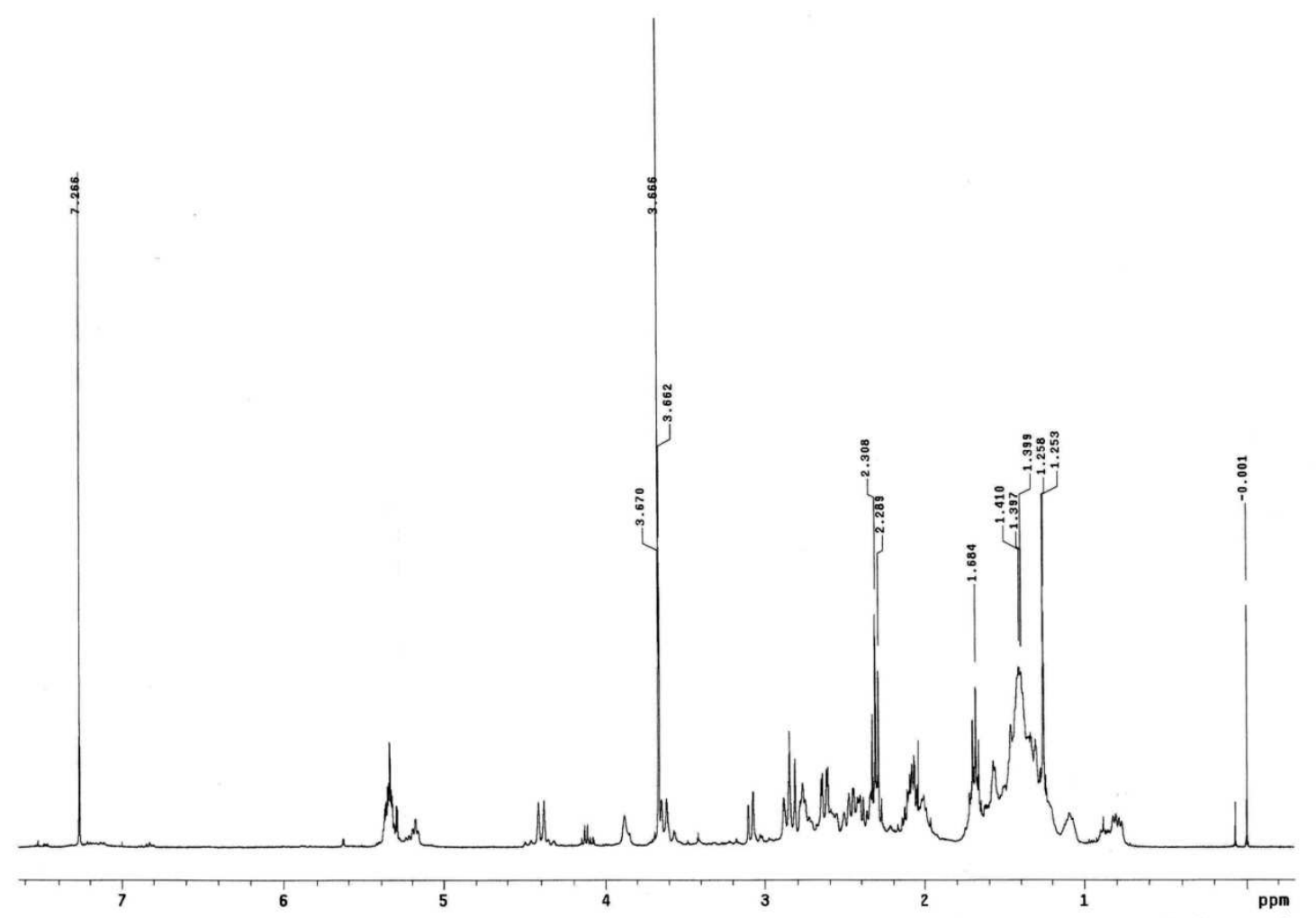




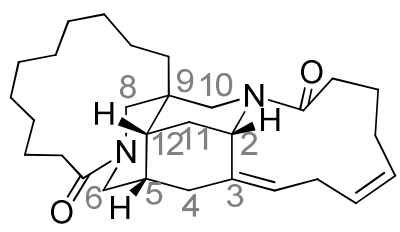

(madangamine numbering)

\section{Pentacyclic dilactam (138).}

First step: An aqueous solution of $\mathrm{LiOH}(1 \mathrm{M}, 3.7 \mathrm{~mL}, 3.7 \mathrm{mmol})$ was added to a solution of the above secondary amine (45 $\mathrm{mg}, 0.092 \mathrm{mmol})$ in anhydrous THF $(3 \mathrm{~mL})$, and the resulting mixture was stirred at room temperature for $3.5 \mathrm{~h}$. The reaction was quenched by addition of saturated aqueous $\mathrm{NaCl}$, and the solution was extracted with EtOAc. The combined organic extracts were dried over anhydrous $\mathrm{MgSO}_{4}$, filtered, and concentrated under reduced pressure to afford the corresponding acid, which was used in the next step without purification.

Second step: A solution of the above amino acid (0.092 mmol) in a 9:1 $\mathrm{CH}_{2} \mathrm{Cl}_{2}$-DMF mixture (3.1 $\mathrm{mL}$ ) was slowly added with a syringe pump $(6 \mathrm{~h})$ to a solution of EDCI (84 $\mathrm{mg}, 0.441 \mathrm{mmol}$ ) and HOBt (60 mg, $0.441 \mathrm{mmol}$ ) in 9:1 $\mathrm{CH}_{2} \mathrm{Cl}_{2}$-DMF (1.5 mL). The mixture was stirred overnight at room temperature, and the resulting solution was then concentrated under reduced pressure. Flash chromatography (9:1 to 6:4 hexane-EtOAc) of the residue afforded pentacyclic dilactam 138 (31 mg, 75\%).

$[\alpha]_{D}^{22}=+44.0\left(c 3.5, \mathrm{CHCl}_{3}\right)$.

${ }^{1} \mathrm{H} \mathrm{NMR} \mathrm{(500} \mathrm{MHz,} \mathrm{CDCl}{ }_{3}$ COSY, gHSQC): $\delta 0.75(\mathrm{dm}, J=14.0 \mathrm{~Hz}, 1 \mathrm{H}, \mathrm{H}-31), 1.20-1.50(\mathrm{~m}, 15 \mathrm{H}$, $\left.\mathrm{CH}_{2}, \mathrm{H}-11\right), 1.60\left(\mathrm{~m}, 2 \mathrm{H}, \mathrm{H}-12, \mathrm{CH}_{2}\right), 1.64\left(\mathrm{~m}, 1 \mathrm{H}, \mathrm{CH}_{2}\right), 1.80\left(\mathrm{~m}, 1 \mathrm{H}, \mathrm{CH}_{2}\right), 1.90\left(\mathrm{~m}, 1 \mathrm{H}, \mathrm{CH}_{2}\right), 1.90$ (m, 1H, H-31), 2.00-2.15 (m, 6H, H-5, CH $\mathrm{CH}_{2}, 2.20$ (m, 1H, H-11), 2.28 (ddd, $J=14.0,12.0,3.5 \mathrm{~Hz}$, 1H, H-16), 2.40-2.55 (m, 3H, H-19, 2H-4), 2.60 (ddd, J = 14.0, 10.5, 3.5 Hz, 1H, H-16), 2.69 (dd, J $=13.5,3.5 \mathrm{~Hz}, 1 \mathrm{H}, \mathrm{H}-6), 2.88-3.00(\mathrm{~m}, 2 \mathrm{H}, \mathrm{H}-8, \mathrm{H}-10), 3.16$ (dt, J = 15.0, $9.5 \mathrm{~Hz}, 1 \mathrm{H}, \mathrm{H}-19), 3.65$ (d, J = 13.5 Hz, 1H, H-10), 4.32 (d, J = 13.5 Hz, 1H, H-6), 4.36 (d, J = 14.5 Hz, 1H, H-8), 4.97 (br. s, $1 \mathrm{H}, \mathrm{H}-2), 5.20$ (td, J = 10.5, $4.5 \mathrm{~Hz}, 1 \mathrm{H}, \mathrm{H}-18), 5.40(\mathrm{~m}, 1 \mathrm{H}, \mathrm{H}-17), 5.60(\mathrm{t}, J=8.5 \mathrm{~Hz}, 1 \mathrm{H}, \mathrm{H}-20)$.

${ }^{13} \mathrm{C}$ NMR $\left(125 \mathrm{MHz}, \mathrm{CDCl}_{3}\right): \delta 20.8\left(\mathrm{CH}_{2}\right), 22.6\left(\mathrm{CH}_{2}\right), 23.5\left(\mathrm{CH}_{2}\right), 24.7\left(\mathrm{CH}_{2}\right), 25.4\left(\mathrm{CH}_{2}\right), 25.5$ $\left(\mathrm{CH}_{2}\right), 25.8$ (C-19), $26.5\left(\mathrm{CH}_{2}\right), 26.6\left(\mathrm{CH}_{2}\right), 27.6\left(\mathrm{CH}_{2}\right), 27.9\left(\mathrm{CH}_{2}\right), 30.0(\mathrm{C}-11), 32.1(\mathrm{C}-16), 33.6$ $\left(\mathrm{CH}_{2}\right.$ ), 33.7 (C-4), 34.0 (C-31), 34.2 (C-5), 38.1 (C-9), 38.2 (C-12), 41.9 (C-10), 46.7 (C-6), 48.1 (C2), 53.0 (C-8), 126.2 (C-20), 128.0, 128.1 (C-17, C-18), 136.1 (C-3), 174.0, 174.1 (2NCO). 
HRMS (ESI) calcd for $\left[\mathrm{C}_{29} \mathrm{H}_{44} \mathrm{~N}_{2} \mathrm{O}_{2}+\mathrm{H}\right]^{+}: 453.3476$, found: 453.3467 .
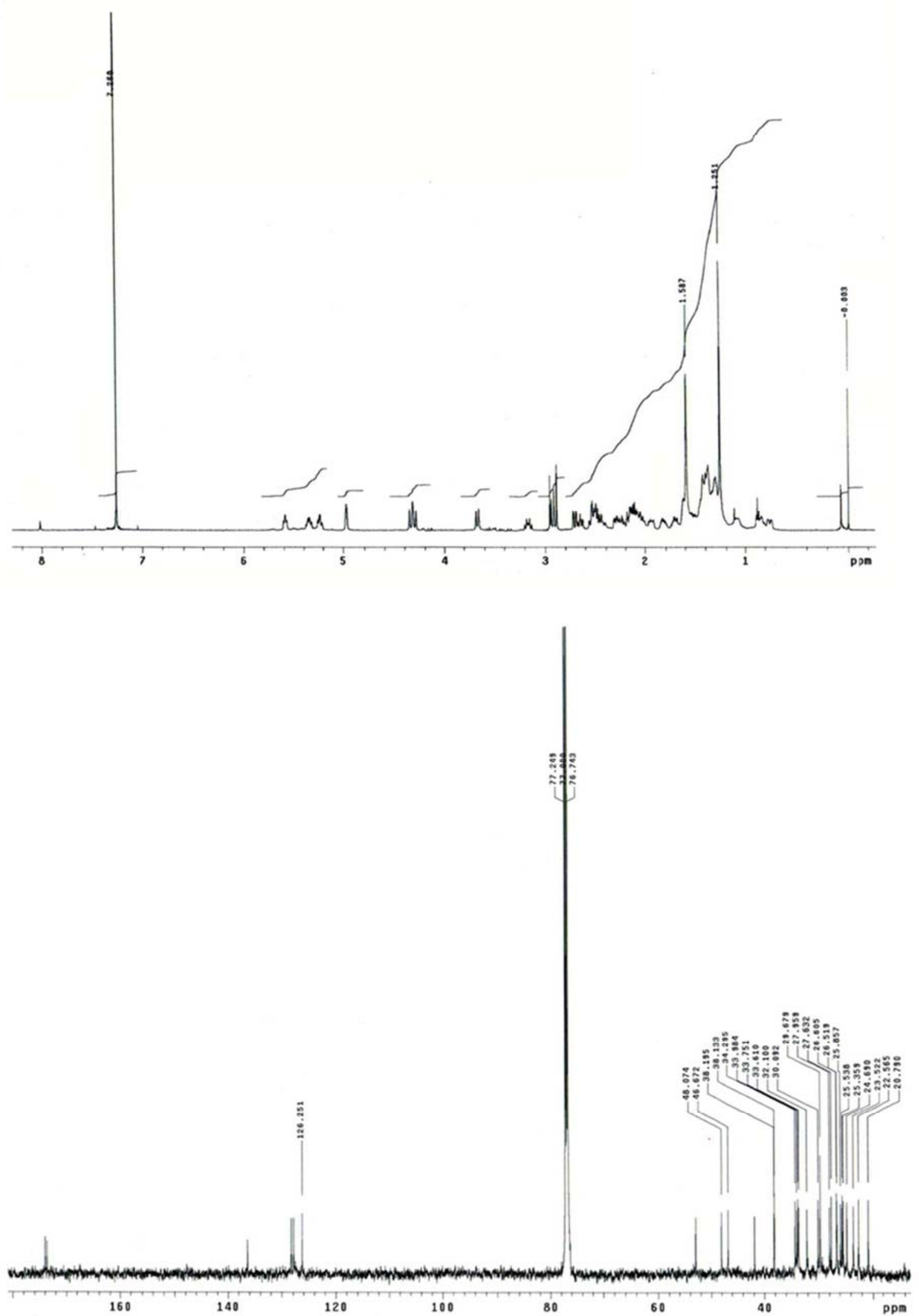


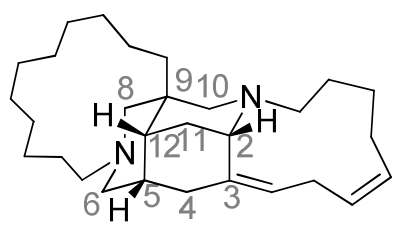

(madangamine numbering)

\section{Madangamine D.}

$\mathrm{LiAlH}_{4}(9 \mathrm{mg}, 0.268 \mathrm{mmol}$ ) was added under an argon atmosphere to a solution of pentacyclic dilactam $138(20 \mathrm{mg}, 0.044 \mathrm{mmol})$ in anhydrous THF $(2 \mathrm{~mL})$. The resulting mixture was stirred at $0{ }^{\circ} \mathrm{C}$ for 40 minutes and at room temperature for 3 hours. The mixture was cooled, and the reaction was quenched with a few drops of distilled water. The resulting suspension was dried over anhydrous $\mathrm{MgSO}_{4}$, filtered, and concentrated under reduced pressure. Flash chromatography (hexane to $8: 2$ hexane-EtOAc) of the residue afforded pure madangamine $D$ (13 mg, 68\%).

$[\alpha]_{D}^{22}=+101.3\left(c 0.29, \mathrm{CHCl}_{3}\right)$.

${ }^{1} \mathrm{H}$ NMR $\left(600 \mathrm{MHz}, \mathrm{C}_{6} \mathrm{D}_{6}, \mathrm{COSY}, \mathrm{gHSQC}\right): \delta 1.01(\mathrm{dm}, J=14.0 \mathrm{~Hz}, 1 \mathrm{H}, \mathrm{H}-31), 1.20-1.45(\mathrm{~m}, 23 \mathrm{H}$, $\left.\mathrm{CH}_{2}, \mathrm{H}-11, \mathrm{H}-12\right), 1.69(\mathrm{~m}, 1 \mathrm{H}, \mathrm{H}-14), 1.75(\mathrm{~m}, 1 \mathrm{H}, \mathrm{H}-5), 1.79$ (d, J = 11.2 Hz, 1H, H-8), 1.80 (m, $1 \mathrm{H}, \mathrm{H}-16), 2.19$ (dd, $J=11.2,3.6 \mathrm{~Hz}, 1 \mathrm{H}, \mathrm{H}-6), 2.20-2.30$ (m, 4H, H-31, H-16, H-4, H-21), 2.33 (m, $2 \mathrm{H}, \mathrm{H}-19, \mathrm{H}-6), 2.38(\mathrm{dt}, J=12.6,3.6 \mathrm{~Hz}, 1 \mathrm{H}, \mathrm{H}-11), 2.45(\mathrm{~d}, J=12.0 \mathrm{~Hz}, 1 \mathrm{H}, \mathrm{H}-10), 2.61(\mathrm{~m}, 1 \mathrm{H}$, $\mathrm{H}-13), 2.66(\mathrm{~m}, 1 \mathrm{H}, \mathrm{H}-21), 2.69$ (d, J = 11.2 Hz, 1H, H-8), 2.82 (ddd, J = 14.0, 11.2, $6.0 \mathrm{~Hz}, 1 \mathrm{H}, \mathrm{H}-$ 13), $3.09(\mathrm{tm}, J=13.2 \mathrm{~Hz}, 1 \mathrm{H}, \mathrm{H}-4), 3.30(\mathrm{~d}, J=12.0 \mathrm{~Hz}, 1 \mathrm{H}, \mathrm{H}-10), 3.35(\mathrm{t}, J=13.2 \mathrm{~Hz}, 1 \mathrm{H}, \mathrm{H}-19)$, 3.71 (br. s, $1 \mathrm{H}, \mathrm{H}-2$ ), 5.20 (dm, $J=12.0 \mathrm{~Hz}, 1 \mathrm{H}, \mathrm{H}-20$ ), 5.40 (tdd, $J=11.4,6.0,1.2 \mathrm{~Hz}, 1 \mathrm{H}, \mathrm{H}-17$ ), $5.46(\mathrm{td}, J=11.4,4.2 \mathrm{~Hz}, 1 \mathrm{H}, \mathrm{H}-18)$.

${ }^{13} \mathrm{C}$ NMR (150 MHz, $\left.\mathrm{C}_{6} \mathrm{D}_{6}\right): \delta 21.6\left(\mathrm{CH}_{2}\right), 24.6(\mathrm{C}-14), 25.2(\mathrm{C}-15), 25.4(\mathrm{C}-16), 26.0\left(\mathrm{CH}_{2}\right), 26.2$ $\left(\mathrm{CH}_{2}\right), 26.3\left(\mathrm{CH}_{2}\right), 26.5\left(\mathrm{CH}_{2}\right), 26.8\left(\mathrm{CH}_{2}\right), 27.0(\mathrm{C}-19), 27.3\left(\mathrm{CH}_{2}\right), 28.5\left(\mathrm{CH}_{2}\right), 30.2\left(\mathrm{CH}_{2}\right), 32.2(\mathrm{C}-$ 11), 35.8 (C-31), 36.8 (C-5), 37.5 (C-9), 37.7 (C-12), 38.6 (C-4), 51.8 (C-2), 54.1 (C-10), 56.3 (C13), 57.4 (C-21), 59.6 (C-6), 61.0 (C-8), 121.9 (C-20), 129.0 (C-17), 129.1 (C-18), 139.3 (C-3).

HRMS (ESI) calcd for $\left[\mathrm{C}_{29} \mathrm{H}_{48} \mathrm{~N}_{2}+\mathrm{H}\right]^{+}: 425.3887$, found: 425.3890 . 


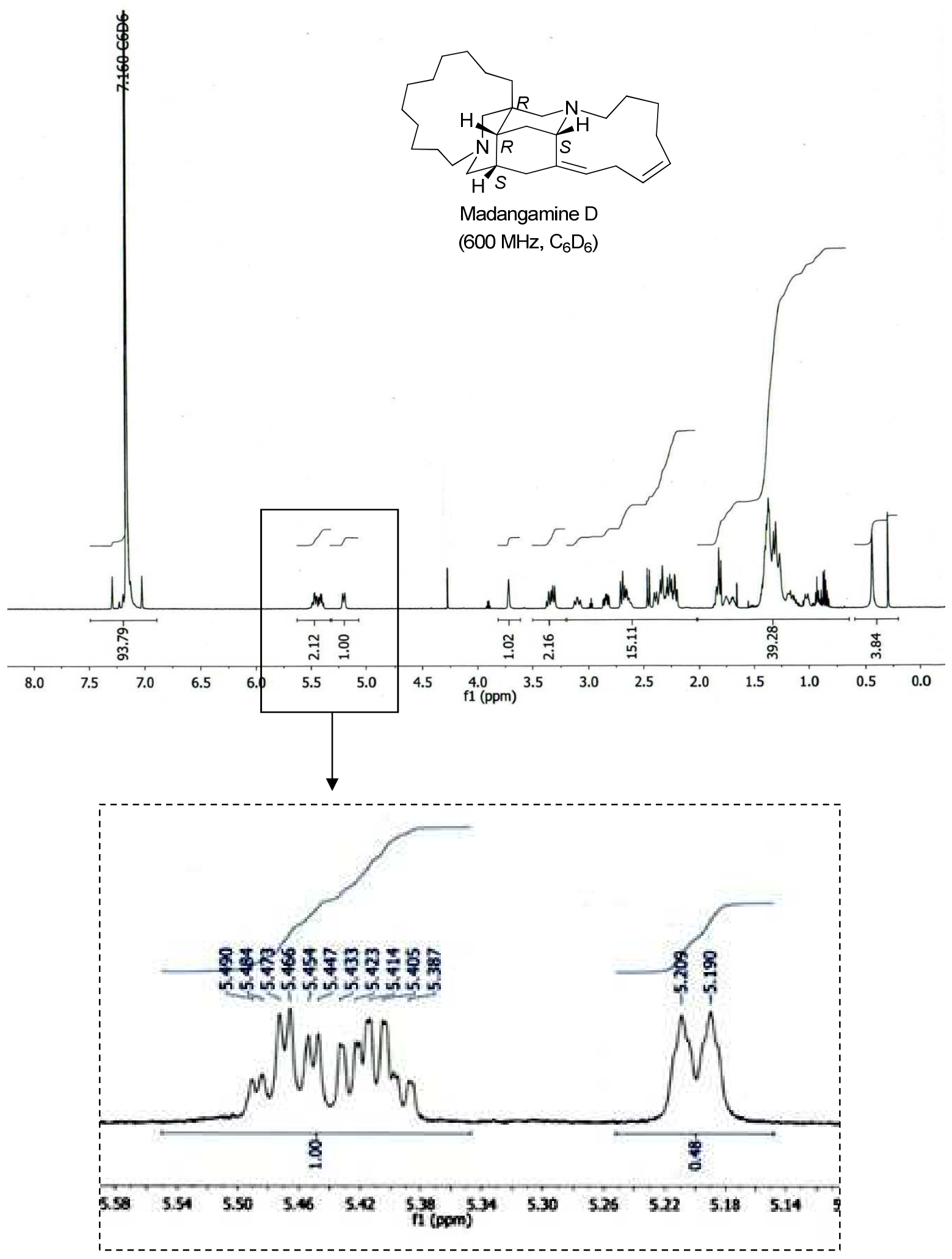



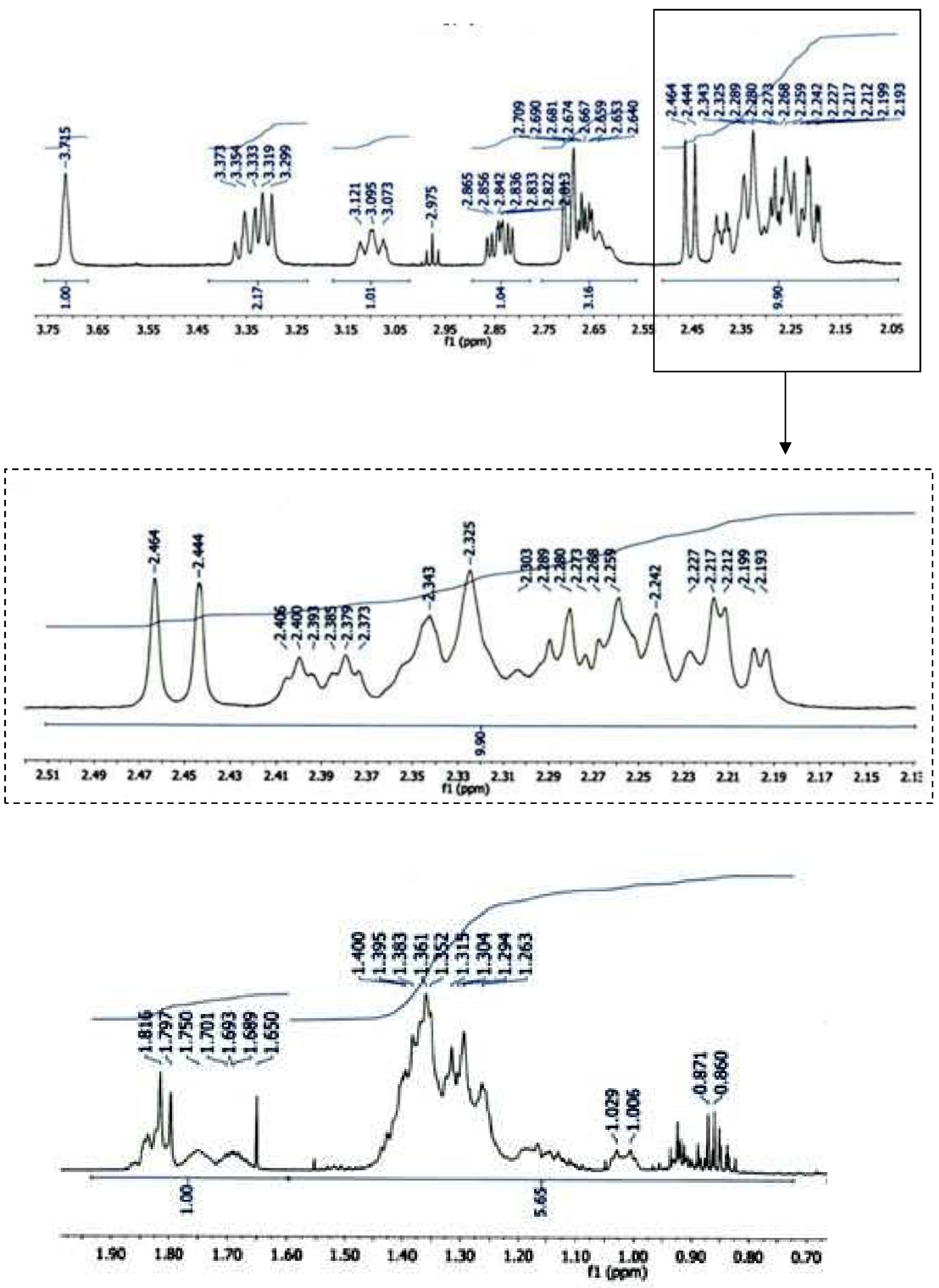

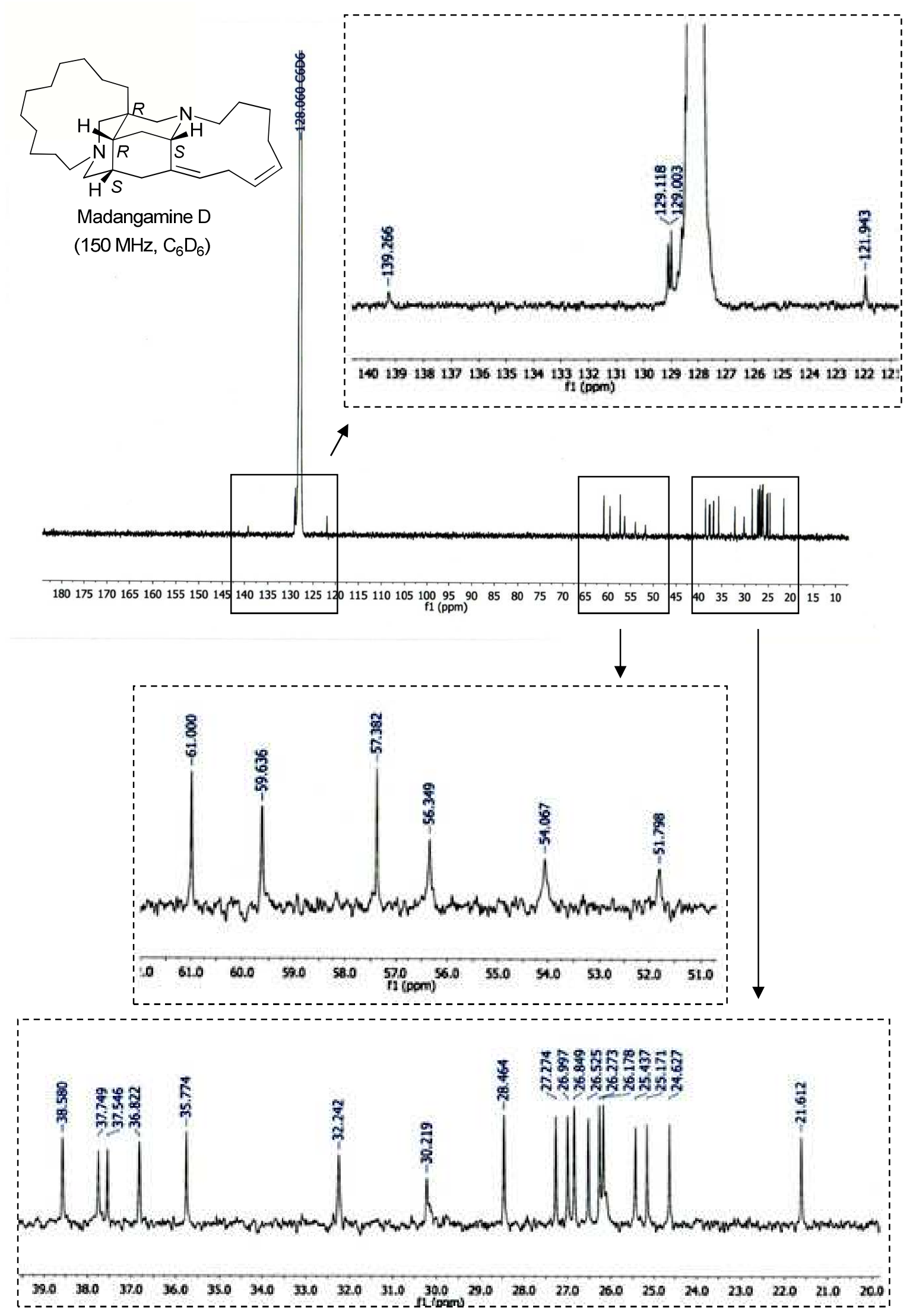


\section{Madangamine $D$}

${ }^{1} \mathrm{H}-{ }^{1} \mathrm{H}$ Cosy, $600 \mathrm{MHz}, \mathrm{C}_{6} \mathrm{D}_{6}$

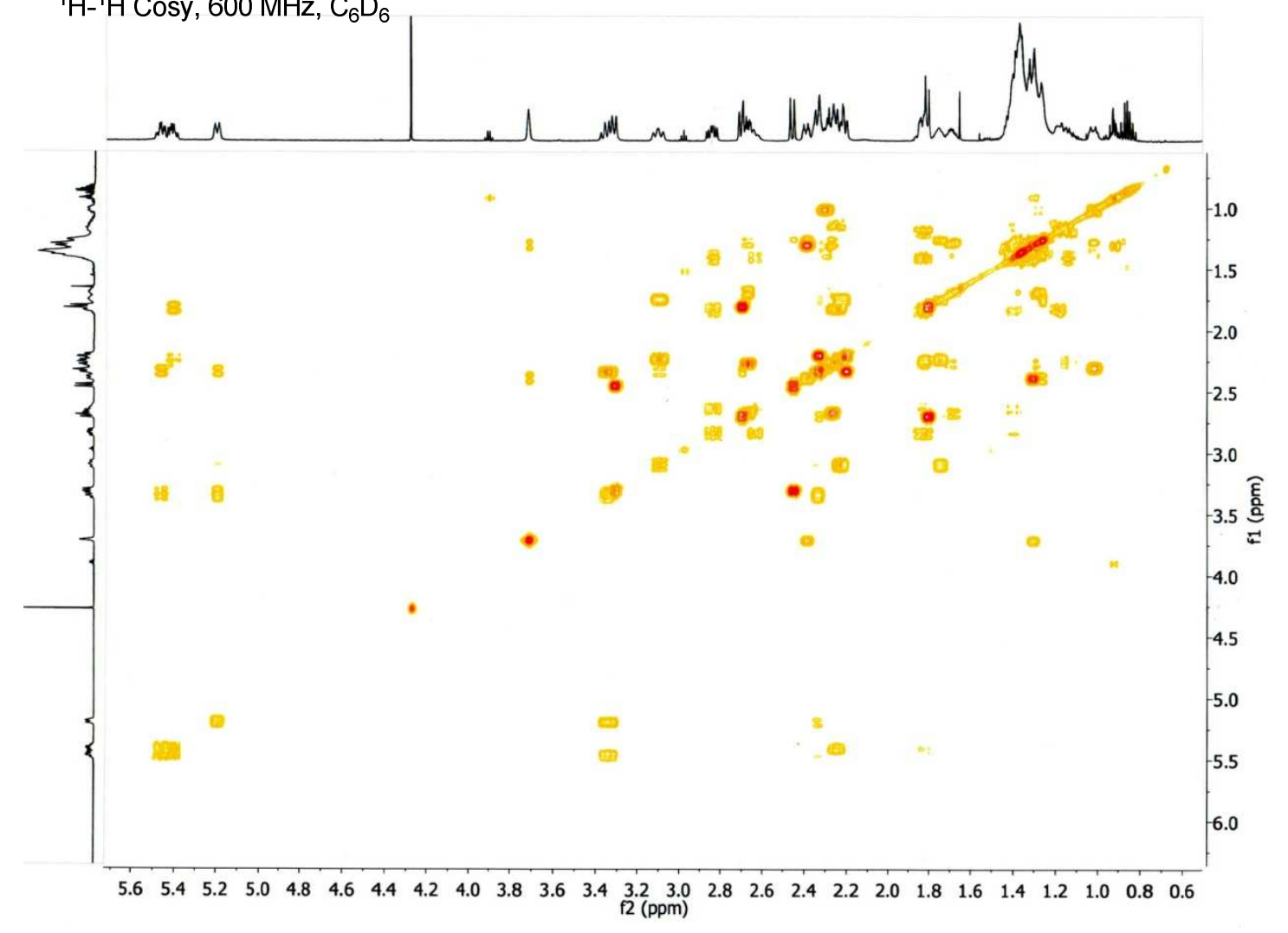

Madangamine $\mathrm{D}$

${ }^{1} \mathrm{H}-{ }^{1} \mathrm{H}$ Noesy, $400 \mathrm{MHz}, \mathrm{C}_{6} \mathrm{D}_{6}$

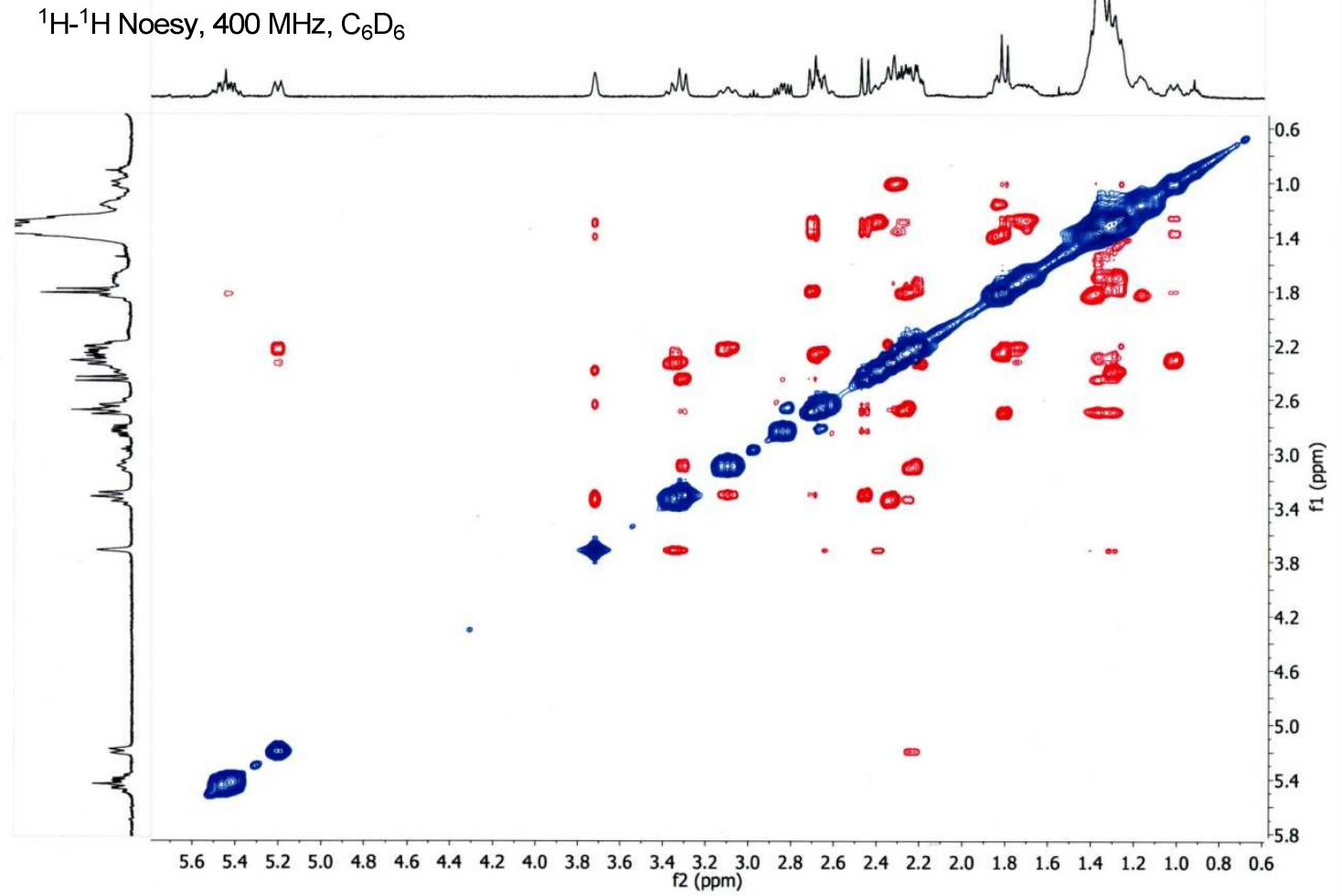


Madangamine $\mathrm{D}$

${ }^{1} \mathrm{H}_{-}{ }^{13} \mathrm{C}$ gHSQC, $600 \mathrm{MHz}, \mathrm{C}_{6} \mathrm{D}_{6}$
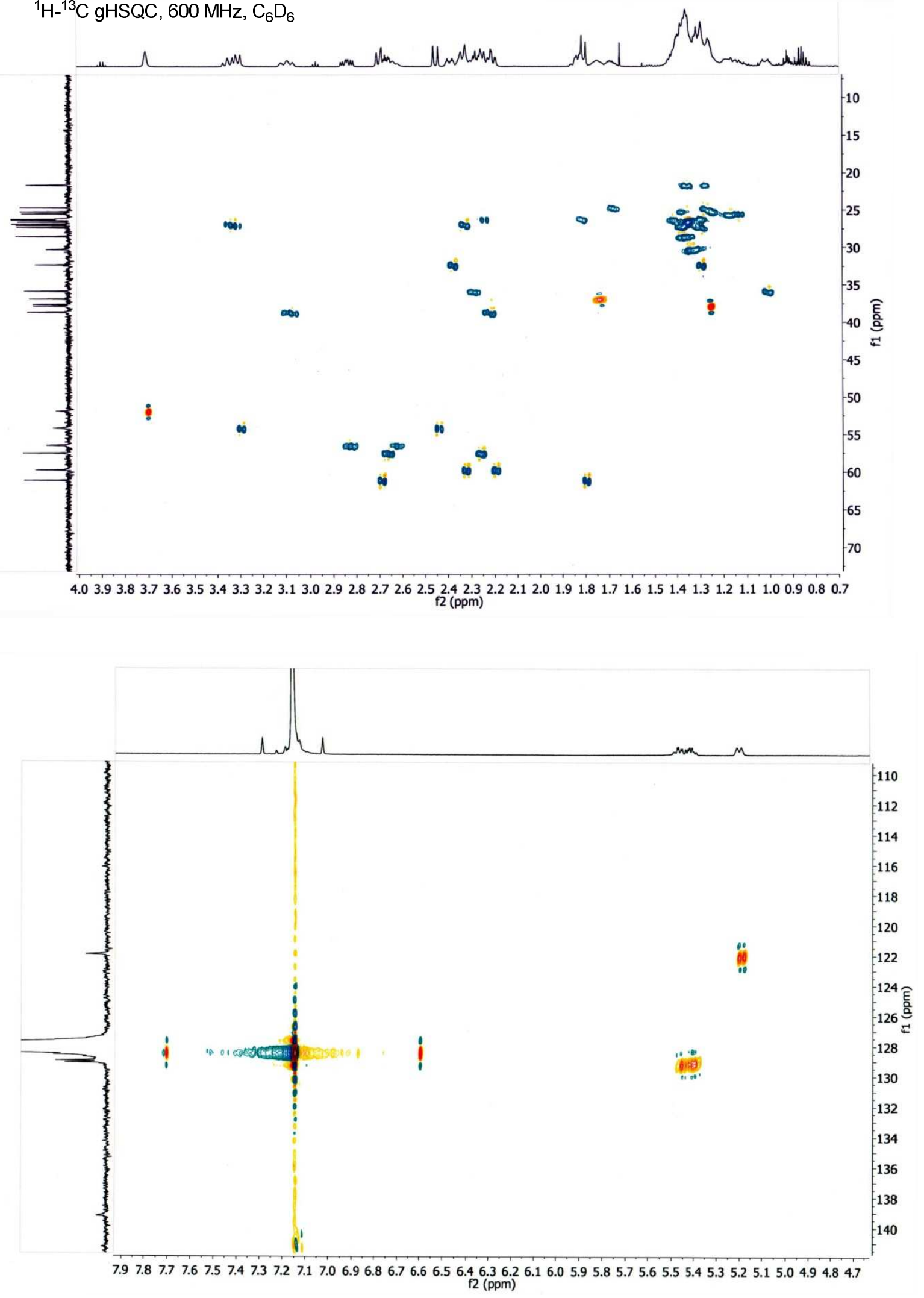
<smiles>C1CNCC(CCC2OCCO2)C1</smiles>

\section{3-[2-(1,3-Dioxolan-2-yl)ethyl]piperidine (57).}

Alkylation step: $n$-BuLi $(25.8 \mathrm{~mL}$ of a $1.6 \mathrm{M}$ solution in hexane, $45.6 \mathrm{mmol}$ ) was added to a solution of $\delta$-valerolactam $(2.26 \mathrm{~g}, 22.8 \mathrm{mmol})$ in anhydrous THF $(75 \mathrm{~mL})$ at $-78{ }^{\circ} \mathrm{C}$ under inert atmosphere. The resulting mixture was stirred at $0{ }^{\circ} \mathrm{C}$ for $1 \mathrm{~h}$. 2-(2-bromoethyl)-1,3-dioxolane $(2.73 \mathrm{~mL}, 22.8 \mathrm{mmol})$ and TBAI $(1.6 \mathrm{~g}, 4.5 \mathrm{mmol})$ were then added at $0{ }^{\circ} \mathrm{C}$ and the mixture was stirred overnight at the same temperature. Saturated aqueous $\mathrm{NaCl}$ was added and the mixture was extracted with $\mathrm{CH}_{2} \mathrm{Cl}_{2}$. The combined organic extracts were dried, filtered and concentrated under reduced pressure. Flash chromatography (neutral alumina, EtOAc) afforded lactam 56 (4.5 g, quant.)

Reduction step: $\mathrm{LiAlH}_{4}(201 \mathrm{mg}, 5.3 \mathrm{mmol})$ was added at room temperature and under inert atmosphere to a solution of lactam $56(527 \mathrm{mg}, 2.6 \mathrm{mmol})$ in anhydrous THF ( $25 \mathrm{~mL})$ and the mixture was stirred at reflux overnight. The resulting suspension was cooled to $0{ }^{\circ} \mathrm{C}$, and the reaction was quenched with distilled water. The aqueous layer was extracted with $\mathrm{Et}_{2} \mathrm{O}$, and the combined organic extracts were dried, filtered, and concentrated to afford the corresponding amine 57 (327 mg, 64\%) which was used in the next step without purification.

${ }^{1} \mathrm{H}$ NMR (400 MHz, CDCl 3 , COSY, gHSQC): $\delta 1.03$ (qd, $\left.J=12.4,3.6 \mathrm{~Hz}, 1 \mathrm{H}, \mathrm{H}-4\right), 1.23-1.34(\mathrm{~m}, 2 \mathrm{H}$, $\left.2 \mathrm{H}-1^{\prime}\right), 1.37-1.49$ (m, 2H, H-3, H-5), 1.67 (m, 3H, 2H-2', H-5), 1.76 (br. s, 1H, NH), 1.95-2.06 (dm, $J=12.4 \mathrm{~Hz}, 1 \mathrm{H}, \mathrm{H}-4), 2.23(\mathrm{dd}, J=12.0,10.4 \mathrm{~Hz}, 1 \mathrm{H}, \mathrm{H}-2), 2.51$ (td, $J=11.6,2.8 \mathrm{~Hz}, 1 \mathrm{H}, \mathrm{H}-6)$, $3.00(\mathrm{dm}, J=11.6 \mathrm{~Hz}, 2 \mathrm{H}, \mathrm{H}-6), 3.03(\mathrm{dm}, J=12.0 \mathrm{~Hz}, 2 \mathrm{H}, \mathrm{H}-2), 3.85\left(\mathrm{~m}, 2 \mathrm{H}, \mathrm{CH}_{2} \mathrm{O}\right), 3.96(\mathrm{~m}, 2 \mathrm{H}$, $\left.\mathrm{CH}_{2} \mathrm{O}\right), 4.83\left(\mathrm{t}, \mathrm{J}=5.2 \mathrm{~Hz}, 1 \mathrm{H}, \mathrm{CHO}_{2}\right.$ ), 6.25 (br. s, $1 \mathrm{H}, \mathrm{NH}$ ).

${ }^{13} \mathrm{C} \mathrm{NMR}\left(100.6 \mathrm{MHz}, \mathrm{CDCl}_{3}\right.$ ): $\delta 26.6(\mathrm{C}-5), 28.7\left(\mathrm{C}-1^{\prime}\right), 31.1$ (C-2'), 31.5 (C-4), 37.7 (C-3), 47.1 (C6), $53.0(\mathrm{C}-2), 64.8\left(2 \mathrm{CH}_{2} \mathrm{O}\right), 104.7\left(\mathrm{CHO}_{2}\right)$. 

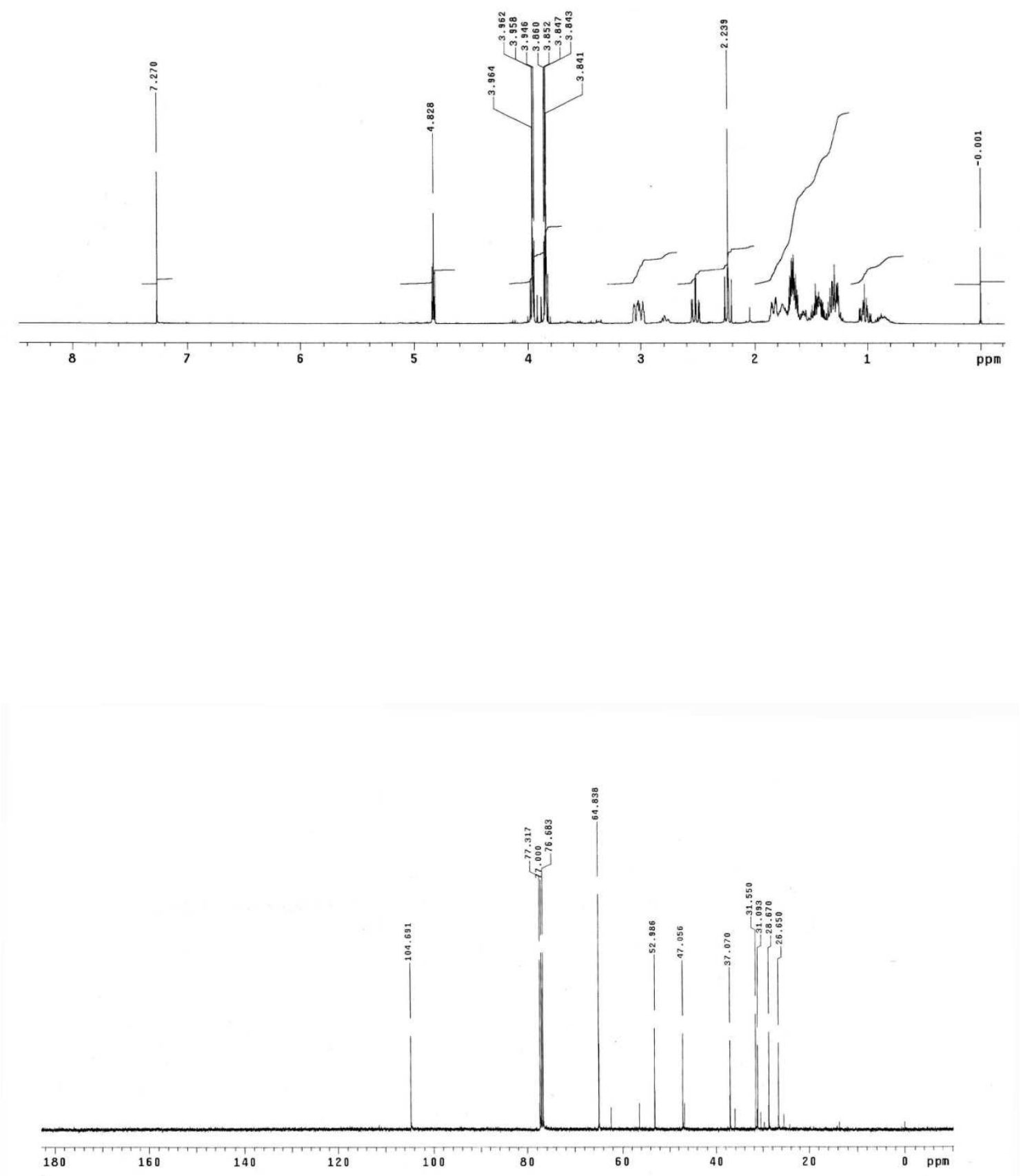
<smiles></smiles>

\section{1-(5-hexenoyl)-3-piperidine propionaldehyde (58)}

First step: Oxalyl chloride ( $4.73 \mathrm{~mL}$ of a $2 \mathrm{M}$ solution in $\mathrm{CH}_{2} \mathrm{Cl}_{2}, 9.47 \mathrm{mmol}$ ) were added to a solution of 5-hexenoic acid (5.64 mmol) with 1 drop of DMF at $0{ }^{\circ} \mathrm{C}$ and, after the evolution of gas subsided, the resulting mixture was stirred for $15 \mathrm{~min}$ then diluted with $\mathrm{Et}_{2} \mathrm{O}$, filtered and concentrated under reduced pressure to give a clear oil.

To a solution of amine $57(292 \mathrm{mg}, 1.5 \mathrm{mmol})$ in $\mathrm{CH}_{2} \mathrm{Cl}_{2}(13 \mathrm{~mL})$ was added $\mathrm{Et}_{3} \mathrm{~N}(4.5 \mathrm{mmol})$. The solution was stirred for $15 \mathrm{~min}$ and then cooled at $0{ }^{\circ} \mathrm{C}$. A solution of 5 -hexanoil chloride (2.55 mmol) in $\mathrm{CH}_{2} \mathrm{Cl}_{2}(5 \mathrm{~mL})$ was slowly added. The reaction mixture was stirred at $0{ }^{\circ} \mathrm{C}$ for $1 \mathrm{~h}$, then gradually warmed to room temperature and stirred overnight. The reaction mixture was cooled at $0{ }^{\circ} \mathrm{C}$, distilled water was then added and the mixture was extracted with $\mathrm{CH}_{2} \mathrm{Cl}_{2}$. The combined organic extracts were dried over anhydrous $\mathrm{Na}_{2} \mathrm{SO}_{4}$, filtered and concentrated under reduced pressure. Flash chromatography (9:1 to 7:3 hexane-EtOAc) afforded acylated compound (83\%).

Second step: $\mathrm{HCl}\left(6 \mathrm{~mL}\right.$ of a $10 \%$ solution in $\mathrm{H}_{2} \mathrm{O}$ ) was added to a solution of the above amide (112 $\mathrm{mg}, 0.4 \mathrm{mmol})$ in acetone $(15 \mathrm{~mL})$. The resulting mixture was stirred at room temperature for $48 \mathrm{~h}$ and then poured into $\mathrm{NaHCO}_{3}$ saturated solution. The acetone was removed under reduced pressure and the aqueous phase was extracted several times with $\mathrm{CH}_{2} \mathrm{Cl}_{2}$. The combined organic extracts were dried over anhydrous $\mathrm{Na}_{2} \mathrm{SO}_{4}$, filtered, and concentrated under reduced pressure to give aldehyde $58(93 \mathrm{mg}, 98 \%)$ which was used in the next step without purification.

${ }^{1} \mathrm{H}$ NMR (400 MHz, CDCl 3 , COSY, gHSQC): $\delta 1.16(\mathrm{~m}, 1 \mathrm{H}, \mathrm{H}-4), 1.44(\mathrm{~m}, 2 \mathrm{H}, \mathrm{H}-5, \mathrm{H}-3), 1.55(\mathrm{~m}$, $\left.2 \mathrm{H}, 2 \mathrm{H}-1^{\prime}\right), 1.73\left(\mathrm{~m}, 3 \mathrm{H}, \mathrm{H}-5,2 \mathrm{H}-3^{\prime \prime}\right), 1.87(\mathrm{dm}, J=12.4 \mathrm{~Hz}, 1 \mathrm{H}, \mathrm{H}-4), 2.10\left(\mathrm{~m}, 2 \mathrm{H}, 2 \mathrm{H}-4^{\prime \prime}\right), 2.32$ $\left(\mathrm{t}, J=7.6 \mathrm{~Hz}, 2 \mathrm{H}, 2 \mathrm{H}-2^{\prime \prime}\right), 2.42,2.57(2 \mathrm{td}, J=12.8,2.8 \mathrm{~Hz}, 1 \mathrm{H}, \mathrm{H}-6 \mathrm{ax}), 2.52\left(\mathrm{~m}, 2 \mathrm{H}, 2 \mathrm{H}-2^{\prime}\right), 2.70$, 
$3.02(2 \mathrm{dd}, J=13.2,10.4 \mathrm{~Hz}, 1 \mathrm{H}, \mathrm{H}-2 \mathrm{ax}), 3.71,3.75(2 \mathrm{~d}, J=13.2 \mathrm{~Hz}, 1 \mathrm{H}, \mathrm{H}-2 \mathrm{eq}), 4.38,4.44$ (2d, J $=12.8 \mathrm{~Hz}, 1 \mathrm{H}, \mathrm{H}-6 \mathrm{eq})$, 4.97-5.05 (m, 2H, CH2=), $5.80(\mathrm{~m}, 1 \mathrm{H}, \mathrm{CH}=)$, 9.78, $9.80(2 \mathrm{~s}, 1 \mathrm{H}, \mathrm{CHO})$.

${ }^{13} \mathrm{C}$ NMR (100.6 MHz, CDCl $)$ ): $\delta 24.4$ (C-3") 25.5 (C-5, C-1'), 29.2 (C-4), 30.7, 30.8 (C-2") , 32.4, 33.5 (C-4"), 33.3 (C-3), 41.1, 41.3 (C-2'), 42.3, 47.0 (C-6), 46.3, 51.5 (C-2), $115.1\left(\mathrm{CH}_{2}=\right), 138.1$ $(\mathrm{CH}=), 171.3$ (NCO), 201.5, $201.9(\mathrm{CHO})$.
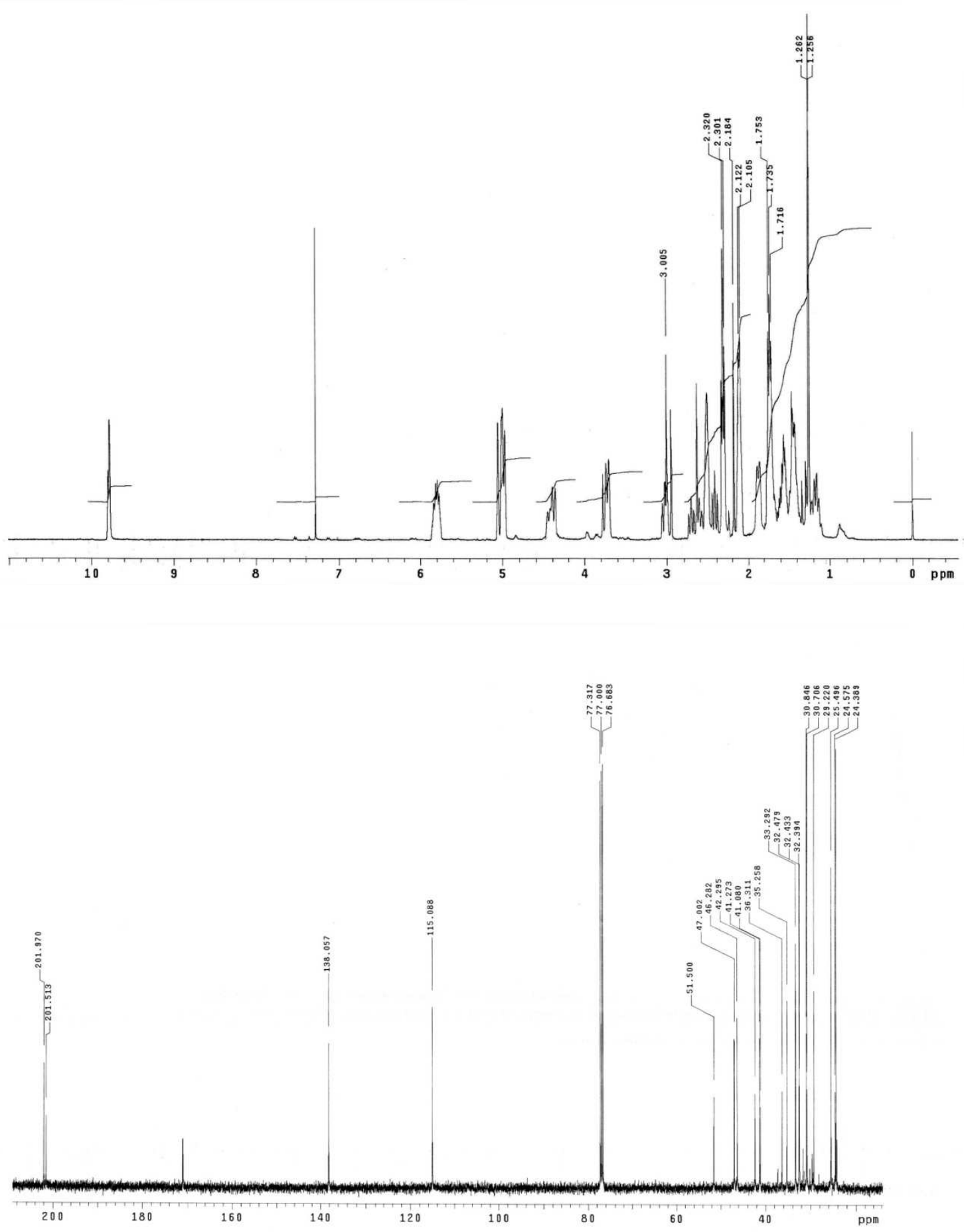


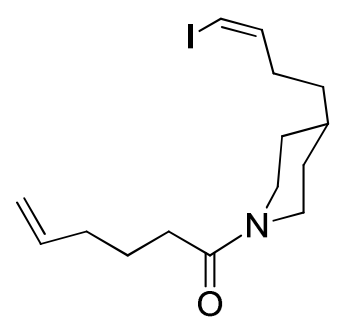

(Z)-1-(5-hexenoyl)-3-(4-iodo-3-butenyl)piperidine (59).

A suspension of the phosphonium iodide ( $362 \mathrm{mg}, 0.683 \mathrm{mmol}$ ) in $5 \mathrm{~mL}$ of THF and $0.12 \mathrm{~mL}$ anhydrous HMPA was added to a solution of NaHMDS (1.0 M in THF, $683 \mu \mathrm{L}, 0.683 \mathrm{mmol}$ ) in THF (10 mL) at $10-15{ }^{\circ} \mathrm{C}$ (ice-water bath). After $1 \mathrm{~min}$, the mixture was cooled to $-78^{\circ} \mathrm{C}$ and a solution of aldehyde 58 (108 mg, $0.455 \mathrm{mmol})$ in THF $(5.0 \mathrm{~mL})$ was added dropwise. After being stirred at $-78{ }^{\circ} \mathrm{C}$ for $20 \mathrm{~min}$, the reaction was quenched by addition of aqueous saturated $\mathrm{NH}_{4} \mathrm{Cl}$ and water. When the mixture was warmed to room temperature, the insoluble solids were filtered off, the layers of the filtrate were separated and the aqueous layer was extracted with diethyl ether. The combined organic phases were washed with brine, dried over $\mathrm{MgSO}_{4}$, filtered, and concentrated. Flash chromatography (2:8 hexane-EtOAc) of the resulting residue afforded vinyl iodide 59 (76 mg, 46\%).

${ }^{1} \mathrm{H}$ NMR (400 MHz, CDCl, COSY, gHSQC): $\delta 1.15(\mathrm{~m}, 1 \mathrm{H}, \mathrm{H}-4), 1.39\left(\mathrm{~m}, 2 \mathrm{H}, 2 \mathrm{H}-1^{\prime}\right), 1.44(\mathrm{~m}, 2 \mathrm{H}$, $\mathrm{H}-3, \mathrm{H}-5), 1.73\left(\mathrm{~m}, 3 \mathrm{H}, \mathrm{H}-5,2 \mathrm{H}-3^{\prime \prime}\right), 1.90$ (dt, J = $\left.13.2 \mathrm{~Hz}, 3.36 \mathrm{~Hz}, 1 \mathrm{H}, \mathrm{H}-4\right), 2.11\left(\mathrm{~m}, 2 \mathrm{H}, \mathrm{H}-4^{\prime \prime}\right)$, $2.18\left(m, 2 \mathrm{H}, \mathrm{H}-2^{\prime}\right), 2.33\left(\mathrm{~m}, 2 \mathrm{H}, 2 \mathrm{H}-2^{\prime \prime}\right), 2.34,2.61\left(\mathrm{~m}, 2 \mathrm{H}, \mathrm{H}-6 \mathrm{ax}, 2-\mathrm{H} 2^{\prime \prime}\right), 2.68,2.99(\mathrm{~m}, 1 \mathrm{H}, \mathrm{H}-$ 2ax), 3.72, $3.76(\mathrm{~d}, \mathrm{~J}=14 \mathrm{~Hz}, \mathrm{H}-2 \mathrm{eq}), 4.39,4.43(\mathrm{~m}, \mathrm{~J}=, 1 \mathrm{H}, \mathrm{H}-6 \mathrm{eq}), 4.97,5.05\left(\mathrm{~m}, 2 \mathrm{H}, \mathrm{CH}_{2}=\right)$, $5.79(\mathrm{~m}, 1 \mathrm{H}, \mathrm{CH}=), 6.24\left(\mathrm{~d}, J=7.2 \mathrm{~Hz}, 1 \mathrm{H}, \mathrm{H}-4^{\prime}\right), 6.16\left(\mathrm{~m}, 1 \mathrm{H}, \mathrm{H}-3^{\prime}\right)$.

${ }^{13} \mathrm{C} \mathrm{NMR}\left(100.6 \mathrm{MHz}, \mathrm{CDCl}_{3}\right): \delta 24.4$ (C-3"), 25.7 (C-5), 30.7 (C-4), 31.6 (C-1'), 31.9 (C-2'), 32.5 (C-2"), 33.2 (C-4"), 35.2 (C-3), 42.3, 47.4 (C-6), 46.3, 51.4 (C-2), 115.0 (C-6"), 138.0 (C-5"), 140.4 (C-3'), 140.7 (C-4'), 171.0 (NCO). 

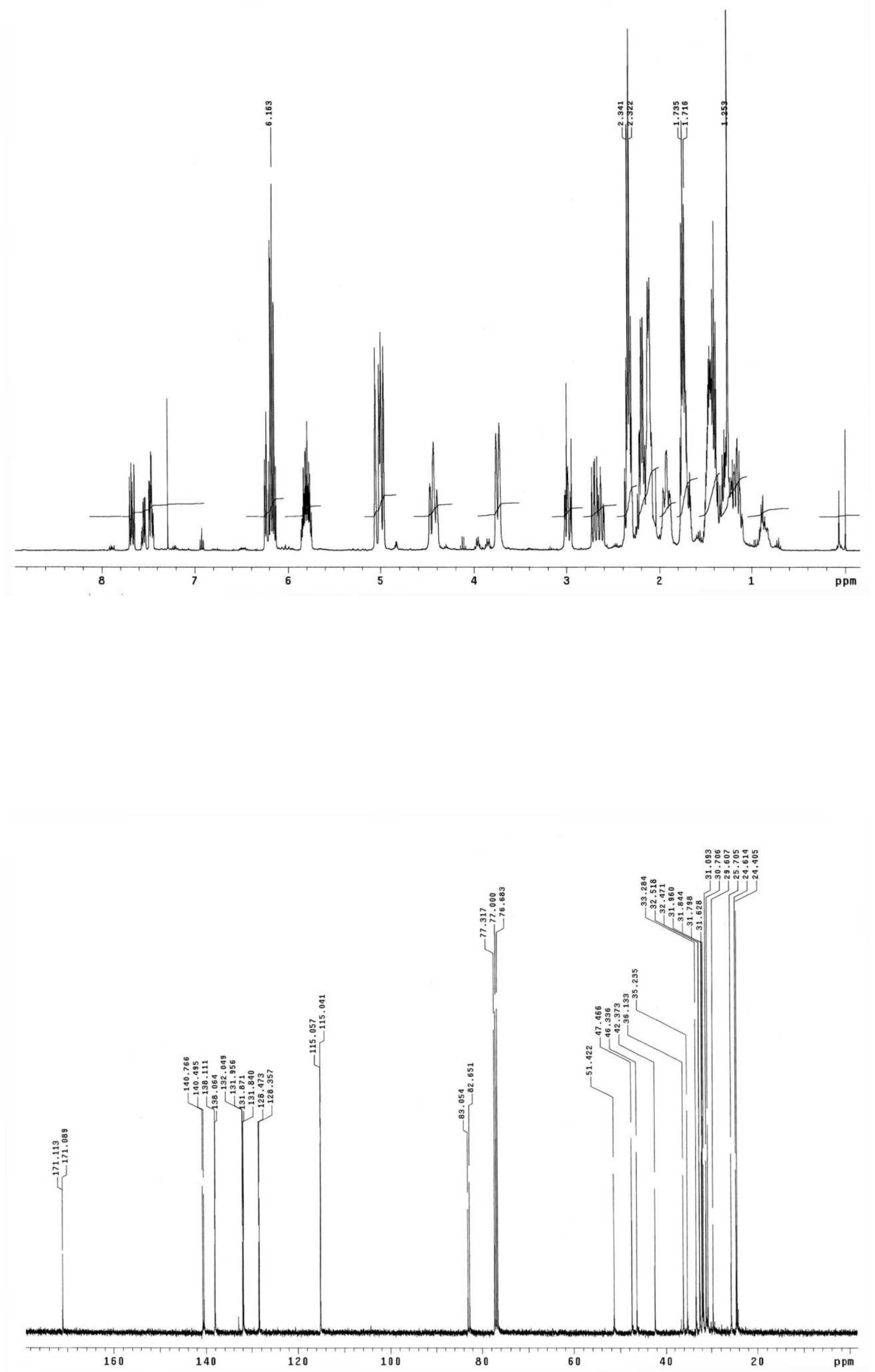
<smiles>C=CCCC1(C(=O)OC)CCCNC1=O</smiles>

\section{3-(3-Butenyl)-3-(methoxycarbonyl)-2-piperidone (64).}

A solution of $\mathrm{NaH}$ (127 mg of a $60 \%$ dispersion in mineral oil, $3.17 \mathrm{mmol}$ ) in DMF ( $5 \mathrm{~mL}$ ) was added via transfer to a solution of 3-(methoxycarbonyl)-2-piperidone (415 mg, $2.64 \mathrm{mmol})$ in DMF $(13 \mathrm{~mL})$ at $0{ }^{\circ} \mathrm{C}$, and the resulting mixture was stirred at $0{ }^{\circ} \mathrm{C}$ for $1 \mathrm{~h}$. 4-Bromo-1-butene (1.34 $\mathrm{mL}, 13.2 \mathrm{mmol}$ ) and tetrabutylammonium iodide (195 $\mathrm{mg}, 0.52 \mathrm{mmol})$ were then added, and the mixture was stirred at room temperature for $4 \mathrm{~h}$. The reaction was quenched with saturated aqueous $\mathrm{NH}_{4} \mathrm{Cl}$ solution, and the resulting mixture was extracted with $\mathrm{CH}_{2} \mathrm{Cl}_{2}$. The combined organic extracts were dried over anhydrous $\mathrm{Na}_{2} \mathrm{SO}_{4}$, filtered, and concentrated under reduced pressure. Flash chromatography (9:1 to 1:9 hexane-EtOAc) afforded compound 64 (506 mg, 91\%).

IR (film): 1731 (COO), 1665 (NCO) $\mathrm{cm}^{-1}$.

${ }^{1} \mathrm{H}$ NMR (400 MHz, CDCl 3 , COSY, gHSQC): $\delta 1.83(\mathrm{~m}, 3 \mathrm{H}, 2 \mathrm{H}-4, \mathrm{H}-5), 1.95-3.25\left(\mathrm{~m}, 4 \mathrm{H}, \mathrm{H}-1^{\prime}, \mathrm{H}-\right.$ $\left.2^{\prime}\right), 2.21(\mathrm{~m}, 1 \mathrm{H}, \mathrm{H}-5), 3.33(\mathrm{~m}, 2 \mathrm{H}, \mathrm{H}-6), 3.74\left(\mathrm{~s}, 3 \mathrm{H}, \mathrm{CH}_{3} \mathrm{O}\right), 4.95(\mathrm{dd}, \mathrm{J}=10.0,1.2 \mathrm{~Hz}, 1 \mathrm{H}, \mathrm{CH}=)$, 5.03 (dd, $J=16.0,1.2 \mathrm{~Hz}, 1 \mathrm{H}, \mathrm{CH}_{2}=$ ), 5.80 (ddt, $\left.J=16.0,10.0,5.6 \mathrm{~Hz}, 1 \mathrm{H}, \mathrm{CH}=\right), 6.85$ (d, $J=5.2$ $\mathrm{Hz}, 1 \mathrm{H}, \mathrm{NH})$.

${ }^{13} \mathrm{C}$ NMR (100.6 MHz, CDCl $)$ ): $\delta 19.5$ (C-4), 28.8 (C-2'), 29.5 (C-5), 34.7 (C-1'), 42.1 (C-6), 52.4 $\left(\mathrm{CH}_{3} \mathrm{O}\right), 53.5(\mathrm{C}-3), 114.7\left(\mathrm{CH}_{2}=\right), 137.8(\mathrm{CH}=), 170.9(\mathrm{NCO}), 173.2(\mathrm{COO})$.

HRMS (ESI) calcd for $\left[\mathrm{C}_{11} \mathrm{H}_{17} \mathrm{NO}_{3}+\mathrm{H}\right]^{+}:$:212.1281, found: 212.1281 . 

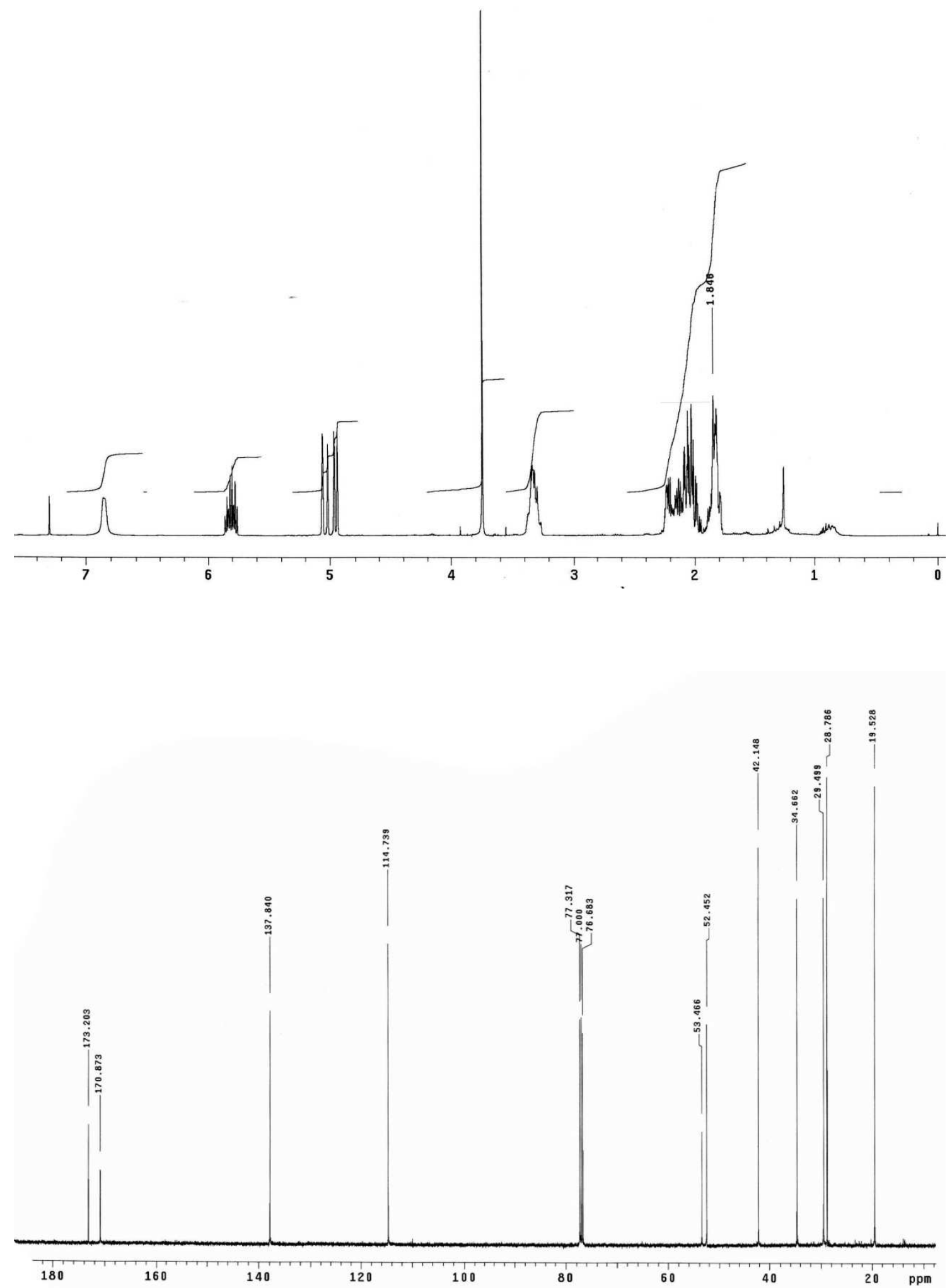
<smiles>C=CCCCC(C(C)=O)C(=O)NCC</smiles>

3-(Methoxycarbonyl)-3-(4-pentenyl)-2-piperidone (65).

Operating as described above, from a solution of 3-methoxycarbonyl-2-piperidone $(231 \mathrm{mg}$, $1.47 \mathrm{mmol}$ ) in DMF (5 mL), a suspension of $\mathrm{NaH}$ (71 $\mathrm{mg}$ of a $60 \%$ dispersion in mineral oil, 1.76 $\mathrm{mmol}$ ) in DMF (5 mL), 5-bromo-1-pentene $(0.87 \mathrm{~mL}, 7.35 \mathrm{mmol})$, and tetrabutylammonium iodide (108 mg, $0.29 \mathrm{mmol}$ ), compound 65 was obtained (380 mg, 99\%) after flash cromatography.

IR (film): 1725 (COO), 1665 (NCO) $\mathrm{cm}^{-1}$.

${ }^{1} \mathrm{H}$ NMR (400 MHz, $\left.\mathrm{CDCl}_{3}, \mathrm{COSY}, \mathrm{gHSQC}\right): \delta 1.39\left(\mathrm{ttd}, J=12.4,12.4,7.2,7.2,4.8 \mathrm{~Hz}, 1 \mathrm{H}, \mathrm{H}-2^{\prime}\right)$, $1.46\left(m, 1 \mathrm{H}, \mathrm{H}-2^{\prime}\right), 1.79(\mathrm{dd}, J=10.8,3.6 \mathrm{~Hz}, 1 \mathrm{H}, \mathrm{H}-5), 1.82-2.02\left(\mathrm{~m}, 4 \mathrm{H}, 2 \mathrm{H}-1^{\prime}, 2 \mathrm{H}-4\right), 2.07(\mathrm{~m}$, $\left.2 \mathrm{H}, \mathrm{H}-3^{\prime}\right), 2.21(\mathrm{~m}, 1 \mathrm{H}, \mathrm{H}-5), 3.33(\mathrm{~m}, 2 \mathrm{H}, \mathrm{H}-6), 3.74\left(\mathrm{~s}, 3 \mathrm{H}, \mathrm{CH}_{3} \mathrm{O}\right), 4.93-5.04\left(\mathrm{~m}, 2 \mathrm{H}, \mathrm{CH}_{2}=\right), 5.79$ (dddd, $J=16.8,10.0,6.4,6.4 \mathrm{~Hz}, 1 \mathrm{H}, \mathrm{CH}=$ ).

${ }^{13} \mathrm{C}$ NMR $\left(100.6 \mathrm{MHz}, \mathrm{CDCl}_{3}\right.$ ): $\delta 19.7$ (C-4), 23.9 (C-2'), 29.5 (C-5), $33.9\left(\mathrm{C}-3^{\prime}\right), 35.1$ (C-1'), 42.4 (C6), $52.6\left(\mathrm{CH}_{3} \mathrm{O}\right), 53.9(\mathrm{C}-3), 114.8\left(\mathrm{CH}_{2}=\right), 138.3(\mathrm{CH}=), 170.7(\mathrm{NCO}), 173.4(\mathrm{COO})$. 

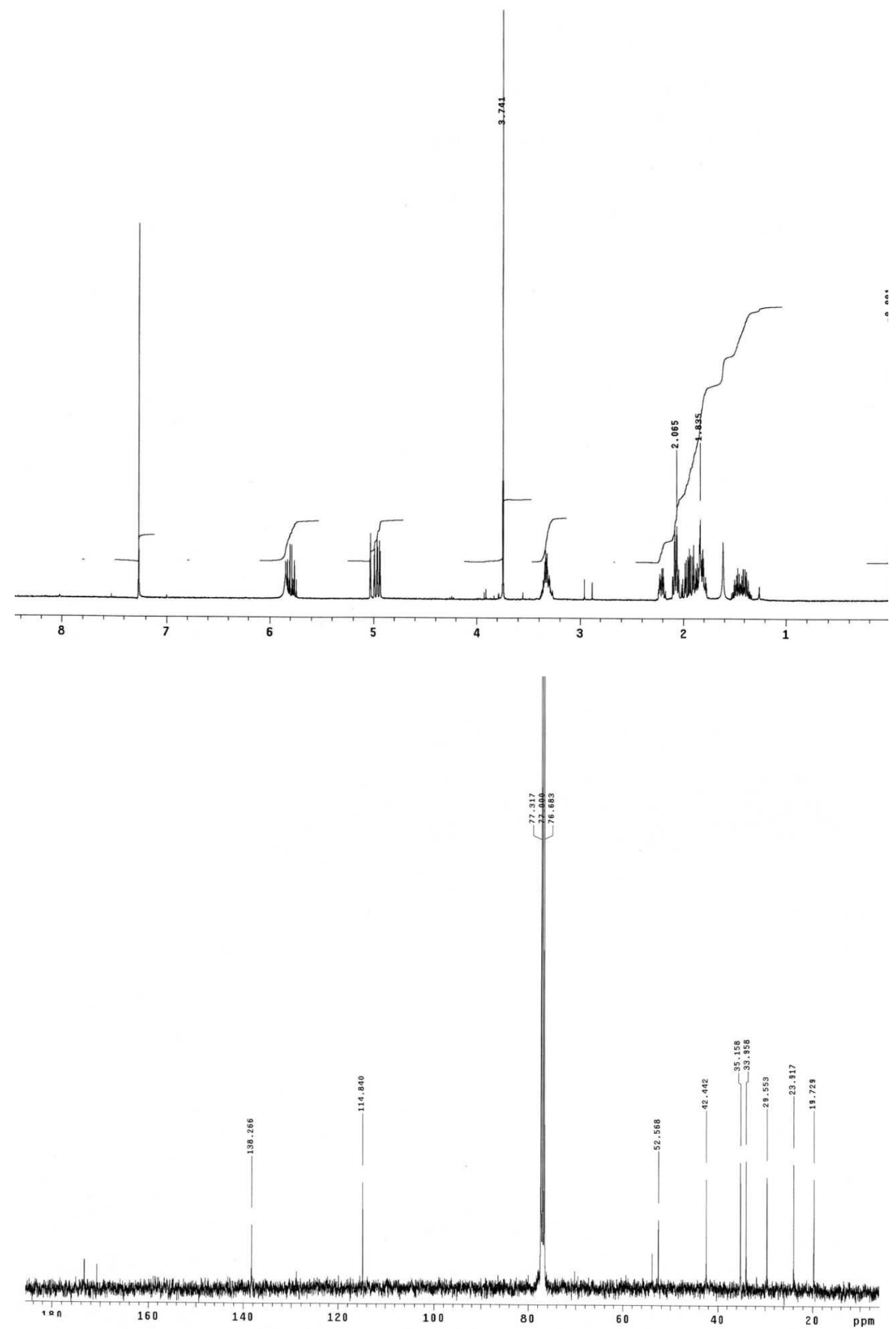


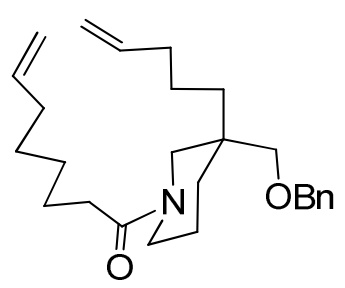

3-(Benzyloxymethyl)-3-(4-pentenyl)-1-(7-octenoyl) piperidine (70).

Reduction step: Lithium aluminum hydride $(90 \mathrm{mg}, 2.38 \mathrm{mmol})$ was added to a solution of compound 65 (302 mg, $1.34 \mathrm{mmol})$ in anhydrous THF (20 mL) under argon atmosphere, and the mixture was heated at reflux for $4 \mathrm{~h}$. The resulting suspension was cooled to room temperature, and the reaction was quenched with distilled water. The aqueous layer was extracted with $\mathrm{Et}_{2} \mathrm{O}$, and the combined organic extracts were dried, filtered, and concentrated to give 3-(hydroxymethyl)-3-(4-pentenyl)piperidine (67) $(220 \mathrm{mg})$, which was used in the next step without purification.

IR (film): $3400(\mathrm{NH}, \mathrm{OH}) \mathrm{cm}^{-1}$.

${ }^{1} \mathrm{H}$ NMR (400 MHz, CDCl 3 , COSY, gHSQC): $\delta 1.15\left(\mathrm{~m}, 2 \mathrm{H}, \mathrm{H}-1^{\prime}\right), 1.24-1.36\left(\mathrm{~m}, 3 \mathrm{H}, 2 \mathrm{H}-2^{\prime}, \mathrm{H}-4\right)$, 1.53 (ddd, $J=17.2,8.4,4.4 \mathrm{~Hz}, 1 \mathrm{H}, \mathrm{H}-5$ ), 1.68 (dt, $J=14.0,3.6 \mathrm{~Hz}, 1 \mathrm{H}, \mathrm{H}-4), 1.89(\mathrm{~m}, 1 \mathrm{H}, \mathrm{H}-5$ ), $2.00\left(\mathrm{~m}, 2 \mathrm{H}, \mathrm{H}-3^{\prime}\right), 2.55(\mathrm{~d}, J=11.6 \mathrm{~Hz}, 1 \mathrm{H}, \mathrm{H}-2), 2.61(\mathrm{td}, J=11.2,3.2 \mathrm{~Hz}, 1 \mathrm{H}, \mathrm{H}-6), 2.84$ (br. s, $1 \mathrm{H}, \mathrm{OH}), 2.95(\mathrm{dd}, J=11.2,4.4 \mathrm{~Hz}, 1 \mathrm{H}, \mathrm{H}-6), 2.97(\mathrm{~d}, J=11.6 \mathrm{~Hz}, 1 \mathrm{H}, \mathrm{H}-2), 3.67\left(\mathrm{~s}, 2 \mathrm{H}, \mathrm{CH}_{2} \mathrm{OH}\right.$ ), 4.93-5.02 (2m, 2H, $\left.\mathrm{CH}_{2}=\right), 5.78(\mathrm{ddt}, J=16.8,10.4,6.8,6.8 \mathrm{~Hz}, 1 \mathrm{H}, \mathrm{CH}=)$.

${ }^{13} \mathrm{C} \mathrm{NMR}\left(100.6 \mathrm{MHz}, \mathrm{CDCl}_{3}\right): \delta 22.2\left(\mathrm{C}-2^{\prime}\right), 22.9$ (C-5), 33.4 (C-4), 34.5 (C-3'), 35.7 (C-3), 37.2 (C$\left.1^{\prime}\right), 46.8(\mathrm{C}-6), 55.6(\mathrm{C}-2), 71.1\left(\mathrm{CH}_{2} \mathrm{OH}\right), 114.6\left(\mathrm{CH}_{2}=\right), 138.7(\mathrm{CH}=)$.

Acylation step: Oxalyl chloride $\left(1.0 \mathrm{~mL}\right.$ of a $2 \mathrm{M}$ solution in $\mathrm{CH}_{2} \mathrm{Cl}_{2}, 2.0 \mathrm{mmol}$ ) was added to a solution of 7-octenoic acid $(0.15 \mathrm{~mL}, 1.0 \mathrm{mmol})$ in $\mathrm{CH}_{2} \mathrm{Cl}_{2}(4 \mathrm{~mL})$ and two drops of DMF, and the resulting mixture was stirred for $3 \mathrm{~h}$. $\mathrm{Et}_{2} \mathrm{O}$ was added, and the resulting mixture was filtered and concentrated under reduced pressure to give crude 7-octenoyl chloride.

A solution of the above chloride $(1.0 \mathrm{mmol})$ in chloroform $(5 \mathrm{~mL})$ was added to a suspension of $67(157 \mathrm{mg}, 0.859 \mathrm{mmol})$ and $\mathrm{K}_{2} \mathrm{CO}_{3}(273 \mathrm{mg}, 1.975 \mathrm{mmol})$ in chloroform $(10 \mathrm{~mL})$, and the mixture was stirred for $1.5 \mathrm{~h}$ at room temperature. Distilled water was then added, and the resulting mixture was stirred for 20 minutes. The aqueous layer was extracted with chloroform, and the combined organic extracts were dried over anhydrous $\mathrm{Na}_{2} \mathrm{SO}_{4}$, filtered, and concentrated under reduced pressure. Flash chromatography (8:2 to 1:1 hexane-EtOAc) 
afforded 3-(hydroxymethyl)-1-(7-octenoyl)-3-(4-pentenyl)piperidine (130 mg, 50\% overall yield for the two steps).

${ }^{1} \mathrm{H}$ NMR (400 MHz, CDCl 3 , COSY, gHSQC): $\delta 1.14\left(\mathrm{td}, J=16.0,16.0,4.0 \mathrm{~Hz}, 1 \mathrm{H}, \mathrm{H}-1^{\prime}\right), 1.29-1.47$ $\left(\mathrm{m}, 7 \mathrm{H}, 2 \mathrm{H}-2^{\prime}, 2 \mathrm{H}-4^{\prime \prime}, 2 \mathrm{H}-5^{\prime \prime}, \mathrm{H}-4\right), 1.49-1.58\left(\mathrm{~m}, 4 \mathrm{H}, 2 \mathrm{H}-5, \mathrm{H}-4, \mathrm{H}-\mathrm{1}^{\prime}\right), 1.64\left(\mathrm{~m}, 2 \mathrm{H}, \mathrm{H}-3^{\prime}\right), 2.05$ (m, 4H, H-6", H-3'), 2.35 (t, J = 7.2 Hz, 2H, H-2') 2.40 (d, $J=13.2 \mathrm{~Hz}, 1 \mathrm{H}, \mathrm{H}-2), 3.06(\mathrm{dm}, J=$ $14.4 \mathrm{~Hz}, 1 \mathrm{H}, \mathrm{H}-6), 3.22$ (d, J = 11.2 Hz, 1H, $\mathrm{CH}_{2} \mathrm{OH}$ ), 3.25 (br. $\mathrm{s}, 1 \mathrm{H}, \mathrm{OH}$ ), 3.39 (d, J = $11.2 \mathrm{~Hz}, 1 \mathrm{H}$, $\left.\mathrm{CH}_{2} \mathrm{OH}\right), 3.78(\mathrm{dm}, J=14.4 \mathrm{~Hz}, 1 \mathrm{H}, \mathrm{H}-6), 4.25(\mathrm{~d}, J=13.2 \mathrm{~Hz}, 1 \mathrm{H}, \mathrm{H}-2), 4.92-5.03\left(4 \mathrm{~m}, 4 \mathrm{H}, \mathrm{CH}_{2}=\right)$, 5.80 (ddt, $J=17.2,10.0,6.8,6.8 \mathrm{~Hz}, 2 \mathrm{H}, \mathrm{CH}=$ ).

${ }^{13} \mathrm{C}$ NMR (100.6 MHz, CDCl $): \delta 22.2$ (C-2'), 22.6 (C-5), 25.3 (C-3'), 28.6, 28.9 (C-4", C-5"), 30.7 (C-4), 33.0 (C-2"), 33.5 (C-6") 34.4 (C-3'), 35.5 (C-1'), 38.5 (C-3), 47.2 (C-6), 48.9 (C-2), 62.4 $\left(\mathrm{CH}_{2} \mathrm{OH}\right), 114.4,114.5\left(\mathrm{CH}_{2}=\right), 138.7,138.8(\mathrm{CH}=), 172.9$ (NCO).

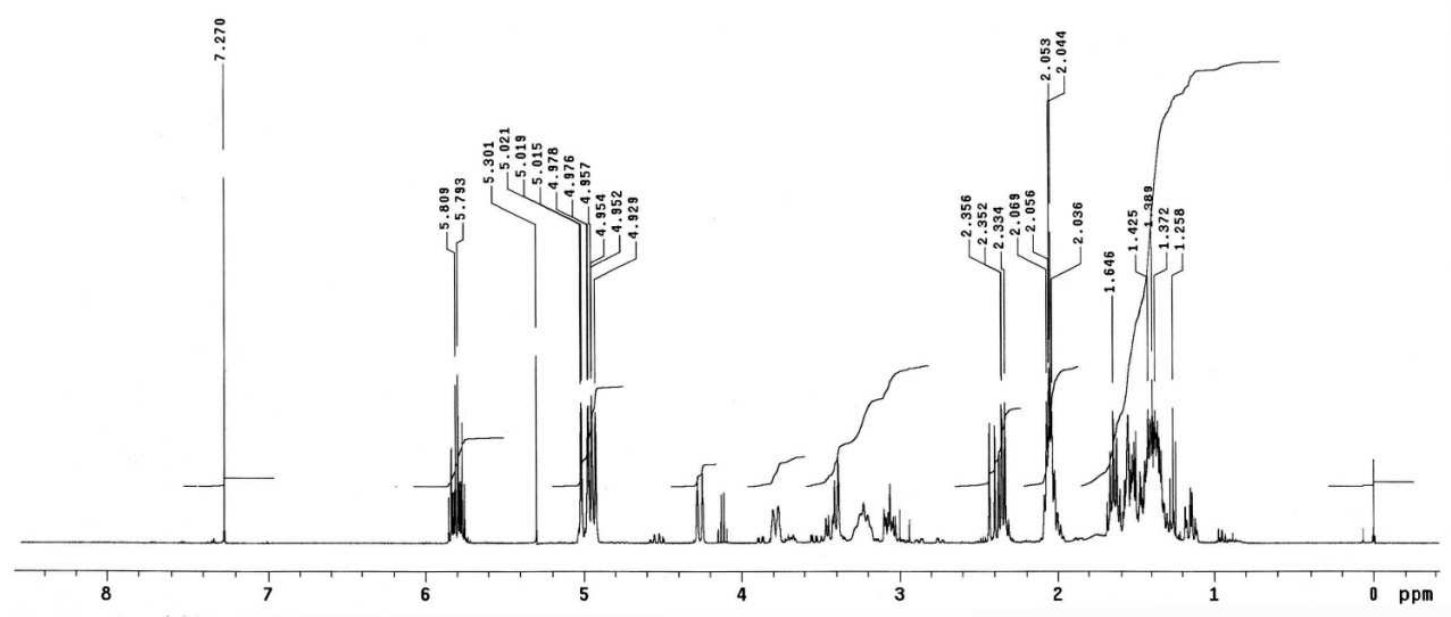


Protection step: A solution of 3-(hydroxymethyl)-1-(7-octenoyl)-3-(4-pentenyl) piperidine (265 $\mathrm{mg}, 0.86 \mathrm{mmol})$ in anhydrous DMF (6 mL) was added under inert atmosphere to a suspension of $\mathrm{NaH}$ ( $83 \mathrm{mg}$ of a $60 \%$ dispersion in mineral oil, $2.09 \mathrm{mmol}$ ) in DMF (6 mL). The resulting mixture was stirred at $0{ }^{\circ} \mathrm{C}$ for $1 \mathrm{~h}$, and then benzyl bromide $(410 \mu \mathrm{L}, 3.47 \mathrm{mmol})$ and tetrabutylammonium iodide $(51 \mathrm{mg}, 0.14 \mathrm{mmol}$ ) were added. The resulting white suspension was stirred at room temperature for $4 \mathrm{~h}$. The reaction was quenched with saturated $\mathrm{NH}_{4} \mathrm{Cl}$ solution, and the resulting mixture was extracted with $\mathrm{Et}_{2} \mathrm{O}$ and $\mathrm{CH}_{2} \mathrm{Cl}_{2}$. The combined organic extracts were dried over anhydrous $\mathrm{MgSO}_{4}$, filtered, and concentrated under reduced pressure. Flash chromatography ( $98: 2$ to $7: 3$ hexane-EtOAc) gave compound 70 ( $327 \mathrm{mg}, 95 \%$ ) as a colourless oil.

${ }^{1} \mathrm{H}$ NMR (400 MHz, CDCl 3 , COSY, gHSQC): $\delta 1.25-1.41\left(\mathrm{~m}, 9 \mathrm{H}, 2 \mathrm{H}-5^{\prime \prime}, 2 \mathrm{H}-4 ", 2 \mathrm{H}-6\right.$ "', 2H-2', H-4), $1.49(\mathrm{~m}, 3 \mathrm{H}, \mathrm{H}-4,2 \mathrm{H}-5), 1.59\left(\mathrm{t}, J=8.4 \mathrm{~Hz}, 2 \mathrm{H}, 2 \mathrm{H}-3^{\prime \prime}\right), 2.01\left(\mathrm{~m}, 4 \mathrm{H}, \mathrm{H}-3^{\prime}, \mathrm{H}-1^{\prime}\right), 2.30(\mathrm{t}, J=8.4$ $\left.\mathrm{Hz}, 2 \mathrm{H}, \mathrm{H}-2^{\prime \prime}\right), 2.99,3.32(\mathrm{~d}, J=13.6 \mathrm{~Hz}, 1 \mathrm{H}, \mathrm{H}-2), 3.18\left(\mathrm{~d}, J=8.8 \mathrm{~Hz}, 1 \mathrm{H}, \mathrm{CH}_{2} \mathrm{OBn}\right), 3.21(\mathrm{~m}, 1 \mathrm{H}$, $\mathrm{H}-6), 3.29$ (d, $J=8.8 \mathrm{~Hz}, 1 \mathrm{H}, \mathrm{CH}_{2} \mathrm{OBn}$ ), 3.48, 3.52 (d, $J=13.6 \mathrm{~Hz}, 1 \mathrm{H}, \mathrm{H}-2$ ), $3.84(\mathrm{~d}, J=13.2 \mathrm{~Hz}$, $1 \mathrm{H}, \mathrm{H}-6), 4.40\left(\mathrm{~d}, J=12.4 \mathrm{~Hz}, 1 \mathrm{H}, \mathrm{CH}_{2} \mathrm{Bn}\right), 4.49\left(\mathrm{~d}, J=12.4 \mathrm{~Hz}, 1 \mathrm{H}, \mathrm{CH}_{2} \mathrm{Bn}\right), 4.96\left(4 \mathrm{H}, \mathrm{CH}_{2}=\right)$, $5.78(\mathrm{~m}, 2 \mathrm{H}, \mathrm{CH}=), 7.28-7.32\left(\mathrm{~m}, 5 \mathrm{H}, \mathrm{H}-0, m, p \mathrm{C}_{6} \mathrm{H}_{5}\right)$.

${ }^{13} \mathrm{C}$ NMR $\left(100.6 \mathrm{MHz}, \mathrm{CDCl}_{3}\right): \delta 20.8,21.9$ (C-5), 22.1, 22.4 (C-2'), 25.0, 25.3 (C-3"), 28.6, 28.9, (C-4", C-5"), 30.7, 30.9 (C-4), 32.7 (C-2"), 33.4-34.3 (C-3', C-6"', C-1'), 37.5, 37.7 (C-3), 42.2, 46.3 (C-6), 48.2, 52.6 (C-2), 71.7, $72.9\left(\mathrm{CH}_{2} \mathrm{OBn}\right), 73.1\left(\mathrm{CH}_{2} \mathrm{Bn}\right), 114.2-114.6\left(\mathrm{CH}_{2}=\right), 126.8-128.2$ $\left(\mathrm{C}-0, m, p \mathrm{C}_{6} \mathrm{H}_{5}\right), 138.2-138.8\left(\mathrm{CH}=, \mathrm{C}-i \mathrm{C}_{6} \mathrm{H}_{5}\right), 171.7,172.2$ (NCO). 

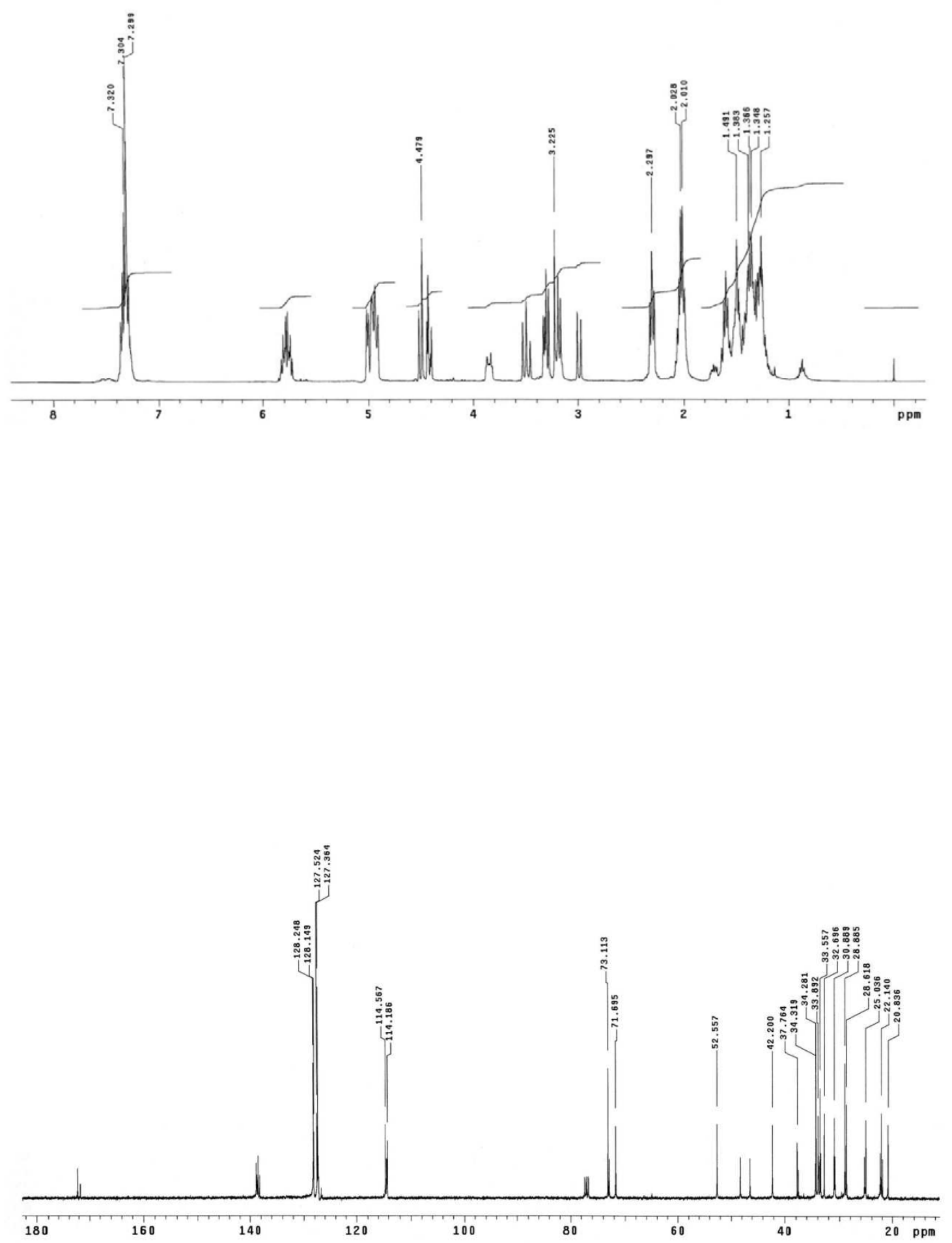


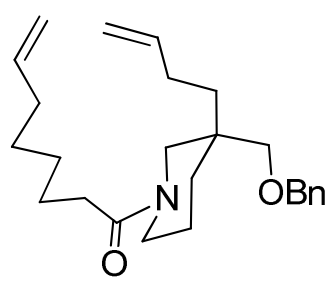

\section{3-(Benzyloxymethyl)-3-(3-butenyl)-1-(1-oxo-7-octenyl)-piperidine (68).}

Reduction step: Lithium aluminum hydride $(88 \mathrm{mg}, 2.34 \mathrm{mmol})$ was added to a solution of compound 64 (306 mg, $1.44 \mathrm{mmol})$ in anhydrous THF (25 mL) under argon atmosphere, and the mixture was heated at reflux for $3 \mathrm{~h}$. The resulting suspension was cooled to room temperature, and the reaction was quenched with distilled water. The aqueous layer was extracted with $\mathrm{Et}_{2} \mathrm{O}$, and the combined organic extracts were dried, filtered, and concentrated to give 3-(3-butenyl)-3-(hydroxymethyl)piperidine (66) (200 mg), which was used in the next step without purification.

IR (film) $3400(\mathrm{NH}, \mathrm{OH}) \mathrm{cm}^{-1}$.

${ }^{1} \mathrm{H}$ NMR (400 MHz, CDCl 3 , COSY, gHSQC): $\delta 1.25\left(\mathrm{~m}, 2 \mathrm{H}, \mathrm{H}-1^{\prime}\right), 1.33(\mathrm{td}, J=13.2,13.2,5.5 \mathrm{~Hz}$, $1 \mathrm{H}, \mathrm{H}-4), 1.55(\mathrm{dt}, J=13.2,4.0,4.0 \mathrm{~Hz}, 1 \mathrm{H}, \mathrm{H}-5), 1.67(\mathrm{tm}, J=13.2 \mathrm{~Hz}, 1 \mathrm{H}, \mathrm{H}-4), 1.85-2.05(\mathrm{~m}$, $\left.3 \mathrm{H}, \mathrm{H}-5,2 \mathrm{H}-2^{\prime}\right), 2.56(\mathrm{~d}, J=12.0 \mathrm{~Hz}, 1 \mathrm{H}, \mathrm{H}-2), 2.62(\mathrm{td}, J=10.8,4.0,4.0 \mathrm{~Hz}, 1 \mathrm{H}, \mathrm{H}-6), 3.00$ (d, J = $12.0 \mathrm{~Hz}, 1 \mathrm{H}, \mathrm{H}-2), 3.65$ (d, J = 11.6 Hz, $1 \mathrm{H}, \mathrm{CH}_{2} \mathrm{OH}$ ), 3.69 (d, J = 11.6 Hz, $1 \mathrm{H}, \mathrm{CH}_{2} \mathrm{OH}$ ), 4.91-5.03 $\left(4 \mathrm{~m}, 2 \mathrm{H}, \mathrm{CH}_{2}=\right), 5.78$ (ddt, $J=16.8,10.4,6.8,6.8 \mathrm{~Hz}, 1 \mathrm{H}, \mathrm{CH}=$ ).

${ }^{13} \mathrm{C} \mathrm{NMR}\left(100.6 \mathrm{MHz}, \mathrm{CDCl}_{3}\right): \delta 22.7$ (C-5), $27.3\left(\mathrm{C}-2^{\prime}\right), 33.0$ (C-4), 35.7 (C-3), 36.7 (C-1'), 46.6 (C6), $55.0(\mathrm{C}-2), 70.1\left(\mathrm{CH}_{2} \mathrm{OH}\right), 114.3\left(\mathrm{CH}_{2}=\right), 139.1(\mathrm{CH}=)$.

Acylation step: Oxalyl chloride ( $1.09 \mathrm{~mL}$ of a $2 \mathrm{M}$ solution in $\mathrm{CH}_{2} \mathrm{Cl}_{2}, 2.18 \mathrm{mmol}$ ) was added to a solution of 7-octenoic acid $(0.17 \mathrm{~mL}, 1.09 \mathrm{mmol})$ in $\mathrm{CH}_{2} \mathrm{Cl}_{2}(3 \mathrm{~mL})$ and two drops of DMF, and the resulting mixture was stirred for $3 \mathrm{~h}$. $\mathrm{Et}_{2} \mathrm{O}$ was added, and the resulting mixture was filtered and concentrated under reduced pressure to give crude 7-octenoyl chloride.

A solution of the above chloride $(1.09 \mathrm{mmol})$ in $\mathrm{CH}_{2} \mathrm{Cl}_{2}(5 \mathrm{~mL})$ was added to a suspension of piperidine 66 (162 mg, $0.99 \mathrm{mmol}$ ) and $\mathrm{K}_{2} \mathrm{CO}_{3}(314 \mathrm{mg}, 2.27 \mathrm{mmol})$ in $\mathrm{CH}_{2} \mathrm{Cl}_{2}(10 \mathrm{~mL})$, and the mixture was stirred for $3 \mathrm{~h}$ at room temperature. Distilled water was then added, and the resulting mixture was stirred for 20 minutes. The aqueous layer was extracted with chloroform, and the combined organic extracts were dried over anhydrous $\mathrm{Na}_{2} \mathrm{SO}_{4}$, filtered, and concentrated under reduced pressure. Flash chromatography (8:2 to 1:1 hexane-EtOAc) 
afforded 3-(3-butenyl)-3-(hydroxymethyl)-1-(7-octenoyl)piperidine (90 mg, 31\%, overall yield for the two steps).

${ }^{1} \mathrm{H} \mathrm{NMR} \mathrm{(400} \mathrm{MHz,} \mathrm{CDCl} 3$, COSY, gHSQC) $\delta 1.25\left(\mathrm{~m}, 1 \mathrm{H}, \mathrm{H}-6{ }^{\prime \prime}\right), 1.38\left(\mathrm{~m}, 5 \mathrm{H}, \mathrm{H}-4,2 \mathrm{H}-4 ", 2 \mathrm{H}-5^{\prime \prime}\right)$, 1.52-1.66 (m, 6H, 2H-3", 2H-5, H-6"', H-4), $2.05\left(\mathrm{~m}, 2 \mathrm{H}, \mathrm{H}-3^{\prime}\right), 2.36\left(\mathrm{t}, \mathrm{J}=7.2 \mathrm{~Hz}, 2 \mathrm{H}, \mathrm{H}-2^{\prime \prime}\right), 2.43$ (d, $J=13.2 \mathrm{~Hz}, 1 \mathrm{H}, \mathrm{H}-2$ ), 3.09 (d, J=13.6 Hz, 1H, H-6), 3.22 (d, J = 11.6 Hz, 1H, CH $\mathrm{CH}_{2} \mathrm{OH}$ ), 3.42 (d, J $\left.=11.6 \mathrm{~Hz}, 1 \mathrm{H}, \mathrm{CH}_{2} \mathrm{OH}\right), 3.79(\mathrm{dm}, J=13.6 \mathrm{~Hz}, 1 \mathrm{H}, \mathrm{H}-6), 4.28(\mathrm{~d}, J=13.2 \mathrm{~Hz}, 1 \mathrm{H}, \mathrm{H}-2), 4.92-5.05$ $\left(4 \mathrm{~m}, 4 \mathrm{H}, \mathrm{CH}_{2}=\right), 5.81$ (ddddd, $\left.J=16.8,10.0,6.0,6.0,4.0 \mathrm{~Hz}, 2 \mathrm{H}, \mathrm{CH}=\right)$.

${ }^{13} \mathrm{C} \mathrm{NMR}\left(100.6 \mathrm{MHz}, \mathrm{CDCl}_{3}\right) \delta 22.6$ (C-5), 25.3 (C-3"), 28.6, 28.9 (C-4", C-5"), 30.7 (C-4), 33.0 (C-2") 33.6 (C-3'), 35.2 (C-6") 38.6 (C-3), 47.2 (C-6), 48.8 (C-2), $62.3\left(\mathrm{CH}_{2} \mathrm{OH}\right), 114.3,114.4$ $\left(\mathrm{CH}_{2}=\right), 138.8,139.0(\mathrm{CH}=), 173.0(\mathrm{NCO})$.

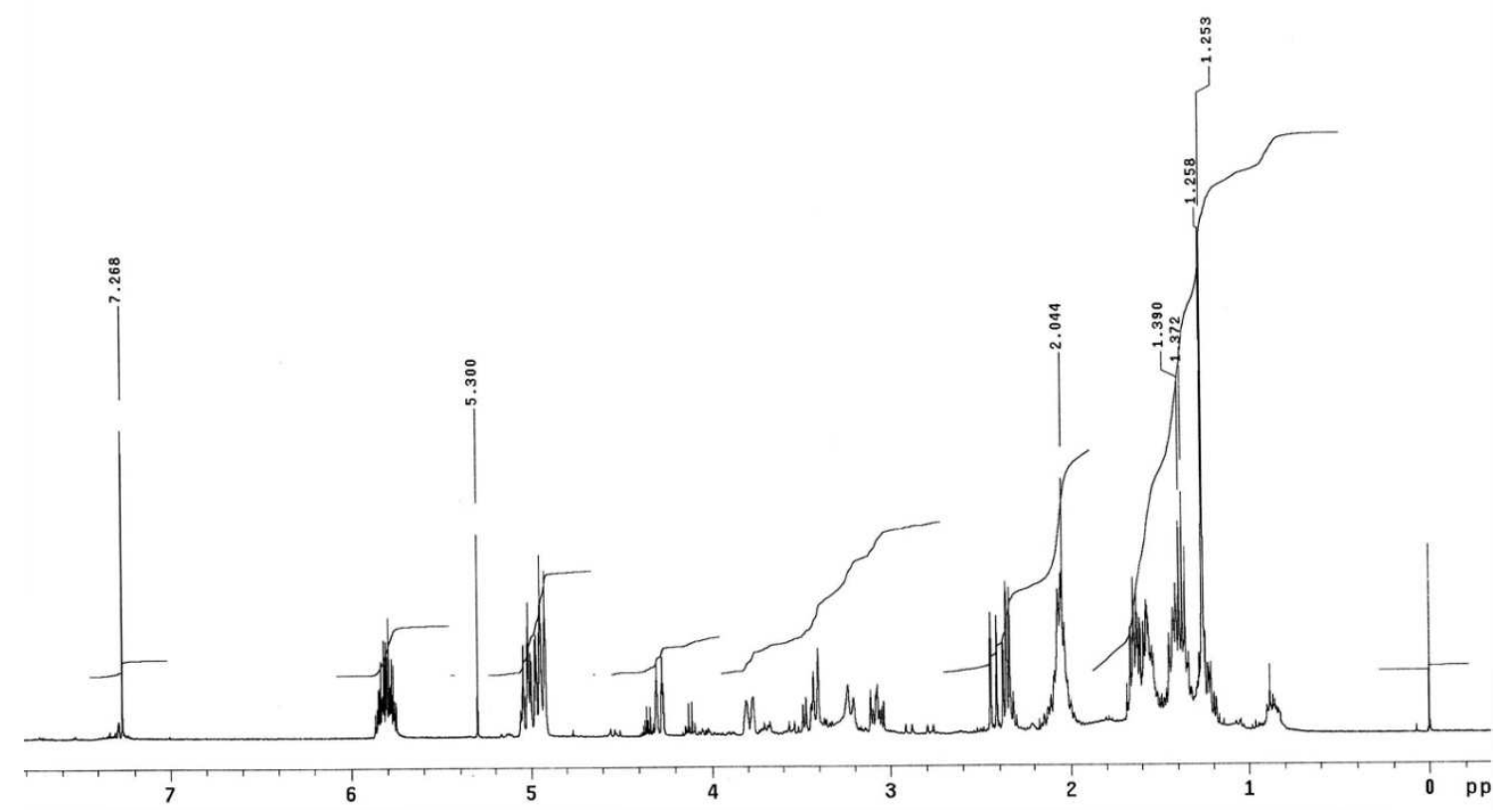


Protection step: A solution of the above alcohol ( $186 \mathrm{mg}, 0.63 \mathrm{mmol}$ ) in anhydrous DMF (4 mL) was added under inert atmosphere to a suspension of $\mathrm{NaH}(76 \mathrm{mg}$ of a $60 \%$ dispersion in mineral oil, $1.9 \mathrm{mmol})$ in DMF $(8 \mathrm{~mL})$. The resulting mixture was stirred at $0{ }^{\circ} \mathrm{C}$ for $1 \mathrm{~h}$, and then benzyl bromide ( $370 \mu \mathrm{L}, 3.17 \mathrm{mmol}$ ) and tetrabutylammonium iodide ( $46 \mathrm{mg}, 0.13 \mathrm{mmol}$ ) were added. The resulting white suspension was stirred at room temperature for $4 \mathrm{~h}$. The reaction was quenched with saturated $\mathrm{NH}_{4} \mathrm{Cl}$ solution, and the resulting mixture was extracted with $\mathrm{Et}_{2} \mathrm{O}$ and $\mathrm{CH}_{2} \mathrm{Cl}_{2}$. The combined organic extracts were dried over anhydrous $\mathrm{MgSO}_{4}$, filtered, and concentrated under reduced pressure. Flash chromatography (98:2 to 7:3 hexane-EtOAc) gave compound 68 (216 $\mathrm{mg}, 89 \%)$ as a colourless oil.

${ }^{1} \mathrm{H}$ NMR (400 MHz, CDCl ${ }_{3}$ COSY, gHSQC): $\delta 1.25-1.44$ (m, 5H, 2H-5", 2H-6", H-4), 1.49-1.62 (m, $\left.5 \mathrm{H}, 2 \mathrm{H}-5,2 \mathrm{H}-3^{\prime \prime}, \mathrm{H}-4\right), 1.95\left(\mathrm{~m}, 2 \mathrm{H}, \mathrm{H}-4^{\prime \prime}\right), 2.03\left(\mathrm{~m}, 2 \mathrm{H}, \mathrm{H}-3^{\prime}\right), 2.30$ (t, J = 8.4 Hz, 2H, H-2') 3.01 (d, $J=13.6 \mathrm{~Hz}, 1 \mathrm{H}, \mathrm{H}-2), 3.19,3.32(\mathrm{~m}, 1 \mathrm{H}, \mathrm{H}-6), 3.22\left(\mathrm{~d}, J=13.2 \mathrm{~Hz}, 1 \mathrm{H}, \mathrm{CH}_{2} \mathrm{OBn}\right), 3.31$ (d, $J=$ 12.3 Hz, 1H, CH $\mathrm{CH}_{2} \mathrm{OBn}$ ), 3.50, 3.53 (d, J = 13.6 Hz, 1H, H-2), 3.86 (d, J=13.2 Hz, 1H, H-6), 4.42 (d, $\left.J=12.0 \mathrm{~Hz}, 1 \mathrm{H}, \mathrm{CH}_{2} \mathrm{Bn}\right), 4.51\left(\mathrm{~d}, J=12.0 \mathrm{~Hz}, 1 \mathrm{H}, \mathrm{CH}_{2} \mathrm{Bn}\right), 4.90-5.02\left(4 \mathrm{H}, \mathrm{CH}_{2}=\right), 5.72-5,84(\mathrm{~m}$, $2 \mathrm{H}, \mathrm{CH}=), 7.30-7.34\left(\mathrm{~m}, 5 \mathrm{H}, \mathrm{H}-0, m, p \mathrm{C}_{6} \mathrm{H}_{5}\right)$.

${ }^{13} \mathrm{C}$ NMR (100.6 MHz, CDCl $): \delta 20.8,21.9$ (C-5), 25.1, 25.3 (C-3"'), 27.4, 27.6 (C-4") , 28.6, 29.4 (C-5") , 30.8, 30.9 (C-4), 32.8 (C-2") , 33.6 (C-3'), 33.7 (C-6", 1'), 37.8, 37.6 (C-3), 42.2, 46.4 (C-6), 48.2, 52.6 (C-2), 71.6, $72.8\left(\mathrm{CH}_{2} \mathrm{OBn}\right), 73.2\left(\mathrm{CH}_{2} \mathrm{Bn}\right), 114.1-114.3\left(\mathrm{CH}_{2}=\right)$, 126.9-128.3 (C-o, $m$, p), 138.2-139.1 ( $\mathrm{CH}=, \mathrm{C}-\mathrm{i}), 171.7,172.9(\mathrm{NCO})$. 

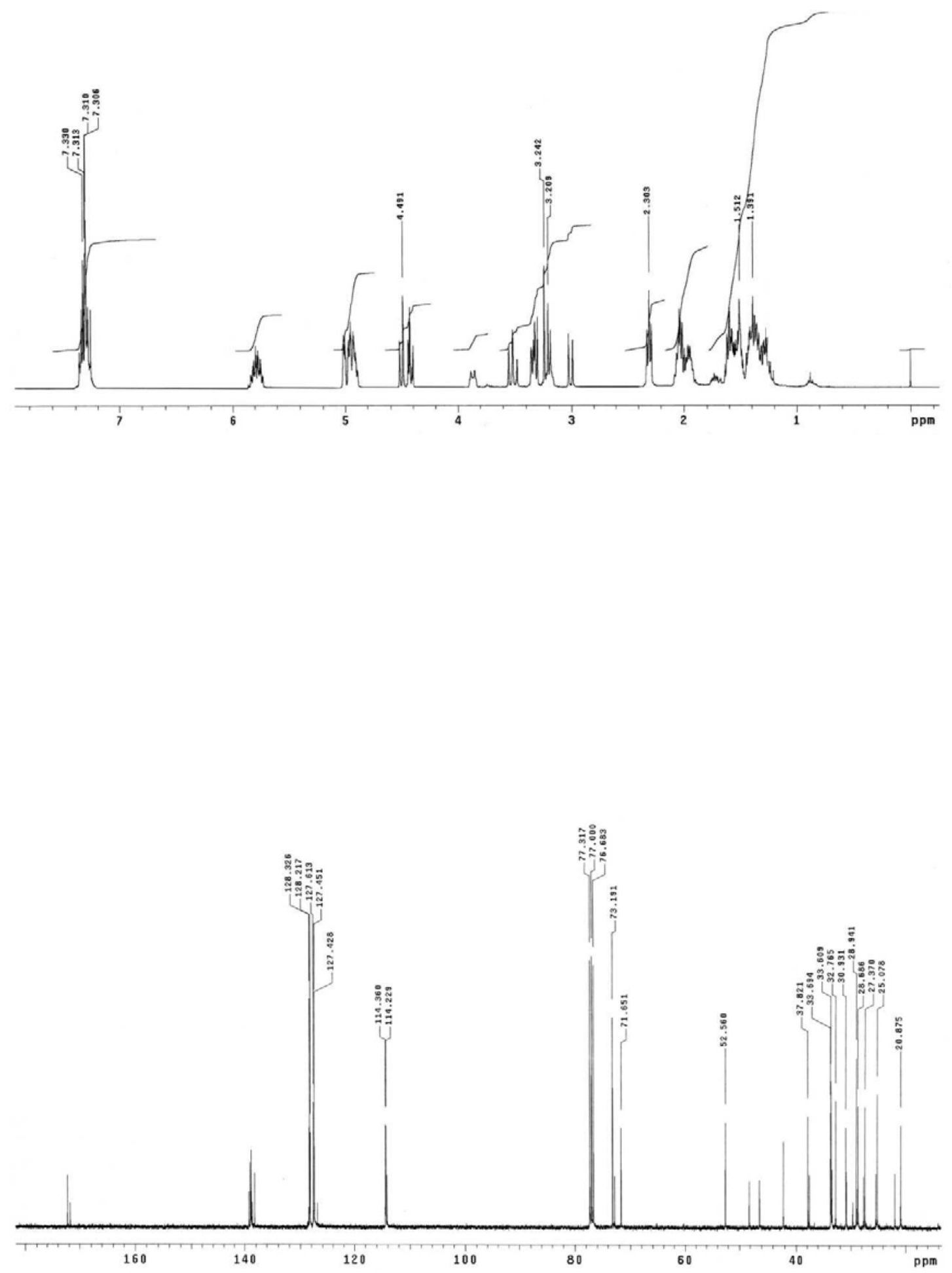


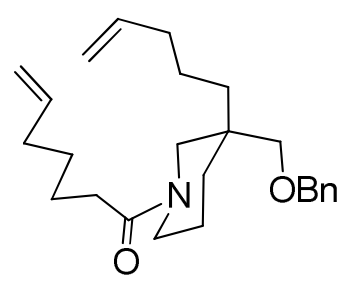

3-(Benzyloxymethyl)-3-(4-pentenyl)-1-(6-heptenoyl) piperidine (69).

Oxalyl chloride ( $2.9 \mathrm{~mL}$ of a $2 \mathrm{M}$ solution in $\mathrm{CH}_{2} \mathrm{Cl}_{2}, 5.89 \mathrm{mmol}$ ) was added to a solution of 6heptenoic acid $(0.39 \mathrm{~mL}, 2.9 \mathrm{mmol})$ in $\mathrm{CH}_{2} \mathrm{Cl}_{2}(7 \mathrm{~mL})$ and two drops of $D M F$, and the resulting mixture was stirred for $3 \mathrm{~h}$. $\mathrm{Et}_{2} \mathrm{O}$ was added, and the resulting mixture was filtered and and concentrated under reduced pressure to give crude 6-heptenoyl chloride.

A solution of the above chloride $(2.9 \mathrm{mmol})$ in chloroform $(5 \mathrm{~mL})$ was added to a suspension of piperidine 67 (540 mg, $2.95 \mathrm{mmol}$ ) and $\mathrm{K}_{2} \mathrm{CO}_{3}(937 \mathrm{mg}, 6.78 \mathrm{mmol})$ in chloroform $(20 \mathrm{~mL})$, and the mixture was stirred for $3 \mathrm{~h}$ at room temperature. Distilled water was then added, and the resulting mixture was stirred for 20 minutes. The aqueous layer was extracted with chloroform, and the combined organic extracts were dried over anhydrous $\mathrm{Na}_{2} \mathrm{SO}_{4}$, filtered, and concentrated under reduced pressure. Flash chromatography (8:2 to $1: 1$ hexane- EtOAc) afforded 1-(6-heptenoyl)-3-(hydroxymethyl)-3-(4-pentenyl)piperidine (200 mg, 31\% overall yield for the two steps).

${ }^{1} \mathrm{H}$ NMR $\left(400 \mathrm{MHz}, \mathrm{CDCl}_{3}, \mathrm{COSY}, \mathrm{gHSQC}\right): \delta 0.88\left(\mathrm{t}, J=7.5 \mathrm{~Hz}, 1 \mathrm{H}, \mathrm{H}-1^{\prime}\right), 1.13-1.46\left(\mathrm{~m}, 5 \mathrm{H}, 2 \mathrm{H}-2^{\prime}\right.$, $\left.2 \mathrm{H}-4^{\prime \prime}, \mathrm{H}-4\right), 1.49-1.58\left(\mathrm{~m}, 4 \mathrm{H}, 2 \mathrm{H}-5, \mathrm{H}-4, \mathrm{H}-1^{\prime}\right), 1.65$ (m, 2H, H-3"'), 2.02-2.12 (m, 4H, 2H-3', 2H$\left.5^{\prime \prime}\right), 2.36\left(\mathrm{t}, J=7.8 \mathrm{~Hz}, 2 \mathrm{H}, \mathrm{H}-2^{\prime \prime}\right), 2.43(\mathrm{~d}, J=13.5 \mathrm{~Hz}, 1 \mathrm{H}, \mathrm{H}-2), 3.09(\mathrm{~m}, 1 \mathrm{H}, \mathrm{H}-6), 3.22(\mathrm{~d}, J=$ $10.8 \mathrm{~Hz}, 1 \mathrm{H}, \mathrm{CH}_{2} \mathrm{OH}$ ), 3.30 (br. $\mathrm{s}, 1 \mathrm{H}, \mathrm{OH}$ ), 3.40 (d, $J=10.8 \mathrm{~Hz}, 1 \mathrm{H}, \mathrm{CH}_{2} \mathrm{OH}$ ), 3.75 (d, $J=13.2 \mathrm{~Hz}$, $1 \mathrm{H}, \mathrm{H}-6), 4.24$ (dd, J = 13.5, $1.5 \mathrm{~Hz}, 1 \mathrm{H}, \mathrm{H}-2$ ), 4.92-5.04 (m, 4H, $\mathrm{CH}_{2}=$ ), 5.80 (ddt, J=17.4, 10.5, $6.6 \mathrm{~Hz}, 2 \mathrm{H}, \mathrm{CH}=)$.

${ }^{13} \mathrm{C} \mathrm{NMR}\left(100.6 \mathrm{MHz}, \mathrm{CDCl}_{3}\right.$ ): $\delta 22.2$ (C-2'), 22.6 (C-5), 24.9 (C-3"), 28.4 (C-4"), 30.6 (C-4), 32.9 (C-2'), 33.4 (C-5"), 34.4 (C-3'), 35.4 (C-1'), 38.5 (C-3), 47.2 (C-6), 48.9 (C-2), $62.4\left(\mathrm{CH}_{2} \mathrm{OH}\right)$, 114.5, $114.6\left(\mathrm{CH}_{2}=\right), 138.3,138.7(\mathrm{CH}=), 172.8$ (NCO). 


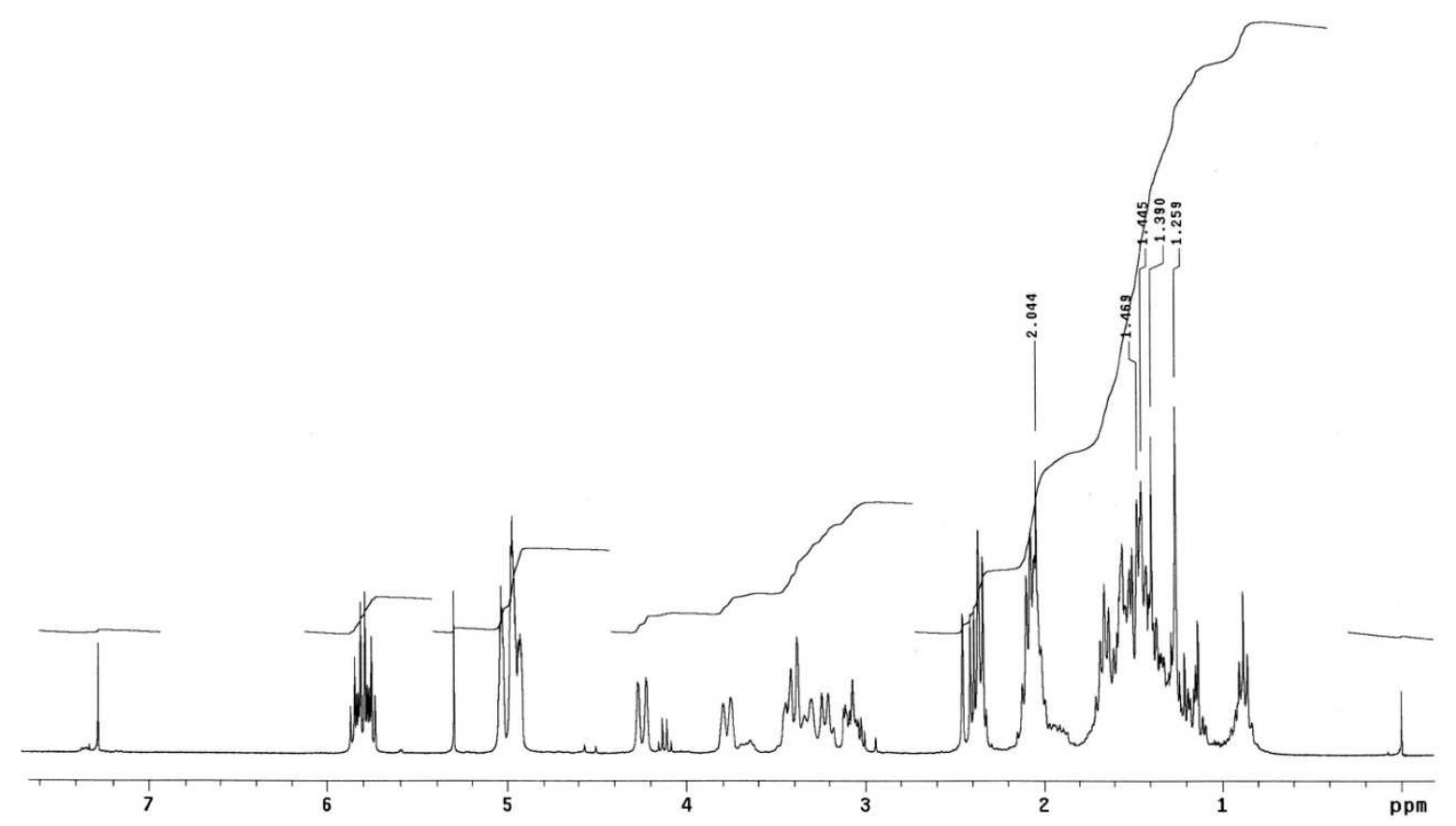


Protection step: A solution of the above alcohol (198 mg, $0.67 \mathrm{mmol})$ in anhydrous DMF ( $6 \mathrm{~mL}$ ) was added under inert atmosphere to a suspension of $\mathrm{NaH}(131 \mathrm{mg}$ of a $60 \%$ dispersion in mineral oil, $2.02 \mathrm{mmol}$ ) in DMF (19 mL). The resulting mixture was stirred at $0{ }^{\circ} \mathrm{C}$ for $1 \mathrm{~h}$, and then benzyl bromide ( $390 \mu \mathrm{L}, 3.37 \mathrm{mmol}$ ) and tetrabutylammonium iodide $(51 \mathrm{mg}, 0.13 \mathrm{mmol})$ were added. The resulting white suspension was stirred at room temperature for $4 \mathrm{~h}$. The reaction was quenched with saturated $\mathrm{NH}_{4} \mathrm{Cl}$ solution, and the resulting mixture was extracted with $\mathrm{Et}_{2} \mathrm{O}$ and $\mathrm{CH}_{2} \mathrm{Cl}_{2}$. The combined organic extracts were dried over anhydrous $\mathrm{MgSO}_{4}$, filtered, and concentrated under reduced pressure. Flash chromatography (98:2 to 7:3 hexaneEtOAc) gave compound 69 (205 mg, 80\%) as a colourless oil.

${ }^{1} \mathrm{H}$ NMR (400 MHz, CDCl 3 , COSY, gHSQC): $\delta 1.21-1.52\left(\mathrm{~m}, 10 \mathrm{H}, 2 \mathrm{H}-2^{\prime}, 2 \mathrm{H}-4^{\prime \prime}, 2 \mathrm{H}-4,2 \mathrm{H}-1^{\prime}, 2 \mathrm{H}-5\right)$, $1.60\left(\mathrm{~m}, 2 \mathrm{H}, \mathrm{H}-3^{\prime \prime}\right), 1.98-2.10\left(\mathrm{~m}, 4 \mathrm{H}, 2 \mathrm{H}-3^{\prime}, 2 \mathrm{H}-5^{\prime \prime}\right), 2.30\left(\mathrm{~m}, 2 \mathrm{H}, \mathrm{H}-2^{\prime \prime}\right), 2.98(\mathrm{~d}, \mathrm{~J}=13.2 \mathrm{~Hz}, \mathrm{H}-$ 2), $3.19\left(\mathrm{~s}, 1 \mathrm{H}, \mathrm{CH}_{2} \mathrm{OH}\right), 3.23\left(\mathrm{~s}, 1 \mathrm{H}, \mathrm{CH}_{2} \mathrm{OH}\right), 3.20(\mathrm{~d}, J=13.2 \mathrm{~Hz}, \mathrm{H}-6), 3.29(\mathrm{~d}, J=13.2 \mathrm{~Hz}, 1 \mathrm{H}$, H-6), $3.21(\mathrm{~d}, J=13.2 \mathrm{~Hz}, 1 \mathrm{H}, \mathrm{H}-2), 3.51,3.48(\mathrm{~d}, J=13.2 \mathrm{~Hz}, 1 \mathrm{H}, \mathrm{H}-2), 3.84(\mathrm{dt}, J=13.2,4.8 \mathrm{~Hz}$, $1 \mathrm{H}, \mathrm{H}-6), 4.41\left(\mathrm{~d}, J=12.0 \mathrm{~Hz}, 1 \mathrm{H}, \mathrm{CH}_{2} \mathrm{Bn}\right), 4.50\left(\mathrm{~d}, J=12.0 \mathrm{~Hz}, 1 \mathrm{H}, \mathrm{CH}_{2} \mathrm{Bn}\right), 4.91-5.03(\mathrm{~m}, 4 \mathrm{H}$, $\left.\mathrm{CH}_{2}=\right)$, 5.73-5.84 (m, 2H, CH=), 7.26-7.33 (m, 5H, $\left.\mathrm{H}-0, m, p \mathrm{C}_{6} \mathrm{H}_{5}\right)$.

${ }^{13} \mathrm{C}$ NMR $\left(100.6 \mathrm{MHz}, \mathrm{CDCl}_{3}\right): \delta 20.9-22.4\left(\mathrm{C}-2^{\prime}, \mathrm{C}-5\right), 24.7,24.9$ (C-3") 28.7 (C-4") , 30.8, 30.9 (C4), 33.3, 33.6 (C-2") , 33.4-34.4 (C-5', C-3', C-1'), 37.6, 37.8 (C-3), 42.2, 46.4 (C-6), 48.3, 52.6 (C2), 71.8, $72.9\left(\mathrm{CH}_{2} \mathrm{OBn}\right), 73.2\left(\mathrm{CH}_{2} \mathrm{Bn}\right), 114.4,114.6\left(\mathrm{CH}_{2}=\right), 127.4,128.3\left(\mathrm{C}-0, m, p \mathrm{C}_{6} \mathrm{H}_{5}\right), 138.5$, $138.8\left(\mathrm{C}-i \mathrm{C}_{6} \mathrm{H}_{5}\right), 138.6(\mathrm{CH}=), 170.0,172.1$ (NCO). 

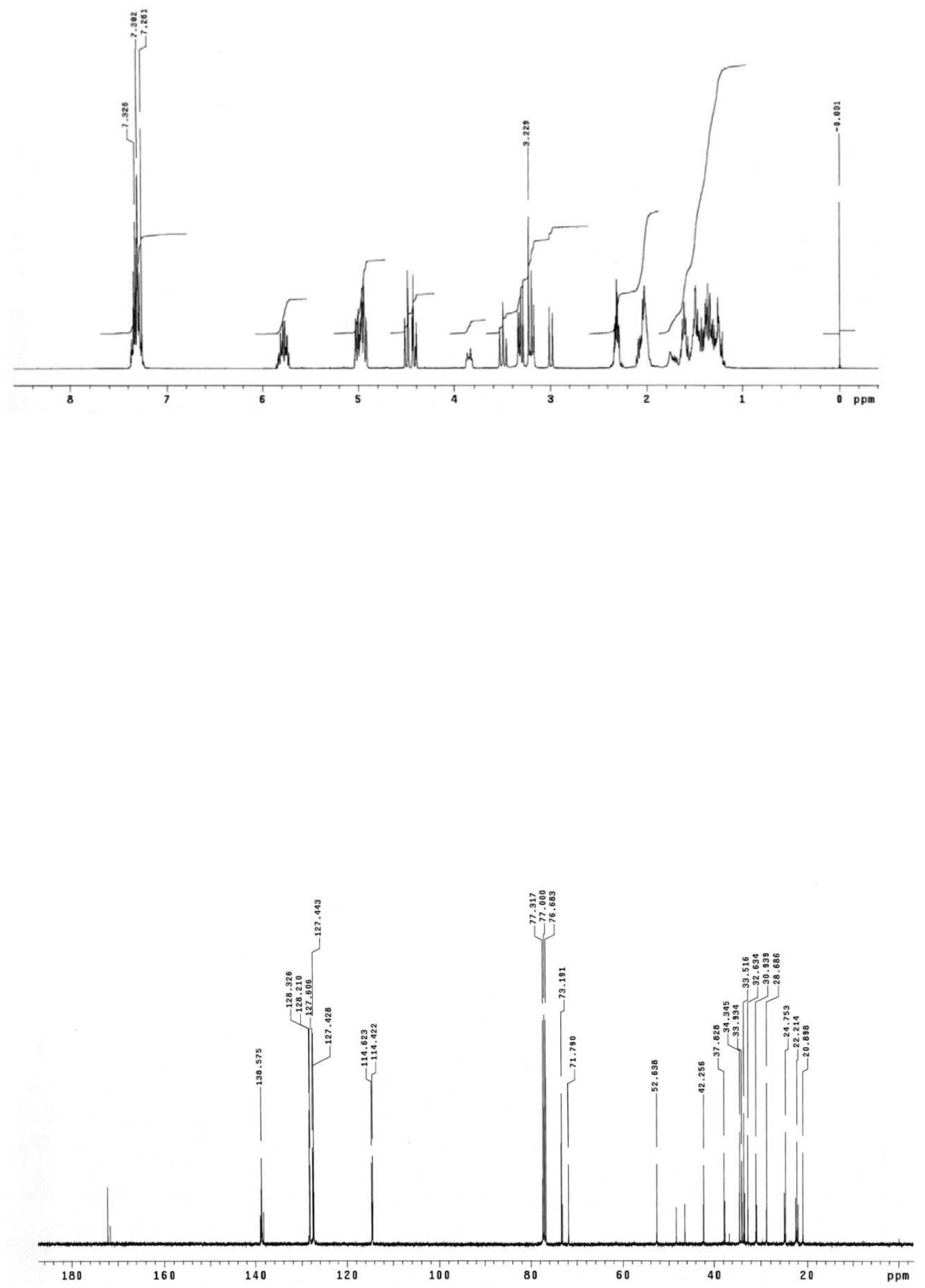


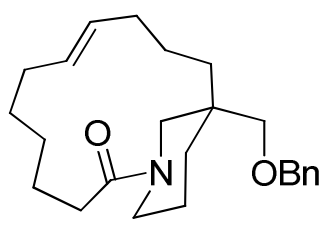

\section{3-(Benzyloxymethyl)-2-oxo-1-azabicyclo[11.3.1] heptadec-8-ene (73).}

A solution of diene 70 (158 mg, $0.396 \mathrm{mmol})$ in $\mathrm{CH}_{2} \mathrm{Cl}_{2}(10 \mathrm{~mL})$ was slowly added via a syringe pump ( $2 \mathrm{~h}$ ) to a solution of Grubbs second generation catalyst (68 $\mathrm{mg}, 0.08 \mathrm{mmol}$ ) in refluxing $\mathrm{CH}_{2} \mathrm{Cl}_{2}(80 \mathrm{~mL})$. The resulting mixture was heated at reflux for $4 \mathrm{~h}$ and concentrated under reduced pressure. Flash chromatography (alumina, 95:5 to 3:7 hexane-EtOAc) afforded macrocyclic compound $\mathbf{7 3}$ (115 mg, 84\%). Only traces of the $Z$ isomer were detected.

\section{E isomer:}

${ }^{1} \mathrm{H}$ NMR (400 MHz, CDCl 3 , COSY, gHSQC): $\delta 1.08-1.52\left(\mathrm{~m}, 9 \mathrm{H}, 2 \mathrm{H}-15, \mathrm{H}-14, \mathrm{CH}_{2}\right), 1.64(\mathrm{~d}, \mathrm{~J}=12.8$ $\mathrm{Hz}, 1 \mathrm{H}, \mathrm{H}-14), 1.78\left(\mathrm{~m}, 1 \mathrm{H}, \mathrm{CH}_{2}\right), 1.91(\mathrm{~m}, 2 \mathrm{H}, \mathrm{H}-7, \mathrm{H}-10), 2.11$ (m, 3H, H-7, H-10, H-3), 2.45 (d, J $=12.8 \mathrm{~Hz}, 1 \mathrm{H}, \mathrm{H}-17), 2.66(\mathrm{t}, J=11.2 \mathrm{~Hz}, 1 \mathrm{H}, \mathrm{H}-3), 2.89(\mathrm{~m}, 1 \mathrm{H}, \mathrm{H}-16), 3.21\left(\mathrm{~s}, 2 \mathrm{H}, \mathrm{CH}_{2} \mathrm{OBn}\right)$, $3.94(\mathrm{~d}, J=12.8 \mathrm{~Hz}, \mathrm{H}-16), 4.45\left(\mathrm{~d}, J=12.4 \mathrm{~Hz}, 1 \mathrm{H}, \mathrm{CH}_{2} \mathrm{Bn}\right.$ ), $4.49\left(\mathrm{~d}, J=12.4 \mathrm{~Hz}, 1 \mathrm{H}, \mathrm{CH}_{2} \mathrm{Bn}\right.$ ), $4.51(\mathrm{~d}, J=12.8 \mathrm{~Hz}, 1 \mathrm{H}, \mathrm{H}-17$ ), 5.17 (ddd, $J=15.2,7.6,4.4 \mathrm{~Hz}, 1 \mathrm{H}, \mathrm{CH}=), 5.24$ (ddd, $J=15.2,7.2$, $4.8 \mathrm{~Hz}, 1 \mathrm{H}, \mathrm{CH}=), 7.32\left(\mathrm{~m}, 5 \mathrm{H}, \mathrm{H}-0, m, p \mathrm{C}_{6} \mathrm{H}_{5}\right)$.

${ }^{13} \mathrm{C} \mathrm{NMR}\left(100.6 \mathrm{MHz}, \mathrm{CDCl}_{3}\right): \delta 21.6,21.6\left(\mathrm{C}-15, \mathrm{CH}_{2}\right), 25.7,26.6,28.1,28.7\left(\mathrm{CH}_{2}\right), 29.8(\mathrm{C}-14)$, 31.2, 32.2 (C-7, C-10), 32.0 (C-3), 37.8 (C-13), 47.0 (C-16), 48.7 (C-17), 73.2 ( $\mathrm{CH}_{2} \mathrm{Bn}$ ), 76.2 $\left(\mathrm{CH}_{2} \mathrm{OBn}\right), 127.3,128.2\left(\mathrm{C}-0, m \mathrm{C}_{6} \mathrm{H}_{5}\right), 127.4\left(\mathrm{C}-p \mathrm{C}_{6} \mathrm{H}_{5}\right), 131.6,131.8(\mathrm{CH}=), 138.6\left(\mathrm{C}-i \mathrm{C}_{6} \mathrm{H}_{5}\right)$, 172.2 (NCO).

HRMS (ESI) calcd for $\left[\mathrm{C}_{24} \mathrm{H}_{35} \mathrm{NO}_{2}+\mathrm{H}\right]^{+}: 370.2741$, found: 370.2741 . 

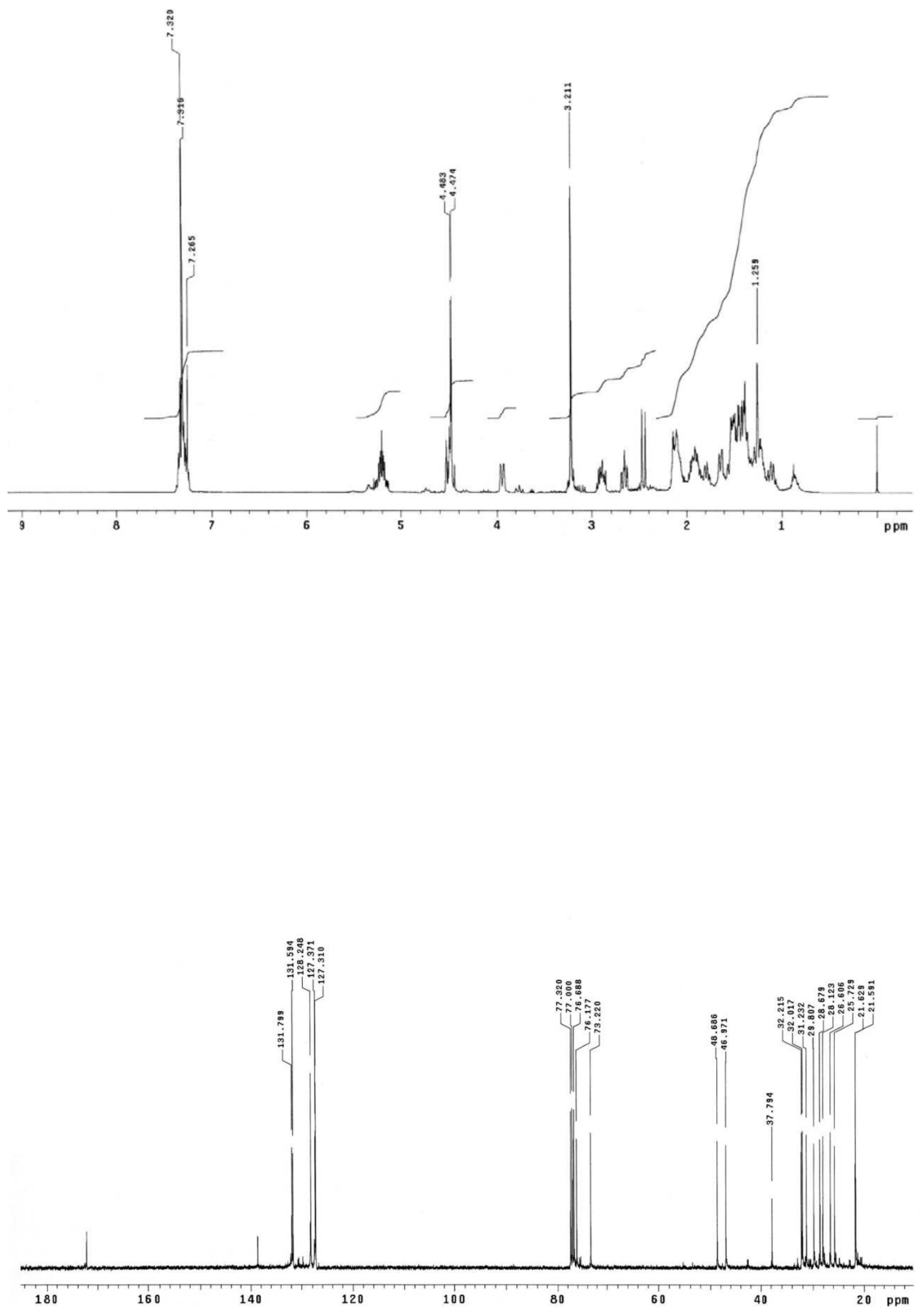
<smiles>O=C1CCCCC/C=C\CCC(CO)(COc2ccccc2)C1</smiles>

13-(Benzyloxymethyl)-2-oxo-1-azabicyclo[10.3.1] hexadec-8-ene (71).

A solution of diene $68(216 \mathrm{mg}, 0.456 \mathrm{mmol})$ in $\mathrm{CH}_{2} \mathrm{Cl}_{2}(20 \mathrm{~mL})$ was slowly added via a syringe pump ( $4 \mathrm{~h}$ ) to a solution of Grubbs second generation catalyst $(77 \mathrm{mg}, 0.09 \mathrm{mmol}$ ) in refluxing $\mathrm{CH}_{2} \mathrm{Cl}_{2}(60 \mathrm{~mL})$. The resulting mixture was heated at reflux for $8 \mathrm{~h}$ and concentrated under reduced pressure. Flash chromatography (alumina, 95:5 to 1:1 hexane-EtOAc) afforded macrocyclic compound 71 (86 $\mathrm{mg}, 53 \%$ ) as an inseparable $2: 1$ mixture of $E / Z$ isomers and a dimer (40 mg, 25\%).

$E$ isomer of compound $\mathbf{7 1}$ (spectral data from a 2:1 E/Z mixture of isomers):

${ }^{1} \mathrm{H}$ NMR (400 MHz, CDCl 3 , COSY, gHSQC): $\delta 1.30-2.15\left(\mathrm{~m}, 14 \mathrm{H}, \mathrm{CH}_{2}\right), 2.45(\mathrm{~d}, J=13.2 \mathrm{~Hz}, 1 \mathrm{H}, \mathrm{H}-$ 16), $2.58(\mathrm{~m}, 2 \mathrm{H}, \mathrm{H}-3), 2.94(\mathrm{td}, J=13.2,2.8 \mathrm{~Hz}, 1 \mathrm{H}, \mathrm{H}-15), 3.22\left(\mathrm{~d}, J=8.8 \mathrm{~Hz}, 1 \mathrm{H}, \mathrm{CH}_{2} \mathrm{Bn}\right.$ ), 3.28 (d, J=8.8 Hz, 1H, CH $\mathrm{CH}_{2} \mathrm{Bn}$ ), $3.89(\mathrm{dm}, J=13.2 \mathrm{~Hz}, 1 \mathrm{H}, \mathrm{H}-15), 4.49\left(\mathrm{~s}, 2 \mathrm{H}, \mathrm{CH}_{2} \mathrm{OBn}\right), 4.53(\mathrm{~d}, J=$ $13.2 \mathrm{~Hz}, 1 \mathrm{H}, \mathrm{H}-16), 5.30$ (ddd, $J=15.2,7.2,3.2 \mathrm{~Hz}, 1 \mathrm{H}, \mathrm{CH}=), 5.36(\mathrm{~m}, 1 \mathrm{H}, \mathrm{CH}=), 7.32(\mathrm{~m}, 5 \mathrm{H}, \mathrm{H}-$ $\left.o, m, p \mathrm{C}_{6} \mathrm{H}_{5}\right)$.

${ }^{13} \mathrm{C} \mathrm{NMR}\left(100.6 \mathrm{MHz}, \mathrm{CDCl}_{3}\right): \delta 21.3(\mathrm{C}-14), 22.0-35.0\left(\mathrm{CH}_{2}\right), 32.8(\mathrm{C}-3), 38.6$ (C-12), $46.4(\mathrm{C}-16)$, 47.8 (C-15), $73.2\left(\mathrm{CH}_{2} \mathrm{OBn}\right), 76.3\left(\mathrm{CH}_{2} \mathrm{Bn}\right), 127.3-128.3$ (C-o, $\left.m, p \mathrm{C}_{6} \mathrm{H}_{5}\right), 130.0,130.9$ ( $\left.\mathrm{CH}=\right)$, $138.5\left(\mathrm{C}-i \mathrm{C}_{6} \mathrm{H}_{5}\right), 171.7(\mathrm{C}=\mathrm{O})$.

$Z$ isomer of compound $\mathbf{7 1}$ (spectral data from a 2:1 E/Z mixture of isomers):

${ }^{1} \mathrm{H}$ NMR (400 MHz, CDCl 3 , COSY, gHSQC): $\delta 1.30-2.15\left(\mathrm{~m}, 14 \mathrm{H}, \mathrm{CH}_{2}\right), 2.40(\mathrm{dm}, J=11.6 \mathrm{~Hz}, 1 \mathrm{H}$, $\mathrm{H}-16), 2.58(\mathrm{~m}, 2 \mathrm{H}, \mathrm{H}-3), 2.84(\mathrm{~d}, J=13.2 \mathrm{~Hz}, 1 \mathrm{H}, \mathrm{H}-15), 3.20$ (d, J = 9.2 Hz, 1H, CH $\mathrm{Bn}$ ), 3.24 (d, $\left.J=9.2 \mathrm{~Hz}, 1 \mathrm{H}, \mathrm{CH}_{2} \mathrm{Bn}\right), 3.67(\mathrm{~d}, J=13.2 \mathrm{~Hz}, 1 \mathrm{H}, \mathrm{H}-15), 4.49\left(\mathrm{~s}, 2 \mathrm{H}, \mathrm{CH}_{2} \mathrm{OBn}\right), 4.62(\mathrm{dm}, J=11.6$ $\mathrm{Hz}, 1 \mathrm{H}, \mathrm{H}-16), 5.36(\mathrm{~m}, 1 \mathrm{H}, \mathrm{CH}=), 5.44(\mathrm{tm}, J=9.2 \mathrm{~Hz}, 1 \mathrm{H}, \mathrm{CH}=), 7.32\left(\mathrm{~m}, 5 \mathrm{H}, \mathrm{H}-\mathrm{o}, m, p \mathrm{C}_{6} \mathrm{H}_{5}\right)$.

${ }^{13} \mathrm{C} \mathrm{NMR}\left(100.6 \mathrm{MHz}, \mathrm{CDCl}_{3}\right): \delta 20.3$ (C-14), 22.2-35.0 $\left(\mathrm{CH}_{2}\right), 32.8$ (C-3), 37.7 (C-12), 42.2 (C-16), 49.1 (C-15), $73.4\left(\mathrm{CH}_{2} \mathrm{OBn}\right), 75.7\left(\mathrm{CH}_{2} \mathrm{Bn}\right), 127.3-128.3$ (C-o, $\left.m, p \mathrm{C}_{6} \mathrm{H}_{5}\right), 131.8,132.9$ ( $\left.\mathrm{CH}=\right)$, $138.3\left(\mathrm{C}-i \mathrm{C}_{6} \mathrm{H}_{5}\right), 172.2(\mathrm{C}=\mathrm{O})$.

HRMS (ESI) calcd for $\left[\mathrm{C}_{23} \mathrm{H}_{33} \mathrm{NO}_{2}+\mathrm{H}\right]^{+}: 356.2589$, found: 356.2589 . 

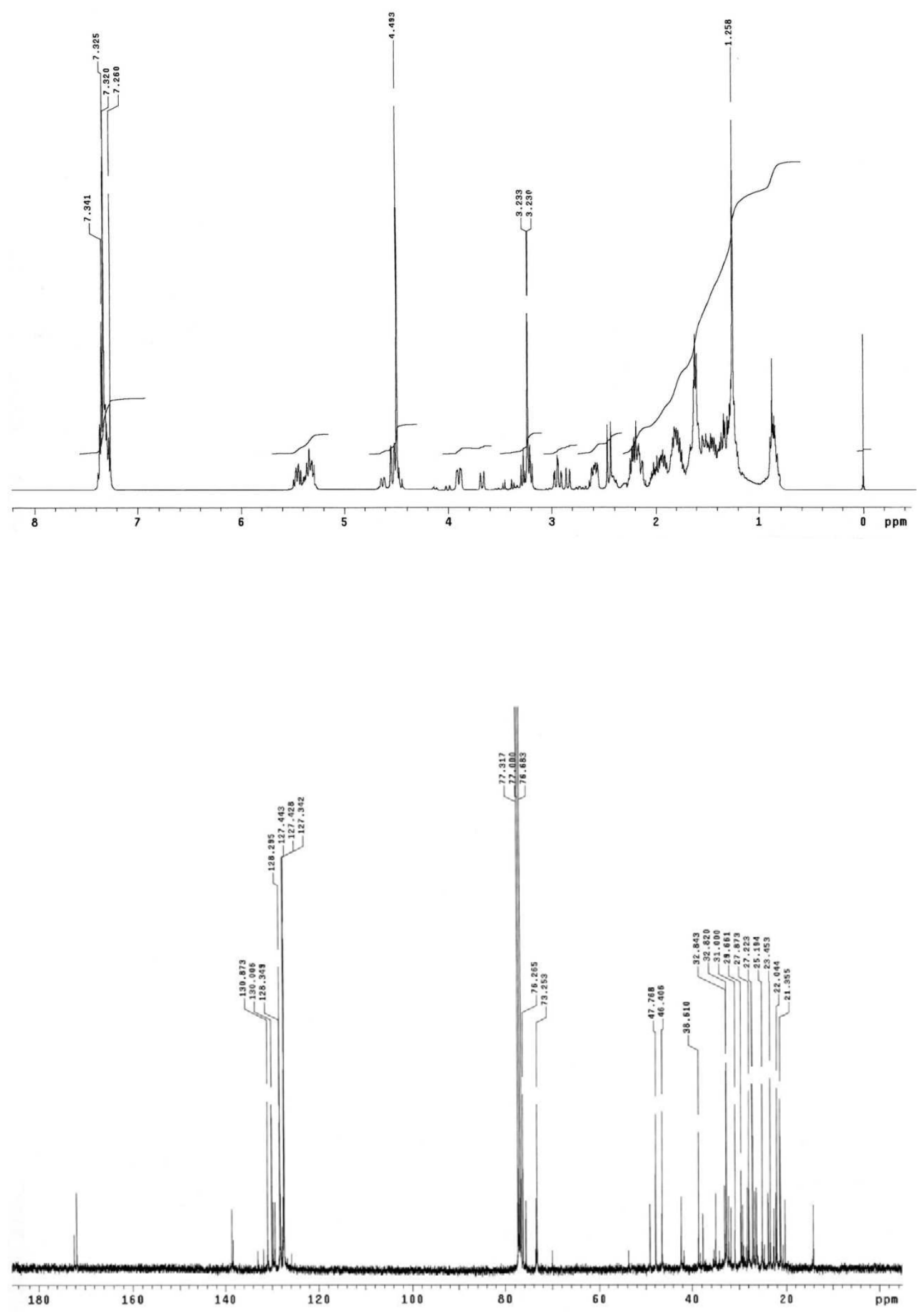


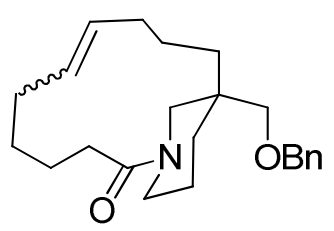

13-(Benzyloxymethyl)-2-oxo-1-azabicyclo[10.3.1]hexadec-8-ene (72).

A solution of diene $69(71 \mathrm{mg}, 0.18 \mathrm{mmol})$ in $\mathrm{CH}_{2} \mathrm{Cl}_{2}(10 \mathrm{~mL})$ was slowly added via a syringe pump ( $4 \mathrm{~h}$ ) to a solution of Grubbs second generation catalyst ( $31 \mathrm{mg}, 0.036 \mathrm{mmol}$ ) in refluxing $\mathrm{CH}_{2} \mathrm{Cl}_{2}$ (35 mL). The resulting mixture was heated at reflux for $6 \mathrm{~h}$ and concentrated under reduced pressure. Flash chromatography (alumina, 95:5 to 1:1 hexane-EtOAc) afforded macrocyclic compound 72 (24 mg, 38\%) as an inseparable 2:1 mixture of $E / Z$ isomers.

$E$ isomer of compound $\mathbf{7 2}$ (spectral data from a 2:1 E/Z mixture of isomers):

${ }^{1} \mathrm{H}$ NMR (400 MHz, CDCl, COSY, gHSQC): $\delta 1.35-2.35\left(\mathrm{~m}, 14 \mathrm{H}, \mathrm{CH}_{2}\right), 2.39(\mathrm{~d}, J=13.2 \mathrm{~Hz}, 1 \mathrm{H}, \mathrm{H}-$ 16), $2.75(\mathrm{~m}, 2 \mathrm{H}, \mathrm{H}-3), 2.89(\mathrm{t}, J=12.4 \mathrm{~Hz}, 1 \mathrm{H}, \mathrm{H}-15), 3.17\left(\mathrm{~d}, J=8.8 \mathrm{~Hz}, 1 \mathrm{H}, \mathrm{CH}_{2} \mathrm{Bn}\right), 3.23(\mathrm{~d}, J=$ $\left.8.8 \mathrm{~Hz}, 1 \mathrm{H}, \mathrm{CH}_{2} \mathrm{Bn}\right), 3.76(\mathrm{dm}, J=12.4 \mathrm{~Hz}, 1 \mathrm{H}, \mathrm{H}-15), 4.34(\mathrm{~d}, J=13.2 \mathrm{~Hz}, 1 \mathrm{H}, \mathrm{H}-16), 4.48(\mathrm{~s}, 2 \mathrm{H}$, $\mathrm{CH}_{2} \mathrm{OBn}$ ), 5.17 (dd, $\left.J=15.2,7.2 \mathrm{~Hz}, 1 \mathrm{H}, \mathrm{CH}=\right), 5.25(\mathrm{~m}, 1 \mathrm{H}, \mathrm{CH}=), 7.32\left(\mathrm{~m}, 5 \mathrm{H}, \mathrm{H}-0, m, p \mathrm{C}_{6} \mathrm{H}_{5}\right)$.

${ }^{13} \mathrm{C} \mathrm{NMR}\left(100.6 \mathrm{MHz}, \mathrm{CDCl}_{3}\right): \delta 20.9$ (C-14), 21.4-32.0 $\left(\mathrm{CH}_{2}\right), 33.5$ (C-3), 38.0 (C-12), 46.8 (C-16), 47.6 (C-15), $73.2\left(\mathrm{CH}_{2} \mathrm{OBn}\right), 76.4\left(\mathrm{CH}_{2} \mathrm{Bn}\right), 127.3-128.3\left(\mathrm{C}-0, m, p \mathrm{C}_{6} \mathrm{H}_{5}\right), 130.5,132.5(\mathrm{CH}=)$, $138.6\left(\mathrm{C}-i \mathrm{C}_{6} \mathrm{H}_{5}\right), 173.0(\mathrm{C}=\mathrm{O})$.

$Z$ isomer of compound 72 (spectral data from a 2:1 E/Z mixture of isomers):

${ }^{1} \mathrm{H}$ NMR $\left(400 \mathrm{MHz}, \mathrm{CDCl}_{3}, \mathrm{COSY}, \mathrm{gHSQC}\right): \delta 1.35-2.35\left(\mathrm{~m}, 14 \mathrm{H}, \mathrm{CH}_{2}\right), 2.39(\mathrm{~d}, J=13.2 \mathrm{~Hz}, 1 \mathrm{H}, \mathrm{H}-$ 16), $2.75(\mathrm{~m}, 2 \mathrm{H}, \mathrm{H}-3), 2.76$ (masked $1 \mathrm{H}, \mathrm{H}-15), 3.10-3.25\left(\mathrm{~m}, 2 \mathrm{H}, \mathrm{CH}_{2} \mathrm{Bn}\right.$ ), $3.40(\mathrm{dm}, J=13.6$ $\mathrm{Hz}, 1 \mathrm{H}, \mathrm{H}-15), 4.47$ (masked, $1 \mathrm{H}, \mathrm{H}-16), 4.47\left(\mathrm{~s}, 2 \mathrm{H}, \mathrm{CH}_{2} \mathrm{OBn}\right), 5.35,5.50(2 \mathrm{~m}, 2 \mathrm{H}, \mathrm{CH}=), 7.32(\mathrm{~m}$, $5 \mathrm{H}, \mathrm{ArH})$.

${ }^{13} \mathrm{C} \mathrm{NMR}\left(100.6 \mathrm{MHz}, \mathrm{CDCl}_{3}\right): \delta 20.9$ (C-14), 21.5-33.6 $\left(\mathrm{CH}_{2}\right), 33.8$ (C-3), 37.8 (C-12), 42.4 (C-16), 48.9 (C-15), $73.3\left(\mathrm{CH}_{2} \mathrm{OBn}\right), 76.2\left(\mathrm{CH}_{2} \mathrm{Bn}\right), 127.3-128.3$ (C-o, $\left.m, p\right), 130.8,134.1$ ( $\left.\mathrm{CH}=\right), 138.2$ (Ci), $172.2(\mathrm{C}=0)$.

HRMS (ESI) calcd for $\left[\mathrm{C}_{23} \mathrm{H}_{33} \mathrm{NO}_{2}+\mathrm{H}\right]^{+}: 356.2589$, found: 356.2582 . 

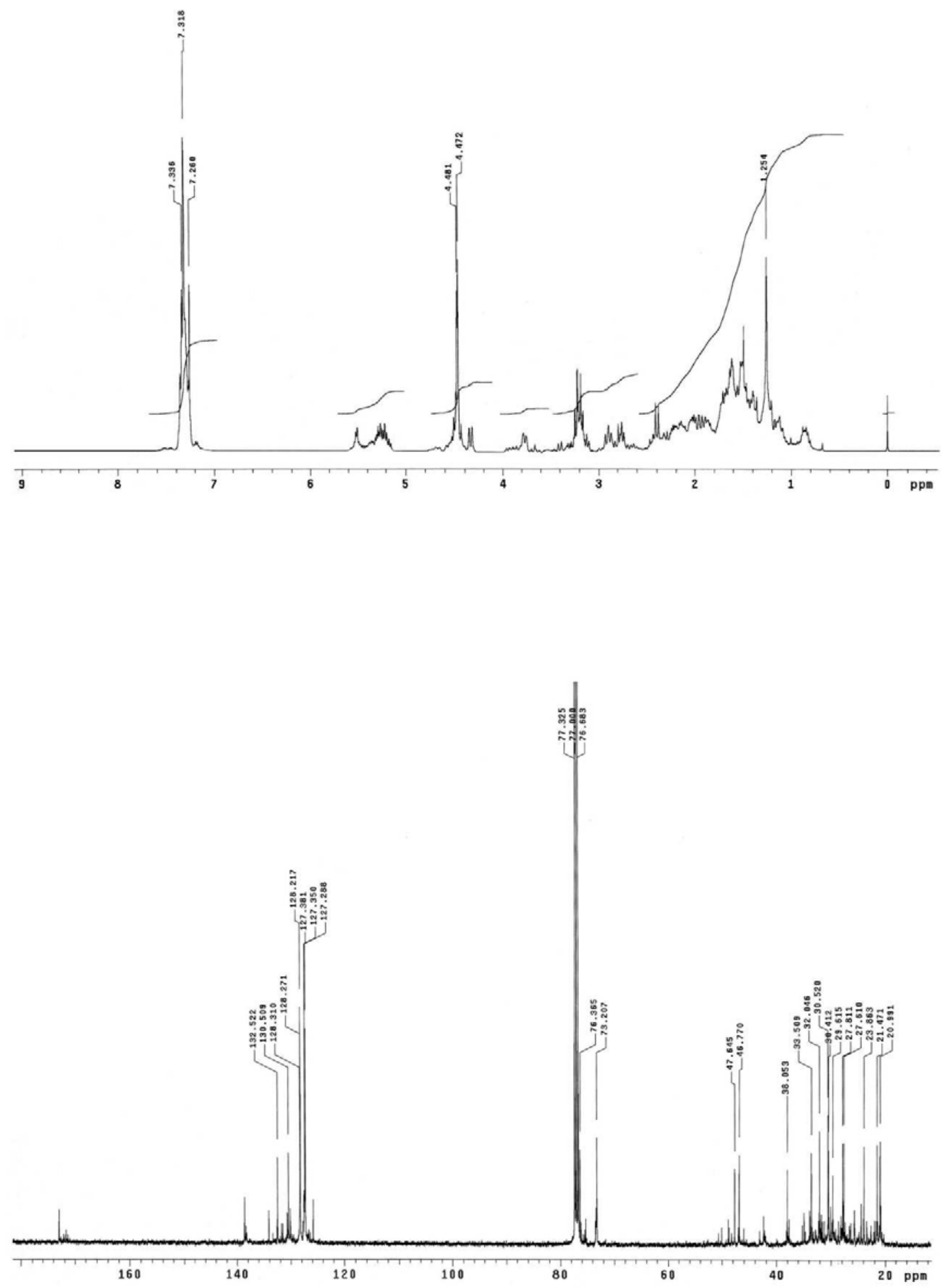


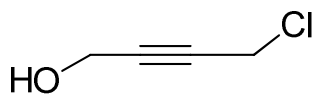

\section{4-Chloro-2-butynol (83).}

Thionyl chloride $(9.3 \mathrm{~mL}, 24.3 \mathrm{~mol})$ was added dropwise over a period of $6 \mathrm{~h}$ to a stirred solution of 2-butyn-1,4-diol (10 g, $11.6 \mathrm{~mol})$ and pyridine $(9.35 \mathrm{~mL}, 12.7 \mathrm{~mol})$ in benzene (12 $\mathrm{mL}$ ) while the temperature was maintained between 10 to $20^{\circ} \mathrm{C}$. The reaction mixture was then stirred overnight at room temperature. The mixture was poured into ice water and the benzene layer was separated. The aqueous phase was extracted with ether and the combined organic extracts were washed with saturated $\mathrm{NaHCO}_{3}$, followed by water and brine, dried over anhydrous $\mathrm{Na}_{2} \mathrm{SO}_{4}$, filtered and concentrated under reduced pressure. Distillation ( $55^{\circ} \mathrm{C} / 0$ tor) provided cloro derivative 83 (4.73 $\mathrm{mg}, 40 \%)$ as colorless oil.

IR (film): $3332(\mathrm{OH}) \mathrm{cm}^{-1}$.

${ }^{1} \mathrm{H} \mathrm{NMR}\left(400 \mathrm{MHz}, \mathrm{CDCl}_{3}, \mathrm{COSY}, \mathrm{gHSQC}\right): \delta 2.80$ (br. s, $\left.1 \mathrm{H}, \mathrm{OH}\right), 4.18(\mathrm{t}, J=2.0 \mathrm{~Hz}, 2 \mathrm{H}, \mathrm{H}-4), 4.33$ $(\mathrm{t}, J=2.0 \mathrm{~Hz}, 2 \mathrm{H}, \mathrm{H}-1)$.

${ }^{13} \mathrm{C} \mathrm{NMR}\left(100.6 \mathrm{MHz}, \mathrm{CDCl}_{3}\right): \delta 30.3$ (C-4), $51.0(\mathrm{C}-1), 80.5,84.6$ (C-2, C-3).

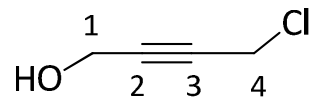



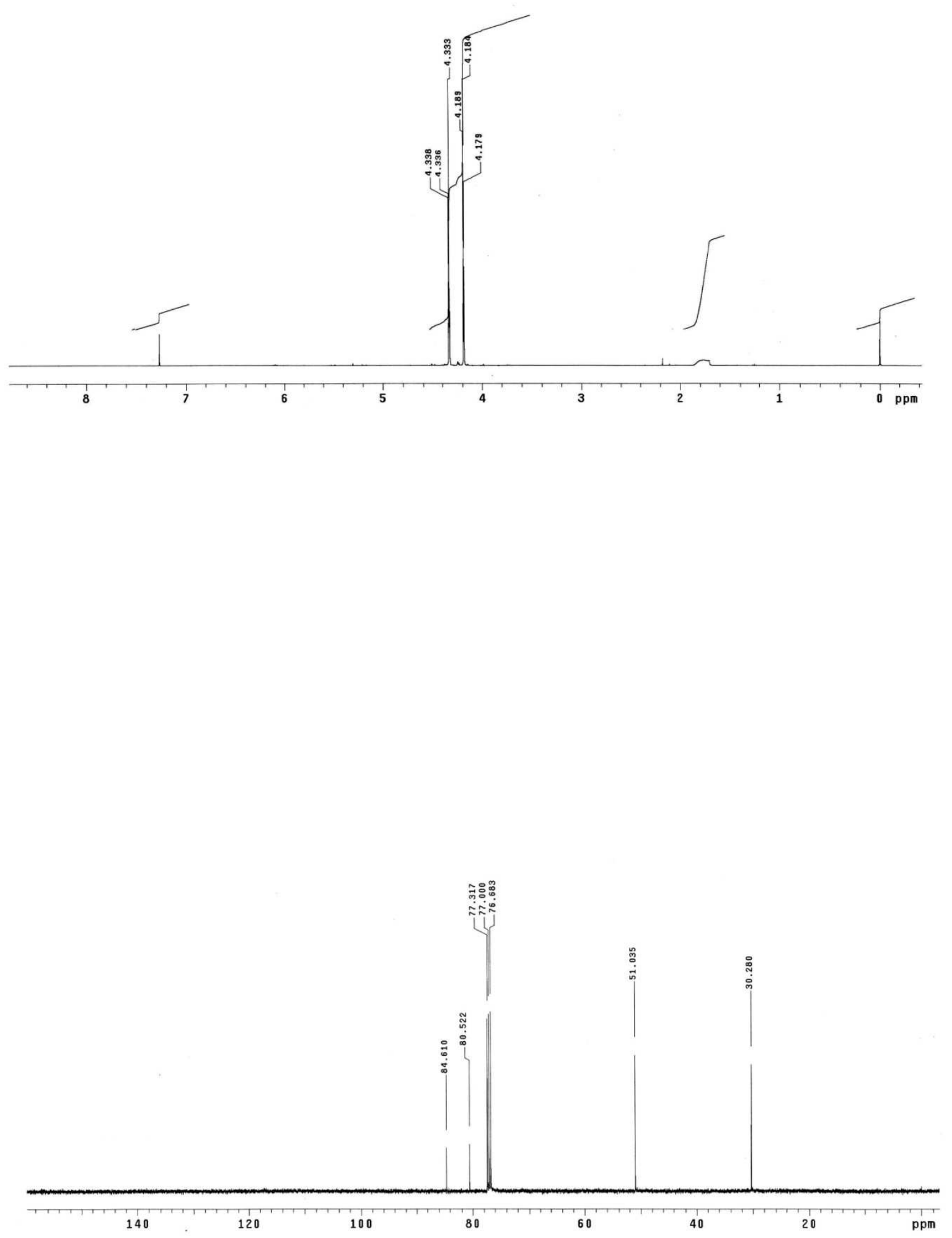


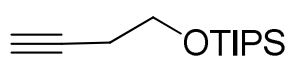

\section{(Triisopropylsilyloxy)-3-butynol (88).}

TipsCl (11 mL, $51.5 \mathrm{mmol}$ ) was added to a stirred solution of imidazole $(3.98 \mathrm{~g}, 58.4 \mathrm{mmol})$ and of 3-butynol (2.6 mL, $34.3 \mathrm{mmol})$ in $\mathrm{CH}_{2} \mathrm{Cl}_{2}(16 \mathrm{~mL})$ and $\mathrm{DMF}(8 \mathrm{~mL})$ at $0{ }^{\circ} \mathrm{C}$, then the mixture was stirred for $4 \mathrm{~h}$ at room temperature. The reaction was quenched by addition of saturated $\mathrm{NH}_{4} \mathrm{Cl}$ and was extracted with $\mathrm{CH}_{2} \mathrm{Cl}_{2}$. The combined organic extracts were dried over $\mathrm{MgSO}_{4}$, filtered and concentrated under reduced pressure. Flash chromatography (hexane) gave silyl derivative 88 as colorless oil $(7.012 \mathrm{~g}, 90 \%)$.

${ }^{1} \mathrm{H}$ NMR $\left(400 \mathrm{MHz}, \mathrm{CDCl}_{3}\right): \delta 1.07\left[2 \mathrm{~S}, 21 \mathrm{H}, \mathrm{CH}\left(\mathrm{CH}_{3}\right)_{2}\right], 1.95(\mathrm{t}, J=2.4 \mathrm{~Hz}, 1 \mathrm{H}, \mathrm{H}-4), 2.44(\mathrm{td}, J=$ $7.2,2.4 \mathrm{~Hz}, 2 \mathrm{H}, \mathrm{H}-2), 3.82(\mathrm{t}, J=7.2 \mathrm{~Hz}, 2 \mathrm{H}, \mathrm{H}-1)$.

${ }^{13} \mathrm{C} \mathrm{NMR}\left(100.6 \mathrm{MHz}, \mathrm{CDCl}_{3}\right): \delta 11.9\left[\mathrm{CH}\left(\mathrm{CH}_{3}\right)_{2}\right], 17.9\left[\mathrm{CH}\left(\mathrm{CH}_{3}\right)_{2}\right], 22.9(\mathrm{C}-2), 62.0$ (C-1), 69.2 (C4), $81.5(C-3)$.

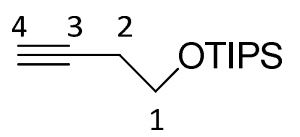



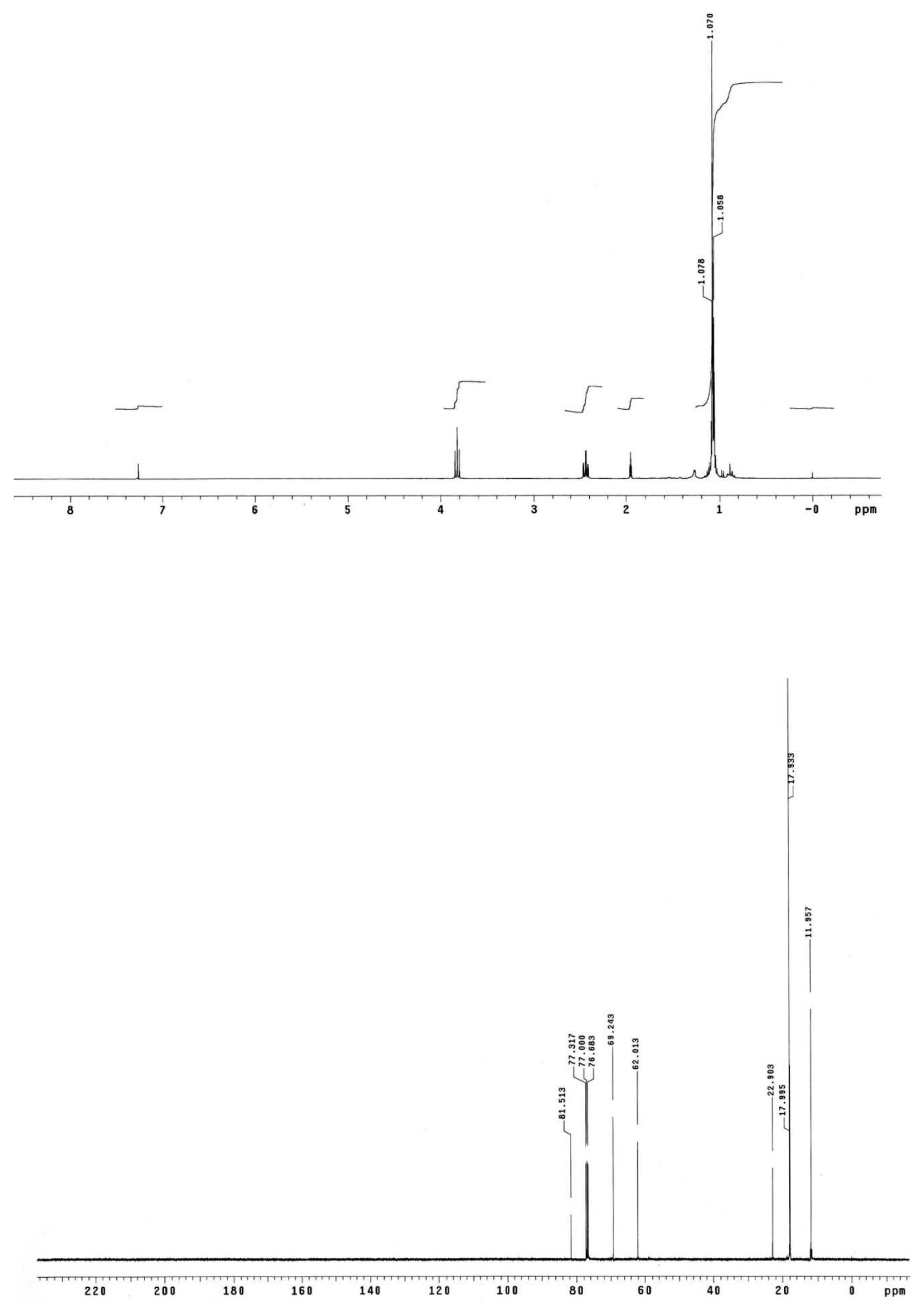


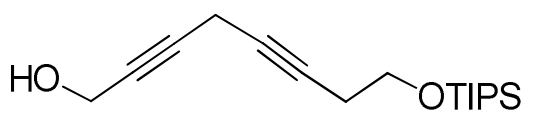

\section{8-[(Triisopropylsilyl)oxy]-2,5-octadiynol (89).}

4-Chloro-2-butyn-1-ol (4.05 g, $38.78 \mathrm{mmol}$ ) and 1-(triisopropylsilyloxy)-3-butyne (11.4 g, 50.5 mmol) were added under inert atmosphere to a suspension of Cul (15.17 g, $77.56 \mathrm{mmol})$, Nal $(11.63 \mathrm{~g}, 77.56 \mathrm{mmol})$, and $\mathrm{K}_{2} \mathrm{CO}_{3}(8.04 \mathrm{~g}, 58.17 \mathrm{mmol})$ in anhydrous DMF $(77 \mathrm{~mL})$. The mixture was stirred overnight at room temperature. Saturated aqueous $\mathrm{NH}_{4} \mathrm{Cl}$ and EtOAc were added, and the resulting mixture was filtered through a Celite ${ }^{\circledR}$ pad. The two phases were separated, and the aqueous layer was extracted with ether. The combined organic extracts were washed with brine, dried over anhydrous $\mathrm{Na}_{2} \mathrm{SO}_{4}$, filtered, and concentrated under reduced pressure. Flash chromatography (hexane to $8: 2$ hexane-EtOAc) gave $89(8.95 \mathrm{~g}, 78 \%)$ as a pale yellow oil. IR (film): $3332(\mathrm{OH}) \mathrm{cm}^{-1}$.

${ }^{1} \mathrm{H}$ NMR $\left(400 \mathrm{MHz}, \mathrm{CDCl}_{3}, \mathrm{COSY}, \mathrm{gHSQC}\right): \delta 1.03,1.04\left[2 \mathrm{~S}, 21 \mathrm{H}, \mathrm{CH}\left(\mathrm{CH}_{3}\right)_{2}\right], 2.39(\mathrm{tt}, J=7.6,2.4$ $\mathrm{Hz}, 2 \mathrm{H}, \mathrm{H}-7), 3.15(\mathrm{~m}, 2 \mathrm{H}, \mathrm{H}-4), 3.76(\mathrm{t}, J=7.6 \mathrm{~Hz}, 2 \mathrm{H}, \mathrm{H}-8), 4.23(\mathrm{t}, J=2.4 \mathrm{~Hz}, 2 \mathrm{H}, \mathrm{H}-1)$.

${ }^{13} \mathrm{C} \mathrm{NMR}\left(100.6 \mathrm{MHz}, \mathrm{CDCl}_{3}\right): \delta 9.5$ (C-4), $11.9\left[\mathrm{CH}\left(\mathrm{CH}_{3}\right)_{2}\right], 17.9\left[\mathrm{CH}\left(\mathrm{CH}_{3}\right)_{2}\right], 23.1$ (C-7), 51.1 (C-1), 62.1 (C-8), 74.5 (C-5), 77.9 (C-3), 78.5 (C-2), 80.3 (C-6).

HRMS calcd for $\left[\mathrm{C}_{17} \mathrm{H}_{30} \mathrm{O}_{2} \mathrm{Si}+\mathrm{H}\right]^{+}: 295.2088$, found: 295.2090 .

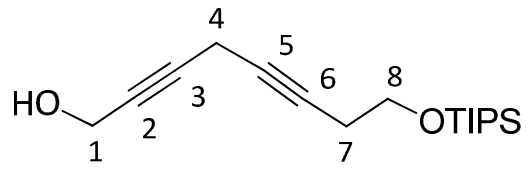



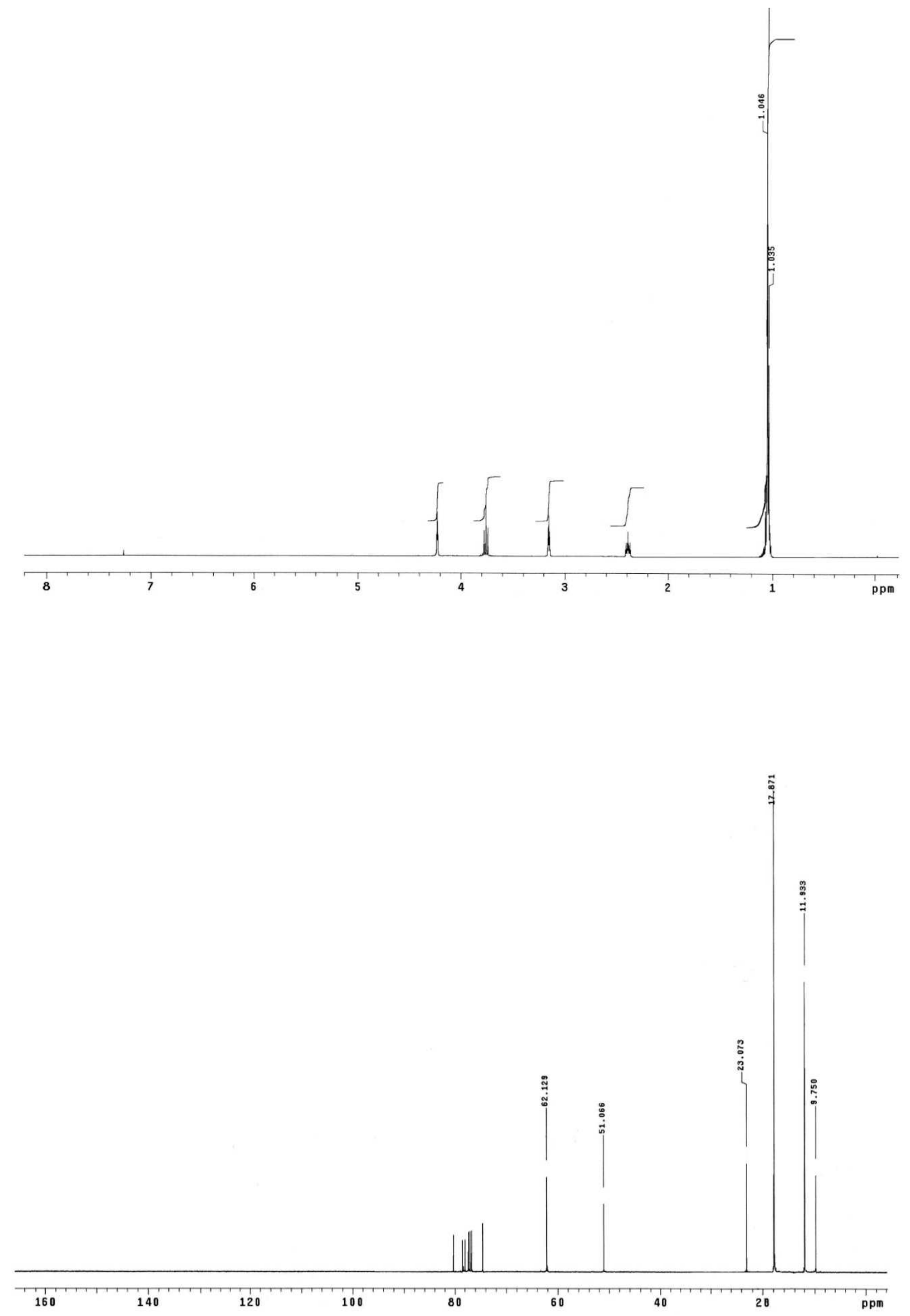


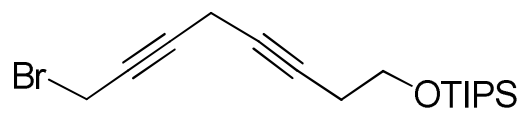

\section{8-Bromo-1-[(triisopropylsilyl)oxy]-3,6-octadiyn (90).}

First step: Triethylamine $(610 \mu \mathrm{L}, 4.38 \mathrm{mmol})$ and mesyl chloride $(454 \mu \mathrm{L}, 5.84 \mathrm{mmol})$ were added dropwise to a solution of alcohol 89 (860 mg, $2.92 \mathrm{mmol})$ in anhydrous $\mathrm{CH}_{2} \mathrm{Cl}_{2}(50 \mathrm{~mL})$ cooled to $0{ }^{\circ} \mathrm{C}$. After the end of addition, the mixture was stirred at $0{ }^{\circ} \mathrm{C}$ for $15 \mathrm{~min}$, thus was stirred for additional $1.5 \mathrm{~h}$ keeping the temperature between 10 and $20^{\circ} \mathrm{C}$. Saturated aqueous $\mathrm{NH}_{4} \mathrm{Cl}$ was added, and the mixture was extracted with $\mathrm{CH}_{2} \mathrm{Cl}_{2}$. The combined organic layers were washed with water, dried over anhydrous $\mathrm{Na}_{2} \mathrm{SO}_{4}$, filtered, and the solvent was removed under reduced pressure to afford compound $\mathbf{9 0}$, which was used in the next step without purification.

Second step: A solution of $\operatorname{LiBr}(2.5 \mathrm{~g}, 29.2 \mathrm{mmol})$ in THF $(20 \mathrm{~mL})$ was added to a solution of mesyl derivative $(2.92 \mathrm{mmol})$ in $\mathrm{CH}_{2} \mathrm{Cl}_{2}(15 \mathrm{~mL})$ at $0{ }^{\circ} \mathrm{C}$ under inert atmosphere. The resulting mixture was stirred at room temperature for $1 \mathrm{~h}$. Distilled water was added, and the mixture was extracted with $\mathrm{CH}_{2} \mathrm{Cl}_{2}$. The combined organic layers were dried over anhydrous $\mathrm{Na}_{2} \mathrm{SO}_{4}$, filtered, and the solvent was removed under reduced pressure. Flash chromatography (hexane to $3: 7$ hexane-EtOAc) afforded bromo derivative 90 (760 $\mathrm{mg}, 73 \%$ yield).

${ }^{1} \mathrm{H}$ NMR $\left(400 \mathrm{MHz}, \mathrm{CDCl}_{3}\right): \delta 1.05,1.06\left[2 \mathrm{~S}, 21 \mathrm{H}, \mathrm{CH}\left(\mathrm{CH}_{3}\right)_{2}\right], 2.41(\mathrm{~m}, J=6.8,2.4 \mathrm{~Hz}, 2 \mathrm{H}, \mathrm{H}-2)$, $3.20(\mathrm{t}, J=2.4 \mathrm{~Hz}, 2 \mathrm{H}, \mathrm{H}-5), 3.78(\mathrm{t}, J=7.2 \mathrm{~Hz}, 2 \mathrm{H}, \mathrm{H}-1), 3.90(\mathrm{t}, J=2.4 \mathrm{~Hz}, 2 \mathrm{H}, \mathrm{H}-8)$.

${ }^{13} \mathrm{C} \mathrm{NMR}\left(100.6 \mathrm{MHz}, \mathrm{CDCl}_{3}\right): \delta 10.1(\mathrm{C}-5), 12.0\left[\mathrm{CH}\left(\mathrm{CH}_{3}\right)_{2}\right], 14.7(\mathrm{C}-8), 17.9\left[\mathrm{CH}\left(\mathrm{CH}_{3}\right)_{2}\right], 23.1$ (C2), 62.1 (C-1), 74.0 (C-4), 75.3 (C-6), 78.3 (C-7), 81.8 (C-3).

HRMS calcd for $\left[\mathrm{C}_{17} \mathrm{H}_{29} \mathrm{BrOSi}+\mathrm{H}\right]^{+}: 357.1244$, found: 357.1232 

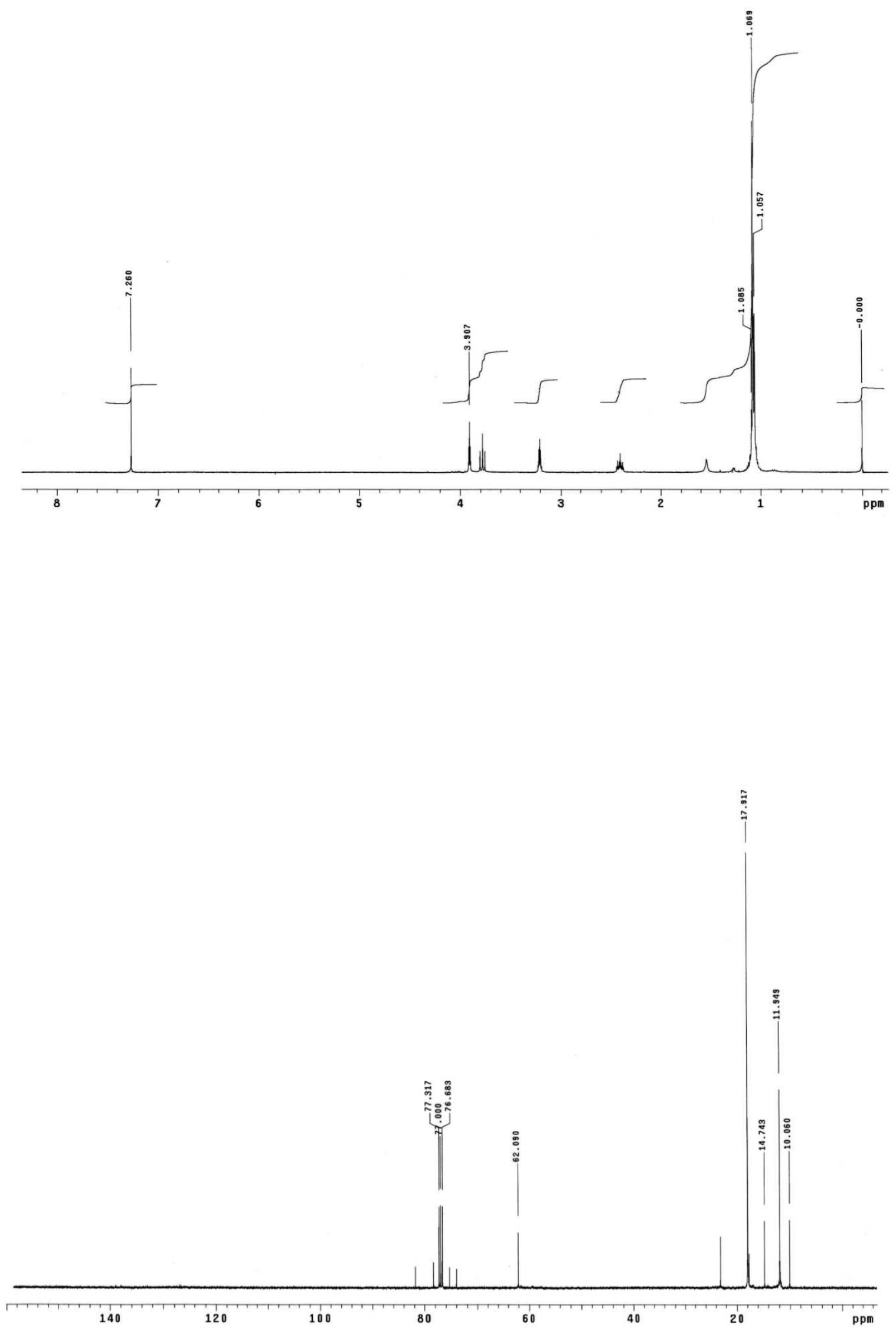


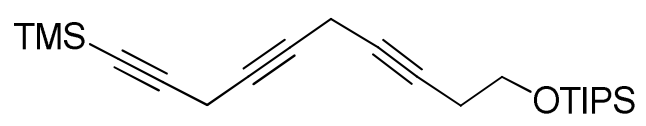

10-(Trimethylsilyl)-1-[(triisopropylsilyl)oxy]-3,6,9-octatriyn (85).

Bromo derivative $90(760 \mathrm{mg}, 2.13 \mathrm{mmol})$ and ethynyltrimethylsilane $(349 \mu \mathrm{L}, 2.04 \mathrm{mmol})$ were added to a suspension of Cul ( $816 \mathrm{mg}, 4.26 \mathrm{mmol})$, Nal $\left(638 \mathrm{mg}, 4.26 \mathrm{mmol}\right.$ ), and $\mathrm{K}_{2} \mathrm{CO}_{3}$ (441 $\mathrm{mg}, 3.10 \mathrm{mmol})$ in of anhydrous DMF $(8 \mathrm{~mL})$ under inert atmosphere. The mixture was stirred overnight at room temperature. Saturated aqueous $\mathrm{NH}_{4} \mathrm{Cl}$ and EtOAc were then added and the mixture was filtered through a celite pad. The aqueous layer of the filtered mixture was extracted with ether $(3 \times 20 \mathrm{~mL})$ and the combined organic layers were washed with brine, dried over anhydrous $\mathrm{Na}_{2} \mathrm{SO}_{4}$, filtered and concentrated under vacuum. Flash chromatography (hexane to 97:3 hexane-EtOAc) gave trialkinyl derivative 85 (685 mg, 86\%) as colorless oil.

${ }^{1} \mathrm{H}$ NMR (400 MHz, CDCl 3 , COSY, gHSQC): $\delta$ 0.06, $0.16\left[2 \mathrm{~s}, 9 \mathrm{H},\left(\mathrm{CH}_{3}\right)_{3} \mathrm{Si}\right], 1.05,1.06[2 \mathrm{~s}, 21 \mathrm{H}$, $\left.\left(\mathrm{CH}_{3}\right)_{2} \mathrm{CH}\right)$ ], $2.41(\mathrm{tt}, J=7.2,2.4 \mathrm{~Hz}, 2 \mathrm{H}, \mathrm{H}-2), 3.13(\mathrm{q}, J=2.4 \mathrm{~Hz}, 2 \mathrm{H}, \mathrm{H}-5), 3.20(\mathrm{t}, J=2.4 \mathrm{~Hz}, 2 \mathrm{H}$, $\mathrm{H}-8), 3.78(\mathrm{t}, J=7.2 \mathrm{~Hz}, 2 \mathrm{H}, \mathrm{H}-1)$.

${ }^{13} \mathrm{C} \mathrm{NMR}\left(100.6 \mathrm{MHz}, \mathrm{CDCl}_{3}\right): \delta-0.1,1.01\left[\left(\mathrm{CH}_{3}\right)_{3} \mathrm{Si}\right], 9.8$ (C-5), 10.9 (C-8), 12.0 [( $\left.\left.\left.\mathrm{CH}_{3}\right)_{2} \mathrm{CH}\right)\right], 17.9$ $\left.\left[\left(\mathrm{CH}_{3}\right)_{2} \mathrm{CH}\right)\right], 23.1$ (C-2), 62.2 (C-1), 73.8, 74.9, 75.1 (C-4, C-6, C-7), 77.7 (C-3), 85.1 (C-9), 99.9 (C10).

HRMS calcd for $\left[\mathrm{C}_{22} \mathrm{H}_{38} \mathrm{OSi}_{2}+\mathrm{H}^{+}: 375.2534\right.$, found: 375.2524 .

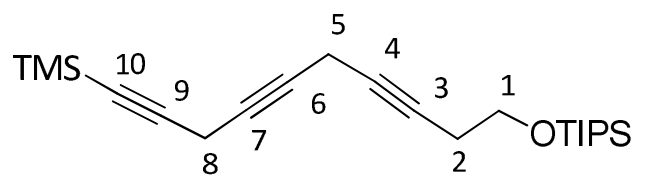



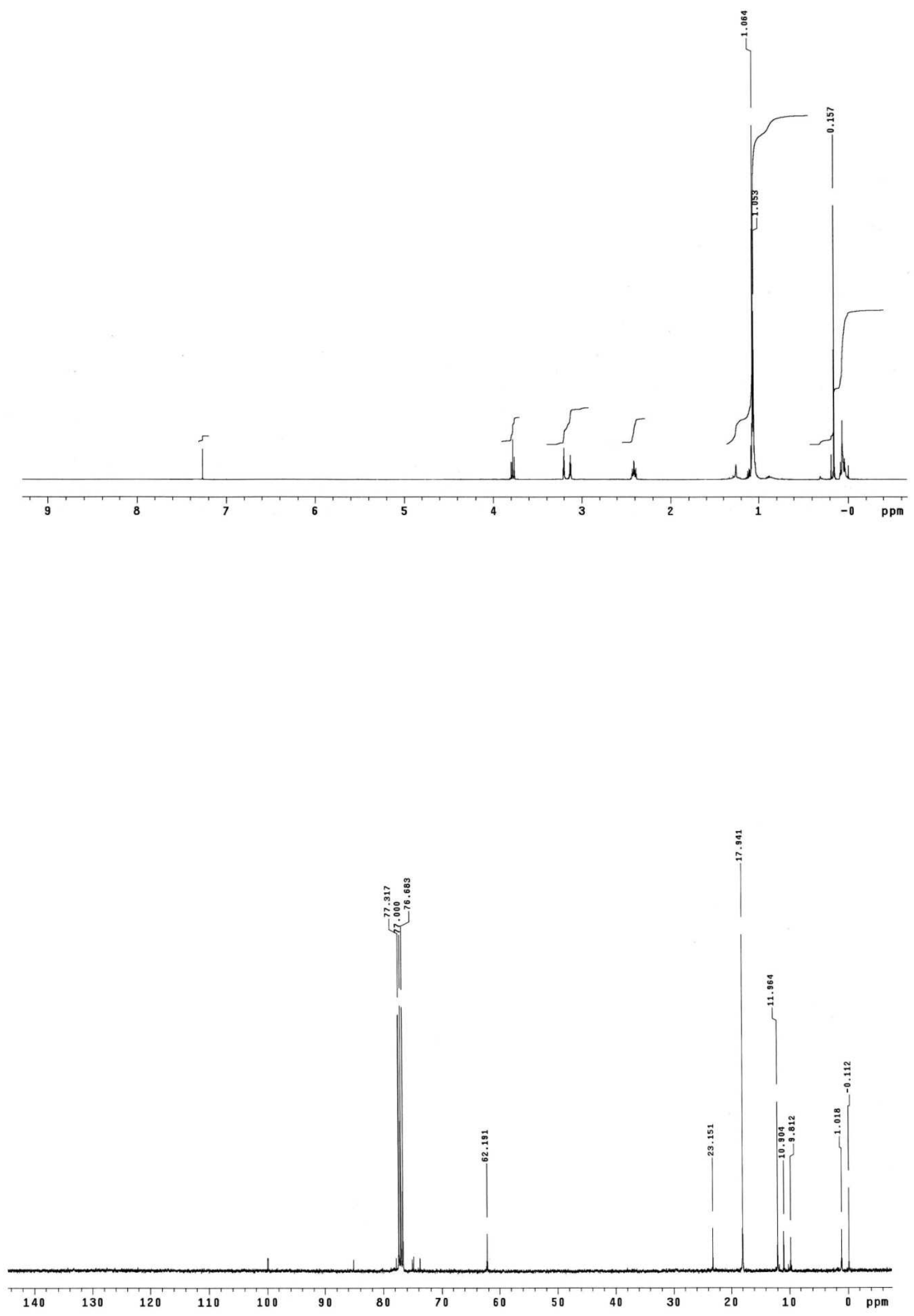


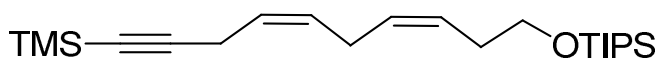

\section{(3Z,6Z)-10-(Trimethylsilyl)-1-[(triisopropylsilyl)oxy]-3,6-octadien-9-yn (86).}

A $1 \mathrm{M}$ solution of $\mathrm{NaBH}_{4}$ in ethanol $(730 \mu \mathrm{L}, 0.73 \mathrm{mmol})$ was added to a stirred suspension of $\mathrm{Ni}(\mathrm{OAc})_{2} \cdot 4 \mathrm{H}_{2} \mathrm{O}(180 \mathrm{mg}, 0.73 \mathrm{mmol})$ in ethanol $(12 \mathrm{~mL})$ under an $\mathrm{H}_{2}$ atmosphere. After the black suspension was stirred for $30 \mathrm{~min}$, ethylenediamine (97 $\mu \mathrm{L}, 1.45 \mathrm{mmol}$ ) was added followed by a solution of trialkinyl $85(544 \mathrm{mg}, 1.45 \mathrm{mmol})$ in ethanol $(1 \mathrm{~mL})$. The reaction flask was purged three times with $\mathrm{H}_{2}$ and the reaction mixture was stirred under $\mathrm{H}_{2}$ atmosphere for $1 \mathrm{~h}$ and $45 \mathrm{~min}$. The resulting mixture was filtered through a pad of silica gel with ether. The filtrate was concentrated and the residue was chromatographed to give diene 86 (411 mg, 75\% yield) as a colorless oil.

$\left.{ }^{1} \mathrm{H} \mathrm{NMR}\left(400 \mathrm{MHz}, \mathrm{CDCl}_{3}, \mathrm{gHSQC}\right): \delta 0.01\left[\mathrm{~s}, 9 \mathrm{H},\left(\mathrm{CH}_{3}\right)_{3} \mathrm{Si}\right], 0.92,0.93\left[2 \mathrm{~s}, 21 \mathrm{H},\left(\mathrm{CH}_{3}\right)_{2} \mathrm{CH}\right)\right], 2.19$ (q, J = 6.9 Hz, 2H, H-2), $2.68(\mathrm{t}, J=7.2 \mathrm{~Hz}, 2 \mathrm{H}, \mathrm{H}-5), 2.87$ (dd, J=4.5, $0.6 \mathrm{~Hz}, 2 \mathrm{H}, \mathrm{H}-8), 3.78(\mathrm{t}, J=$ $6.9 \mathrm{~Hz}, 2 \mathrm{H}, \mathrm{H}-1)$.

${ }^{13} \mathrm{C}$ NMR (100.6 MHz, CDCl $)$ ): $\left.\left.\delta 0.07\left[\left(\mathrm{CH}_{3}\right)_{3} \mathrm{Si}\right], 12.0\left[\left(\mathrm{CH}_{3}\right)_{2} \mathrm{CH}\right)\right], 18.0\left[\left(\mathrm{CH}_{3}\right)_{2} \mathrm{CH}\right)\right], 18.4$ (C-8), 25.7 (C-5), 31.3 (C-2), 63.0 (C-1), 84.2 (C-9), 105.3 (C-10), 124.3, 126.8, 128.7, 129.9 (4CH=).

HRMS calcd for $\left[\mathrm{C}_{22} \mathrm{H}_{42} \mathrm{OSi}_{2}+\mathrm{H}\right]^{+}: 379.2846$, found: 379.2848 .

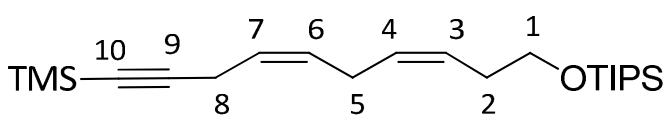



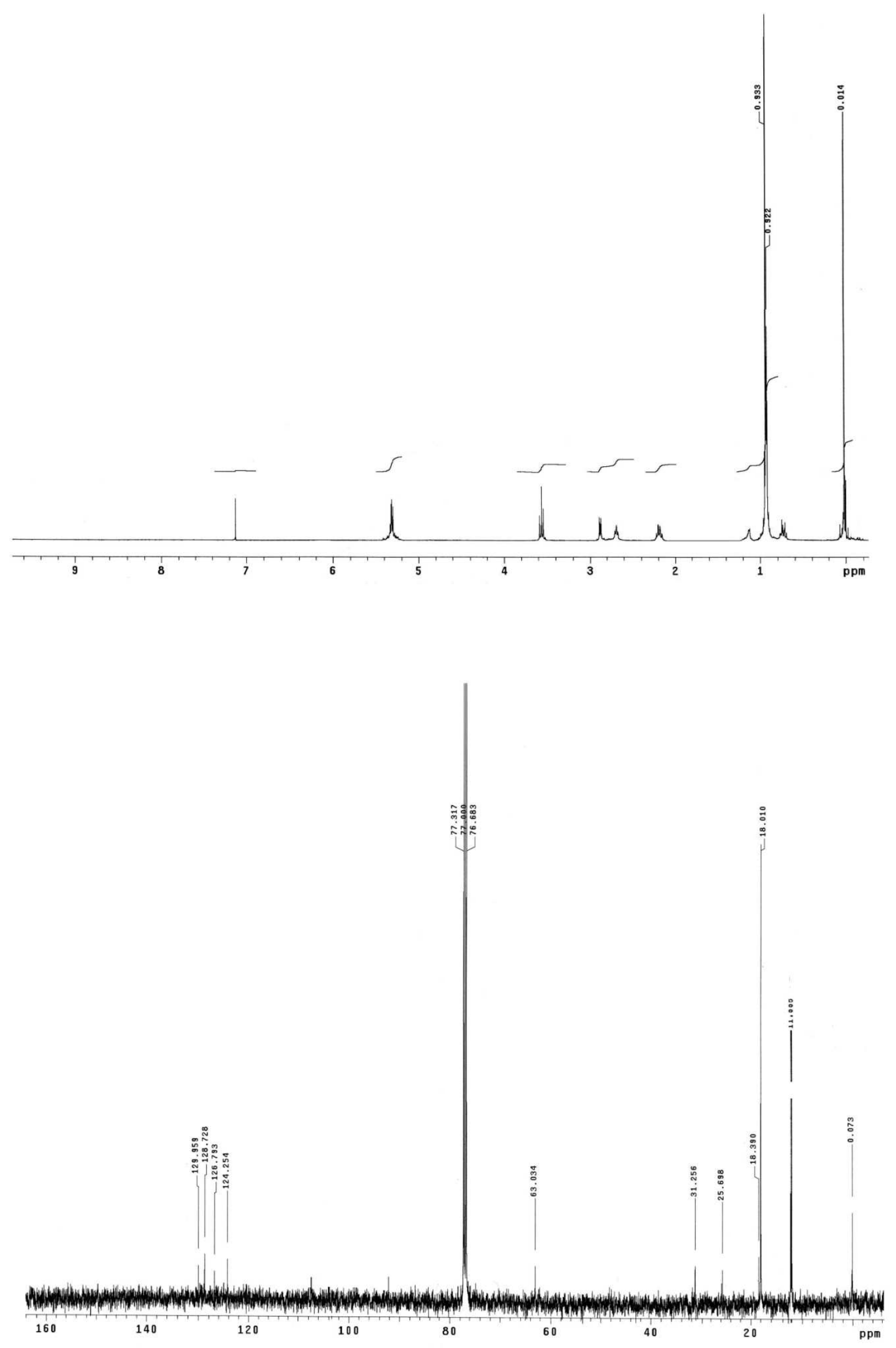


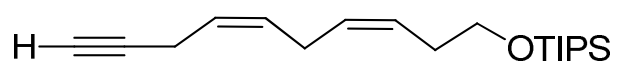

\section{(3Z,6Z)-1-[(Triisopropylsilyl)oxy]-3,6-octadien-9-yn (79).}

$\mathrm{K}_{2} \mathrm{CO}_{3}(80 \mathrm{mg}, 0.579 \mathrm{mmol})$ was added to a stirred solution of diene 86 (146 mg, $\left.0.386 \mathrm{mmol}\right)$ in anhydrous methanol $(2 \mathrm{~mL})$ at $0^{\circ} \mathrm{C}$. The reaction was stirred at room temperature for one hour and then methanol was removed under reduced pressure. EtOAc and saturated aqueous $\mathrm{NH}_{4} \mathrm{Cl}$ were added and the aqueous layer was extracted with EtOAc. The combined organic extracts were dried over $\mathrm{Na}_{2} \mathrm{SO}_{4}$, and the solvent was concentrated under reduced pressure. Flash chromatography (hexane) afforded the unstable alkyne derivate 79 (110 mg, 74\% yield).

${ }^{1} \mathrm{H}$ NMR $\left.\left(300 \mathrm{MHz}, \mathrm{CDCl}_{3}\right): \delta 0.92,0.93\left[2 \mathrm{~s}, 21 \mathrm{H},\left(\mathrm{CH}_{3}\right)_{2} \mathrm{CH}\right)\right], 1.95(\mathrm{~m}, 1 \mathrm{H}, \mathrm{H}-10), 2.25(\mathrm{q}, J=6.9$ $\mathrm{Hz}, 2 \mathrm{H}, \mathrm{H}-2$ ), $2.80(\mathrm{t}, J=7.2 \mathrm{~Hz}, 2 \mathrm{H}, \mathrm{H}-5), 2.97$ (dd, J = 4.5, $0.6 \mathrm{~Hz}, 2 \mathrm{H}, \mathrm{H}-8), 3.65(\mathrm{t}, J=6.9 \mathrm{~Hz}$, $2 \mathrm{H}, \mathrm{H}-1), 5.35-5.45(\mathrm{~m}, 4 \mathrm{H}, \mathrm{CH}=)$.

$\left.\left.{ }^{13} \mathrm{C} \mathrm{NMR}\left(100.6 \mathrm{MHz}, \mathrm{CDCl}_{3}\right): \delta 12.0\left[\left(\mathrm{CH}_{3}\right)_{2} \mathrm{CH}\right)\right], 18.0\left[\left(\mathrm{CH}_{3}\right)_{2} \mathrm{CH}\right)\right], 22.6$ (C-8), 25.5 (C-5), 31.6 (C2), 63.0 (C-1), 68.1 (C-10), 82.3 (C-9), 123.9, 126.9, 128.6, 132.0 (4CH=).

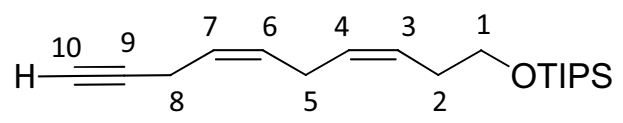



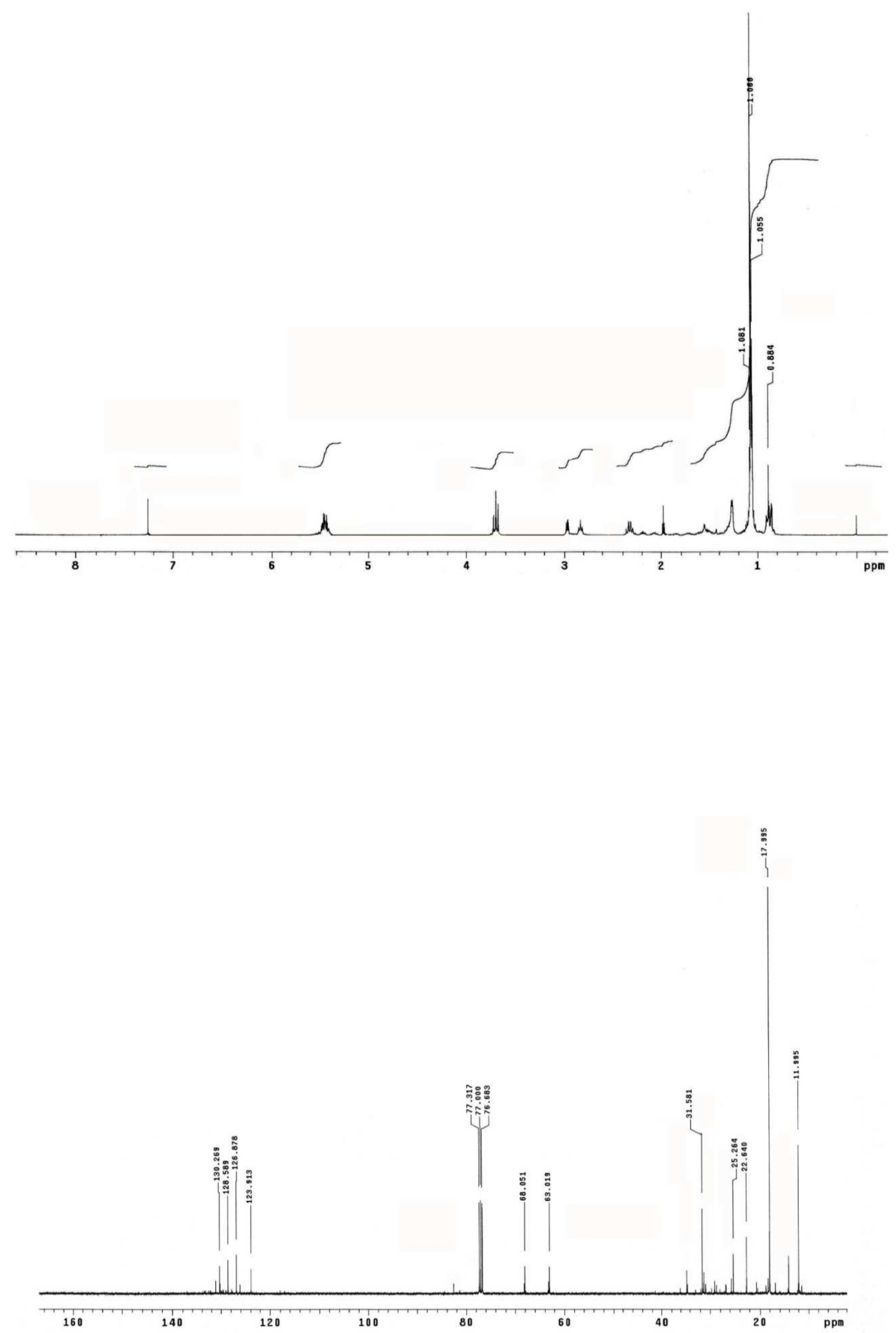


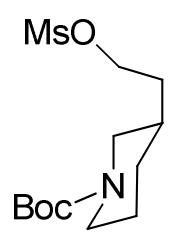

\section{$N$-(tert-Butoxycarbonyl)-3-[(methansulfonyloxy)ethyl]piperidine (92).}

Triethylamine (156 $\mu \mathrm{L}, 1.12 \mathrm{mmol})$ and mesyl chloride (116 $\mu \mathrm{L}, 1.49 \mathrm{mmol})$ were added dropwise to a cooled $\left(0^{\circ} \mathrm{C}\right)$ solution of commercial available $\mathbf{N}$-(tert-butoxycarbonyl)-3-(2hydroxyethyl)piperidine (171 $\mathrm{mg}, 0.746 \mathrm{mmol})$ in anhydrous $\mathrm{CH}_{2} \mathrm{Cl}_{2}(10 \mathrm{~mL})$. After the end of addition, the mixture was stirred at $10{ }^{\circ} \mathrm{C}$ for $15 \mathrm{~min}$, thus was allowed to heat slowly to room temperature and was stirred for additional $1.5 \mathrm{~h}$. Saturated aqueous $\mathrm{NH}_{4} \mathrm{Cl}$ was added, and the mixture was extracted with $\mathrm{CH}_{2} \mathrm{Cl}_{2}$. The combined organic extracts were dried over anhydrous $\mathrm{Na}_{2} \mathrm{SO}_{4}$, filtered, and the solvent was removed under reduced pressure. Flash chromatography (9:1 to 7:3 hexane-EtOAc) afforded methansulfonyl derivative 92 (160 mg, 71\%).

${ }^{1} \mathrm{H}$ NMR (400 MHz, CDCl 3 , COSY, gHSQC): $\delta 1.14(\mathrm{~m}, 1 \mathrm{H}, \mathrm{H}-4), 1.42$ [s, $\left.10 \mathrm{H},\left(\mathrm{CH}_{3}\right)_{3} \mathrm{C}, \mathrm{H}-5\right], 1.61$ (m, 3H, H-5, H-3, H-1') $1.69\left(m, 1 \mathrm{H}, \mathrm{H}-1^{\prime}\right), 1.81(\mathrm{dm}, \mathrm{J}=12.8 \mathrm{~Hz}, 1 \mathrm{H}, \mathrm{H}-4), 2.60,2.83$ (br. s, 2H, H-1ax, H-6ax), 3.02 (s, 3H, CH 3 S), 3.67, $3.80(2 \mathrm{~m}, 2 \mathrm{H}, \mathrm{H}-1$ eq, H-6eq), 4.28 (t, J = 6.8 Hz, 2H, H$\left.2^{\prime}\right)$.

${ }^{13} \mathrm{C} \mathrm{NMR}\left(100.6 \mathrm{MHz}, \mathrm{CDCl}_{3}\right): \delta 24.0(\mathrm{C}-5), 28.4\left[\left(\mathrm{CH}_{3}\right)_{3} \mathrm{C}\right], 30.4$ (C-4), 31.0 (C-3), $32.2\left(\mathrm{C}-1^{\prime}\right), 37.4$ $\left(\mathrm{CH}_{3} \mathrm{~S}\right), 44.5,49.1(\mathrm{C}-1, \mathrm{C}-6), 67.6\left(\mathrm{C}-2^{\prime}\right), 79.5\left[\left(\mathrm{CH}_{3}\right)_{3} \mathrm{C}\right], 154.7$ (NCOO).

HRMS calcd for $\left[\mathrm{C}_{13} \mathrm{H}_{25} \mathrm{NO}_{5} \mathrm{~S}+\mathrm{H}\right]^{+}: 308.1526$, found: 308.1526 . 

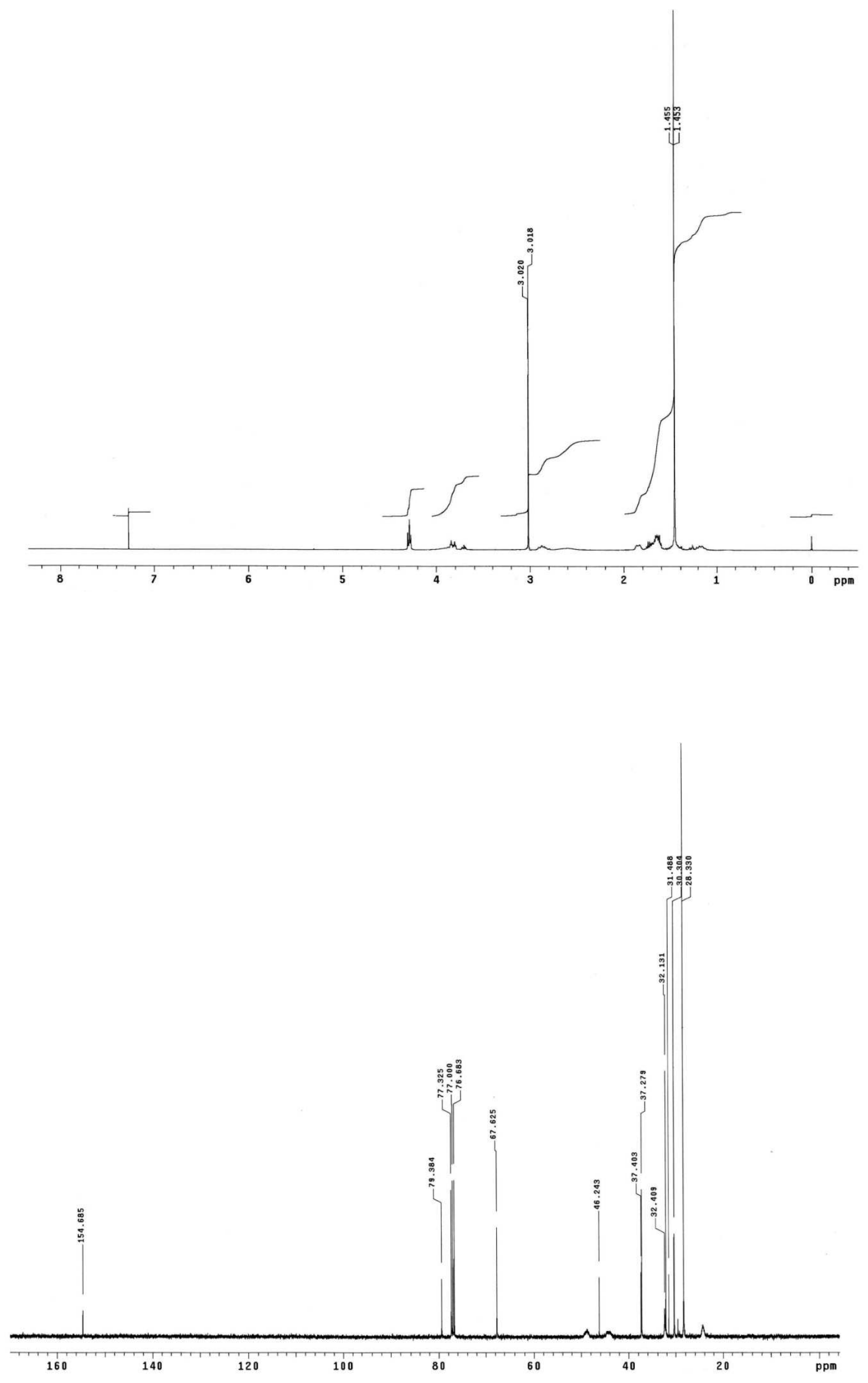
TMS<smiles>O=C1NCCC1CCC#CS</smiles>

3-[4-(Trimethylsilyl)-3-butynyl]-2-piperidinone (96).

$n$-BuLi (9.39 mL of a $1.6 \mathrm{M}$ solution in hexane, $15.0 \mathrm{mmol}$ ) was added to a stirring solution of $\delta$ valerolactam ( $1.49 \mathrm{~g}, 15.0 \mathrm{mmol})$ in anhydrous THF $(38 \mathrm{~mL})$ previously cooled at $-78{ }^{\circ} \mathrm{C}$. The resulting mixture was stirred for 30 minutes, and then TMSCl (1.91 mL, $15.0 \mathrm{mmol})$ was added while the solution was allowed to warm to room temperature. LDA $(8.27 \mathrm{~mL}$ of a $2 \mathrm{M}$ solution in THF, $16.54 \mathrm{mmol}$ ) was then added at $-78{ }^{\circ} \mathrm{C}$ to the above mixture, which was then again allowed to warm to room temperature. After stirring at room temperature for 30 minutes, a solution of 4-iodo-1-(trimethylsilyl)-1-butyne in THF $(10 \mathrm{~mL})$ was added dropwise at $0{ }^{\circ} \mathrm{C}$, and the resulting dark solution was stirred at this temperature for $1 \mathrm{~h}$, and for $3 \mathrm{~h}$ at room temperature. The reaction was quenched with saturated aqueous $\mathrm{NH}_{4} \mathrm{Cl}$, and the mixture was extracted with EtOAc. The combined organic extracts were dried over anhydrous $\mathrm{MgSO}_{4}$, filtered, and concentrated under reduced pressure. Flash chromatography (9:1 to 4:6 hexaneEtOAc) afforded piperidone 96 (1.67 g, 50\%) as a colourless oil.

${ }^{1} \mathrm{H}$ NMR (400 MHz, CDCl 3 , COSY, gHSQC): $\delta 0.13$ [s, 9H, $\left(\mathrm{CH}_{3}\right)_{3} \mathrm{Si}$ ], 1.51 (dddd, $J=13.2,10.0$, 10.0, $2.8 \mathrm{~Hz}, 1 \mathrm{H}, \mathrm{H}-4$ ), 1.62 (dddd, $\left.J=13.6,8.4,8.4,2.8 \mathrm{~Hz}, 1 \mathrm{H}, \mathrm{H}-1^{\prime}\right), 1.73(\mathrm{~m}, 1 \mathrm{H}, \mathrm{H}-5), 1.87$ $(m, 1 \mathrm{H}, \mathrm{H}-5), 1.99(\mathrm{~m}, 1 \mathrm{H}, \mathrm{H}-4), 2.19\left(\mathrm{~m}, 1 \mathrm{H}, \mathrm{H}-1^{\prime}\right), 2.32-2.43\left(\mathrm{~m}, 3 \mathrm{H}, 2 \mathrm{H}-2^{\prime}, \mathrm{H}-3\right), 3.29(\mathrm{~m}, 2 \mathrm{H}$, $\mathrm{H}-6), 6.02$ (br. s, 1H, NH).

${ }^{13} \mathrm{C} \mathrm{NMR}\left(100.6 \mathrm{MHz}, \mathrm{CDCl}_{3}\right): \delta 0.1\left[\left(\mathrm{CH}_{3}\right)_{3} \mathrm{Si}\right], 17.7$ (C-2'), 21.5 (C-5), 26.1 (C-4), 30.3 (C-1'), 40.1 (C-3), 42.4 (C-6), 85.0 (C-3'), 106.6 (C-4'), 174.5 (NCO).

HRMS (ESI) calcd for $\left[\mathrm{C}_{12} \mathrm{H}_{22} \mathrm{NOSi}+\mathrm{H}\right]^{+}: 224.1465$, found: 224.1465. 

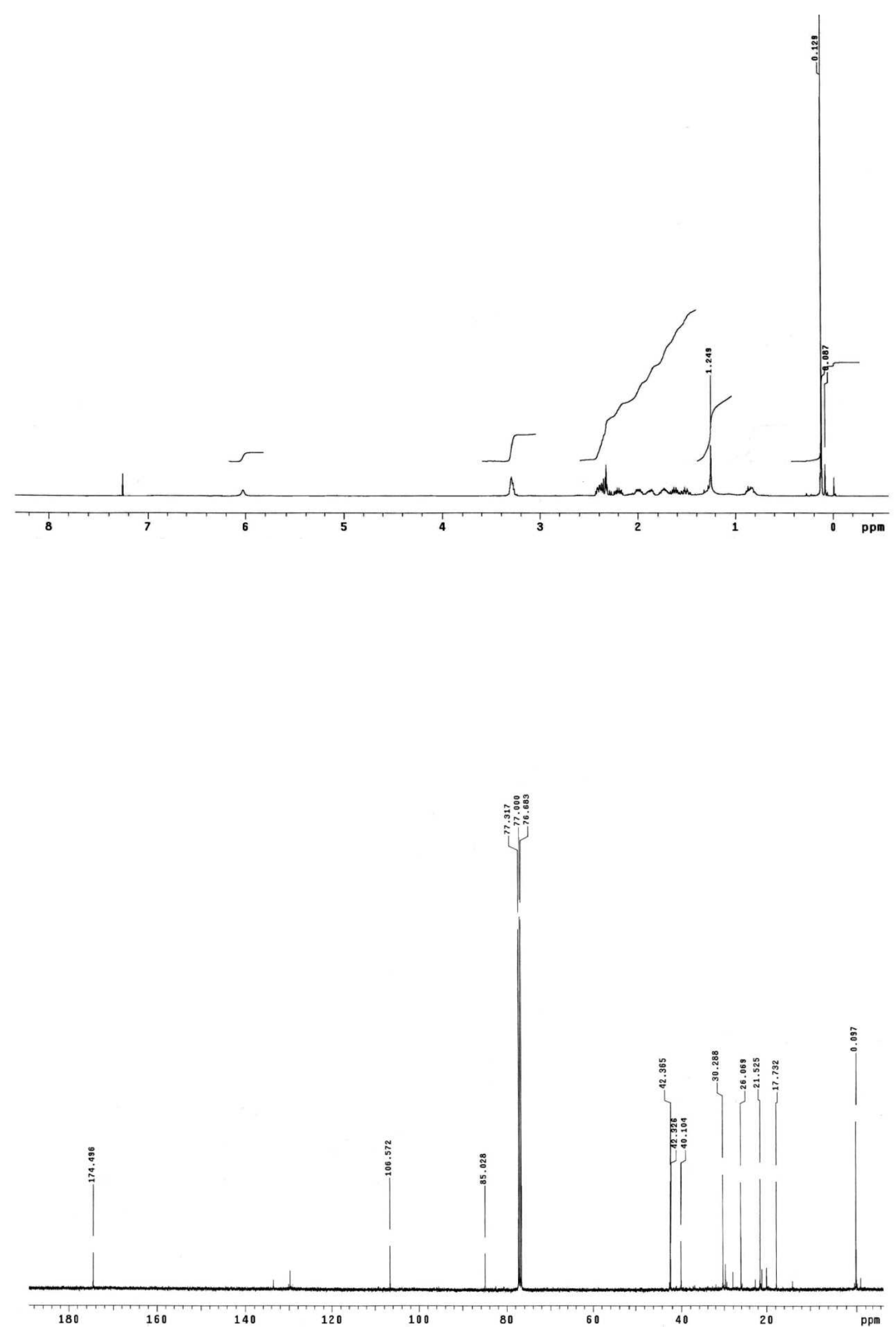


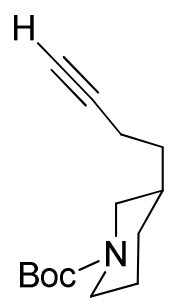

N-(tert-Butoxycarbonyl)-3-[4-(trimethylsilyl)-3-butynyl]piperidine (93).

Reduction step: Lithium aluminum hydride $(1.53 \mathrm{~g}, 38.52 \mathrm{mmol})$ was added under argon atmosphere to a solution of the piperidone $96(2.86 \mathrm{~g}, 12.84 \mathrm{mmol})$ in anhydrous THF (642 $\mathrm{mL}$ ), and the mixture was stirred at room temperature for $3 \mathrm{~h}$. The resulting suspension was cooled to $0{ }^{\circ} \mathrm{C}$, and the reaction was quenched by the slow addition of water $(1.53 \mathrm{~mL})$ and $15 \%$ aqueous $\mathrm{NaOH}(1.53 \mathrm{~mL})$. Anhydrous $\mathrm{MgSO}_{4}$ was added, and the mixture was then filtered through Celite ${ }^{\circledR}$ and concentrated to give 3-[4-(trimethylsilyl)-3-butynyl]piperidine, which was used in the next step without further purification.

N-Boc protection step: A solution of di-tert-butyl dicarbonate $(3.36 \mathrm{~g}, 15.39 \mathrm{mmol})$ in anhydrous $\mathrm{CH}_{2} \mathrm{Cl}_{2}(33 \mathrm{~mL}$ ) was added dropwise at room temperature under inert atmosphere to a solution of the above amino derivative $(12.84 \mathrm{mmol})$ in anhydrous $\mathrm{CH}_{2} \mathrm{Cl}_{2}(180 \mathrm{~mL})$. The resulting mixture was stirred overnight, poured into water, and extracted with $\mathrm{CH}_{2} \mathrm{Cl}_{2}$. The combined organic extracts were dried over anhydrous $\mathrm{Na}_{2} \mathrm{SO}_{4}$, filtered, and concentrated under reduced pressure. Flash chromatography (95:5 hexane-EtOAc) gave the resulting $N$-Boc piperidine derivative $(2.97 \mathrm{~g}, 74 \%)$.

TMS deprotection step: $\mathrm{K}_{2} \mathrm{CO}_{3}(137 \mathrm{mg}, 0.99 \mathrm{mmol})$ was added to a cooled $\left(0^{\circ} \mathrm{C}\right)$ solution of the silyl derivative $(204 \mathrm{mg}, 0.66 \mathrm{mmol})$ in anhydrous methanol $(3.3 \mathrm{~mL})$. The mixture was allowed to warm to room temperature, stirred overnight, and concentrated under reduced pressure. The reaction was quenched by addition of saturated aqueous $\mathrm{NH}_{4} \mathrm{Cl}$, and the resulting aqueous solution was extracted with EtOAc. The combined organic extracts were dried over $\mathrm{Na}_{2} \mathrm{SO}_{4}$, filtered, and concentrated under reduced pressure. Flash chromatography (95:5 hexaneEtOAc) afforded compound 93 (131 mg, 84\%).

${ }^{1} \mathrm{H}$ NMR (400 MHz, CDCl 3 , COSY, gHSQC): $\delta 1.07$ (m, 1H, H-1'), 1.35-1.53 (m, 3H, 2H-4, H-5), $1.44\left[\mathrm{~s}, 9 \mathrm{H},\left(\mathrm{CH}_{3}\right)_{3} \mathrm{C}\right], 1.59(\mathrm{~m}, 2 \mathrm{H}, \mathrm{H}-3, \mathrm{H}-5), 1.83\left(\mathrm{~m}, 1 \mathrm{H}, \mathrm{H}-1^{\prime}\right), 1.93\left(\mathrm{~m}, 1 \mathrm{H}, \mathrm{H}-4^{\prime}\right), 2.21(\mathrm{t}, J=$ $7.2 \mathrm{~Hz}, 2 \mathrm{H}, \mathrm{H}-2^{\prime}$ ), 2.50, 2.80 (2br. s, 2H, H-2ax, H-6ax), 3.82, 3.85 (2br. s, H-2eq, H-6eq).

${ }^{13} \mathrm{C} \mathrm{NMR}(100.6 \mathrm{MHz}, \mathrm{CDCl}$ ) $): \delta 15.8\left(\mathrm{C}-2^{\prime}\right), 25.6(\mathrm{C}-5), 28.4\left[\left(\mathrm{CH}_{3}\right)_{3} \mathrm{C}\right], 30.5\left(\mathrm{C}-1^{\prime}\right), 32.3(\mathrm{C}-4), 34.8$ (C-3), 44.4, 49.5 (C-2, C-6), 68.4 (C-4'), $79.2\left[\left(\mathrm{CH}_{3}\right)_{3} \mathrm{C}\right], 84.1$ (C-3'), 154.8 (NCOO). 
HRMS (ESI) calcd for $\left[\mathrm{C}_{14} \mathrm{H}_{23} \mathrm{NO}_{2}+\mathrm{Na}\right]^{+}: 260.1621$, found: 260.1621 .
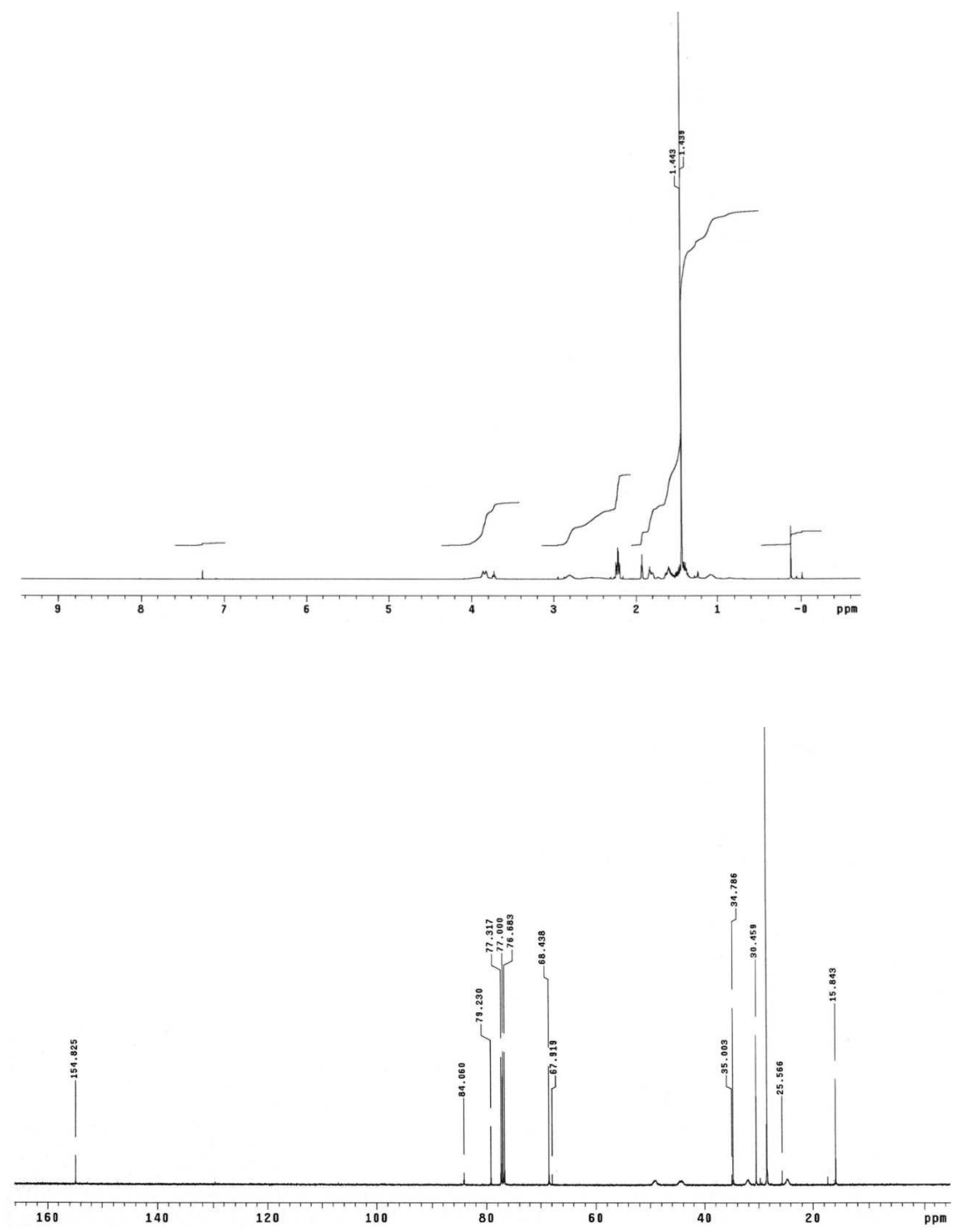
$\mathrm{HO}$<smiles>C/C=C\C/C=C\CCO[InH]</smiles>

(2Z,5Z)-8-[(Triisopropylsilyl)oxy]-2,5-octadienol.

A $1 \mathrm{M}$ solution of $\mathrm{NaBH}_{4}$ in ethanol $(3.1 \mathrm{~mL}, 3.06 \mathrm{mmol})$ was added to a stirred suspension of $\mathrm{Ni}(\mathrm{OAc})_{2} \cdot 4 \mathrm{H}_{2} \mathrm{O}(760 \mathrm{mg}, 3.06 \mathrm{mmol})$ in ethanol $(165 \mathrm{~mL})$ under an $\mathrm{H}_{2}$ atmosphere. After the black suspension was stirred for $30 \mathrm{~min}$, ethylenediamine ( $275 \mu \mathrm{L}, 4.08 \mathrm{mmol}$ ) was added followed by a solution of compound $89(3.0 \mathrm{~g}, 10.2 \mathrm{mmol})$ in ethanol $(5 \mathrm{~mL})$. The reaction flask was purged three times with $\mathrm{H}_{2}$ and then the reaction mixture was stirred under $\mathrm{H}_{2}$ atmosphere for $2 \mathrm{~h}$ with weak continuous hydrogen bubbling. The resulting mixture was filtered through a pad of silica gel with ether. The filtrate was concentrated and the residue was chromatographed (95:5 to 9:1 hexane-EtOAc) to give the corresponding diene (2.36 g, $78 \%)$ as a colorless oil.

${ }^{1} \mathrm{H}$ NMR (400 MHz, CDCl 3 , COSY, gHSQC): $\left.\delta 1.06,1.07\left[2 \mathrm{~s}, 21 \mathrm{H},\left(\mathrm{CH}_{3}\right)_{2} \mathrm{CH}\right)\right], 2.33(\mathrm{~m}, 2 \mathrm{H}, \mathrm{H}-7)$, $2.86(\mathrm{t}, J=6.8 \mathrm{~Hz}, 2 \mathrm{H}, \mathrm{H}-4), 3.70(\mathrm{t}, J=6.4 \mathrm{~Hz}, 2 \mathrm{H}, \mathrm{H}-8), 4.23(\mathrm{~d}, J=6.8 \mathrm{~Hz}, 2 \mathrm{H}, \mathrm{H}-1), 5.40-5.49$ (m, 2H, H-5, H-6), 5.54-5.61 (2m, 2H, H-2, H-3).

$\left.\left.{ }^{13} \mathrm{C} \mathrm{NMR}\left(100.6 \mathrm{MHz}, \mathrm{CDCl}_{3}\right): \delta 12.0\left[\left(\mathrm{CH}_{3}\right)_{2} \mathrm{CH}\right)\right], 18.0\left[\left(\mathrm{CH}_{3}\right)_{2} \mathrm{CH}\right)\right], 25.9(\mathrm{C}-4), 31.2(\mathrm{C}-7), 58.6$ (C1), 63.0 (C-8), 127.0, 128.6, 128.8, 131.1 (4CH=).

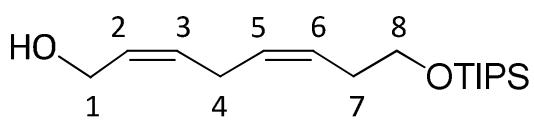



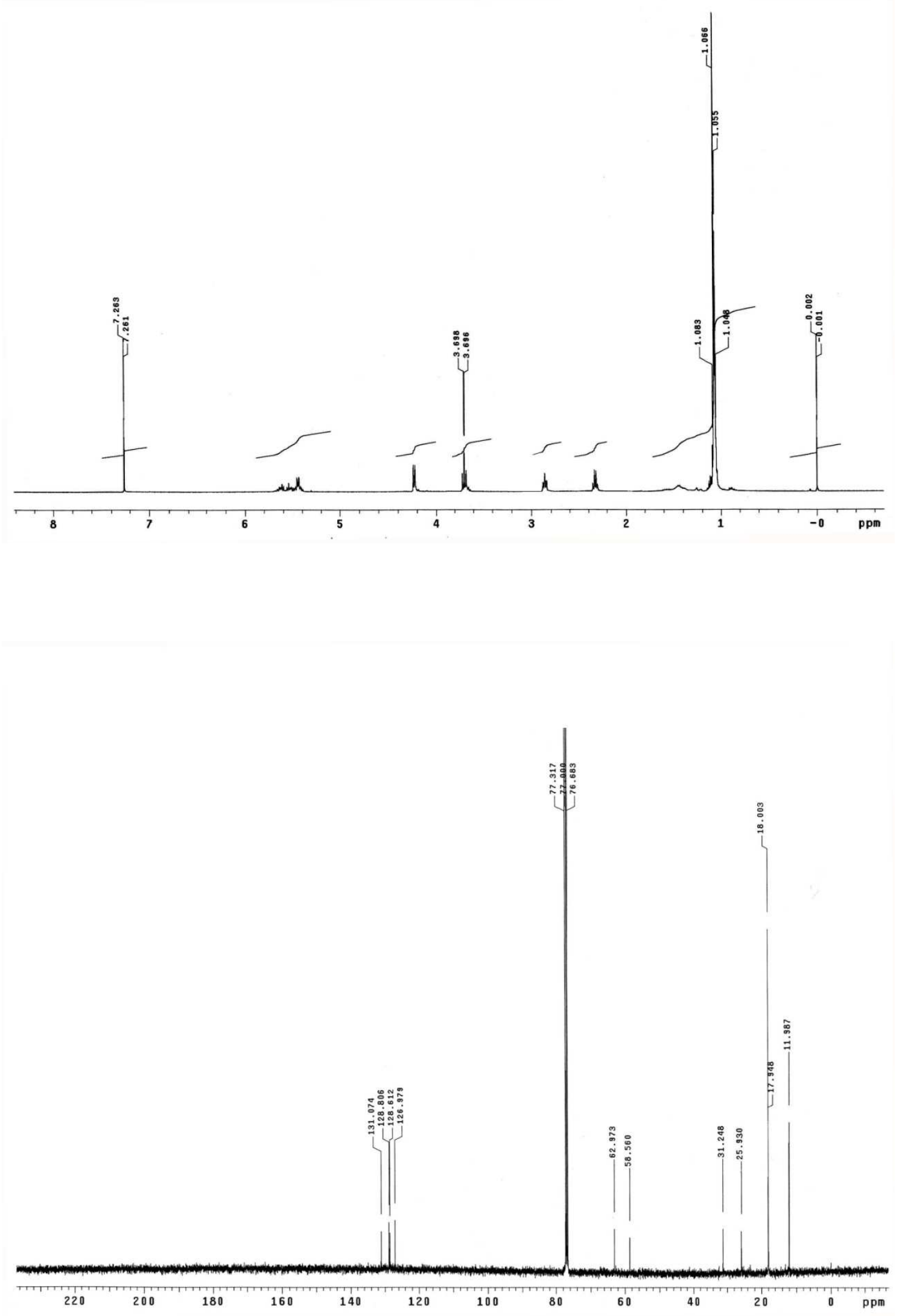
<smiles>BrC/C=C\C/C=C/CCO[SnH3]</smiles>

(3Z,6Z)-8-Bromo-1-[(triisopropylsilyl)oxy]-3,6-octadien (95).

Triethylamine $(350 \mu \mathrm{L}, 2.5 \mathrm{mmol})$ and methansulfonyl chloride $(180 \mu \mathrm{L}, 2.31 \mathrm{mmol})$ were added dropwise to a solution of (2Z,5Z)-8-[(triisopropylsilyl)oxy]-2,5-octadienol (574 mg, 1.93 mmol) in anhydrous $\mathrm{CH}_{2} \mathrm{Cl}_{2}(32 \mathrm{~mL})$ cooled to $0{ }^{\circ} \mathrm{C}$. After the end of addition, the mixture was stirred at $0{ }^{\circ} \mathrm{C}$ for $15 \mathrm{~min}$, thus was stirred for additional $2 \mathrm{~h}$ keeping the temperature between $10{ }^{\circ} \mathrm{C}$ and $20{ }^{\circ} \mathrm{C}$. Saturated aqueous $\mathrm{NH}_{4} \mathrm{Cl}$ was added, and the mixture was extracted with $\mathrm{CH}_{2} \mathrm{Cl}_{2}$. The combined organic extracts were washed with water, dried over anhydrous $\mathrm{Na}_{2} \mathrm{SO}_{4}$, filtered, and concentrated under reduced pressure to afford a methansulfonyl derivative intermediate.

A solution of $\operatorname{LiBr}(1.67 \mathrm{~g}, 19.3 \mathrm{mmol})$ in anhydrous THF $(8 \mathrm{~mL})$ was added to a solution of the above mesyl derivative $(1.93 \mathrm{mmol})$ in anhydrous $\mathrm{CH}_{2} \mathrm{Cl}_{2}(6 \mathrm{~mL})$ at $0{ }^{\circ} \mathrm{C}$ under inert atmosphere. The resulting mixture was stirred at room temperature for $1 \mathrm{~h}$. Distilled water was added, and the mixture was then extracted with $\mathrm{CH}_{2} \mathrm{Cl}_{2}$. The combined organic extracts were dried over anhydrous $\mathrm{Na}_{2} \mathrm{SO}_{4}$, filtered, and the solvent was removed under reduced pressure. Flash chromatography (hexane to $98: 2$ hexane- $\mathrm{Et}_{2} \mathrm{O}$ ) afforded bromo derivative 95 (435 mg, $73 \%)$.

${ }^{1} \mathrm{H}$ NMR $\left.\left(300 \mathrm{MHz}, \mathrm{CDCl}_{3}\right): \delta 1.06,1.07\left[2 \mathrm{~s}, 21 \mathrm{H},\left(\mathrm{CH}_{3}\right)_{2} \mathrm{CH}\right)\right], 2.36(\mathrm{~m}, 2 \mathrm{H}, \mathrm{H}-2), 2.85(\mathrm{t}, J=6.8$ $\mathrm{Hz}, 2 \mathrm{H}, \mathrm{H}-5), 3.70(\mathrm{t}, J=6.4 \mathrm{~Hz}, 2 \mathrm{H}, \mathrm{H}-1), 4.04(\mathrm{~d}, J=6.8 \mathrm{~Hz}, 2 \mathrm{H}, \mathrm{H}-8), 5.40-5.80(\mathrm{~m}, 4 \mathrm{H}, \mathrm{H}-5, \mathrm{H}-$ $6, \mathrm{H}-2, \mathrm{H}-3)$.

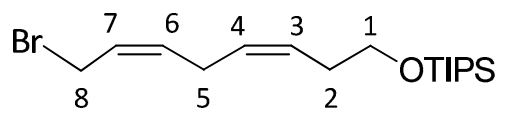




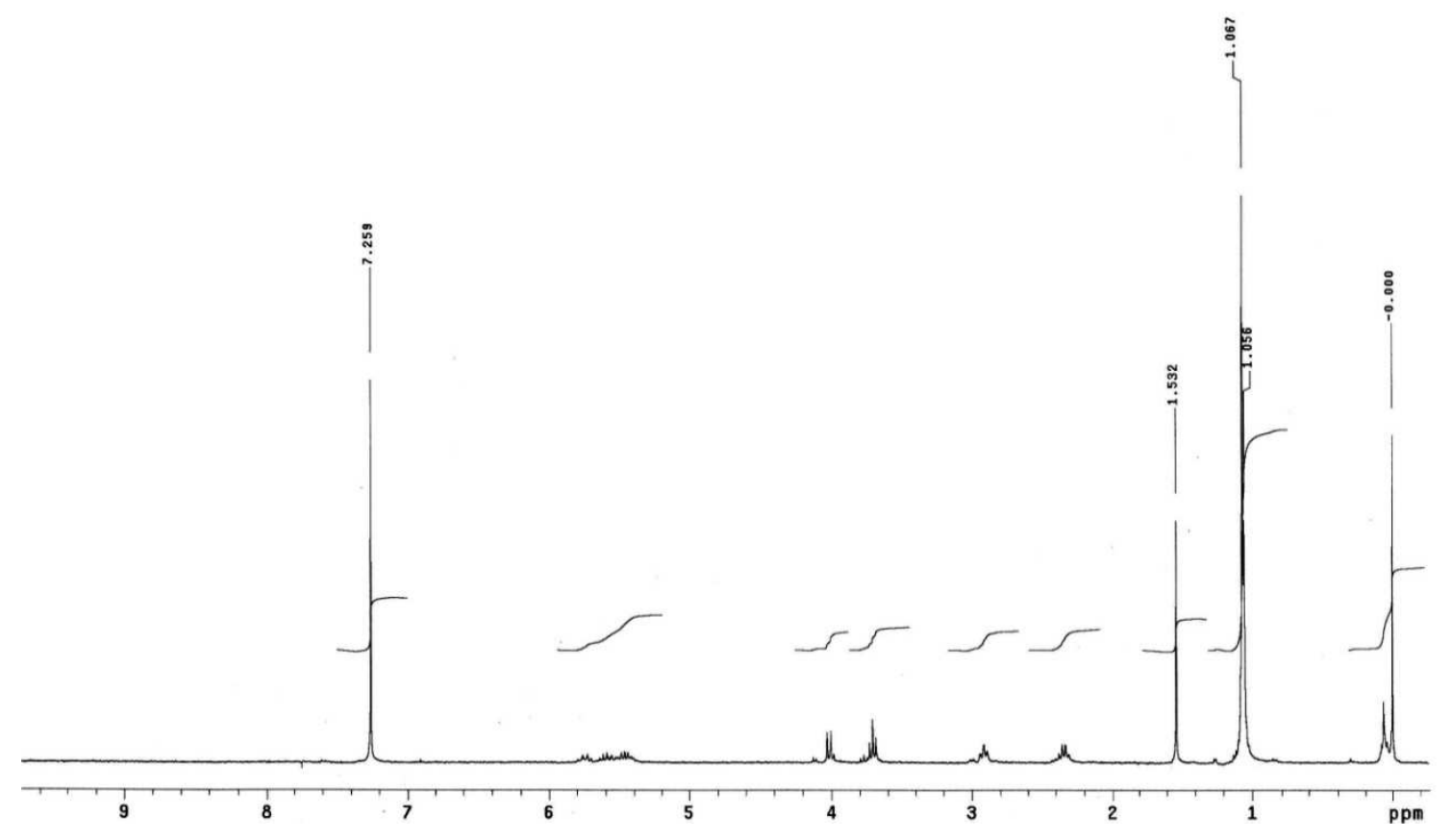




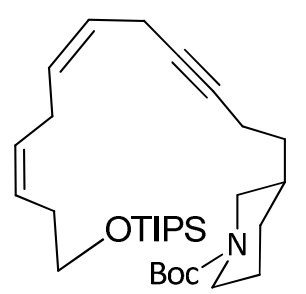

(6Z,9Z)-N-(tert-Butoxycarbonyl)-3-[12-(triisopropylsilyloxy)dodeca-6,9-dien-3-inyl]piperidine (80).

Bromo derivative 95 (500 mg, $1.39 \mathrm{mmol})$ and piperidine 93 (164 mg, 0.70mmol) were added under inert atmosphere to a suspension of Cul (264 mg, $1.39 \mathrm{mmol}$ ), Nal (208 mg, $1.39 \mathrm{mmol}$ ), and $\mathrm{K}_{2} \mathrm{CO}_{3}(143 \mathrm{mg}, 1.05 \mathrm{mmol})$ in of anhydrous $\mathrm{DMF}(1.5 \mathrm{~mL})$. The mixture was stirred overnight at room temperature. Then the mixture was filtered through a Celite ${ }^{\circledR}$ pad and concentrated under vacuum. Flash chromatography (hexane to 97:3 hexane- $\mathrm{Et}_{2} \mathrm{O}$ ) gave piperidine 80 (312 mg, 86\%).

IR (film): 1694 (CO) $\mathrm{cm}^{-1}$.

${ }^{1} \mathrm{H}$ NMR $\left(400 \mathrm{MHz}, \mathrm{CDCl}_{3}, \mathrm{COSY}, \mathrm{gHSQC}\right): \delta 0.99,1.02\left(2 \mathrm{~s}, 22 \mathrm{H}, \mathrm{SiCH}\left(\mathrm{CH}_{3}\right)_{2}, \mathrm{H}-1^{\prime}\right], 1.32(\mathrm{~m}, 1 \mathrm{H}$, $\mathrm{H}-4), 1.39\left[\mathrm{~m}, 11 \mathrm{H}, \mathrm{C}\left(\mathrm{CH}_{3}\right)_{3}, \mathrm{H}-4, \mathrm{H}-5\right], 1.49-1.58(\mathrm{~m}, 2 \mathrm{H}, \mathrm{H}-3, \mathrm{H}-5), 1.76\left(\mathrm{~m}, 1 \mathrm{H}, \mathrm{H}-1^{\prime}\right), 2.15(\mathrm{~m}$, $\left.2 \mathrm{H}, \mathrm{H}-2^{\prime}\right), 2.23\left(\mathrm{~m}, 2 \mathrm{H}, \mathrm{H}-11^{\prime}\right), 2.30$ (masked, $\left.1 \mathrm{H}, \mathrm{H}-6\right), 2.72$ (m, 3H, H-8' H-2), $2.79(\mathrm{~m}, 2 \mathrm{H}, \mathrm{H}-$ $\left.5^{\prime}\right), 3.60\left(\mathrm{t}, J=6.8 \mathrm{~Hz}, 2 \mathrm{H}, \mathrm{H}-12^{\prime}\right), 3.76-3.80(2 \mathrm{~m}, 2 \mathrm{H}, \mathrm{H}-2, \mathrm{H}-6), 5.353-5.46,5.58(3 \mathrm{~m}, 4 \mathrm{H}, \mathrm{CH}=)$.

${ }^{13} \mathrm{C} \mathrm{NMR}\left(100.6 \mathrm{MHz}, \mathrm{CDCl}_{3}\right): \delta 11.8\left[\mathrm{SiCH}\left(\mathrm{CH}_{3}\right)_{2}\right], 16.1\left(\mathrm{C}-2^{\prime}\right), 17.8\left[\mathrm{SiCH}\left(\mathrm{CH}_{3}\right)_{2}\right], 21.8\left(\mathrm{C}-5^{\prime}\right), 24.3$ (C-5), $28.3\left[\mathrm{C}\left(\mathrm{CH}_{3}\right)_{3}\right], 30.0$ (C-8'), 30.3 (C-1'), 31.0 (C-11'), 32.5 (C-4), 34.8 (C-3), 44.4, 49.8 (C-2, C-6), 62.9 (C-12'), 77.3, $78.9\left[\mathrm{C}-3^{\prime}, \mathrm{C}-4^{\prime}, \mathrm{C}\left(\mathrm{CH}_{3}\right)_{3}\right], 125.0,126.6,128.7,129.5(4 \mathrm{CH}=), 154.6$ (NCO).

HRMS (ESI) calcd for $\left[\mathrm{C}_{31} \mathrm{H}_{55} \mathrm{NO}_{3} \mathrm{Si}+\mathrm{H}\right]^{+}: 518.4024$, found: 518.4025 . 

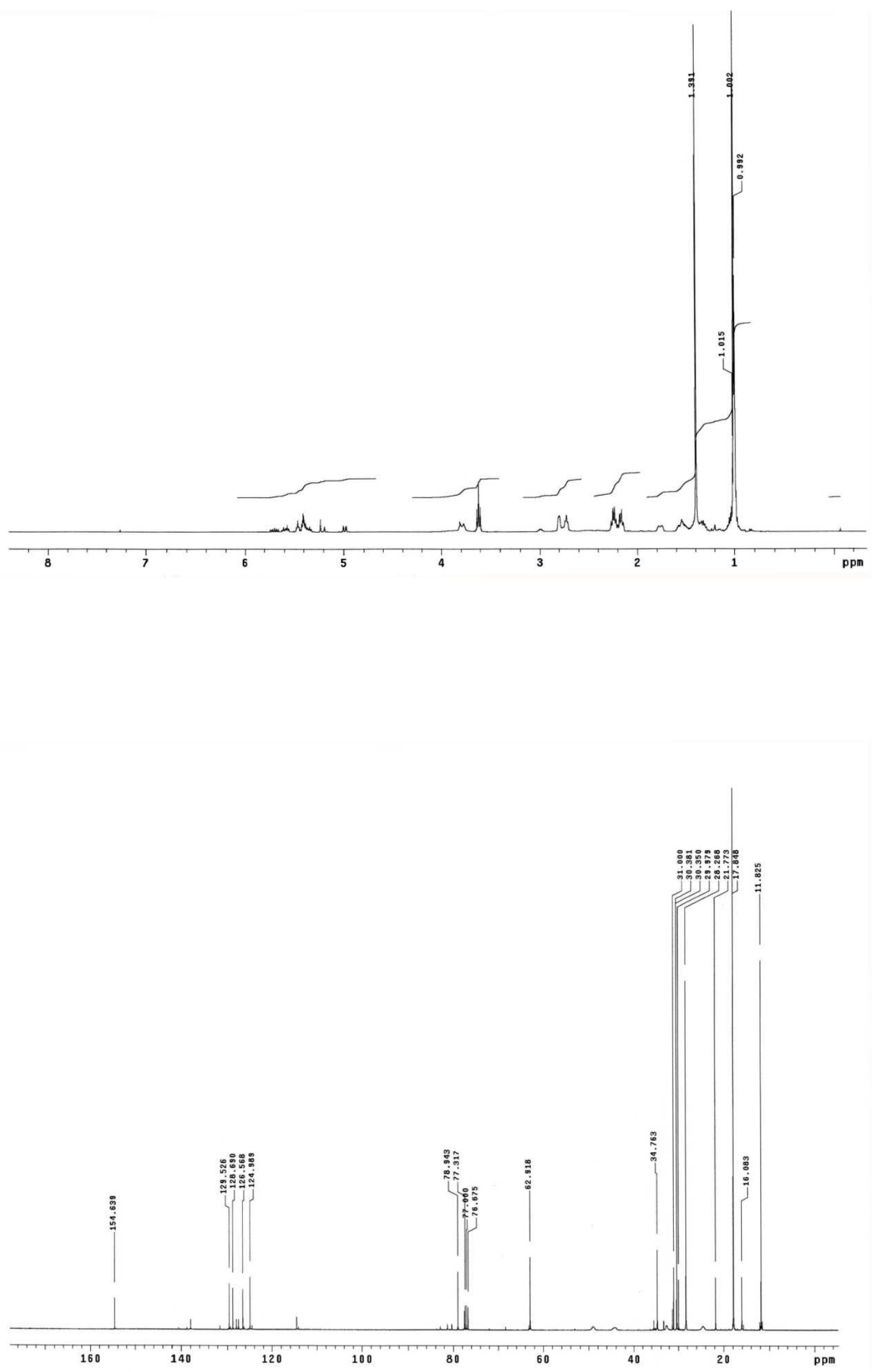


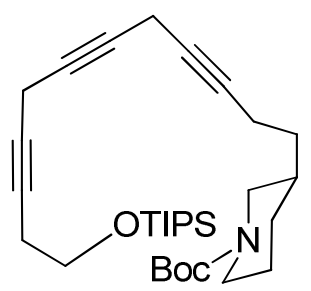

N-(tert-Butoxycarbonyl)-3-[12-(triisopropylsilyloxy)dodeca-3,6,9-triynyl]piperidine (94).

The diyne 90 (500 mg, $1.39 \mathrm{mmol}$ ) and compound 93 (164 mg, $0.70 \mathrm{mmol}$ ) were added under inert atmosphere to a suspension of Cul (264 mg, $1.38 \mathrm{mmol}$ ), Nal (208 mg, $1.38 \mathrm{mmol}$ ), and $\mathrm{K}_{2} \mathrm{CO}_{3}(143 \mathrm{mmg}, 1.05 \mathrm{mmol})$ in anhydrous DMF $(1.5 \mathrm{~mL})$. The resulting mixture was stirred overnight at room temperature. Saturated aqueous $\mathrm{NH}_{4} \mathrm{Cl}$ and EtOAc were added, and the mixture was filtered through a Celite ${ }^{\circledast}$ pad. The two phases are separated, and the aqueous layer was extracted with ether. The combined organic extracts were washed with brine, dried over anhydrous $\mathrm{Na}_{2} \mathrm{SO}_{4}$, filtered, and concentrated under reduced pressure. Flash chromatography (hexane to $94: 6$ hexane-EtOAc) gave the compound 94 (312 mg, 86\%) as a pale yellow oil.

IR (film): 1694 (CO) $\mathrm{cm}^{-1}$.

${ }^{1} \mathrm{H}$ NMR (400 MHz, CDCl 3 , COSY, gHSQC): $\delta 1.08\left[\mathrm{~m}, 22 \mathrm{H}, \mathrm{SiCH}\left(\mathrm{CH}_{3}\right)_{2}, \mathrm{H}-1^{\prime}\right], 1.38(\mathrm{~m}, 1 \mathrm{H}, \mathrm{H}-4)$, $1.49\left[\mathrm{~m}, 11 \mathrm{H},\left(\mathrm{CH}_{3}\right)_{3} \mathrm{C}, \mathrm{H}-4, \mathrm{H}-5\right], 1.55(\mathrm{~m}, 1 \mathrm{H}, \mathrm{H}-3), 1.61(\mathrm{~m}, 1 \mathrm{H}, \mathrm{H}-5), 1.82(\mathrm{dm}, J=11.6 \mathrm{~Hz}, 1 \mathrm{H}$, $\left.\mathrm{H}-1^{\prime}\right), 2.19\left(\mathrm{~m}, 4 \mathrm{H}, \mathrm{H}-5^{\prime}, \mathrm{H}-2^{\prime}\right), 2.35$ (m, 2H, H-11'), 2.80 (m, 2H, H-2ax, H-6ax), 3.12 (s, 2H, H-8'), $3.77\left(t, J=6.8 \mathrm{~Hz}, 2 \mathrm{H}, \mathrm{H}-12^{\prime}\right), 3.84,3.87$ (br. s, 2H, H-2eq, H-6eq).

${ }^{13} \mathrm{C}$ NMR (100.6 MHz, CDCl 3 ): $\delta 9.7\left(\mathrm{C}^{\prime} 8^{\prime}\right), 11.9\left[\mathrm{SiCH}\left(\mathrm{CH}_{3}\right)_{2}\right], 15.8,16.1$ (C-5', C-2'), 17.9 [ $\left.\mathrm{SiCH}\left(\mathrm{CH}_{3}\right)_{2}\right], 23.1\left(\mathrm{C}-11^{\prime}\right), 24.5(\mathrm{C}-5), 28.4\left[\mathrm{C}\left(\mathrm{CH}_{3}\right)_{2}\right], 30.4$ (C-1'), 32.2 (C-4), 34.9 (C-3), 44.6, 49.1

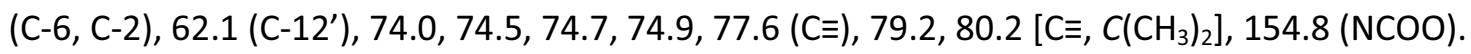
HRMS (ESI) calcd for $\left[\mathrm{C}_{31} \mathrm{H}_{51} \mathrm{NO}_{3} \mathrm{Si}+\mathrm{NH}_{4}\right]^{+}: 531.3976$, found: 531.3974 . 

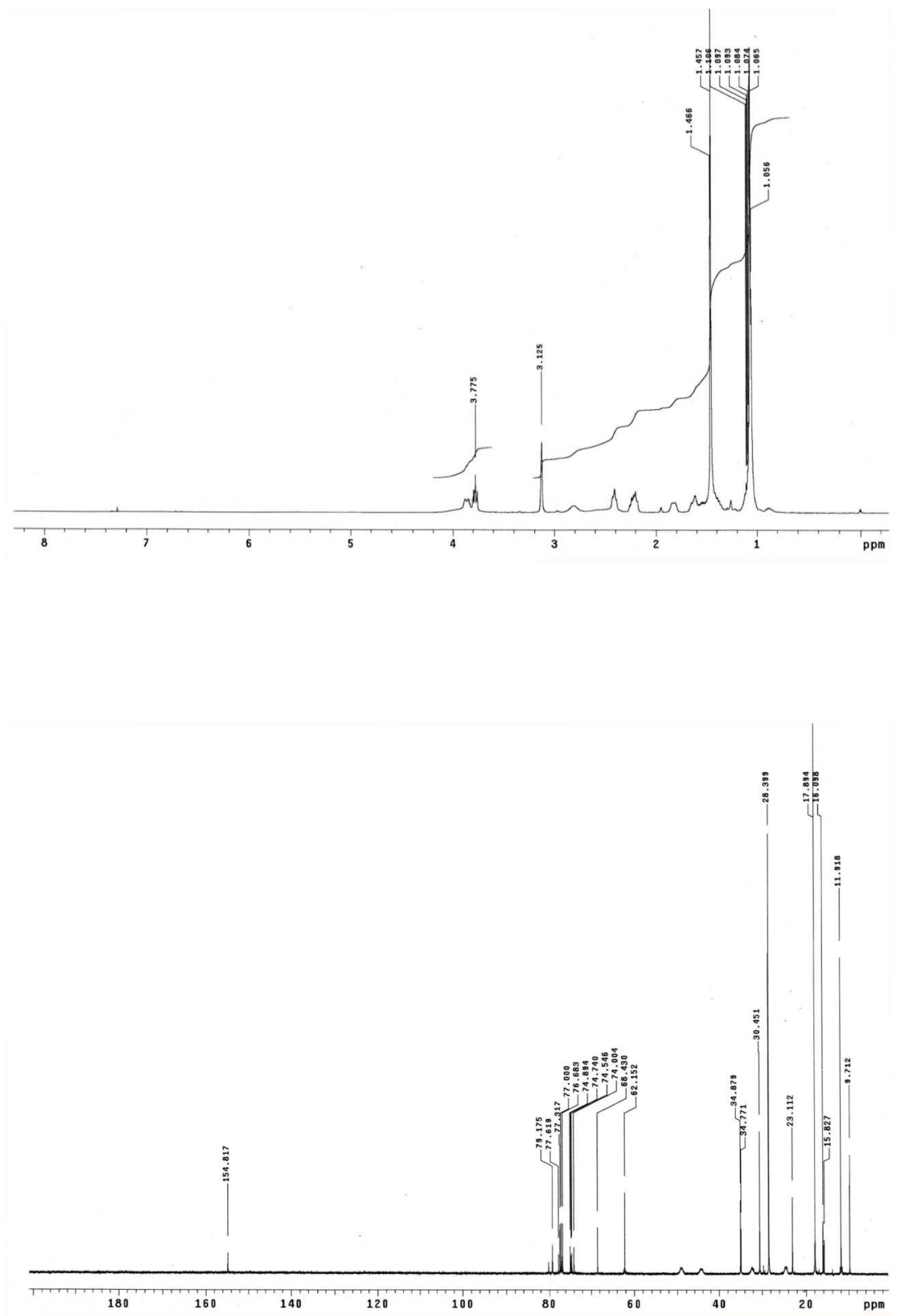


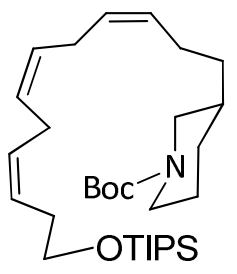

(3Z,6Z,9Z)-N-(tert-Butoxycarbonyl)-3-[12-(triisopropylsilyloxy)dodeca-3,6,9-trienyl]piperidine (81).

A suspension of dicyclohexylborane $(1.14 \mathrm{mmol})$ was prepared at $0{ }^{\circ} \mathrm{C}$ by dropwise addition of cyclohexene ( $232 \mu \mathrm{L}, 2.28 \mathrm{mmol}$ ) to a solution of borane-dimethyl sulfide complex ( $571 \mu \mathrm{L}$ of a $2 \mathrm{M}$ solution in THF, $1.14 \mathrm{mmol}$ ) in THF ( $3 \mathrm{~mL}$ ). The resulting mixture was warmed to $20{ }^{\circ} \mathrm{C}$, stirred for $2 \mathrm{~h}$, and then cooled again at $0{ }^{\circ} \mathrm{C}$. Triyne $94(140 \mathrm{mg}, 0.272 \mathrm{mmol})$ in anhydrous THF $(1.5 \mathrm{~mL})$ was then added, and the mixture was warmed to $20^{\circ} \mathrm{C}$ over $2 \mathrm{~h}$ and was stirred at room temperature for an additional $2 \mathrm{~h}$. Glacial acetic acid ( $435 \mu \mathrm{L})$ was added dropwise, and the resulting solution was stirred overnight. The solution was then cooled to $0{ }^{\circ} \mathrm{C}$ and basified by slow addition of aqueous $\mathrm{NaOH}$ ( $1.63 \mathrm{~mL}$ of a $5 \mathrm{M}$ solution) and $30 \%$ aqueous $\mathrm{H}_{2} \mathrm{O}_{2}$ (435 $\mu \mathrm{L}$ ). Water was then added, and the resulting mixture was extracted with EtOAc. The combined organic extracts were dried over anhydrous $\mathrm{Na}_{2} \mathrm{SO}_{4}$, filtered, and concentrated under reduced pressure. Flash chromatography (hexane to 95:5 hexane-EtOAc) gave compound $81(119 \mathrm{mg}, 84 \%)$ as a colourless oil.

${ }^{1} \mathrm{H}$ NMR (400 MHz, CDCl, COSY, gHSQC): $\delta 1.05\left(\mathrm{~m}, 22 \mathrm{H}, \mathrm{SiCH}\left(\mathrm{CH}_{3}\right)_{2}, \mathrm{H}-1^{\prime}\right], 1.25(\mathrm{~m}, 2 \mathrm{H}, 2 \mathrm{H}-4)$, $1.40(m, 1 \mathrm{H}, \mathrm{H}-5), 1.45\left[\mathrm{~m}, 10 \mathrm{H}, \mathrm{C}\left(\mathrm{CH}_{3}\right)_{3}, \mathrm{H}-3\right], 1.61(\mathrm{~m}, 1 \mathrm{H}, \mathrm{H}-5), 1.81\left(\mathrm{~m}, 1 \mathrm{H}, \mathrm{H}-1^{\prime}\right), 2.08(\mathrm{~m}$, $\left.2 \mathrm{H}, \mathrm{H}-2^{\prime}\right), 2.32\left(\mathrm{~m}, 2 \mathrm{H}, \mathrm{H}-11^{\prime}\right), 2.80\left(\mathrm{~m}, 6 \mathrm{H}, 2 \mathrm{H}-8^{\prime}, 2 \mathrm{H}-5^{\prime}, \mathrm{H}-2 \mathrm{ax}, \mathrm{H}-6 \mathrm{ax}\right), 3.69(\mathrm{t}, \mathrm{J}=6.8 \mathrm{~Hz}, 2 \mathrm{H}, \mathrm{H}-$ $\left.12^{\prime}\right), 3.90(\mathrm{~m}, 2 \mathrm{H}, \mathrm{H}-2 \mathrm{eq}, \mathrm{H}-6 \mathrm{eq}), 5.35,5.43$ ( $\left.\mathrm{CH}=\right)$.

${ }^{13} \mathrm{C} \mathrm{NMR}\left(100.6 \mathrm{MHz}, \mathrm{CDCl}_{3}\right): \delta 12.0\left[\mathrm{SiCH}\left(\mathrm{CH}_{3}\right)_{2}\right], 18.0\left[\mathrm{SiCH}\left(\mathrm{CH}_{3}\right)_{2}\right], 24.4\left(\mathrm{C}-2^{\prime}\right), 24.9$ (C-5), 25.6, $25.8\left(\mathrm{C}-8^{\prime}, \mathrm{C}-5^{\prime}\right), 28.5\left[\mathrm{C}\left(\mathrm{CH}_{3}\right)_{3}\right], 30.9\left(\mathrm{C}-1^{\prime}\right), 31.0\left(\mathrm{C}-11^{\prime}\right), 33.5$ (C-4), 35.4 (C-3), 44.4, 49.8 (C-2, C-6), 63.0 (C-12'), $79.1\left[\mathrm{C}\left(\mathrm{CH}_{3}\right)_{3}\right], 126.3$ (C-10'), 128.0, 128.2, 128.3 (C-4', C-6', C-7'), 129.4, 129.9 (C-3', C-9'), 154.9 (NCO).

HRMS (ESI) calcd for $\left[\mathrm{C}_{31} \mathrm{H}_{57} \mathrm{NO}_{3} \mathrm{Si}+\mathrm{H}\right]^{+}: 520.4199$, found: 520.4180 . 

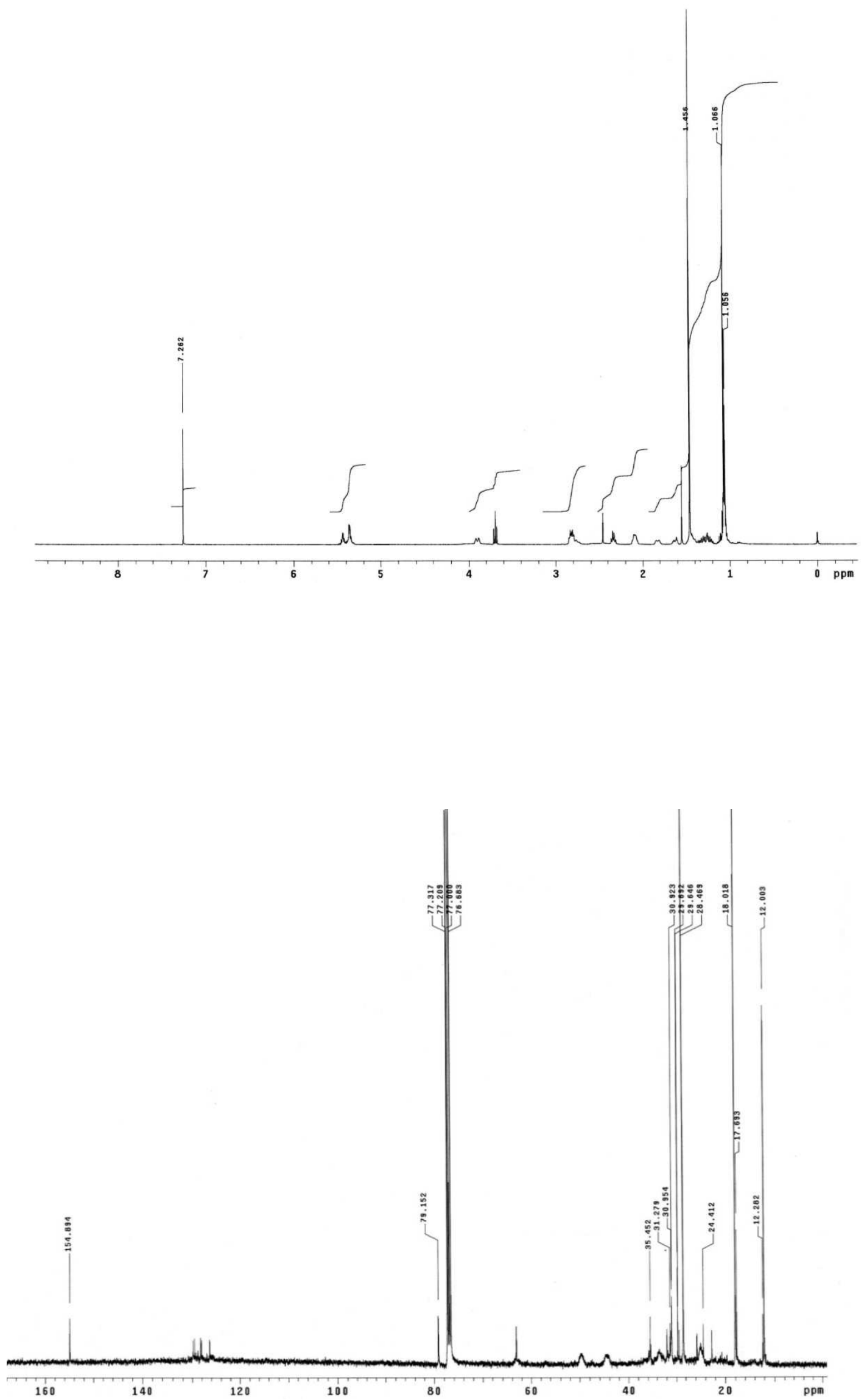


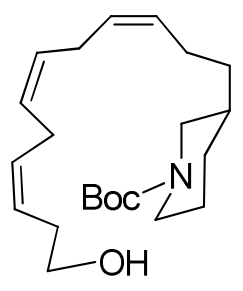

(3Z,6Z,9Z)-N-(tert-Butoxycarbonyl)-3-[12-hydroxydodeca-3,6,9-trienyl]piperidine (97).

$2 \mathrm{~N}$ aqueous $\mathrm{HCl}(3.16 \mathrm{~mL}$ ) was added to a stirred solution of silyl derivative 81 (119 mg, 0.23 $\mathrm{mmol}$ ) in methanol $(23 \mathrm{~mL})$, and the mixture was stirred for $30 \mathrm{~min}$. The solvent was evaporated under reduced pressure, saturated aqueous $\mathrm{NaHCO}_{3}$ was added, and the resulting mixture was extracted with $\mathrm{CH}_{2} \mathrm{Cl}_{2}$. The combined organic extracts were dried over anhydrous $\mathrm{Na}_{2} \mathrm{SO}_{4}$, filtered, and concentrated under reduced pressure to give a white foam. Flash chromatography (hexane to 7:3 hexane-EtOAc) gave alcohol $97(71 \mathrm{mg}, 85 \%)$ as a colourless oil.

${ }^{1} \mathrm{H}$ NMR $\left(400 \mathrm{MHz}, \mathrm{CDCl}_{3}, \mathrm{COSY}, \mathrm{gHSQC}\right): \delta 1.25\left(\mathrm{~m}, 4 \mathrm{H}, \mathrm{H}-2^{\prime}, \mathrm{H}-4\right), 1.45\left[\mathrm{~s}, 11 \mathrm{H}, \mathrm{C}\left(\mathrm{CH}_{3}\right)_{3}, \mathrm{H}-5, \mathrm{H}-\right.$ 3), $1.61(\mathrm{~m}, 1 \mathrm{H}, \mathrm{H}-5), 1.81\left(\mathrm{dm}, J=12.4 \mathrm{~Hz}, 2 \mathrm{H}, \mathrm{H}-1^{\prime}\right), 2.11\left(\mathrm{~m}, 2 \mathrm{H}, \mathrm{H}-5^{\prime}\right), 2.37\left(\mathrm{~m}, 2 \mathrm{H}, \mathrm{H}-11^{\prime}\right)$, $2.75(\mathrm{~m}, 2 \mathrm{H}, \mathrm{H}-2 \mathrm{ax}, \mathrm{H}-6 \mathrm{ax}), 2.83\left(\mathrm{~m}, 2 \mathrm{H}, \mathrm{H}-8^{\prime}\right), 3.65\left(\mathrm{t}, J=8.0 \mathrm{~Hz}, 2 \mathrm{H}, \mathrm{H}-12^{\prime}\right), 3.90(2 \mathrm{~m}, 2 \mathrm{H}, \mathrm{H}-$ 2eq, H-6eq), 5.37 (m, 4H, H-4', H-6', H-7', H-10'), 5.56 (m, 2H, H-3', H-9').

${ }^{13} \mathrm{C} \mathrm{NMR}\left(100.6 \mathrm{MHz}, \mathrm{CDCl}_{3}\right): \delta 24.5$ (C-5'), 25.0 (C-2'), 25.6 (C-5), 25.8 (C-8'), $28.4\left[\mathrm{C}\left(\mathrm{CH}_{3}\right)_{2}\right]$, 30.9 (C-11'), 31.0 (C-1'), 33.7 (C-4), 35.5 (C-3), 44.8, 49.3 (C-2, C-6), 62.1 (C-12'), 79.2 [C( $\left.\left(\mathrm{CH}_{3}\right)_{2}\right]$, 125.7 (C-10'), 127.8, 127.9, 128.5 (C-4', C-6', C-7') 129.9, 130.9 (C-3', C-9'), 154.9 (NCO).

HRMS (ESI) calcd for $\left[\mathrm{C}_{22} \mathrm{H}_{37} \mathrm{NO}_{3}+\mathrm{H}\right]^{+}: 364.2846$, found: 364.2841 . 

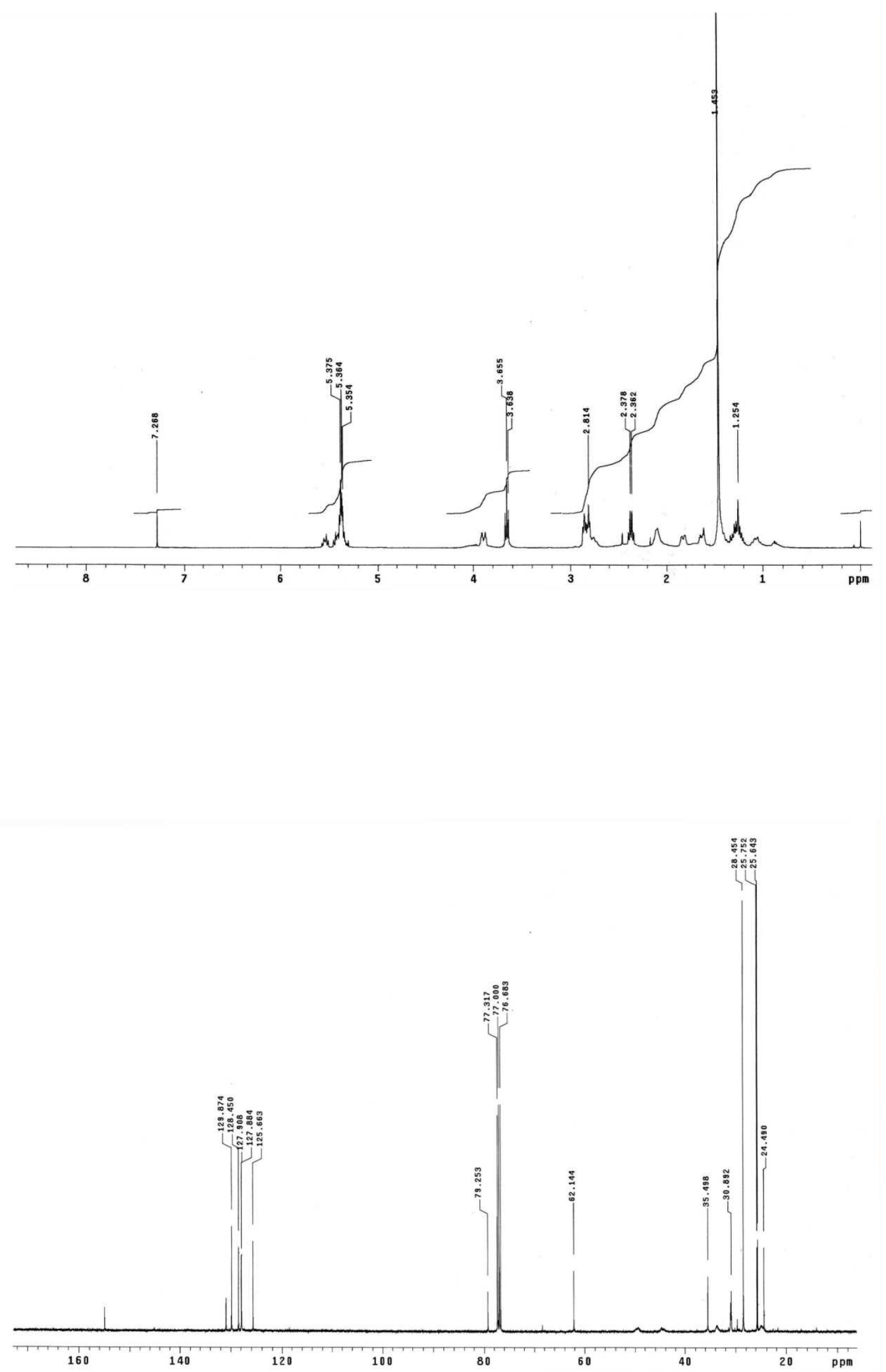


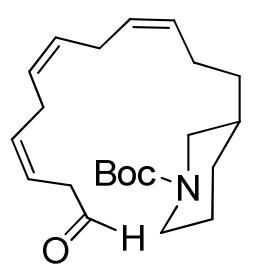

(3Z,6Z,9Z)-N-(tert-Butoxycarbonyl)-3-piperidinedodeca-3,6,9-trienaldehyde (74).

$\mathrm{NaHCO}_{3}(436 \mathrm{mg}, 5.19 \mathrm{mmol})$ and Dess-Martin periodinane $(2.24 \mathrm{~g}, 5.19 \mathrm{mmol})$ were added to a solution of the alcohol $97(630 \mathrm{mg}, 1.73 \mathrm{mmol})$ in $\mathrm{CH}_{2} \mathrm{Cl}_{2}(35 \mathrm{~mL})$. After $18 \mathrm{~h}$ of stirring at room temperature, a saturated aqueous solution of $\mathrm{NaHCO}_{3}-\mathrm{Na}_{2} \mathrm{~S}_{2} \mathrm{O}_{3}$ (1:1) was slow added. The resulting mixture was vigorously stirred for $1 \mathrm{~h}$ and extracted with $\mathrm{CH}_{2} \mathrm{Cl}_{2}$. The combined organic extracts were dried over anhydrous $\mathrm{Na}_{2} \mathrm{SO}_{4}$, filtered, and concentrated under reduced pressure. Flash chromatography (hexane to 7:3 hexane-EtOAc) afforded aldehyde 74 (374 mg, $60 \%)$, which was immediately used in the next step.

${ }^{1} \mathrm{H}$ NMR (400 MHz, CDCl 3 , COSY, gHSQC): $\delta 1.02\left(\mathrm{~m}, 1 \mathrm{H}, \mathrm{H}-\mathrm{1}^{\prime}\right), 1.20(\mathrm{~m}, 2 \mathrm{H}, \mathrm{H}-4), 1.35(\mathrm{~m}, 2 \mathrm{H}, \mathrm{H}-$ 5, H-3), $1.41\left[\mathrm{~s}, 9 \mathrm{H}, \mathrm{C}\left(\mathrm{CH}_{3}\right)_{3}\right], 1.57(\mathrm{~m}, 1 \mathrm{H}, \mathrm{H}-5), 1.77\left(\mathrm{~m}, 1 \mathrm{H}, \mathrm{H}-1^{\prime}\right), 2.04\left(\mathrm{~m}, 2 \mathrm{H}, \mathrm{H}-2^{\prime}\right), 2.76(\mathrm{~m}$, $\left.6 \mathrm{H}, \mathrm{H}-2, \mathrm{H}-6,2 \mathrm{H}-5^{\prime}, 2 \mathrm{H}-8^{\prime}\right), 3.18\left(\mathrm{~d}, J=7.2 \mathrm{~Hz}, 2 \mathrm{H}, \mathrm{H}-11^{\prime}\right), 3.85(\mathrm{dm}, J=12.8 \mathrm{~Hz}, 2 \mathrm{H}, \mathrm{H}-2 \mathrm{ax}, \mathrm{H}-$ $6 a x), 3.99$ (m, 1H, H-2eq, H-6eq), 5.31 (m, 4H, H-4', H-5', H-6', H-7'), 5.63 (m, 2H, H-3', H-9'), $9.63(\mathrm{~s}, 1 \mathrm{H}, \mathrm{CHO})$.

${ }^{13} \mathrm{C}$ NMR $\left.\left(100.6 \mathrm{MHz}, \mathrm{CDCl}_{3}\right): \delta 24.3\left(\mathrm{C}-2^{\prime}\right), 24.8(\mathrm{C}-5), 25.5\left(\mathrm{C}-5^{\prime}\right), 25.8\left(\mathrm{C}-8^{\prime}\right), 28.3\left[\mathrm{C}^{\prime} \mathrm{CH}_{3}\right)_{2}\right]$, 30.8 (C-1'), 33.4 (C-4), 35.3 (C-3), 42.3 (C-11'), 44.7, 49.2 (C-2, C-6), 79.0 [C( $\left.\left.\mathrm{CH}_{3}\right)_{2}\right], 118.5$ (C$\left.10^{\prime}\right), 126.8,127.5,128.8$, (C-4', C-6', C-7'), 129.9, 132.9 (C-3', C-9'), 154.7 (NCO), 199.1 (CHO).

HRMS (ESI) calcd for $\left[\mathrm{C}_{22} \mathrm{H}_{35} \mathrm{NO}_{3}+\mathrm{H}\right]^{+}: 362.2676$, found: 362.2693 . 

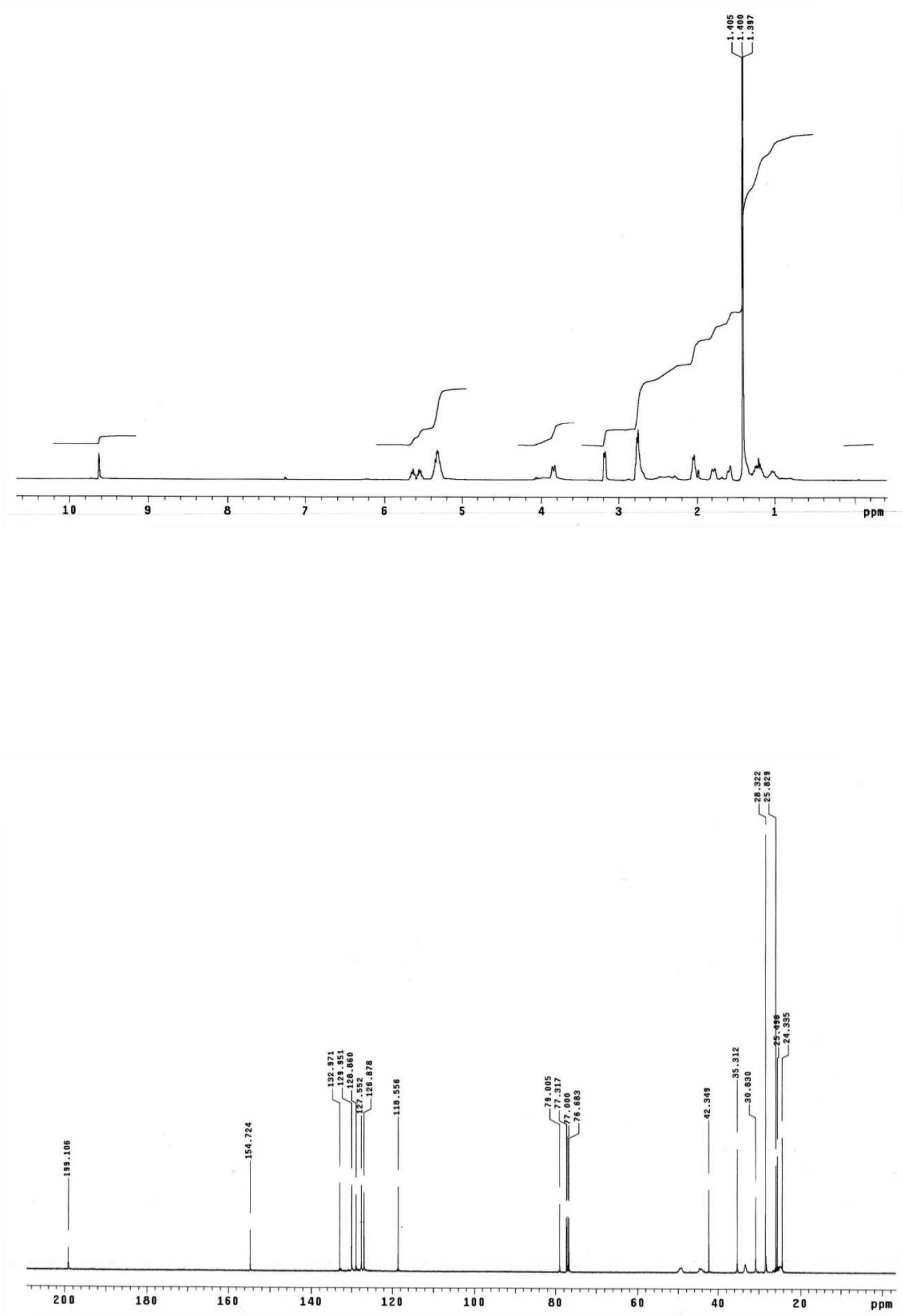
<smiles>O=C(O)/C=C\C/C=C\C/C=C\CCC1CCCC(C(=O)O)N1C(=O)O</smiles>

(3Z,6Z,9Z)-N-(tert-Butoxycarbonyl)-3-piperidinedodeca-3,6,9-trienoic acid (75).

$t$-BuOH $(11 \mathrm{~mL})$ and 1-methyl-1-cyclohexene $(1.5 \mathrm{~mL})$ were added to a stirring solution of the above aldehyde 74 (131 mg, $0.36 \mathrm{mmol})$ in $\mathrm{CH}_{3} \mathrm{CN}(4 \mathrm{~mL})$. A solution of $\mathrm{NaClO}_{2}$ (80\%, $273 \mathrm{mg}$, $2.4 \mathrm{mmol})$ and $\mathrm{NaH}_{2} \mathrm{PO}_{4}(300 \mathrm{mg}, 2.5 \mathrm{mmol})$ in water $(4 \mathrm{~mL})$ was then added, and the mixture was stirred at room temperature for $1.5 \mathrm{~h}$. Then $0.1 \mathrm{M} \mathrm{Na}_{2} \mathrm{~S}_{2} \mathrm{O}_{3}$ and $2 \mathrm{~N}$ aqueous $\mathrm{HCl}$ solutions were added (until $\mathrm{pH}=1$ ), and the aqueous solution was extracted with EtOAc. The combined organic extracts were dried over $\mathrm{MgSO}_{4}$, filtered, and concentrated affording the acid $\mathbf{7 5}$ as a colourless oil, which was used in the next step without further purification.

${ }^{1} \mathrm{H}$ NMR (400 MHz, $\mathrm{CDCl}_{3}$, selected resonances): $\delta 1.05\left(\mathrm{~m}, 1 \mathrm{H}, \mathrm{H}-\mathrm{1}^{\prime}\right), 1.20(\mathrm{~m}, 2 \mathrm{H}, \mathrm{H}-4), 1.35$ (m, 2H, H-5, H-3), $1.45\left(\mathrm{~s}, 9 \mathrm{H},\left[\mathrm{C}\left(\mathrm{CH}_{3}\right)_{2}\right]\right), 1.60(\mathrm{~m}, 1 \mathrm{H}, \mathrm{H}-5), 1.75\left(\mathrm{~m}, 1 \mathrm{H}, \mathrm{H}-1^{\prime}\right), 2.00(\mathrm{~m}, 2 \mathrm{H}, \mathrm{H}-$ $\left.2^{\prime}\right), 2.76\left(\mathrm{~m}, 6 \mathrm{H}, \mathrm{H}-2, \mathrm{H}-6,2 \mathrm{H}-5^{\prime}, 2 \mathrm{H}-8^{\prime}\right), 3.18\left(\mathrm{~d}, J=7.2 \mathrm{~Hz}, 2 \mathrm{H}, \mathrm{H}-11^{\prime}\right), 3.90(\mathrm{dm}, J=12.8 \mathrm{~Hz}, \mathrm{H}-$ 2, H-6), 5.31 (m, 4H, H-4', H-5', H-6', H-7'), 5.60 (m, 2H, H-3', H-9'). 


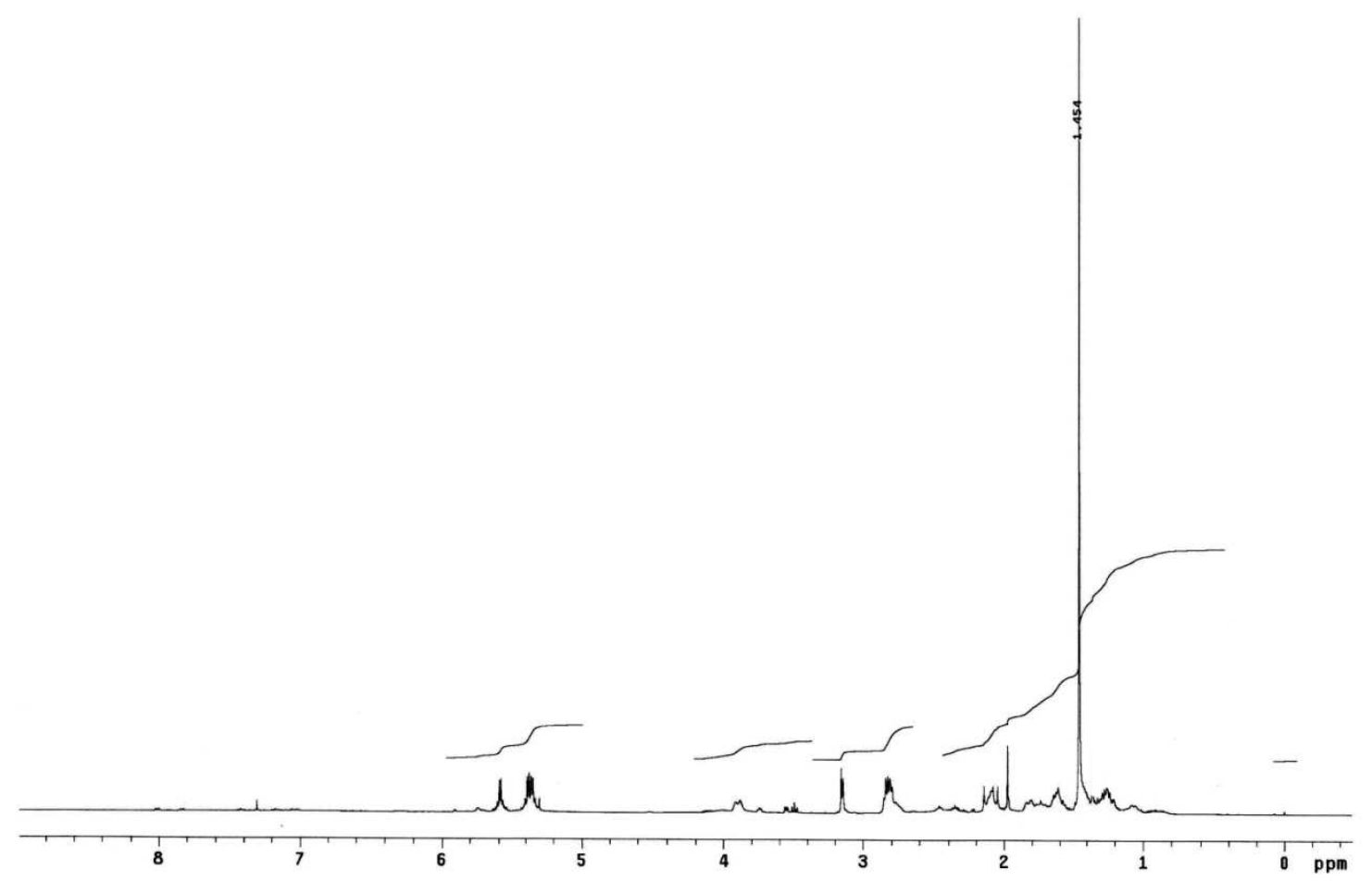




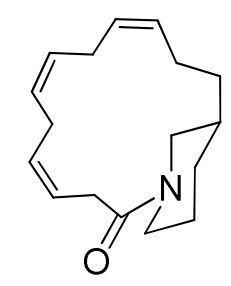

(4Z,7Z,10Z)-2-Oxo-1-azabicyclo[12.3.1]octadeca-4,7,10-triene (76).

TFA ( $3 \mathrm{~mL}$ ) was added to a solution of the acid $75(0.36 \mathrm{mmol})$ in anhydrous $\mathrm{CH}_{2} \mathrm{Cl}_{2}(10 \mathrm{~mL})$, and the mixture was stirred at room temperature for 30 minutes. Toluene $(2 \mathrm{~mL})$ was added, and the solution was concentrated under reduced pressure. A solution of the resulting residue in $\mathrm{CH}_{2} \mathrm{Cl}_{2} / \mathrm{DMF}$ (9:1, $2 \mathrm{~mL}$ ) was added to a solution of $\mathrm{HOBt}$ (364 mg, $1.9 \mathrm{mmol}$ ) and $\mathrm{EDCl}$ (258 $\mathrm{mg}, 1.9 \mathrm{mmol})$ in $\mathrm{CH}_{2} \mathrm{Cl}_{2} / \mathrm{DMF}(9: 1,2 \mathrm{~mL})$. The resulting mixture was stirred overnight at room temperature, and concentrated under reduced pressure. Then $1 \mathrm{~N}$ aqueous $\mathrm{HCl}$ was added. The solution was washed with EtOAc, neutralized $\left(\mathrm{NaHCO}_{3}\right)$, and extracted with $\mathrm{CH}_{2} \mathrm{Cl}_{2}$. The combined organic extracts were dried over anhydrous $\mathrm{Na}_{2} \mathrm{SO}_{4}$, filtered, and concentrated under reduced pressure. Flash chromatography (hexane to 3:7 hexane-EtOAc) gave bicyclic compound 76 ( $33 \mathrm{mg}, 35 \%$ overall yield for two steps).

${ }^{1} \mathrm{H} \mathrm{NMR}\left(400 \mathrm{MHz}, \mathrm{CDCl}_{3}, \mathrm{COSY}, \mathrm{gHSQC}\right): \delta 1.24(\mathrm{~m}, 1 \mathrm{H}, \mathrm{H}-4), 1.26\left(\mathrm{~m}, 2 \mathrm{H}, \mathrm{H}-12^{\prime}\right), 1.42(\mathrm{~m}, 1 \mathrm{H}$, $\mathrm{H}-5), 1.45(\mathrm{~m}, 1 \mathrm{H}, \mathrm{H}-3), 1.64(\mathrm{~m}, 1 \mathrm{H}, \mathrm{H}-5), 1.75(\mathrm{~m}, 1 \mathrm{H}, \mathrm{H}-4), 2.17(\mathrm{~m}, 2 \mathrm{H}, \mathrm{H}-11), 2.70(\mathrm{dd}, J=$ 14.0, 9.6 Hz, 1H, H-2ax), $2.80\left(\mathrm{~m}, 2 \mathrm{H}, \mathrm{H}-5^{\prime}\right), 2.87(\mathrm{~m}, 1 \mathrm{H}, \mathrm{H}-6 \mathrm{ax}), 2.91\left(\mathrm{~m}, 2 \mathrm{H}, 2 \mathrm{H}-8^{\prime}\right), 3.14(\mathrm{dm}, J$ $\left.=5.6 \mathrm{~Hz}, 2 \mathrm{H}, \mathrm{H}-2^{\prime}\right), 3.74(\mathrm{dm}, J=13.2 \mathrm{~Hz}, 1 \mathrm{H}, \mathrm{H}-2 \mathrm{eq}), 4.15$ (dt, $\left.J=13.2,5.6 \mathrm{~Hz}, 1 \mathrm{H}, \mathrm{H}-6 \mathrm{eq}\right), 5.38-$ $5.47\left(m, 4 H, H-6^{\prime}, H-7^{\prime}, H-9^{\prime}, H-10^{\prime}\right)$, 5.63-5.67 (m, 2H, H-3', H-6').

${ }^{13} \mathrm{C}$ NMR (100.6 MHz, CDCl $)_{3}$ ): $\delta 24.5$ (C-5), 25.1 (C-11), 26.1 (C-8'), 26.3 (C-5'), 32.3 (C-12), 32.5 (C-4), 32.4 (C-2'), 42.8 (C-6), 50.7 (C-2), 123.9 (C-3'), 126.7 (C-7'), 127.3 (C-9'), 128.1 (C-6'), 128.9 (C-4'), 129.9 (C-10'), 169.6 (NCO).

HRMS (ESI) calcd for [ $\left.\mathrm{C}_{17} \mathrm{H}_{25} \mathrm{NO}+\mathrm{H}\right]^{+}: 260.2009$, found: 260.2010 . 

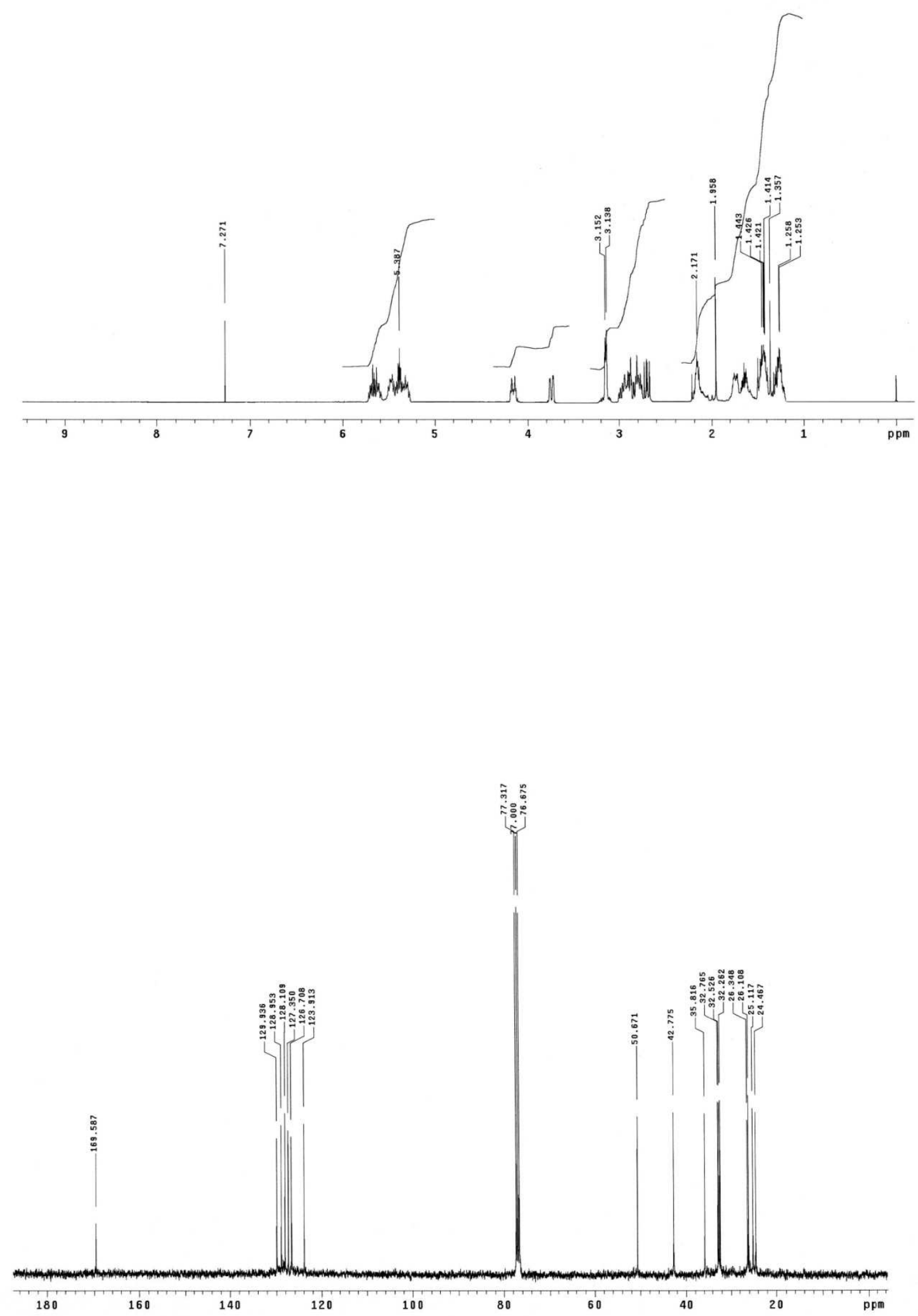

